Research Report

\title{
Current and Projected Characteristics and Unique Health Care Needs of the Patient Population Served by the Department of Veterans Affairs
}

RAND Health

Sponsored by the U.S. Department of Veterans Affairs 
For more information on this publication, visit www.rand.org/t/rr1165z1

Published by the RAND Corporation, Santa Monica, Calif.

(C) Copyright 2015 RAND Corporation

RAND $^{\oplus}$ is a registered trademark.

\section{Limited Print and Electronic Distribution Rights}

This document and trademark(s) contained herein are protected by law. This representation of RAND intellectual property is provided for noncommercial use only. Unauthorized posting of this publication online is prohibited. Permission is given to duplicate this document for personal use only, as long as it is unaltered and complete. Permission is required from RAND to reproduce, or reuse in another form, any of its research documents for commercial use. For information on reprint and linking permissions, please visit www.rand.org/pubs/permissions.html.

The RAND Corporation is a research organization that develops solutions to public policy challenges to help make communities throughout the world safer and more secure, healthier and more prosperous. RAND is nonprofit, nonpartisan, and committed to the public interest.

The analyses upon which this publication is based were performed under a contract for the Department of Veterans Affairs. The content of this publication does not necessarily reflect the views or policies of the Department of Veterans Affairs, nor does the mention of trade names, commercial products, or organizations imply endorsement by the U.S. government. The author assumes full responsibility for the accuracy and completeness of the ideas presented.

\section{Support RAND}

Make a tax-deductible charitable contribution at www.rand.org/giving/contribute

www.rand.org 


\section{Authorship Credits}

\begin{tabular}{|c|c|c|}
\hline $\begin{array}{c}\text { Study Dire } \\
\text { Christine E } \\
\text { Heather K }\end{array}$ & \multicolumn{2}{|c|}{$\begin{array}{c}\text { Veterans Choice Act Study Director } \\
\text { Carrie Farmer }\end{array}$} \\
\hline $\begin{array}{l}\text { Communication } \\
\text { David Adar }\end{array}$ & \multicolumn{2}{|c|}{$\begin{array}{c}\text { Veterans Choice Act Study Co-Director } \\
\text { Susan Hosek }\end{array}$} \\
\hline $\begin{array}{l}\text { Chapter One: } \\
\text { Introduction }\end{array}$ & $\begin{array}{l}\text { Chapter Two: } \\
\text { Conceptual Framework }\end{array}$ & $\begin{array}{c}\text { Chapter Three: } \\
\text { Demographic Projections }\end{array}$ \\
\hline $\begin{array}{c}\text { David Adamson } \\
\text { Christine Eibner } \\
\text { Heather Krull }\end{array}$ & $\begin{array}{l}\text { Christine Eibner } \\
\text { Amii Kress } \\
\text { Andrew Mulcahy } \\
\text { Jaime Hastings } \\
\text { Kristine Brown } \\
\text { Matthew Cefalu } \\
\text { Michael Pollard } \\
\text { Heather Krull }\end{array}$ & $\begin{array}{c}\text { Michael Pollard* } \\
\text { Ernesto Amaral } \\
\text { Joshua Mendelsohn } \\
\text { Matthew Cefalu } \\
\text { Amii Kress } \\
\text { Rachel Ross }\end{array}$ \\
\hline $\begin{array}{l}\text { Chapter Four: } \\
\text { Enrollment and Reliance }\end{array}$ & $\begin{array}{l}\text { Chapter Five: } \\
\text { Health Care Needs Projections }\end{array}$ & $\begin{array}{l}\text { Chapter Six: } \\
\text { Scenarios }\end{array}$ \\
\hline \multirow[t]{3}{*}{$\begin{array}{l}\text { Matthew Cefalu* } \\
\text { Andrew Mulcahy* } \\
\text { Kristine Brown } \\
\text { Jaime Hastings } \\
\text { Amii Kress } \\
\text { Carolyn Rutter } \\
\text { David Adamson } \\
\text { Robin Weinick }\end{array}$} & $\begin{array}{l}\text { Kristine Brown* } \\
\text { Kanaka Shetty* } \\
\text { Trinidad Beleche } \\
\text { Matthew Cefalu } \\
\text { Kandice Kapinos } \\
\text { Carolyn Rutter } \\
\text { Amii Kress } \\
\text { Rachel Ross }\end{array}$ & $\begin{array}{l}\text { Andrew Mulcahy* } \\
\text { Philip Armour } \\
\text { Joshua Mendelsohn } \\
\text { Dulani Woods } \\
\text { Olena Bogdan }\end{array}$ \\
\hline & $\begin{array}{l}\text { Chapter Seven: } \\
\text { Conclusions }\end{array}$ & \\
\hline & $\begin{array}{l}\text { Heather Krull } \\
\text { Kristine Brown } \\
\text { Amii Kress } \\
\text { Andrew Mulcahy } \\
\text { Michael Pollard } \\
\text { Christine Eibner }\end{array}$ & \\
\hline
\end{tabular}





\section{Preface}

Congress enacted and President Barack Obama signed into law the Veterans Access, Choice, and Accountability Act of 2014 (Public Law 113-146) ("Veterans Choice Act"), as amended by the U.S. Department of Veterans Affairs (VA) Expiring Authorities Act of 2014 (Public Law 113-175), to improve access to timely, high-quality health care for Veterans. Under "Title IIHealth Care Administrative Matters," Section 201 called for an independent assessment of 12 facets of VA's health care delivery systems and management processes (Assessments A-L): veteran demographics and health care needs (A), health care capabilities (B), authorities and mechanisms for purchasing care $(\mathrm{C})$, access standards (D), appointment scheduling (E), inpatient clinical workflow $(\mathrm{F})$, staffing and productivity $(\mathrm{G})$, health information technology $(\mathrm{H})$, business processes for purchased care (I), pharmaceuticals and medical supplies (J), construction and capital management $(\mathrm{K})$, and leadership (L).

VA engaged the Centers for Medicare and Medicaid Services (CMS) Alliance to Modernize Healthcare (CAMH), a federally funded research and development center sponsored by CMS and operated by the MITRE Corporation, to serve as the program integrator and as primary developer of 11 of the 12 independent assessments. CAMH subcontracted with the RAND Corporation to conduct three assessments (A, B, and C). CAMH coordinated the assessments, prepared an integrated report for the overall study, and furnished the complete set of reports to the VA Secretary, the House and Senate Veterans Affairs Committees, and the Commission on Care on September 1, 2015. VA made the reports available to the public on its website at www.va.gov/opa/choiceact/factsheets_and_details.asp. This version has been copyedited and reformatted for ease of reading.

This report describes the results of analyses of the demographics and health care needs of the current and future U.S. Veteran population (Assessment A). Specifically, it examines the demographic characteristics of the current and projected population of U.S. Veterans and patients of the VA health care system, the unique health care needs of the patient population currently served by VA, and the extent to which these patients rely on VA for health care. It also projects the health care needs of Veterans who might become patients in the future and how these needs will evolve over time, given current policies.

This research was conducted by RAND Health, a division of the RAND Corporation. Additional information about RAND can be found at www.rand.org. 



\section{Contents}

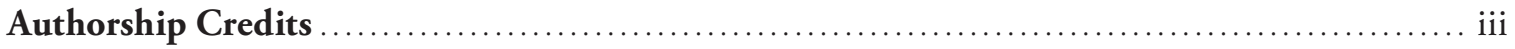

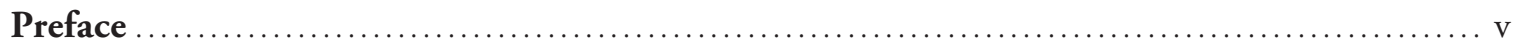

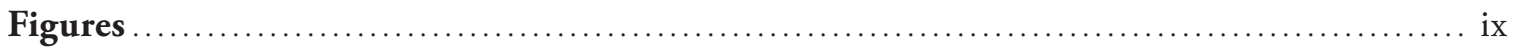

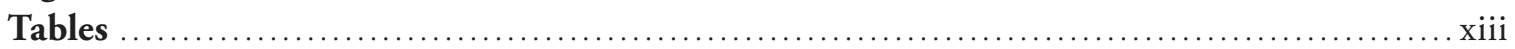

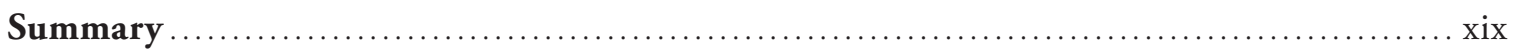

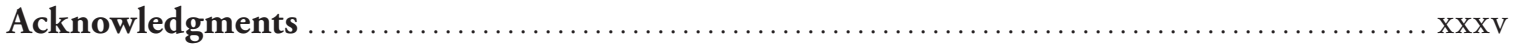

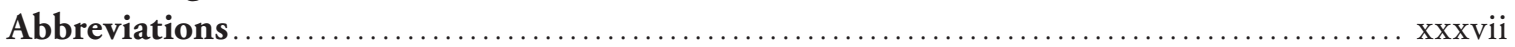

CHAPTER ONE

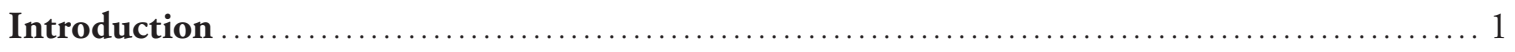

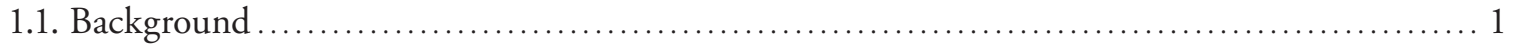

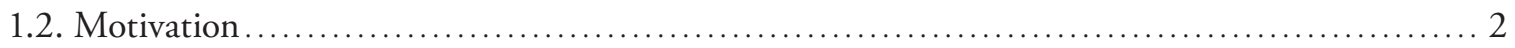

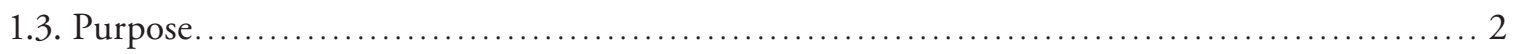

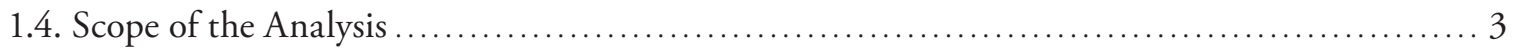

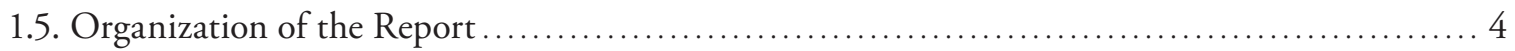

CHAPTER TWO

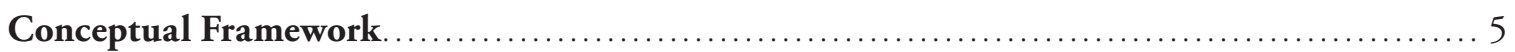

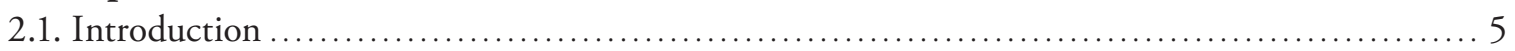

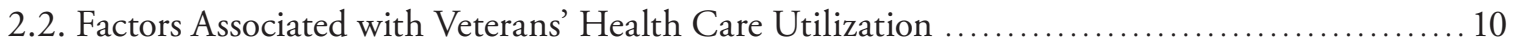

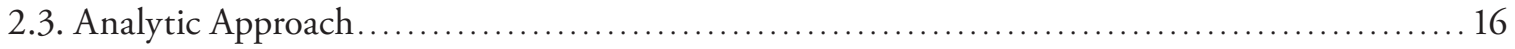

2.4. Existing VA Forecasting Models ................................................. 21

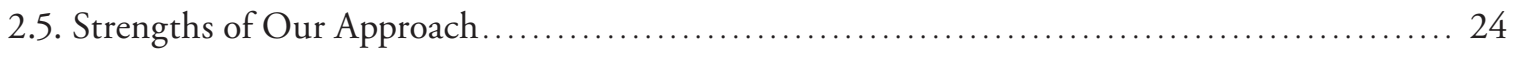

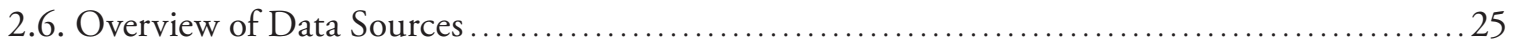

CHAPTER THREE

Current and Future Demographics of the Veteran Population ........................... 31

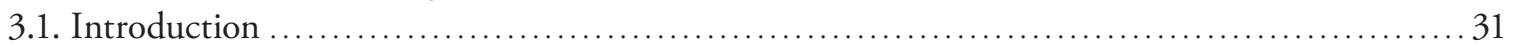

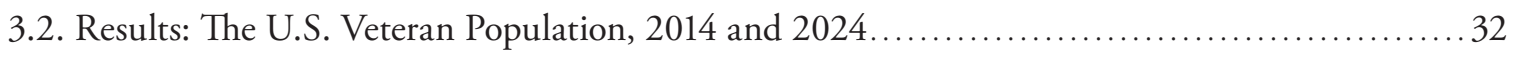

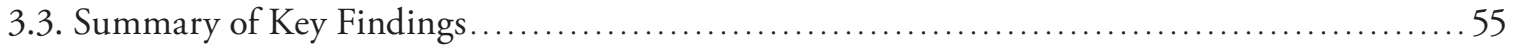

CHAPTER FOUR

Enrollment in and Reliance on the VA Health Care System........................... 57

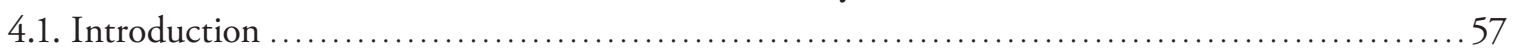

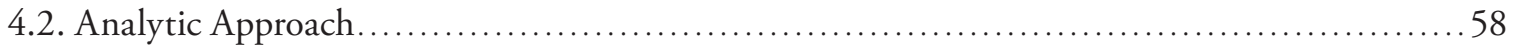

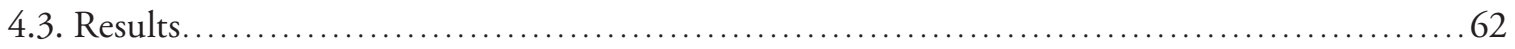


4.4. Reliance Analysis Results from MEPS Data ........................................... 69

4.5. Reliance Analysis Results from EHCPM ............................................ 73

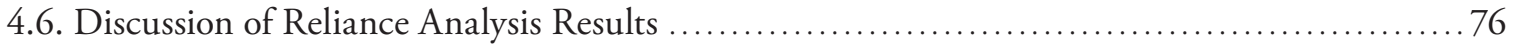

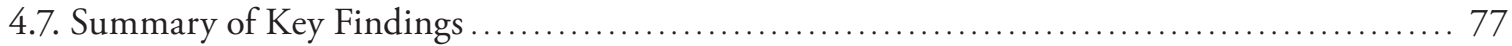

CHAPTER FIVE

Health Care Needs of the Veteran Population ........................................... 79

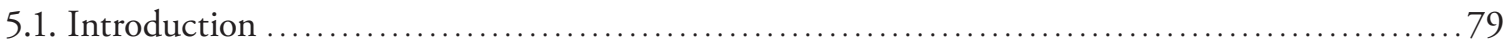

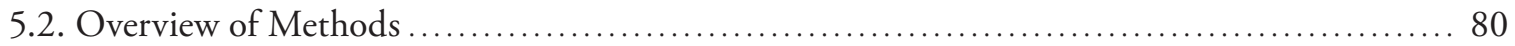

5.3. Current Health Care Needs of Veterans and Non-Veterans ................................ 83

5.4. Current Health Care Needs of Veterans, by VA Patient Status ............................. 96

5.5. Future Health Care Needs of Veterans .................................................. 109

5.6. Future Health Care Needs of VA Patients ............................................. 114

5.7. Summary of Key Findings ..................................................... 118

CHAPTER SIX

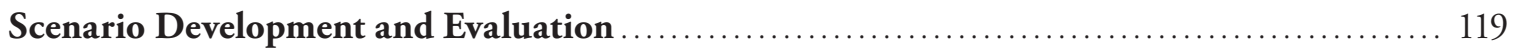

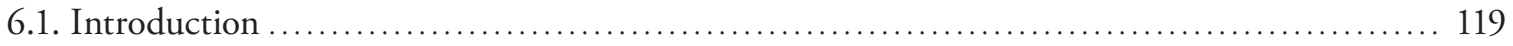

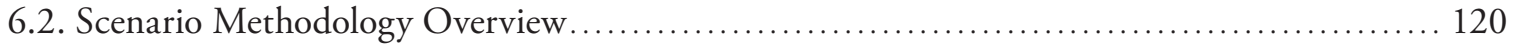

6.3. Scenario Typology and Framework............................................... 121

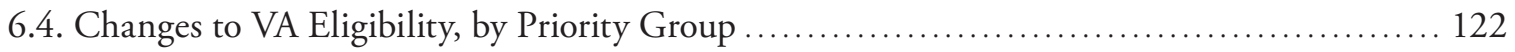

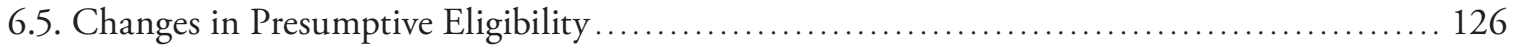

6.6. Potential Impact of Future Military Conflict on VA Use............................. 129

6.7. Improving Access to VA Care ...................................................... 138

6.8. The Affordable Care Act's Coverage Expansion..................................... 143

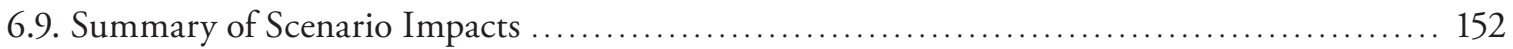

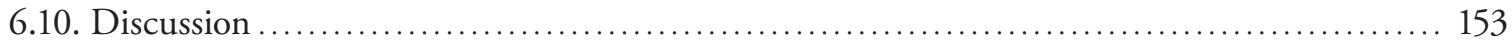

CHAPTER SEVEN

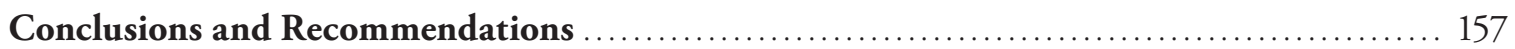

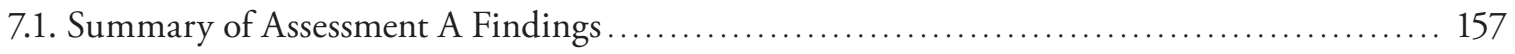

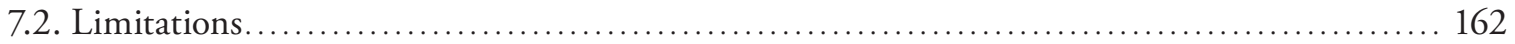

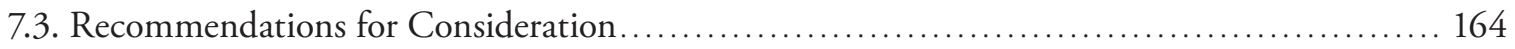

7.4. Overall Assessment A Conclusions...................................................... 168

\section{APPENDIXES}

A. Current and Future Demographic Trends in the Veteran Population ................ 171

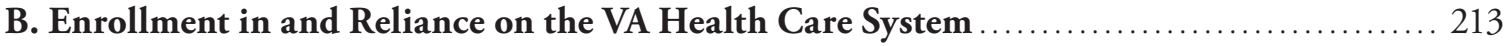

C. Health Care Needs of the Veteran Population ..................................... 223

D. Scenario Development and Evaluation ............................................ 325

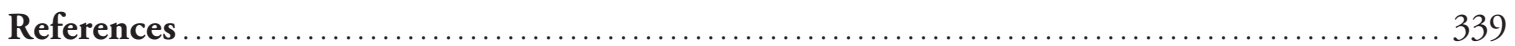




\section{Figures}

S-1. Socioeconomic Characteristics of the Veteran and Non-Veteran Civilian Population,

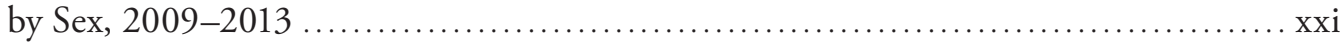

S-2. The Number of U.S. Veterans Will Decline by 19 Percent by $2024 \ldots \ldots \ldots \ldots \ldots \ldots \ldots \ldots$ xxiii

S-3. Veterans Have Higher Diagnosed Prevalence of Several Key Health Conditions

(Unadjusted Prevalence) .............................................. xxvi

S-4. Veterans Have a Higher Prevalence of Several Key Health Conditions (Adjusted

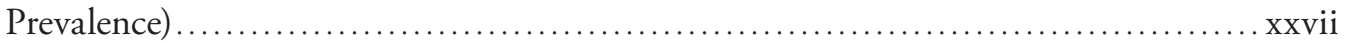

S-5. VA Patients Have a Higher Prevalence of Several Key Health Conditions

(Unadjusted Prevalence) ................................................ xxviii

S-6. Projected Unadjusted Prevalence of Selected Health Conditions Among Veterans

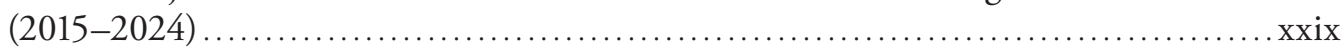

2-1. Conceptual Model of Factors Associated with Veterans' Use of VA Health Care ............. 10

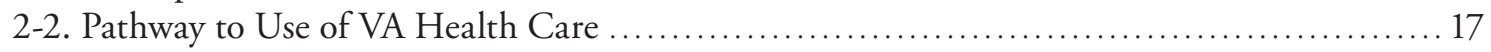

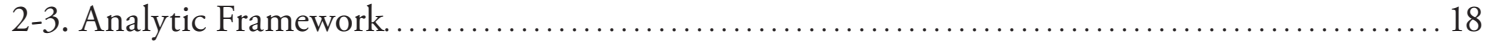

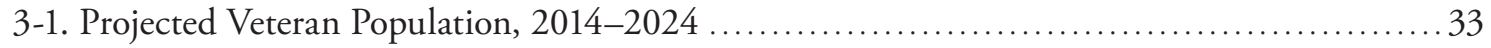

3-2. Race/Ethnicity Composition of the Veteran Population, 2014 and 2024 (Projected) ......... 33

3-3. Total Veteran Population, by Sex, 2014-2024 (Projected) .......................... 34

3-4. Number of New Veterans from Active Component, Total and by Sex .................... 35

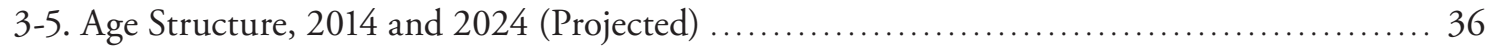

3-6. Mean Age, by Sex, 2014-2024 (Projected) ....................................... 36

3-7. Number of New Veterans from Active Component, by Age ............................. 37

3-8. Conflict-Era Veterans as Percentage of Total Veteran Population, 2014-2024 (Projected) ... 38

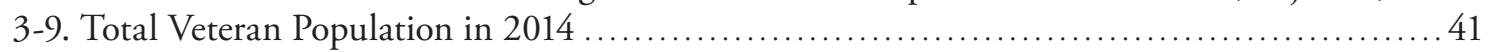

3-10. Total Veteran Population in 2024 (Projected) .................................... 42

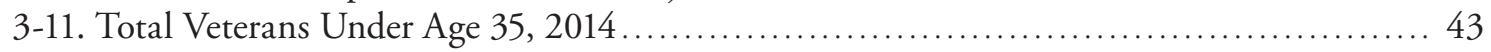

3-12. Total Veterans Under Age 35, 2024 (Projected)................................. 44

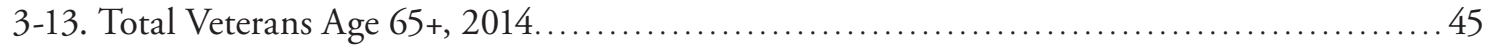

3-14. Total Veterans Age 65+, 2024 (Projected) ....................................... 46

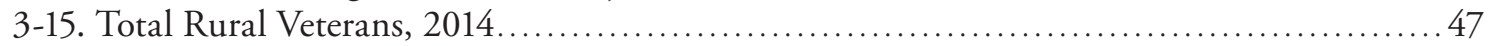

3-16. Total Rural Veterans, 2024 (Projected) ...................................... 48

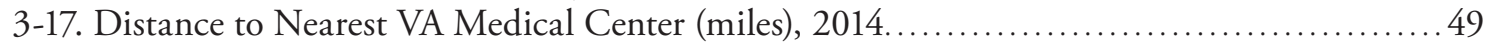

3-18. Percentage of Veterans Living Within a Given Distance of a VAMC, by Urban/Rural Status........................................................... 50

3-19. Percentage of Veterans Living Within a Given Distance of a VAMC, by Region........... 50

3-20. Percentage of Veterans Living Within a Given Distance of the Nearest VA Facility, VAMC Versus CBOC..................................................... 51

3-21. Projected Percent Population Change 2014-2024, with VAMC Locations ................. 54 
4-1. Conceptual Model of Enrollment in the VA Health Care System .......................... 59

4-2. Percentage of Separating Veterans Who Were Deployed .............................6 63

4-3. Percentage of Separating Veterans with 19+ Years of Service Among Those Who

Were Deployed .......................................................... 64

4-4. Number of Veterans Within 5 Years of Separation Among Those Who Were Deployed ... 64

4-5. Probability of New Enrollment, by Age (2009-2014) .............................. 65

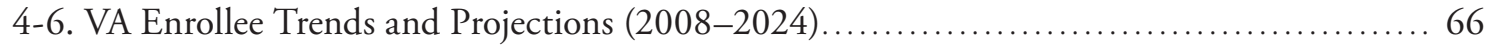

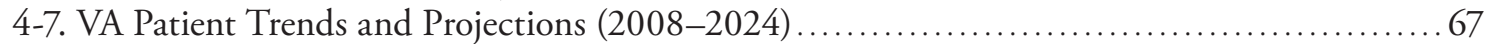

4-8. Percentage of VA Patients Who Served in Afghanistan and Iraq (2008-2024) ............. 68

4-9. Reliance for VA Patients and All Veterans, by Service Category ........................ 69

4-10. Reliance for VA Patients, by Health Care Service Category and Age .................... 70

4-11. Reliance for All Veterans, by Health Care Service Category and Age ........................ 71

4-12. Reliance for VA Patients, by Health Care Service Category, Sex, and Income............... 71

4-13. Reliance for All Veterans, by Health Care Service Category, Sex, and Income .............. 72

4-14. Reliance for VA Patients, by Coverage and Self-Reported Health Status . .................... 72

4-15. Reliance for All Veterans, by Coverage and Self-Reported Health Status.................... 73

4-16. EHCPM-Reported Reliance, by Service Category ................................... 74

4-17. EHCPM-Reported Prescription Drug (30-Day Rx) Reliance, by Age ................... 75

4-18. EHCPM-Reported Evaluation and Management (Visits) Reliance, by Age ................ 75

4-19. MEPS-Based Coverage for Prescription Drug Events, VA Patients ...................... 76

5-1. The Age Distribution for Veterans and Non-Veterans ............................... 85

5-2. Diagnosed (Unadjusted) Prevalence of Selected Health Conditions for Veterans and

Non-Veterans ........................................................ 87

5-3. Adjusted Diagnosed Prevalence of Selected Health Conditions for Veterans and

Non-Veterans ............................................................... 88

5-4. Adjusted Means of Disease Burden Measures for Veterans and Non-Veterans .............. 90

5-5. Adjusted Means of a Comorbid Mental Health Condition (Mental Health Condition +

Another Condition/Limitation) for Veterans and Non-Veterans ...................... 91

5-6. Prevalence of Selected Diagnosed Health Conditions for Veterans and NonVeterans,

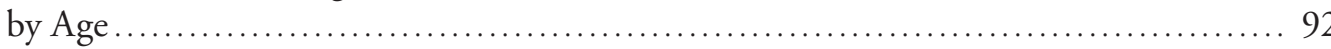

5-7. Difference in the Prevalence of Diagnosed Health Conditions for Veterans and

Non-Veterans: Vulnerable Populations .................................... 94

5-8. Age Distribution of Veterans, by VA Patient Status............................... 97

5-9. Unadjusted Prevalence of Diagnosed High-Prevalence Health Conditions Among Veterans, by VA Patient Status ........................................... 98

5-10. Unadjusted Prevalence of Diagnosed Lower-Prevalence Health Conditions Among Veterans, by VA Patient Status ................................................. 99

5-11. Adjusted Prevalence of Diagnosed High-Prevalence Health Conditions Among Veterans, by VA Patient Status ............................................. 100

5-12. Adjusted Prevalence of Diagnosed Lower-Prevalence Health Conditions Among Veterans, by VA Patient Status ............................................. 101

5-13. Adjusted Means of Disease Burden Measures for Veterans, by VA Patient Status ......... 102

5-14. Adjusted Means of Comorbid Mental Health Condition (Mental Health

Condition + Another Condition/Limitation) for Veterans, by VA Patient Status ...... 103

5-15. Prevalence of Selected Diagnosed Conditions Among Veterans, by VA Patient

Status and Age ......................................................... 104

5-16. VA Encounter-Based Prevalence of Diagnosed Health Conditions Among VA Patients.... 105

5-17. VA Encounter-Based Prevalence of Diagnosed Health Conditions, by Priority Group..... 107

5-18. Health Condition Prevalence in MHS and VA Encounter Data for Young Veterans ....... 109 
5-19. Fraction of Recently Separated Veterans Under Age 35 Who Deployed on Active

Duty .................................................................. 110

5-20. Projected Prevalence of Selected Health Conditions Among Veterans (2015-2024) ....... 112

5-21. Projected Prevalence of Service-Connected Conditions Among Veterans (2015-2024) ... 113

5-22. Projected Prevalence of Selected Health Conditions Among VA Patients (2015-2024) ... 115

5-23. Projected Prevalence of Service-Connected Conditions of VA Patients (2015-2024) ...... 117

5-24. VA Encounter-Based Projections of Health Conditions Among VA Patients

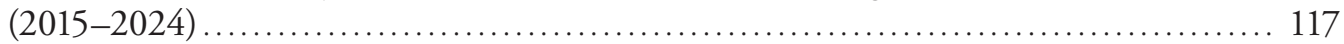

6-1. Assessment A Scenario Framework ............................................ 122

6-2. Progression from Vietnam-Era Veterans to Likely New VA Patients If Hypertension Is Adopted as a Presumptively Service-Connected Condition ....................... 129

6-3. End-Strength Scenarios (total non-civilian U.S. military personnel) .................... 132

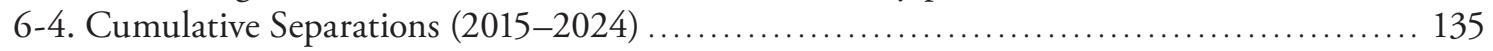

6-5. Cumulative New VA Patients Under Various Scenario Assumptions..................... 136

6-6. Selected Reasons for Not Utilizing VA Care (percentage of non-user Veterans) ............ 140

6-7. Relationship Between Veteran Characteristics and Self-Reported Access Barriers ........... 140

6-8. VA Health Care Use Rates, by Veteran Health Insurance Coverage ..................... 145

6-9. Coverage Expansion Patient Flows ............................................. 147

6-10. Scenario Impacts on Projected VA Patient Counts .................................. 153

7-1. Trends in the Veteran Population, Enrollment, and Use of VA Care .................... 164

A-1. PUMA in Los Angeles, California ................................................ 173

A-2. Coefficients from Zero-Inflated Poisson Regression Models for Number of Migrants

(Dependent Variable) .................................................... 184

A-3. Comparison of RAND and VetPop2014 Projections............................... 188

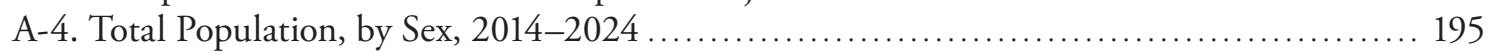

A-5. Average Age, by Sex, 2014-2024 ......................................... 196

A-6. Total Veteran Population Counts: 2014 and 2024 .................................... 197

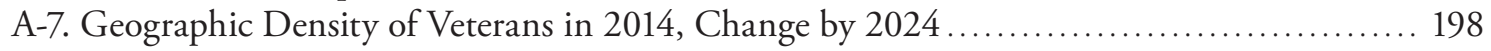

A-8. Percentage of Non-Hispanic White Veterans: 2014 and $2024 \ldots \ldots \ldots \ldots \ldots \ldots \ldots \ldots \ldots \ldots \ldots$

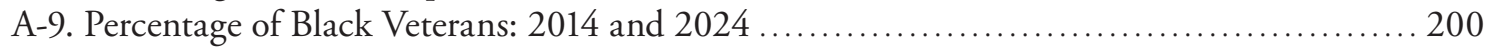

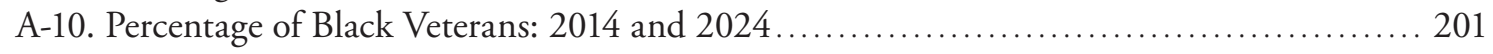

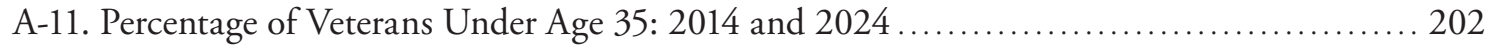

A-12. Percentage of Veterans Under Age 35 in 2014, Change by $2024 \ldots \ldots \ldots \ldots \ldots \ldots \ldots \ldots \ldots \ldots$

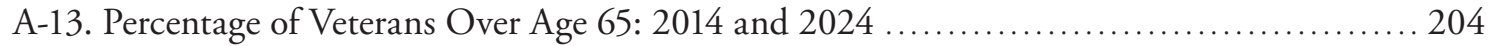

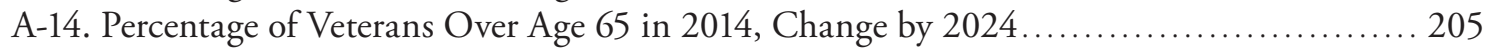

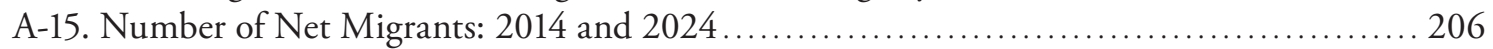

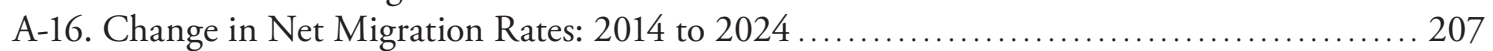

A-17. Model Comparison ............................................................ 208

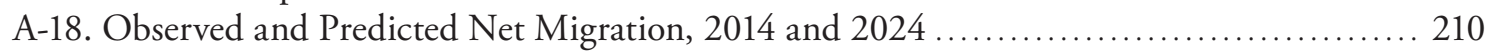

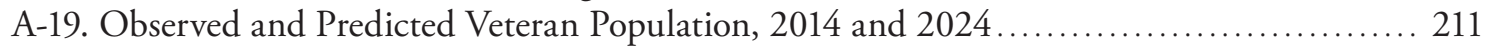

C-1. Predicted Prevalence Goodness of Fit ......................................... 322

C-2. Veteran Prevalence Projections with and without Time Trends for Common Chronic

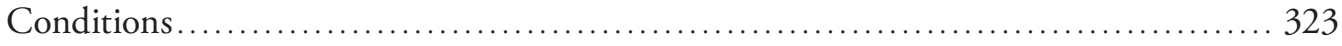

D-1. Maximum and Minimum Non-Civilian U.S. Military Personnel ...................... 330

D-2. Maximum and Minimum Rates of Hostile Deployment............................. 331

D-3. Typical Years of Service for Active Component Service Members, Officers vs. Enlisted ... 332 



\section{Tables}

S-1. Socioeconomic Characteristics of Veterans, by VA Patient Status, 2006-2012............ xxii

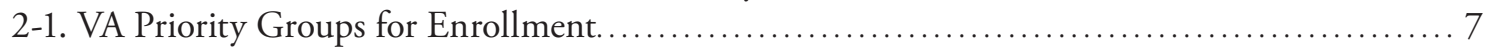

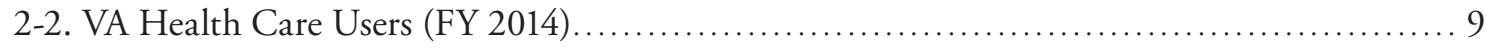

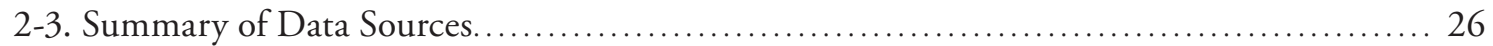

2-4. Survey Definitions of Veteran and VA Patient................................... 30

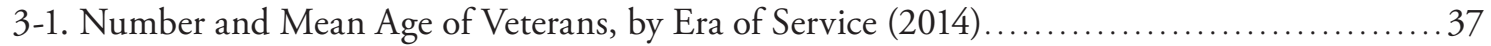

3-2. Socioeconomic Characteristics of the Veteran and Non-Veteran Civilian Population,

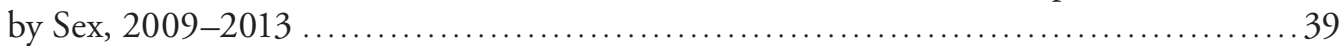

4-1. Data Sources and Category Definitions ........................................... 61

5-1. Socioeconomic Characteristics of Non-Veterans, Veterans, Non-VA Patients, and VA Patients in MEPS ..................................................... 86

5-2. Socioeconomic Characteristics of Veterans, by VA Patient Status, 2006-2012 ............ 97

5-3. Prevalence of Diagnosed Health Conditions among VA Patients, by VA Reliance .......... 108

6-1. VA Patients According to 2014 VA Administrative Data and 2013 ACS Estimates,

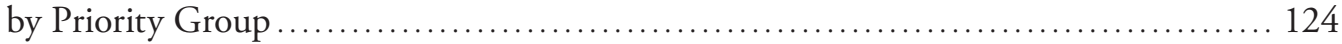

6-2. 2013 ACS Estimates and Use Rates, by Priority Group and Eligibility Status ............. 125

6-3. IOM's Assessment of the Evidence Connecting Certain Health Conditions to Military Service in Vietnam ................................................ 127

6-4. Upper Bound of New Patients Responding to Improved Access ........................ 141

6-5. Upper Bound of New Patients Among Enrolled Veterans Responding to Improved Access ........................................................................ 142

6-6. Distribution of Veterans, by Income and State Medicaid Expansion Decisions ............. 145

6-7. Coverage Changes Under ACA for Previously Uninsured Veterans, by Income Category ... 149

6-8. Eligibility for Previously Uninsured Veterans, by Income Category .................... 149

6-9. Predicted VA Enrollees and Patients ........................................... 150

6-10. Predicted Coverage Changes for Veterans Enrolled in VA Health Care Only, by

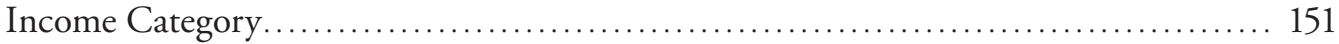

6-11. Scenario Impacts on Projected VA Patient Counts................................ 152

A-1. Demographic and Geographic Characteristics .................................. 172

A-2. Counts of Veterans and Migration Variables, 2014-2024 ......................... 179

A-3. Estimates from Zero-Inflated Poisson Regression Models for Number of Migrants (Dependent Variable) ....................................................... 181

A-4. Migration in 2014 as a Function of VA Facilities ..................................... 186

A-5. Projected Race/Ethnicity of Veteran Population in 2024, RAND and VetPop2014 ........ 188

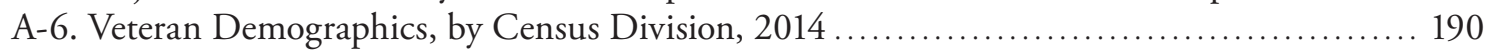

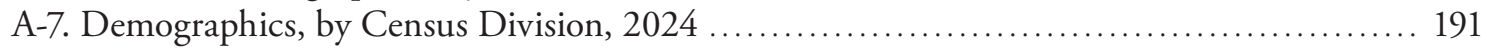

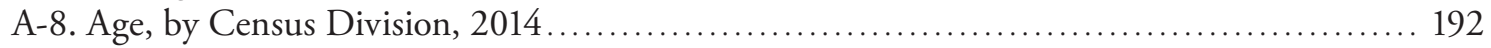

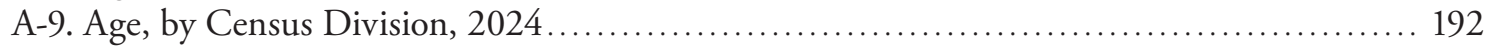




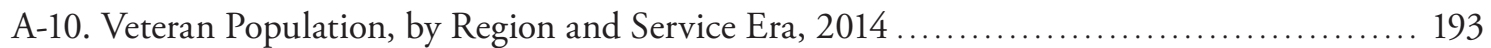

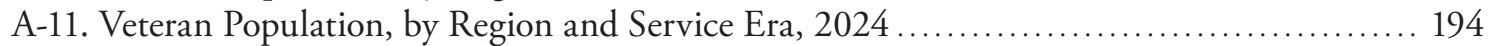

B-1. The Probability That a VA Enrollee Uses VA Health Care: Baseline Model ................. 215

B-2. The Probability That a VA Enrollee Uses VA Health Care, by Demographic

Characteristics............................................................ 216

B-3. The Characteristics of Non-Veterans, Veterans and VA Patients in MEPS ................. 219

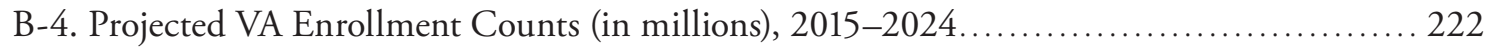

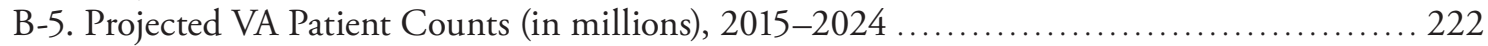

C-1. Demographic and Geographic Characteristics .................................... 224

C-2. Identification of Health Conditions ................................................... 226

C-3. Summary of Data Sources ....................................................... 231

C-4. Summary of Published Studies Comparing Self-Reported Versus Administrative Health Data ................................................................... 233

C-5. Summary of Published Comparisons of Health Conditions Based on Self-Report Versus Administrative Health Data ............................................. 234

C-6. Prevalence of Diagnosed Health Conditions for Veterans and Non-Veterans ............... 245

C-7. Predicted Means of Disease Burden Measures, by Veteran Status ......................... 246

C-8. Adjusted Means of Comorbid Mental Health Condition (Mental Health Condition + Another Condition/Limitation) for Veterans and Non-Veterans .......... 247

C-9. Prevalence of Diagnosed Health Conditions for Veterans, by Demographic Characteristics........................................................... 247

C-10. Difference in the Prevalence of Diagnosed Health Conditions for Veterans and Non-Veterans, by Demographic Characteristics ................................. 249

C-11. Difference in the Prevalence of Diagnosed Health Conditions for Veterans and Non-Veterans, by Poverty/Income and Employment Status....................... 250

C-12. Financial Insecurities, Predicted Means for Veterans and Non-Veterans................... 252

C-13. Prevalence of Diagnosed Health Conditions, by VA Patient Status....................... 253

C-14. Predicted Means of Disease Burden Measures Among, by VA Patient Status .............. 254

C-15. Adjusted Means of Comorbid Mental Health Condition (Mental Health Condition + Another Condition/Limitation) for Veterans, by VA Patient Status ...... 255

C-16. Prevalence of Diagnosed Health Conditions for VA Patients, by Demographic Characteristics....................................................... 255

C-17. Prevalence of Diagnosed Health Conditions for Non-VA Patient Veterans, by Demographic Characteristics............................................... 257

C-18. Characteristics of Non-Veterans, Veterans, and VA Patients in MEPS .................. 258

C-19. Prevalence of Health Conditions in VA Encounter Data .............................. 261

C-20. Prevalence of Health Conditions Among VA Patients, by Priority Group ................ 263

C-21. Prevalence of Diagnosed Health Conditions Among VA Patients, by VA Reliance......... 265

C-22. Prevalence of Health Conditions in MHS and VA Encounter Data for Veterans Younger Than 35 Years Old ................................................ 268

C-23. The Prevalence of Diagnosed Health Conditions for Veterans and Non-Veterans: Alternative Specifications ............................................... 270

C-24. Odds Ratios of Each Disease Condition for Control Variables Included in Model 2: Sample: Veterans and Non-Veterans......................................... 272

C-25. Odds Ratios of Each Disease Condition for Control Variables Included in Model 2: Sample: Veterans and Non-Veterans (cont.) .................................. 273

C-26. Odds Ratios of Each Disease Condition for Control Variables Included in Model 2: Sample: Veterans and Non-Veterans (cont.) ....................................... 274 
C-27. The Prevalence of Diagnosed Health Conditions by VA Patient Status: Alternative Specifications .................................................................. 275

C-28. Odds Ratios of Each Disease Condition for Control Variables Included in Model 2: Sample: VA Patients and Non-VA Patients (Veterans Only)........................ 277

C-29. Odds Ratios of Each Disease Condition for Control Variables Included in Model 2: Sample: VA Patients and Non-VA Patients (Veterans Only) (continued) .............. 278

C-30. Odds Ratios of Each Disease Condition for Control Variables Included in Model 2: Sample: VA Patients and Non-VA Patients (Veterans Only) (continued) ............... 279

C-31. Prevalence of Self-Reported Health Conditions for Veterans and Non-Veterans in NHIS ............................................................ 280

C-32. Prevalence of Self-Reported Health Outcomes for Veterans and Non-Veterans in BRFSS ................................................................... 282

C-33. Projected Prevalence of Diagnosed Asthma Among Veterans, by Sex, 2014-2024 ....... 283

C-34. Projected Prevalence of Diagnosed Asthma Among Veterans, by Age, 2014-2024 ....... 283

C-35. Projected Prevalence of Diagnosed Asthma Among Veterans, by Race/Ethnicity,

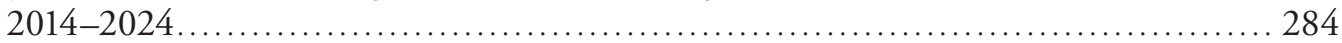

C-36. Projected Prevalence of Diagnosed CHF Among Veterans, by Sex, 2014-2024 .......... 284

C-37. Projected Prevalence of Diagnosed CHF Among Veterans, by Age, 2014-2024 .......... 285

C-38. Projected Prevalence of Diagnosed CHF Among Veterans, by Race/Ethnicity,

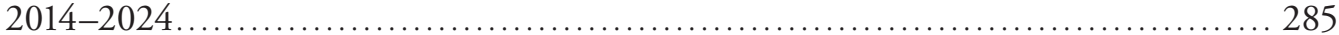

C-39. Projected Prevalence of Diagnosed COPD Among Veterans, by Sex, 2014-2024 ........ 286

C-40. Projected Prevalence of Diagnosed COPD Among Veterans, by Age, 2014-2024 ........ 286

C-41. Projected Prevalence of Diagnosed COPD Among Veterans, by Race/Ethnicity,

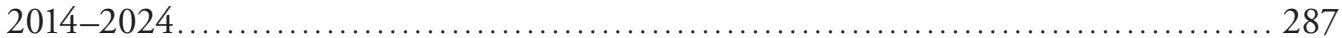

C-42. Projected Prevalence of Diagnosed Diabetes Among Veterans, by Sex, 2014-2024 ...... 287

C-43. Projected Prevalence of Diagnosed Diabetes Among Veterans, by Age, 2014-2024 ...... 288

C-44. Projected Prevalence of Diagnosed Diabetes Among Veterans, by Race/Ethnicity,

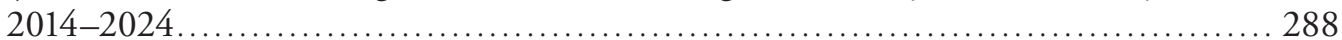

C-45. Projected Prevalence of Diagnosed GERD Among Veterans, by Sex, 2014-2024 ........ 289

C-46. Projected Prevalence of Diagnosed GERD Among Veterans, by Age, 2014-2024 ........ 289

C-47. Projected Prevalence of Diagnosed GERD Among Veterans, by Race/Ethnicity,

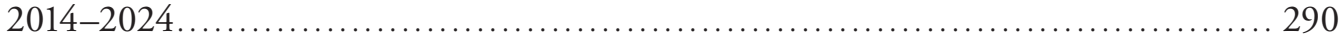

C-48. Projected Prevalence of Diagnosed Hearing Loss Among Veterans, by Sex,

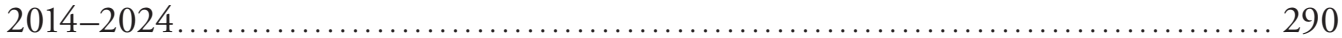

C-49. Projected Prevalence of Diagnosed Hearing Loss Among Veterans, by Age, 2014-2024.

C-50. Projected Prevalence of Diagnosed Hearing Loss Among Veterans, by Race/Ethnicity, 2014-2024

C-51. Projected Prevalence of Diagnosed Hypertension Among Veterans, by Sex, 2014-2024

C-52. Projected Prevalence of Diagnosed Hypertension Among Veterans, by Age, 2014-2024

C-53. Projected Prevalence of Diagnosed Hypertension Among Veterans, by Race/Ethnicity, 2014-2024

C-54. Projected Prevalence of Diagnosed Ischemic Heart Disease Among Veterans,

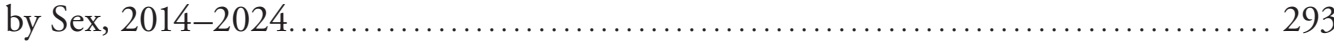

C-55. Projected Prevalence of Diagnosed Ischemic Heart Disease Among Veterans, by Age, 2014-2024 
C-56. Projected Prevalence of Diagnosed Ischemic Heart Disease Among Veterans, by Race/Ethnicity, 2014-2024

C-57. Projected Prevalence of Diagnosed Lipid Disorder Among Veterans, by Sex, 2014-2024.

C-58. Projected Prevalence of Diagnosed Lipid Disorder Among Veterans, by Age, 2014-2024

C-59. Projected Prevalence of Diagnosed Lipid Disorder Among Veterans, by Race/Ethnicity, 2014-2024

C-60. Projected Prevalence of Diagnosed Lower Back Pain Among Veterans, by Sex, 2014-2024.

C-61. Projected Prevalence of Diagnosed Lower Back Pain Among Veterans, by Age, 2014-2024.

C-62. Projected Prevalence of Diagnosed Lower Back Pain Among Veterans, by Race/Ethnicity, 2014-2024

C-63. Projected Prevalence of Diagnosed Malignant Cancers Among Veterans, by Sex, 2014-2024.

C-64. Projected Prevalence of Diagnosed Malignant Cancers Among Veterans, by Age, 2014-2024.

C-65. Projected Prevalence of Diagnosed Malignant Cancers Among Veterans, by Race/Ethnicity, 2014-2024

C-66. Projected Prevalence of Diagnosed Asthma Among VA Patients, by Sex, 2014-2024 _... 299

C-67. Projected Prevalence of Diagnosed Asthma Among VA Patients, by Age, 2014-2024 .... 300

C-68. Projected Prevalence of Diagnosed Asthma Among VA Patients, by Race/Ethnicity,

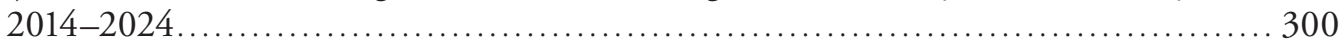

C-69. Projected Prevalence of Diagnosed CHF Among VA Patients, by Sex, 2014-2024 ....... 301

C-70. Projected Prevalence of Diagnosed CHF Among VA Patients, by Age, 2014-2024 ....... 301

C-71. Projected Prevalence of Diagnosed CHF Among VA Patients, by Race/Ethnicity,

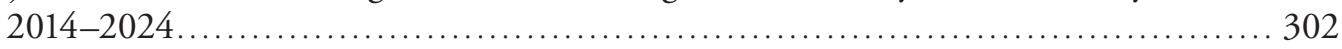

C-72. Projected Prevalence of Diagnosed COPD Among VA Patients, by Sex, 2014-2024 ..... 302

C-73. Projected Prevalence of Diagnosed COPD Among VA Patients, by Age, 2014-2024 ..... 303

C-74. Projected Prevalence of Diagnosed COPD Among VA Patients, by Race/Ethnicity,

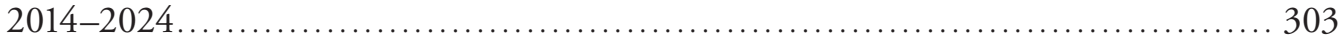

C-75. Projected Prevalence of Diagnosed Diabetes Among VA Patients, by Sex, 2014-2024.... 304

C-76. Projected Prevalence of Diagnosed Diabetes Among VA Patients, by Age, 2014-2024 ... 304

C-77. Projected Prevalence of Diagnosed Diabetes Among VA Patients, by Race/Ethnicity, 2014-2024

C-78. Projected Prevalence of Diagnosed Gastroesophageal Reflux Disease Among VA Patients, by Sex, 2014-2024.

C-79. Projected Prevalence of Diagnosed Gastroesophageal Reflux Disease Among VA Patients, by Age, 2014-2024 .............................................. 306

C-80. Projected Prevalence of Diagnosed Gastroesophageal Reflux Disease Among VA Patients, by Race/Ethnicity, 2014-2024 .................................... 306

C-81. Projected Prevalence of Diagnosed Hearing Loss Among VA Patients, by Sex,

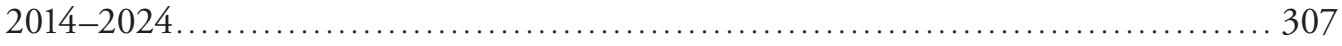

C-82. Projected Prevalence of Diagnosed Hearing Loss Among VA Patients, by Age, 2014-2024.

C-83. Projected Prevalence of Diagnosed Hearing Loss Among VA Patients, by Race/Ethnicity, 2014-2024 
C-84. Projected Prevalence of Diagnosed Hypertension Among VA Patients, by Sex, 2014-2024

C-85. Projected Prevalence of Diagnosed Hypertension Among VA Patients, by Age, 2014-2024.

C-86. Projected Prevalence of Diagnosed Hypertension Among VA Patients, by Race/Ethnicity, 2014-2024

C-87. Projected Prevalence of Diagnosed Ischemic Heart Disease Among VA Patients,

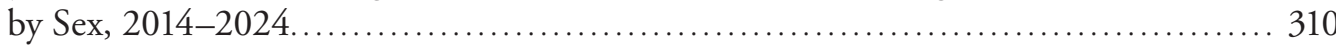

C-88. Projected Prevalence of Diagnosed Ischemic Heart Disease Among VA Patients,

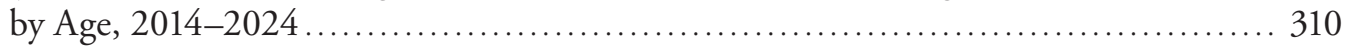

C-89. Projected Prevalence of Diagnosed Ischemic Heart Disease Among VA Patients, by Race/Ethnicity, 2014-2024 ........................................... 311

C-90. Projected Prevalence of Diagnosed Lipid Disorder Among VA Patients, by Sex, 2014-2024................................................................... 311

C-91. Projected Prevalence of Diagnosed Lipid Disorder Among VA Patients, by Age,

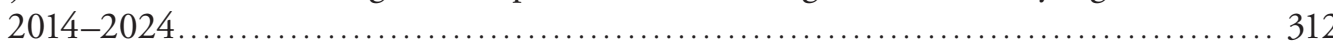

C-92. Projected Prevalence of Diagnosed Lipid Disorder Among VA Patients, by Race/Ethnicity, 2014-2024 ................................................ 312

C-93. Projected Prevalence of Diagnosed Lower Back Pain Among VA Patients, by

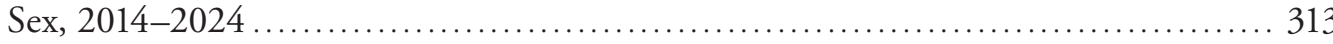

C-94. Projected Prevalence of Diagnosed Lower Back Pain Among VA Patients, by

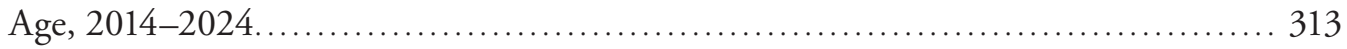

C-95. Projected Prevalence of Diagnosed Lower Back Pain Among VA Patients, by Race/Ethnicity, 2014-2024 ............................................... 314

C-96. Projected Prevalence of Diagnosed Malignant Cancers Among VA Patients, by

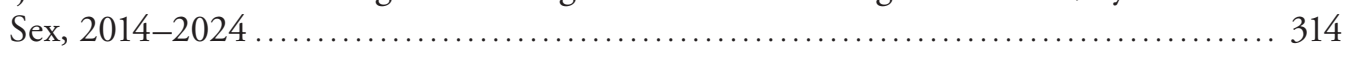

C-97. Projected Prevalence of Diagnosed Malignant Cancers Among VA Patients, by

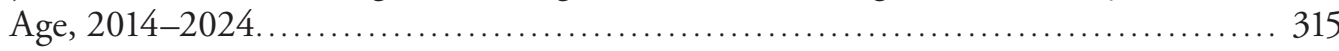

C-98. Projected Prevalence of Diagnosed Malignant Cancers Among VA Patients, by Race/Ethnicity, 2014-2024 ............................................... 315

C-99. Projected Prevalence of Diagnosed Mental Health Conditions Among Veterans,

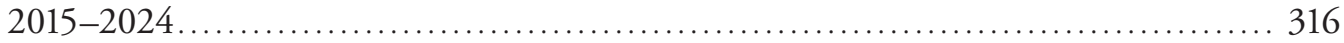

C-100. Projected Prevalence of Diagnosed Posttraumatic Stress Disorder Among

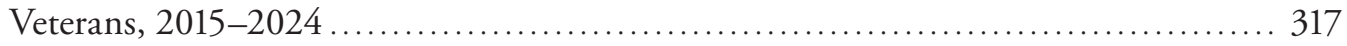

C-101. Projected Prevalence of Diagnosed Mental Health Conditions Among VA Patients, 2015-2024 ....................................................... 318

C-102. Projected Prevalence of Diagnosed Posttraumatic Stress Disorder Among VA Patients, 2015-2024.

C-103. Projected Prevalence of Diagnosed Traumatic Brain Injury Among VA Patients, 2015-2024.

C-104. Projected Prevalence of Diagnosed Musculoskeletal Conditions Associated with Chronic Pain Among VA Patients, 2015-2024

D-1. Estimate for New VA Patients If Hypertension Becomes a Presumptively Service-Connected Condition for Vietnam Veterans (thousands)....

D-2. Estimated Number of Uninsured Veterans Who Were Not VA Patients and Predicted to Gain or Not Gain Other Coverage by 2016.

D-3. Estimated Number of Uninsured Veterans Who Were Not VA Patients, Predicted to Be Eligible to Enroll in VA Health Care, and Predicted to Gain or Not Gain Other Coverage by 2016 
xviii Characteristics and Unique Health Care Needs of the Patient Population Served by VA

D-4. Thousands of Estimated New VA Enrollees at Different Enrollment Rate Assumptions ................................................................ 336

D-5. Thousands of Estimated New VA Patients at Different Enrollment Rate Assumptions ............................................................... 337

D-6. Estimated Number of Veterans with Only VA Coverage and Predicted to Gain or Not Gain Other Coverage by 2016 


\section{Summary}

The Department of Veterans Affairs (VA) provides health care to eligible veterans. In this report, we describe the current and projected characteristics and health care needs of the U.S. veteran population as whole, as well as the population of veterans who receive health care from VA. This analysis was conducted in response to the Veterans Access, Choice, and Accountability Act of 2014 (Section 201), which mandated "an independent assessment of current and projected demographics and unique health care needs of the patient population served by the Department."

\section{Study Purpose and Approach}

We examined the demographic characteristics of the current and projected population of U.S. Veterans and patients of the VA health care system. In addition, we examined the unique health care needs of the patient population currently served by VA and projected the health care needs of Veterans who might become patients in the future. We use the term Veteran to describe all Veterans, whether or not they use VA health care services, and the term VA patients to describe Veterans who received at least some health care from VA in the past year.

This report addresses four overarching research questions:

- What are the demographic characteristics of the U.S. Veteran population, and how are these projected to change between 2015 and 2024?

- To what extent do Veterans, including VA patients, rely on VA for their health care?

- What are the current health care needs of the Veteran population, including both VA patients and non-VA patients, and how do these compare with the needs of the nonVeteran population? How will the needs of Veterans in general and the VA patient population specifically evolve over time given current policies?

- How might the projected number of Veterans and VA patients change because of external forces or changes in VA policies?

To address the research questions, we conducted a series of analytic activities: Using a cohort-based approach, we estimated the size and demographic composition of the Veteran population; using the projected number of Veterans as a baseline, we estimated future enrollment in the VA health care system, the future size of the VA patient population, and the share of health care services that current Veterans receive from VA; we combined several data sources to assess the unique health care needs of Veterans and VA patients compared with nonVeterans; we used a modeling approach to assess how the number of VA patients and their health conditions might evolve over time; and we conducted scenario testing to understand 
how VA policies and external factors might affect the size of the Veteran population and the number of VA patients.

\section{Background}

VA provides health care services to enrolled Veterans who seek care at VA facilities, or-in some cases - through contracted care purchased from the civilian sector. Eligibility for VA health care has evolved over time, and today's eligibility rules are rooted in the Veterans Health Care Eligibility Reform Act of 1996. The law mandated health care for service-connected health conditions and for Veterans with a service-connected disability rated at 50 percent or higher. The Secretary of Veterans Affairs has legal discretion over the provision of all other care, but VA must maintain specialized treatment and rehabilitation programs for spinal injuries, blindness, amputations, mental illness, and other serious service-connected health conditions.

In general, a Veteran must have served in the U.S. military for at least 24 months and received an honorable discharge to enroll for VA health care. Some exceptions are permitted; for example, Veterans serving less than 24 months may be eligible if they were medically retired from military service due to a service-connected condition. To implement the 1996 law, VA established a priority system for determining which groups of Veterans will be authorized for care within the authorized budget. This structure places Veterans in one of eight priority groups based on their service-connected disability rating, income, and other factors. A Veteran's priority group designation affects his or her eligibility to receive care through VA, as well as his or her cost-sharing requirements (that is, whether co-payments are required and, if so, how much). Currently, enrollment is limited to recent combat Veterans, Veterans with qualifying incomes, and Veterans with service-connected or other disabilities. Based on our analysis of VA administrative data, about 9 million Veterans ( 42 percent of all Veterans) were enrolled in 2014. Non-enrolled Veterans include a mix of Veterans who are ineligible to enroll and Veterans who are eligible to enroll but choose not to do so.

Use of VA health care depends on a number of factors, including the total number of Veterans in the population, Veterans' eligibility to enroll for services, Veterans' enrollment decisions when eligible, and Veterans' decisions to seek VA health care services when enrolled. Because many Veterans have access to health care through other sources, such as employer insurance or Medicare, not all will choose to enroll, and those who do enroll may choose not to use VA for all of their health care needs. In addition, both VA policy and factors external to VA can affect Veterans' use of services. For example, a policy change enabling higher-income Veterans to enroll could increase demand for VA services. Similarly, a future military conflict could increase the number of Veterans in the pipeline and affect their health care needs. In our analysis, we distinguish VA enrollees from VA patients; a VA patient is an enrollee who has used VA health care in the past year.

In this assessment, we used data from VA and from other federal sources, such as the U.S. Census Bureau, to estimate the total number of Veterans and VA patients, to project the size of these populations over time, and to estimate the health care needs of these populations. Our baseline estimates and projections assumed that VA policies and other factors that might affect Veterans' demand for services are constant, with adjustments for policy changes that have 
already been announced (such as the President's plan to reduce the size of the U.S. military). In scenario testing, we considered how uncertain future events, such as a future conflict or a change in VA eligibility policy, might affect the size and health care needs of the Veteran and VA patient populations.

\section{Key Findings}

\section{Current and Projected Demographic Trends in the Veteran Population}

Today's Veterans generally enjoy favorable socioeconomic outcomes relative to their nonVeteran counterparts. Using data from the American Community Survey (ACS), we find that Veterans are less likely to be unemployed, less likely to be living below the poverty line, and more likely to have graduated from high school, on average, than non-Veterans (Figure S-1). Veterans are also more likely than non-Veterans to have medical insurance; only 7 percent of female Veterans and 6 percent of male Veterans were uninsured during the 2009 to 2013 time period, according to the ACS. In contrast, 15 percent of female non-Veterans and 22 percent of male non-Veterans were uninsured during this time period. Rates of uninsurance among the Veteran population may be low in part because many Veterans have access to free insurance through VA. Insurance rates in the United States have increased since 2013, due to the implementation of the Patient Protection and Affordable Care Act (ACA) (Office of the Assistant Secretary for Planning and Evaluation, 2015a). We did not have data, however, that allowed us to compare post-ACA insurance rates between Veterans and non-Veterans.

Figure S-1

Socioeconomic Characteristics of the Veteran and Non-Veteran Civilian Population, by Sex, 2009-2013

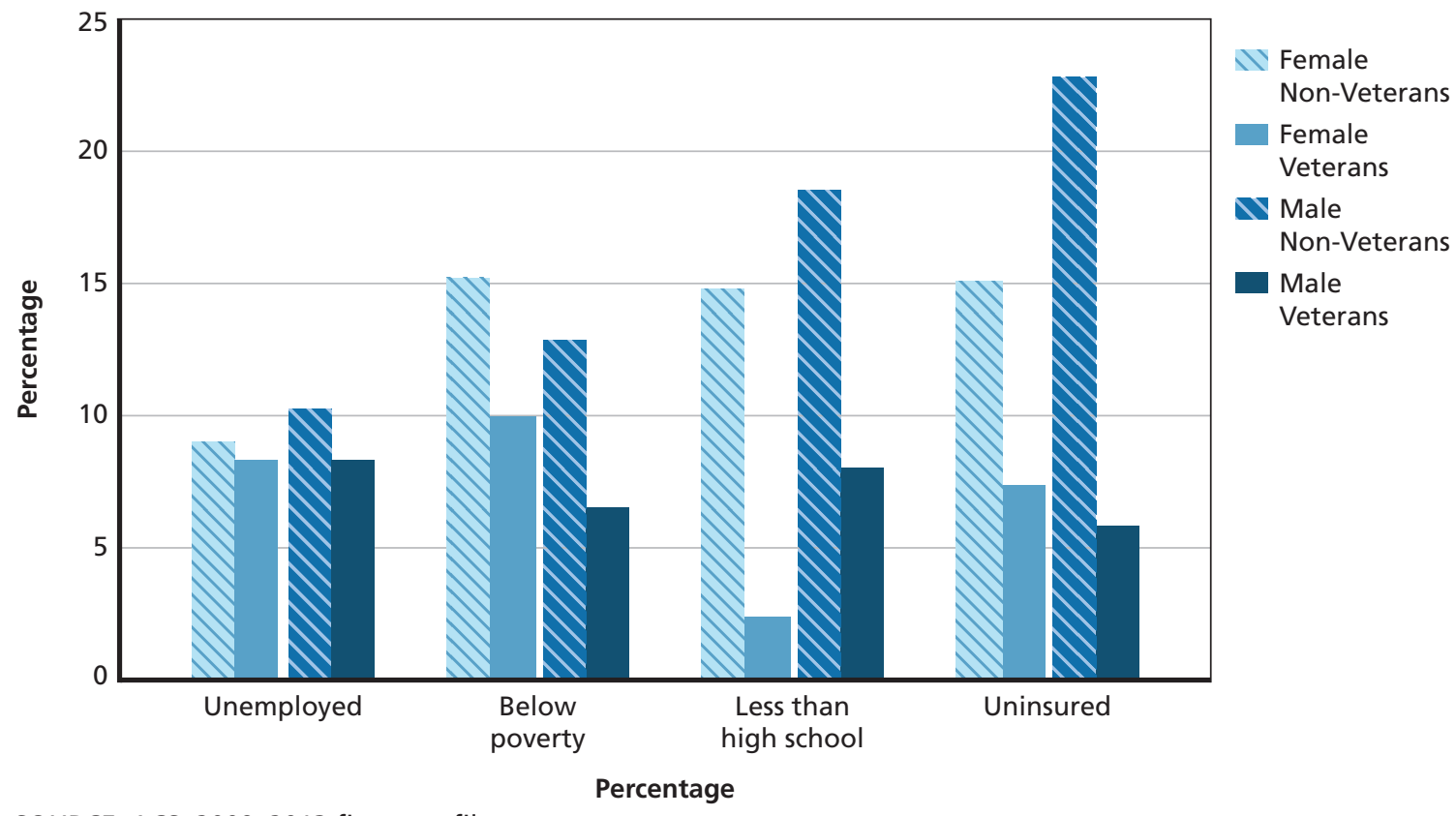

SOURCE: ACS, 2009-2013 five-year file.

RAND RR1165z1-S.1 
Homelessness is declining among Veterans. Homelessness remains a significant problem among Veterans. Veterans are overrepresented in the U.S. adult homeless population: In 2010, Veterans accounted for approximately 10 percent of the adult population; however, they represented a disproportionate share of the homeless adult (16 percent) and sheltered homeless adult (13 percent) populations (National Center for Veterans Analysis and Statistics, 2012b). Notwithstanding this, the rate of homelessness among Veterans has declined since 2010. According to the U.S. Department of Housing and Urban Development, there were 49,933 homeless Veterans in 2014, representing less than 0.25 percent of the total Veteran population. Between 2010 and 2014, the number of homeless Veterans declined by 33 percent (U.S. Department of Housing and Urban Development, 2014).

VA patients tend to be older and less socioeconomically well off than Veterans who do not rely on VA for care. Using data from the Medical Expenditure Panel Survey (MEPS), we are able to compare Veterans who use VA care with Veterans who do not use VA care (Table S-1). VA patients are older and less well-off from a socioeconomic standpoint than Veterans who do not use VA for care. For example, 9 percent of VA patients have less than a high school education, compared with 6 percent of Veterans. VA patients' average household incomes are more than 20 percent lower than incomes for non-patient Veterans. VA patients are also far less likely to be employed than non-VA patients. ${ }^{1}$ Partly, these differences are by design, because higher-income Veterans may not be eligible for VA services.

We project that the population of U.S. Veterans will decrease by 19 percent over the next 10 years. The U.S. Veteran population has been decreasing for the past three decades, and this trend will continue. There were 27.5 million Veterans in the United States as of the 1990 Census; we estimate that there were 21.6 million Veterans in 2014. Over the next 10 years, our projections, drawing on VA, U.S. Census, and U.S. Department of Defense (DoD) data, show that the Veteran population will decline to 17.5 million, a decrease of 19 percent relative to 2014 levels (Figure S-2). Given the strong preexisting trends and the President's ongoing drawdown in the size of the active duty military population (Hagel, 2014; Parrish, 2011; Office of

Table S-1

Socioeconomic Characteristics of Veterans, by VA Patient Status, 2006-2012

\begin{tabular}{lcc}
\hline Characteristic & Veterans, VA Patients & Veterans, Non-VA Patients \\
\hline Over age 65 & $52.2 \%$ & $38.7 \%$ \\
Married & $62.6 \%$ & $68.0 \%$ \\
Less than high school education & $9.1 \%$ & $5.8 \%$ \\
Employed* & $41.3 \%$ & $62.8 \%$ \\
Average household income & $\$ 35,981$ & $\$ 45,278$ \\
\hline
\end{tabular}

SOURCE: RAND analysis of MEPS, 2006-2012.

NOTES: Veterans, VA patients and Veterans, non-VA patients are mutually exclusive categories. Sample size, VA patients $=4,871$, and sample size, non-VA patients $=7,442$.

* Non-employed individuals include both people who are unemployed and people who are out of the labor force, such as retirees.

1 The remaining 37.2 percent of non-VA patients and 58.7 percent of VA patients who are not employed include both unemployed individuals and people who are out of the labor force because, for example, they are retired or disabled and unable to work. 
Figure S-2

The Number of U.S. Veterans Will Decline by 19 Percent by 2024

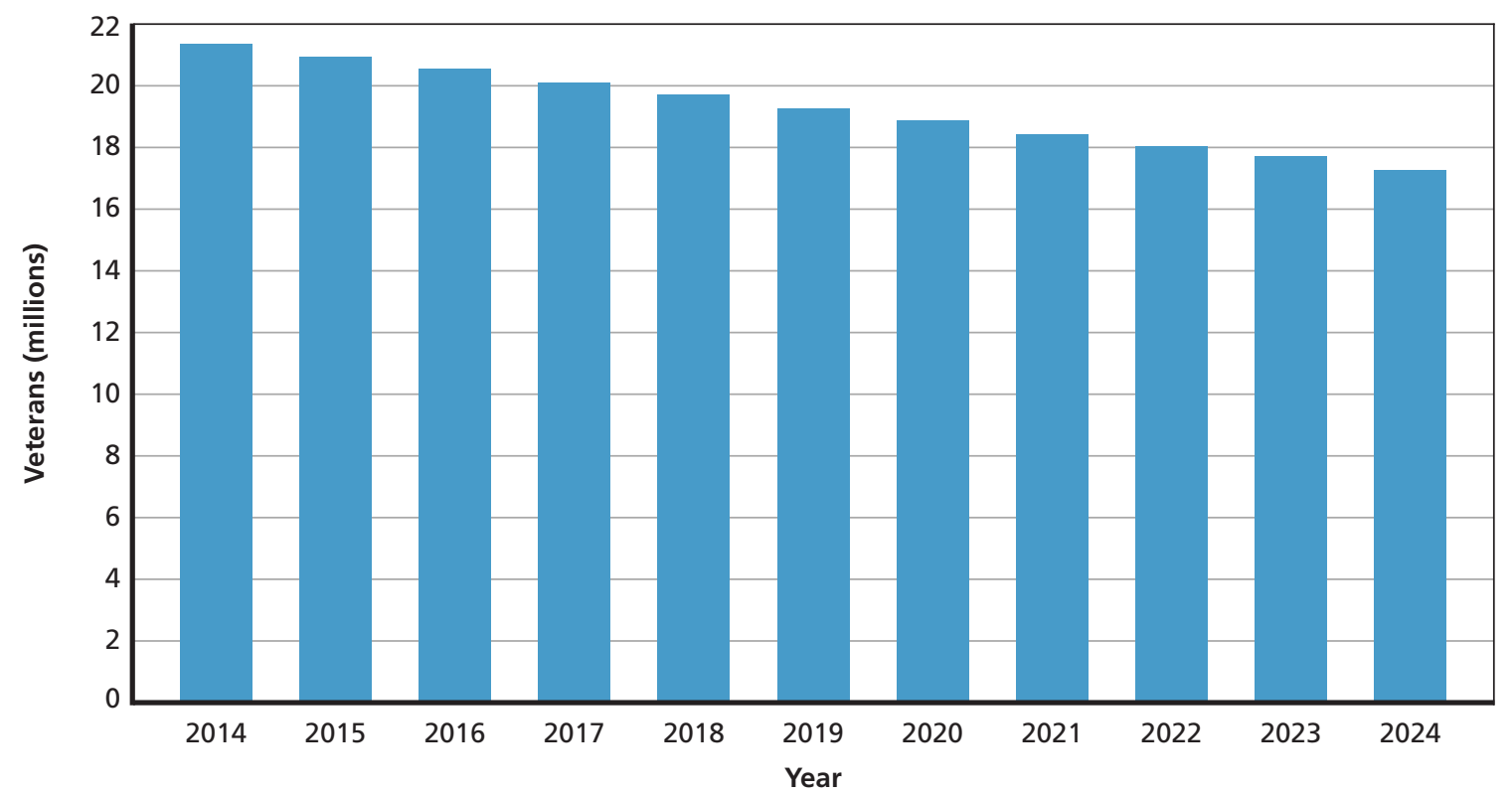

SOURCE: RAND analysis of VA, DoD, and Census data.

RAND RR1165z1-S.2

the Under Secretary of Defense [Comptroller], 2015), the reduction in the size of the Veteran population is inevitable, absent a major policy change to increase the size of the military (for example, if an unanticipated large-scale conflict were to materialize).

Geographic distribution of Veterans will shift slightly. We estimate that, geographically, the Veteran population will become more concentrated in urban areas, and the relative proportion of the Veteran population in the Ohio River Valley region will diminish.

There will be modest changes in the demographic mix, by sex and race/ethnicity. Currently, Veterans are more likely than non-Veterans to be male, and are on average much older. We estimate that approximately 92 percent of the Veteran population was male in 2014. We also estimate that 75 percent of Veterans were age 55 or older, compared with only 34 percent of the non-Veteran population. By 2024, this will shift somewhat: The proportion of female Veterans will increase 3 percentage points, from 8 to 11 percent, by 2024, and the share of non-Hispanic white males will decrease from 80 to 74 percent over the same period. Mean age will increase slightly; the population will have a higher proportion of both older and younger Veterans.

These projections are based on historic separation rates, the anticipated decrease in military end-strength over the next several years, and an assumption that there are no significant new conflicts during the projection period.

\section{Enrollment and Reliance on VA Health Care}

The number of Veterans receiving VA health care is projected to level off over the next 10 years. While the Veteran population is projected to decline by 19 percent over the next 10 years, the number of VA patients is projected to increase until 2019. Use of VA health care has increased across all demographic groups since 2005, and the fraction of Veterans under 
age 35 who are VA patients has increased threefold. The growth of VA use by Veterans may be related to outreach efforts on the part of VA, policies that have expanded the list of conditions granting presumptive eligibility for VA services, and streamlined enrollment processes. Continued increases in the rates of VA use are expected to slow the decline in the number of VA patients. Nevertheless, in years beyond 2019, VA may begin to experience slight declines in the volume of patients. Because VA will be coming off a period of more than a decade of expanded use, careful monitoring and new policies may be necessary to address the leveling-off and possible reduction in demand for services that could occur after 2019.

Health care planning for VA is complicated by the fact that most Veterans have more than one possible source of health coverage. The extent to which Veterans use VA care as opposed to care from other sources is captured in the concept of reliance, by which we mean the fraction of Veterans' total care that is provided by or paid for by VA. Reliance on VA versus other sources of care varies by type of care, but it averages below 50 percent for many routine services. Across all types of care, Veterans under age 30 are the most reliant on VA, and those over age 65 are least reliant.

Both VA policy, such as policies to enhance Veterans' access to VA services, and external trends, such as the cost and availability of private health insurance, can affect Veterans' reliance on VA. However, VA has limited visibility into patients' reliance. While VA has access to data on care obtained at VA facilities, it is difficult to track how much care Veterans consume outside the VA system-for example, through private health insurance. Yet understanding reliance is critical for planning, because shifts in reliance can affect the total amount of care that Veterans obtain from VA facilities.

We analyzed reliance using data from MEPS and compared these estimates with reliance estimates used in VA's Enrollee Health Care Projection Model (EHCPM). MEPS is a survey of health care utilization and spending conducted by the Agency for Healthcare Research and Quality (AHRQ). The EHCPM is a forecasting model sponsored by VA, which relies on VA survey data, Medicare claims data, and proprietary data from the actuarial firm Milliman.

Using MEPS data, we found that younger Veterans, lower-income Veterans, Veterans in rural areas, Veterans without other access to health insurance coverage, and Veterans with poorer self-reported health status rely more than other Veterans on VA. However, the estimated share of care obtained through VA is generally lower in the MEPS estimates than in the EHCPM estimates. For example, MEPS indicates that VA patients obtain 30 percent of their prescription drugs through VA, compared with ECHPM's estimate that enrollees obtain 66 percent of their prescriptions from VA. Because the EHCPM estimates are in part based on proprietary methods, we were unable to ascertain fully the reasons for these differences. However, a general conclusion is that VA might benefit from validating current reliance estimates and investing in survey approaches to better understand Veterans' total health care needs.

\section{Unique Health Care Needs of Veterans and VA Patients}

To identify the unique health care needs of Veterans and the VA patient population, ${ }^{2}$ we first compared the prevalence of key health conditions among the current Veteran population with those among the non-Veteran population. We then compared the prevalence of key health

2 We define the unique health care needs of Veterans as those that disproportionately affect Veterans relative to nonVeterans. These include both service-connected conditions, such as posttraumatic stress disorder (PTSD), and other conditions that are more prevalent among Veterans than non-Veterans, including diabetes and cancer. 
conditions among VA patients with those among Veterans who do not use VA health care and analyzed which characteristics (including the presence of particular health conditions) were associated with receiving care at VA facilities. Our analysis relied on MEPS, which collects information on all care received, regardless of payer, and information on Veteran status and use of VA services. With MEPS data, we can analyze all of a Veteran's diagnosed health conditions, regardless of whether the Veteran used VA health services. We can also use MEPS data to compare Veterans with non-Veterans. While the numbers reported in this summary are from MEPS, we also present in the body of the report findings from analysis of VA administrative data on patient encounters.

We examined both unadjusted prevalence rates of these health conditions and adjusted prevalence rates, which accounted for key demographic characteristics, such as age and sex. Both rates provide unique information with relevance to policy issues.

Unadjusted prevalence rates provide a snapshot of the overall Veteran population and enable us to compare how Veterans and VA patients may differ from civilians in terms of their health care needs. Unadjusted rates, however, do not account for the fact that Veterans are typically older and more likely to be male than civilians. Nevertheless, these numbers are useful for planning purposes. For example, the fact that Veterans have a much higher rate of diabetes than non-Veterans is useful for determining the types of providers and services that Veterans need, even if most of the difference between Veterans and non-Veterans can be explained by factors such as age and sex.

Adjusted prevalence rates help us understand how Veterans' and VA patients' health care needs may differ from the needs of demographically similar non-Veterans. As a result, these comparisons inform our understanding of how the experience of being a Veteran affects health. However, because they already account for demographic differences, without careful interpretation, the adjusted prevalence rates may appear to understate key differences in health care needs between Veterans and non-Veterans at the population level.

We also projected the prevalence of the health conditions of Veterans and VA patients forward over the next 10 years, accounting for predicted changes in their demographic composition and their service experiences.

Veterans have a higher unadjusted prevalence of diagnosed health conditions than non-Veterans. The diagnosed prevalence of many common chronic health conditions, unadjusted for differences in demographic characteristics, is higher among Veterans than nonVeterans. For example, the prevalence of diabetes and gastroesophageal reflux disease (GERD) disorders among Veterans is substantially higher than for non-Veterans (Figure S-3). Veterans are more likely than non-Veterans to be diagnosed with cancer, hearing loss, and PTSD. Mental health conditions, generally, are equally prevalent in the Veteran and non-Veteran populations. Because Veterans are more likely to have insurance than non-Veterans, some of these differences could reflect that Veterans are more likely to receive diagnoses than non-Veterans. Nevertheless, understanding differences in diagnosed conditions sheds light on differences in conditions that Veterans and non-Veterans are being treated for under existing policies. As such, these analyses inform our understanding of whether Veteran providers are likely to treat a different mix of conditions than civilian providers. Our analyses suggest that VA providers are likely to be treating a sicker population with more chronic conditions, such as cancer, diabetes, and chronic obstructive pulmonary disease (COPD), than the population expected by civilian providers. 
Figure S-3

Veterans Have Higher Diagnosed Prevalence of Several Key Health Conditions (Unadjusted Prevalence)

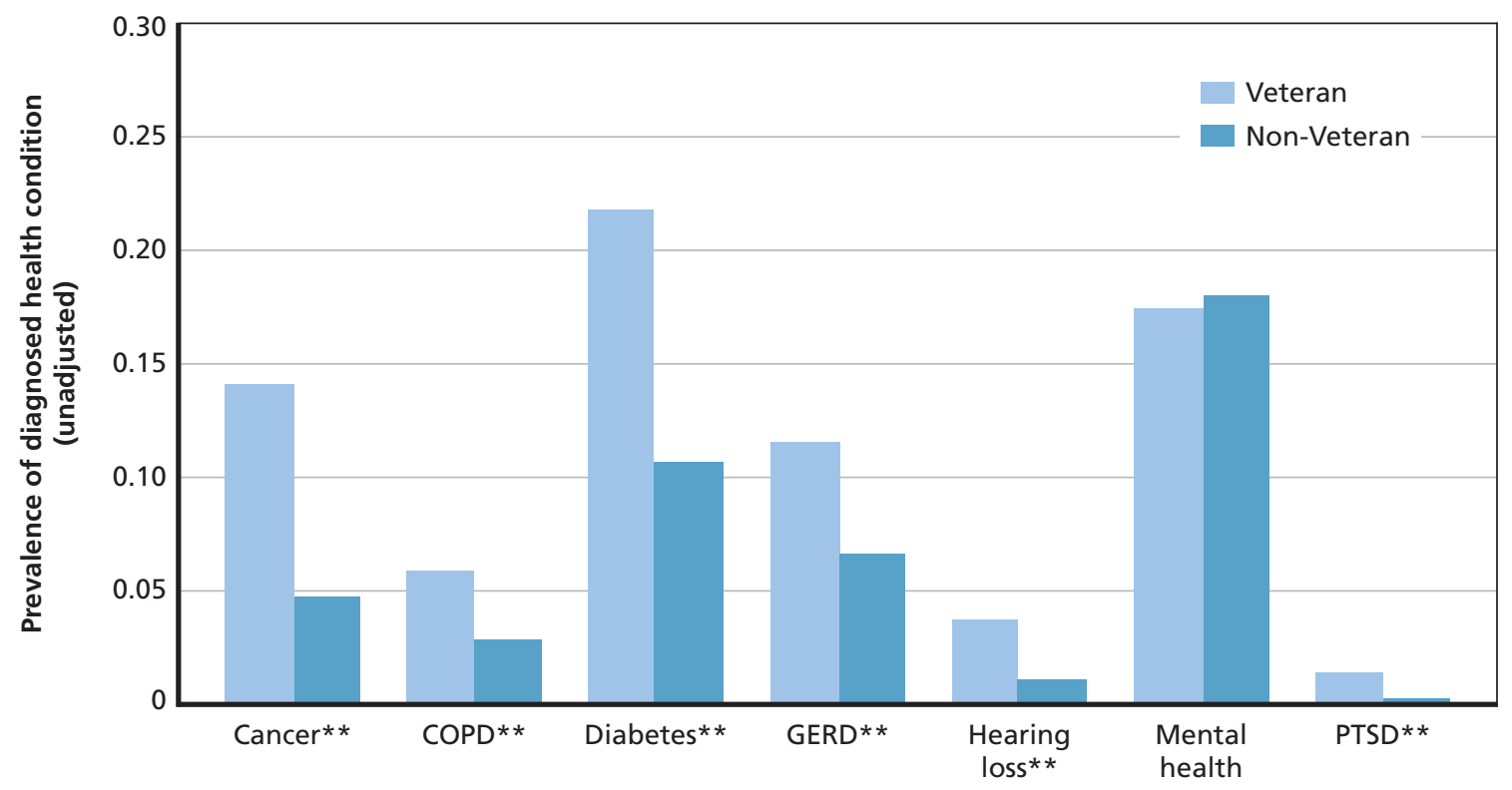

SOURCE: RAND analysis of MEPS, 2006-2012.

NOTES: ** indicates a statistically significant difference between Veterans and non-Veterans at $\mathrm{p}$-value $<0.05$. Sample size, non-Veterans $=150,225$, and sample size, Veterans $=12,313$. Sample sizes may be smaller for some conditions due to missing values. Cancer includes any malignancy, and Mental Health includes any mental health condition.

RAND RR1165z1-S.3

Veterans also have a higher adjusted prevalence of key health conditions than nonVeterans. For some conditions, adjusting for demographic characteristics substantially reduces the difference in prevalence rates between Veterans and non-Veterans. For example, Veterans are twice as likely to have diabetes as non-Veterans in the unadjusted model, but after adjusting for demographic characteristics, the prevalence rate among Veterans is only 13 percent higher. In the adjusted model, Veterans are more likely to have mental health conditions than non-Veterans, while differences were not statistically significant in the unadjusted model. Even though fewer than 5 percent of Veterans are diagnosed with PTSD, it is even rarer in the nonVeteran population. After controlling for age and other factors (Figure S-4), Veterans are 13.5 times more likely than non-Veterans to be diagnosed with PTSD.

VA patients are typically less healthy than Veterans who do not use VA health care. Compared with Veterans seen by private health care providers, Veterans who received treatment from VA had higher rates of cancer, diabetes, hypertension, PTSD, ischemic heart disease (IHD), and other conditions (Figure S-5). These differences reflect VA patients' older age, and also reflect the eligibility criteria for enrolling in VA care, which depend in part on health status. Among VA patients, the unadjusted prevalence of common chronic conditions (e.g., diabetes, cancer) is 51 to 96 percent higher for VA patients relative to Veterans who do not use VA care; however, rates of PTSD are several orders of magnitude higher among VA patients relative to non-patients. Adjusting for demographic characteristics slightly reduces differences in prevalence rates between patients and non-patients (results shown in Chapter Five). 
Figure S-4

Veterans Have a Higher Prevalence of Several Key Health Conditions (Adjusted Prevalence)

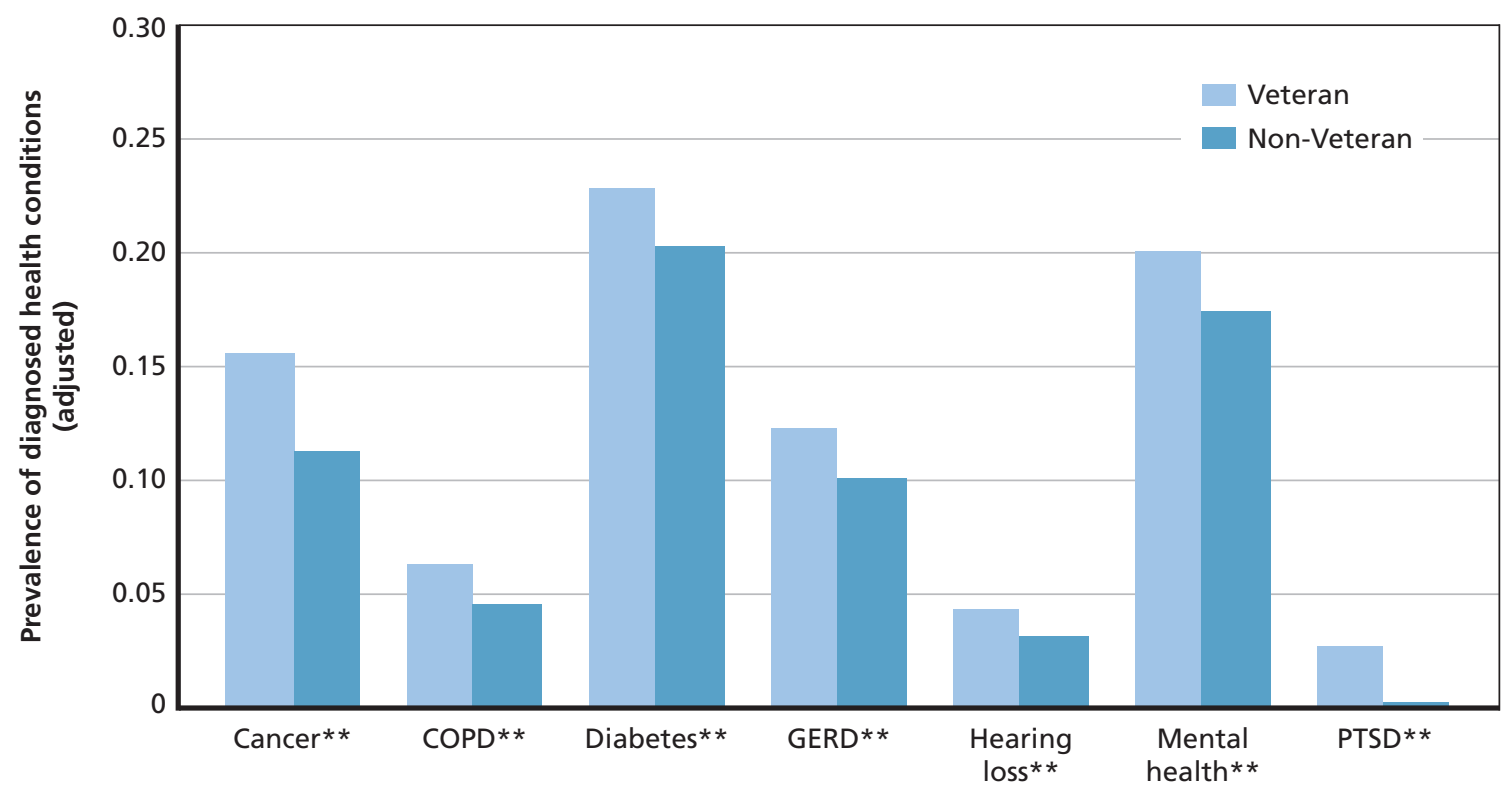

SOURCE: RAND analysis of MEPS, 2006-2012.

NOTES: ** indicates a statistically significant difference between Veterans and non-Veterans at $p$-value $<0.05$. Sample size, non-Veterans $=150,225$, and sample size, Veterans $=12,313$. Sample sizes may be smaller for some conditions due to missing values. The prevalence rate of each health condition is the predicted prevalence in 2014 for the populations of Veterans and non-Veterans, both with age, sex, race/ethnicity, region, and urbanicity, adjusted to match the demographic composition of Veterans in 2012. Cancer includes any malignancy, and Mental Health includes any mental health condition.

RAND RR1165z1-S.4

According to the MEPS data in Figure S-5, about 25 percent of all patients who accessed care at VA had a mental health condition, and 3.3 percent had PTSD. ${ }^{3}$ Rates of PTSD are substantially higher among Veterans under age 35 (see Chapter Five). When combined with the otherwise rare conditions related to combat-amputation, traumatic brain injury (TBI), blindness, and severe burns - and the vulnerable circumstances of some patients, VA handles a patient mix that differs from what community providers typically see.

The prevalence of many common conditions is projected to increase among Veterans over the next 10 years. We estimate that aging in the Veteran population will lead to increases in the prevalence of several common health conditions among Veterans over the next 10 years. Figure $S-6$ shows projected unadjusted prevalence for hypertension, diabetes, IHD, and mental health conditions; in Chapter Five, we report similar charts for a more extensive set of conditions. Among all Veterans, we estimate that the prevalence rates for diabetes and hypertension will increase by about 12 and 8 percent, respectively, between 2015 and 2024 . However, while aging will tend to increase the prevalence of IHD, we estimate that prevalence rates for IHD will decline during 2015-2024. This finding is consistent with long-standing

3 As shown in more detail in Chapter Five, PTSD prevalence rates for VA patients are higher in the VA administrative encounter data than in the MEPS data. The higher prevalence in the encounter data could reflect that these data are more recent than MEPS, especially because we pool six years of data (2006-2012) in our MEPS analysis. In addition, MEPS data could be biased downward if people are reluctant to report mental health conditions in surveys, while VA encounter data could be biased upward if some encounters reflect "rule-out" diagnoses. 
Figure S-5

VA Patients Have a Higher Prevalence of Several Key Health Conditions (Unadjusted Prevalence)

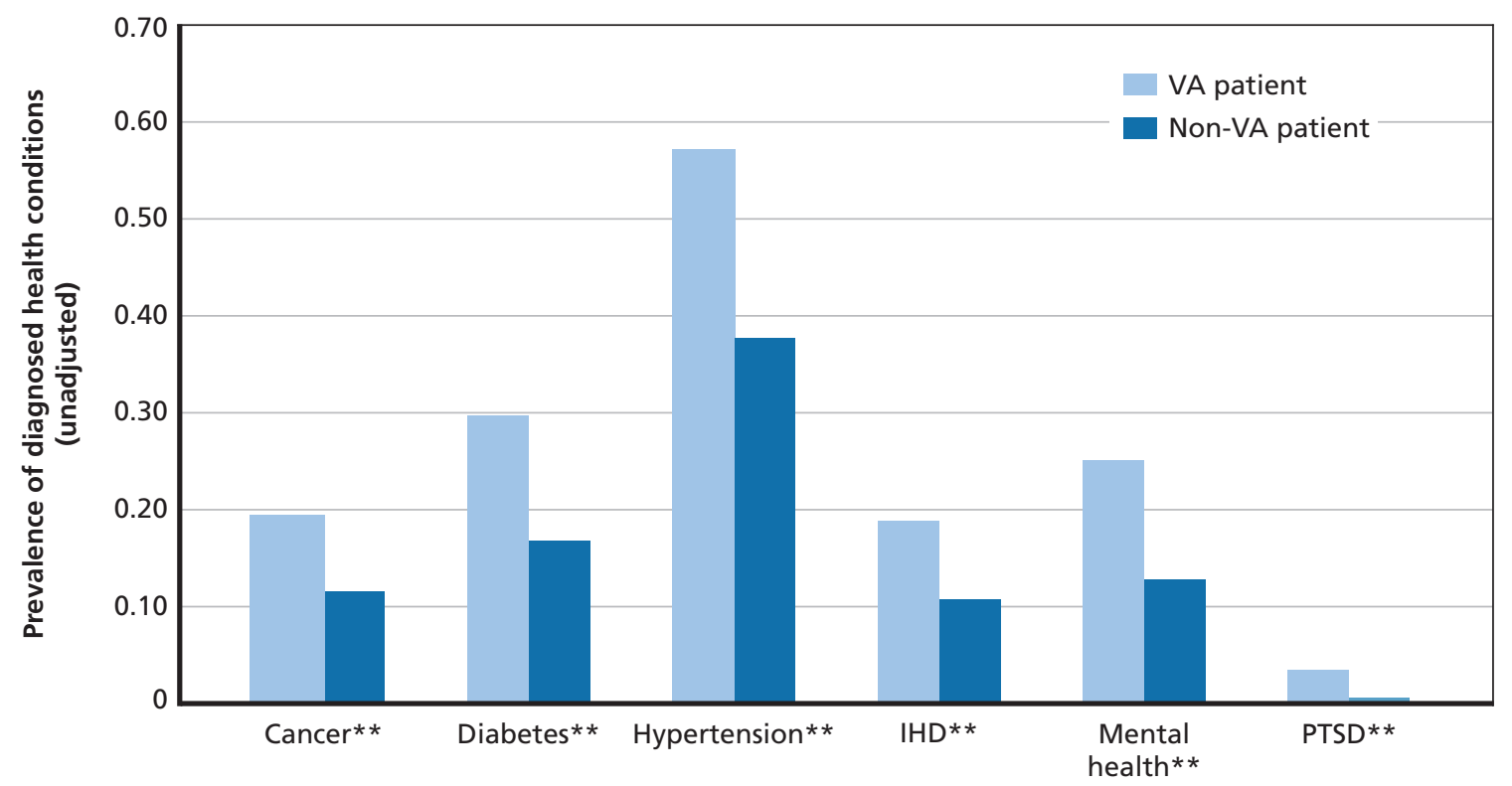

SOURCE: RAND analysis of MEPS, 2006-2012.

NOTES: ** indicates a statistically significant difference between VA patients and Veterans who are not VA patients at $p$-value $<0.05$. Sample size, VA patients $=4,871$, and sample size, non-VA patients $=7,442$. Sample sizes may be smaller for some conditions due to missing values. Cancer includes any malignancy, and Mental Health includes any mental health condition.

RAND RR1165z1-S.5

trends toward decreasing prevalence of acute coronary syndrome across all age groups in the U.S. population (Krumholz, Normand, \& Wang, 2014; Talbott et al., 2013). However, this decline largely represents an extrapolation of recent declines in the prevalence of IHD noted in MEPS. The relatively large confidence bands suggest that the trend is uncertain and actual prevalence may not decline as sharply. Mental health conditions increase moderately over time, with prevalence rates rising by about 6.8 percent.

Figure S-6 reports prevalence rates among all Veterans, which we estimated using a combination of data sources, including MEPS and MHS data, on service members who recently converted from active duty to Veteran status. In Chapter Five, we provide more detail on the analytic approach that we used to generate these estimates, and present additional results for VA patients (as opposed to all Veterans). In general, prevalence rates among VA patients increase somewhat more than prevalence rates for all Veterans. As a result, the gap in prevalence rates between VA patients and Veterans who do no use VA health care is projected to increase over time.

\section{Scenarios}

We examined five scenarios, based on hypothetical future changes to VA policy or to the environment surrounding VA health care.

Scenario 1: Broader VA eligibility. Higher-income Veterans without disabilities are currently ineligible to enroll for VA coverage or to receive care at VA. Expanding eligibility to currently excluded groups could lead to more than 4.8 million newly eligible Veterans and as 
Figure S-6

Projected Unadjusted Prevalence of Selected Health Conditions Among Veterans (2015-2024)
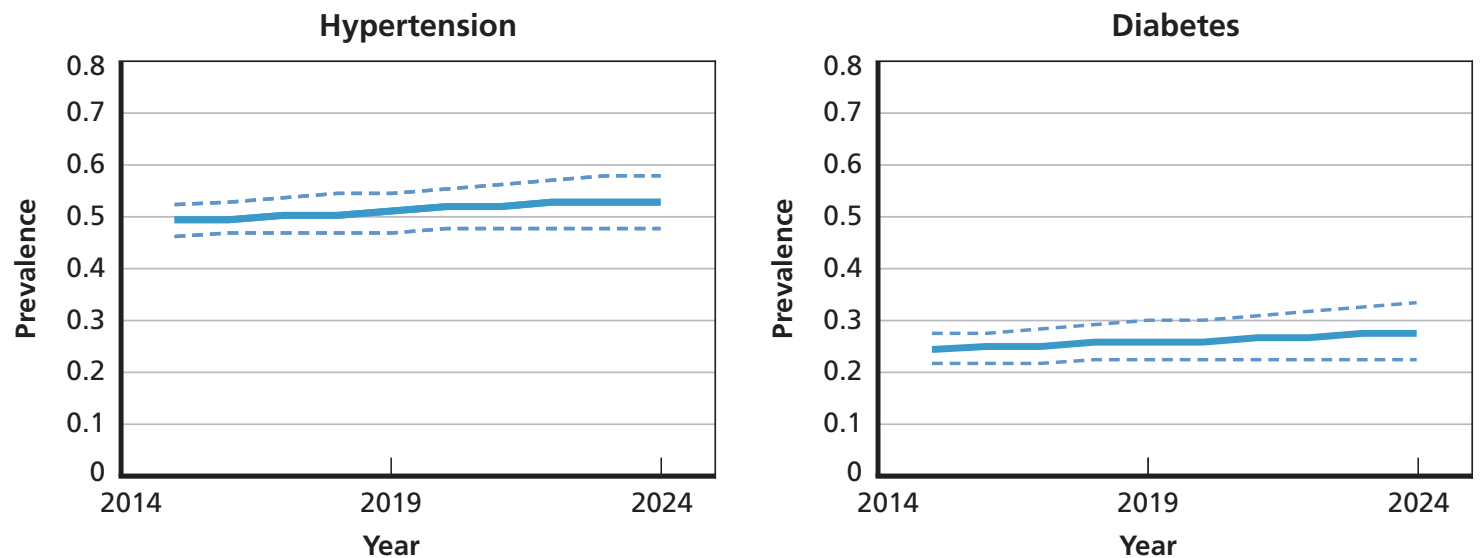

Ischemic heart disease
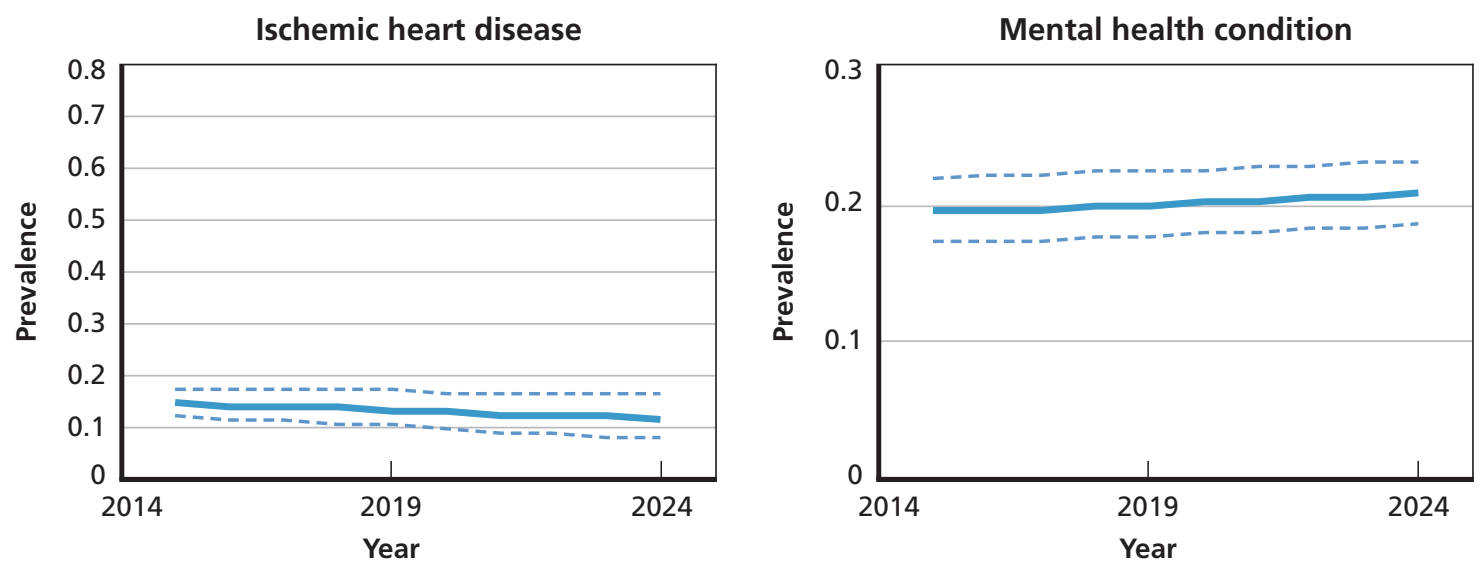

SOURCE: RAND analysis of VA, DoD, and Census data (2005-2014, Section 3), and MEPS (2006-2012).

NOTES: Solid lines indicate the projected prevalence for each health condition, which accounts for the changes in the composition of the VA patient populations by age, sex, race/ethnicity, Census region, and metropolitan statistical area (MSA). The dashed lines indicate upper and lower bounds for the projected prevalence rates. (See Appendix C.1.5 for methodological details.)

RAND RR1165z1-S. 6

many as 2.1 million new VA patients, amounting to a 35-percent increase in the size of VA's patient population.

Scenario 2: Including hypertension presumptively as a service-connected condition for Vietnam Veterans. According to the Institute of Medicine of the National Academies $(\mathrm{IOM})$, there is increasingly solid evidence that hypertension among Vietnam-era Veterans is related to service in the Vietnam Theater of Operations. As yet, VA has not added hypertension to the list of presumptive conditions for Vietnam-era Veterans. If hypertension were included, we estimate that this would translate into 363,000 new VA patients, an increase of 6.4 percent in VA's total patient population.

Scenario 3: Hypothetical future conflict. How would demand for VA health care services be affected by future military conflict? In examining 36 possible scenarios, we found that the vast majority of them project between 500,000 and 925,000 new VA patients by 2024. However, most low-conflict scenarios anticipate 500,000 and 600,000 new patients, while most high-conflict scenarios predict between 750,000 and 925,000. This suggests that 
even moderate levels of deployment could substantially increase the size of the incoming cohort of VA patients. In fact, our projections suggest that, for every new patient that would have entered the VA system in more-peaceful times, approximately 1.5 new patients will enter the VA system following a major conflict. However, previous cohorts, especially the Vietnam cohort, were much larger than recent cohorts, so the difference will be small relative to the entire VA patient population.

Scenario 4: Expanding access to VA care by extending the Veterans Choice Act or by other means. Surveys have shown that 1.8 million Veterans reported not using VA care due at least in part to access barriers. We estimate that if these barriers were removed, at most an additional 235,000 Veterans per year might use VA.

Scenario 5: Effects of the ACA. Policy changes associated with the ACA could have conflicting effects on Veterans' use of VA health care. The individual mandate, which requires most individuals to obtain health insurance coverage, could increase Veterans' propensity to enroll in the VA system. However, ACA's coverage expansions, which include expanded Medicaid eligibility and subsidies to buy individual market insurance, could cause some current enrollees to use fewer VA services. Our analysis found that the net impact of ACA coverage expansions on the number of VA patients is relatively modest: We estimate 98,000 fewer VA patients under base assumptions, although other plausible assumptions result in increases in VA patient counts.

\section{Conclusions and Recommendations}

\section{Overall Conclusions}

The number of Veterans has been declining for three decades, and our analysis of the Veteran population over the next 10 years suggests that this trend will continue. The total number of Veterans is expected to decrease by 19 percent between 2014 and 2024. The median age of the population will continue to increase, and Veterans are projected to become more geographically concentrated over this period.

Veterans are more likely than non-Veterans to be diagnosed with health conditions, including those that are chronic and in some cases linked to service in the military. Although some of these differences may be explained by the age and sex characteristics of Veterans compared with non-Veterans, differences remain after these characteristics are taken into account. The higher adjusted prevalence rates may be related to the challenges of serving in the military and in combat.

Among all Veterans, those who receive at least some of their health care from VA are generally more likely than Veterans who do not use VA health care to be diagnosed with many of the conditions we examined in this assessment. This result may be related to the fact that eligibility to receive VA services is based, in part, on Veterans' disability status and whether they are rated for service-connected conditions.

Assessing trends in Veterans' health care needs is complicated, because assessing the need or demand for health care requires an understanding of the extent to which Veterans rely on VA to meet those needs. During the past three decades, the number of Veterans has decreased while the number of VA patients has increased. This is due in part to an increase in the number 
of Veterans who have been rated for service-connected disabilities, as well as policy changes that made more Veterans eligible for VA health care benefits (due to presumptive eligibility), that made it easier for Veterans to apply for benefits, and that gave the benefit of the doubt to Veterans in cases where there was uncertainty. Looking to the future, our patient projection models suggest that the number of VA patients will continue to increase through 2019, but could level off or decline in subsequent years.

In addition to VA policies, external policies (such as the ACA) and other trends (such as the cost of civilian health care) may influence the way Veterans interact with VA's health care system. Our analysis of five potential future scenarios illustrates the extent to which policy may affect the projected number of Veterans and VA patients. Among them, expanding eligibility rules to include higher-income Veterans, entering future conflicts, and improving access to VA health care generated the largest increases in new Veterans and VA patients.

Finally, VA data systems and U.S. data collection efforts more broadly have significant limitations that hinder planners' ability to assess how demand for VA services might change over time. For example, there has not been a full accounting of the U.S. Veteran population since the 2000 Census. In addition, current VA data collection systems do not assess detailed information on Veterans' health care conditions and health care utilization patterns. Important data gaps include that data are often completely unavailable for Veterans who are not currently eligible or enrolled in VA health programs. Even among those who use VA care regularly, VA has detailed information only on care paid for or provided by VA, or paid for by the federal Medicare program. Additional data collection would be needed to fully understand Veterans' total health care needs, including use of care currently provided by the civilian sector. Understanding these gaps is important because shifts in patient reliance and changes in eligibility rules could cause more Veterans to seek care at VA and could change the mix of care sought from VA versus civilian providers.

\section{Recommendations for Consideration \\ Prepare for a Changing Veteran Landscape}

The number of VA patients has been increasing since 2005, despite the three-decades-long decline in the size of the Veteran population. We estimate that this increase will continue through 2019. However, in 2020 and beyond, it is likely that the size of the VA patient population will level off or even decrease. Total demand for VA services during this time period will be heavily influenced by utilization patterns among patients; if the health care needs of the population are significant or the cost of outside options is high, patients may use more care than they have in previous years. Nonetheless, there is a possibility that demand for services will level off or decline as the continued growth in the patient population slows or even reverses. The likely short-term growth in demand, followed by a leveling-off or decline in the next decade, may make it difficult to ensure that the size of the VA health system is tailored to fully meet the needs of the population in the near term without becoming inefficiently large in the long run. Increasing the use of care purchased from the civilian sector may enable VA to meet short-run increases in demand without requiring costly investment in facilities, infrastructure, and personnel that could become less needed in the future. 


\section{Improve Tracking of Some Veteran Populations}

Because the 2010 Census did not capture information on Veteran status, there has not been a full-scale accounting of the U.S. Veteran population since 2000. As a result, VA must estimate the size of the Veteran population using data from more than 15 years ago, coupled with smaller surveys and information on personnel losses from DoD. While ACS provides information on a sample of Veterans (1,197,923 Veterans in the 2009-2013 sample), this is not a full accounting of the Veteran population. An updated census of the Veteran population would enable a definitive count of all Veterans, while also helping to refine sampling procedures for the yearly surveys of samples of the population. Given that the events of September 11, 2001, set off prolonged U.S. engagement in oversees conflict and changed DoD accession and personnel retention policies that affect the flow of service members from active duty to Veteran status, it seems that the nation is overdue for an updated census of the Veteran population. We recommend asking about Veteran status in the 2020 Census.

In addition, little is currently known about how the utilization patterns and health care needs of Veterans from the conflicts in Afghanistan and Iraq will evolve over time. Yet, Afghanistan and Iraq Veterans are more likely to have service-connected disabilities than other Veterans and are automatically eligible for VA health care for five years after leaving the military. Historically, Veterans have relied less on VA health care as they age, gain access to other health insurance (e.g., through an employer), and start families. However, it is not clear the extent to which these patterns will hold for newer Veterans, who have different exposures and enhanced eligibility relative to previous cohorts. Closely monitoring this population may help VA planners to prepare as these Veterans age and their health care needs and utilization patterns shift.

\section{Anticipate Potential Shifts in the Geographic Distribution of Veterans, and Align VA Facilities and Services to Meet These Needs}

While our estimates suggest that the geographic distribution of Veterans will remain relatively stable over time, there may be several opportunities to streamline or shift VA resources to ensure adequate capacity in all parts of the country. Given projected declines in the size of the Veteran population living in the Ohio River Valley and upper Midwest, it may be possible to consolidate relatively proximal VA facilities in those regions. At the same time, some areas of projected Veteran population growth-including Montana, Wyoming, and Colorado-are not currently well covered by VA facilities. While the absolute size of the Veteran population in these areas will remain small, there may be opportunities to use telehealth and communitybased outpatient clinic (CBOC) services to meet Veterans' needs in these areas. There may be a more pressing need to expand VA coverage in the Southwest, where Veterans Affairs Medical Centers (VAMCs) are currently widely spaced, and where growth in the Veteran population is expected to be significant. Finally, we estimate that the Veteran population under age 35 will increase in the regions around Los Angeles; Dallas; Washington, D.C.; and northern New Jersey by 2024. VA facilities in these areas might monitor growth in utilization among younger Veterans to ensure that they are able to meet the needs of this group.

\section{Improve Collection of Data on Veteran Health Care Utilization and Reliance}

Fully understanding the needs of the patient population served by VA will require data that do not currently exist. These data would capture information on all sources of health care that are used by an individual-including when and where care is delivered, what diagnoses are 
recorded and procedures performed, and who pays for the services—as well as what needs for care are unmet, and why. The data would also require a large enough sample of Veterans to ensure that it is representative of the population, and to allow VA to track the prevalence of relatively rare service-connected conditions. Creating these data would enable an analysis of the extent to which Veterans currently rely on VA for health care, as well as how that reliance may change as a result of internal VA policies or external factors. It would also provide insight into where VA succeeds in meeting the health care needs of its patient population and what types of obstacles exist in delivering needed care. In addition, by collecting information on Veterans who are not currently patients, the data would enable VA to better plan for changes in the demand for services that might occur if VA eligibility rules changed, or if additional Veterans chose to enroll.

Current surveys of Veterans do not capture comprehensive information on health care use, particularly among Veterans who are not currently eligible for or enrolled in the VA system. While MEPS contains information on all the care that respondents receive regardless of payer, the survey contains only a small sample of Veterans, and this sample may not be adequately representative of the population. VA might consider fielding a comprehensive survey of all Veterans, aimed at assessing their total health care use patterns, including use of non-VA care. Such a survey could be modeled on the MEPS Household Component (MEPS-HC), which collects utilization data across all sources of care for the general population. Potentially, VA could work with AHRQ (the organization that fields MEPS-HC) to include a more robust sample of Veterans in its survey.

\section{Incorporate Separation Patterns and Health Care Needs of Current Service Members into Projections}

In this assessment, we incorporated data on current service members-who will become Veterans in the future - in several of our analyses, including (1) counts of service member separations in our demographic analysis to augment Census data of Veterans from 2000, (2) diagnosed health conditions of separating service members who received care through the Military Health System (MHS), and (3) estimated number of service members who would separate and become Veterans in the case of a hypothetical future conflict.

At present, VA does not have access to DoD MHS encounter data. Such data could enable VA planners to analyze health care needs among current active duty service personnel who may become Veterans in the future. For this study, we utilized MHS data from 2008 to 2014 to explore whether current service members (future Veterans) have different health care needs from current Veterans. We estimate that service members are much more likely than current Veterans to have a diagnosed musculoskeletal condition or asthma at the time of separation from service. On the other hand, the prevalence of mental health conditions is higher in the existing Veteran population than among separating service members. This result may reflect a disincentive to seek care for mental health conditions while serving in the military. To the extent that individuals who separate from the military and become Veterans during the 2015-2024 projection window have different health care needs from the patients currently being served by VA, adding MHS data is critical for projecting the needs that VA must meet in the future. 


\section{Develop an Analytic Framework to Perform Scenario Testing}

Our analysis of five future scenarios highlights the importance of developing methods and models that can respond quickly and agilely to policy changes. While some of the policy changes we considered resulted in modest changes in number of new Veterans and new VA patients, others estimated as many as hundreds of thousands of new Veterans and patients. The VA Office of the Actuary (OACT) has a Veteran Healthcare Scenario Model that is able to estimate, for instance, how changes in demographic characteristics or economic conditions (such as employment or income) may affect demand for VA services and related costs. Expanding this model to include such events as changes in the civilian health sector, unanticipated changes in perceptions about health care quality, and groundbreaking new technologies, to name a few, will enable VA to address the types of uncertainties that current models may not address. Having methods in place to estimate the effects of these types of changes on Veteran demand for health care services will improve VA's efforts to meet the health care needs of its patient population. 


\section{Acknowledgments}

We gratefully acknowledge the support of Alfonso Rivera Illingworth, who provided superb project coordination; Jill Gurvey, Andrew Madler, Craig Martin, Teague Ruder, and Mark Totten, who provided research programming support; and Christian Lopez, Marc PunKay, and Rachel Ross for research assistance. We are grateful for the support and guidance from the project's management team, including Robin Weinick and Terri Tanielian, and the strong organizational support from Clare Stevens. We appreciate the comments provided by our reviewers, Beth Asch, Daniel Ginsberg, Carole Gresenz, Katherine Kahn, Kenneth Kizer, Paul Koegel, and Allison Percy. We addressed their constructive critiques, as part of RAND's rigorous quality assurance process, to improve the quality of this report. We are grateful to Meg Harrell for coordinating the quality assurance process for this project. We thank Stacy Fitzsimmons and Anna Hansberry for excellent administrative assistance, and Allison Kerns for careful edits in the preparation of this report. We also acknowledge the support of our MITRE colleague, Sandy Sinay, along with her team. We are grateful to the subject-matter experts at VA who helped us develop an understanding of their existing and ongoing projection models. 



\section{Abbreviations}

ACA Patient Protection and Affordable Care Act

ACS American Community Survey

ADL activity of daily living

AHRQ Agency for Healthcare Research and Quality

AIDS acquired immune deficiency syndrome

AMI acute myocardial infarction

BRFSS Behavioral Risk Factor Surveillance System

$\mathrm{CAMH}$ CMS Alliance to Modernize Healthcare

CBOC community-based outpatient clinic

CCS Clinical Classifications Software

CDC Centers for Disease Control and Prevention

CHAMPVA Civilian Health and Medical Program of the Department of Veterans Affairs

$\mathrm{CHF} \quad$ congestive heart failure

CMS Centers for Medicare \& Medicaid Services

COPD chronic obstructive pulmonary disease

CTS Contingency Tracking System

DMDC Defense Manpower Data Center

DoD U.S. Department of Defense

EHCPM Enrollee Health Care Projection Model

FFRDC federally funded research and development center

FPL federal poverty level

FY fiscal year

GERD gastroesophageal reflux disease

GMT geographic means test 
HAIG Healthcare Analysis and Information Group

HBP hypertension (high blood pressure)

HIV human immunodeficiency virus

IADL instrumental activity of daily living

ICD International Classification of Diseases

IHD ischemic heart disease

IOM Institute of Medicine of the National Academies

IRS Internal Revenue Service

IS insufficient sample size

MEPS Medical Expenditure Panel Survey

MEPS-HC Medical Expenditure Panel Survey Household Component

MHS Military Health System

MSA metropolitan statistical area

NHIS National Health Interview Survey

NSV National Survey of Veterans

OACT Office of the Actuary

PG priority group

POW prisoner of war

PPV positive predictive value

PTSD posttraumatic stress disorder

PUMA Public Use Microdata Area

PUMS Public Use Microdata Sample

SoE Survey of Veteran Enrollees' Health and Reliance Upon VA

SSDI Social Security Disability Insurance Trust Fund

TBI traumatic brain injury

VA

U.S. Department of Veterans Affairs

VAMC

VA Medical Center

VCAA

Veterans Claims Assistance Act

VetPop2014 Veteran Population Projection Model 2014

VHA Veterans Health Administration

VHSM Veteran Healthcare Scenario Model

WEX Work Experience files 


\subsection{Background}

The Veterans Access, Choice, and Accountability Act of 2014 ("Veterans Choice Act") was signed into law on August 7, 2014. In addition to expanding the criteria through which Veterans can access civilian providers for their health care, the legislation called for an independent assessment of the VA health care system across a broad array of topics related to the delivery of health care services to Veterans in VA-owned and -operated facilities, as well as those under contract to VA.

In September 2014, CAMH - the MITRE Corporation's federally funded research and development center-entered into a contract with VA to serve as the overall integrator for the independent assessment. MITRE subcontracted with the RAND Corporation to conduct three of the 11 specific assessment tasks (Assessments A, B, and C) and to furnish reports on the findings and recommendations from these assessments for inclusion in an integrated report to VA and Congress.

This report presents findings from Assessment A (identified under Title II-Health Care Administrative Matters, Section 201 of the Veterans Choice Act), which examined the current and projected demographics and unique health care needs of Veterans and the patient population served by VA. In this report, we use the term Veteran to describe all Veterans, whether or not they use VA health services, and VA patient to describe an individual who received at least some health care from VA in the previous year.

The Veterans Choice Act language required that Assessment A address "current and projected demographics and unique health care needs of the patient population served by the Department." We interpreted this language to require an independent analysis of the current characteristics and unique health care needs of the Veteran population and VA patients, and estimates of how the characteristics and needs of these populations will evolve over time. To generate these estimates, we compiled data from VA administrative files, DoD administrative files, surveys conducted by the U.S. Census Bureau and other federal agencies, and surveys conducted by VA to develop a comprehensive picture of U.S. Veterans, VA patients, and the demographic characteristics and health care needs of these populations. We then used established statistical methods to project these characteristics and needs forward over time. Finally, we considered the extent to which uncertain future events, including changes to VA policy, possible future military conflicts, and other external factors, might affect the size and characteristics of the Veteran population overall and of VA patients specifically. 


\subsection{Motivation}

U.S. Veterans represent a special population of individuals who have served their country and may have faced extraordinary health risks during deployment to combat areas. Part of the promise that the United States makes to its armed forces is to provide for their health care needs during their service and afterward, when it is the mission of VA to meet Veterans' needs. Because many Veterans have served on overseas missions, including combat, Veterans are a clinically complex and vulnerable population. Meeting the needs of this population requires a clear understanding of Veterans' distinctive characteristics in comparison with non-Veterans, in terms of both their demographic and health characteristics, and how these are likely to evolve.

The Veterans Choice Act and its congressionally mandated assessments were further motivated by challenges faced by VA patients in recent years, including long wait times to receive services, poor patient outcomes, and a million-person backlog among Veterans waiting for disability claims to be evaluated. While this report does not directly address these specific challenges, understanding the size, demographic composition, and health care needs of the population eligible for VA services is critically important to ensure that VA has the capacity to meet Veterans' needs in the future.

\subsection{Purpose}

This report responds to the Veterans Choice Act requirement for an independent assessment of the demographics and health care needs of the Veteran population. Specifically, it examines the demographic characteristics of the current and projected population of U.S. Veterans and VA patients. In addition, it delineates the unique health care needs of the patient population currently served by VA, as well as the projected needs of Veterans who might become patients in the future. The findings presented here will inform future VA efforts to plan, budget, and staff, and, along with the findings from Assessment B (health care capabilities and resources of VA; RAND Health, 2015), will highlight areas where assets do not meet current or projected needs. This report addresses four overarching research questions:

- What were the demographic characteristics of the U.S. Veteran population in 2014, and how are these projected to change between 2015 and 2024?

- What are the current health care needs of the Veteran population, including both VA patients and non-VA patients, and how do these compare with the needs of the nonVeteran population? How will the needs of Veterans in general and the patient population specifically evolve over time given current policies?

- To what extent do Veterans rely on VA for their health care?

- How might external forces or changes to VA policy affect the characteristics of Veterans, their eligibility to use VA health care, and their health care needs over time?

Defining the "unique" health care needs of the VA population is challenging, because many common health conditions affecting Veterans also affect non-Veteran populations. Further, even though there are many combat-related conditions that are important to VA— such as amputations, burns, and TBIs - these conditions are not, strictly speaking, "unique" to Veter- 
ans; they can occur among civilians as well. We therefore define the unique health care needs of Veterans as those that disproportionately affect Veterans relative to non-Veterans. These include both service-connected conditions, such as PTSD, and other conditions that are more prevalent among Veterans than non-Veterans, including diabetes and cancer.

An additional challenge that we faced in this analysis was that not all Veterans are currently eligible to receive care at VA, not all eligible Veterans opt to enroll for services, and not all enrollees receive all or even the majority of their care from VA. From a conceptual standpoint, estimates of the future VA patient population must account for the possibility that Veterans' enrollment and use patterns may change over time due to external factors, such as the cost of private health insurance. From a data standpoint, estimates must address the fact that the VA health system's data include only information on VA patients, and will not necessarily include comprehensive data on patients' total health care utilization and diagnoses. In Chapter Two, we provide a conceptual discussion of these issues. The analyses reported in Chapters Four and Five identify differences between VA patients and non-patients and to determine the health care needs of these populations without relying solely on information reported in VA patient data.

\subsection{Scope of the Analysis}

We recognize that VA has developed several models, including the Veterans Population Projection Model 2014 (VetPop2014) and the EHCPM, that forecast the size and characteristics of the VA population and the health care utilization of VA patients. In keeping with Congress's desire for an independent assessment, we generated our own estimates of the Veteran and patient populations, without relying on existing VA models. However, our work is not meant to replace these models, which are extremely sophisticated tools that VA has spent years refining. Rather, our goal was to take a fresh look at population and patient projections, in some cases using new methodologies and data. The analyses presented here may be useful to VA planners when considering future updates or refinements to existing VA models.

As part of our analysis, we reviewed the methods used to develop VetPop2014 and EHCPM, and we discuss these methods in the report. We did not, however, conduct a systematic evaluation of the strengths and weaknesses of existing VA models. Our focus was on understanding the Veteran population rather than evaluating VA's current modeling approaches.

Our baseline estimates consider the Veteran population in 2014. To estimate trends over time, we chose a 10-year time frame, from 2015 through 2024. In selecting this period, we aimed to balance the need for a long-term understanding of how the Veteran and VA patient populations might evolve against the reality that predictive models become less reliable when forecasting far into the future. We settled on a 10-year projection window, which aligns with the time frame used by the Congressional Budget Office when scoring legislation.

Finally, it is worth noting that this analysis was conducted under constraints due to the short time frame allotted for the assessments and the limited availability of data. The entire assessment was conducted in an eight-month time frame. Many of the VA and DoD data sources that we used in this analysis required special permissions that took months to obtain and further limited the time in which we could conduct our analysis. 


\subsection{Organization of the Report}

The rest of this report is organized as follows: Chapter Two presents an overview of the Veteran population and the VA enrollment process. This chapter also provides an overview of our modeling approach and a brief description of methods. In Chapter Three, which responds to the congressional requirement to analyze Veterans' "current and projected" demographics, we discuss our model of the Veteran population, its demographic characteristics, and how the size and characteristics of this population might evolve over time. One key attribute we consider in analyzing demographics is where Veterans are geographically located, both now and in the future. Chapter Four discusses Veterans' decisions to enroll in VA health care and reliance on VA when enrolled. Chapter Five responds to the requirement to assess the "unique health care needs of the patient population served by the Department" and focuses on understanding the current needs of both Veterans and VA patients, and how these needs will evolve over time under current policy. In Chapter Six, we consider hypothetical future scenarios that might affect the size and composition of the Veteran population, Veterans' propensity to use VA health services, and the health care needs of future Veterans. This chapter builds on the analyses presented in Chapters Three, Four, and Five by recognizing the inherent uncertainty in attempting to predict future outcomes and providing alternative estimates of future trends. Chapter Seven presents the study's conclusions and recommendations. 


\subsection{Introduction}

While we present a more detailed summary of specific methods in subsequent chapters and appendixes, this chapter focuses on our conceptual approach. Below, we briefly describe the distinction between a Veteran and a VA patient, and outline steps that a Veteran must take to enroll and begin using VA health care. In addition, we provide an overview of the approach we used to model how the population of Veterans, and correspondingly VA patients, might evolve over time. Finally, we discuss a framework for analyzing how patient needs may change in the future.

\subsubsection{Definition of a Veteran and Eligibility to Use VA Health Care Services}

A Veteran is defined by law as "a person who served in the active military, naval, or air service, and who was discharged or released therefrom under conditions other than dishonorable" (Definitions, 2011). We estimate that there were 21.6 million Veterans in the U.S. population in 2014. However, there are additional eligibility requirements for enrolling in the VA health care system. Current eligibility rules for VA health care were established in the Veterans Health Care Eligibility Reform Act of 1996. The legislation revised Section 1710 in Title 38 to mandate health care for:

- Veterans with service-connected health conditions

- Veterans with a compensable service-connected disability or who received a disability discharge from the military

- Veterans unable to pay for their health care

- Certain other specific groups.

This mandate, however, is subject to the appropriated funding. For the first time, the 1996 legislation also gave VA discretionary authority to provide health care to all other Veterans "to the extent and in the amount provided in advance in appropriations Acts for such purposes." The Secretary of Veterans Affairs has legal discretion over the provision of all other care, but VA must maintain specialized treatment and rehabilitation programs for spinal injuries, blindness, amputations, mental illness, and other serious service-connected health conditions. Under current policy, all enrolled Veterans have access to VA's comprehensive health care benefits package, but a Veteran may also receive certain benefits (e.g., dental care) based on his or her unique eligibility status (U.S. Department of Veterans Affairs, 2014c).

Consistent with the legal definition of a Veteran, eligibility for health care is limited to individuals who are former active duty service members, or current or former reservists or 
national guardsmen who (a) were called to active duty by a federal order and (b) completed the full period of that active service. Veterans also must have served either 24 continuous months or the full period for which they were called to active duty. Exceptions to this minimum service duty requirement apply to Veterans who were discharged for a disability incurred or aggravated in the line of duty, discharged for a hardship or "early out," or served prior to September 7, 1980 (U.S. Department of Veterans Affairs, 2015c). The VA website notes that there are numerous additional exceptions to the minimum duty requirements, which can be evaluated during the enrollment process. In Chapter Six, we estimate that 17.8 million (81 percent) of all individuals with any military service meet these basic enrollment criteria. However, as we discuss below, not all of these Veterans are currently eligible to enroll and use VA health care.

Under certain extenuating circumstances (for example, if a Veteran is rated permanently and totally disabled due to a service-connected disability, or if a Veteran dies while on active duty), a Veteran's family members may also be eligible for VA health benefits. However, VA health care for Veterans is prioritized over that for Veteran dependents.

\subsubsection{Enrollment Priority Groups}

VA uses Veterans' service-connected disability ratings, along with income and other factors, to assign Veterans to one of eight priority groups through its enrollment system (Congressional Budget Office, 2007, 2009). Priority groups are used to determine a Veteran's enrollment priority, a Veteran's access to certain additional health care benefits, and whether co-pays are required. The three highest priority groups (priority groups 1-3) are for Veterans with a service-connected disability rated at 10 percent or higher. Table 2-1 lists all of the current VA priority groups, including a brief description of each. Eligibility determinations and assignments to priority groups are made by the Health Eligibility Center in Atlanta, Georgia (U.S. Department of Veterans Affairs, 2015g).

In 2003, VA sought to improve access to services for higher-priority Veterans by restricting enrollment of higher-income Veterans without service-connected disabilities (Goldberg, 2015). In part, this policy was created because VA is funded through annual appropriations, and restricting enrollment helped to keep spending within VA's budget. The policy also was created to "recognize the higher obligation owed to Veterans requiring care for their serviceconnected disabilities, and to lower-income Veterans" (Enrollment-Provision of Hospital and Outpatient Care to Veterans Subpriorities of Priority Categories 7 and 8 and Annual Enrollment Level Decision, 2003). Based on this policy, VA continued to treat Veterans in all priority groups and treat new enrollees in priority groups $1-7$, but suspended the enrollment of additional Veterans in priority group 8.

The most recent change in enrollment eligibility, in May 2009, relaxed the enrollment restrictions on Veterans in priority group 8 (U.S. Department of Veterans Affairs, 2009a). Under the new policy, additional subpriorities were added for priority group 8 for Veterans whose income exceeded the current VA national means test or geographical means test income thresholds ${ }^{1}$ by 10 percent or less (Expansion of Enrollment in the VA Health Care System, 2009). The new regulations went into effect in June 2009, and VA then began enrolling (for VA health care benefits) those Veterans who were eligible in subgroups $8 \mathrm{a}$ through $8 \mathrm{~d}$, while continuing to exclude Veterans in subgroups 8e and 8g (Congressional Budget Office, 2009).

1 These thresholds are established by the Department of Housing and Urban Development. 
Table 2-1

VA Priority Groups for Enrollment

\begin{tabular}{|c|c|}
\hline $\begin{array}{l}\text { Priority } \\
\text { Group }\end{array}$ & Description \\
\hline 1 & $\begin{array}{l}\text { - Service-connected disability } 50 \% \text { or more disabling } \\
\text { - Veterans determined unemployable due to service-connected disabilities }\end{array}$ \\
\hline 2 & - Service-connected disability $30 \%$ or $40 \%$ disabling \\
\hline 3 & $\begin{array}{l}\text { - Service-connected disability } 10 \% \text { or } 20 \% \text { disabling } \\
\text { - Other: former prisoners of war (POWs); Purple Heart medal awardees; Medal of Honor awardees; } \\
\text { Veterans discharged for disability incurred during/aggregated by military service; other }\end{array}$ \\
\hline 4 & $\begin{array}{l}\text { - Catastrophically disabled (ability to complete activities of daily living is permanently compro- } \\
\text { mised; Veterans require personal/mechanical assistance to leave home/bed or require constant } \\
\text { assistance) } \\
\text { - Recipients of VA aid/attendance or housebound benefits }\end{array}$ \\
\hline 5 & $\begin{array}{l}\text { - Low income: annual income below zip-code-based adjusted income limits; Veterans receiving VA } \\
\text { pensions; Veterans eligible for Medicaid }\end{array}$ \\
\hline 6 & $\begin{array}{l}\text { - Service-connected disability } 0 \% \text {, compensable } \\
\text { - Veterans with occupational exposures } \\
\text { - } \text { lonizing radiation from atmospheric testing, Hiroshima, or Nagasaki } \\
\text { - } \text { Project } 112 / \text { SHAD participants } \\
\text { - Service in Vietnam (January } 9,1962-M a y ~ 7,1975 \text { ) } \\
\text { - Service in Persian Gulf War (August } 2,1990-\text { November } 11,1998) \\
\text { - Service in theater of combat operations after November 11, 1998, and discharged after January } \\
28,2003\end{array}$ \\
\hline 7 & $\begin{array}{l}\text { - Low income: gross household income below geographically adjusted income limits; agree to } \\
\text { co-pays }\end{array}$ \\
\hline 8 & $\begin{array}{l}\text { - Veterans with gross household income above VA and the geographically adjusted income limits } \\
\text { for their resident location and who agree to pay co-pays }\end{array}$ \\
\hline \multicolumn{2}{|c|}{ Priority Group 8 Veterans Who Are Currently Eligible to Enroll for VA Health Care } \\
\hline $8 a$ & $\begin{array}{l}\text { Veterans with noncompensable } 0 \% \text { service-connected disabilities who enrolled as of January } 16 \text {, } \\
2003 \text {, and who have remained enrolled since that date and/or placed in this subpriority due to } \\
\text { changed eligibility status }\end{array}$ \\
\hline $8 b$ & $\begin{array}{l}\text { - Veterans with noncompensable } 0 \% \text { service-connected disabilities who enrolled on or after June } \\
15,2009, \text { whose income exceeds the current VA or geographic income limits by } 10 \% \text { or less }\end{array}$ \\
\hline $8 c$ & $\begin{array}{l}\text { - Veterans without a service-connected disability who enrolled as of January } 16,2003 \text {, and who } \\
\text { have remained enrolled since that date and/or placed in this subpriority due to changed eligibility } \\
\text { status }\end{array}$ \\
\hline $8 d$ & $\begin{array}{l}\text { - Veterans without a service-connected disability who enrolled on or after June } 15,2009 \text {, whose } \\
\text { income exceeds the current VA or geographic income limits by } 10 \% \text { or less }\end{array}$ \\
\hline \multicolumn{2}{|c|}{ Priority Group 8 Veterans Who Are Not Currently Eligible to Enroll for VA Health Care } \\
\hline $8 \mathrm{e}$ & $\begin{array}{l}\text { - Veterans with noncompensable } 0 \% \text { service-connected disabilities who do not meet the criteria } \\
\text { for priority groups } 8 a-8 d\end{array}$ \\
\hline $8 f$ & $\begin{array}{l}\text { - Veterans without a service-connected disability who do not meeting the criteria for priority } \\
\text { groups } 8 a-8 d\end{array}$ \\
\hline
\end{tabular}

SOURCE: U.S. Department of Veterans Affairs, 2015c.

NOTE: Veterans assigned to priority group 8 who do not meet the criteria listed in the table are currently ineligible to receive VA care. 
Of the 17.8 million who we estimate meet the basic eligibility criteria, 13 million ( 73 percent) are in priority groups 1 through $8 \mathrm{~d}$ or in groups $8 \mathrm{e}$ and $8 \mathrm{~g}$ but enrolled prior to the most recent eligibility change in 2009.

For many Veterans, enrollment eligibility hinges on income. Veterans whose incomes fall below the national income test, who are Medicaid eligible, or who receive a disability pension are classified into priority group 5, unless they qualify for a higher priority group based on service-connected conditions or other disabilities. In some cases, there is a geographic means test (GMT) that exceeds the national income threshold. Veterans whose income is above the national income threshold but below the geographic means threshold are classified in priority group 7. Those with higher incomes may qualify for priority groups $8 \mathrm{a}$ or $8 \mathrm{~d}$, if their incomes are within 10 percent of the relevant threshold (either the national income threshold or the GMT, whichever is binding). Both the national income threshold and the geographic means threshold vary depending on whether a Veteran has dependents.

Veterans who served in a combat theater after 1998 have automatic eligibility to enroll for up to five years after they leave the military. ${ }^{2}$ These Veterans are placed in priority group 6 unless they qualify for a higher priority group (e.g., have a disability rating of 10 percent or higher or income below the threshold for priority group 5). Veterans with qualifying occupational exposures are also placed in priority group 6, unless they qualify for a higher priority group based on income or disabilities. A Veteran may apply for VA health care benefits at any time after separation (U.S. Department of Veterans Affairs, 2009b).

\subsubsection{Use of VA Services Conditional on Enrollment}

Once Veterans enroll and are deemed eligible to receive care, Veterans must make a choice about whether to use VA health care and how much care to consume. The majority of enrolled Veterans have access to other health care coverage, and approximately half of enrolled Veterans are also enrolled in Medicare or Medicaid (Congressional Budget Office, 2009; Goldberg, 2015). Enrolled Veterans receive the majority of their health care outside of VA (Goldberg, 2015). The term reliance refers to the fraction of a Veteran's total care that is provided by or paid for by VA. Based on the EHCPM, VA estimates that current VA patients have on average about 21 percent of their total physical medicine (that is, physical therapy and occupational therapy) visits with VA, 38 percent of their emergency room visits with VA, and 66 percent of their prescriptions from VA.

However, reliance rates can vary substantially based on Veterans' health care needs and underlying characteristics. Veterans enrolled in priority group 1 (those with a service-connected disability rated 50-percent or more disabling) and in priority group 4 (those with a nonserviceconnected catastrophic disability) face the highest overall health care costs and rely on VA for a greater percentage of their medical care than other Veterans (Congressional Budget Office, 2009). In general, VA enrollees with private insurance are less likely to use VA care than other Veterans (Shen, Hendricks, Wang, Gardner, \& Kazis, 2008). Veterans who utilize VA for all of their health care are more likely to be from disadvantaged (poor, less-educated, minority) groups (Nelson, Starkebaum, \& Reiber, 2007). Among Veterans with Medicare, those Veterans who are disability-eligible for Medicare have more VA primary and specialty care visits

2 On February 12, 2015, the Clay Hunt Suicide Prevention for American Veterans Act provided a one-year window of enhanced enrollment for combat Veterans who were discharged or released from active service after January 1, 2009, and before January 1, 2011, who did not enroll within the original five-year window. 
than Veterans age-eligible for Medicare (Liu et al., 2012). The increased utilization of both primary and specialty care among disability-eligible Veterans with Medicare is likely due to greater health care need (Liu et al., 2012). Other factors, such as access constraints and perceived quality, may also affect Veterans' decisions to use care.

In fiscal year (FY) 2014, 6.2 million unique patients used VA health care services or received VA reimbursed treatment (Table 2-2). The majority of VA users were Veterans (5.9 million), and 4.6 million of these Veterans were in priority groups 1 through 6 . The table focuses on Veterans who sought any care, regardless of how much care they obtained at VA.

Between 2000 and 2011, the number of Veterans seeking inpatient treatment increased by 131 percent, and the number of Veterans seeking outpatient care increased by 201 percent (National Center for Veterans Analysis and Statistics, 2011b). The number of Veterans seeking VA care continued to increase through 2012 (National Center for Veterans Analysis and Statistics, 2014e). These increases occurred despite a substantial decline in the size of the Veteran population. For example, the U.S. Census Bureau estimates that there were more than 26 million U.S. Veterans in 2000 (Richardson \& Waldrop, 2003), compared with just under 22 million today according to both our analysis and VA's (National Center for Veterans Analysis and Statistics, 2014b).

This increase in use of VA health care is likely due in part to the close to 300 percent increase in the number of pre-9/11, Gulf War-era Veterans receiving health care from FY 2000 to FY 2009 and extended enrollment for Afghanistan and Iraq Veterans (National Center for Veterans Analysis and Statistics, 2011b). The increases are also due in part to policy changes that have expanded VA's outreach to Veterans, eased the diagnostic criteria used to determine service-connected PTSD, and implemented the use of online application forms (Congressional Budget Office, 2014b). In addition, over time, VA has expanded the list of conditions that are considered to be presumptively related to service and therefore automatically render the Veteran eligible for benefits. For example, in 2001, VA granted presumptive eligibility status to Vietnam Veterans with type 2 diabetes, due to links to Agent Orange exposure (Congressional Budget Office, 2014b).

Table 2-2

VA Health Care Users (FY 2014)

\begin{tabular}{lc}
\hline Type of VA Health Care User & FY 2014 Unique Patients \\
\hline Priority groups 1-6 & $4,612,915$ \\
Priority groups 7-8 & $1,256,610$ \\
Priority group not assigned & 2,241 \\
Veterans & $\mathbf{5 , 8 7 1 , 7 6 6}$ \\
Non-Veterans & 308,805 \\
Total unique patients & $6,180,571$ \\
\hline
\end{tabular}

SOURCE: Authors' analysis of FY 2014 data from the Veterans Health Administration (VHA) Support Service Center.

NOTES: Unique patients are unique individuals who were treated by VA or received treatment paid for by VA; Non-Veterans include active duty military and reserve, family members of Veterans who qualify to enroll, VA employees receiving occupational health care, and so on. 


\subsection{Factors Associated with Veterans' Health Care Utilization}

A Veteran's decision to use VA health care depends on a number of factors, which are summarized in Figure 2-1, a conceptual model of health care utilization among Veterans, adapted from Aday and Andersen (1974). At a macro level, health care utilization is influenced by organizational policies and characteristics of the health care system. Health policy, especially in a large national health care system like VA's, is one of the primary drivers of health care utilization. As described in more detail below, VA has implemented numerous policies since the 1990s that have directly influenced Veterans' access to and use of VA health care. VA's health care delivery system also directly influences utilization. VA provides many services that are not available through non-VA sources, due in part to the needs of the unique patient population and to VA's social mission. Access, frequently measured in terms of wait times by VA, is also associated with use of health care services.

At the patient level, health care utilization is typically determined by three factors: predisposing factors (e.g., age), enabling factors (e.g., health insurance), and need for health care services, perceived or actual. Veterans, especially those using VA health care, have a different sociodemographic, health insurance, and health status than their peers who do not use VA health care. Finally, Veteran satisfaction with health care, including perceptions of quality, access, and stigma associated with certain treatments or care settings, also influences their health care utilization.

\subsubsection{Policies That Affect VA Use}

Federal law authorizes VA to provide medically necessary health care services to eligible Veterans (U.S. Government Accountability Office, 2009). According to VA officials, federal laws

Figure 2-1

Conceptual Model of Factors Associated with Veterans' Use of VA Health Care

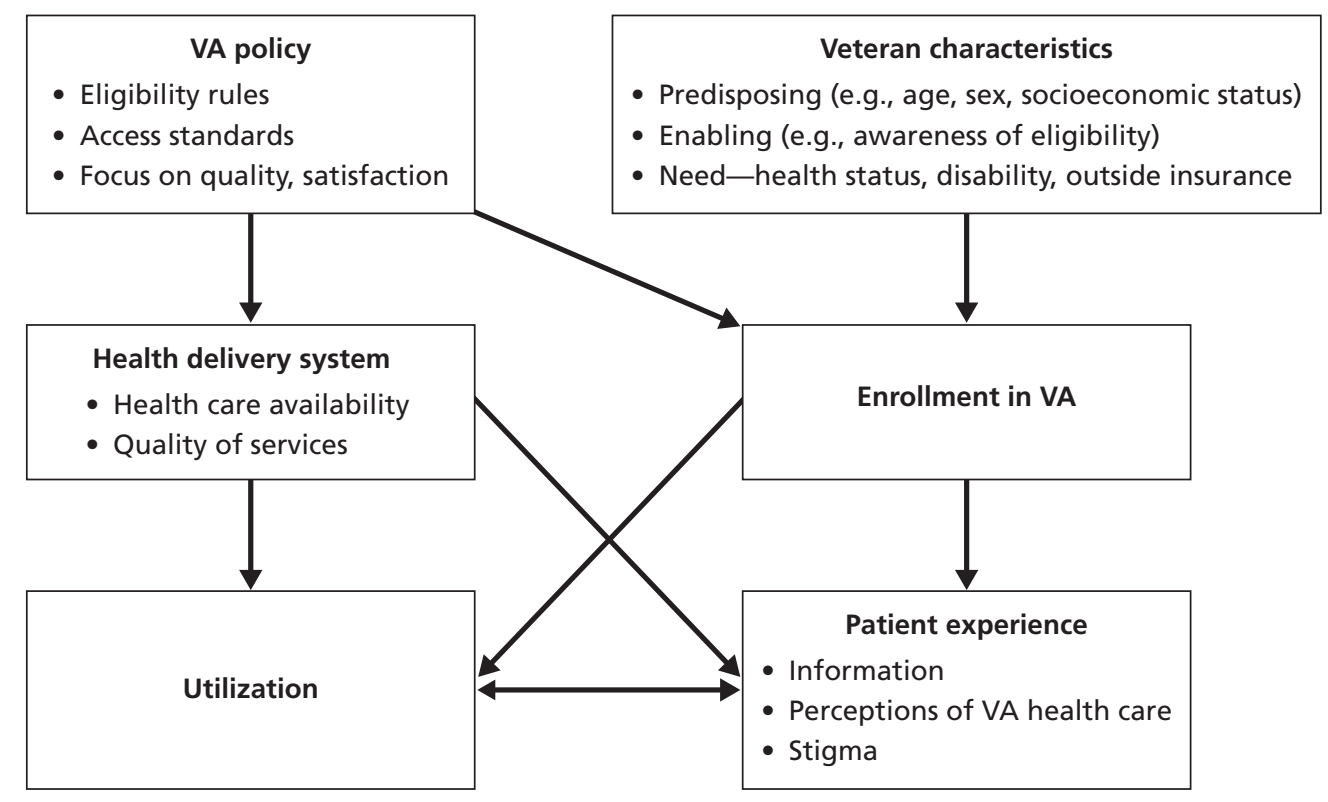

SOURCE: Based on Aday \& Andersen, 1974.

RAND RR1165z1-2.1 
and court decisions over the past decade have expanded Veterans' entitlement to a variety of VA benefits, not just health care (U.S. Government Accountability Office, 2013c). In the area of VA health benefits, legal and policy changes have attempted to expand and improve services provided to eligible Veterans. The foundation for this expansion began in the mid-1990s.

VA enrollment policies directly determine the number and composition of Veterans who are eligible to receive VA health care services. Veterans who have service-connected disabilities (including presumptive diseases) are given higher priority for VA health care, skewing the VA patient population toward Veterans with greater health care needs. VA-specific policies may also lead to a higher rate of diagnosis for some health conditions, such as mental health conditions. ${ }^{3}$ Factors external to VA may also affect the number of Veterans who are eligible to receive VA health care and the choices of those eligible to use VA health services. Deployment and combat experiences are linked to higher rates of service-connected disability (Congressional Budget Office, 2014b), which translates into a larger number of Veterans eligible to enroll in VA and in part determines the prevalence of the health conditions among the newest Veterans.

In the 1990s, there was a "reengineering" of VA's health care system, with the purpose of improving performance, quality, and innovation (Congressional Budget Office, 2007). Prior to these changes, higher-income Veterans without service-connected disabilities were eligible for limited medical services, which did not include outpatient care (Congressional Budget Office, 2009). The Veterans' Health Care Eligibility Reform Act of 1996 revised VA eligibility rules, lifted the restriction on outpatient care, and expanded outpatient services in an effort to provide comprehensive health care to each enrolled Veteran (Veterans' Health Care Eligibility Reform Act of 1996, 1996). After the law, all enrolled Veterans were eligible for VA's full range of health care benefits, including outpatient services (Congressional Budget Office, 2009). Consequently, there was an increase in Veterans' use of VA outpatient care (Congressional Budget Office, 2009).

In 2000, the Veterans Claims Assistance Act (VCAA) clarified the duty of VA to assist benefits claimants, including actions to be taken for and information to be provided to Veterans (Veterans Claims Assistance Act of 2000, 2000). For example, VCAA required VA to assist a Veteran filing a claim in obtaining evidence to substantiate that claim before making a decision on benefits (Veterans Claims Assistance Act of 2000, 2000). VCAA also required VA to give the benefit of the doubt to Veterans in making benefits determinations. The "Combat Veteran" Authority of 2008 extended enhanced eligibility and expedited enrollment for VA health care for Veterans of Afghanistan and Iraq.

Recent years also have seen changes in VA policies related to women's health. These include the following requirements: each VAMC must have a Women Veterans program manager, who is responsible for assessing the needs of female Veterans (U.S. Government Accountability Office, 2009); all VAMCs and CBOCs must make comprehensive primary care available from one primary care provider at one site for all eligible female Veterans, including routine detection and management of acute and chronic illness, preventive care, basic gender-specific care, ${ }^{4}$ and basic mental health care (U.S. Government Accountability Office, 2009); and all VAMCs

\footnotetext{
3 For example, in 2004, VA finalized a five-year Mental Health Strategic Plan, which emphasized mental health as an important part of Veterans' overall health, and its objectives included increasing early mental health screening and assessments.

4 Gender-specific care is minimally defined as cervical cancer screening, breast examination, management of menopause, mammography, obstetric care, and infertility evaluation (U.S. Government Accountability Office, 2009).
} 
and CBOCs must ensure the privacy ${ }^{5}$ of female Veterans in all care settings. Federal law also now requires VA to provide mental health screening, counseling, and treatment for eligible Veterans who have experienced military sexual trauma (Counseling and Treatment for Sexual Trauma, 2011). Recent statistics show that 21 percent of female Veterans screened positive for military sexual trauma (U.S. Government Accountability Office, 2009). Even though the military sexual trauma law is particularly relevant to female Veterans, it applies to all Veterans.

\subsubsection{Characteristics of Veterans}

\subsubsection{Predisposing Factors}

By predisposing factors, we mean demographic and socioeconomic characteristics that affect individuals' chances of using health care. Two demographic characteristics that set Veterans apart from the general population are age and sex, both of which are highly related to health care utilization. Veterans are significantly older and more likely to be male than non-Veterans (Goldberg, 2015; National Center for Veterans Analysis and Statistics, 2014d). Not only does an aging Veteran population face greater health care needs, but users of VA health care tend to be older than non-users (Congressional Budget Office, 2009; Elhai, Grubaugh, Richardson, Egede, \& Creamer, 2008; Zeber, Copeland, \& Grazier, 2006). Conditions such as hypertension and diabetes are more prevalent in older age. Large older cohorts of Veterans will continue to require additional services, including regular monitoring of health conditions, periodic diagnostic testing, and regular use of pharmaceuticals or other medical services, all of which will tax a burdened system.

In recent years, there has been a dramatic increase in the number of female Gulf Warera (pre-9/11 and post-9/11) Veterans seeking care (U.S. Government Accountability Office, 2009; Frayne et al., 2014). The number of female Veterans utilizing VA health care has doubled since 2003, and female Veterans accounted for 7 percent of patients seen in the VA health care system in FY 2013 (Frayne et al., 2014). Female Veterans face a burden of physical and mental illness similar to male Veterans (Frayne et al., 2006), are more likely than male Veterans to use VA health care exclusively (National Center for Veterans Analysis and Statistics, 2014d), and have higher health care expenditures than male Veterans (Bertakis \& Azari, 2010; Bertakis, Azari, Helms, Callahan, \& Robbins, 2000). The specific health care needs of female Veterans vary by service era (Washington, Bean-Mayberry, Hamilton, Cordasco, \& Yano, 2013), with recent Veterans (of Afghanistan and Iraq) experiencing more health care encounters at VA than earlier eras.

Other demographic and socioeconomic characteristics, such as income, education, and employment, may also affect health care utilization decisions. In Chapter Three, we present results showing that Veterans and VA patients have higher average incomes than non-Veterans, that Veterans are more likely to be employed than non-Veterans, and that Veterans are more likely to have graduated from high school than non-Veterans. The relationship between socioeconomic status and health care use in the general population is complex; those with higher socioeconomic status are more likely to be insured and to have a usual source of care (Agency

\footnotetext{
5 Privacy requirements include auditory and visual privacy at check-in and interview areas; the location of examination rooms, presence of privacy curtains, and orientation of examination tables; access to private restrooms in outpatient, inpatient, and residential care settings; and the availability of sanitary products in public restrooms at VA facilities (U.S. Government Accountability Office, 2009).
} 
for Healthcare Research and Quality, 2014), but those with lower socioeconomic status tend to have poorer health outcomes and greater need for health services (Centers for Disease Control and Prevention, 2013).

\subsubsection{Enabling Characteristics}

In the context of this model, enabling characteristics are the means that individuals have available to them for use of services, which include individual-specific resources (such as income) and characteristics of the environment in which the Veteran lives (such as rurality). Veterans may not fully understand their eligibility for VA health care benefits, services available at VA, how to apply for services, and the need for treatment for their condition (U.S. Government Accountability Office, 2011; Weeks et al., 2004; Wittrock, Ono, Stewart, Reisinger, \& Charlton, 2015). One study found that the most common reason that potentially eligible rural Veterans did not use VA care was that they were unaware of their eligibility (Wittrock et al., 2015). Among rural non-enrolled Veterans, it was a common perception that VA enrollees were poor and were required to have experienced combat and a subsequent injury or disability (Wittrock et al., 2015). These findings are cause for concern because rural Veterans tend to have more physical health comorbidities and likely a greater need for VA health care services than other Veterans (Weeks et al., 2004). Some Veterans believe that VA services are focused on certain groups, such as older male Veterans, and are not available for women and younger Veterans (U.S. Government Accountability Office, 2011).

\subsubsection{Need}

Veterans with greater health care needs are both more likely to qualify for VA coverage and more likely to seek care at VA. Prior research has shown that Veterans with severe service-connected disabilities are particularly likely to seek health care from VA (Goldberg, 2015; McGeary, Ford, McCutchen, \& Barnes, 2007). Similarly, mental health conditions and substance use disorders are associated with higher use of VA care (Virgo, Price, Spitznagel, \& Ji, 1999). Afghanistan and Iraq Veterans with PTSD are using VA health care more frequently than Veterans of other eras (Elbogen et al., 2013; Shiner, Drake, Watts, Desai, \& Schnurr, 2012). Data suggest that Afghanistan and Iraq Veterans with PTSD may also be at increased risk for poorer physical health in terms of medical disease burden (Possemato, Wade, Andersen, \& Ouimette, 2010).

\subsubsection{Health Delivery System}

The availability of specific health care services through VA directly influences health care utilization. VA offers several services that are not readily available through other public or private insurance. For instance, VA offers extensive specialized mental health coverage and social worker services (Congressional Budget Office, 2009; Goldberg, 2015). Veterans are more likely to use VA for outpatient care and VA-emphasized services, including specialized mental health care for PTSD and outpatient psychiatric substance abuse counseling (Congressional Budget Office, 2009; Goldberg, 2015). These services may not be covered by private insurance, or the coverage may not be as extensive as VA coverage, which usually provides these services to Veterans with no or minimal cost-sharing (Congressional Budget Office, 2009; Goldberg, 2015).

At the same time, the lack of specific services at VA facilities may cause Veterans to seek care outside of the VA health care system or go without important treatment. For example, VA health care services for female Veterans are not widely available. Based on VA-provided workforce data, the Veterans Choice Act's Assessment B team estimates that only 81 out of 141 
local VA health systems had an obstetrician or gynecologist on staff (RAND Health, 2015). Gynecologists were not available in one-third of VAMCs (Disabled American Veterans, 2015), and most CBOCs refer women Veterans to VAMCs, which may be up to 130 miles away, for gender-specific services (U.S. Government Accountability Office, 2009). Several other gaps in VA health care services have been identified, including limited PTSD interventions in some VAMCs (Institute of Medicine of the National Academies, 2014b) and limited specialized PTSD programs for women Veterans (Disabled American Veterans, 2015; U.S. Government Accountability Office, 2009). Veterans tend to rely on non-VA providers for emergency services, inpatient care, and outpatient surgery (Congressional Budget Office, 2009).

\subsubsection{Costs}

Veterans enrolled in VA health care benefits do not pay enrollment fees, monthly premiums, or deductibles (Goldberg, 2015; U.S. Department of Veterans Affairs, 2015b). Some Veterans are required to submit co-payments for VA health care, which vary by priority group (U.S. Department of Veterans Affairs, 2015a). In general, Veterans in priority group 1 have no copays, Veterans in priority groups 2 through 6 face co-pays for some services, and Veterans in priority groups 7 and 8 face co-pays for most or all services. ${ }^{6}$ Veterans who have co-pays are charged only one co-pay per day regardless of the number of appointments on that day. VA estimated that the annual out-of-pocket costs for VA health care among Veterans in priority groups with co-pays were \$320 in 2014 (U.S. Department of Veterans Affairs, 2014b). This is likely an underestimate of the total costs to Veterans for their health care, given that enrolled Veterans receive most (70 percent) of their health care outside of VA (Goldberg, 2015).

Because many VA enrollees have access to other health insurance, costs associated with using VA services must be considered against the costs and convenience of external options. Growth in civilian health care costs, such as shifts toward high-deductible health plans in the employer health insurance market, may cause some Veterans to rely more heavily on VA. At the same time, some reforms implemented under the ACA, such as expanded Medicaid eligibility and access to premium and cost-sharing subsidies, may reduce Veterans' demand for VA care.

\subsubsection{Quality}

Studies have demonstrated that VA provides high-quality medical care overall (Congressional Budget Office, 2009) and for specific clinical populations (U.S. Department of Veterans Affairs, 2012). In fact, a 2005 article found that VA patients receive two-thirds of the care recommended by national standards, while patients in other health care systems receive about half of the recommended care (S. M. Asch et al., 2004). VA has certain initiatives in place, such as reviewing providers' actions following an adverse event, in an effort to improve the quality of care provided (U.S. Government Accountability Office, 2013b). Additionally, VA tracks the quality of its health care through numerous metrics, including patient satisfaction (Congressional Budget Office, 2007), adherence to clinical guidelines, patient use of VA Virtual Care Modality, geographic access, and wait times (Veterans Health Administration, 2015).

More-recent analysis conducted by RAND in Assessment B (part of this congressionally mandated study) concluded that VA health care quality was good overall; however, quality was uneven across the VA health care system, with marked differences between the highest-

\footnotetext{
6 For additional details, see U.S. Department of Veterans Affairs, 2015a.
} 
and lowest-performing VA facilities (RAND Health, 2015). VA outpatient care outperformed non-VA outpatient care on most quality measures; however, inpatient quality measures varied. VA has served as a leader in the quality measurement arena and uses many systems for monitoring quality, but there are mixed opinions within VA on the impact of the quality measures.

Positive reports about quality may lead more Veterans to seek care through VA. However, it is unclear whether studies that have shown strong clinical quality outcomes in the VA health care system are widely known or understood among the Veteran population. Veterans may be influenced by findings that are reported in major newspapers, and could potentially be deterred from seeking care by recent reports of long wait times and other access problems. We discuss Veterans' perceptions about the VA health care system in Section 2.2.4.

\subsubsection{Access}

Under the Veterans Choice Act, timely access is defined as care provided within 30 days of the Veteran's desired date for an appointment. VA measures and routinely reports wait times for both primary and specialty care appointments for new and existing patients (Veterans Health Administration, 2015). However, wait time measures have been shown in recent years to be unreliable. Schedulers do not always record the desired appointment date in the scheduling system correctly, and scheduling policies are not always adhered to, resulting in the potential for inaccurate wait time calculations (U.S. Government Accountability Office, 2012). In other cases, wait times are not tracked, such as for VA-purchased care, which prevents VA from assessing whether non-VA care is meeting the wait time standards that apply to VA facilitybased care (U.S. Government Accountability Office, 2014a).

Assessment B evaluated access to VA care and noted considerable variability across the dimensions of access. Geographic access varied by region and by access standard used (drivetime versus straight-line distance). Overall, most appointments met VA timeliness standards (30 days of preferred date); however, the average number of days that Veterans wait varies tremendously across the VA health care system. Veterans are less likely than patients in the private sector to report getting appointments as soon as needed. (RAND Health, 2015)

In recent years, VA wait times have increased, resulting in a slight decrease in utilization, as well as adverse health outcomes among vulnerable Veterans (Pizer \& Prentice, 2011). Delays in accessing care have occurred for patients seeking outpatient primary and specialty care within VA (U.S. Government Accountability Office, 2012, 2013a, 2014b). Veterans who received care at VA facilities with longer wait times were at increased risk of adverse long-term health outcomes (e.g., preventable hospitalizations) and intermediate outcomes (e.g., worse hemoglobin A1C levels) than Veterans receiving care at facilities with shorter wait times (Pizer $\&$ Prentice, 2011). The increase in wait times and associated adverse health outcomes were responsible, in part, for the greater use of technological methods to deliver care (e.g., messaging between patients and providers, telehealth) (U.S. Government Accountability Office, 2013a), and the development of legislation that expanded VA coverage to allow enrolled Veterans to seek VA-purchased care from community providers. In addition to the long wait times for VA patients, as recently as last year, nearly 1 million Veterans were stuck in a backlog waiting for their disability determinations to be evaluated (Hicks, 2014; Zoroya, 2014). Some of these individuals have an initial disability determination, but are seeking re-evaluation to increase their disability ratings, which in turn could affect their co-payment requirements. Such backlogs may affect Veterans' ability to get timely access to care, because some will be unable to enroll, and others may face higher cost-sharing payments until their disability claims are adjudicated. 
Long wait times and other access challenges likely deter Veterans from seeking care in two ways. First, long wait times and backlogs in processing disability claims pose barriers to accessing care among those who make an attempt to use VA services. Second, reports about long wait times, inconveniently located facilities, and other challenges may make some Veterans less likely to enroll or attempt to use VA services.

\subsubsection{Patient Experience}

The Aday-Andersen framework defines consumer satisfaction, or patient experience, as the "attitudes toward the medical care system of those who have experienced a contact with it." Veterans perceptions about the VA health system are also important predictors of health care use (Congressional Budget Office, 2009). One study found that media coverage of adverse events that occurred in VA facilities was associated with lower enrollment rates and subsequent Veteran disenrollment (Weeks \& Mills, 2003). Facilities that had published reports of adverse events had lower enrollment rates after publication of the report (Weeks \& Mills, 2003). Veterans' negative perceptions about VA health care influence Veterans' use of VA health care; these negative perceptions include both overall negative perceptions about VA and the treatment of Veterans by VA and more-specific perceptions that VA cannot meet their health care needs (U.S. Government Accountability Office, 2011). Veterans dissatisfied with their VA health care are more likely to seek care outside of VA (Stroupe et al., 2005).

Having considered the theoretical factors that influence a Veteran's decision to enroll in and use VA health care services, we now turn to our empirical approach, which makes use of a variety of DoD and VA data that capture experiences, characteristics, and environmental factors correlated with these decisions.

\subsection{Analytic Approach}

\subsubsection{Overview}

The goal of our assessment is to estimate the current and projected demographic characteristics and unique health care needs of the patient population served by VA. To accomplish this goal, we selected and synthesized data to estimate the size of the current Veteran population, their characteristics, the probability of using VA health care services, and trends over time in both the number of Veterans and their probability of health care use. Our baseline projections incorporated known trends and policy changes, such as DoD's ongoing "drawdown" aiming to reduce the size of the active duty force (Hagel, 2014; Parrish, 2011; Office of the Under Secretary of Defense [Comptroller], 2015). However, because many factors that could affect the size of the VA patient population are unknown and difficult to predict, we also considered uncertain future scenarios. These scenarios included both policies that are within VA's control, such as a change in conditions that qualify for presumptive eligibility, and policies external to VA, such as the rollout of the ACA.

The number of Veterans who will ultimately seek care is determined by several factors, including the number of Veterans in the pipeline, Veterans' eligibility for enrollment, Veterans' decisions to enroll conditional on eligibility, and Veterans' decisions to use care when enrolled (Figure 2-2). These factors are influenced not only by VA policies but also DoD policies, trends in the generosity and availability of civilian health insurance coverage, geopolitical issues (such 
Figure 2-2

Pathway to Use of VA Health Care

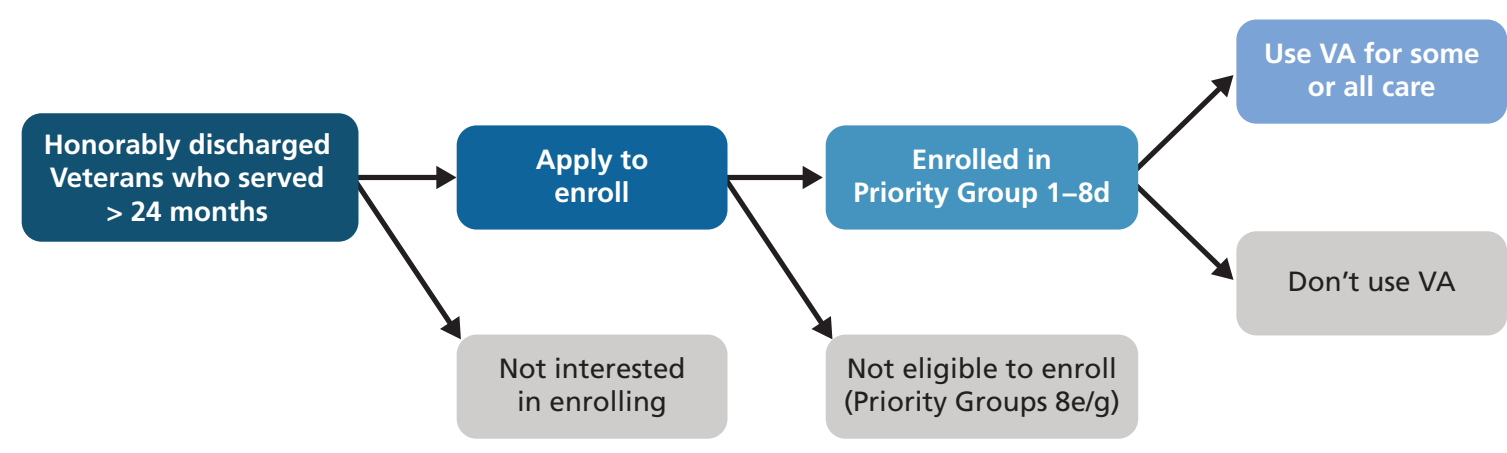

RAND RR1165z1-2.2

as the emergence of conflicts and the U.S. response), and Veterans' perceptions about the VA health system. Approaches to estimating future demand for VA health services must therefore consider previous utilization trends and current policies, as well as how these uncertain future scenarios could affect decisions. While our main analyses held policies and other factors that affect VA utilization constant (with an exception for the announced trends in the drawdown of the size of the military force), our scenario testing analyzed the impact of changes in Veterans' proclivity to use VA health services.

Figure 2-3 describes the analytic approach that we undertook for this project. Briefly, the approach involved estimating the current number of Veterans and the share of Veterans who are currently VA patients, using data from sources such as the Census, ACS, and MEPS. We then projected both the size of the Veteran population and use of VA care over time, accounting for historical trends and known policy changes on the horizon. Finally, we considered how uncertain future scenarios may affect the size of the population and Veterans' proclivity to use VA services. The use rate refers to the probability of an individual becoming a VA patient given that he or she is a Veteran. A critical step to becoming a VA patient is the decision to enroll in VA health services. However, few available data sources combine information on Veterans, enrollment, and use of care. Because enrollment is an intermediate step that is not critical to answering all of the questions posed by Congress, we did not necessarily consider enrollment in all of our analyses. In analyses that required estimating en rollment, we predicted the enrollment rate and the conditional use rate, which is the use rate among enrollees (as opposed to the overall use rate among all Veterans). The overall use rate is illustrated by the curved arrows in Figure 2-3, and the enrollment and conditional use rates are illustrated with straight, horizontal arrows. Mathematically, the overall use rate represents the product of the enrollment rate and the conditional use rate.

When considering uncertain future scenarios, we modified the enrollment and the use rates among those enrolled to account for potential changes that may occur due to future events. For example, a policy that would loosen restrictions on enrollment among Veterans in priority groups $8 \mathrm{e}$ and $8 \mathrm{~g}$ would affect both the probability of enrollment and the probability of using care conditional on enrollment. 
Figure 2-3

Analytic Framework

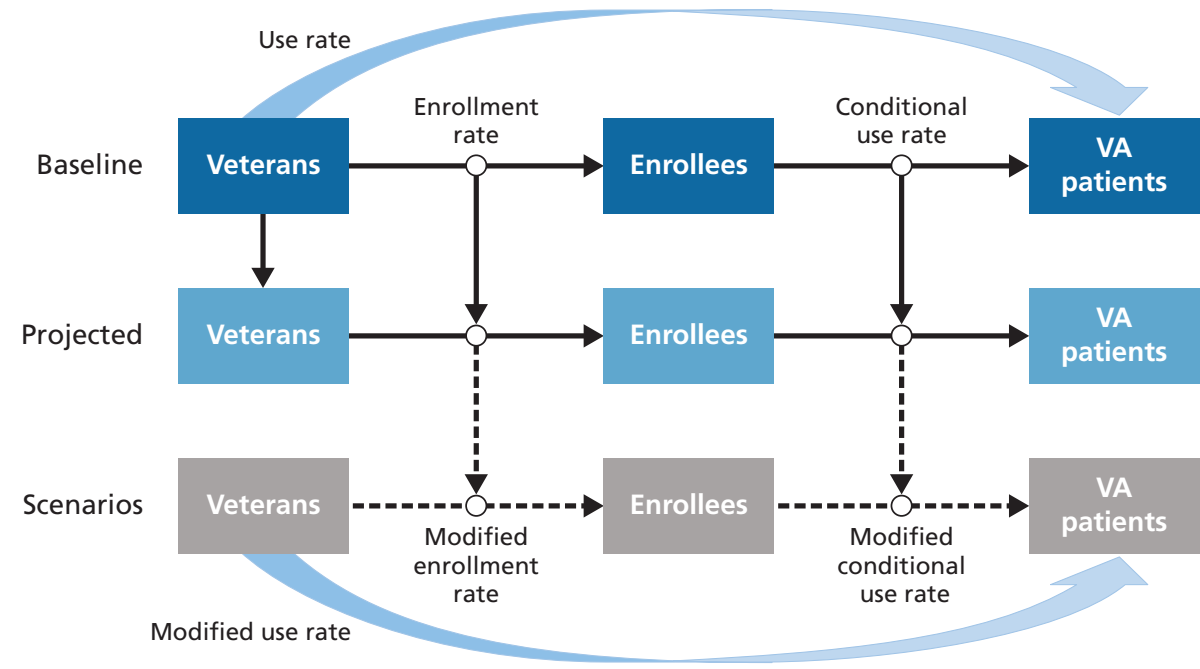

RAND RR1165z1-2.3

\subsubsection{Baseline 2014 Population Estimates}

To develop our baseline estimates, we started with data from the 2000 Census (the most recent national accounting of Veterans; the 2010 Census did not assess whether respondents were Veterans), and appended that data with more-recent information on Veterans from ACS, an annual survey conducted by the U.S. Census Bureau that collects information on Veteran status, demographic characteristics, and insurance coverage. To account for newly discharged Veterans and reservists since the 2000 Census, we incorporated data from DoD, including the Defense Manpower Data Center (DMDC) Active Duty Loss files, the Work Experience (WEX) files, and the Contingency Tracking System files. These data sources are described in more detail later in this chapter and in Appendix A. With this information, we were able to develop a comprehensive picture of the current Veteran population, including demographic characteristics such as age, sex, geographic location, race/ethnicity, and service era.

Because the demographic files described above do not contain information on Veterans' health status or use of health care, we turned to other data to assess this information. One important source of information was MEPS, which collects information on health care conditions and use of services for the U.S. noninstitutionalized population, including Veterans. With these data, we could identify whether an individual is a Veteran, whether the individual reports VA as a source of health coverage, whether the individual received any health care paid for by VA, what types of conditions and illnesses the individual experienced in the past year, and treatments received in the past year. Because MEPS collects comprehensive information on both VA and non-VA care, we could use the data to understand Veterans' health care needs regardless of whether they sought treatment from VA sources. In addition, because MEPS collects information on both Veterans and non-Veterans, we could use the data to compare Veterans' health conditions and comorbidities with non-Veterans' health conditions and comorbidities. A drawback of MEPS, however, is that its relatively small sample size precluded us from using these data to investigate extremely rare conditions, such as amputations, that are of high interest to VA. In addition, because diagnoses are based on self-reports, MEPS could miss key 
conditions, especially those associated with stigma (e.g., mental health conditions) (Agency for Healthcare Research and Quality, 2004).

As an additional source of data on the health status of the VA patient population, we turned to encounter data from the VA health system. The encounter data record every patient interaction that a Veteran has with the VA health system, enabling us to get a comprehensive picture of care and diagnoses delivered by VA. With these data, we could count the total number of VA patients and analyze treated Veterans' recorded diagnoses, including diagnoses for rare conditions such as amputations, burns, blindness, TBI, and spinal cord injury. Further, because the encounter data record all patient interactions, they are not subject to recall bias or other reporting biases. However, the encounter data do not contain information on care or diagnoses received outside of VA, and the administrative nature of the data can make it difficult to separate true diagnoses from diagnosis associated with encounters to "rule out" disease (e.g., if an individual receives a test for diabetes but the results are negative, this visit may nevertheless have an associated diabetes diagnosis code in the encounter data). ${ }^{7}$ Further, the data contain no information on non-Veterans (other than a small number of Veterans' family members who are eligible for care), making it impossible to use VA encounter data to compare Veterans' diagnoses with non-Veterans' diagnoses.

Despite these limitations, the combination of the MEPS data and the VA data allowed us to develop a nuanced understanding of Veterans' health care needs. With MEPS, we could assess needs of all Veterans whether or not they use the VA health system, and we could compare these needs with the needs of non-Veterans. With the VA encounter data, we could access more-detailed information on the unique health care needs of the current VA patient population.

To understand transitions between becoming an enrollee and becoming a VA patient at baseline, we relied on administrative data from VA that record the total number of enrollees overall and by subgroup (e.g., based on priority status). We also used the encounter data to determine the number of unique VA patients in a given year. However, there was no definitive source of information on the total Veteran population living in the United States. Like the VA OACT, we therefore had to rely on Census and survey data to estimate the size of the total population.

\subsubsection{Population Projections}

We projected the future size and demographic characteristics of the Veteran population by combining several sources of information, including:

- Past trends in the size and characteristics of the Veteran population observed in historical data

- Statistical models of migration patterns used by demographers, known as "gravity models" (defined in Chapter Three)

\footnotetext{
7 In our analysis with the VA encounter data, we attempted to identify conditions with greater accuracy by requiring that one inpatient encounter be labeled with the associated conditions or that two outpatient encounters were labeled with the associated conditions; this is a common approach for identifying conditions within VA encounter data (Park et al., 2014). We made exceptions for TBI, acute coronary syndrome, and acute myocardial infarction (AMI). We identified TBI cases even if there was only a single outpatient or inpatient diagnosis flagged because acute causes of TBI (concussion, skull fracture, etc.) may be described only at the initial visit, per guidance from DoD (U.S. Department of Defense, 2010). In contrast, we identified acute coronary syndrome and AMI if one or more inpatient stays had a principal diagnosis code associated with those conditions (Petersen, Wright, Normand, \& Daley, 1999).
} 
- Information on the characteristics and size of incoming Veteran cohorts, based on DoD data on the current active duty population, annual discharge patterns, and projected plans to reduce the size of the force

- Projected mortality rates among Veterans derived from the Centers for Disease Control and Prevention (CDC) and the VA OACT.

We combined this information to develop detailed annual estimates of how the Veteran population will change between 2015 and 2024, accounting for trends in demographics, migration patterns, and projected mortality rates. For example, our approach accounted for the fact that newly discharged Veterans are more likely to be female and more likely to be Hispanic than the existing population of Veterans (although, despite these trends, Veterans are still disproportionately male and primarily non-Hispanic).

To project VA patient status over time, we estimated the use rate, or the probability that a future Veteran will use VA for health services. We used administrative data from VA to model the probability that a Veteran will use VA health services, accounting for demographic characteristics and historical time trends in the proclivity to access care at VA. The projected use rate was combined with the Veteran population projections to estimate the number of VA patients in each year from 2015 to 2024.

We used statistical models to estimate the health conditions of the total Veteran population and the VA patient population, taking into account such factors as age, sex, race/ethnicity, Census region, and historical time trends. This information was appended to our projections of the size and demographic composition of the Veteran and VA patient populations, and allowed us to project the health conditions of Veterans and VA patients in each year between 2015 and 2024. In projecting the future health conditions of Veterans and VA patients, we also considered the health conditions of the current active duty force, using encounter data from MHS.

\subsubsection{Scenarios}

The projected trends in the Veteran population and VA patient population described above are based on a "steady state" and are driven by predicted changes in Veteran population demographics and observed trends in health care needs; they do not account for uncertain future scenarios that might affect the composition of the Veteran population and Veterans' health care use. In Chapter Six, we consider the impact of uncertain policy changes and external trends that could affect demand for VA services. The scenarios we consider focus on factors that could affect the number of Veterans in the pipeline (e.g., possible future conflicts), factors that could affect Veterans' eligibility to receive care (e.g., changes in enrollment prioritization, changes in conditions that grant presumptive eligibility status), and factors that could affect Veterans' use of care when eligible (e.g., expansions to the Veterans Choice Act; eligibility for new, non-Veteran programs due to the ACA).

The scenario tests build from our baseline projections, incorporating possible changes in the size of the population and Veterans' propensity to use VA services. An inherent limitation of the scenario testing section is that, because we were modeling uncertain future events, we did not always have access to reliable historical information that we could use to estimate effect sizes, and instead needed to rely on relatively strong assumptions. For example, in estimating the effects of a potential future conflict on the size of the VA population, it is unclear that we could draw meaningful insight from experiences of prior conflicts (e.g., Vietnam, World War II) because technologies, methods of fighting, and military strategic approaches have changed 
dramatically over time. For the future conflict scenario, we relied on military staffing patterns observed during the most-recent conflicts (Afghanistan and Iraq), but we recognize that even these recent conflicts could be quite different from what might occur in a hypothetical future conflict. As a result, the output of our scenario testing is by definition highly uncertain. Nevertheless, this type of evaluation is important for contingency planning and understanding the possible magnitudes of demand shifts that VA could face if large-scale changes should occur. While any given scenario is unlikely to come to fruition in exactly the manner we have posited, analyzing possible changes in demand may help VA to respond quickly when unforeseen circumstances arrive. For example, a modeling strategy could be altered or updated as better data or more-specific policy details emerge.

\subsection{Existing VA Forecasting Models}

For the purposes of this independent assessment, we implemented our own modeling approaches to estimate the current and future demographics of the Veteran population and their health care needs. However, VA has three existing modeling tools that it uses to estimate future demand: VetPop (a model of the Veteran population), EHCPM, and the VA OACT's Veteran Healthcare Scenario Model (VHSM). In this section, we briefly describe each of these models. However, the amount of available documentation for the models varies. We had access to detailed documentation for the EHCPM but not the other two models. As a result, we are able to offer a more thorough description of the EHCPM model.

\subsubsection{VetPop}

The VetPop2014 model was developed by OACT for Veteran population projections from 2014 to 2043. The model provides Veteran counts by age, sex, service era, and race/ethnicity at the county level. VetPop2014 is the culmination of years of work and refinement by OACT, representing the seventh iteration of the projection model (the previous iteration was developed in 2011). Documentation for the VetPop2014 model is scarce, and we rely on information contained in an online two-page abstract and discussions with the VetPop2014 team at OACT (Office of the Actuary, 2014).

VetPop2014 starts with a baseline population, then applies mortality rates and adds new Veterans over time. The model also accounts for Veterans' migration patterns throughout the forecasting period. The baseline population for the VetPop2014 model comes from the 2000 U.S. Census. VetPop2014 then applies age- and sex-specific mortality rates derived from mortality data that include Veteran-specific information from VA administrative data and U.S. population data from the Social Security Administration and the Internal Revenue Service (IRS). Mortality rates in VetPop2014 vary by age and sex. VetPop2014 assumes a slight mortality improvement for older Veterans due to expected increases in life expectancy.

VetPop2014 projects annual separations from the military (i.e., new Veterans) by age and sex, and by active and reserve component, using DoD data. The abstract states that "[b]ased on DoD's annual military separation data from FY1980 to FY2013 . . VetPop2014 ... developed a set of Time Series Models to project annual separations for various age and gender groups. ... VetPop2014 Model then used historical county separation data based on VA administrative records along with migration information from the IRS to project the county level separation from FY2014 to FY2043 using predictive modeling techniques." VetPop2014 
assumes that conflicts in the Gulf end by 2018 and that there are no other major conflicts in the next 30 years. Finally, VetPop2014 models migration at the county level using historical data from VA, IRS, and ACS. Predictive migration models are developed for various age (five groups) and sex cohorts.

\subsubsection{Enrollee Health Care Projection Model}

EHCPM is a model for projecting how Veterans will interact with the VA health care system, and what consequences VA will experience as a result. The model is organized into three stages, which predict (1) eligibility and enrollment of Veterans in VA health care, (2) Veteran demand for health care services and reliance on VA health care for those services, and (3) total cost to VA of providing services. Each stage is contingent on projections from the previous stages, as well as external data and models.

At the highest level, EHCPM calculates costs as the product of Veterans, demand per patient, a reliance factor representing the proportion of care that Veterans receive from VA, and a unit cost:

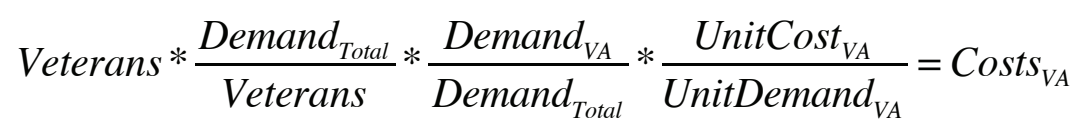

This process is repeated for many Veteran subpopulations (defined by geography, demographics, priority group, and other characteristics) and for many categories of health care services.

EHCPM is a policy and planning model. It serves three functions for VA. First, the model provides a principled way to anticipate future demand for VA services, so that administrators can plan accordingly. Second, the model enables VA to provide Congress with an estimate of future costs, which, in turn, helps to ensure that its congressionally apportioned budget is sufficient to meet service demand. Third, because political, cultural, and demographic factors strongly shape the VA operating environment, EHCPM enables administrators to understand the operational consequences of factors both within and beyond VA's control.

In this regard, a key feature of the model is its ability to produce estimates for hypothetical scenarios, such as new legislation. EHCPM decomposes model outcomes into a series of joint probabilities, each of which can be altered to simulate the impact of scenarios and project the consequences for VA health care. For example, the probability that a Veteran uses services can be decomposed as the probabilities of enrollment in VA care $\times$ reliance on VA over alternative sources of insurance $\times$ patient demand for health care services. If a new piece of legislation had the potential to raise Veteran co-pays, that might lower the probability that Veterans rely on VA care instead of alternative insurance options. The model could be recalculated with an artificially lowered reliance rate, enabling planners to understand how the proposed legislation would affect patient demand for VA services.

The joint probability strategy greatly increases the volume, variety, and specificity of the data required. To project enrollment of Veterans in VA health care, EHCPM relies on (1) actual enrollee and user data from internal records; (2) estimates of the characteristics of the total Veteran population, which is itself a multidata-source model projection from the VA OACT; (3) DoD data on deployment and separation of military personnel; and (4) actuarial mortality tables. To project health care demand and Veteran reliance on VA for it, EHCPM 
relies on (1) user data from internal records on Veteran characteristics and diagnoses; (2) the VA Survey of Veteran Enrollees' Health and Reliance Upon VA (SoE); (3) Medicare data for Veterans age 65 or older; (4) Chronic Illness \& Disability Payment System data from the University of California, San Diego, on the relationship between diagnoses and usage patterns for chronic illness/disability; (5) data from the actuarial firm Milliman's Health Cost Guidelines on health care utilization and cost averages by geographic area; and (6) various proprietary scores developed by Milliman. To project health care costs, EHCPM relies on (1) VA unit cost, workload, and budget obligations data; (2) CMS data on the work required to provide various physician services; and (3) Milliman data on the workload burden of providing various outpatient hospital services. Each stage of the model relies on outputs from the previous stage. In many instances, EHCPM uses linear modeling, with some analyst discretion in model specifications, ${ }^{8}$ to extrapolate needed figures with no suitable data source. Finally, many model inputs are smoothed to account for temporal or categorical progression and trends.

At each step, model projections are calibrated against the previous year's actual statistics. This involves running the model for the previous year, and then calculating an adjustment statistic for each model output to make model outputs identical to the previous year's actual figures. These adjustment statistics are then applied to the future projections, which predict various aspects of VA health care over the next 20 years. Analysts also examine these adjustment figures for trends, as trending adjustments may be the first sign of an underlying change in the health care system.

\subsubsection{Office of the Actuary Veterans Health Care Scenario Model}

Based on conversations with the VA OACT, VA uses VHSM for strategic planning and specifically to evaluate the budget impact of various business scenarios. By applying multiple analytic methods, VHSM produces analysis of key drivers and three types of model outputs: (1) baseline models, (2) scenario models, and (3) stochastic models. The key-drivers analysis is used to evaluate the trends and important factors influencing eligibility and demand for VA services, utilization of these services, and corresponding costs. The baseline model can then identify gaps in VA services in terms of demand and associated costs, while the scenario model can be used, for example, to understand how the changes in Veteran population demographic characteristics or economic conditions (such as income or employment) can influence the demand for VA services and related costs. The stochastic models are the Monte Carlo Simulations of the likelihood that VA will actually meet the demand for its services, given its budget limits, if the scenario under consideration is realized.

OACT relies on many data sources in its analysis: (1) VA administrative data (enrollment files, patient utilization files, benefits data), (2) public data (MEPS for medical expenditures, CMS data on Medicare and Medicaid, U.S. Census for socioeconomic demographic data, DoD data for new Veterans, and the Social Security Administration for mortality and disability data), and (3) industry and commercial data (Society of Actuaries health care trend analysis, United Health Group claim data for utilization and cost benchmarks, and Acxiom data for socioeconomic analysis).

8 These adjustments are reviewed by another actuary at Milliman who is not directly involved with the EHCPM work. 


\subsection{Strengths of Our Approach}

VA has made substantial investments in the models described above and refined them over the years. Our analysis, which was done on an eight-month time frame with limited access to data, was not meant to replace current VA models. Rather, we aimed to provide an independent assessment of the current and future Veteran population and patients' need for health services. In addition, while our models are prototypes and have not been refined to the same extent as VetPop and EHCPM, we incorporated several features into these model that could be useful for future VA planning. Strengths of our approach include the following.

Use of DoD data: We incorporated several sources of data from DoD into our models, to enable us to refine our estimates of the pipeline of future Veterans and their health care needs. By using the combination of the DMDC loss files, WEX files, and Contingency Tracking System files, we were able to account for age, sex, race, branch, active duty status, and activated reserve status in our demographic projections. To the best of our understanding, the VetPop model relies on aggregate rather than micro-level DoD data, and accounts for only age, sex, and service era in projection models, although race/ethnicity estimates are ultimately presented among the characteristics of future Veterans.

In addition, we incorporated health status information from MHS into our estimates of Veterans' future health care needs. We do not believe that MHS data or other data on current military populations are incorporated into EHCPM. Better anticipating the needs of incoming Veterans could improve model accuracy.

Effort to incorporate racelethnicity: The DoD data we used to enhance our projections include information on race and ethnicity, enabling us to estimate how changes in the racial and ethnic composition of the current military force may affect the demographics and health care needs of future Veterans. In addition, we incorporated race/ethnic-specific mortality rates from CDC into our model, adjusted to account for overall differences between Veteran and non-Veteran mortality. In contrast, mortality rates in VetPop account only for sex. A more nuanced focus on race and ethnicity may help VA to better understand the evolving needs of the VA population, especially because the number of Hispanic Veterans is increasing.

Use of MEPS to estimate reliance: VA faces a significant challenge in estimating future demand for health services due to the issue of reliance, or the fact that many Veterans receive only a portion of their total health care from VA. Reliance raises two important sources of uncertainty in estimating future demand: Reliance patterns may shift over time, and existing VA administrative data do not provide visibility into the total health care utilization and needs of the patient population. EHCPM appends VA data with Medicare claims data to estimate reliance for patients age 65 and older, and uses a combination of VA survey data and proprietary commercial utilization benchmarks to estimate reliance among Veterans under age 65 .

We took a different approach, using MEPS to estimate Veterans' reliance and propensity to use VA health care. MEPS is a survey of the noninstitutionalized civilian population (including Veterans) that aims to understand the U.S. population's total demand for health services and use of care across all possible settings. Because the survey identifies Veterans and assesses how they pay for health care, we were able to use this data to infer total utilization, whether or not care was provided by VA, as discussed in Chapter Five. Relative to current surveys conducted by VA, MEPS provides greater detail on the use of health services and specific diagnoses. Further, MEPS data suggest that Veterans over age 65 use other sources of care 
besides VA and Medicare (e.g., private insurance, self-pay), raising questions about VA's current approach to estimating reliance among this population.

MEPS has some significant drawbacks in terms of its utility for VA planning. Most importantly, the sample size of Veterans in MEPS is small, and the survey is not currently designed to ensure that the demographic distribution of Veterans is accurately preserved. For these reasons, it is unclear whether relying on MEPS in its current form would significantly enhance VA's forecasting capability. However, it is possible that the methods employed by MEPS to estimate health care use for the general population could be tailored to support a similar survey of Veterans.

Scenario testing: We tested the sensitivity of our estimates to alternative (but plausible) scenarios in which the number of eligible Veterans and Veterans' proclivity to use health care were altered relative to our initial projections. Our analysis considered both the impact of policy changes that VA could enact on its own, as well as the impact of policy changes and external trends that are beyond VA's control. The scenarios helped us to understand what types of policy change and events might significantly change the demand for VA services, and hence require changes to planning approaches. While it is unlikely that any of the specific policy scenarios will unfold in exactly the manner we have modeled, the approach is useful to understand the potential impacts of uncertain future events. Conducting regular sensitivity analyses related to potential changes in Veterans' eligibility and utilization patterns could help VA to be better prepared when unforeseen circumstances arise.

We reiterate that our analysis was not meant to replace ongoing VA modeling efforts. Rather, we aimed to provide a fresh perspective on potential modeling approaches, and to provide insight into how different methodologies might affect results.

\subsection{Overview of Data Sources}

Throughout this report, we use a variety of data sources that provide information on populations (civilians, service members, Veterans, VA enrollees, VA patients) and their characteristics. Table 2-3 summarizes the populations in each data set, including the National Health Interview Survey (NHIS), the Behavioral Risk Factor Surveillance System (BRFSS), and the National Survey of Veterans (NSV), among others; indicates whether health and geographic information is included; and denotes specifically whether we could determine if an individual was eligible for VA services, was enrolled in VA, and was a VA patient. The passages following Table 2-3 briefly discuss the main advantages and limitations of each available data source.

The Medical Expenditure Panel Survey (MEPS), sponsored by AHRQ, is a recurring nationally representative longitudinal survey of the U.S. civilian noninstitutionalized population drawn from the NHIS sampling frame. The Household Component of MEPS collects information on medical utilization, expenditures, and sources of payment for care obtained by households in all care settings outside of nursing homes. It also includes detailed information obtained via questionnaires provided to households and separately to their medical providers. Demographic information includes age, sex, race/ethnicity, marital status, education, and income, but does not include information on service era. Health information is derived from responses to survey questions about several common medical conditions and responses to open-ended questions about medical conditions or procedures. These open-ended questions are translated into International Classification of Diseases, 9th revision (ICD-9), codes that are 
Table 2-3

Summary of Data Sources

\begin{tabular}{|c|c|c|c|c|c|c|}
\hline Data File & Population & Health & $\begin{array}{c}\text { Geographic } \\
\text { Location }\end{array}$ & $\begin{array}{l}\text { Veteran } \\
\text { Status }\end{array}$ & VA Enrolled & $\begin{array}{c}\text { VA } \\
\text { Patient }\end{array}$ \\
\hline \multicolumn{7}{|c|}{ Nationally Representative Surveys } \\
\hline MEPS ${ }^{a}$ & $\begin{array}{l}\text { Civilians, Veterans, } \\
\text { VA patients }\end{array}$ & Yes & Census region & Yes & Yes & Yes \\
\hline NHIS & $\begin{array}{l}\text { Civilians, Veterans, } \\
\text { VA enrollees }\end{array}$ & Yes & Census region & Yes & Yes & Yes \\
\hline BRFSSC & $\begin{array}{l}\text { National Veterans, } \\
\text { civilians }\end{array}$ & Yes & State & Yes & Yes & Yes \\
\hline U.S. Census & Civilians, Veterans & No & Yes & No & No & No \\
\hline $\mathrm{ACS}^{\mathrm{d}}$ & $\begin{array}{l}\text { Civilians, Veterans, } \\
\text { eligible Veterans, } \\
\text { enrollees }\end{array}$ & Limited & Yes & Yes & Yes & No \\
\hline \multicolumn{7}{|l|}{ Veteran/VA Surveys } \\
\hline NSV & Veterans & Limited & No & Yes & Yes & Yes \\
\hline SoE & VA enrollees & Limited & Yes & Yes & Yes & Yes \\
\hline \multicolumn{7}{|l|}{ Administrative Data } \\
\hline VA Encounter & VA patients & Yes & Yes & Yes & Yes & Yes \\
\hline VA Enrollment & $\begin{array}{l}\text { VA patients, VA } \\
\text { enrollees }\end{array}$ & No & Yes & Yes & Yes & Yes \\
\hline TRICARE/MHS & Active duty military & Yes & Yes & N/A & N/A & $\mathrm{N} / \mathrm{A}$ \\
\hline $\begin{array}{l}\text { Active Duty Master } \\
\text { and Loss }\end{array}$ & Active duty military & No & No & $N / A$ & $\mathrm{~N} / \mathrm{A}$ & $\mathrm{N} / \mathrm{A}$ \\
\hline Work Experience & Service members & No & No & N/A & $\mathrm{N} / \mathrm{A}$ & N/A \\
\hline $\begin{array}{l}\text { Contingency } \\
\text { Tracking System }\end{array}$ & Service members & No & Yes & N/A & $\mathrm{N} / \mathrm{A}$ & $N / A$ \\
\hline
\end{tabular}

NOTE: For the survey data sources, details vary regarding how Veteran status, enrollee states, and patient status is assessed. Table 2-4 reports details on how Veteran status is determined. Enrollee status is typically based on a self-report of enrollment or use of VA insurance. Patient status may be inferred in some cases based on utilization of VA services and/or payment for services by the VA.

a See Medical Expenditure Panel Survey, undated.

b See Centers for Disease Control and Prevention, 2012.

' See Centers for Disease Control and Prevention, 2014a.

d See U.S. Census Bureau, 2015.

used for analysis. MEPS does not contain sufficient information to precisely determine eligibility or en rollment in VA services. Given these limitations, we define Veterans as those respondents who report being honorably discharged from the military, ${ }^{9}$ and we define active VA

9 Prior to 2006, the survey instrument asked whether anyone in the household had ever served on active duty in the Armed Forces of the United States [emphasis added]. 
patients to be those respondents who had any payment by VA for services used. ${ }^{10}$ The period of analysis covered data from 2006 to 2012.

MEPS has several strengths relative to other possible data sources that we could have used for this analysis. First, the goal of the assessment was to analyze the health care needs of the patient population served by VA. We interpreted this to include all health care needs, not just health care needs addressed by VA providers or paid for by VA. Because many VA patients receive a large portion of their health care needs from non-VA sources, we felt it was important to use a data source that captured non-VA care. MEPS allows us to identify all care consumed by a VA patient, not just care provided by VA. The fact that use of VA care is influenced by access barriers makes it even more critical to consider episodes of care provided outside of VA. Focusing only on care provided by VA would understate both the total needs of the VA population and the underlying need for VA services.

Second, MEPS allows us to identify the health care needs of Veterans who do not currently use VA for any care. This population is important because many of these Veterans are potential VA patients and could opt to use VA care in the future. We recognize that some of these Veterans are not currently eligible to enroll in the VA system. However, VA enrollment policy is fluid and changes over time, and VA has discretionary authority to provide care for all Veterans. For example, VA suspended enrollment for Veterans in priority group 8 in 2003, and then relaxed some of these restrictions in 2009. Thus, fully understanding the needs of the future patient population requires considering the needs of Veterans who are not currently eligible to enroll.

Third, MEPS allows us to compare the health care needs of Veterans with the health care needs of non-Veterans using a single data source that collects information using the same methods for both groups. This comparison is important to understand the unique health care needs of the VA population, a requirement identified by Congress.

The National Health Interview Survey (NHIS), sponsored by CDC, is an individuallevel annual survey that includes information on demographics, Census region, general health, cancer screening, self-reported medical conditions (including asthma, arthritis, cancer, diabetes), health behaviors (including alcohol use, smoking, exercise), physical or functional limitations, and mental health (adult mental health, stress). We defined eligible Veterans to be those who self-reported having served in active military duty. It is not possible to identify VA patients in these data. We analyzed data from 2011 to 2013, which covers the period when military service information is available. We used NHIS to estimate the prevalence of the selected health conditions among Veterans and non-Veterans. Where possible, we compared these estimates with those derived using MEPS, which were based on ICD-9-coded health conditions.

The Behavioral Risk Factor Surveillance System (BRFSS), sponsored by CDC, is a nationally representative survey fielded monthly by state health departments that collects individual-level information on health behaviors and risk factors associated with the leading causes of premature mortality and morbidity among adults in the United States. We used data covering 2013. The survey allows for periodic experimental modules and for states to ask additional questions beyond the core instrument. The survey asks whether respondents have ever served in active military duty in the core instruments, and we used this response to identify Veterans. There are also several experimental modules (for certain states) that have asked more-

10 Methodology for identifying Veterans is discussed in Roemer, 2012. 
detailed questions about deployment, service, and a few specific Veterans' health issues (e.g., PTSD, TBI), but we did not use this information because the sample sizes were small and the populations were not nationally representative. We used BRFSS to estimate the prevalence of the self-reported health conditions among Veterans and non-Veterans. BRFSS was also used to examine differences in health behaviors (e.g., smoking) and other health indicators (e.g., obesity) between Veterans and non-Veterans.

The U.S. Census collected information about the 115.9 million housing units and 281.4 million people in the United States on April 1, 2000. A 5-percent sample of people and housing units received a more detailed long-form survey that contained questions about Veteran status and periods of service. As a starting point, the team used the 5-percent sample 2000 Census data to assess the baseline Veteran population in 2000. The 2010 Census did not include a long form and did not collect information on Veteran status. The 2010 Census short form included only basic demographic questions (e.g., name, relationship with head of household, age, sex, Hispanic origin, race) and household information (e.g., number of people in the household, whether the home is owned or rented). The 2000 Census long form asked detailed demographic and household questions, including Veteran status and time that the person served on active duty in the U.S. armed forces. As of the 2010 Census, detailed sociodemographic and other information is collected in ACS, rather than the Census.

The American Community Survey (ACS) is an ongoing mandatory survey conducted by the U.S. Census Bureau that collects data each year to bridge intercensal periods and provide detailed information about the population, including Veteran status. ACS also includes information on current location and location in the previous year. Our analysis uses ACS to determine Veteran geographic distribution and migration patterns. It was not possible to use ACS to accurately measure the number of Veterans in the population; ACS is generally acknowledged to undercount Veterans, though it is assumed to accurately capture the distribution of Veteran characteristics (age, sex, race/ethnicity, service cohort, location). For example, the 2013 ACS estimates of total Veteran population are roughly equivalent to our own estimates of the number of Veterans observed in the 2000 Census who are estimated to still be living in 2013 - that is, ACS estimates effectively undercount by the number of new Veterans who entered the population from 2000 to 2013. For this reason, the team produced a set of population projections using a combination of Census 2000 data, DoD data, and ACS data. More specifically, the team used the 2005-2009 and 2009-2013 ACS five-year estimates available through the American FactFinder website (U.S. Census Bureau, undated). ACS data prior to 2005 do not have information about residence in previous year, which is necessary for migration estimates.

The National Survey of Veterans (NSV), sponsored by VA, is a recurring nationwide survey of Veterans, military service members, and their families. Our analysis used the 2010 NSV survey. NSV data include individual-level demographic and socioeconomic characteristics, general health status, branch of service and period of active duty, and self-reported enrollment in VA and use of VA benefits and services. NSV data were used to estimate characteristics of current Veterans who use VA medical services and how these differ from Veterans who are not VA patients.

The VA Survey of Veteran Enrollees' Health and Reliance Upon VA (SoE) is a recurring nationwide survey of more than 40,000 Veterans enrolled in the VA system. The survey includes each enrollee's demographic and socioeconomic characteristics, general health status, period of military service, and priority group (from administrative record). It also includes 
information relevant to enrollees' relationships with the VA system, including when and why individuals enrolled in VA, health insurance coverage, long-term care insurance, use of VA and non-VA services, and payer information. We used this survey to determine the characteristics of Veteran VA enrollees predicting use (or non-use) of VA services and, in combination with demographic projections, future rates of VA use.

VA encounter data include individual-level information on diagnoses, demographic characteristics, and geographic location (state). VA encounter data were used to estimate current and prior condition prevalence patterns among active VA patients. These data include all health care encounters provided or paid for by VA, which allowed us to estimate the prevalence of service-connected health conditions that exhibit very low prevalence in the national population. A limitation of these data is that they do not capture Veterans who did not use VA care. Moreover, the database includes only health conditions of VA patients that were treated at VA. This may represent only a subset of total health conditions if VA patients also seek care from non-VA providers. Most Veterans with service-connected disabilities use VA (RAND analysis of FY 2014 VHA Support Service Center Current Enrollment Cube data), so the prevalence estimates for these conditions are expected to be more representative of the overall prevalence in the Veteran population.

VA enrollment data are derived from the Health Eligibility Center enrollment files via the VA Business Intelligence System Current Enrollment Cube and the Assistant Deputy Under Secretary for Health enrollment file. These sources include the counts of VA patients and VA enrollees by state of residence, sex, age group, and Iraq-Afghanistan deployment status. We used data covering 2005 to 2014.

Military Health System (TRICARE) encounter data include information on diagnoses and demographic characteristics of active military personnel. We used TRICARE data to estimate the prevalence of health conditions among separating personnel, which may be useful in predicting health conditions among future VA service users and in determining variation in demand for VA services by health condition.

The Active Duty Master and Loss files provide an inventory of all individuals on active duty (excluding reservists on active duty for training) for the Army, Navy, Marine Corps, Air Force, Coast Guard, Public Health Service, and National Oceanic and Atmospheric Administration Commissioned Corps at a point in time. Relevant personal data elements include date of birth, sex, race, and ethnic group. Relevant military data elements include months of service and basic active service date, as well as anticipated service contract end date. The Active Duty Military Personnel Transaction file contains a transaction record for every individual entrance, separation, or reenlistment in the Army, Navy, Air Force, Marine Corps, and Coast Guard within a specific time frame. The Active Duty Loss files are subsets of the Master/Transaction file. The team used these data to supplement the 2000 Census counts for April 2000 to December 2014. Each separation, or "loss," indicates an incoming Veteran to the civilian population.

The Work Experience (WEX) file contains a longitudinal record for each individual who has served in the active or reserve forces since September 1990. For those individuals, the WEX includes information on service back to 1975 . The file is organized by "transactions"; in other words, a new record is generated whenever there is a change in the key variablesservice/component/reserve category, pay grade, occupation (primary, secondary, or duty), and unit identification code. The WEX is built from information in DMDC's Active Duty Master Personnel Edit file, equivalent reserve files, and the underlying service files. 
The Contingency Tracking System is an administrative data set that contains one record for every activation or deployment in support of the conflicts in Afghanistan and Iraq. These deployment data were linked to the DMDC Loss files in many analyses throughout this report to track the portion of separating service members who were ever deployed over time.

Table 2-4 contains definitions of Veteran and VA patient in the nationally representative survey data used in the analyses in this report. The two VA surveys (NSV and SoE) and VA administrative data (VA encounter, VA enrollment) define the two terms according to the legal definition described above. The DoD administrative data (MHS TRICARE, Active Duty Master and Loss files, and Contingency Tracking System) are designed around service members and therefore do not explicitly contain Veteran and VA patient data. We define Veterans in these data to mean those who have separated from the military after serving two or more years, and for reservists, a period of activation of 30 days or more is required. We do not exclude former service members who received other-than-honorable discharges.

Table 2-4

Survey Definitions of Veteran and VA Patient

\begin{tabular}{lll}
\hline Data File & \multicolumn{1}{c}{ Veteran } & VA Patient \\
\hline MEPS & Self-report of being honorably discharged by the military & $\begin{array}{l}\text { Respondents who had } \\
\text { any payment by VA } \\
\text { for services used }\end{array}$ \\
\hline NHIS & Self-report of having served in active military duty & N/A \\
\hline BRFSSF & Self-report of having ever served in active military duty & N/A \\
\hline ACSF & $\begin{array}{l}\text { Once an individual has ceased to "ever serve on active duty } \\
\text { in the U.S. Armed Forces, military Reserves or National } \\
\text { Guard. Active duty does not include training for the } \\
\text { Reserves or National Guard, but does include activation, for } \\
\text { example, for the Persian Gulf War" }\end{array}$ & \\
\hline NSV & VA definition & VA definition \\
\hline
\end{tabular}




\section{Current and Future Demographics of the Veteran Population}

\subsection{Introduction}

Although many national surveys collect information on Veterans, there has not been a full national accounting of all Veterans since the 2000 Census. This chapter presents estimates of the number of Veterans and their demographic characteristics. Motivated directly by the language of the Veterans Choice Act, this chapter describes the current population of Veterans in the United States, categorizes them according to age, sex, race/ethnicity, service cohort, and geographic location, and projects how this population will change by 2024 . These estimates are intended to be informative in their own right, as well as to support analyses in Chapter Four to predict VA enrollment, in Chapter Five to predict health care needs, and in Chapter Six to conduct scenario testing.

The team derived these estimates using standard demographic techniques. We estimated the national population using cohort component population projection methods, and estimated migration flows using gravity models. Cohort component population projection is a method that estimates future population sizes by applying mortality rates specific to age, sex, and race/ethnicity to a baseline population. The projection method also accounted for new Veterans entering the population as they leave the military throughout the projection period, and applied the same mortality rates to them moving forward. The team used data from DoD to determine the number and characteristics of new Veterans entering the population from 2000 to 2014 . The team further assumed that total military end-strength would decline and that there would be no significant new conflicts over the projection period. ${ }^{1}$ We used data from the U.S. Census Bureau to initially distribute our 2013 national projections, then used gravity models to estimate the migration flows of Veterans through $2024 .^{2}$ For complete details of the methods used to derive the population projections, see Appendix A.

This chapter defines Veteran according to information available in the ACS and DoD data. ACS characterizes Veterans as those who "ever served on active duty in the U.S. Armed Forces, military Reserves or National Guard. Active duty does not include training for the Reserves or National Guard, but does include activation, for example, for the Persian Gulf War." Once individuals have ceased to serve on active duty in any of these capacities, they are considered Veterans for the purposes of the projections. Note that ACS does not have information on length of service (only eras of active duty) or on status of discharge. Thus, not all Veterans in the projection exercise may qualify for VA services. For example, Veterans who

\footnotetext{
1 Refer to Chapter Six for more discussion on how future conflicts would affect these results.

2 Gravity models are statistical models of migration that take into account a variety of factors, including age, sex, race/ ethnicity, service era, population size of sending and receiving areas, and distance between areas.
} 
served less than two years or Veterans with "bad paper" discharges (dishonorable, other-thanhonorable, and bad conduct discharges) are all ineligible for VA services but are included in the definition of Veteran in this chapter. Additionally, in projections of future Veteran counts, DoD separation data were used to identify individuals who separate from the military. Here, because personnel files were used, we were able to account for years served and whether an individual deployed during his or her time in service.

\section{Overview of Methods and Data for Demographic Analysis}

- We estimated the 2014 and 2024 population of U.S. Veterans using a cohort component population projection method.

- Data for this analysis came from the U.S. Census for baseline national projections; we then factored in estimates of mortality, adjusted for demographic characteristics, and added data from DoD on Veterans entering the population.

- We estimated migration flows of Veterans using gravity models.

- Supplementary data came from ACS and accounted for a variety of factors, including age, sex, race/ethnicity, service era, population size of sending and receiving areas, and distance between areas.

- For complete details of the methods used to derive the population projections, see Appendix A.

\subsection{Results: The U.S. Veteran Population, 2014 and $2024^{3}$}

\subsubsection{Total Population}

We estimate that the U.S. Veteran population will shrink over the next decade-declining from 21.6 million in 2014 to 17.5 million by 2024, a 19-percent decrease (Figure 3-1). Over a longer time frame, VA estimates that the total number of Veterans will continue to decline, by 37 percent between 2008 and 2033 (U.S. Government Accountability Office, 2009) due to the continued aging of the Veteran population and military downsizing. This projected decrease suggests that in 2032, there will be fewer than 15 million living Veterans (McGeary et al., 2007). Detailed comparisons with VA estimates are discussed in Appendix A.

\subsubsection{Race/Ethnicity}

We estimate that the proportion of Veterans who are non-Hispanic white will decline slightly, from 80 percent in 2014 to 76 percent by 2024. The literature shows that the racial and ethnic composition of the Veteran population varies by age. Older Veterans are primarily nonHispanic white, while younger Veterans are more likely to be racially and ethnically diverse (Lee \& Beckhusen, 2012). Gulf War-era Veterans are more racially and ethnically diverse than prior cohorts of Veterans (Holder, 2014). In 2012, minorities accounted for 20 percent of the male Veteran population and 38 percent of male non-Veterans (National Center for Veterans Analysis and Statistics, 2014c). Among Veterans, the two largest minority groups were Veterans who were black or Hispanic (National Center for Veterans Analysis and Statistics, 2011a, 2014a). Figure 3-2 shows our estimates of the racial and ethnic composition of the Veteran population in 2014 and in 2024. Like VA, we estimate that the largest minority groups represented in the Veteran population are Veterans who are black or Hispanic.

3 Detailed tables and figures are presented in Appendix B, Table B-3. Here we highlight the main features of the results. 
Figure 3-1

Projected Veteran Population, 2014-2024

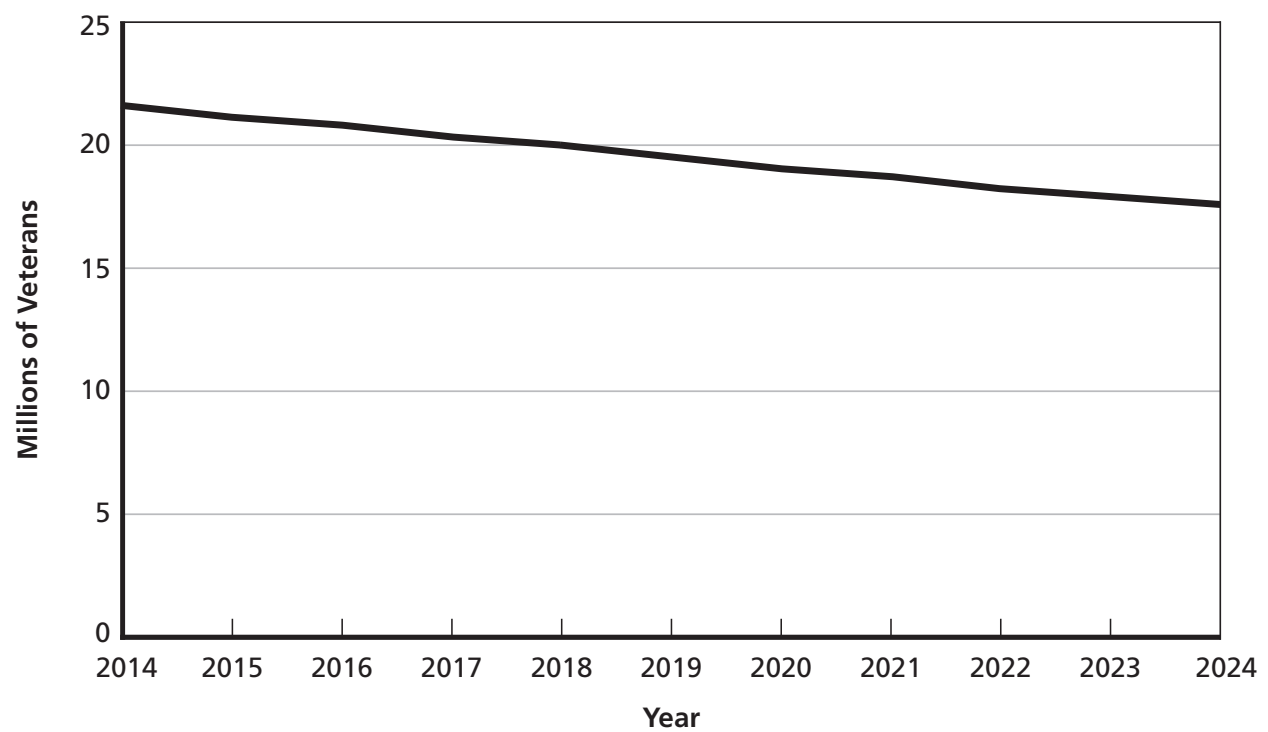

SOURCE: RAND analysis of VA, DoD, and Census data RAND RR1165z1-3.1

Figure 3-2

Race/Ethnicity Composition of the Veteran Population, 2014 and 2024 (Projected)

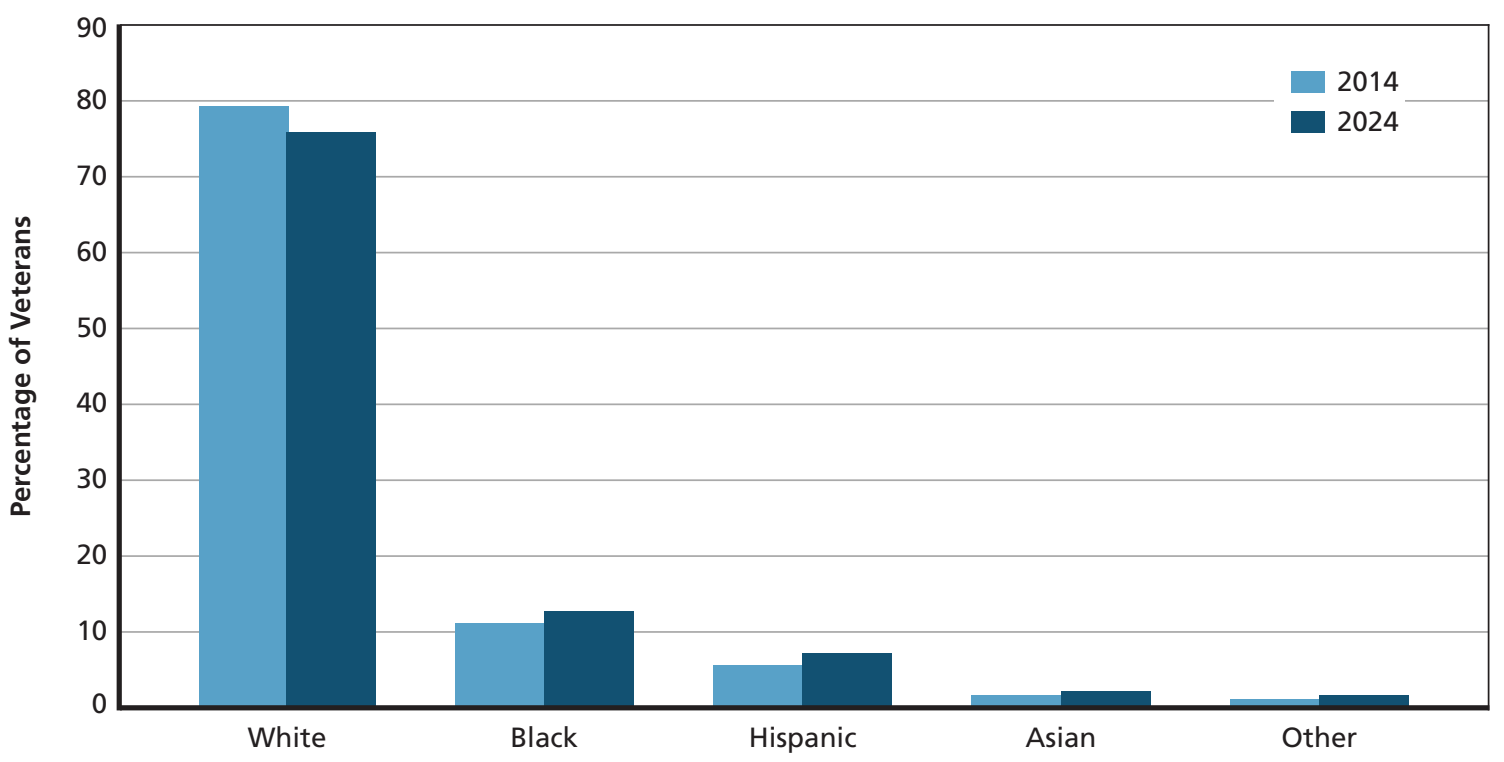

SOURCE: RAND analysis of VA, DoD, and Census data. RAND RR1165z1-3.2 


\subsubsection{Sex}

We estimate that the proportion of male Veterans will decline from 92 percent to 89 percent by 2024 . Based on the growth trend observed since 2000, the total number of female Veterans is projected to increase very slightly at the same time (Figure 3-3), leading to a 38-percent increase in the relative share of female Veterans, from 8 percent to 11 percent of the Veteran population by 2024 . Despite this increase, the Veteran population remains predominately male throughout the projection period.

Our estimates are in line with the existing literature on this topic, which confirms that the majority of Veterans will continue to be men, but the proportion of women is growing (U.S. Government Accountability Office, 2009). In 2008, 15 percent of active duty military and 7.7 percent (1.8 million) of Veterans were women (National Center for Veterans Analysis and Statistics, 2011a; U.S. Census Bureau, 2014). Approximately 33 percent of female Veterans were minorities in 2012 (National Center for Veterans Analysis and Statistics, 2014a). Female Veterans were more likely than non-Veterans to be non-Hispanic black (19 percent versus 12 percent) or non-Hispanic white (69 percent versus 67 percent) (National Center for Veterans Analysis and Statistics, 2011a).

In Figure 3-4, we display the number of new service members who separate from the active component to become Veterans. We estimate that the total number of new Veterans will decrease from approximately 192,000 in 2015 to 162,000 in 2024 . The decrease in the total number of new Veterans is driven mostly by separations of male service members, down from 164,000 in 2015 to 138,000 in 2024. We note that new Veterans from the active component represented approximately 1 percent of all Veterans in 2014 (roughly 224,000 out of 21.6 million).

Figure 3-3

Total Veteran Population, by Sex, 2014-2024 (Projected)

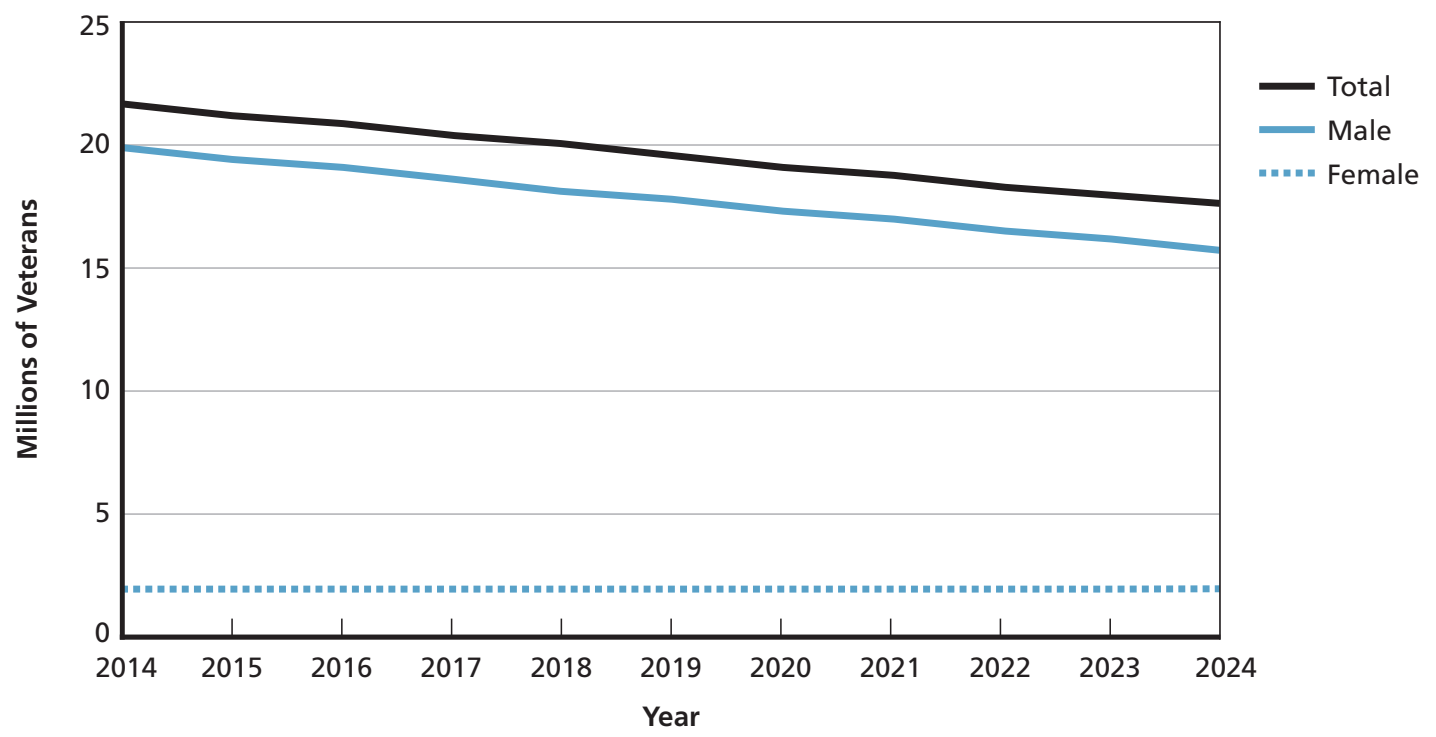

SOURCE: RAND analysis of VA, DoD, and Census data. RAND RR1165z1-3.3 
Figure 3-4

Number of New Veterans from Active Component, Total and by Sex

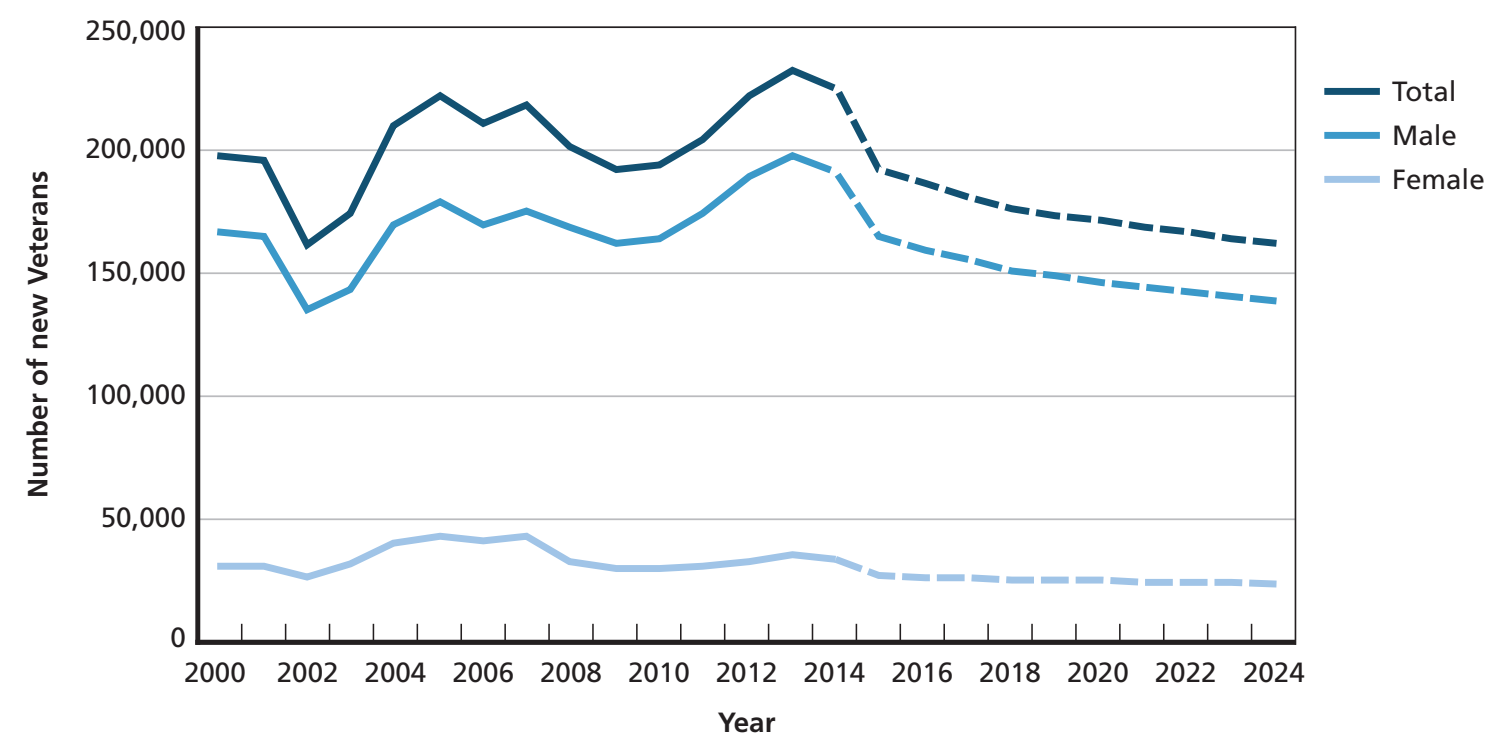

SOURCE: RAND analysis of DoD data.

RAND RR1165z1-3.4

\subsubsection{Age}

We estimate that the age structure of Veterans will shift between 2014 and 2024, as shown in Figure 3-5. We estimate that the share of Veterans ages 45-64 will decline from 34 percent to 31 percent of the Veteran population, while the share of both younger and older Veterans will increase; the share of Veterans age 65+ will increase from 49 percent to 51 percent by 2024; and the share of all Veterans at the oldest ages (85+) will increase from 9 percent to 10 percent. As shown in Figure 3-6, Veterans' mean age will increase slowly throughout the period. Male Veterans' mean age will rise much more slowly than female Veterans' mean age, although female Veterans are substantially younger than male Veterans overall, and will continue to be so during the projection period.

We also examined the age profile of newly separated active component service members and plot the results in Figure 3-7. While the number of service members who are projected to separate between 2015 and 2024 is relatively constant for those in age groups 35-44 and 45+, the number of new Veterans under age 35 is expected to decrease throughout the 10-year projection horizon. We estimate that 146,000 new Veterans will be under age 35 in 2015, and that number is expected to decrease to 123,000 by 2024 .

A profile of the current age structure shows that in 2014, the largest conflict-era cohort-Vietnam-era Veterans_-averaged 67 years of age, while the second-largest conflictera cohort_Gulf War-era Veterans_-averaged 47 years of age (see Table 3-1). 
Figure 3-5

Age Structure, 2014 and 2024 (Projected)

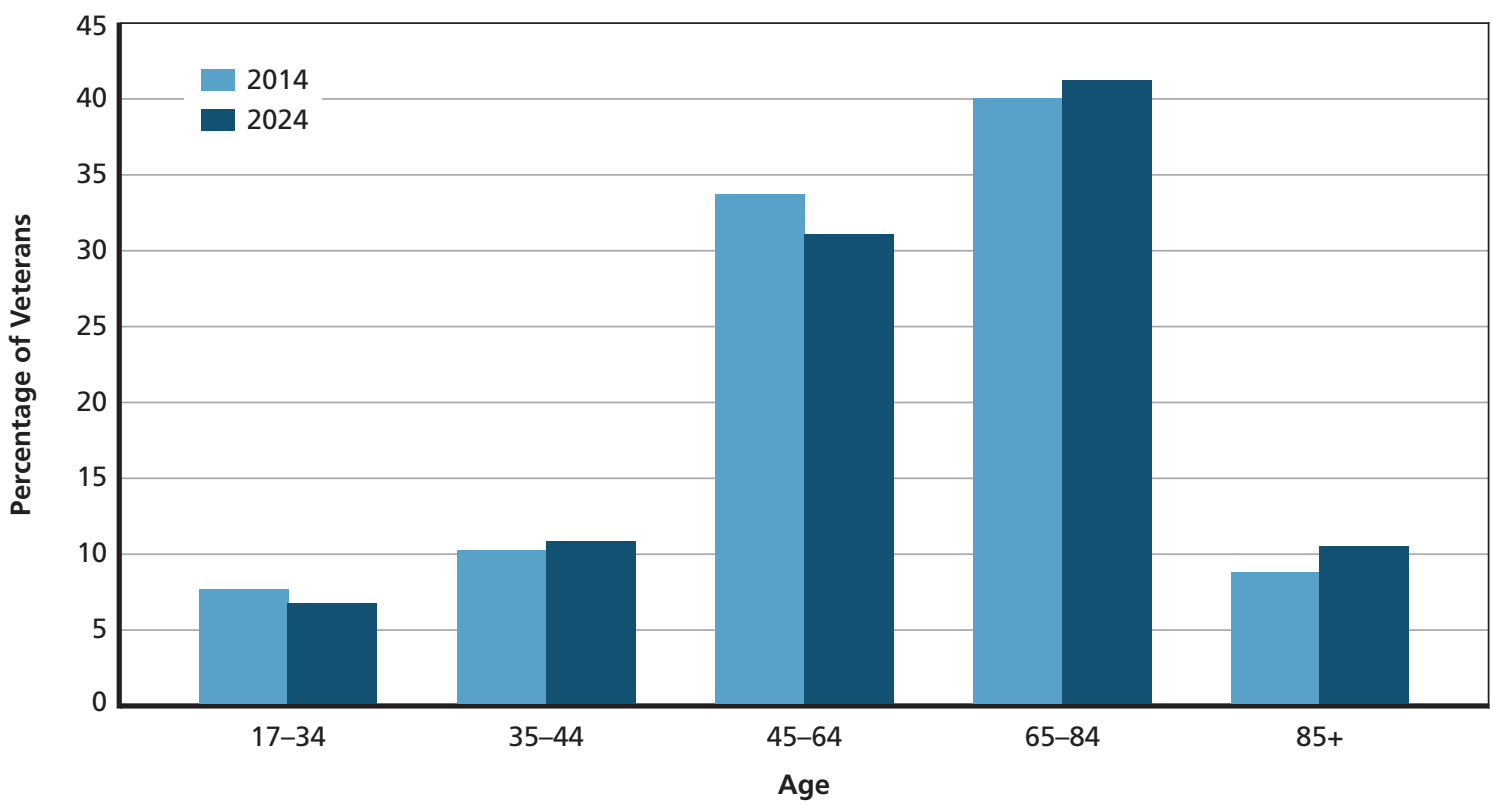

SOURCE: RAND analysis of VA, DoD, and Census data.

NOTE: Population in 2014: 21.6 million. Population in 2024: 17.5 million.

RAND RR1165z1-3.5

Figure 3-6

Mean Age, by Sex, 2014-2024 (Projected)

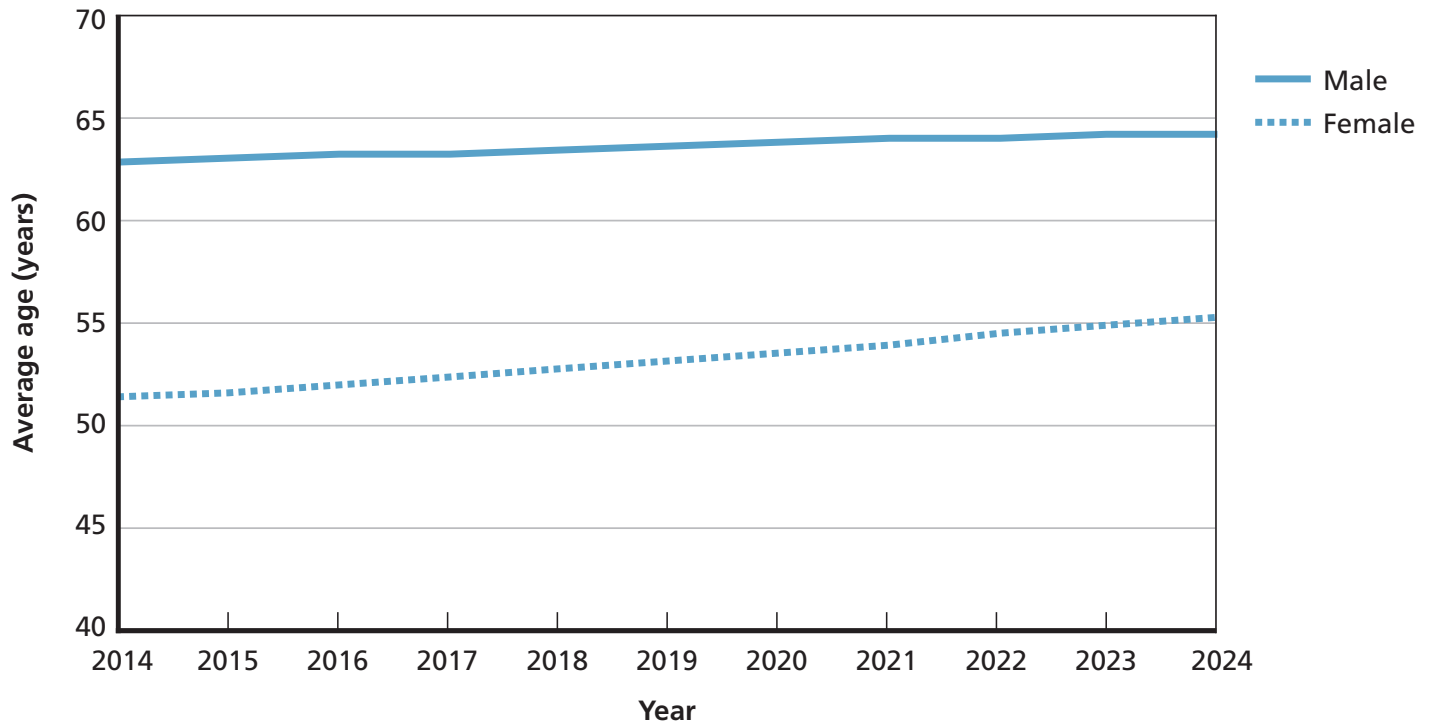

SOURCE: RAND analysis of VA, DoD, and Census data.

RAND RR1165z1-3.6 
Figure 3-7

Number of New Veterans from Active Component, by Age

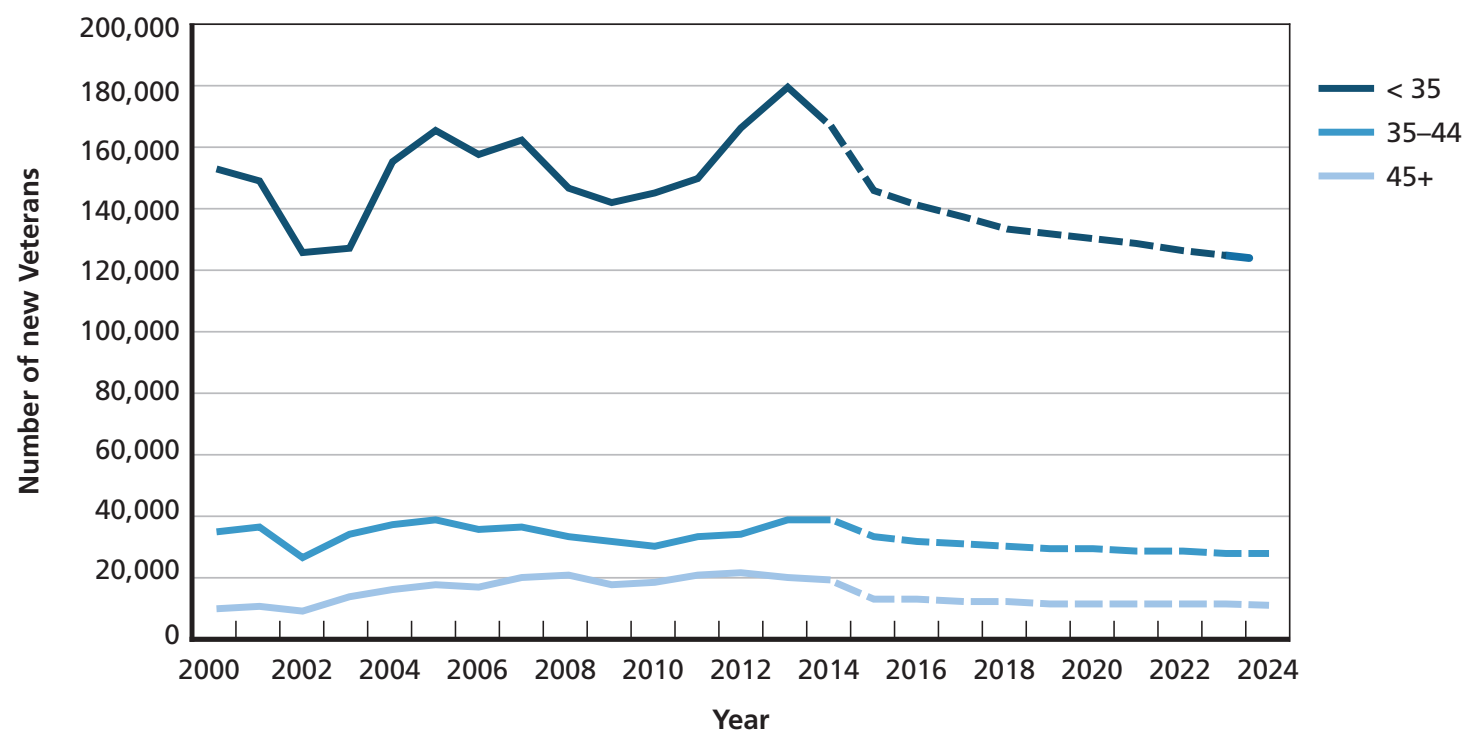

SOURCE: RAND analysis of DoD data.

RAND RR1165z1-3.7

Table 3-1

Number and Mean Age of Veterans, by Era of Service (2014)

\begin{tabular}{lcc}
\hline Era of Service & Mean Age & Veterans (millions) \\
\hline Pre-1950 & 86 & 1.6 \\
Korean conflict & 82 & 2.0 \\
Pre-Vietnam peace & 75 & 2.1 \\
Vietnam & 67 & 6.7 \\
Post-Vietnam peace & 53 & 3.3 \\
Gulf War & 47 & 3.2 \\
Post-9/11 & 36 & 2.6 \\
\hline
\end{tabular}

SOURCE: RAND analysis of VA, DoD, and Census data.

NOTE: Individuals are grouped into the most recent active duty wartime era they served in (if they report multiple periods of service), or if they only served during peacetime, they are grouped into their most recent peacetime era.

\subsubsection{Service Cohort}

The team estimates that pre-Vietnam-era Veterans constituted 25 percent of the total Veteran population in 2014 , but by 2024 , their share is projected to fall to 13 percent of the total. The analysis also estimates that the share of Vietnam-era Veterans (1964-1975) will decline slightly from 32 percent to 29 percent of the Veteran population by 2024. These estimates are consistent with earlier numbers; in 2000, Vietnam-era Veterans were estimated to account for 31.7 percent of the Veteran population (Richardson \& Waldrop, 2003). The RAND projection estimates that the proportion of Gulf War-era and post-9/11-era Veterans will grow from 
26 percent to 41 percent of the total Veteran population by 2024; post-9/11 Veterans alone will account for 24 percent of all Veterans in 2024. Figure 3-8 presents the projected serviceera cohort composition changes in the Veteran population over time (pre- and post-Vietnam peacetime-only service eras are not presented), highlighting the rapid proportional growth of the post-9/11 era.

\subsubsection{Socioeconomic Status}

\subsubsection{Employment}

Educational and economic measures are routinely considered in the examination of demographic events and processes (O'Hare, Pollard and Ritualo, 2004). Our review of the literature found that in 2014, approximately 5 percent of Veterans were unemployed compared with 6 percent of non-Veterans (Bureau of Labor Statistics, 2015). There was notable variation in unemployment by service era. Approximately 7 percent of Veterans who deployed to Afghanistan and Iraq were unemployed in 2014 (Bureau of Labor Statistics, 2015). In 2014, there were approximately 573,000 unemployed Veterans (Bureau of Labor Statistics, 2015). The majority of the unemployed Veterans (59 percent) were age 45 or older (Bureau of Labor Statistics, 2015). Comparisons between Veterans and civilians that account for disability status show that Veteran status and being female are associated with higher rates of unemployment, though disability is the strongest predictor of being unemployed (Smith, 2014).

In Table 3-2, we present data on the socioeconomic status of the Veteran and non-Veteran civilian populations using ACS data from 2009 to 2013. The data in the table are interpreted as the mean throughout the 2009-2013 period. ${ }^{4}$ According to ACS, unemployment was lower

Figure 3-8

Conflict-Era Veterans as Percentage of Total Veteran Population, 2014-2024 (Projected)

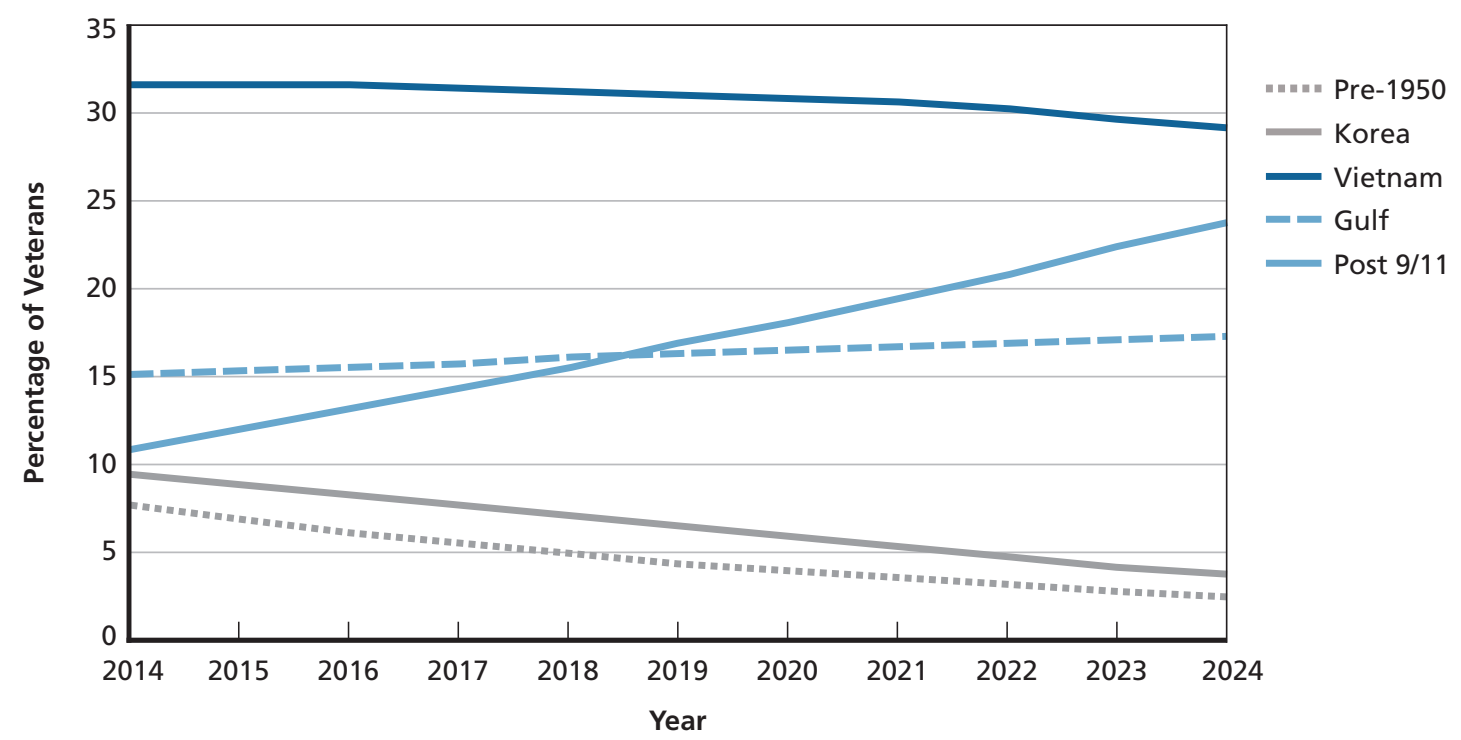

SOURCE: RAND analysis of VA, DoD, and Census data.

RAND RR1165z1-3.8

4 ACS estimates are period estimates; when precision of estimates is more important than currency of estimates, the U.S. Census Bureau recommends using the five-year ACS estimates rather than the one-year estimates. Thus, we rely on the fiveyear ACS estimates throughout this section. See Beaghen \& Weidman, 2008. 
Table 3-2

Socioeconomic Characteristics of the Veteran and Non-Veteran Civilian Population, by Sex, 2009-2013

\begin{tabular}{|c|c|c|c|c|c|c|}
\hline \multirow{2}{*}{$\begin{array}{l}\text { Socioeconomic } \\
\text { Characteristics }\end{array}$} & \multicolumn{2}{|c|}{ Non-Veteran Civilians } & \multicolumn{2}{|c|}{ Veterans } & \multicolumn{2}{|c|}{ Veterans with VA Insurance } \\
\hline & Female & Male & Female & Male & Female & Male \\
\hline$\%$ unemployed & 9.07 & 10.26 & 8.30 & 8.43 & 12.15 & 12.58 \\
\hline Avg. total family income & $\$ 86,335$ & $\$ 92,117$ & $\$ 89,547$ & $\$ 87,533$ & $\$ 79,576$ & $\$ 73,717$ \\
\hline Avg. total personal income & $\$ 27,113$ & $\$ 44,456$ & $\$ 38,304$ & $\$ 50,964$ & $\$ 35,775$ & $\$ 41,514$ \\
\hline$\%$ income $<100 \% \mathrm{FPL}$ & 15.25 & 12.94 & 9.98 & 6.63 & 12.33 & 8.52 \\
\hline$\%$ income $100-250 \%$ FPL & 27.38 & 25.87 & 24.25 & 23.50 & 28.52 & 29.77 \\
\hline$\%$ income $250-400 \% \mathrm{FPL}$ & 21.32 & 21.92 & 23.85 & 24.68 & 24.30 & 25.71 \\
\hline$\%$ income $>400 \% \mathrm{FPL}$ & 36.05 & 39.27 & 41.92 & 45.19 & 34.84 & 36.00 \\
\hline$\%$ Less than high school & 14.81 & 18.51 & 2.41 & 8.05 & 2.45 & 9.57 \\
\hline$\%$ HS graduate or GED & 27.47 & 28.29 & 19.07 & 30.50 & 16.81 & 30.98 \\
\hline$\%$ Some college & 23.85 & 21.69 & 31.60 & 27.43 & 32.65 & 29.11 \\
\hline$\%$ College + & 33.86 & 31.51 & 46.92 & 34.02 & 48.09 & 30.34 \\
\hline$\%$ With medical insurance & 84.90 & 77.24 & 92.62 & 94.15 & 100.00 & 100.00 \\
\hline
\end{tabular}

SOURCE: RAND analysis of Census data.

NOTES: All Veteran means are statistically different (at $p<0.001)$ from non-Veteran civilian means by sex. Unemployment does not include those who are not in the labor force (e.g., retired).

$\mathrm{FPL}=$ federal poverty level, which varies by size of the household.

for Veteran than non-Veteran civilians, but it was slightly higher throughout this period than the level reported by the Bureau of Labor Statistics. The table also indicates that Veterans tend to have higher incomes and education levels than their non-Veteran counterparts. Both male and female Veterans have higher average personal incomes than non-Veterans, and female Veterans have higher average family incomes than non-Veterans. Male Veterans, however, report lower average total family incomes than their non-Veteran civilian male counterparts. For both sexes, Veterans are less likely to live below the poverty line than non-Veterans, and more likely to have graduated from high school. Relative to non-Veterans, Veterans are also more likely to have at least a college degree. Characteristics of Veterans with VA medical insurance are also presented; 5 unemployment is higher, and income is lower, as expected by design.

\subsubsection{Income}

Corresponding to the analysis described above, the literature also suggests that Veterans are less likely to live below the poverty line than non-Veterans, and that Veterans have higher median incomes $(2000,2009$, 2012) (National Center for Veterans Analysis and Statistics, 2011c, 2014a, 2014d). Veterans working full-time had higher median earnings and personal incomes than non-Veterans in 2012 (National Center for Veterans Analysis and Statistics,

5 Individuals' insurance status is reported by the primary householder responding to the ACS survey, who responds on behalf of all members of the household. 
2014c). Compared with male Veterans, female Veterans are more likely to have no health insurance coverage, have no income, and live in poverty (National Center for Veterans Analysis and Statistics, 2014d). VA patients are more likely to have a lower household income compared with non-patients. However, these differences are not surprising, given that eligibility for VA care is partly dependent on income (National Center for Veterans Analysis and Statistics, 2014e).

\subsubsection{Homelessness}

In 2010, Veterans accounted for approximately 10 percent of the adult population; however, they represented a disproportionate share of the homeless adult (16 percent) and sheltered homeless adult (13 percent) populations (National Center for Veterans Analysis and Statistics, 2012b). Approximately 10 percent of homeless Veterans are women (U.S. Department of Housing and Urban Development, 2014), and female Veterans are three to four times more likely than non-Veteran women to become homeless (Washington et al., 2010). Among female Veterans, sexual assault during military service, unemployment, disability, and poor physical and mental health are associated with being homeless (Washington et al., 2010). While homelessness is a significant problem among the Veteran population, the total size of the homeless Veteran population has decreased over time. The U.S. Department of Housing and Urban Development reported that there were 49,933 homeless Veterans in 2014, representing less than 0.25 percent of the total Veteran population. Between 2010 and 2014, the number of homeless Veterans declined by 33 percent (U.S. Department of Housing and Urban Development, 2014).

\subsubsection{Geographic Distribution of Veterans}

This section presents a series of maps that show the geographic distribution of the U.S. Veteran population by a variety of characteristics, and how this distribution is expected to change between 2014 and 2024. Understanding the geographic distribution of Veterans is an important consideration for policies that attempt to align the availability of health care services with the Veteran population. The maps on each topic are paired: The first of the two presents information for 2014, and the second presents information for 2024. We report geographic detail using Public Use Microdata Areas (PUMAs), which are geographic units used by the U.S. Census Bureau. Typically, each PUMA contains roughly 100,000 residents. PUMAs respect state borders, but not necessarily county or municipality borders. We provide more-complete information on PUMAs in Appendix A.

Each map shading indicates the total number of Veterans living in all PUMAs within 40 miles of the center of the shaded PUMA. ${ }^{6}$ The text in the lower left corner reports the total number of Veterans depicted in the map, as well as the percentage of the total Veteran population they represent. The bar chart on the lower right serves the dual purpose of reporting how each shade corresponds to the number of Veterans, as well as what portion of the depicted population lives in the PUMA shaded with each color.

\subsubsection{Geographic Concentration of Veterans}

Figure 3-9 depicts the geographic distribution of the Veteran population as a whole in 2014. Like the U.S. population as a whole, the majority of Veterans are concentrated in a small number of heavily urbanized regions. The "Bos-Wash" corridor, a stretch of heavily urbanized

\footnotetext{
6 For an explanation of why we shaded based on all Veterans living near each PUMA rather than just those living in each PUMA, please see Appendix A.
} 
Figure 3-9

Total Veteran Population in 2014

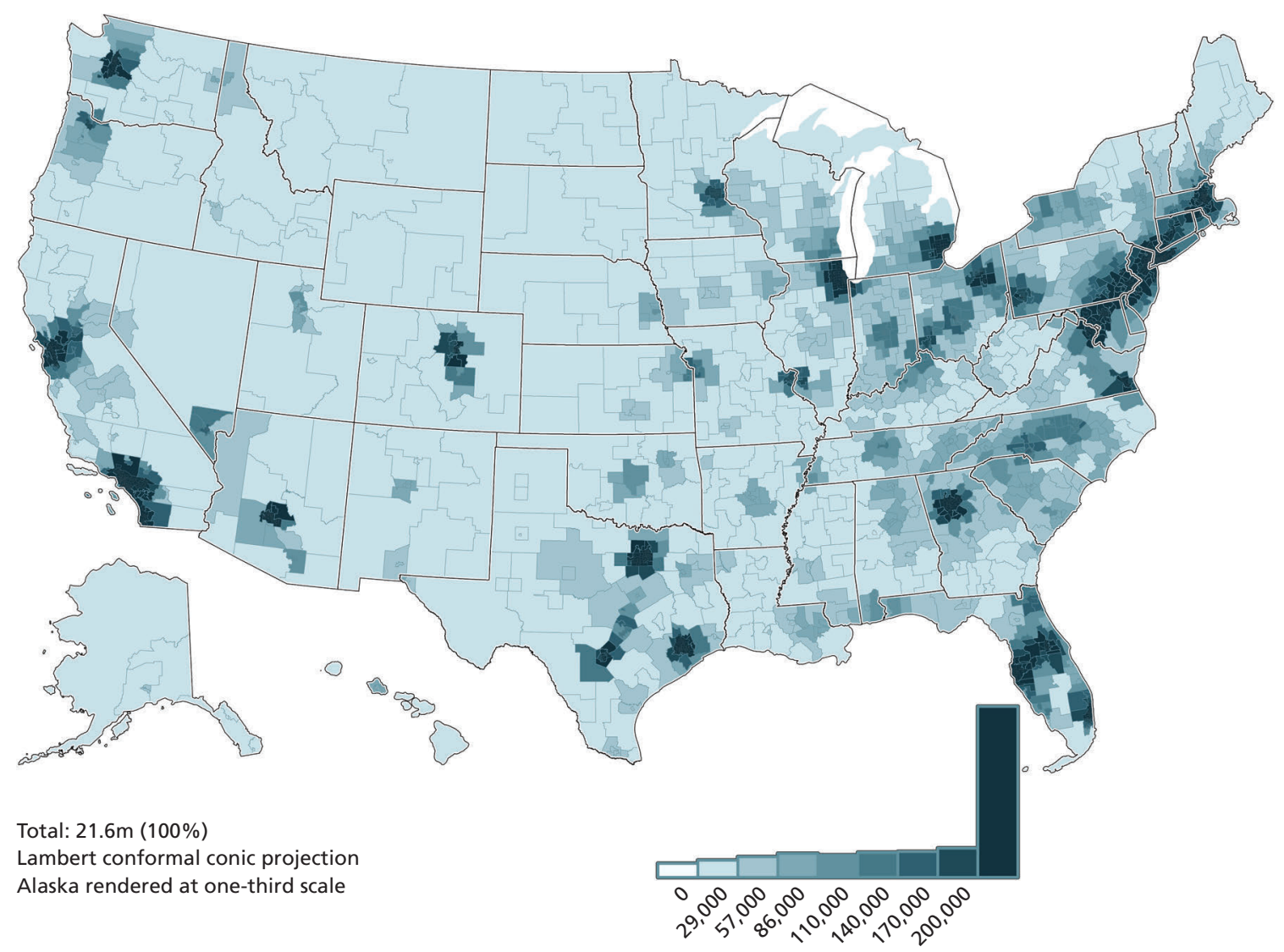

SOURCE: RAND analysis of VA, DoD, and Census data.

RAND RR1165z1-3.9

area that runs from Boston, Massachusetts, to Washington, D.C., contains 30-50 million people and 1.43 million Veterans. ${ }^{7}$ Southern California, including the Los Angeles, Riverside, and San Diego metropolitan areas, contains more than 20.97 million people and 1 million Veterans. Other large cities, such as Chicago (population: $9.55 \mathrm{~m}$, Veterans: $423 \mathrm{k}$ ), Dallas $(6.95 \mathrm{~m}, 360 \mathrm{k})$, Houston $(6.49 \mathrm{~m}, 332 \mathrm{k})$, Atlanta $(5.61 \mathrm{~m}, 405 \mathrm{k})$, Miami $(5.93 \mathrm{~m}, 262 \mathrm{k})$, and the San Francisco Bay Area $(6.55 \mathrm{~m}, 346 \mathrm{k})$, account for another 41.08 million people, including 2.08 million Veterans. Taken together, these eight urbanized regions account for 35 percent of the American population and 20 percent of the Veteran population.

Of the 318.9 million people residing in the United States in 2014, 21.6 million, or 6.8 percent, were Veterans. Slightly more Veterans than expected based on this national average live in Virginia Beach (14 percent), Boston (14 percent), central Florida (10 percent), Cleveland (10 percent), Washington, D.C. (9 percent), and San Antonio (9 percent). Slightly fewer Veterans than expected live in Chicago ( 4 percent), Miami (4 percent), Dallas (5 percent), Los Angeles (5 percent), Houston (5 percent), San Francisco (5 percent), Minneapolis (5 percent), and New York City (3 percent).

\footnotetext{
7 Veteran estimates include all Veterans living in a PUMA within 40 miles of the named city centers. Total population estimates include the entire metropolitan population, not just the population of the named cities proper.
} 
Figure 3-10 displays how the Veteran population is expected to look in 2024. Overall, we expect the population to decline to 17.5 million as older cohorts of Veterans experience high rates of age-related mortality. For the most part, these losses will not change the geographic distribution of Veterans. However, we estimate that the share of Veterans in cities in the Ohio River Valley (the Great Lakes region spanning from western New York through the Midwest), including Buffalo, Cincinnati, Cleveland, Columbus, Detroit, Indianapolis, and Pittsburgh, will decline.

\subsubsection{Age Patterns in Geographic Distribution}

Over time, there will be more diversity in the geographic distribution of Veterans by age. Veteran mean age will grow older over time, but the increases in the proportion of Veterans at both the younger and older ages will alter the geographic distribution of Veterans by age. We estimate that Veterans under age 35 will be concentrated in areas surrounding Los Angeles; Dallas; Washington, D.C.; and northern New Jersey by 2024. Over time, Veterans under age 35 will constitute a greater proportion of the population in Northern California, central Washington state, the Midwest, and Wyoming and Utah. Other portions of the Southwest and much of the Southeastern seaboard, from Virginia Beach through the coast of Georgia, will see a decrease in the proportion of the population that is under age 35. See Figures 3-11 and 3-12.

Figure 3-10

Total Veteran Population in 2024 (Projected)

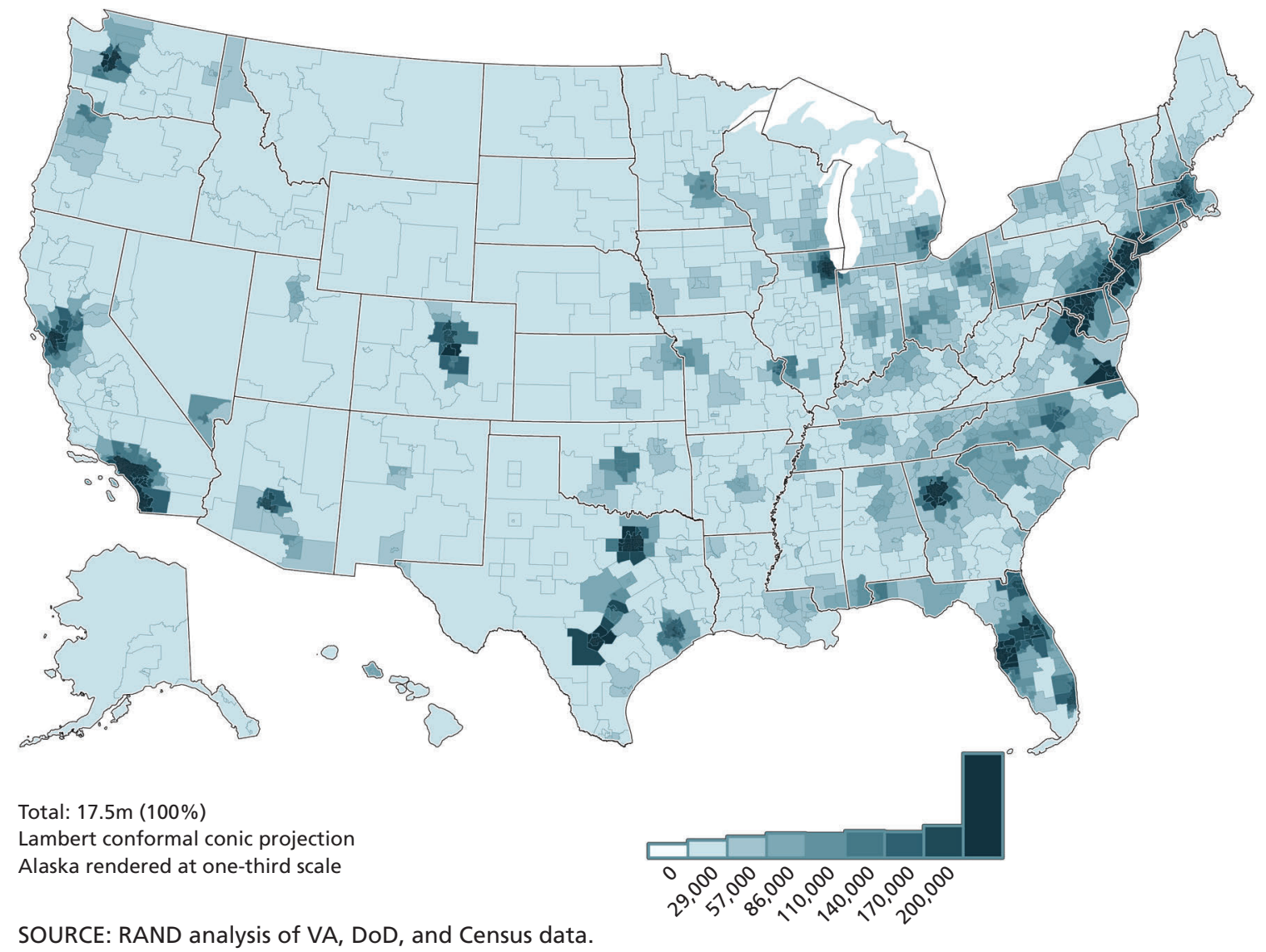


Figure 3-11

Total Veterans Under Age 35, 2014

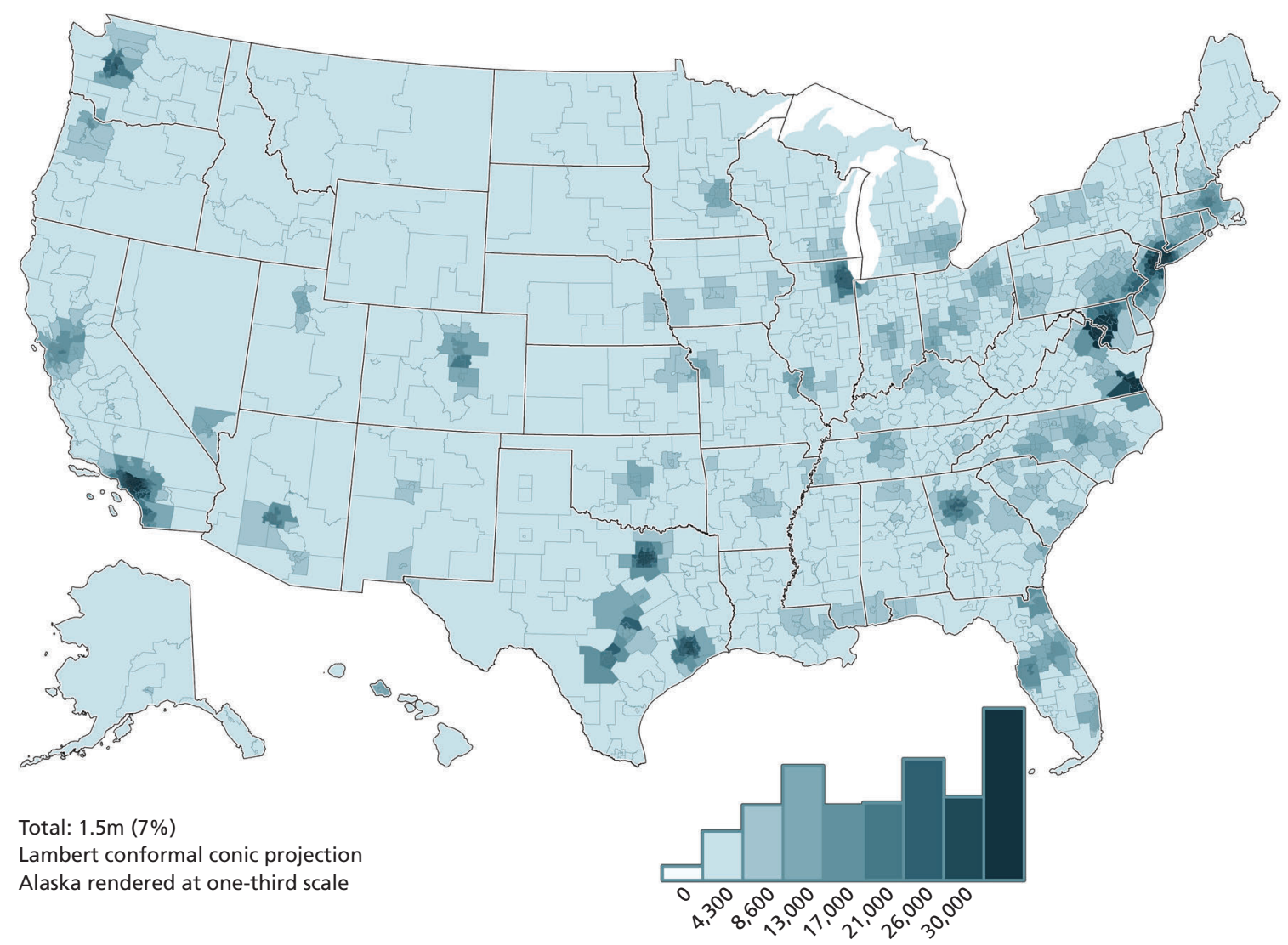

SOURCE: RAND analysis of VA, DoD, and Census data.

RAND RR1165z1-3.11

Concentration of older Veterans in areas of current higher prevalence will continue to 2024 (see Figures 3-13 and 3-14). San Francisco; Los Angeles; Denver; southwestern Texas; much of Florida; Washington, D.C.; western Pennsylvania; northern New Jersey; New York City; and western Massachusetts are currently places in which the share of older Veterans is high, and they are predicted to remain high through 2024. At the same time, we estimate that the share of older Veterans living in much of the Northeast and Florida (especially the panhandle), the Midwest, Wyoming, Utah, and southwestern Alabama will decline.

Trends in geographic distribution by age are likely to reflect cohort changes in where Veterans reside, rather than trends in migration per se. Areas where older Veterans decline in proportion are most likely to be areas where they are not being replaced by incoming cohorts of Veterans. Similarly, areas with proportionate growth in Veterans over age 65 are likely areas where currently middle-aged Veterans live and will continue to live as they age. Florida is an exception to this, as older Veterans will also tend to migrate there (although in relatively low numbers in comparison with the local populations). See Section 3.2.9 for more details. 
Figure 3-12

Total Veterans Under Age 35, 2024 (Projected)

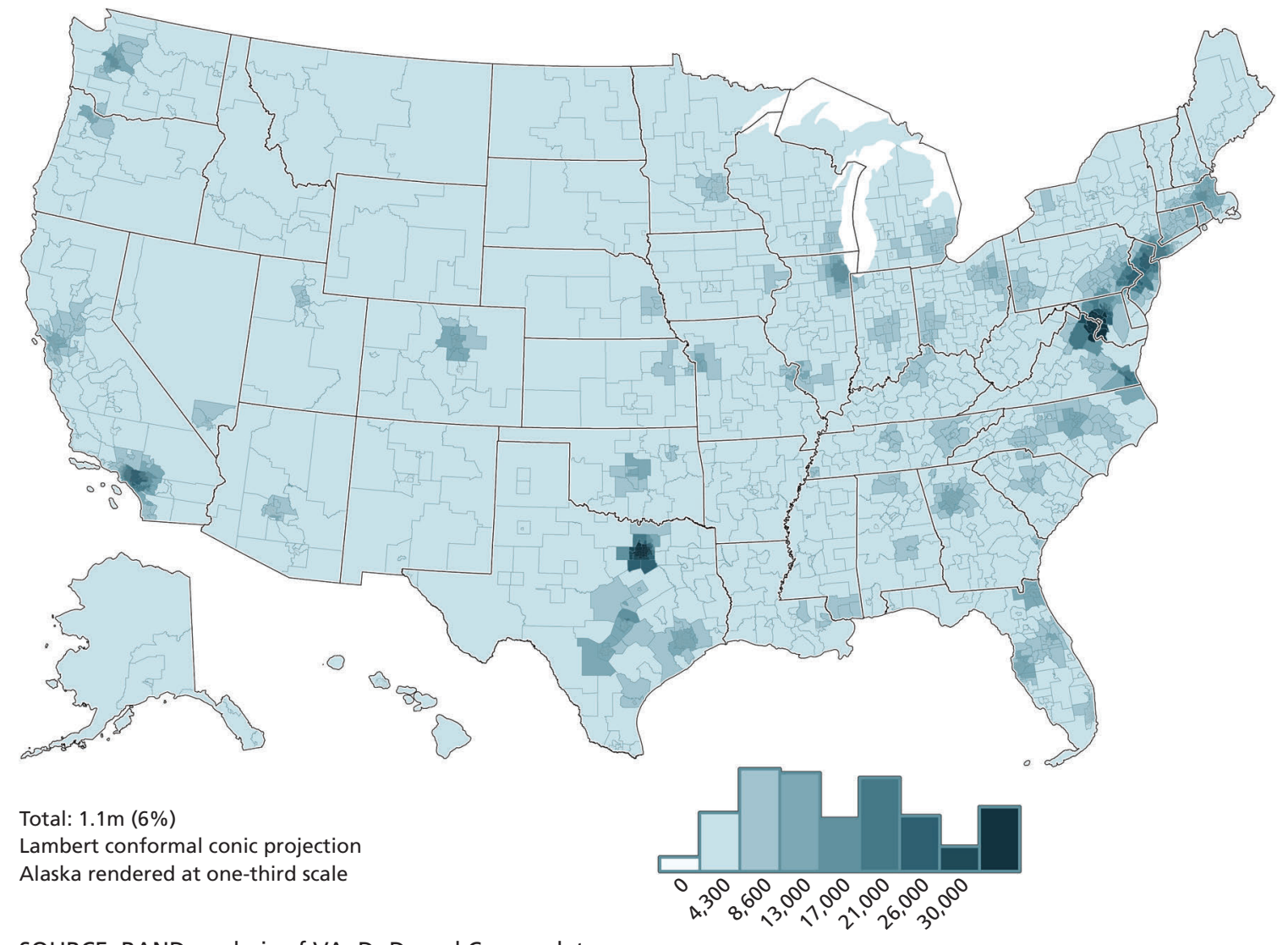

SOURCE: RAND analysis of VA, DoD, and Census data.

RAND RR1165z1-3.12 
Figure 3-13

Total Veterans Age 65+, 2014

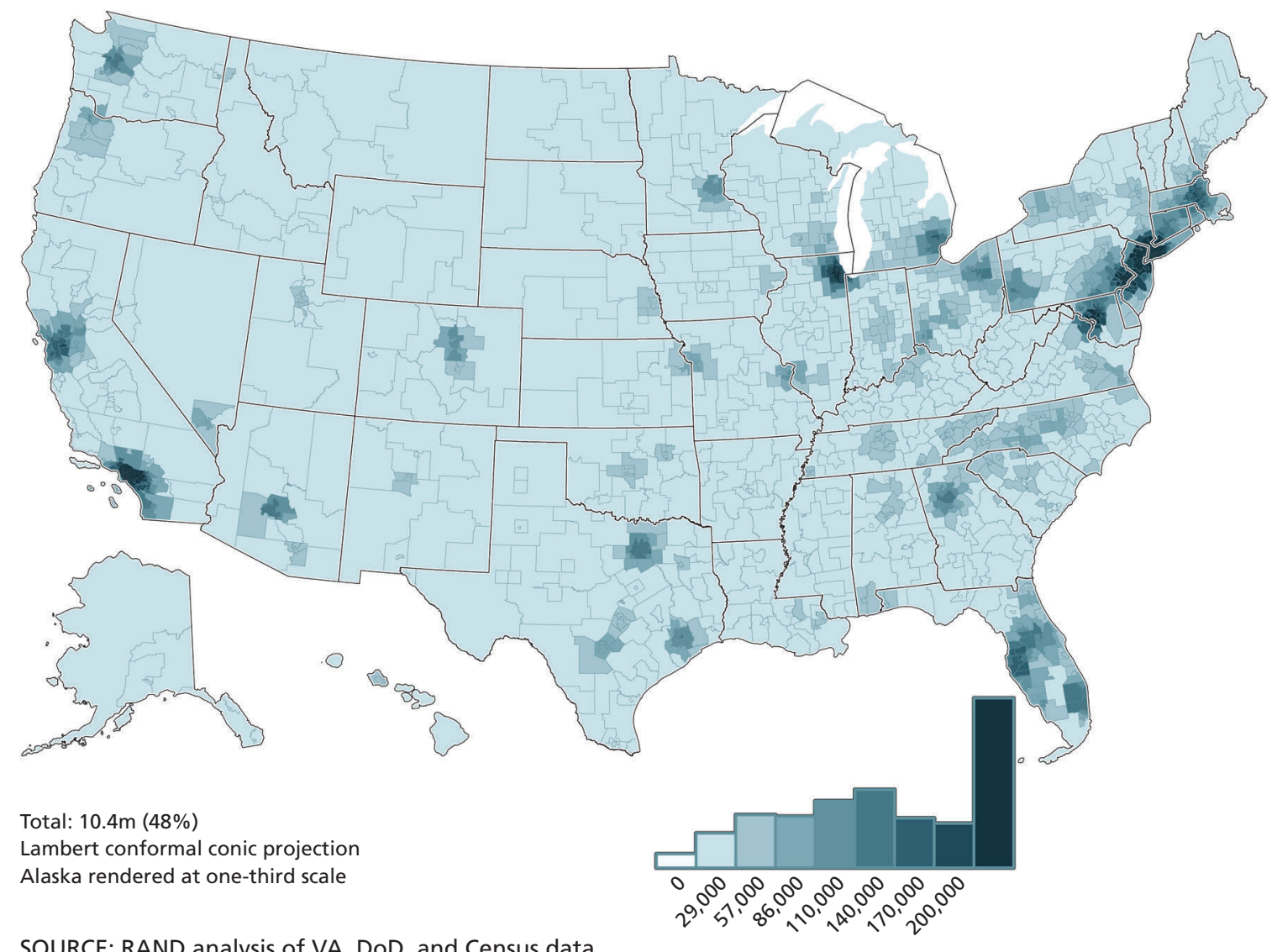

SOURCE: RAND analysis of VA, DoD, and Census data.

RAND RR1165z1-3.13 
Figure 3-14

Total Veterans Age 65+, 2024 (Projected)

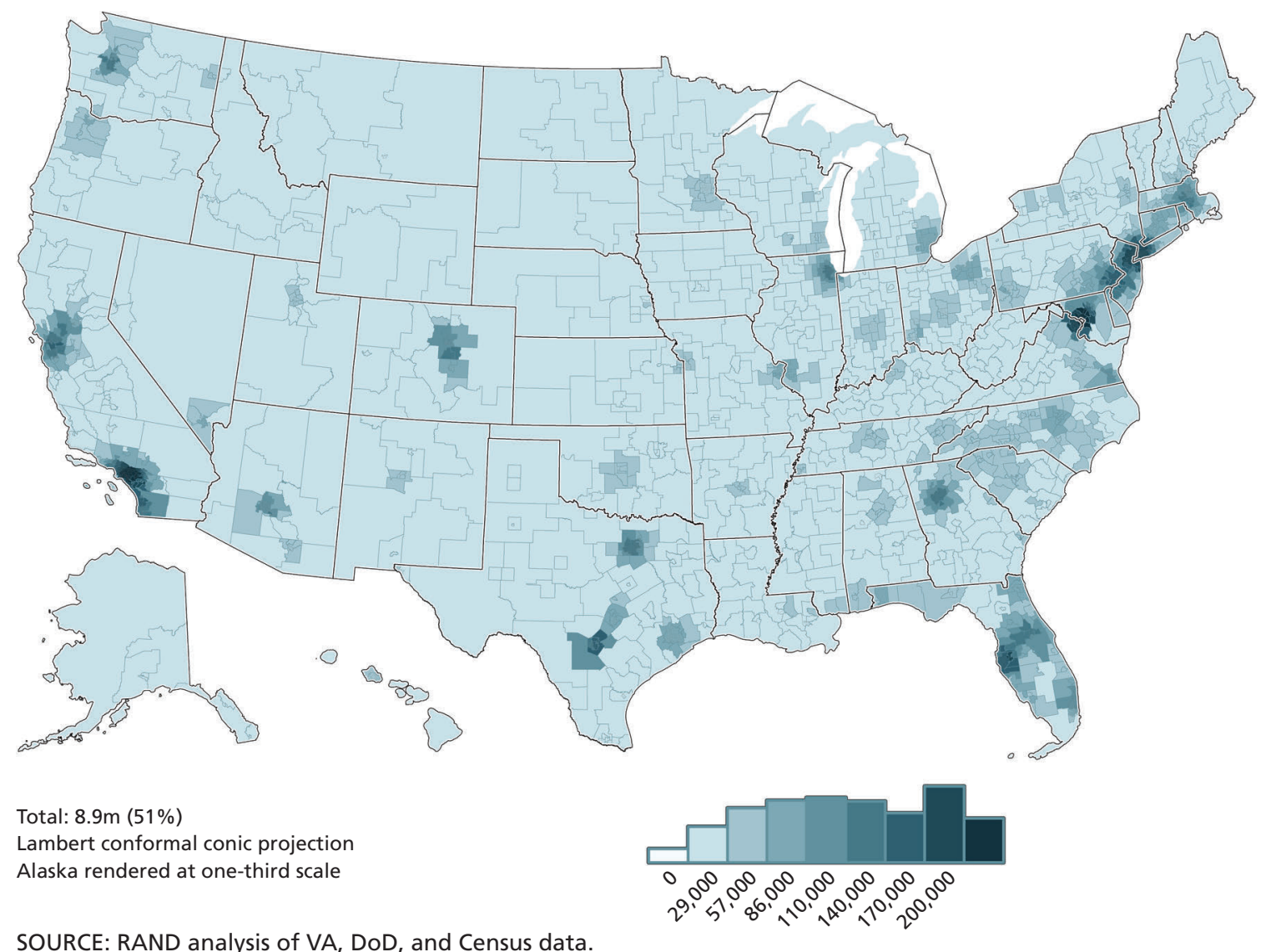

SOURCE: RAND analysis of VA, DoD, and Census data.

RAND RR1165z1-3.14

\subsubsection{The Changing Urban-Rural Distribution}

Over time, fewer Veterans are going to be located in rural areas, ${ }^{8}$ reflecting both the overall national population trend of movement away from rural areas and absence of younger Veterans replacing older rural Veterans. However, northwestern Washington state, a belt running through Montana to Wisconsin, parts of Northern Michigan, much of Maine, Alaska, and northern Texas (Amarillo outskirts) will remain areas of rural Veteran populations by 2024. See Figures 3-15 and 3-16.

\subsubsection{Distance to Nearest Veteran Facility}

The VA medical system relies primarily on two kinds of facilities: VAMCs and CBOCs. VAMCs are full-service medical centers, offering both primary care and specialty care. CBOCs are satellite clinics that provide primary, preventative, and behavioral health services. While VAMCs are the heart of the system, CBOCs provide a cost-effective way to increase access to

8 Rural status is based on rural-urban commuting area codes, which are based on measures of population density, urbanization, and daily commuting from the 2010 Census and 2006-2010 ACS. Our analysis assumes that the classifications will remain the same in 2024 as in 2014, and thus the 2024 maps may be best interpreted as "based on areas that were rural in 2014." Refer to the U.S. Department of Agriculture Economic Research Service for more information on rural-urban commuting area codes (U.S. Department of Agriculture, 2014). 
Figure 3-15

Total Rural Veterans, 2014

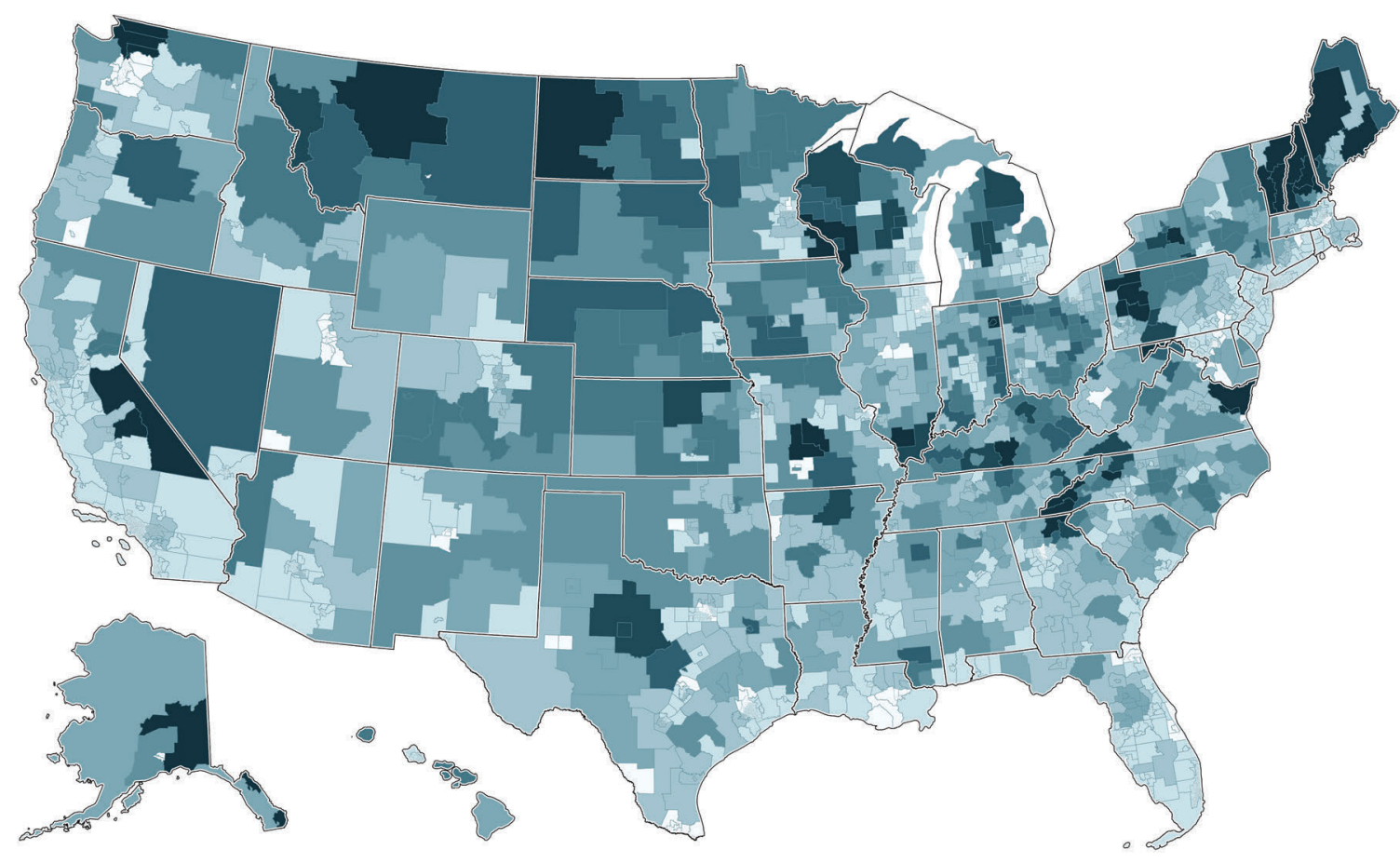

Total: $1.7 \mathrm{~m}(8 \%)$

Lambert conformal conic projection

Alaska rendered at one-third scale

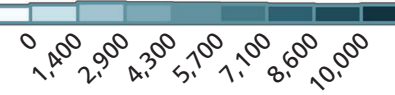

SOURCE: RAND analysis of VA, DoD, and Census data.

RAND RR1165z1-3.15

basic services in rural areas, reduce travel time to primary care services, and serve as a flexible option for adapting to changes in demand for VA services.

\subsubsection{VA Medical Centers}

Figure 3-17 displays the straight-line distance from the center of each PUMA to the nearest VAMC. In general, VAMCs are more prevalent and closer spaced in the Northeast, and most Veterans live within a relatively short distance of their nearest facility. However, coverage is uneven by region, especially the more sparsely populated noncoastal Western states.

Figure 3-18 shows the percentages of the Veteran population living in PUMAs within a given distance of the nearest VAMC in 2014, for Veterans living in urban and rural areas. Figure 3-19 shows the same for Veterans by region. The darker lines indicate the percentage of the Veteran population living in urban areas, while the lighter lines indicate the percentage of Veterans living in rural areas. The solid lines report the percentages for 2014, while the dashed lines indicate the percentages for 2024, based on our projections.

In 2014, 70 percent of the urban Veteran population live within 40 miles of the nearest VAMC, and 90 percent live within 80 miles. By 2024, this distribution is projected to change relatively little, with perhaps a 1-2 percentage point increase in those living farther away from the nearest VAMC. 
Figure 3-16

Total Rural Veterans, 2024 (Projected)

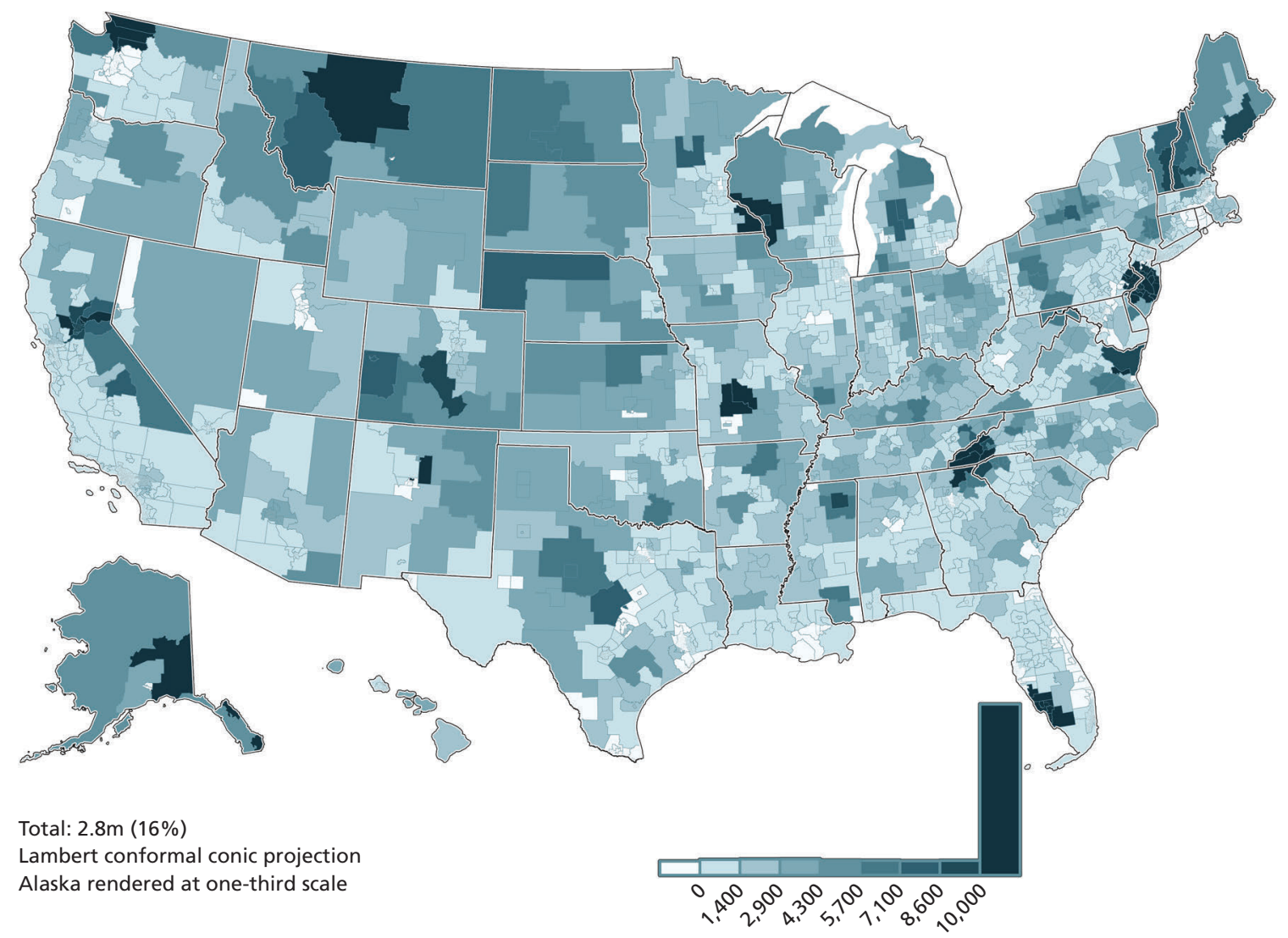

SOURCE: RAND analysis of VA, DoD, and Census data.

RAND RR1165z1-3.16 
Figure 3-17

Distance to Nearest VA Medical Center (miles), 2014

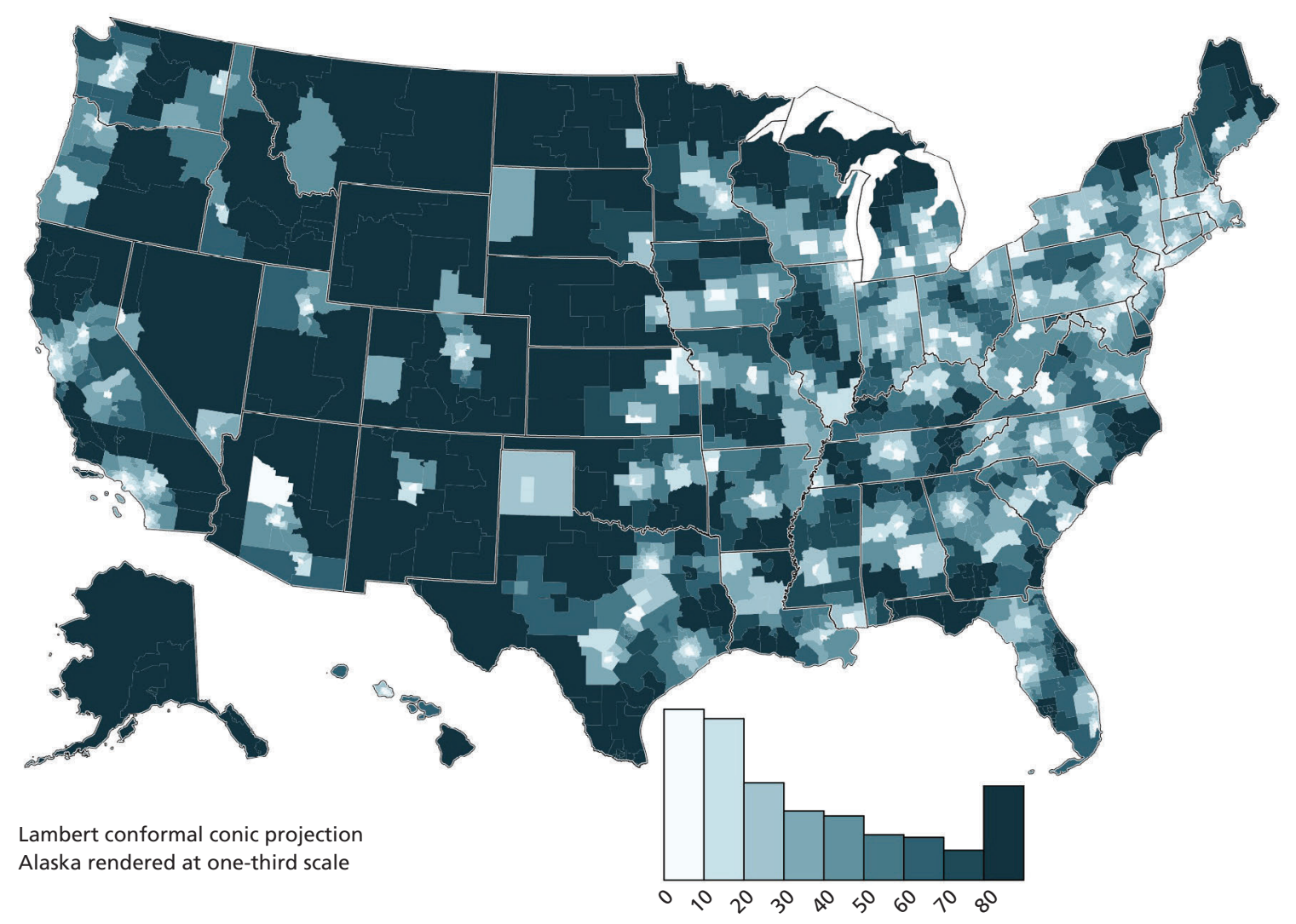

SOURCE: RAND analysis of VA, DoD, and Census data.

RAND RR116521-3.17 
Figure 3-18

Percentage of Veterans Living Within a Given Distance of a VAMC, by Urban/Rural Status

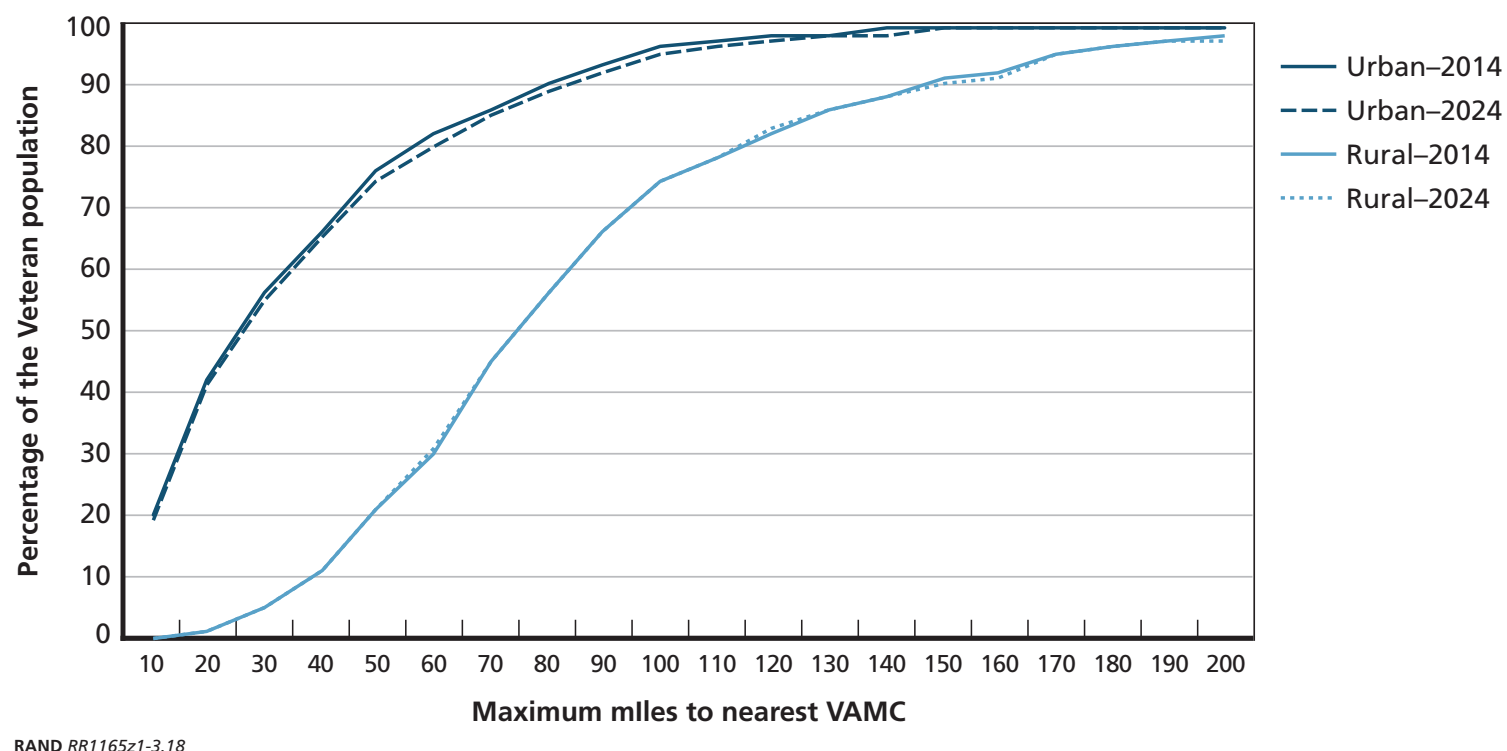

Figure 3-19

Percentage of Veterans Living Within a Given Distance of a VAMC, by Region

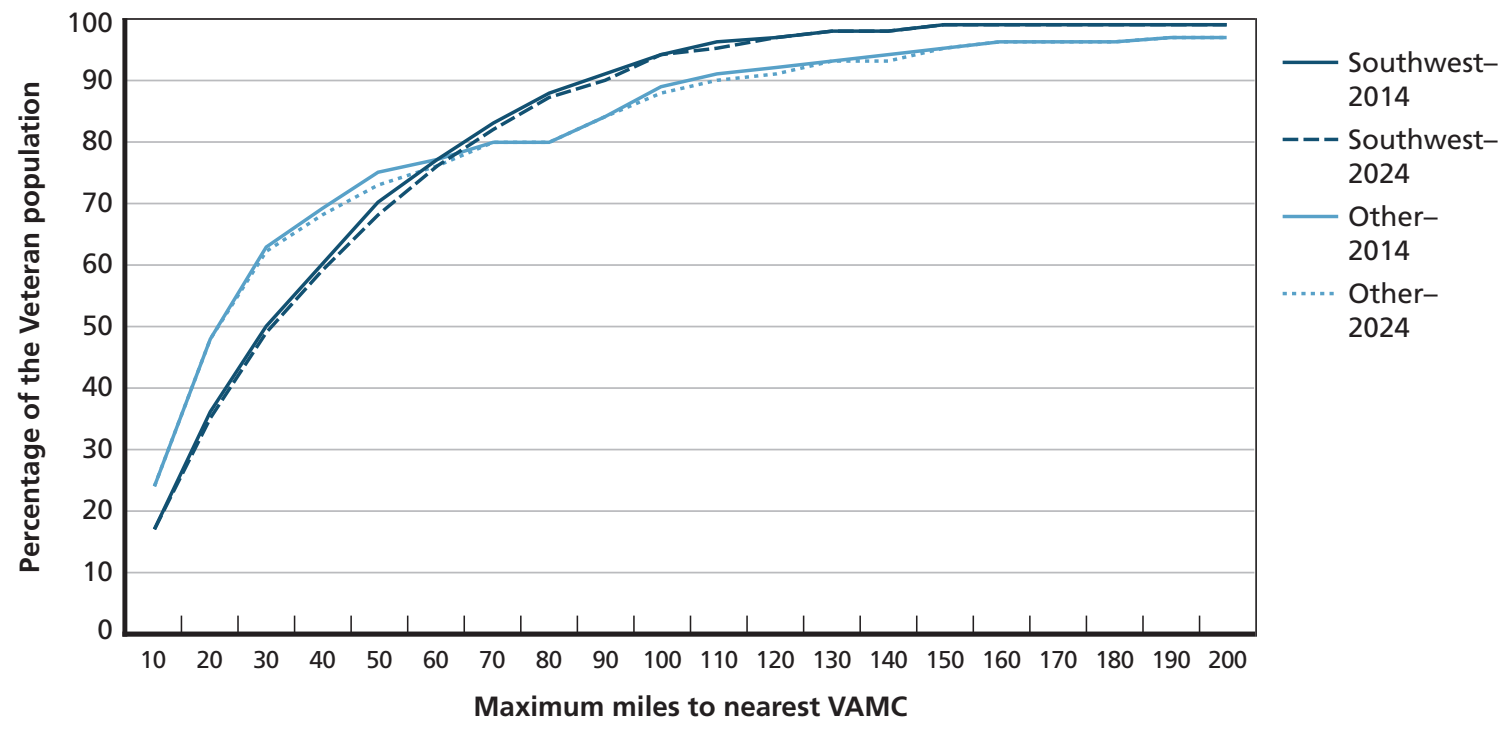

RAND RR1165z1-3.19

As expected, the rural Veteran population tends to be much farther from the nearest VAMC. While more than 70 percent of urban Veterans live within 40 miles of a VAMC, less than 20 percent of rural Veterans do. The differences persist when we consider a much wider radius. While nearly all urban Veterans live within 100 miles of a VAMC, less than 80 percent of rural Veterans live within a similar radius. Many of these Veterans live in relatively remote areas. Just under 40 percent live more than 200 miles from the nearest large ( $>2$ million) citymostly in the Great Plains and the Southwest. 
For the most part, the urban Veteran population is projected to stay closely aligned with the location of current VAMC and population distribution. However, the Veteran population in the Southwest has the most uneven alignment and most risk of future misalignment. In 2014, 10 percent more Southwestern Veterans lived within 40 miles of a VAMC, but 10 percent less lived within 75 miles. By 2024, the situation is not projected to improve. The Southwest has witnessed particularly strong population growth rates in recent decades, including growth in the Veteran segments. New centers of population have emerged, and the construction of VAMCs has not yet caught up. Moreover, because VAMCs in the Southwest are far more widely spaced apart, emerging population centers in the Southwest are less likely to fall within a short distance of an existing facility, and less likely to have an alternative VAMC in proximity. ${ }^{9}$ This combination of factors - above-average rates of population change and wider spacing of VA facilities - places Southwestern VAMCs at higher risk of becoming geographically misaligned with the Veteran population.

\subsubsection{Community-Based Outpatient Clinics}

Figure 3-20 charts the percentage of the Veteran population living in PUMAs within a given radius from different types of VA facilities. While VAMCs offer a wide array of medical services, CBOCs offer the most commonly used basic services-primary, preventive, and counseling services. The darker line reports the maximum distance to the nearest VAMC, while the lighter line reports the equivalent statistics for the nearest CBOC.

\section{Figure 3-20}

Percentage of Veterans Living Within a Given Distance of the Nearest VA Facility, VAMC Versus CBOC

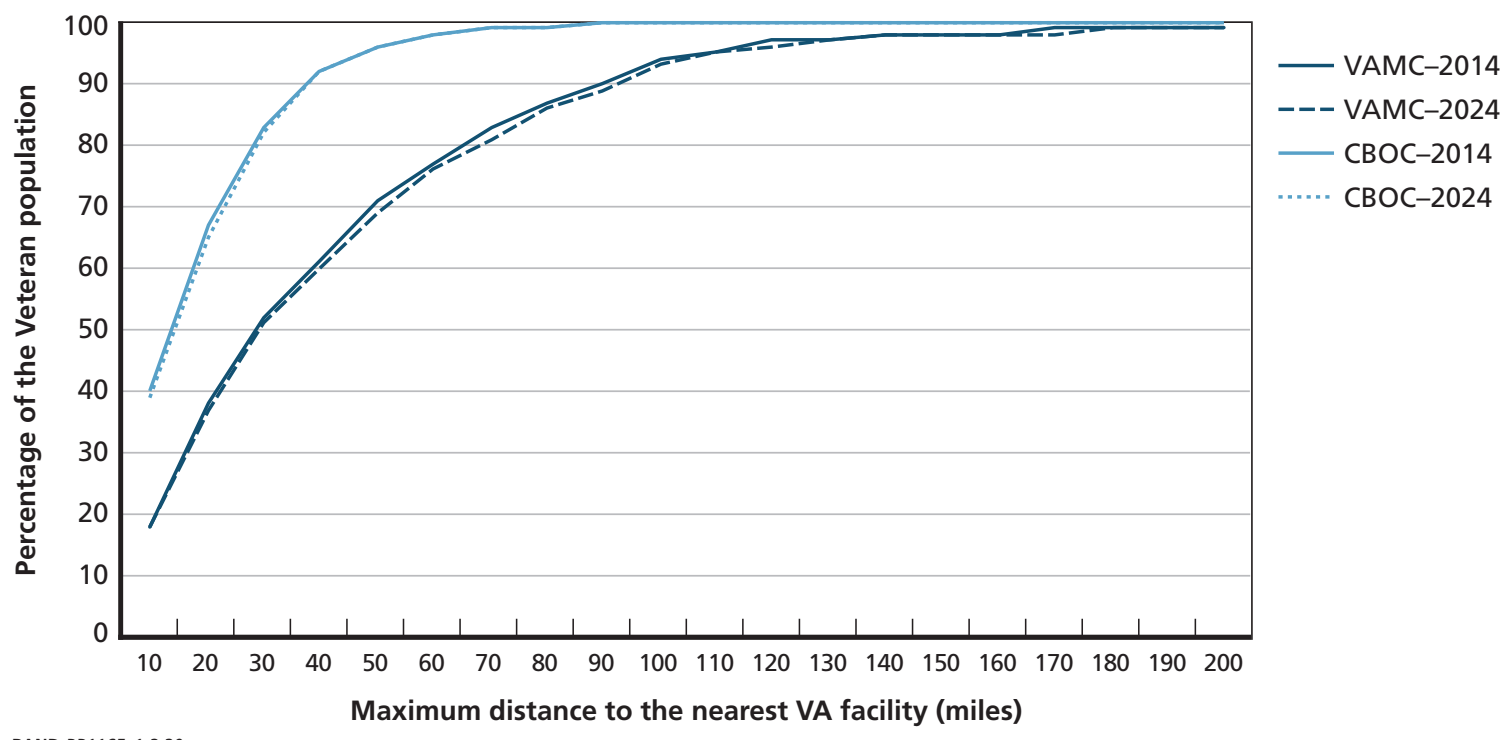

RAND RR1165z1-3.20

9 This is in marked contrast to the Ohio River Valley region. The Ohio River Valley is also experiencing significant shifts in the distribution of the population, but spacing of VAMCs generally means that emerging population centers still fall within a short distance of the a facility. 
More than 90 percent of all Veterans live in a PUMA that falls within 40 miles of a CBOC, compared with just under 70 percent for a VAMC. Because of the wider geographic coverage of the CBOC network, this pattern is unlikely to change by 2024 , despite projected change in the distribution of the Veteran population. That is, while Veterans may be closest to a different set of CBOCs in 2024, they will still be relatively close to a CBOC.

\subsubsection{Veteran Net Migration}

Veterans, like other civilians, move around the country for work, family, retirement, and many other reasons. ${ }^{10}$ In order to inform projections of where Veterans will live over the next 10 years, it is important to consider their migration patterns throughout the entire period. We estimated net migration for each year between 2014 and 2024 based on predicted in-migration and out-migration rates from gravity models, which utilized information on sex, service era, age, race/ethnicity, distance between PUMAs, and population of Veterans in areas of origin and destination to estimate the number of migrants. Results suggest that men are less likely to migrate than women, consistent with migration trends in the national population, according to ACS and other data sources. Previous research has linked greater female residential mobility and desire to move to greater residential satisfaction (Mateyka, 2012) and notes that women's migration was significantly less affected by the Great Recession than men's (Benetsky \& Fields, 2015). ${ }^{11}$ All else equal, older Veterans (especially those 70 and older) are more likely to migrate compared with younger Veterans (25-29 age group), likely reflecting retirement moves as in the rest of the civilian population. However, all else equal, Veterans in older service cohorts are less likely to migrate compared with those in the $9 / 11$ era. Thus, within each service cohort, older Veterans are more likely to migrate, especially among the most recent service cohorts. All race/ethnicity groups other than whites are more likely to migrate than whites. Migration is less likely to occur between PUMAs that are farther apart - that is, migration over longer distances is less likely than migration over shorter distances. Longer distances between PUMAs have negative impacts on the likelihood of migrating. The overall net migration rates vary from 2.97 percent in 2014 to 1.61 percent in 2024. Based on ACS data, migration between PUMAs is around 4 percent for the American population, which indicates that Veterans are less likely to migrate than non-Veterans. A previous study about interregional population flows in the United States (Raymer \& Rogers, 2007) suggests that migration rates are higher among those between ages 20 and 39, which is related to labor migration. Since only 11.86 percent of Veterans were within this age range in 2014 , gravity models suggest that they are more likely to migrate at older, near-retirement ages. Thus, net migration is relatively small and not likely to be a major factor in Veteran demographics. Prior research also suggests that migrating Veterans do not have a noticeable impact on VA health care use (Cowper \& Longino, 1992).

It is important to note that this is a discussion of net migration, not in- and out-migration separately (that is, the churn in Veteran residents). Outflows of migrants to areas generally closely match inflows that replace them, resulting in relatively low levels of net migration. It is

\footnotetext{
10 Here we refer to residential migration, not vacation or other travel.

11 Mateyka (2012) used data from the Survey of Income and Program Participation, conducted by the U.S. Census Bureau, to identify greater mobility and greater desire to move among women in 2010-2011. Desire to move was measured using the following questions: (1) Are conditions in your home undesirable enough that you would like to move? (2) Overall, is the threat of crime where you live undesirable enough that you would like to move? (3) Is your neighborhood undesirable enough that you would like to move? (4) Are the public services undesirable enough that you would like to move?
} 
also important to note that the estimation process does not treat the initial entry of Veterans to the civilian population as migration; in the projection method, Veterans are assumed to initially enter the civilian population according to historical geographic distribution of Veterans with the same age, sex, race/ethnicity, and service-era characteristics, as described in Appendix A. In these projections, migration refers only to movement after the initial entry to the civilian population; we do not include the movement between the initial location of service members when they exit the military and where they are initially distributed as an incoming Veteran. We interpret this type of population change as cohort change, rather than change resulting from migration. In this way, some areas may see relatively increasing populations due to cohort change, but negative net migration (e.g., incoming Veterans may initially locate in Los Angeles, but subsequently move elsewhere).

We used predicted rates from gravity models to estimate net migration. Areas with the highest net migration (the result of in-migrants subtracted by out-migrants at the end of the year) are in Texas, Arizona, Utah, southern Colorado, Wyoming, western Montana, Idaho, Washington state (except the interior), coastal Oregon, Northern California, and northwestern and southwestern Florida. Areas with greater negative net migration are in the interior of Washington state; Southern California; Phoenix, Arizona; San Antonio, Houston, and Dallas, Texas; and Jacksonville, Florida. By 2024, Nevada is also expected to experience general negative net migration, and Southern California will see marginal net in-migration, although net migration overall is low.

\subsubsection{Net Migration and Presence of VA Facilities}

In order to examine the hypothesis that Veterans choose to move to areas where they are closer to VA facilities, we used spatial regression models to examine how growth and movement in the Veteran population is related to the location of a range of VA facilities. Complete details of the estimation methods and results are provided in Appendix A.1.5. Results indicate that in net, migrating Veterans are tending to move farther away from, rather than closer to, VA facilities (VAMCs and CBOCs). This suggests that Veterans' migration decisions are not strongly driven by the presence of VA facilities, and indeed, that they are choosing to move to areas that are not as well covered with VA facilities. Recall that migration is only a small contributor to how Veterans are distributed around the country; although net migration patterns suggest movement away from VA facilities, this does not mean an overall trend of Veterans being farther from facilities. As Section 3.2.8 describes, overall distance to facilities is not likely to change substantially over the next 10 years.

\subsubsection{Changing Population Size and VAMC Location}

The RAND projections indicate an overall 19-percent decline in the Veteran population over the next decade. While the majority of the country will see shrinking Veteran populations, some areas will lose proportionately more than others, and several areas are projected to see growth in the number of Veterans. For a sense of the regions in which VA facilities will face particularly steep population declines (or growth), and regions without VA facilities that will face growth, see Figure 3-21, which presents the projected percent change in Veteran population size for each PUMA between 2014 and 2024, with VAMC facilities indicated on the map as black dots.

Population loss is the norm, but the greatest population losses over time are expected in the Ohio River Valley and upper Midwest areas, as well as rural regions of the West. Several 
Figure 3-21

Projected Percent Population Change 2014-2024, with VAMC Locations

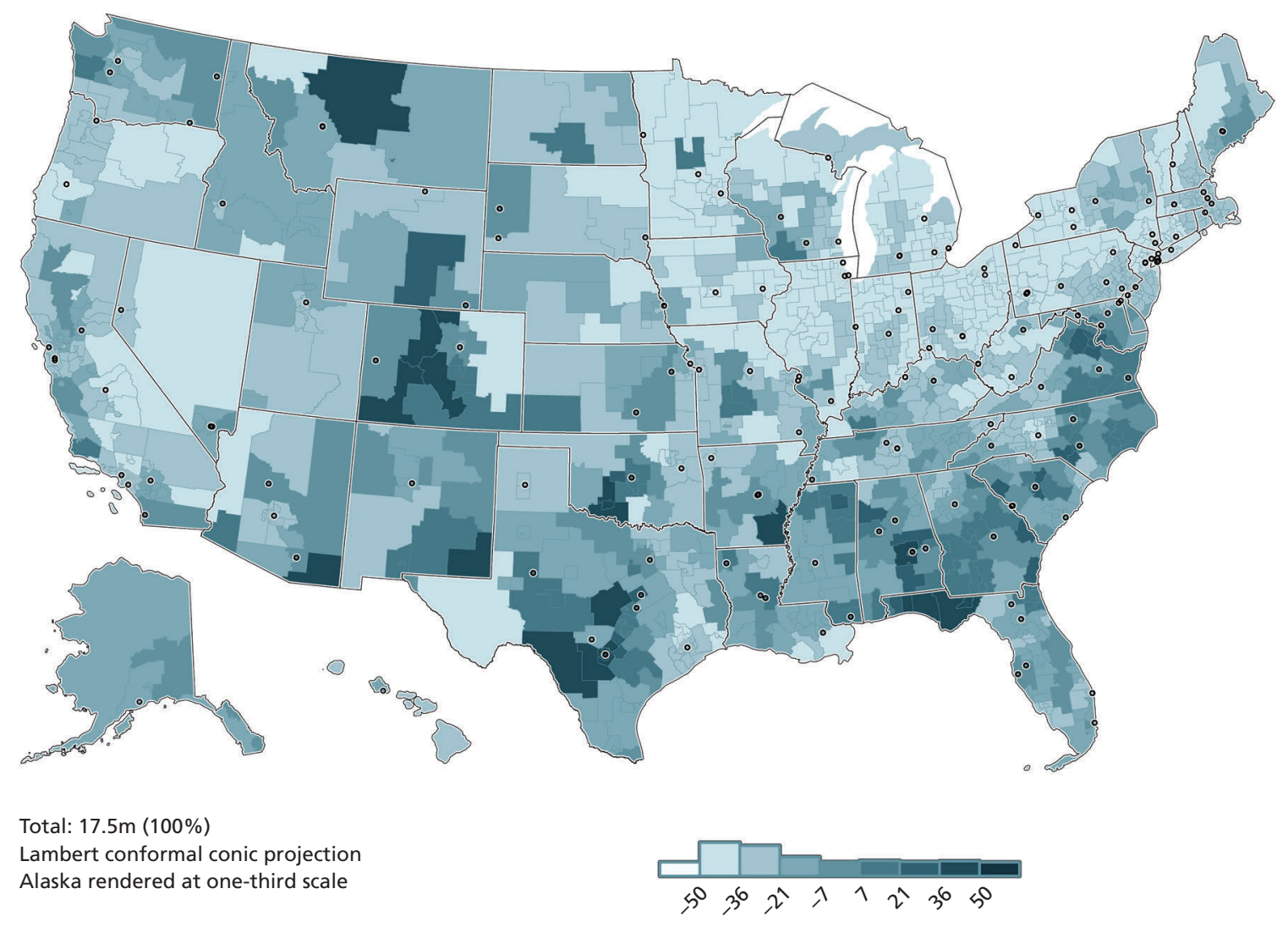

SOURCE: RAND analysis of VA, DoD, and Census data.

RAND RR116521-3.21

regions are expected to see population gains, however. Washington, D.C.; Charlotte, North Carolina; Columbia, South Carolina; Tallahassee/Panama City, Florida; San Antonio and Austin, Texas; and Montgomery, Alabama, are all particularly notable, as population gains are projected for the cities themselves, whereas most other population growth is projected to occur in areas encircling cities.

One implication of the projected population growth is that some of these areas currently do not have local VAMC facilities; growth in Montana, Wyoming, and Colorado occurs where VAMCs do not currently exist. Similarly, growth in the Florida Panhandle is in the absence of any VAMCs. It is important to note that (1) these areas have relatively small Veteran populations to begin with, and that higher percentage growth in these regions may not translate to large absolute increases, and (2) at least part of the growth in the North Dakota, Montana, South Dakota, and Wyoming High Plains region is related to current trends in the shale oil boom that will be sensitive to the economics of oil production. Regardless, the projected growth trends are worth noting, given their relatively stark differences to the overall national population loss trend. 


\subsection{Summary of Key Findings}

The population of U.S. Veterans will decrease by 19 percent over the next 10 years. The Veteran population has been decreasing for more than three decades, and this trend will continue. According to the U.S. Census, in 1970, there were 28.1 million Veterans; in 1990, there were 27.5 million Veterans. We estimate that there were 21.6 million Veterans in 2014. Over the next 10 years, our projections, drawing on Census, VA, and DoD data, show that the Veteran population will decline to 17.5 million. This represents a 19-percent decrease and is in keeping with declines in the size of the total military end-strength since the 1980s. The large cohorts that served prior to the all-volunteer military in 1973 are aging and dying off. The newer Veterans entering the system reflect smaller, all-volunteer service cohorts.

Vietnam Veterans will no longer constitute the largest service cohort by 2024. The share of Vietnam-era Veterans (1964-1975), currently the largest service cohort, will decline slightly from 32 percent of the Veteran population in 2014 to 29 percent by 2024 . Pre-Vietnamera Veterans constituted 25 percent of the total Veteran population in 2014, but by 2024, their share is projected to fall to 13 percent. The proportion of Veterans from the Gulf War and post9/11 eras will grow from 26 percent in 2014 to 41 percent of the total Veteran population by 2024; post-9/11 Veterans alone will account for 24 percent of Veterans in 2024.

The age mix among Veterans will shift slightly. One consequence of this declining service cohort replacement is that the age mix among Veterans will shift slightly over the next 10 years; Veterans will become somewhat older on average. This is particularly pronounced for female Veterans; male Veterans' average age will rise slightly from 62.8 in 2014 to 64.3 in 2024, while female Veterans' average age will rise from 51.4 to 55.2 over the same period. Middle-age Veterans will decline in share; the share of Veterans ages 45-64 will decline from 34 percent to 31 percent of the Veteran population, while the share of both younger and older Veterans will increase.

The racial/ethnic mix will also change modestly. Another consequence of the changing cohort mix will be that the race/ethnic mix of the Veteran population will change modestly. The proportion of Veterans who are non-Hispanic white will decline slightly from 80 percent in 2014 to 76 percent by 2024, while all other race/ethnic groups see slight increases in proportion (with the largest gain among Hispanics).

The Veteran population will become more concentrated in urban areas, and the relative share of the Veteran population in the Ohio River Valley region will diminish. Another consequence of the changing cohort mix will be that the Veteran population will shift away from the largest cities of the Ohio River Valley region, while becoming more concentrated in the major urban centers in other regions. However, migration is less frequent among Veterans than non-Veterans and will not play a substantial role in the geographic distribution of Veterans between 2014 and 2024. While migration rates vary with a range of demographic characteristics, the overall trend is one of slow decline in migration rates generally. VAMC facilities in the Ohio River Valley region will face more-rapid declines in the total Veteran population base they serve than the Southwest region, where our projections suggest that relative Veteran population concentration will not be as well matched in 2024 to existing VAMC locations.

The 2024 projections indicate a 19-percent decline in overall Veteran population. Despite this decline, the 2024 geographic distribution will not be drastically different from the current distribution, and we do not project that overall distance to existing VA facilities will increase 
substantially. The existing CBOC coverage puts almost all Veterans ( 92 percent) within 40 miles of some type of VA facility in 2024. However, the total numbers and characteristics of Veterans will change within the overall geographic distribution. Looking forward, it does not necessarily seem to be a matter of building new facilities, but rather anticipating the types of services that will need to be provided at existing VA facilities.

First, in terms of the total Veteran populations to be served there are some anticipated changes to plan for. The Ohio River Valley and upper Midwest will see the greatest declines in Veteran population; it may be possible to consolidate the relatively proximal VA facilities in those regions as the population shrinks. At the same time, several regions are expected to see population gains. Most regions with gains, such as near Washington, D.C.; Charlotte, North Carolina; and San Antonio and Austin, Texas, are currently near VA facilities. Other growth areas, such as Montana, Wyoming, and Colorado, are not currently well covered. In these latter growth areas the population will remain relatively small, and access to telehealth and CBOC services may be important ways to meet Veteran needs. Similarly, rural areas in northwestern Washington State, northern Michigan, and northern Texas will remain areas with rural Veteran populations in 2024 that could also benefit from continued or expanded services.

Second, while the total Veteran population will be growing older on average through 2024, and health services related to aging will be needed everywhere, Veterans under age 35 will be concentrating in areas surrounding Los Angeles, Dallas, Washington, D.C., and northern New Jersey by 2024, and they will also constitute a greater proportion of the population in Northern California, central Washington state, the Midwest, and Wyoming and Utah. In these areas, it may be of particular importance to provide services relevant to younger Veterans compared with elsewhere. Much of the Southeastern seaboard, from Virginia Beach through the coast of Georgia, will see a decrease in the proportion of the population that is under age 35 at the same time, and focus on services for younger Veterans may need to be shifted West and North.

Overall, the Veteran population in the Southwest has the most uneven alignment and most risk of future geographic misalignment by 2024. The Southwest has seen, and will continue to see, relatively strong population growth (Veteran and non-Veteran civilian alike). Because VAMCs in the Southwest are far more widely spaced apart, population centers in the Southwest are less likely to fall within a short distance of an existing facility, and less likely to have an alternative VAMC in proximity. Combined with the relatively younger Veteran population anticipated in this region relative to others by 2024, particular awareness of potential future service demand by this population is important for planning. Detailed analysis of access to specific types of services and VA facilities is presented in Assessment B, Chapter Four (RAND Health, 2015b). 


\subsection{Introduction}

Understanding the volume and mix of VA health care services that Veterans will use in the future is critical for VA's resource and capability planning. The main challenge in measuring and describing Veterans' use of health care services is that many Veterans obtain some or all of their health care from non-VA sources, as described in Chapter Two. Understanding the future volume of VA health care services requires calculating (1) the number of Veterans enrolled in the VA health care system; (2) the number of Veterans who seek health care at a VA facility; and (3) Veterans' reliance on the VA health care system (i.e., the share of health care services that VA patients receive from VA versus from other sources).

The conceptual model of VA health care use, discussed in Chapter Two, highlights Veteran characteristics and VA policy as the main determinants of enrollment in and use of the VA health care system. Veteran characteristics that affect enrollment and use include the Veteran's sociodemographic characteristics, health care needs (including service-connected disabilities), and access to other health insurance. The "Combat Veteran" Authority of 2008, which extended enhanced eligibility and expedited enrollment for VA health care for Veterans of Afghanistan and Iraq, is an important policy affecting current patterns of enrollment and use among Veterans. This policy allows Veterans who served in a theater of combat operations after November 11, 1998, to enroll in VA without first establishing their priority group for a period of five years post-discharge. These Veterans are placed in priority group 6 unless they qualify for a higher priority group and, at the end of the five-year period, may be shifted to priority group 7 or 8 .

Once Veterans enroll and are deemed eligible to receive care, they must make a choice about whether to use VA health care and how much care to consume. The majority of enrolled Veterans have access to other health care coverage, and approximately half of enrolled Veterans are also enrolled in Medicare or Medicaid (Congressional Budget Office, 2009; Goldberg, 2015). Enrolled Veterans receive the majority of their health care outside of VA (Goldberg, 2015). Based on EHCPM, VA estimates that current VA patients have on average about 21 percent of their total physical medicine (that is, physical therapy and occupational therapy) visits with VA, 38 percent of their emergency room visits with VA, and 66 percent of their prescriptions from VA.

In this chapter, we project future enrollment in the VA health care system and the future size of the VA patient population. We include the factors outlined in the conceptual model to the extent possible given the available data. These projections are used in Chapter Five to project the future health care needs of the VA patient population. Our primary task was to describe the current and projected demographics and unique health care needs of Veterans 
rather than current and future VA health care use. There are significant practical data and analytic challenges in measuring and projecting the health care services that Veterans demand or use. Nonetheless, we measure reliance for a select set of health care service categories in MEPS data and compare these reliance estimates with those used in EHCPM. We also introduce a new reliance concept - population reliance — to convey the share of health care services that all Veterans (rather than only VA patients) receive from VA.

\subsection{Analytic Approach}

\section{Overview of Methods and Data for Enrollment and Reliance Analysis}

- We estimated the probability of a Veteran enrolling in the VA health care system taking into account age, sex, deployment status, service era, and a time trend using aggregated data from multiple sources.

- We estimated the number of VA patients from 2014 through 2024 by multiplying the number of enrollees by the probability of use among enrollees, which was calculated using the SoE.

- We estimated VA patients' reliance on VA health care using MEPS data from the household survey, the Prescribed Medicines file, the Office-Based Medical Provider Visits file, and the Inpatient Stays file.

- To compare our reliance estimates with VA's, we evaluated reliance factors used by VA by combining three separate EHCPM files.

\subsubsection{Projecting the Number of Veterans Enrolled in the VA Health Care System}

As described in the conceptual model of VA health care use in Chapter Two, the decision to use VA for health care services is dependent on a number of factors, including VA policies, Veteran characteristics, cost, access, and perceptions of the care available through VA's health care system. The first step in the process of receiving VA health care services is enrollment in the VA health care system. Just as with the decision to seek care, the decision to enroll is a complex process influenced by many of the same factors. Ideally, we would have modeled an individual Veteran's decision to enroll in the VA health care system based on the factors included in the conceptual model, but we did not have access to a single data source linking all of these factors. We instead used data aggregated by age, sex, and deployment status (with respect to Afghanistan and Iraq) to model enrollment rates.

We estimated the probability of new enrollment in the VA health care system (given that a Veteran has yet to enroll) using a logistic regression model that incorporated age, sex, deployment status, service era (e.g., Vietnam War era), and a time trend. ${ }^{1}$ Starting with 2015, predicted enrollment probabilities based on this model were applied to the population of yetto-enroll Veterans to estimate the number of new enrollees. These new enrollees were added to the previous year's surviving population of enrolled Veterans. This process was repeated for each subsequent year. In Figure 4-1, we present a conceptual model of enrollment in the VA health care system that depicts this process.

\footnotetext{
1 We estimated and projected VA enrollment using RAND Veteran population projections, DoD data, and VA administrative data. These data sources do not uniformly include information on income (or priority group), so we could not include income as a predictor of enrollment.
} 
Figure 4-1

Conceptual Model of Enrollment in the VA Health Care System

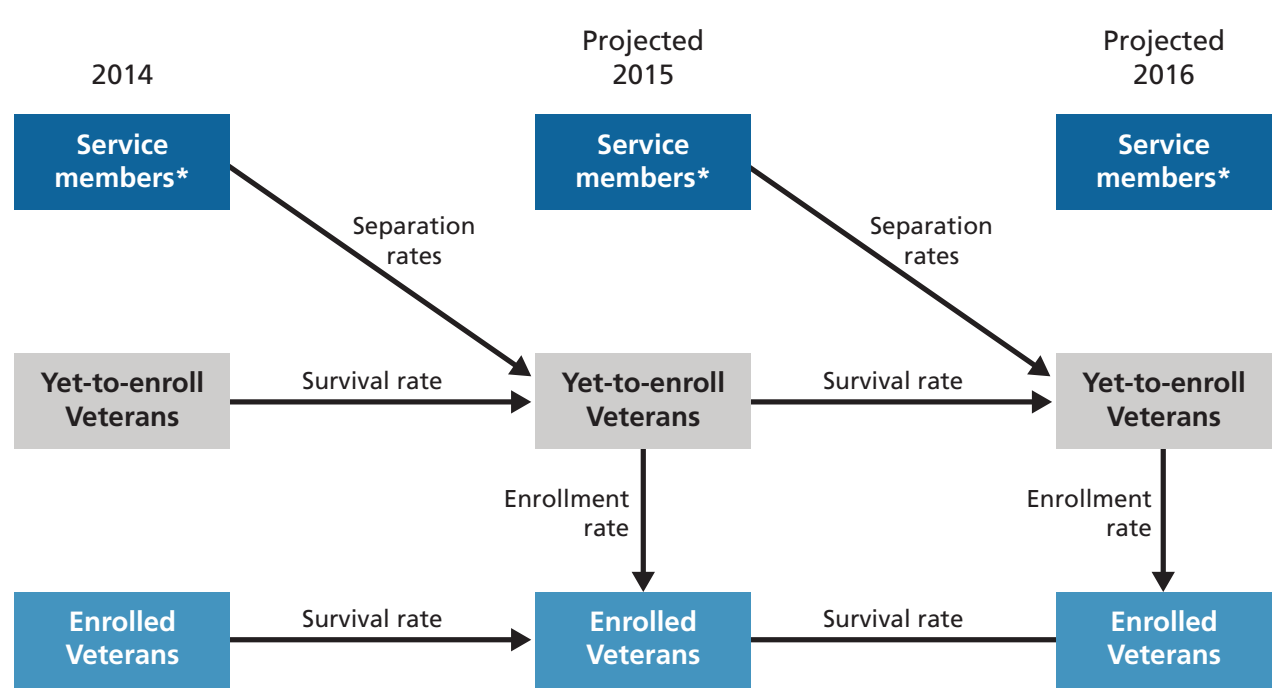

NOTES: Survival rate is the probability that an individual survives to the next year. *Number of separations among service members are projected in Chapter Three.

RAND RR1165z1-4.1

We considered three different sets of assumptions about the probability of new enrollment in future years and compared the projected number of enrollees under each of these assumptions. First, we assumed that the probability of enrollment will continue to follow the recent pattern of enrollment, which we refer to as the status quo assumption. Second, we assumed that the probability of enrollment among Veterans who were deployed to Afghanistan and Iraq will follow the recent pattern of enrollment among Veterans who never deployed to Afghanistan or Iraq, which we refer to as the never deployed assumption. Third, we assumed that the probability of enrollment among Veterans who deployed to Afghanistan and Iraq will decrease over time by allowing the effect of deployment on future enrollment to decrease by 5 percent each year, which we refer to as the decreasing enrollment assumption. The never deployed and decreasing enrollment assumptions reflect the fact that we do not anticipate enrollment to remain at the current historical levels as we move away from the operations in Afghanistan and Iraq. There are several reasons why we postulate that this may occur: (1) the number of separating service members with deployment experience will decrease over time; (2) the number of all deployed Veterans within the five-year window of enhanced enrollment eligibility will decrease over time; (3) the percentage of separating service members with more than 19 years of experience (i.e., retiring and eligible for TRICARE) and with deployment experience will increase over time; and (4) the health status of separating service members who were deployed will improve because military personnel with more-serious deployment-related medical conditions are likely to leave earlier than those without such conditions. Each of these factors, among others, is expected to decrease enrollment rates among future Veterans who deployed. Further discussion is provided in Section 4.5.

Our projections of enrollment in the VA health care system do not account for the effects of the Great Recession. We used VA administrative data from 2008 to 2014 to model new enrollment, which does not provide us with sufficient data before the recession to properly account for its impact on enrollment. Assuming that the Great Recession increased the probability of enrollment in the VA health care system, our projected enrollee counts are too high. 


\subsubsection{Projecting the Number of VA Patients}

We projected the number of VA patients from 2014 to 2024 by multiplying the number of enrollees by the probability of use among enrollees. These VA patient projections account for mortality because they are based on the VA enrollee projections, which account for mortality as described in Section 4.2.1.

We estimated the probability of VA use among enrollees using the SoE from 2010 through 2014. We fit a logistic regression model for the probability of VA use that included age, sex, service era, Iraq-Afghanistan deployment status, and a linear time trend. ${ }^{2}$ These characteristics were chosen to model VA use among enrollees because they aligned with the characteristics that were used to project enrollment. We present the predicted probabilities of using VA health care services using the estimated model in Appendix B, Table B-1. We also present the results of an extended model in Table B-2.

Ideally, we would have modeled an individual Veteran's decision to use VA health care services based on the factors included in the conceptual model, but using factors that were not included in our enrollment projections would have required additional modeling. For example, a Veteran's income is related to VA use; if we wanted to include income in our projections of VA use, it would have been necessary to project Veterans' future income. There are significant practical data and analytic challenges in projecting the future income of Veterans, which is why we decided against this approach.

Veterans who served in a theater of combat operations after November 11, 1998, are eligible for an enhanced period of enrollment for five years post-discharge. We checked the sensitivity of our results by including an indicator that a Veteran who deployed is within five years of separation in our VA use model; however, this indicator was not associated with use.

\subsubsection{MEPS Analyses of Reliance}

We calculated reliance using the 2008 to 2012 MEPS data, specifically data from the household survey, from the Prescribed Medicines file listing all prescriptions filled by MEPS respondents, the Office-Based Medical Provider Visits file, and the Inpatient Stays file. Prescribed medicine, office visit, and inpatient stay data from MEPS are based on self-reported utilization by survey respondents. MEPS staff audit a sample of prescriptions and visits. Our general approach followed four steps:

1. Identify Veterans and VA patients in the MEPS household files.

2. Identify in the MEPS data the prescribed medicines, office-based visits, and inpatient stays that were provided or paid for by VA.

3. Aggregate the number of services received by VA patients overall and those that were provided by or paid for by VA for each health care service category (e.g., physical and occupational therapy).

4. Calculate reliance by dividing the sum of services provided by or paid for by VA by the total number of services received.

We also calculated a population reliance factor (described below) for all Veterans in addition to our measure that is restricted to VA patients only. Further, we report reliance for mul-

2 We projected the number of VA patients from 2015 through 2024 by multiplying the number of projected enrollees by the probability of VA use among enrollees. Enrollees are not projected with income (or priority group) information, so we could not incorporate income-specific probabilities of VA use into the projections. 
tiple health care service categories: prescribed medicines, all office visits, specific office visit categories (evaluation and management, laboratory tests, radiology, kidney dialysis, and physical and occupational therapy), and inpatient stays involving surgical procedures. This selection of health care service categories is meant to give a sense of the variation in reliance across broad categories of health care services. Our selections were driven by the feasibility of roughly aligning health care service categories in MEPS with service categories in EHCPM; however, we do not imply that the MEPS and EHCPM health care service categories are identical. We also recognize that the unit of measurement in MEPS and EHCPM are not always consistentfor example, EHCPM counts prescriptions normalized to a 30-day supply, whereas we simply count prescription drug fills in MEPS.

For this analysis, we identified VA patients in MEPS by inferring whether an individual in MEPS is a VA patient based on the respondents' source of payment for health care. Specifically, we defined active VA patients as those respondents who had any payment by VA for services used. Unfortunately, MEPS data do not enable us to identify all Veterans who are eligible for VA services; we can identify only those eligible Veterans who use VA services. We defined health care service categories and identified services that were provided by or paid for by VA, as described in Table 4-1. The remaining services in each category were assumed to be provided and paid for by some other source. For each category and population, we defined reliance

Table 4-1

Data Sources and Category Definitions

\begin{tabular}{|c|c|c|}
\hline Service Category & Data Source & Criteria \\
\hline Prescription drugs & $\begin{array}{l}\text { MEPS Prescribed } \\
\text { Medicines files, 2008- } \\
2012\end{array}$ & $\begin{array}{l}\text { All prescription events with VA paid amount }>\$ 0 \text {. We did } \\
\text { not adjust MEPS prescribed medicine events to account for } \\
\text { differences in days supplied across events. While this might } \\
\text { be feasible for some MEPS events (e.g., events with } 90 \\
\text { days supplied), a significant share of MEPS events have an } \\
\text { "unknown" number of days supplied. }\end{array}$ \\
\hline Office visits & $\begin{array}{l}\text { MEPS Office-Based } \\
\text { Medical Provider Visits } \\
\text { files, 2008-2012 }\end{array}$ & $\begin{array}{l}\text { All office visits with place of service = "VA facility" OR VA paid } \\
\text { amount }>\$ 0\end{array}$ \\
\hline $\begin{array}{l}\text { Office-based evaluation } \\
\text { and management visits }\end{array}$ & $\begin{array}{l}\text { MEPS Office-Based } \\
\text { Medical Provider Visits } \\
\text { files, 2008-2012 }\end{array}$ & $\begin{array}{l}\text { All office visits with visit type = "General checkup" or "Follow- } \\
\text { up or post-operative visit" AND (place of service = "VA facility" } \\
\text { OR VA paid amount }>\$ 0 \text { ) }\end{array}$ \\
\hline $\begin{array}{l}\text { Office-based laboratory } \\
\text { services }\end{array}$ & $\begin{array}{l}\text { MEPS Office-Based } \\
\text { Medical Provider Visits } \\
\text { files, 2008-2012 }\end{array}$ & $\begin{array}{l}\text { All office visits with "this visit patient had lab tests" }=1 \text { AND } \\
\text { (place of service = "VA facility" OR VA paid amount }>\$ 0 \text { ) }\end{array}$ \\
\hline $\begin{array}{l}\text { Office-based dialysis } \\
\text { services }\end{array}$ & $\begin{array}{l}\text { MEPS Office-Based } \\
\text { Medical Provider Visits } \\
\text { files, 2008-2012 }\end{array}$ & $\begin{array}{l}\text { All office visits with "this visit patient had dialysis" = } 1 \text { AND } \\
\text { (place of service = "VA facility" OR VA paid amount }>\$ 0 \text { ) }\end{array}$ \\
\hline $\begin{array}{l}\text { Office-based radiology } \\
\text { services }\end{array}$ & $\begin{array}{l}\text { MEPS Office-Based } \\
\text { Medical Provider Visits } \\
\text { files, 2008-2012 }\end{array}$ & $\begin{array}{l}\text { All office visits with "this visit patient had..." one of the } \\
\text { following = 1: sonogram, } x \text {-rays, mammography, or MRI, AND } \\
\text { (place of service = "VA facility" OR VA paid amount }>\$ 0 \text { ) }\end{array}$ \\
\hline $\begin{array}{l}\text { Office-based physical/ } \\
\text { occupational therapy } \\
\text { services }\end{array}$ & $\begin{array}{l}\text { MEPS Office-Based } \\
\text { Medical Provider Visits } \\
\text { files, 2008-2012 }\end{array}$ & $\begin{array}{l}\text { All office visits with ("this visit patient had physical therapy" = } \\
1 \text { OR "this visit patient had occupational therapy") AND (place } \\
\text { of service = "VA facility" OR VA paid amount > \$0) }\end{array}$ \\
\hline $\begin{array}{l}\text { Inpatient stays involving } \\
\text { surgical procedures }\end{array}$ & $\begin{array}{l}\text { MEPS Hospital Inpatient } \\
\text { Stays files, 2008-2012 }\end{array}$ & $\begin{array}{l}\text { All inpatient stays with imputed VA facility or provider } \\
\text { payments }>\$ 0 \text { and an indicator for "any operation or surgery } \\
\text { performed while the respondent was in the hospital" }\end{array}$ \\
\hline
\end{tabular}


as the ratio of the sum of services provided by or paid for by VA to the sum of total services received by the population, weighted appropriately.

Our reliance measures can be interpreted as the proportion of care in a service category that VA patients receive from VA. We calculate a second reliance measure, a population reliance estimate, that is identical to the formula above but calculates reliance for all Veterans, including Veterans who are not VA patients. The interpretation of population reliance is the proportion of care in a service category that all Veterans (rather than VA patients) receive from VA. We focused on utilization-measured in events - rather than spending, in part for consistency with the VA definition of reliance and in part to control for differences in health care costs or prices across payers and systems.

\subsubsection{EHCPM Analyses of Reliance}

While we did not have access to the underlying data used to replicate the EHCPM reliance calculations from the ground up, we were able to evaluate reliance factors provided by VA. We combined three separate EHCPM files to adjust VA-provided reliance factors so that they are more analogous to those that we calculated in MEPS. These files contained the following:

- Reliance estimates for 25 health care service categories by priority group, age category, whether or not the Veteran enrolled prior to eligibility reform, sex, and submarket (subdivisions of Veterans Integrated Service Networks)

- Utilization, measured in units relevant to individual health care service categories and defined using the same criteria as the reliance factors

- Counts of unique Veterans who used at least some services in each health care service category.

Because different VA-provided EHCPM output files were formatted differently, we applied a set of adjustments so that the different input files used the same age categories and priority groups.

\subsection{Results}

\subsubsection{Projection of Veterans Enrolled in the VA Health Care System}

Currently, the most important driver of enrollment in the VA health care system is the flow of separating Veterans who were deployed in Afghanistan and Iraq. This is occurring, in part, because of the enhanced period of eligibility for deployed service members and an increased need for health care due to combat experience. As of the end of FY 2014, 67 percent of eligible Afghanistan and Iraq Veterans were enrolled in the VA health care system, and 60 percent of these eligible Veterans had obtained VA health care (Epidemiology Program Office of Public Health, 2015). Both of these indicate that current enrollment rates among Veterans who were deployed in Afghanistan and Iraq are high.

We estimate that the percentage of all Veterans who deployed to Afghanistan or Iraq will increase from 9.1 percent in 2014 to 15.1 percent in 2024, corresponding to an increase of approximately 700,000 Veterans. Veterans who served in Afghanistan or Iraq will make up an increasing share of the total Veteran population and, as already stated, are much more likely to enroll in the VA health care system. Understanding the future pattern of enrollment among 
these 700,000 yet-to-separate Veterans who deployed is the first step in understanding the volume and mix of VA health care services that Veterans will use in the future. Assuming that all of these yet-to-separate Veterans join the VA patient population would increase the population by more than 10 percent.

As we move away from these combat operations, fewer separating Veterans will have deployment experience, and the overall new enrollment rates in the VA health care system will likely decrease. Figure 4-2 illustrates that the percentage of separating service members who were deployed is estimated to decrease from 61.7 percent in 2014 to 12.8 percent in 2024.

In addition, the characteristics of separating Veterans with deployment experience will change as we get further away from the operations in Afghanistan and Iraq. We describe three specific examples. First, separating Veterans will become older and more experienced between 2015 and 2024. These Veterans are more likely to be retiring from the military and are eligible for TRICARE. 85 percent of military retirees under age 65 have no private health coverage and are reliant on TRICARE (Office of the Assistant Secretary of Defense (Health Affairs), 2015). In 2010, 23 percent of Veterans with TRICARE-only coverage used some VA health care; an unknown amount of this care was provided through a DoD-VA sharing agreement. Figure 4-3 illustrates that, by 2024, nearly 50 percent of separating Veterans who were deployed are separating with at least 19 years of experience and are likely to have TRICARE, compared with less than 25 percent in 2014. Although the flow of separating Veterans eligible for enhanced enrollment will continue, they will be fewer in number, less dependent on VA health care services due to eligibility for TRICARE, and less likely to enroll in the VA health care system.

Second, fewer Veterans will be within the five-year window of enhanced enrollment eligibility. Figure 4-4 illustrates a steep decline in the projected number of Veterans who were deployed and are within five years of separation. It is expected that enrollment rates will decline as a result.

Figure 4-2

Percentage of Separating Veterans Who Were Deployed

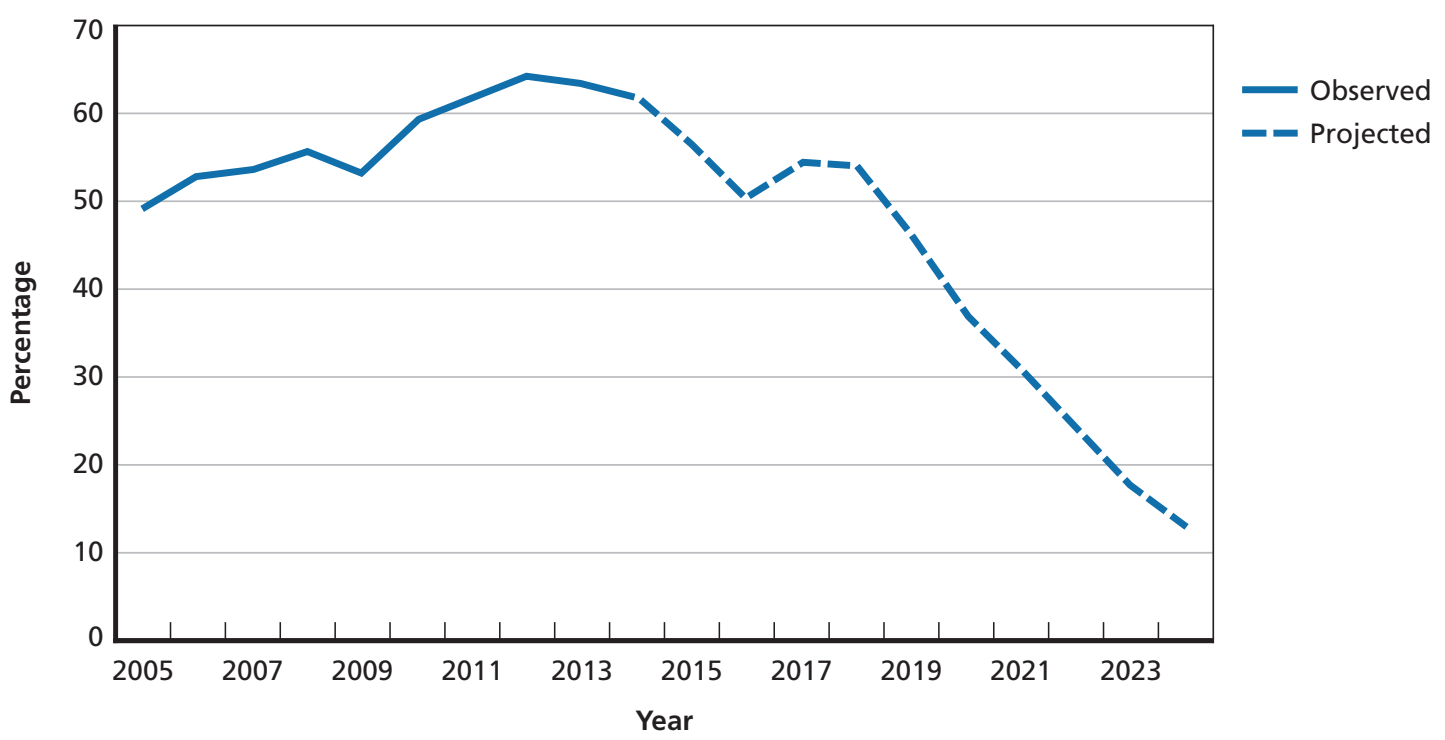

SOURCE: RAND analysis of DoD data.

RAND RR1165z1-4.2 
Figure 4-3

Percentage of Separating Veterans with 19+ Years of Service Among Those Who Were Deployed

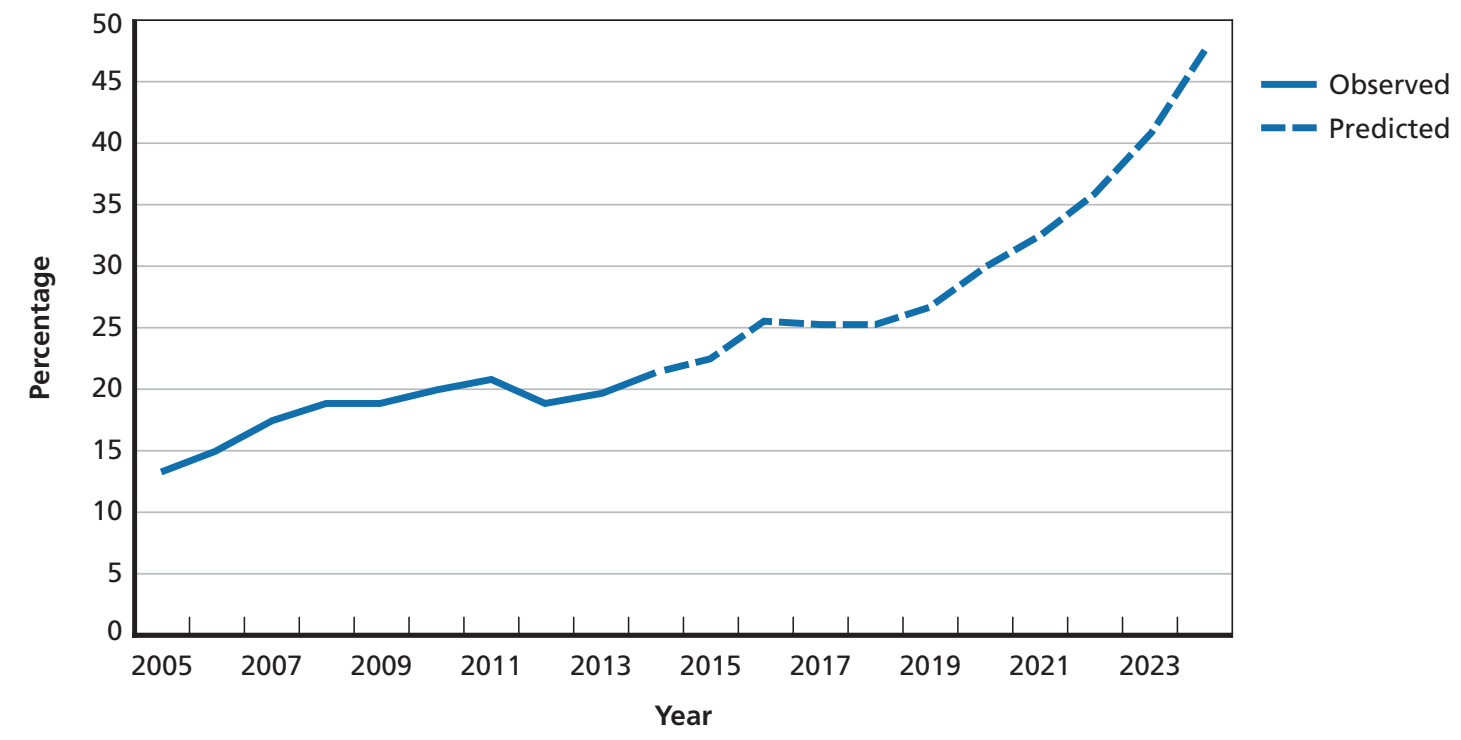

SOURCE: RAND analysis of DoD data.

RAND RR1165z1-4.3

Figure 4-4

Number of Veterans Within 5 Years of Separation Among Those Who Were Deployed

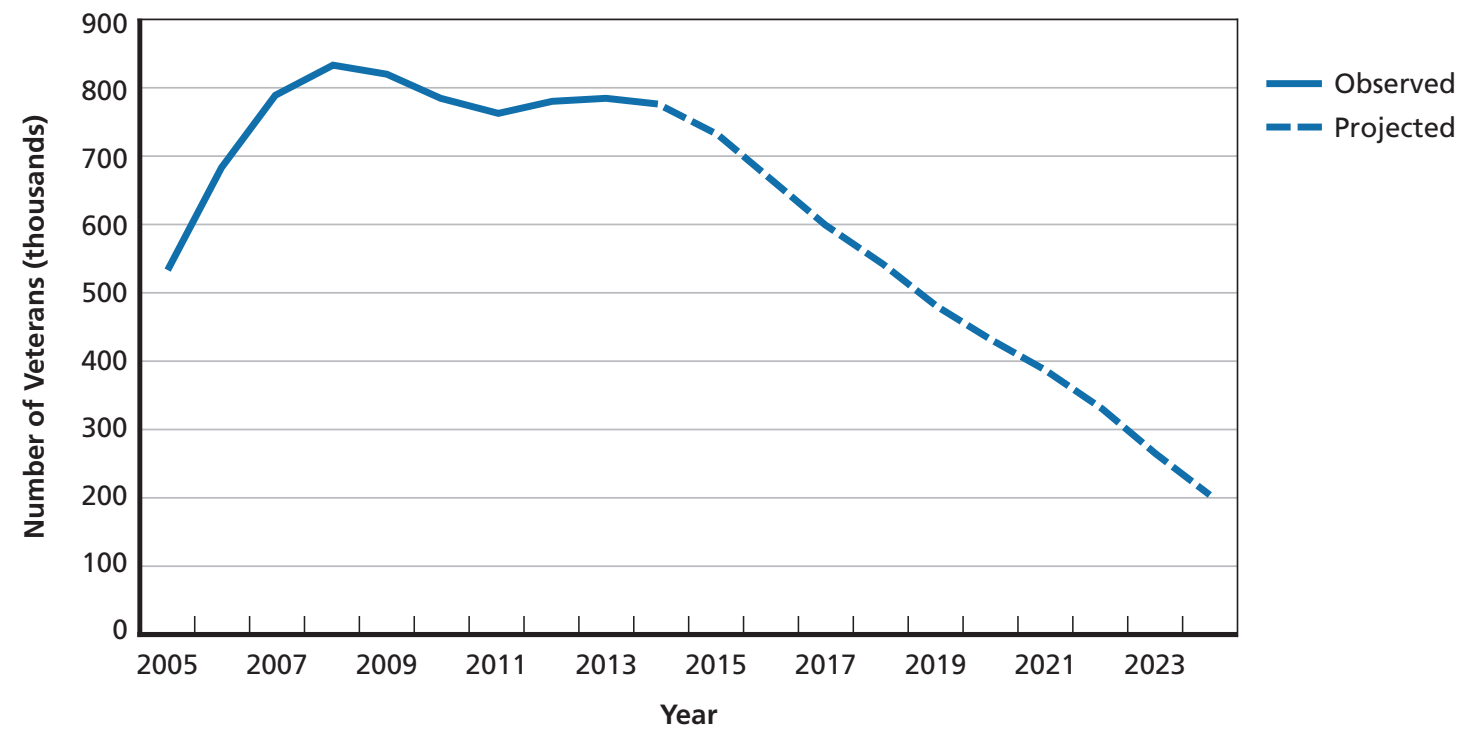

SOURCE: RAND analysis of DoD data.

RAND RR1165z1-4.4

Third, we expect the health status of separating Veterans to improve over time. Veterans with service-connected health care needs as a result of deployment are likely to separate earlier if they do not meet medical retention standards and, once separated, are more likely to seek care for the service-connected health need that contributed to their separation. In addition, selection of service members to continue in military service may be based on their overall 
health due to the military health and fitness standards. We expect that as the number of active duty service members who were deployed decreases, the overall health status of these service members will improve. If true, the overall health status of future separating Veterans who were deployed will improve over time. As indicated in the conceptual model in Chapter Two, a Veteran's need of health care is associated with his or her decision to seek care. If separating Veterans' health statuses are improving over time, we expect lower enrollment rates as a result.

We did not have access to data that would allow us to incorporate this type of information into our projections. Therefore, we took a sensitivity analysis approach when projecting the number of enrollees. Our three sets of assumptions provide us with a range of plausible projections and inform us on the sensitivity of our final results to these assumptions. Recall that our status quo assumption is that the recent pattern of enrollment will continue in the future. While a reasonable baseline, we expect this assumption to overestimate the number of enrolled Veterans moving forward due to the previously stated reasons. The never deployed assumption attempts to account for the expected decline in enrollment among Veterans who were deployed by aligning their pattern of en rollment with Veterans who were never deployed. This can be considered as a lower bound, as the probability of new enrollment among yet-toenroll Veterans is much lower among Veteran who were never deployed (in 2014, 2.7 percent versus 18.3 percent). The decreasing enrollment assumption is a trade-off between the first and second. We assume that the effect of deployment on enrollment rates will decrease by 5 percent each year and reflects the previously described changes in the characteristics of separating Veterans over time.

Note that these assumptions focus on the enrollment rates of Veterans who were deployed. This is because the probability of enrollment among Veterans who never deployed is much lower than their deployed counterparts, as demonstrated in Figure 4-5. In addition, the probability of new enrollment appears to be much more consistent for non-deployed Veterans among those age 30 and older.

\section{Figure 4-5}

Probability of New Enrollment, by Age (2009-2014)

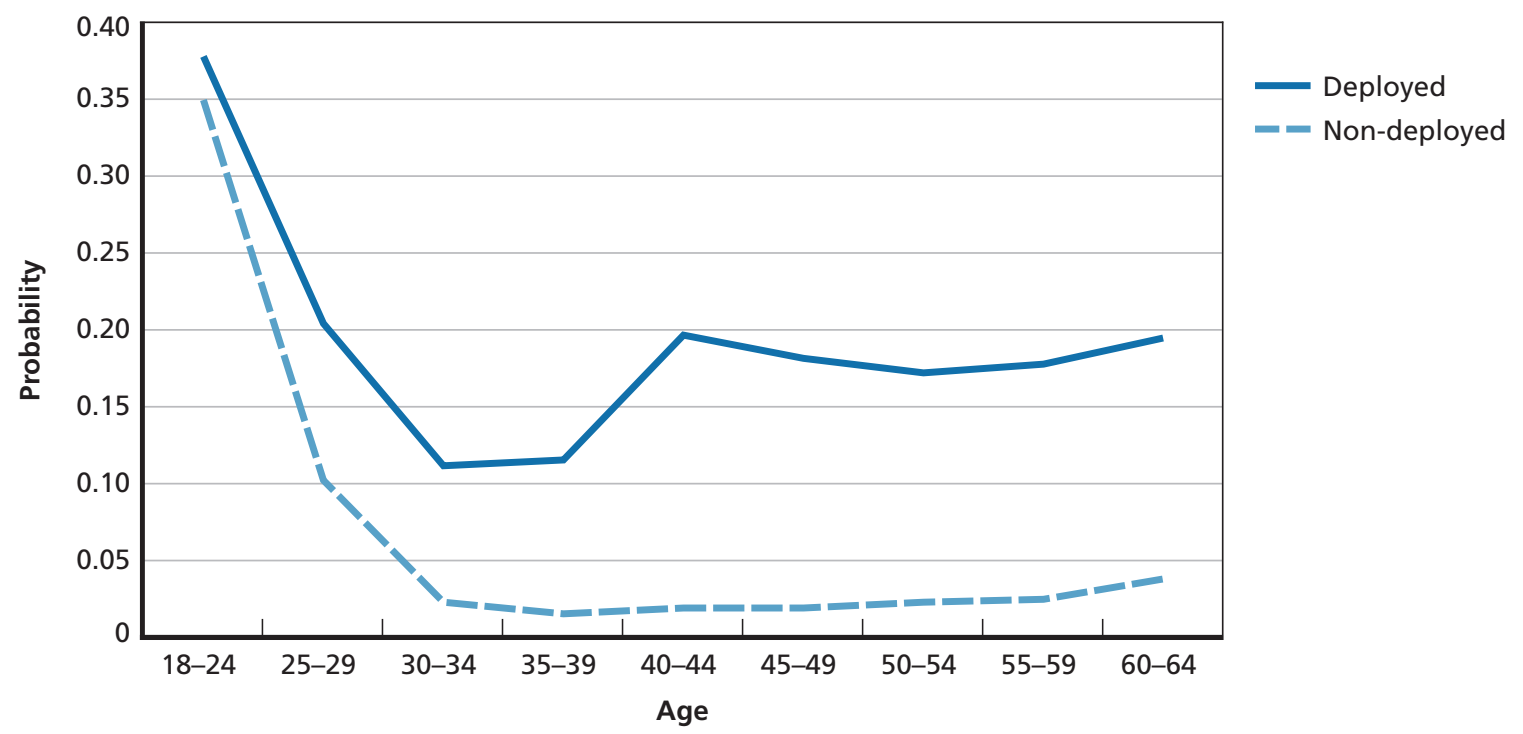

SOURCE: RAND analysis of VA, DoD, and Census data.

RAND RR1165z1-4.5 
Figure 4-6 illustrates the historical trend and projected number of VA enrollees under our three sets of assumptions. We project the number of VA enrollees to increase under all three of our projection assumptions, but by varying degrees. The number of VA enrollees in 2014 was 9.1 million Veterans, and our projections predict increases to 10.0 million, 9.8 million, and 9.3 million in 2024. All three sets of assumptions show an increase in the projected number of enrollees for several years, but these increases are projected to level off or reverse around 2020. This will occur as enrollment rates among younger Veterans are unable to offset the mortality rates of older Veterans and as most Veterans who were deployed in Afghanistan or Iraq have already made their initial decision on enrollment.

The difference between the projection under the status quo assumption and the never deployed assumption is about 670,000 enrollees by 2024, amounting to about 7 percent of the current enrollee population. This difference is driven by the pattern of enrollment among Veterans who deployed in Afghanistan and Iraq and serves as a range of plausible projections. That is, we expect the actual pattern of enrollment to fall in between these two projections. The difference between the projected enrollee population under the status quo assumption and the decreasing enrollment assumption is even smaller, approximately 200,000 enrollees by 2024 .

Enrollment in the VA health care system is only the first step toward understanding the volume of VA health care services that Veterans will use in the future.

\subsubsection{Projection of VA Patients}

We projected the number of VA patients by multiplying the number of enrollees by the probability of use among enrollees. While we estimate only one model for the probability of VA use conditional on enrollment, we apply these estimates to the enrollee projections under each of the three sets of assumptions to produce three sets of projections for the number of VA patients. The differences in the projected number of VA patients are caused by differences in the total

Figure 4-6

VA Enrollee Trends and Projections (2008-2024)

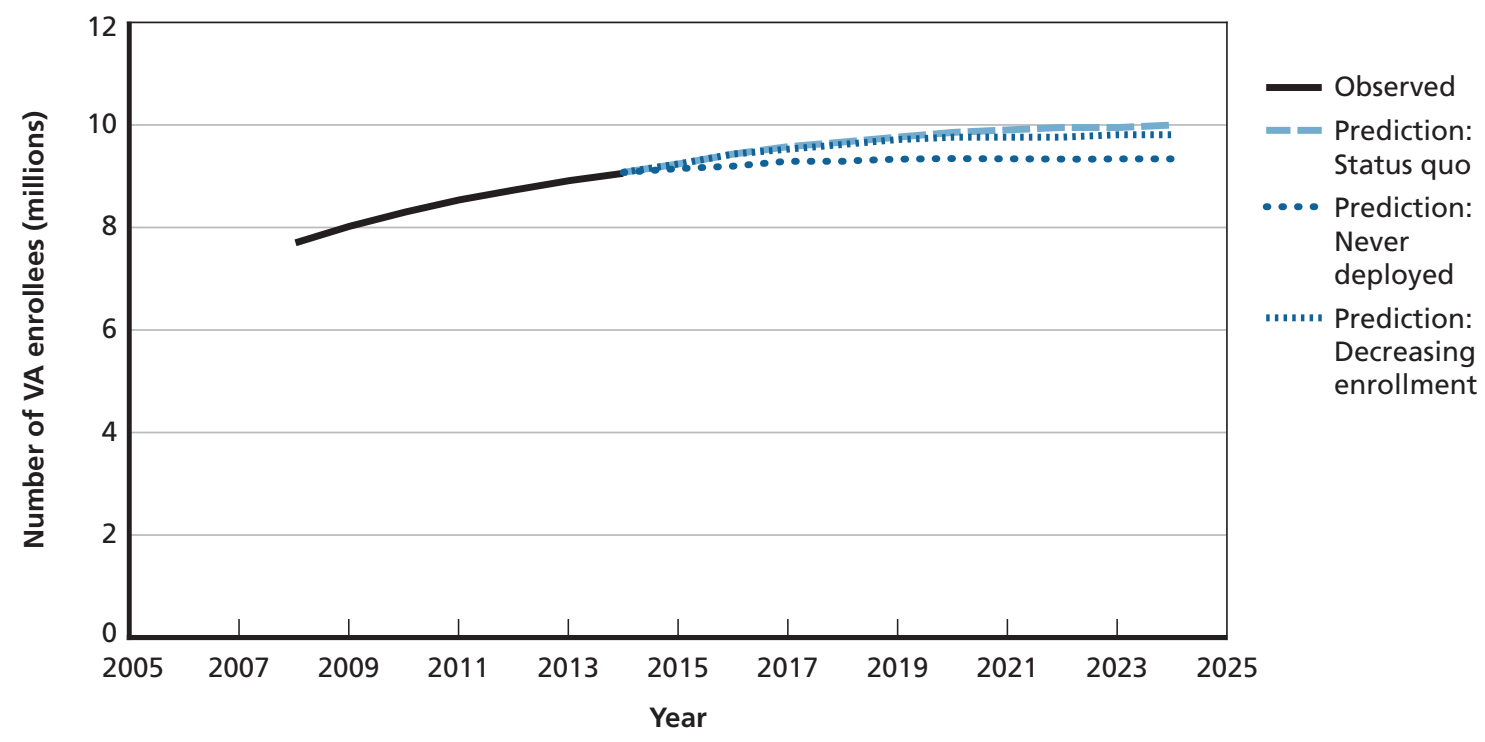

SOURCE: RAND analysis of VA, DoD, and Census data.

RAND RR1165z1-4.6 
number of projected enrollees and the demographic composition of projected enrollees. The probability of using VA health care services conditional on enrollment, age, sex, service era, deployment status, and year does not differ.

Figure 4-7 illustrates the historical trend in the number of VA patients and our projections. We project little change in the total number of VA patients. The number of VA patients in 2014 was 5.9 million, and we project the number of VA patients will be between 5.8 million and 6.2 million in 2024. The pattern in the observed and projected number of VA patients roughly follows the pattern in enrollment. While the number of VA patients increases nearly 20 percent between 2008 and2014, we project that this trend will flatten out and reverse over the next 10 years. This predicted trend is largely driven by the trend in the number of VA enrollees.

The difference between the projected number of VA patients under the status quo assumption and under the never deployed assumption is about 370,000 patients by 2024, amounting to 6.5 percent of the current VA patient population. The difference between the projected enrollee population under the status quo assumption and the decreasing enrollment assumption is even smaller, only 110,000 patients by 2024 .

The percentage of VA patients who served in Afghanistan is projected to increase over the next decade, as illustrated in Figure 4-8. By 2024, 19 percent of VA patients are projected to have served in Afghanistan and Iraq. The long-term health effects of service in Afghanistan and Iraq are unknown, and it is imperative that VA monitor trends in health care utilization among these Veterans to ensure adequate resources and capabilities to meet their unique and changing health care demands.

We explored whether or not the period of enhanced eligibility was related to VA use among enrollees. Veterans who enrolled in priority group 6 during the period of enhanced eligibility are most likely to be shifted to priority group 7 or 8 at the end of the enhanced eligibility period. Among VA enrollees who were deployed in Afghanistan and Iraq, there is no

Figure 4-7

VA Patient Trends and Projections (2008-2024)

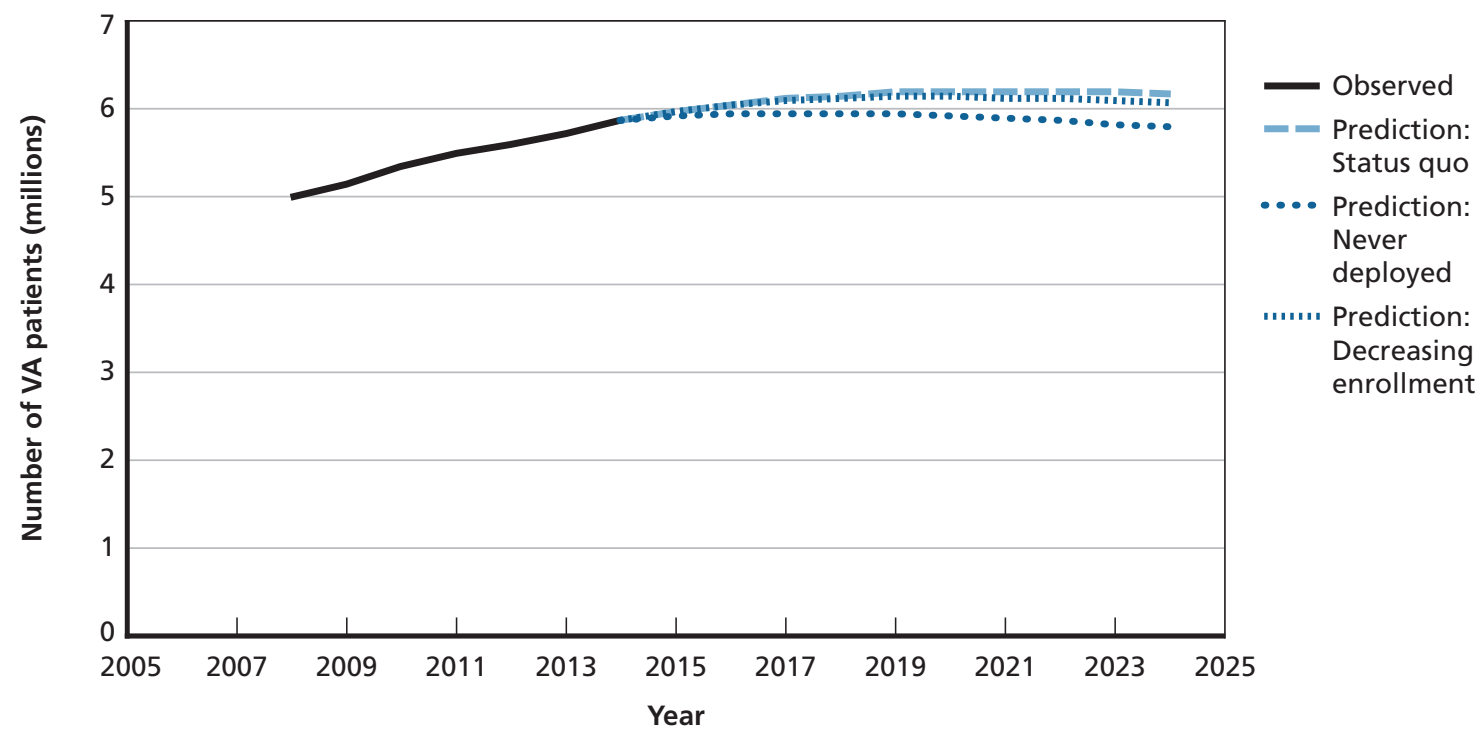

SOURCE: RAND analysis of VA, DoD, and Census data. 
Figure 4-8

Percentage of VA Patients Who Served in Afghanistan and Iraq (2008-2024)

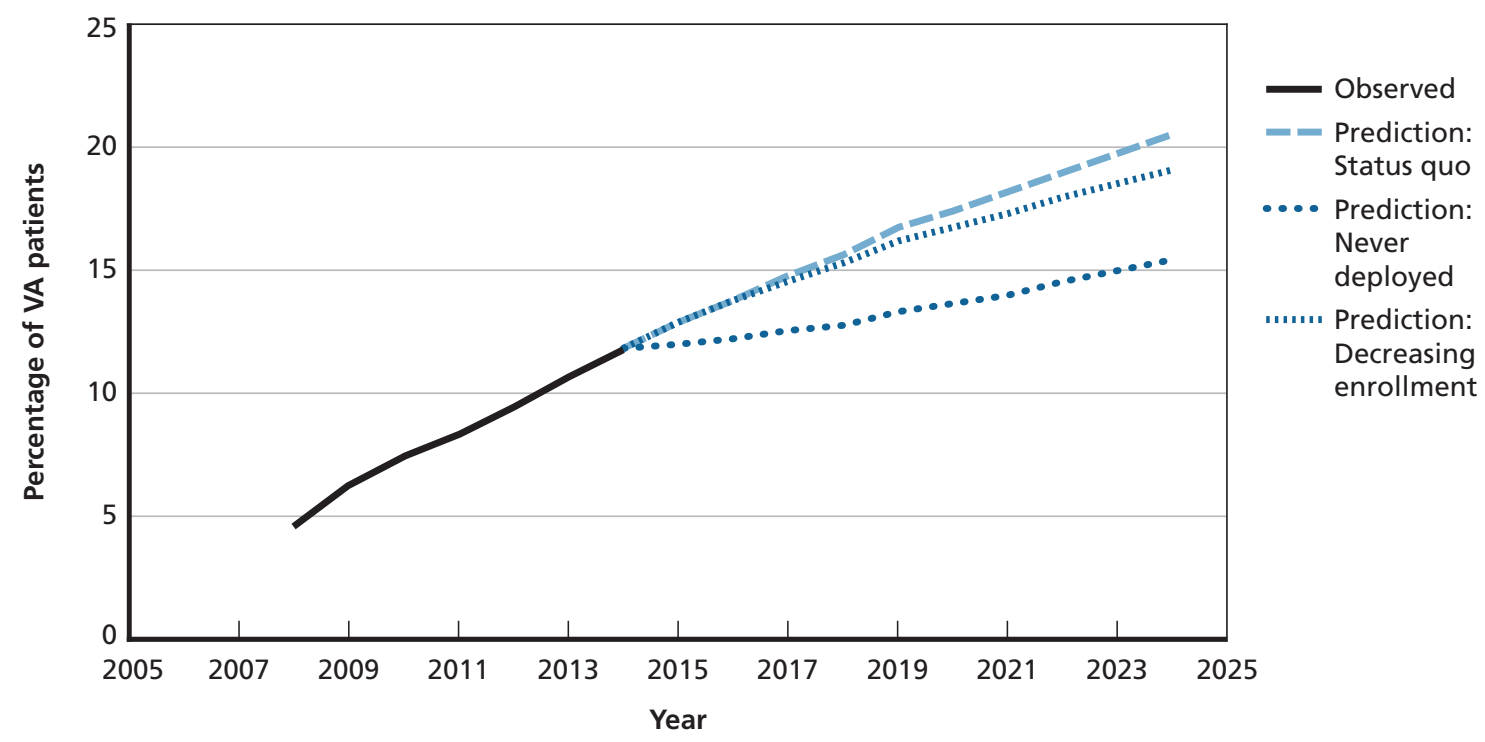

SOURCE: RAND analysis of VA, DoD, and Census data.

RAND RR1165z1-4.8

difference in the percentage of Veterans using VA health care services between priority group 6 enrollees within the period of enhanced eligibility (35 percent) and priority group 7 or 8 enrollees outside the period of enhanced eligibility (37 percent). This indicates that the probability of VA use is not different between enrollees within and outside the period of enhanced eligibility, but it does not provide any information on how reliance may change when the period of enhanced eligibility ends. Veterans who move to a lower priority group will have co-pays, but these are modest; otherwise, there is little to no change in access to care or service availability. One explanation of the continued use of VA health care is that the enhanced eligibility policy gets Veterans to enroll in the VA health care system who would have otherwise not enrolled. Once these Veterans enroll and begin receiving VA health care, they are satisfied with the quality of care that they are receiving and continue seeking VA health care.

In addition to the factors and trends that affect the health care needs of the Veteran population overall, the health care needs in the VA patient population are determined in part by who uses VA health care services. Changes in VA policies-such as new priority group cutoffs for enrollment eligibility, additions of new presumptive service-connected conditions, or changes to the enhanced eligibility benefits for new combat Veterans-will directly affect the number and composition of Veterans who are eligible to receive VA health care services. External factors that affect access to affordable health care, such as fluctuations in the economic climate or changes in the eligibility rules for other public health programs (e.g., Medicare) have the potential to affect VA use rates and the composition of the VA patient population. We assumed that these factors remain constant over the next 10 years in these baseline projections. The effects of policy changes on future Veteran, enrollee, and patient counts are considered in the scenario analyses in Chapter Six. 


\subsection{Reliance Analysis Results from MEPS Data}

\subsubsection{Overall Veteran and VA Patient Reliance on VA}

Reliance on VA varies across the select health care service categories that we examined using MEPS data, ranging from 15 percent for all office-based visits to 34 percent for office-based laboratory services (see Figure 4-9). Because many Veterans do not use care provided or paid for by VA, there are also important differences between reliance calculated only for VA patients and population reliance calculated across all Veterans. Adding non-patient Veterans to the reliance calculation increases the reliance denominator but not the reliance numerator. As a result, each population reliance estimate is lower than the corresponding VA patient-only reliance factor. For example, while VA patients obtain 30 percent of prescription fills from VA (according to MEPS), across all Veterans, the prescription drug reliance rate falls to 16 percent. Both reliance factors may be relevant to policymakers depending on the context.

\subsubsection{Veteran and VA Patient Reliance on VA by Age, Demographics, and Health Status}

There are important differences in reliance across Veterans with different demographic, health status, and insurance coverage characteristics. Figures 4-10 through 4-15 report prescription drug, office visit, and inpatient surgery reliance rates for VA patients and all Veterans, both overall and across a range of Veteran subpopulations. Notably, reliance for all three health care service categories is significantly higher for younger age groups (who are less likely to have

Figure 4-9

Reliance for VA Patients and All Veterans, by Service Category

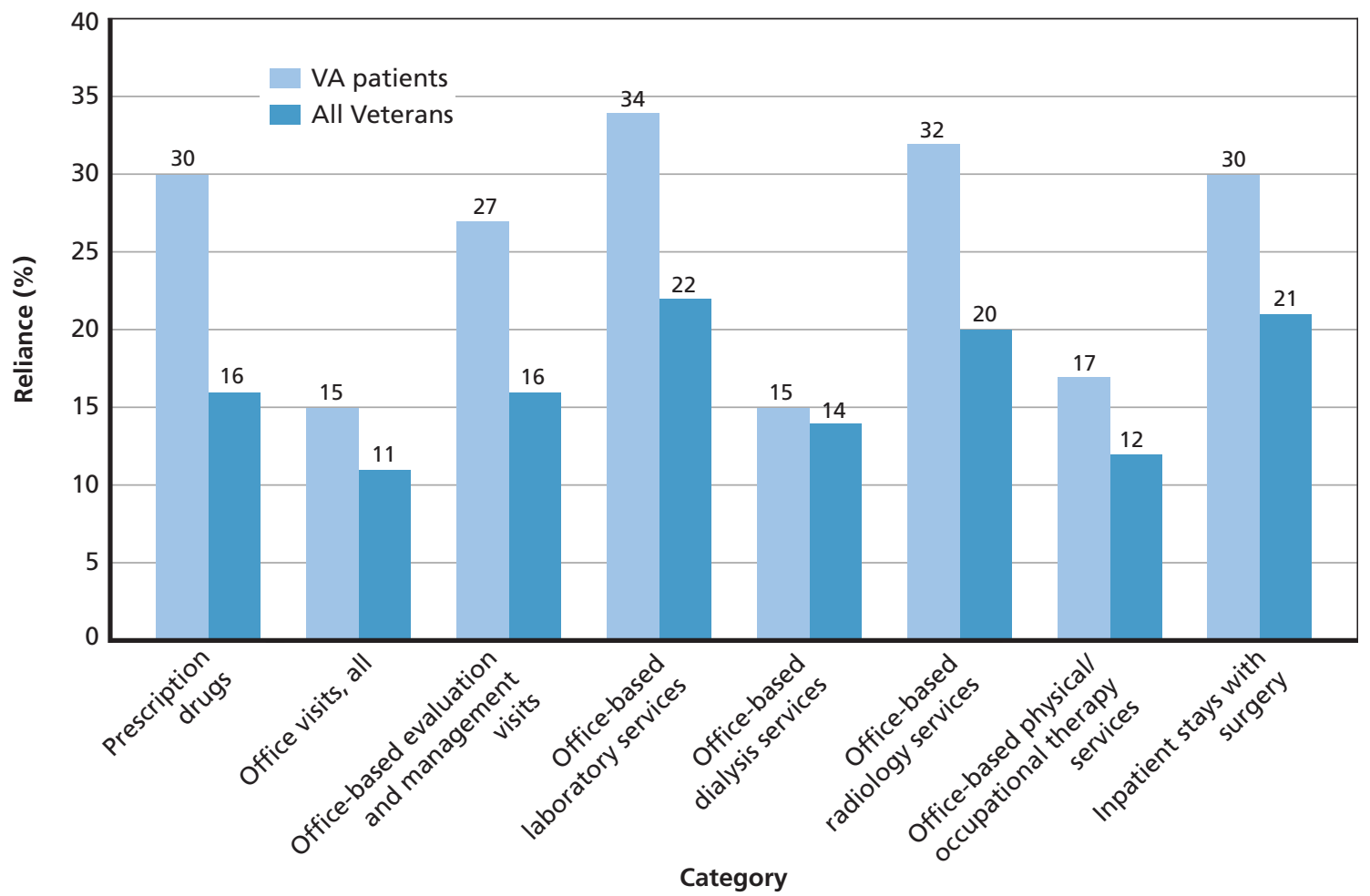

SOURCE: RAND analysis of 2008-2012 MEPS data. 
other health insurance) than for older Veterans who are likely to have access to other sources of coverage, such as employer-sponsored insurance or Medicare.

While reliance for office visits is similar across male and female Veterans, male Veterans have higher prescription drug reliance than female Veterans (35 percent versus 26 percent), and female Veterans have considerably higher inpatient surgery reliance than male Veterans. The inpatient surgery result-as well as several large swings in reliance across age groups, such as the change in inpatient stays with surgical procedures across the mid-30s to late-40s age categories - could reflect cohort effects, availability of other coverage, changing health care needs, or small sample sizes. Lower-income Veterans have generally higher reliance rates across health care service categories. Finally, Veterans who self-report being in fair or poor health or who are uninsured have relatively high reliance rates compared with other Veterans.

Reliance rates for all Veterans (Figures 4-11, 4-13, and 4-15) are lower than reliance rates calculated just among VA patients (Figures 4-10, 4-12, and 4-14).

Figure 4-10

Reliance for VA Patients, by Health Care Service Category and Age

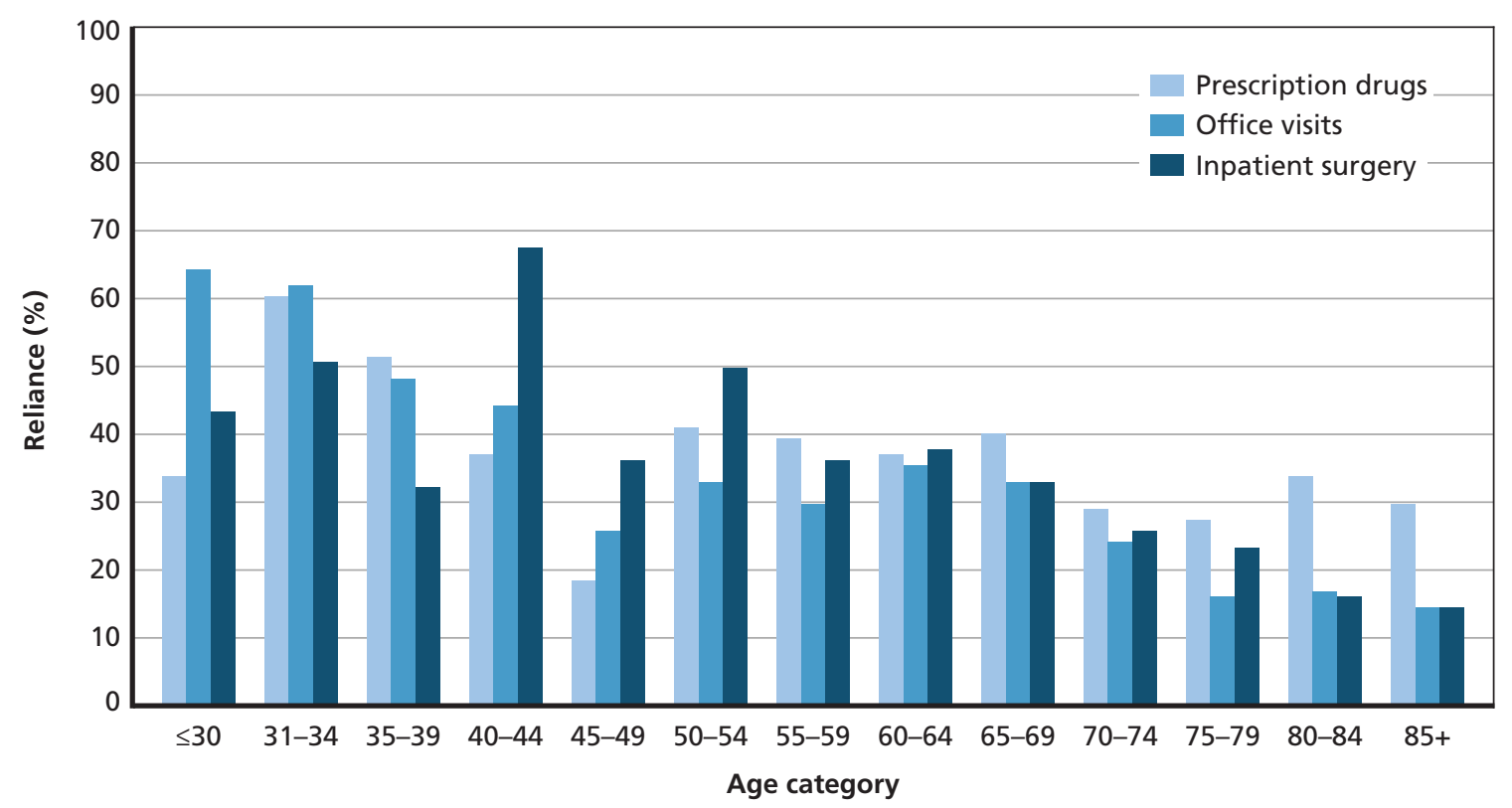

SOURCE: RAND analysis of 2008-2012 MEPS data.

RAND RR1165z1-4.10 
Figure 4-11

Reliance for All Veterans, by Health Care Service Category and Age

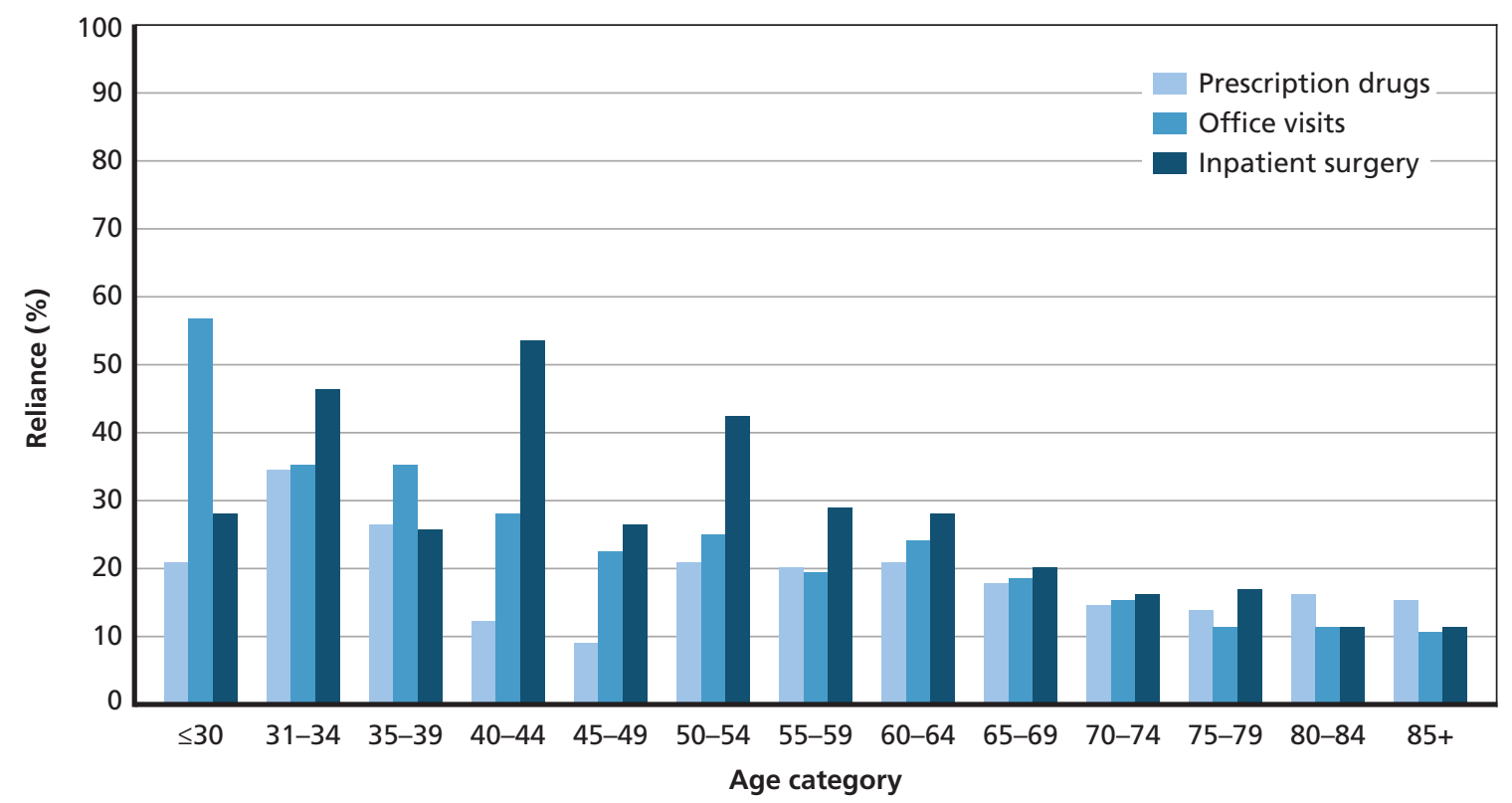

SOURCE: RAND analysis of 2008-2012 MEPS data.

RAND RR1165z1-4.11

Figure 4-12

Reliance for VA Patients, by Health Care Service Category, Sex, and Income

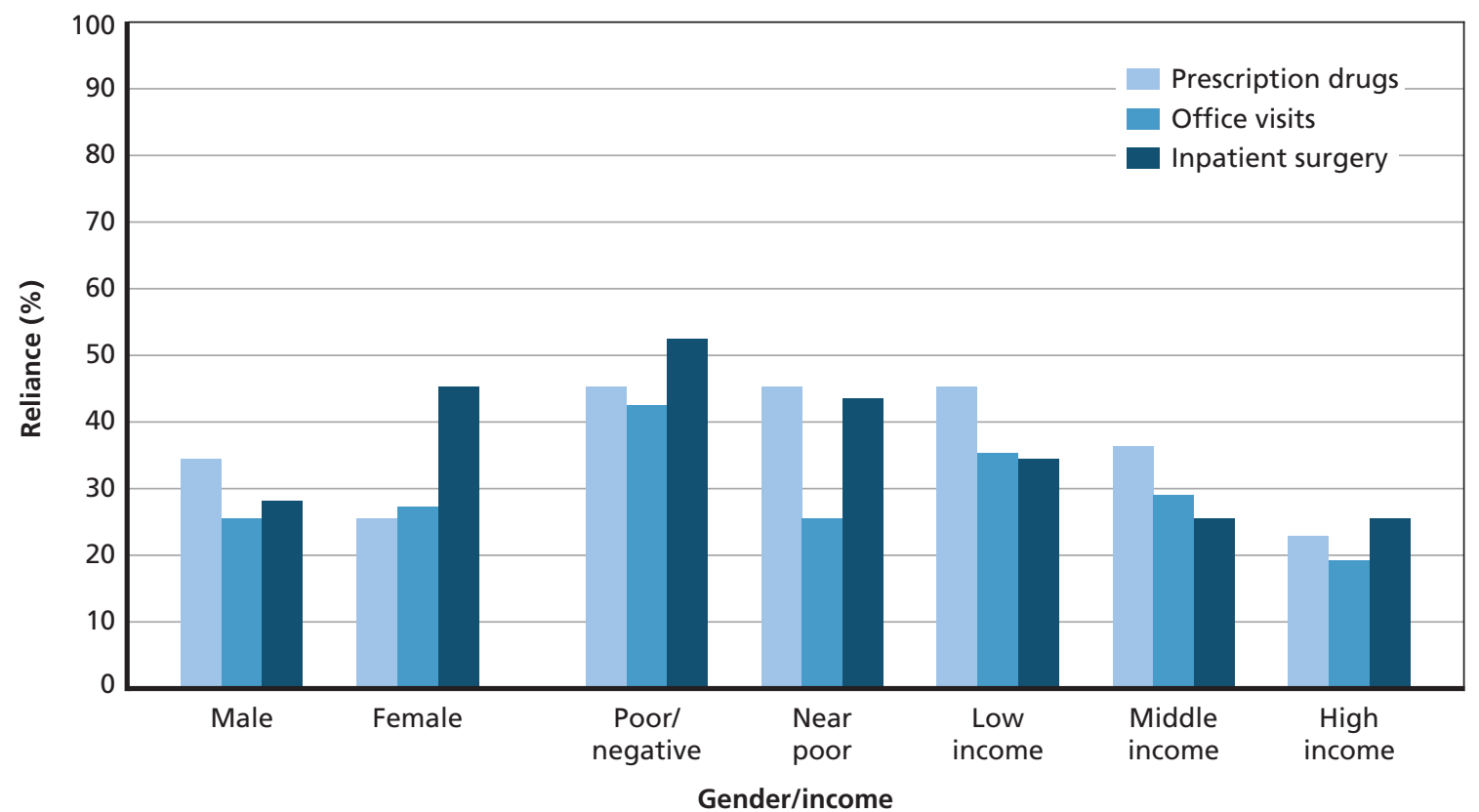

SOURCE: RAND analysis of 2008-2012 MEPS data.

NOTE: Income categories are defined as follows: Negative or poor: Less than $100 \%$ of the poverty line based on family size and composition; Near poor: $100 \%$ to less than $125 \%$ of the poverty line; Low income: $125 \%$ to less than $200 \%$ of the poverty line; Middle income: $200 \%$ to less than $400 \%$ of the poverty line; High income: $400 \%$ of the poverty line or higher.

RAND RR1165z1-4.12 
Figure 4-13

Reliance for All Veterans, by Health Care Service Category, Sex, and Income

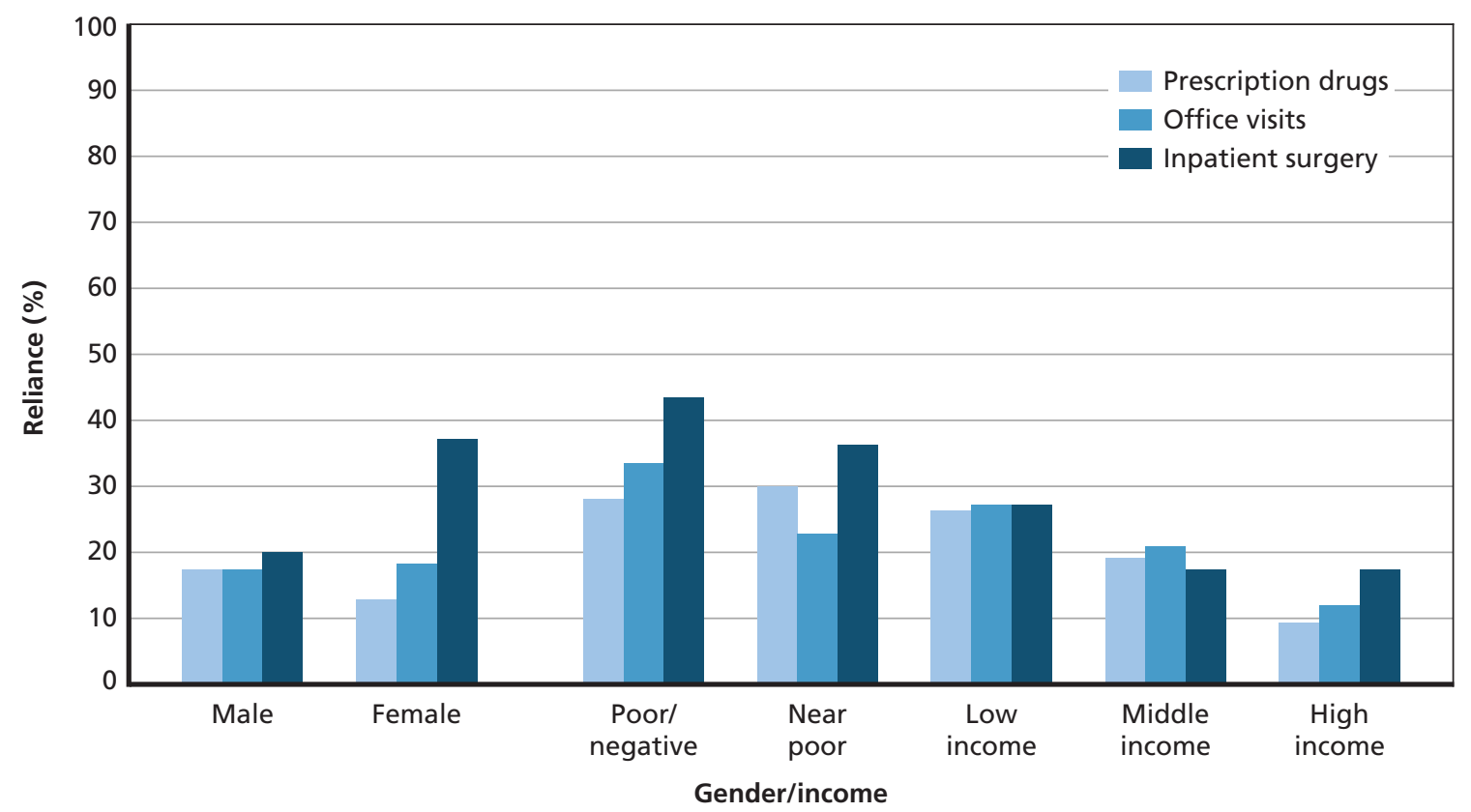

SOURCE: RAND analysis of 2008-2012 MEPS data.

NOTE: Income categories are defined as follows: Negative or poor: Less than $100 \%$ of the poverty line based on family size and composition; Near poor: $100 \%$ to less than $125 \%$ of the poverty line; Low income: $125 \%$ to less than $200 \%$ of the poverty line; Middle income: $200 \%$ to less than $400 \%$ of the poverty line; High income: $400 \%$ of the poverty lineor higher.

RAND RR1165z1-4.13

Figure 4-14

Reliance for VA Patients, by Coverage and Self-Reported Health Status

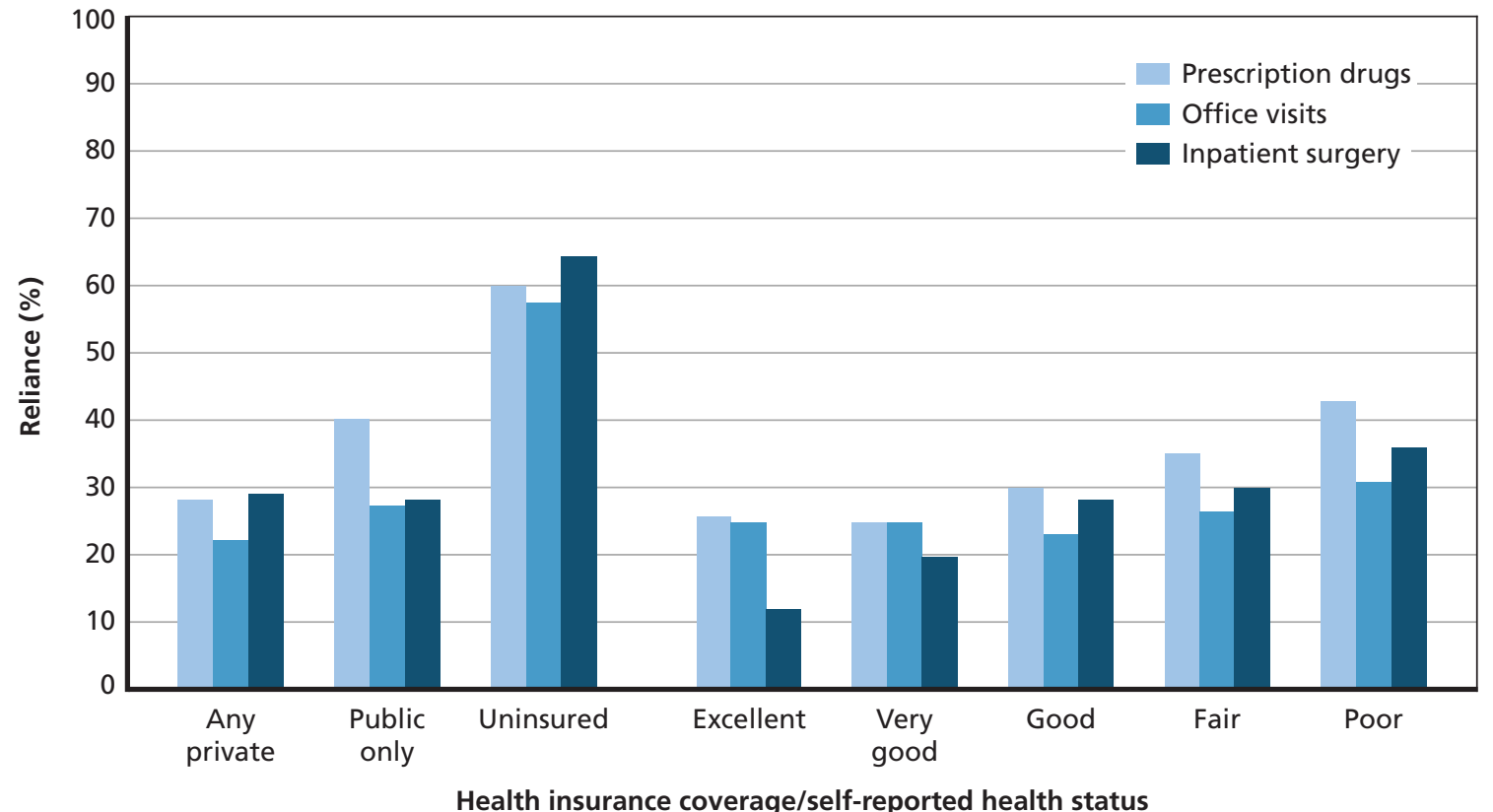

SOURCE: RAND analysis of 2008-2012 MEPS data.

RAND RR1165z1-4.14 
Figure 4-15

Reliance for All Veterans, by Coverage and Self-Reported Health Status

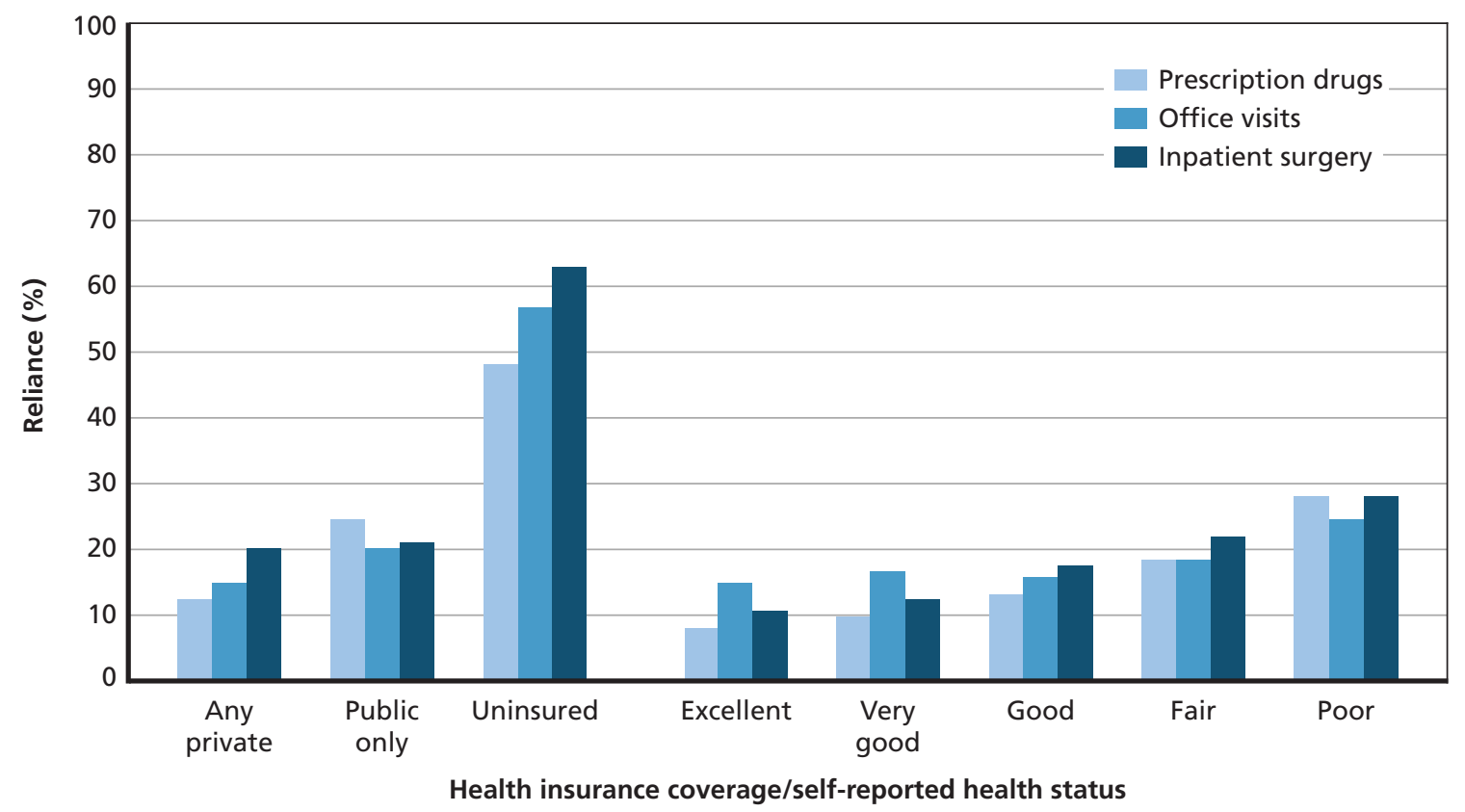

SOURCE: RAND analysis of 2008-2012 MEPS data.

RAND RR1165z1-4.15

\subsection{Reliance Analysis Results from EHCPM}

This analysis, which draws on output from the ECHPM, addresses VA enrollees rather than patients. Figure 4-16 presents reliance rates among enrollees for each health care service category that is projected in EHCPM using information from the private sector rather than only information from VA. We omit other health care service categories that are projected based on VA historical utilization patterns, such as outpatient mental health, because EHCPM does not separately estimate reliance for these categories. Reliance ranges from a high of 66 percent for prescription drugs to a low of 3 percent for chiropractic service visits.

There is significant variation in reliance rates among different Veteran subpopulations. For example, reliance for prescription drugs and for outpatient evaluation and management visits (i.e., outpatient visits that do not involve any procedures) generally decreases across Veteran age categories, with a consistent "bump" in reliance for Veterans at age 45 through Medicare eligibility at age 65 (Figures 4-17 and 4-18). 
Figure 4-16

EHCPM-Reported Reliance, by Service Category

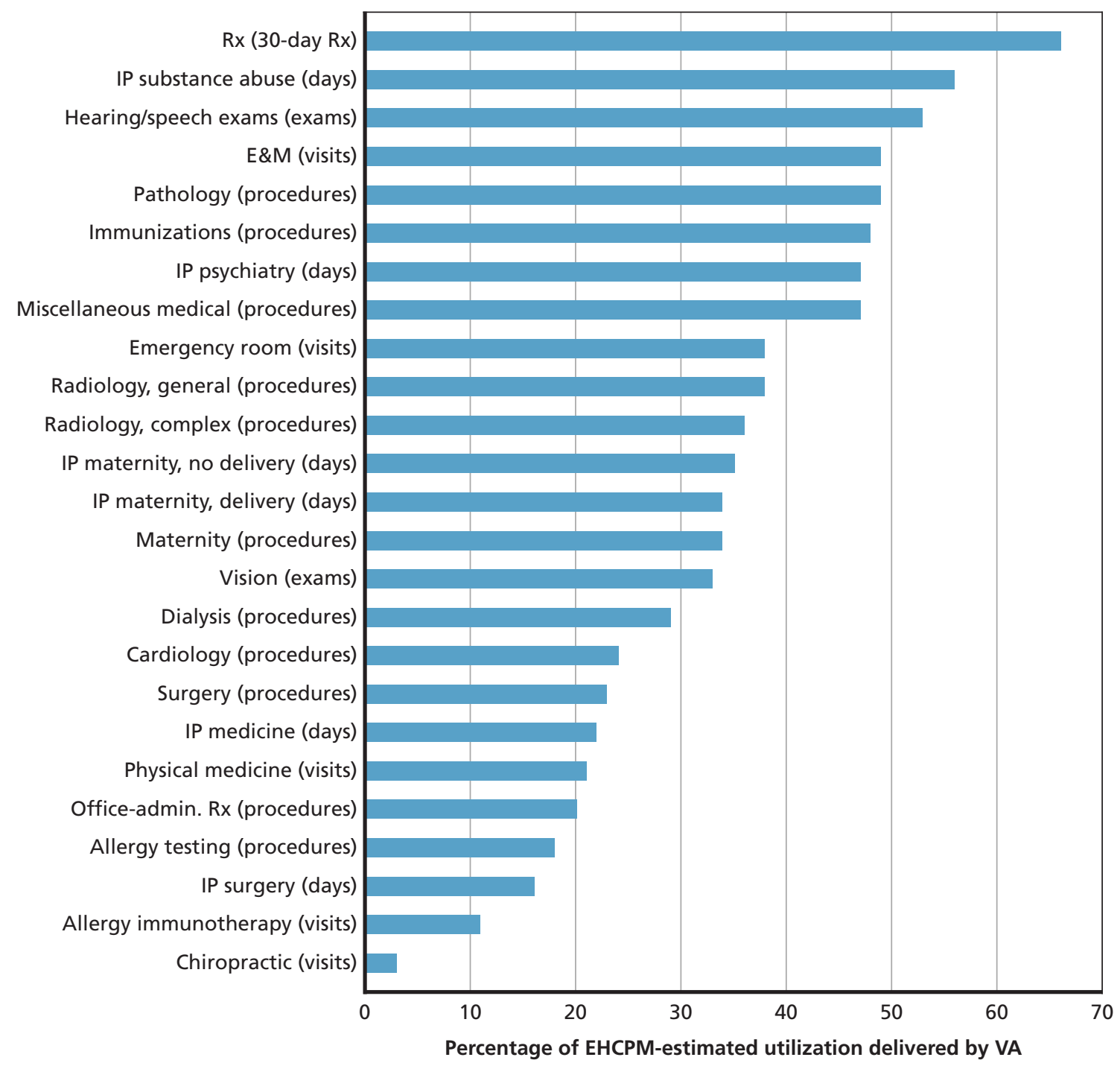

SOURCE: RAND analysis of EHCPM reliance factors and data.

NOTES: $\mathrm{Rx}=$ prescription; IP = inpatient; $\mathrm{E} \& \mathrm{M}=$ evaluation and management.

RAND RR1165z1-4.16 
Figure 4-17

EHCPM-Reported Prescription Drug (30-Day Rx) Reliance, by Age

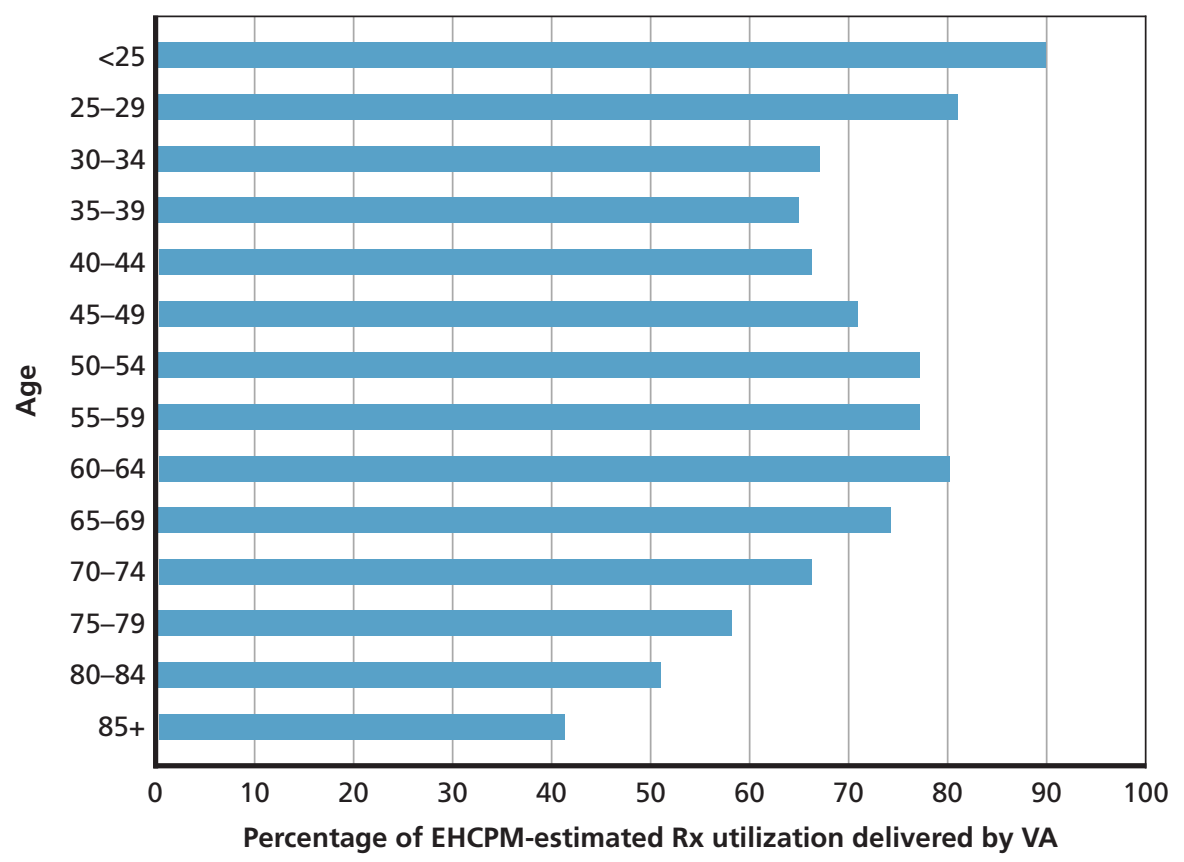

SOURCE: RAND analysis of EHCPM reliance factors and data.

NOTE: $R x=$ prescription.

RAND RR1165z1-4.17

Figure 4-18

EHCPM-Reported Evaluation and Management (Visits) Reliance, by Age

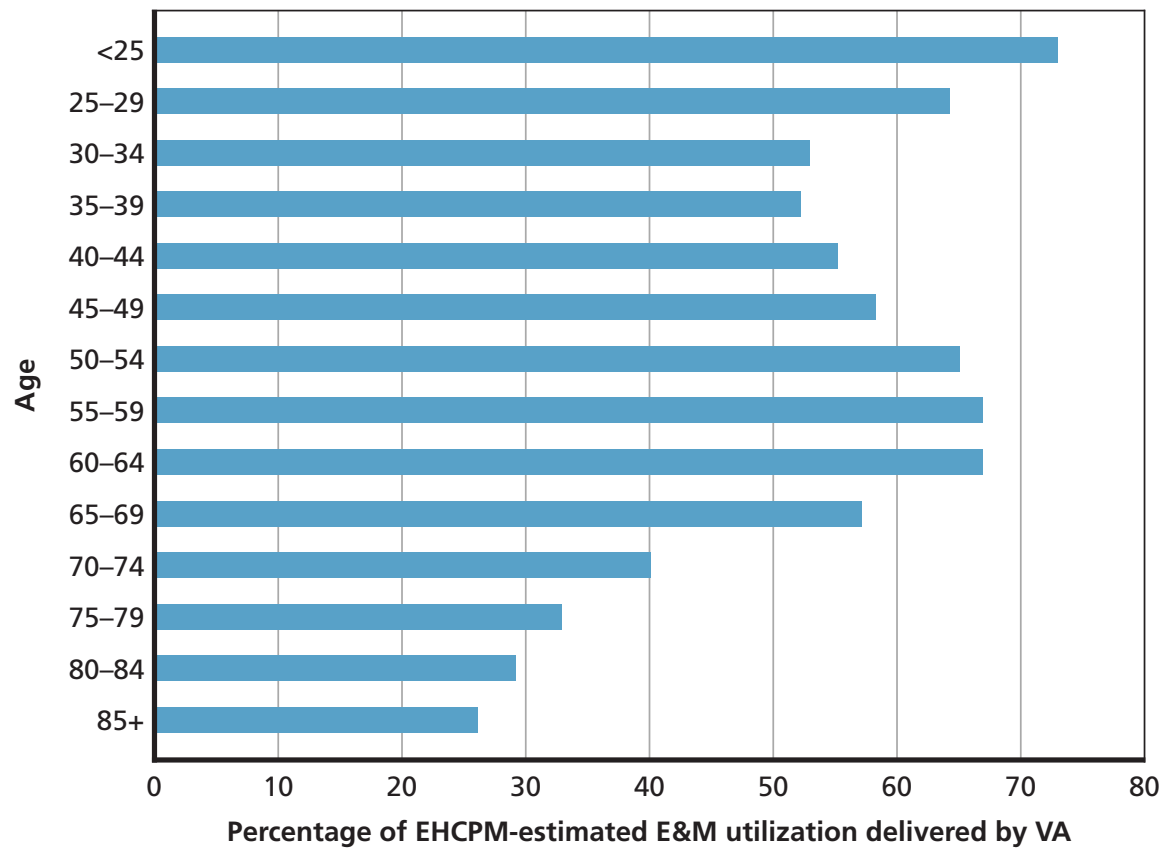

SOURCE: RAND analysis of EHCPM reliance factors and data.

NOTE: $E \& M=$ evaluation and management.

RAND RR1165z1-4.18 


\subsection{Discussion of Reliance Analysis Results}

When measured using external data from MEPS, reliance of VA patients on VA care tends to be lower than reliance calculated from EHCPM. For example, for prescription medications, reliance measured in MEPS is roughly 30 percent, compared with 66 percent in EHCPMmeaning that using the MEPS analysis, VA patients use VA services for a smaller proportion of their prescription medications. The differences are particularly stark given that the EHCPM focuses on enrollees, while the MEPS analysis focuses on VA patients. Because some enrollees do not use VA health care at all, one might expect that reliance rate would be higher among patients than enrollees. The one exception to this finding is inpatient stays with surgical procedures-where reliance measured with MEPS is nearly twice as high as that measured with EHCPM. This difference could be due in part to the different unit of measurement across these two data sources-days in EHCPM and stays in MEPS. A lower EHCPM reliance rate could be explained by shorter VA stays on average compared with the average length of stay across all of Veterans' surgical inpatient stays.

For prescribed medicines, whether or not drugs paid entirely out of pocket are included in the reliance denominator has a significant impact on the reliance estimate. In MEPS, prescriptions paid entirely out of pocket account for more than one-third of VA patient prescription drug events overall, and nearly half of prescription drug events for VA patients over age 85 (Figure 4-19). These prescriptions may be low-cost generic drugs (such as $\$ 5$ generics offered at chain pharmacies and "big box" retailers). For the health care service categories that we analyzed in this chapter, reliance in EHCPM is calculated using inputs from Medicare for the over- 65 population and from commercial benchmarks and the SoE for the under- 65 population. Based on these data sources, it is not clear whether the significant share of cash transactions observed in MEPS is accounted for in VA's reliance estimates.

Figure 4-19

MEPS-Based Coverage for Prescription Drug Events, VA Patients

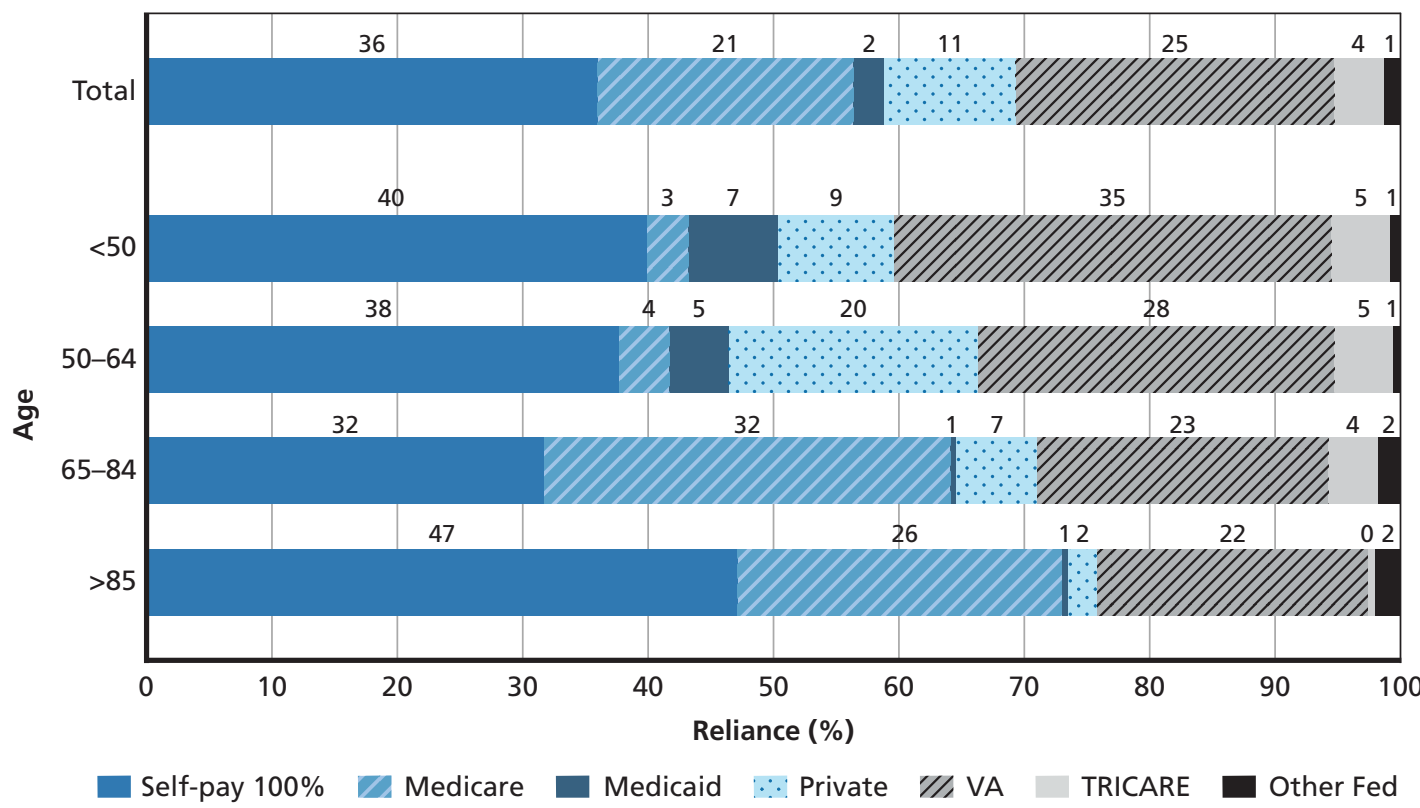

SOURCE: RAND analysis of 2008-2012 MEPS data.

RAND RR1165z1-4.19 
MEPS-based reliance estimates across health care service categories suggest that lowerincome Veterans, Veterans in rural areas, Veterans without other sources of coverage, and Veterans with poorer self-reported health status have higher reliance rates than other Veterans. That is, these groups obtain a larger proportion of their care from VA than other groups of Veterans.

The MEPS reliance estimates capture all health care utilization regardless of payer and regardless of whether the service is captured in VA encounter and claims data. However, there are important limitations when using MEPS to calculate reliance. First, MEPS contains relatively limited information on the specific health care services that patients receive. Second, the MEPS sample is small and for some health care service categories includes few Veterans, especially when restricting to specific Veteran subpopulations defined by age or other characteristics. Third, it is not always fully apparent in MEPS when services were (a) delivered by VA or (b) paid for by VA and delivered by private providers (i.e., purchased care). Fourth, MEPS estimates of health care utilization are based on respondents' recollections of the care they received, not on transactional data. Finally, some severely ill, high-utilization patients may drop out of MEPS when they die or experience an extended hospitalization. As a result, MEPS may omit utilization for the sickest patients.

Calculating reliance across the full range of health care service categories and for all Veteran subpopulations - as is done in EHCPM - is currently a considerable undertaking due to the lack of data sets describing the complete health care utilization of Veterans. Despite these limitations, MEPS provides comprehensive data that can be used to estimate reliance for Veterans directly rather than through analogy to non-Veteran populations. Future VA surveys or partnerships between VA and AHRQ (the organization that runs MEPS) could be designed to generate data for this purpose.

\subsection{Summary of Key Findings}

The number of Veterans receiving VA health care is projected to peak over the next 10 years. While the Veteran population is projected to decline by 20 percent over the next 10 years, the VA patient population is projected to reach its peak level in 2019. Use of VA has increased across all demographic groups since 2005, and the portion of Veterans under age 35 who are VA patients has increased threefold. The growth of VA use by Veterans may be related to outreach efforts on the part of VA, policies that have expanded the list of conditions granting presumptive eligibility for VA services, and streamlined enrollment processes. Continued increases in the rates of VA use are expected to slow the decline in the number of VA patients.

Understanding the future demand of health care services among Veterans who served in Iraq or Afghanistan is critical. By 2024, about 19 percent of VA patients are projected to have served in Afghanistan or Iraq. The long-term health impacts of service in Afghanistan and Iraq are unknown. It is imperative that VA monitor trends in health care utilization among these Veterans to ensure adequate resources and capabilities to meet the unique and changing health care demands of these Veterans

Veterans continue to seek VA health care after the period of enhanced eligibility closes. Among VA enrollees who were deployed in Afghanistan or Iraq, there is no difference in the percentage of Veterans using VA health care services between priority group 6 enrollees within the period of enhanced eligibility and priority group 7 or 8 enrollees outside the period 
of enhanced eligibility. This indicates that Veterans continue to use VA health care services after being shifted to a lower priority group at the end of the enhanced eligibility period. One plausible explanation for this pattern is that the enhanced eligibility policy encourages some Veterans who otherwise would not have used VA to seek care there. Then, if their needs are being met, or if employment or other circumstances do not present other health care options, they may continue to use VA beyond the enhanced eligibility period.

Health status and demographic factors influence reliance. Reliance estimates from MEPS, a nationally representative survey of the noninstitutionalized population, suggest that lower-income Veterans, Veterans in rural areas, Veterans without other sources of coverage, and Veterans with poorer self-reported health status have higher reliance rates than other Veterans.

Estimates from MEPS data show lower rates of reliance than those derived from VA's EHCPM model. For example, for prescription drugs, reliance measured in MEPS indicates that Veterans rely on VA to obtain roughly 30 percent of their prescription drugs compared with an estimate of 66 percent from EHCPM, although there are important differences between the two sources of reliance estimates.

The decision to include or exclude Veterans who are not VA patients influences reliance rates. Including Veterans who are not VA patients in reliance estimates yields lower "population reliance" rates. For example, reliance for prescription drugs among VA patients is 30 percent, while reliance for prescription drugs across all Veterans is 16 percent. Both statistics may be useful to decisionmakers. 


\subsection{Introduction}

Health care needs are an important determinant of demand for health care services. The aim of this chapter is to examine the health care needs of all Veterans, and of Veterans who use VA health care services in particular, so that VA can better anticipate and meet Veterans' needs.

Using the most recent data available, we first assessed the health care needs of all Veterans and of Veterans who have used VA health care services in the past year. We then projected the health care needs of both populations forward over a 10-year time horizon. We discuss the findings in three main sections:

An assessment of the unique health care needs of current Veterans relative to nonVeterans. As in earlier chapters, we define the unique health care needs of Veterans as those that disproportionately affect Veterans relative to non-Veterans. These include both serviceconnected conditions, such as PTSD, and other conditions that are more prevalent among Veterans than non-Veterans, including diabetes and cancer. Veterans with such conditions may be better served by specialists located at VA facilities if the prevalence in the national population is low and if non-VA providers are less equipped to address these conditions. To identify the unique non-combat-related health care needs of Veterans, we compared the prevalence of key health conditions among the current Veteran population with those among the non-Veteran population.

An assessment both of the health care needs of VA patients relative to Veterans who are not VA patients and of factors related to VA use. Veterans who meet VA basic eligibility and minimum service duty requirements (U.S. Department of Veterans Affairs, 2015c) can apply to claim VA benefits. Veterans, therefore, must choose to apply, enroll if deemed eligible, and finally choose to use VA services (take-up) if enrolled. Although we cannot observe all of this information in the data sources we analyzed, we can differentiate between Veterans who use VA services (whom we define as VA patients) and those who do not in a given year. An understanding of why Veterans become VA patients is needed to predict how changes in VA policy and other factors external to VA will affect the size and composition of the patient population. We therefore compared the prevalence of health conditions among VA patients with Veterans who were non-VA patients and analyzed which Veteran characteristics (including the presence of particular health conditions) were associated with receiving care at VA facilities.

Projections of the future health care needs of Veterans and VA patients for the years 2015-2024. The population of Veterans and VA patients may change substantially in the next decade as the current population ages and as new Veterans with different demographic charac- 
teristics and military service experiences choose to use VA services. We projected the prevalence of the health conditions of Veterans and VA patients forward over the next 10 years, accounting for predicted changes in their demographic composition and their service experiences.

\subsection{Overview of Methods}

\section{Overview of Methods and Data for Veteran Health Care Needs Analysis}

- We compared the unadjusted prevalence of diagnosed health conditions among Veterans with the unadjusted prevalence of the same conditions among non-Veterans.

- We then compared the same prevalence rates adjusted for demographic characteristics, including age, sex, and race/ethnicity.

- Similarly, we compared the unadjusted and adjusted prevalence of diagnosed health conditions among VA patients with the prevalence of the same conditions among Veterans who do not use VA health care services.

- We projected the prevalence of diagnosed health conditions among Veterans and VA patients by applying our prevalence estimates to the projected Veteran population (Chapter Three) and the projected VA patient population (Chapter Four).

- Data for this analysis came from MEPS, supplemented with encounter data from VA and MHS.

\subsubsection{Data Sources}

Our analyses relied on several data sources, including Veteran and nationally representative survey data. We highlight some of the primary sources of health data here. Further details about the data used are available in Chapter Two and Appendix C.1.3.

We relied primarily on MEPS. The individual-level data contain information on Veteran status (but do not include information on service era), age, sex, race/ethnicity, marital status, education, and income. Health information is obtained using open-ended questions about medical conditions present during the past year. These open-ended responses are then collapsed into ICD-9 codes.

MEPS is the only publicly available data source from which we could estimate diagnosed prevalence rates for all Veterans (who are defined based on self-report of being honorably discharged by the military). We inferred whether an individual in MEPS is a VA patient based on the respondents' source of payment for health care. Specifically, we defined active VA patients as those respondents who had any payment by VA for services used. Unfortunately, MEPS data did not enable us to identify all Veterans who are eligible for VA services; we could identify only those eligible Veterans who use VA services. There were also some specific conditions for which MEPS was incapable of providing reliable estimates due to sample size limitations; these primarily consisted of relatively rare conditions, such as polytrauma, TBI, and medically unexplained illness.

We augmented MEPS with two administrative data sources. VA encounter data include individual-level information on diagnoses, demographic characteristics, and geographic location (state). VA encounter data are used to estimate current and prior condition prevalence patterns among active VA patients. This data set has larger sample sizes than MEPS, which allowed us to estimate the prevalence of service-connected health conditions that have a low prevalence rate in the national population. A limitation of the VA encounter data is that the 
data include information only on VA patient encounters and may miss diagnoses and conditions that were treated in non-VA settings. This includes Veterans not enrolled in VA, Veterans who were enrolled but did not use VA services in the survey year, and Veterans who were only partially reliant on VA. We therefore consulted the existing literature to find estimates of the prevalence of particular conditions. These instances are noted in the text. We also used MHS encounter data, which include information on the diagnoses and demographic characteristics of active component military personnel. The prevalence of health conditions among separating personnel predicts the health conditions of the newest Veteran cohorts and VA patients.

\subsubsection{Analytic Approach}

The main analyses in this section focus on comparisons of the prevalence of diagnosed health conditions between populations and on projections of health conditions among Veterans. We compared diagnosed prevalence rates between Veterans and non-Veterans, and between VA patients and non-VA patients. We made projections for both the general Veteran population and the VA patient population. A full description of the methods can be found in Appendix C.1.5.

Ideally, to understand the relative health care needs of Veterans versus non-Veterans and VA patients versus non-VA patients, we would estimate the underlying ("true") prevalence of health conditions for each population. Such estimates would allow us to assess more accurately the unique health care needs of Veterans and to understand how changes in where Veterans access care will affect the care they receive and the demands on health care service providers, including VA. However, it is not possible to measure underlying health status and undiagnosed conditions, so we focus on the prevalence of diagnosed health conditions.

The diagnosed prevalence rates are determined by the underlying prevalence of health conditions, access to/use of health care, and the propensity of the health care providers to diagnose particular conditions. For example, if Veterans are more likely to seek health care services than non-Veterans because they are more likely to have health insurance coverage (Chapter Three), their underlying health conditions will be diagnosed at higher rates. On the provider side, VA specializes in Veterans' health care, so VA staff may be more likely to recognize health conditions that are significant for the Veteran population but are relatively rare in the non-Veteran population (e.g., PTSD) and diagnose these at a higher rate. This would cause the diagnosed prevalence rates of such conditions to be higher among Veterans who use VA health care services than among Veterans (and non-Veterans) who seek health care from other providers, even if the underlying prevalence was the same.

With these limitations in mind, we consider three alternative estimates of diagnosed prevalence: (1) unadjusted observed prevalence rates, (2) prevalence rates adjusted to account for the demographic differences between the two populations, and (3) prevalence rates adjusted to account for demographic differences and differences in access to health care. The unadjusted observed prevalence rates (alternative 1) are estimated as the proportion of the population with a particular health condition. The adjusted prevalence rates (alternatives 2 and 3) are estimated with a generalized linear regression model applied to individual-level data (MEPS, 20062012). The demographic-adjusted model includes age, sex, race/ethnicity, census region of residence, an indicator for residence in a metropolitan area (the MSA), and a time trend as predictors of the probability of having each diagnosed health condition. The health access-adjusted model extends the demographic-adjusted model to include educational attainment, health insurance coverage, marital status, and employment/full-time student status. The appropriate approach for estimating prevalence of diagnosed health conditions depends on the question 
being addressed. We describe what can be learned from each approach for the comparison of Veterans to non-Veterans and VA patients to non-VA patients below.

We first compare disease prevalence among Veterans with non-Veterans to assess the unique health care needs of the Veteran populations. The simple comparison of the observed diagnosed prevalence rates among Veterans and non-Veterans provides insights about actual differences in health care needs across the two populations. The differences in diagnosed prevalence rates do not necessarily reflect the differences in the underlying health care needs of Veterans and non-Veterans, but they do represent differences in the conditions that Veterans and non-Veterans are being treated for, and thus shed light on differences in the types of conditions that community and VA health care providers need to be prepared to treat, given status quo policies.

The simple comparison of diagnosed prevalence rates between Veterans and non-Veterans does not allow us to disentangle differences in health status that are due to the different demographic composition of Veterans and non-Veterans or differential access to health care from differences due to other factors. For example, because Veterans are older on average, we would expect higher prevalence rates among Veterans in conditions correlated with age, such as hypertension. For a complete summary of the demographic differences between Veterans and non-Veterans, see Section 5.3.1 and Appendix C.2.3.

In order to identify the unique health care needs of Veterans, it is therefore necessary to adjust statistically for these differences in demographic characteristics. Adjusted rates allow us to compare prevalence among non-Veterans and Veterans who "look alike" based on their demographic characteristics (age, sex, race/ethnicity, and residential location, as described above). We did this by predicting the prevalence of each health condition among Veterans and non-Veterans with the same demographic characteristics. Adjusted estimates use the Veteran population as our reference population, so that adjusted non-Veteran prevalence rates are predicted "as if" non-Veterans had the same demographic characteristics as Veterans. Therefore, we can attribute any differences remaining after adjustment to factors other than the demographics we include in our model (e.g., military service or deployment, environmental risks, occupational health risks, and unobserved individual-level characteristics that underlie the decision to join the armed forces, such as sense of duty to the country). This provides insight into health conditions that are unique to Veterans and not simply attributable to the demographic composition of the Veteran population.

Finally, to try to estimate diagnosed prevalence rates that are closer to the underlying prevalence of each health condition, we also adjust for such factors as health insurance, employment, and education (as described above), some of which are likely related to health care access and use. However, the results from this approach are difficult to interpret. Unlike the basic demographic characteristics, these additional controls are potentially influenced by the experience of being a Veteran, by DoD and VA policies, and by individual health status. For example, access to health care coverage may be associated with better or worse health, and differentially so across Veterans and non-Veterans. VA specifically targets health coverage to Veterans with worse health outcomes (e.g., service-connected disabilities), ensuring that health coverage is available. On the other hand, health insurance coverage is often tied to employment, so non-Veterans who find it difficult to work due to a health condition will be less likely to have health insurance coverage. For these reasons, we focus on the adjusted model that controls for demographic characteristics only. However, we provide results for the extended model 
that also controls for health care access in Appendix C.3. In practice, the two adjusted models produce very similar results.

Similarly, comparisons between VA patients and non-VA patients are made using both unadjusted and adjusted prevalence estimates. The reference population for the adjusted prevalence remains the entire Veteran population. All comparisons made between VA patients and non-VA patients using adjusted prevalence account for the different demographic composition of the two populations. Therefore, we can attribute any remaining differences after adjustment to factors other than the demographics we include in our model. As with Veterans, the unadjusted prevalence rates are the best indicators of the current health care needs among each population and the needs facing the health care providers that serve these populations. The adjusted prevalence rates allow us to better understand the disproportionate prevalence of health conditions among VA patients after controlling for predisposing demographic characteristics.

We projected the future prevalence (and counts) of key health conditions among Veterans in three main steps. First, we projected forward the Veteran population, categorized by demographic group, as described in Chapter Three. Second, we projected forward the prevalence of key health conditions, categorized by demographic group using MEPS. We also incorporated a nonlinear trend to account for unobservable trends in risk factors. See Appendix C.1.5 for details. Finally, we multiplied the number of Veterans in each demographic group by the corresponding prevalence to yield the projected number of Veterans with a particular health condition from 2015 through 2024.

We also projected the future prevalence (and counts) of key health conditions among $V A$ patients in four main steps. First, we projected the Veteran population forward, categorized by demographic group, as described in Chapter Three. Second, we projected forward the number of VA patients, categorized by demographic group in Chapter Four. Third, we projected forward the prevalence of key health conditions, categorized by demographic groups using MEPS and VA encounter data. We also incorporated a nonlinear trend to account for unobservable trends in risk factors. Finally, we multiplied the number of VA patients in each demographic group by the corresponding prevalence to get the projected number of VA patients with a particular health condition from 2015 through 2024. See Appendix C.1.5 for details.

\subsection{Current Health Care Needs of Veterans and Non-Veterans}

In this section, we present the estimates of the prevalence of select health conditions among Veterans and non-Veterans using the most recent data available. There are several data limitations that should be kept in mind when viewing the results. Most of the results reported here use ICD-9 diagnosis codes in MEPS data to determine each individual's health conditions. These ICD-9 codes were derived from professional coders' abstractions of respondents' interviews; they are not derived from claims. Prevalence rates may be underestimated in respondents with limited access to health care, who may not know which conditions they have (see Appendix A for further discussion). In addition, ICD-9 codes do not indicate severity of illness or the complexity of a patient's situation. Fully analyzing the severity and complexity of patients' conditions would have required abstracting medical records, a task that was not possible given data constraints and the time frame available to complete this report. MEPS does not include information about service era or service-connected disability, so we are not able to 
look at differences in the prevalence of health conditions along these dimensions. ${ }^{1}$ However, MEPS is the only data source that provides health condition information for all Veterans independent of health care provider.

\subsubsection{Demographic Differences Between Veterans and Non-Veterans}

Veterans and non-Veterans differ not only by military service experiences but also by demographic characteristics that are associated with the prevalence of particular health conditions. This section evaluates the state of Veterans' health relative to that of non-Veterans. In addition, we identified conditions that disproportionately affect Veterans after accounting for demographic differences between the populations.

Using MEPS data, we also examined the demographic profile of Veterans and nonVeterans (see also Table C-18 in Appendix C), which may explain differences in disease prevalence. We found significant differences in age, sex, and race/ethnicity composition. Veterans and non-Veterans also differ in their geographic distribution.

Figure 5-1 shows the age distribution among Veterans and non-Veterans in the MEPS sample. These data demonstrate that Veterans are older; nearly 70 percent of Veterans are age 55 or older, compared with 31 percent of non-Veterans. When we examined the sex composition among Veterans and non-Veterans in our sample, we found that Veterans are predominantly male; more than 93 percent of Veterans are men, compared with 40 percent of non-Veterans. ${ }^{2}$ These differences are consistent with those reported by VA (Smith, 2014; U.S. Census Bureau, 2014; U.S. Department of Veterans Affairs, 2014a; U.S. Government Accountability Office, 2009).

When we compared the race/ethnicity composition of Veterans and non-Veterans, we found that, consistent with tabulations from other sources (Lee \& Beckhusen, 2012; National Center for Veterans Analysis and Statistics, 2014c), Veterans are predominantly non-Hispanic white men. In our sample, more than 82 percent of Veterans identified themselves as nonHispanic white, compared with 66 percent of non-Veterans. Because access to medical services can vary by geographic area, we also included adjustments for Census region of residence. For example, VA has noted that the largest populations of Veterans are in the South (9.9 million) and Midwest (6.1 million) (National Center for Veterans Analysis and Statistics, 2012a). MEPS data include an indicator for whether each respondent resides in an MSA. We used this as a proxy for rural versus urban residence, because previous studies have shown that rural Veterans are different from Veterans living in urban areas. For example, rural Veterans are more likely than urban Veterans to have at least one disability or to have a service-connected disability rating of 50 percent or more (National Center for Veterans Analysis and Statistics, 2012a). MEPS data indicate that about 81 percent of Veterans live in a metropolitan area versus about 85 percent of non-Veterans.

In the next section, we present the prevalence of diagnosed health conditions among Veterans and non-Veterans as estimated in MEPS. Differences in demographic characteristics of

\footnotetext{
1 MEPS began including service era in 2011; however, the Veteran and VA patient sample sizes for 2011 forward were not large enough for this analysis.

2 The proportion of the non-Veteran population that is male is lower than the proportion of the U.S. population that is male (49 percent) (U.S. Census Bureau, 2014) because it excludes Veterans, who are about 7 percent of the population and 93 percent male (U.S. Census Bureau, 2012a). MEPS also excludes individuals in military and correctional institutions, juvenile institutions, military housing, and other institutions, who are more likely to be male.
} 
Figure 5-1

The Age Distribution for Veterans and Non-Veterans

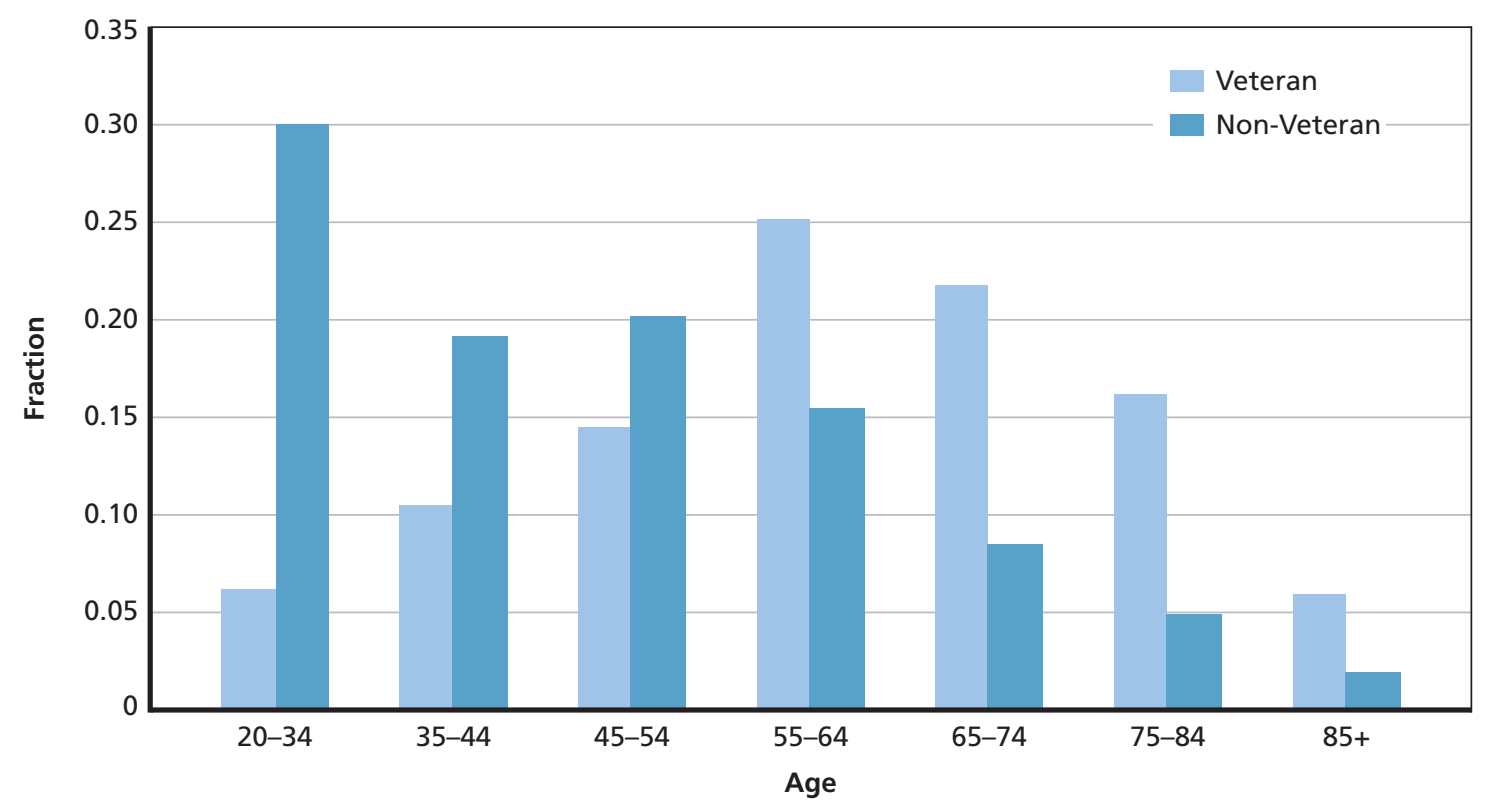

SOURCE: MEPS, 2006-2012.

NOTES: ** indicates a statistically significant difference between Veterans and non-Veterans at $\mathrm{p}$-value $<0.05$.

Sample size, non-Veterans $=222,178$, and sample size, Veterans $=12,953$.

RAND RR1165z1-5.1

Veterans and non-Veterans likely account for a substantial proportion of the unadjusted differences in health care needs between the two populations. This comparison provides insights about actual differences in health care needs across the two populations, as discussed in Section 5.2.2. However, given that we aim to identify the unique health care needs of Veterans that result from the total experience of military service, it is important to estimate differences between Veterans and non-Veterans that are not driven by differences in the demographic characteristics of the two groups. Thus, in the remainder of Section 5.3, all comparisons made between Veterans and non-Veterans will use "adjusted" estimates, which are statistically corrected to account for the different demographic composition of the two populations.

There are also socioeconomic characteristics that differ between Veterans and nonVeterans and that may be related to diagnosed disease prevalence through impacts on underlying prevalence, access to health care, or provider type. For example, Veterans are more likely to be married than non-Veterans, a finding that is consistent with previous research documenting higher rates of marriage among military relative to civilian populations (Karney, Loughran, \& Pollard, 2012). These additional characteristics are summarized by Veteran and VA patient status in Table 5-1. As discussed in Section 5.2, we do not adjust for these additional differences between Veterans and non-Veterans in our main adjusted model because they may be affected by the experience of being a Veteran and by DoD and VA policies, making the results difficult to interpret. However, we provide results for the extended model that controls for these additional characteristics in Appendix C.3.1. Our baseline adjusted model and the extended model produce very similar results. 
Table 5-1

Socioeconomic Characteristics of Non-Veterans, Veterans, Non-VA Patients, and VA Patients in MEPS

\begin{tabular}{|c|c|c|c|c|}
\hline \multirow[b]{2}{*}{ Demographic Group } & \multicolumn{4}{|c|}{$\begin{array}{l}\text { Distribution by Demographic Characteristics } \\
\text { (Standard Error) }\end{array}$} \\
\hline & Veterans & Non-Veterans & $\begin{array}{l}\text { Veterans, VA } \\
\text { Patients }\end{array}$ & $\begin{array}{l}\text { Veterans, Non-VA } \\
\text { Patients }\end{array}$ \\
\hline \multicolumn{5}{|l|}{ Marital status } \\
\hline Married & $\begin{array}{c}0.659 \\
(0.009)\end{array}$ & $\begin{array}{c}0.523 \\
(0.004)\end{array}$ & $\begin{array}{c}0.626 \\
(0.010)\end{array}$ & $\begin{array}{c}0.680 \\
(0.011)\end{array}$ \\
\hline \multicolumn{5}{|l|}{ Student status } \\
\hline Student or currently in school & $\begin{array}{c}0.008 \\
(0.001)\end{array}$ & $\begin{array}{c}0.094 \\
(0.001)\end{array}$ & $\begin{array}{c}0.008 \\
(0.001)\end{array}$ & $\begin{array}{c}0.008 \\
(0.001)\end{array}$ \\
\hline \multicolumn{5}{|l|}{ Educational attainment } \\
\hline Less than high school & $\begin{array}{c}0.071 \\
(0.004)\end{array}$ & $\begin{array}{c}0.176 \\
(0.003)\end{array}$ & $\begin{array}{c}0.091 \\
(0.006)\end{array}$ & $\begin{array}{c}0.058 \\
(0.004)\end{array}$ \\
\hline High school diploma or GED & $\begin{array}{c}0.340 \\
(0.009)\end{array}$ & $\begin{array}{c}0.308 \\
(0.004)\end{array}$ & $\begin{array}{c}0.350 \\
(0.011)\end{array}$ & $\begin{array}{c}0.333 \\
(0.010)\end{array}$ \\
\hline Some college & $\begin{array}{c}0.210 \\
(0.006)\end{array}$ & $\begin{array}{c}0.180 \\
(0.002)\end{array}$ & $\begin{array}{c}0.203 \\
(0.009)\end{array}$ & $\begin{array}{c}0.214 \\
(0.007)\end{array}$ \\
\hline College & $\begin{array}{c}0.380 \\
(0.008)\end{array}$ & $\begin{array}{c}0.336 \\
(0.005)\end{array}$ & $\begin{array}{c}0.356 \\
(0.010)\end{array}$ & $\begin{array}{c}0.396 \\
(0.010)\end{array}$ \\
\hline Employment status & & & & \\
\hline Employed (not on active duty) ${ }^{a}$ & $\begin{array}{c}0.546 \\
(0.009)\end{array}$ & $\begin{array}{c}0.707 \\
(0.003)\end{array}$ & $\begin{array}{c}0.413 \\
(0.012)\end{array}$ & $\begin{array}{c}0.628 \\
(0.010)\end{array}$ \\
\hline Income & & & & \\
\hline Total household income (\$) & $\begin{array}{c}41,708 \\
(541.29)\end{array}$ & $\begin{array}{c}33,546 \\
(304.78)\end{array}$ & $\begin{array}{c}35,981 \\
(753.76)\end{array}$ & $\begin{array}{c}45,278 \\
(646.53)\end{array}$ \\
\hline
\end{tabular}

SOURCE: RAND analysis of MEPS, 2006-2012.

NOTES: Veterans, VA patients and Veterans, non-VA patients are mutually exclusive categories of Veterans. Sample size, Veterans $=12,313$; sample size, non-Veterans $=150,225$; sample size, VA patients $=4,871$; and sample size, non-VA patients $=7,442$.

${ }^{a}$ Non-employed individuals includes both people who are unemployed and people who are out of the labor force, such as retirees. 


\subsubsection{Prevalence of Health Conditions for Veterans and Non-Veterans}

Analysis of MEPS data showed that the diagnosed prevalence of many health conditions is greater for Veterans than non-Veterans. In Appendix C, Table C-6, we list the health conditions examined and report adjusted and unadjusted prevalence rates for Veterans and nonVeterans. Differences in unadjusted prevalence rates vary by condition. For example, the diagnosed prevalence of asthma among Veterans is about 1.4 percentage points lower than among non-Veterans, but the prevalence of hypertension and lipid disorders among Veterans is more than 20 percentage points higher than for non-Veterans. We illustrate the pattern of our findings in Figure 5-2 for a subset of conditions examined. Differences that are statistically different from zero at $\mathrm{p}<0.05$ are marked with two asterisks $(* *)$. Except for mental health conditions, Veterans exhibit higher unadjusted prevalence rates than non-Veterans. However, demographic characteristics affect the prevalence of many health conditions; the prevalence of hypertension and many other chronic conditions increases with age, and some conditions are more prevalent in men than in women. In the next section, we adjust the prevalence estimates for the demographic differences between Veterans and non-Veterans described in the previous section. The difference between the unadjusted and adjusted rates reflects the portion of the differences in prevalence rates that can be explained by demographic differences.

Figure 5-2

Diagnosed (Unadjusted) Prevalence of Selected Health Conditions for Veterans and Non-Veterans

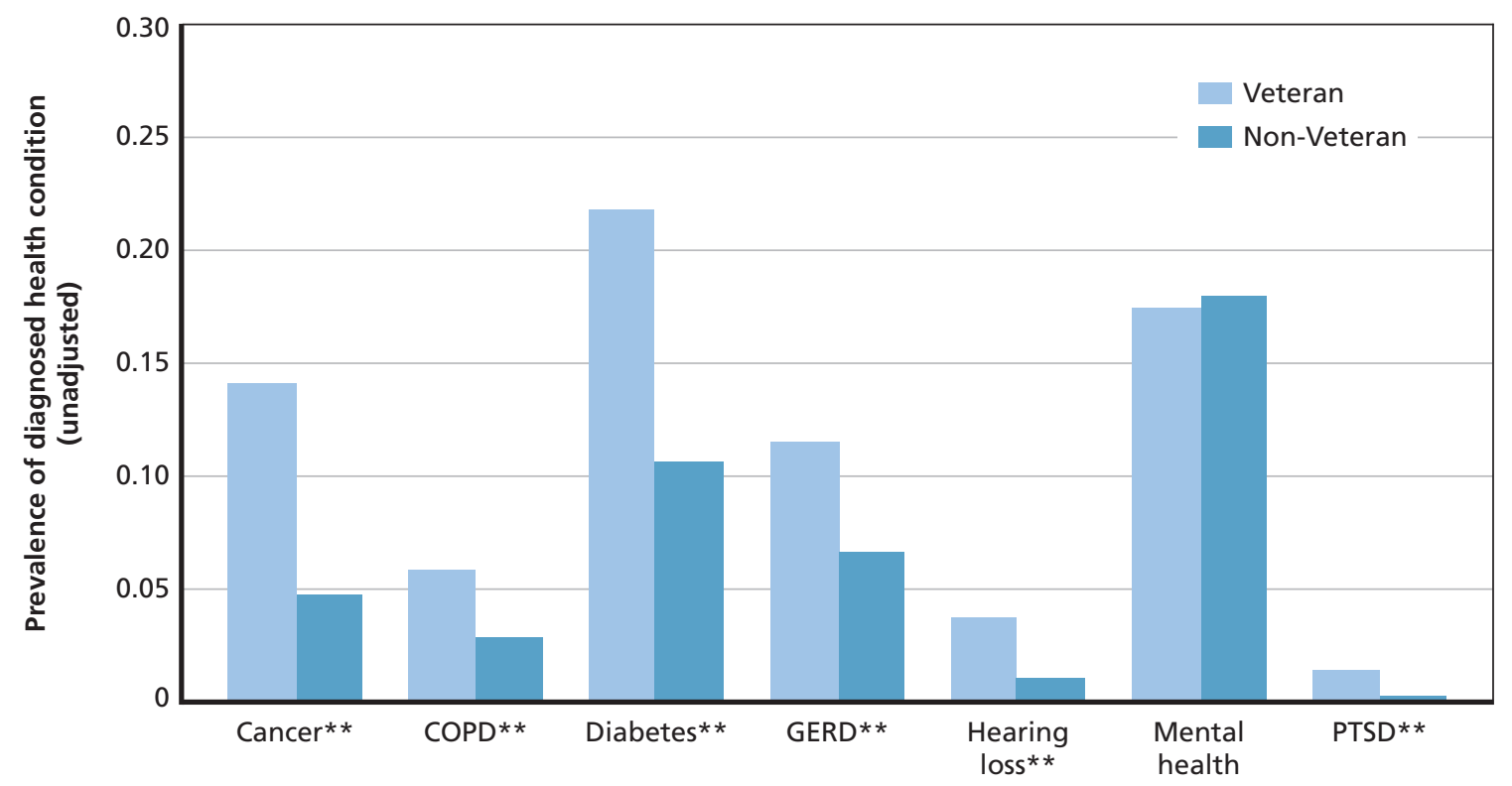

SOURCE: RAND analysis of MEPS, 2006-2012.

NOTES: ** indicates a statistically significant difference between Veterans and non-Veterans at p-value $<0.05$. Sample size, non-Veterans $=150,225$, and sample size, Veterans $=12,313$. Sample sizes may be smaller for some conditions due to missing values. Cancer includes any malignancy, and Mental Health includes any mental health condition.

RAND RR1165z1-5.2 


\subsubsection{Prevalence of Health Conditions for Veterans and Non-Veterans, Adjusting for Demographic Differences}

In this section, we present estimates of the prevalence of health conditions for Veterans and non-Veterans, adjusting for a set of demographic characteristics and time trends (changes in disease prevalence over time). We adjusted for age, sex, race/ethnicity, Census region, whether an individual resided in an MSA, and calendar year. We adjusted for age to account for changes in the development of health conditions over the life cycle and for the differential age composition of Veterans and non-Veterans, as seen in Figure 5-3. We adjusted for Census region of residence and whether an individual resided in an MSA to account for the differences in where Veterans and non-Veterans reside. We adjusted for sex and race/ethnicity to account for the demographic differences of Veterans and non-Veterans. Finally, we adjusted for differences in health conditions over time due to secular changes in disease prevalence. For example, a public health campaign to increase awareness of preventive treatments for a particular condition could cause a decline in prevalence that is not related to Veteran status (see Appendix C.2). We refer to this baseline model of adjusted disease prevalence as Model 1.

Overall, we found smaller differences in adjusted diagnosed disease prevalence rates in Model 1 relative to the unadjusted differences, but results still suggest that the adjusted prevalence of many chronic conditions is higher for Veterans than it is for non-Veterans of the same age, sex, and race/ethnicity. Figure 5-3 shows the results for cancer, COPD, diabetes, GERD, hearing loss, any mental health condition, and PTSD (see full results in Table C-6). The largest

Figure 5-3

Adjusted Diagnosed Prevalence of Selected Health Conditions for Veterans and Non-Veterans

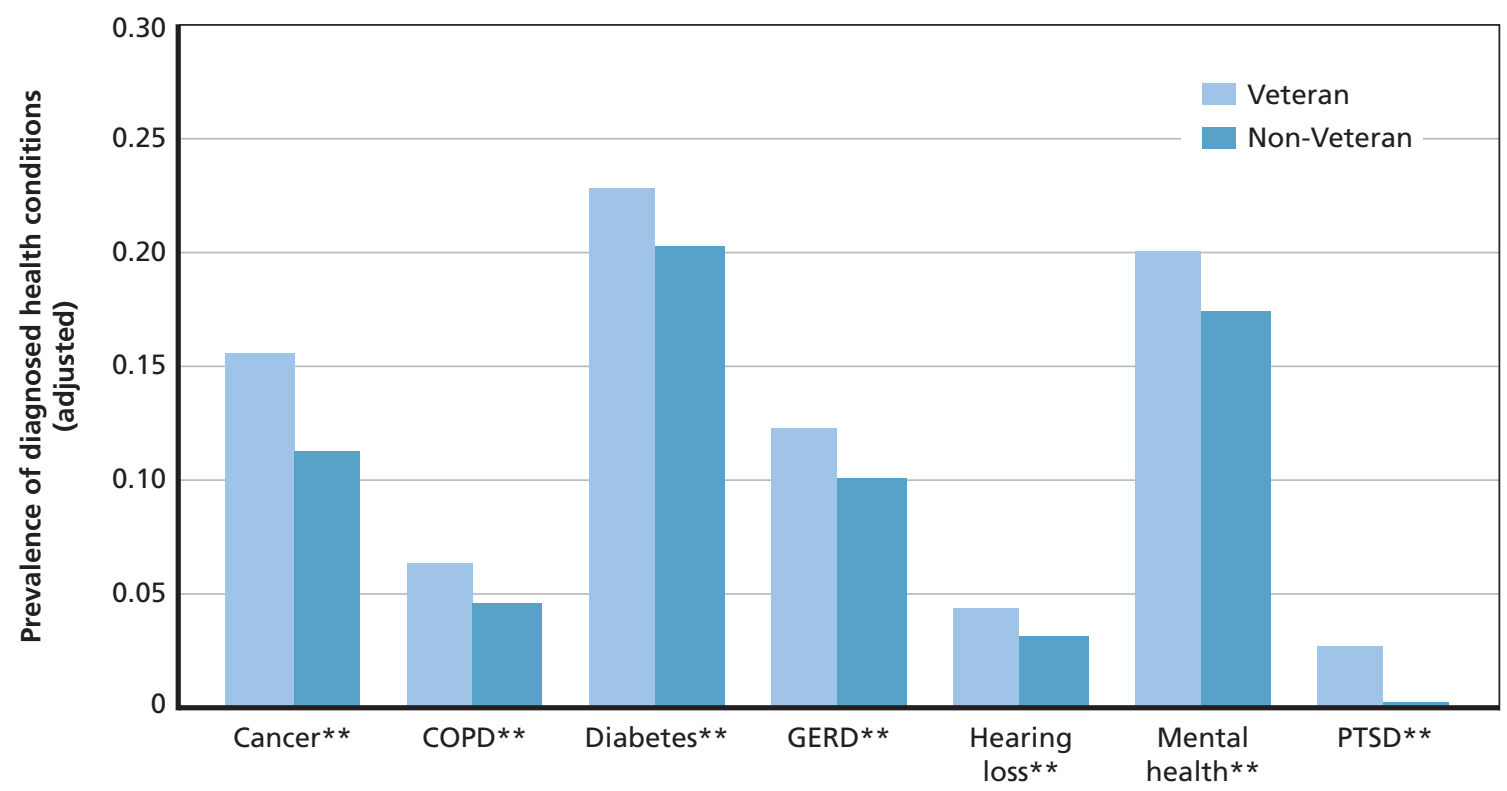

SOURCE: RAND analysis of MEPS, 2006-2012.

NOTES: ** indicates a statistically significant difference between Veterans and non-Veterans at p-value $<0.05$. Sample size, non-Veterans $=150,225$, and sample size, Veterans $=12,313$. Sample sizes may be smaller for some conditions due to missing values. The prevalence rate of each health condition is the predicted prevalence in 2014 for the populations of Veterans and non-Veterans, both with age, sex, race/ethnicity, region, and urbanicity, adjusted to match the demographic composition of Veterans in 2012. Cancer includes any malignancy, and Mental Health includes any mental health condition.

RAND RR1165z1-5.3 
absolute difference in prevalence is for cancer at 4.3 percentage points, followed by diabetes, mental health conditions, PTSD, and GERD at 2.5 percentage points.

Another way to compare prevalence rates is to examine relative risk of being a Veteran; that is, the prevalence rate for Veterans divided by the prevalence rate for non-Veterans. The largest relative risk in prevalence is for PTSD, which is 13.5 times more prevalent among Veterans than non-Veterans. The prevalence of cancer, hearing loss, and COPD are more than 1.3 times more prevalent for Veterans than non-Veterans. Hypertension, which is excluded from Figure 5-3 due to scale, has the highest prevalence (at 47 percent) for both Veterans and nonVeterans (see Table C-6.)

We examined the full set of estimable health conditions in MEPS. The pattern of results is qualitatively similar to those reported here (see Table C-6). Moreover, the differences in the prevalence of health conditions between Veterans and non-Veterans are not sensitive to different specifications of the statistical model. In a second model, Model 2, we adjusted for additional individual factors, including marital status, education level, employment and student status, health insurance coverage, and interactions between race/ethnicity and sex (see Appendix C.3.1). Differences in predicted prevalence rates are qualitatively similar to those reported here. Model 2 results showed that marriage, college completion, and employment are associated with a lower probability of having been diagnosed or treated for most health conditions. Health insurance coverage is associated with higher probability of having been diagnosed or treated for most health conditions. This finding is consistent with the notion that insured Veterans and non-Veterans use more health care services than uninsured persons because, in MEPS, measures of health conditions identified by providers reflect only those conditions for which individuals received health care.

Next, we examined the extent to which Veterans and non-Veterans differ in disease burden and comorbidities, based on adjusted differences in the Charlson Comorbidity Index, measures of functional status, and the prevalence of comorbid mental health (a mental health diagnosis in addition to one other diagnosis). Our results are descriptive and not indicative of a causal relationship between Veteran status and comorbidity.

The Charlson Comorbidity Index is a measure that assigns weights to chronic conditions based on their severity (adjusted risk of mortality) or resource use and then is summed to produce an index ranging from zero to 41 (Charlson, Pompei, Ales, \& MacKenzie, 1987); having fewer or lower-risk chronic conditions translates into a lower Charlson score, which is in turn correlated with lower risk of death. ${ }^{3}$ In Figure 5-4, we report the predicted likelihood that Veterans and non-Veterans have multiple comorbid or co-occurring conditions based on the Charlson Comorbidity Index. We found that Veterans' and non-Veterans' health had similar index values. This finding holds when we use an indicator of having a Charlson Comorbidity Index greater than one rather than the index value (see Table C-7).

To assess the extent to which Veterans and non-Veterans differ in disease burden, we also examined adjusted differences in functional status. Measures of functional status included the ability to perform self-care tasks, such as bathing and dressing — that is, activities of daily living (ADLs) — and the ability to complete tasks necessary for living independently, includ-

3 The 16 Charlson conditions included in our index are as follows (points/weights in parentheses): myocardial infarction (1), congestive heart failure (CHF) (1), peripheral vascular disease (1), cerebrovascular disease (1), COPD (1), dementia (1), paralysis (1), diabetes (1), chronic renal failure (2), mild liver disease (1), moderate/severe liver disease (3), ulcers (1), rheumatic disease (1), malignant cancer (3), metastatic carcinoma (6), and human immunodeficiency virus (HIV)/acquired immune deficiency syndrome (AIDS) (6). 
Figure 5-4

Adjusted Means of Disease Burden Measures for Veterans and Non-Veterans

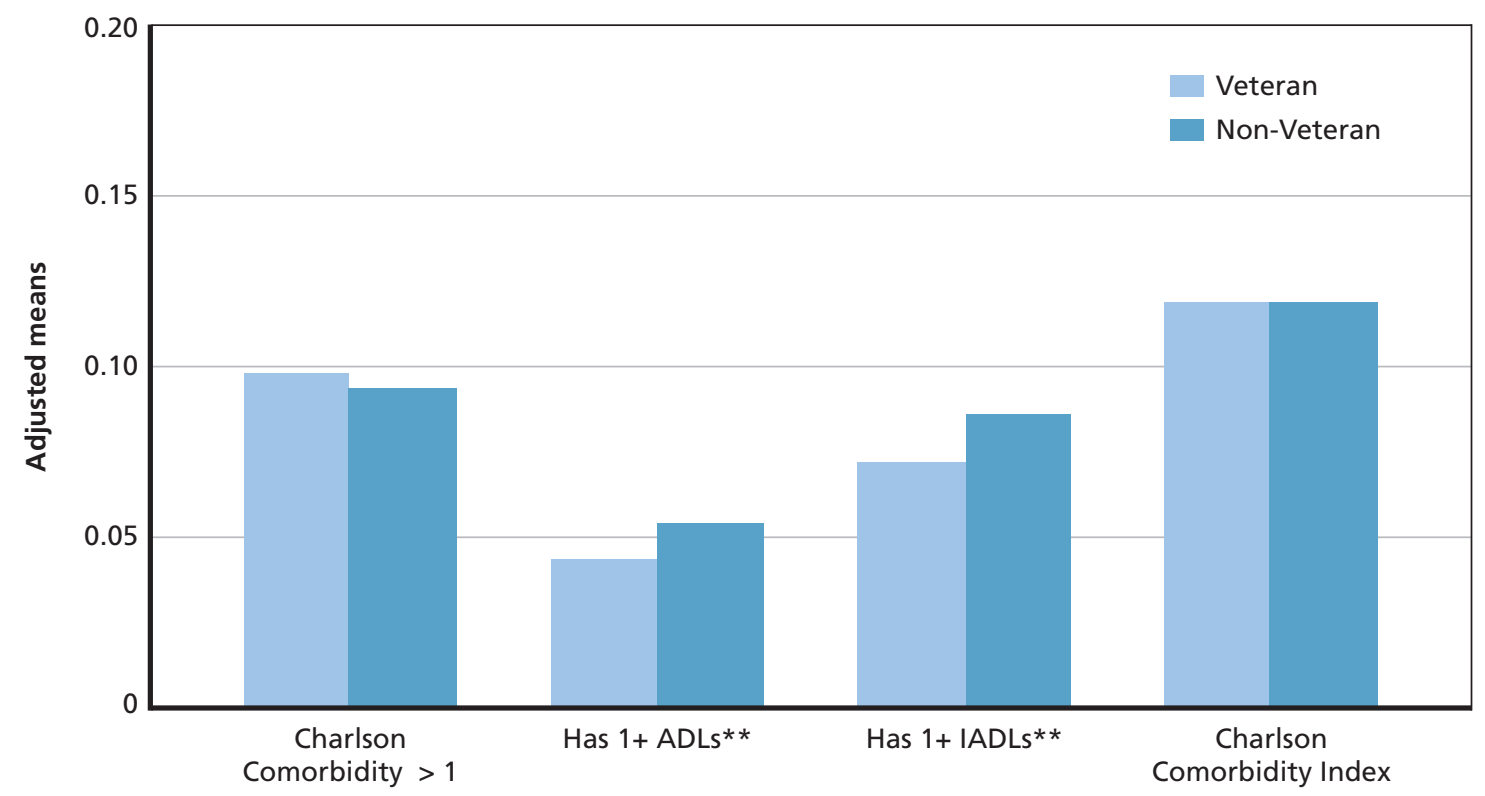

SOURCE: RAND analysis of MEPS, 2006-2012.

NOTES: ** indicates a statistically significant difference between Veterans and non-Veterans at $p$-value $<0.05$. Sample size, non-Veterans $=150,225$, and sample size, Veterans $=12,313$. Sample sizes may be smaller for some conditions due to missing values. Predicted or adjusted means were obtained from estimating logistic regressions with the following additional covariates included: sex (male is the omitted category), five race/ ethnicity categories, 14 age categories, four Census regions, an MSA indicator, and year fixed effects using the margins command in Stata treating Veterans as though they had similar observable characteristics as civilians. The Charlson Comorbidity Index model was estimated using a Poisson regression.

RAND RR1165z1-5.4

ing housework, using the phone, and buying groceries - that is, instrumental activities of daily living (IADLs). Veterans were less likely to need assistance with one or more ADLs or with one or more IADLs relative to non-Veterans. Although it may seem counterintuitive, our finding that Veterans are less likely to report an ADL or IADL limitation than non-Veterans is consistent with other studies using data from the Census and the Panel Study of Income Dynamics. For example, one study reported that although Veterans were more likely to have any type of disability or limitation, they were less likely to have a memory, personal care (similar to ADL measure), mobility, or work (precluding) disability than non-Veterans (Wilmoth, London, \& Parker, 2011). Another study found that non-combat Veterans actually have significantly lower rates of disability than both non-Veterans and combat Veterans (MacLean, 2010). We are unable to distinguish between non-combat and combat Veterans in the MEPS data, but if our sample contains a disproportionate share of non-combat Veterans, this could also explain why we find lower rates of ADL and IADL limitations.

Finally, we investigated adjusted differences in comorbid mental health, the likelihood of having both a mental health diagnosis and any other diagnosis or limitation. Specifically, we estimated whether Veterans were more or less likely to have a mental health diagnosis and (1) any of the 16 Charlson conditions, (2) any of the other 29 conditions we examined in preparing this section (see Table C-2 for the full list), (3) any ADL limitation, or (4) any IADL limitation. We found that Veterans were nearly 3 percentage points more likely than non- 
Veterans to have a diagnosed mental health condition and any of the 29 other conditions we examined in this section, but there were no other statistically significant differences. We report these results in Figure 5-5.

Our findings are consistent with previous literature that suggests that, compared with non-Veterans, Veterans have worse overall health and higher rates of many health conditions. For instance, male Veterans ages 45-54 are significantly more likely to report being in fair or poor health and to report serious psychological distress than non-Veteran males (Kramarow, 2012). Other studies have suggested that Veterans tend to consume more alcohol, are more likely to smoke, and are less likely to exercise (Bohnert et al., 2012; Centers for Disease Control and Prevention, 2014b; Lehavot, Hoerster, Nelson, Jakupcak, \& Simpson, 2012). ${ }^{4}$

We assessed the robustness of our findings that the prevalence of many chronic conditions is higher among Veterans than non-Veterans by carrying out similar analyses of BRFSS and NHIS under both the basic model and alternative specifications (Appendix C.3). Results from BRFSS and NHIS corroborate findings from analysis of MEPS data.

Figure 5-5

Adjusted Means of a Comorbid Mental Health Condition (Mental Health Condition + Another Condition/Limitation) for Veterans and Non-Veterans

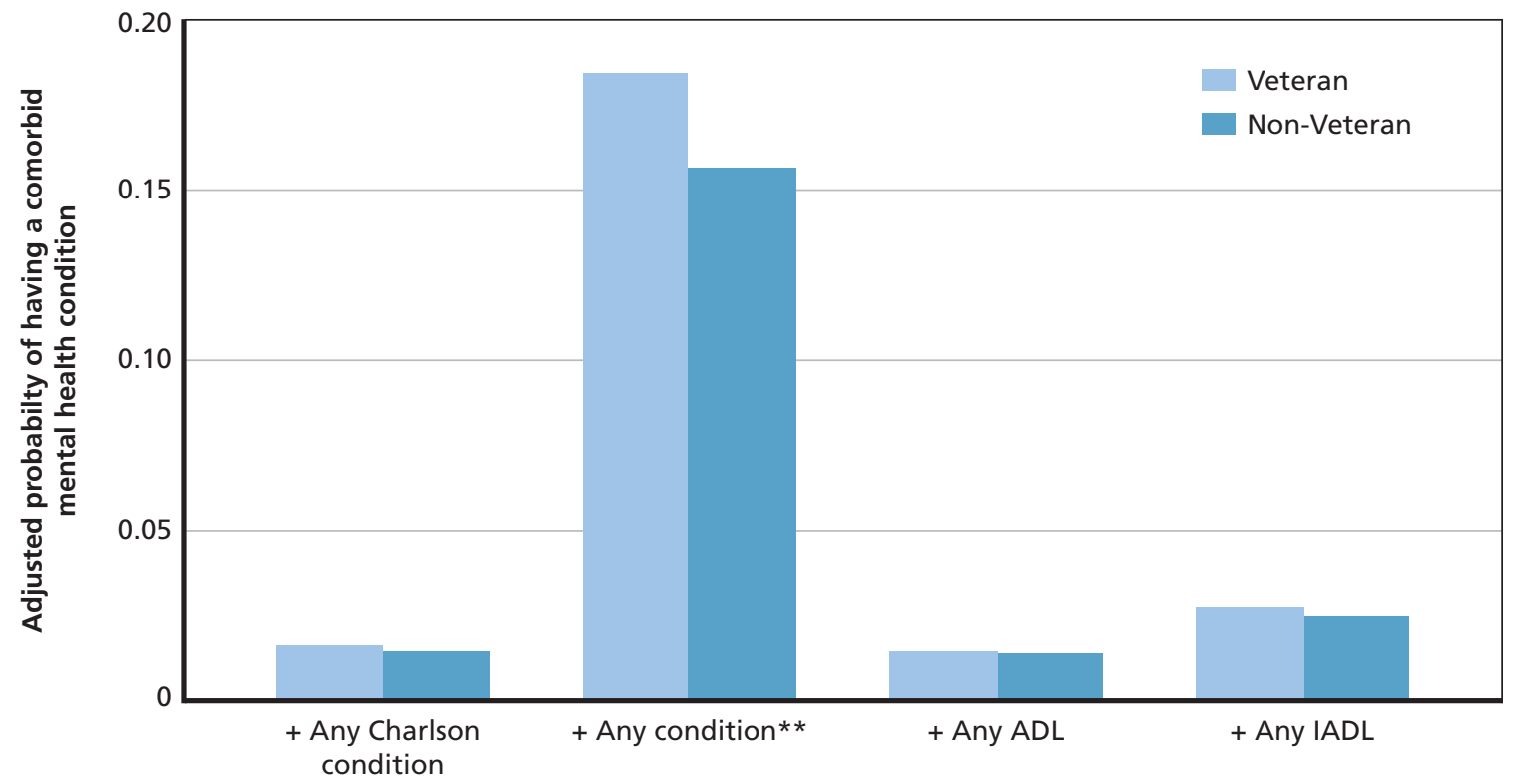

SOURCE: RAND analysis of MEPS, 2006-2012.

NOTES: ** indicates a statistically significant difference between Veterans and non-Veterans at $p$-value $<0.05$ Sample size, non-Veterans $=150,225$, and sample size, Veterans $=12,313$. Sample sizes may be smaller for some conditions due to missing values. Predicted or adjusted means were obtained from estimating logistic regressions with the following additional covariates included: sex (male is the omitted category), five race/ ethnicity categories, 14 age categories, four Census regions, an MSA indicator, and year fixed effects using the margins command in Stata treating Veterans as though they had similar observable characteristics as civilians. The Charlson Comorbidity Index model was estimated using a Poisson regression.

RAND RR1165z1-5.5

4 One exception is that our estimates, using BRFSS (2013), show that Veterans are more likely to have exercised in the past 30 days (see Appendix C.3.3.3). 


\subsubsection{Heterogeneity by Demographic Characteristics}

As discussed, there are significant differences in the health care needs of Veterans and nonVeterans, some of which can be explained by differences in demographic characteristics. In this section, we examine the extent to which diagnosed disease prevalence rates differ across Veterans and non-Veterans within a given age, sex, or race/ethnicity category. In Figure 5-6, we illustrate how differences in the prevalence of chronic conditions between Veterans and non-Veterans vary by age. The selected conditions are those for which the overall prevalence for Veterans is statistically higher than the prevalence for non-Veterans and for which there is evidence that the difference varies across age groups. Asthma exhibits similar patterns for both Veterans and non-Veterans, except that it is slightly lower for younger Veterans ( $<45$ years).

Figure 5-6

Prevalence of Selected Diagnosed Health Conditions for Veterans and NonVeterans, by Age
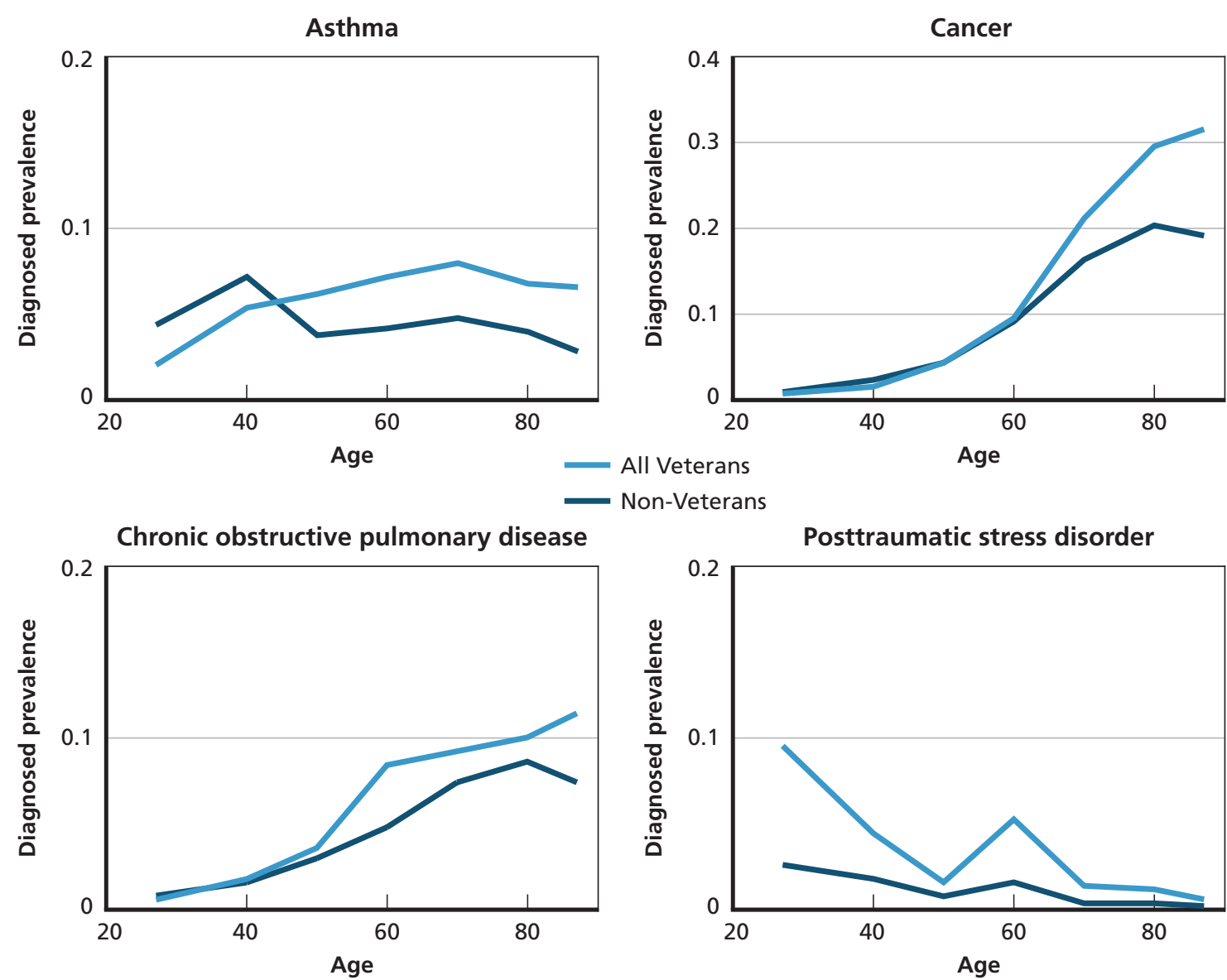

SOURCE: RAND analysis of MEPS, 2006-2012.

NOTES: Sample size, non-Veterans $=150,225$, and sample size, Veterans $=12,313$. Sample sizes may be smaller for some conditions due to missing values. The adjusted prevalence rates are the predicted prevalence from a logit estimation that included indicators for sex, five race/ethnicity categories, seven age categories, four Census regions, residential location in an MSA, and a nonlinear time trend. These estimated differences control for the demographic differences between Veterans and non-Veterans and across age groups. Cancer includes any malignancy.

RAND RR1165z1-5.6 
Differences in diagnosed disease prevalence across Veterans and non-Veterans across age categories reflect both the changing relative health status with age and differences by service cohort, which is highly correlated with age. The lower prevalence of asthma observed among younger Veterans is consistent with the physical requirements for enlisting in the military (Boyle, 2014). The difference in the prevalence of cancer between Veterans and non-Veterans is statistically higher for those 65 and older, but not in younger age groups. The difference in the prevalence of COPD appears to grow with age, but the trend is not statistically significant. For PTSD, the difference in prevalence (statistically significant) is highest for the 20-34 agegroup, and it decreases for the 35-44 and 55-64 cohorts.

We also found differences in disease prevalence by sex, race/ethnicity, and geographic area of residence (see Appendix C.2.1, Tables C-9 and C-10 for the full set of results). Among men, diagnosed disease prevalence rates are typically greater for Veterans relative to non-Veterans, whereas this is not consistently the case among women. For most health conditions, the prevalence rates are similar across race/ethnicity groups. We observe differences for cancer and mental health conditions, including PTSD. Veterans living in a metropolitan area tended to have greater diagnosed disease prevalence rates relative to their non-Veteran counterparts for most conditions. Whether these differences exist because access to care may be different across rural and urban areas or because sicker Veterans needing care may live in urban areas is unclear.

Previous studies have suggested that while the overall difference in unemployment rates for Veterans and non-Veterans is similar, there is notable variation by era of services and age; for example, 59 percent of unemployed Veterans are younger than 45 (Bureau of Labor Statistics, 2015). Veterans, accounting for approximately 10 percent of the adult population, represent a disproportionate share of the homeless adult (16 percent) and sheltered homeless adult (13 percent) populations (National Center for Veterans Analysis and Statistics, 2012b). Thus, we considered whether poverty, income, and employment status were associated with differences in prevalence of health conditions between Veterans and non-Veterans (Table C-11). We used the MEPS categorical measure of poverty based on family income as a percentage of the poverty line (poor or negative income, near poor, low income, middle income, and high income). In Figure 5-7, we show that unemployed Veterans tend to have higher prevalence rates of most conditions relative to unemployed non-Veterans, but employed Veterans also tend to have greater disease prevalence relative to their employed non-Veteran counterparts. The figure presents the results for mental health and PTSD. The prevalence of any mental health condition and PTSD were higher by approximately 4 percentage points for Veterans who were categorized as poor based on family income.

Using NHIS data, we investigated the extent to which there were significant differences in financial insecurities for Veterans and non-Veterans. Specifically, we examined the differences in the probability of being moderately to severely worried about paying bills, health care costs, and housing costs, and in participation in the Temporary Assistance for Needy Families program for Veterans and non-Veterans. Generally, the probability of being moderately to severely worried about financial insecurities was lower for Veterans than non-Veterans (Panel A of Table C-12). These findings persisted when we focused on those individuals reporting any chronic condition (Panel B of Table C-12) or a health problem requiring special equipment (Panel C of Table C-12). 
Figure 5-7

Difference in the Prevalence of Diagnosed Health Conditions for Veterans and Non-Veterans: Vulnerable Populations
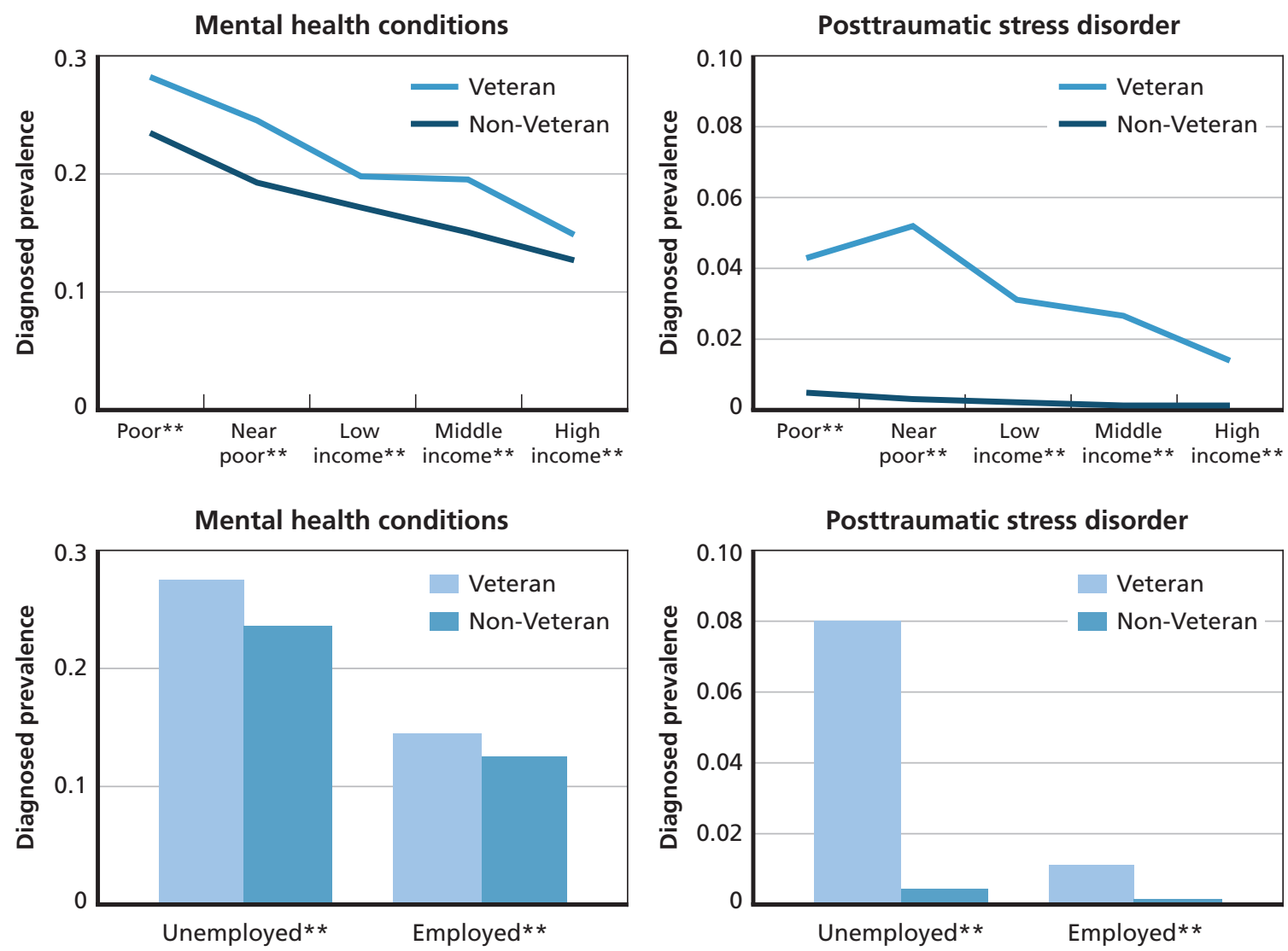

SOURCE: RAND analysis of MEPS, 2006-2012.

NOTES: ** indicates a statistically significant difference between Veterans and non-Veterans at $p$-value $<0.05$. Sample size, non-Veterans $=150,225$, and sample size, Veterans $=12,313$. We use the MEPS categorical measure of poverty based on family income as a percentage of the poverty line. The adjusted prevalence rates are the predicted prevalence from a logit estimation that included indicators for sex, five race/ethnicity categories, seven age categories, four Census regions, residential location in an MSA, and a nonlinear time trend. These estimated differences control for the demographic differences between Veterans and non-Veterans and across age groups. RAND RR1165z1-5.7

\subsubsection{Prevalence of Rare Health Conditions and Other Risks Among Veterans}

Due to the relatively small sample size of Veterans in MEPS, we could not include rare conditions in our analysis. To fill this gap in the preceding analysis, we briefly discuss the results of other studies to provide a more complete picture of the unique health care needs of Veterans; of note, the studies did not compare prevalence rates between Veterans and non-Veterans, so we cannot comment on whether these conditions disproportionately affect Veterans.

We highlight injuries and conditions that are more likely to be prevalent among Veterans due to their association with military service. The unique nature of combat in Afghanistan and Iraq, including improvised explosive devices, is associated with severe combat injuries, including amputation, burns, spinal cord injury, and TBI. The survival of injured Afghanistan and Iraq Veterans is approximately 90 percent, due in part to improvements in medical care and protective gear (Golding, 2011). The prevalence of serious injuries remains low; however, these Veter- 
ans have complex long-term health care needs. ${ }^{5}$ From 2001 to 2010, there were approximately 1,500 amputations among service members who served in Afghanistan, Iraq, and unaffiliated conflicts, 1,200 of which were major limb amputations (Fischer, 2010). TBI has been labeled a "signature injury" for the Afghanistan and Iraq conflicts (Taylor et al., 2012), and while there may be a deployment-related risk for experiencing a TBI (such as being exposed to blast explosions), service members may also experience a TBI in non-deployed settings. Between 2000 and 2014, more than 300,000 service members were diagnosed with TBI (U.S. Defense and Veterans Brain Injury Center, 2014). TBI frequently occurs in conjunction with polytrauma and other disabling conditions, such as amputation, burns, spinal cord injury, auditory and visual damage, spinal cord injury, and PTSD. ${ }^{6}$ Based on an analysis of VHA administrative data from 2004 to 2009, approximately 5 percent of Veterans treated by VA were diagnosed with both PTSD and TBI. This polytrauma occurred in 75 percent of diagnosed TBI cases and in approximately 20 percent of diagnosed PTSD cases (Congressional Budget Office, 2012).

Medically unexplained illnesses_-also referred to as "chronic multisymptom illness" and formerly known as Gulf War Syndrome-are a critical concern for many Veterans who served during the 1990-1991 Gulf War. Medically unexplained illnesses involve a cluster of medically unexplained chronic symptoms — such as fatigue, headaches, joint pain, indigestion, insomnia, dizziness, respiratory disorders, and memory problems - that Veterans attribute to their deployment (Institute of Medicine of the National Academies, 2014a). Survey data from 1999 and 2001 showed a prevalence of 28.9 percent of Veterans deployed during the Gulf War period, compared with a prevalence of 15.8 percent among Veterans who were not deployed (Blanchard et al., 2006). There are also several diseases that warrant special attention among Veterans. In 2011, VA cared for more than 25,000 Veterans with HIV/AIDS (U.S. Department of Veterans Affairs, 2012), and VA is the nation's largest single provider of HIV health care (U.S. Department of Veterans Affairs, 2011). Wang et al. (2015) found a total of 25,648 VA users ages 18-64 who had been diagnosed with HIV from 2007-2012, of which 11,371 had not been previously treated with anti-retroviral therapy. Chronic hepatitis C, caused by the hepatitis $\mathrm{C}$ virus, is a recognized public health issue among Veterans (Zuniga, Chen, Lane, Allmer, \& JimenezLucho, 2006). Among the 5.6 million Veterans accessing care in the VA system in 2008, prevalence was 2.6 percent (Office of Public Health and Environmental Hazards, 2010).

Suicide and substance abuse are two important risks for the Veteran population. Suicide risk is elevated for those who have participated in military service, particularly for males, who are at a higher risk of suicide compared with non-Veterans in all age groups except the oldest (Kaplan, McFarland, Huguet, \& Valenstein, 2012). A population-based study of pre-9/11 male Veterans also found that Veterans were at an increased risk of suicide compared with non-Veterans (Kaplan, Huguet, McFarland, \& Newsom, 2007). Veterans engage in higher

5 Several DoD databases record information on battlefield injuries or medical care that is delivered in theater that would allow for a study of the type of care that is needed for patients who would not have survived their injuries in previous conflicts. However, examination of these sources was beyond the scope of this analysis. In some cases, additional clinical work would be required to determine whether the patient would have died from injuries in previous conflicts. In all cases, data constraints in this particular study prohibited a linked analysis of theater records with subsequent care delivered by VA. For instance, DMDC maintains data on casualties that occurred during the conflicts in Iraq and Afghanistan. Joint Trauma System also maintains a DoD Trauma Registry that contains information on health care delivered to trauma cases in theater.

6 Polytrauma occurs when a person experiences injuries to multiple body parts and organ systems, often as a result of a blast. 
rates of alcohol use than civilians. The prevalence of heavy drinking (consuming on average at least 15 drinks per week in the prior year) and smoking is higher among Veterans ages 25-74 compared with non-Veterans (Centers for Disease Control and Prevention, 2014b, 2015; Lehavot et al., 2012).

\subsection{Current Health Care Needs of Veterans, by VA Patient Status}

Veterans' use of VA health care depends on many factors, including service experience, socioeconomic status, and health insurance options. This section considers how VA patients ${ }^{7}$ differ from other Veterans in their health care needs to understand current demand for VA health care services and to project the health care needs of the VA patient population through 2024. As in Section 5.3, we present estimates of the prevalence of selected health conditions using the most recent data available, and most of the results reported here use ICD-9 diagnosis codes in MEPS data to determine each individual's health conditions, which may underestimate prevalence among respondents with limited access to health care. We supplement the MEPS analysis with analysis of VA encounter data and MHS encounter data. Like MEPS, VA encounter data do not include information about service era. ${ }^{8}$ We cannot address differences in health care needs by service-connected disability using MEPS, but we do provide results by priority group using VA encounter data. A limitation of the estimates using VA encounter data is that many Veterans use non-VA providers of health care services. The VA administrative data include only conditions diagnosed or treated at VA, so comorbidity analysis would be difficult to interpret.

\subsubsection{Demographic Differences by VA Patient Status}

There are some differences in the demographic composition of the VA patient population and the population of Veterans who do not use VA services. Most notably, the VA patient population is older, as seen in Figure 5-8. The difference in the proportion of VA patients and non-VA patients in each age group is statistically different in all but the 55-64 age group. We briefly highlight the key findings here, but we report differences across other demographic characteristics in Appendix C.2.3, Table C-18. The percentage of VA patients who are male (94 percent) is not statistically different from the percentage of non-VA patients who are male (93 percent). The percentage of the VA patient population that is black (non-Hispanic) is slightly higher and the percentage that is Asian is slightly lower than the non-VA patient population. It is unlikely that differences in disease prevalence by race/ethnicity or sex are driving the large differences in the disease prevalence by VA patient status.

\footnotetext{
7 VA patients are Veterans identified in MEPS as having any health care expenditures paid for by VA or the Civilian Health and Medical Program of the Department of Veterans Affairs (CHAMPVA). Medical expenditures in the MEPS data are derived from both household survey information and information provided from the medical provider component (if available). They include direct payments for care provided during the year (payments both out of pocket and by insurance or other sources). As noted, the medical provider component includes data collected from a sample of office-based visits to physicians, hospital visits, and prescription drugs. If this information was incomplete or a respondent did not consent to contacting providers, then expenditures were based on household reports. In this analysis, we denote Veterans (honorably discharged and not on active military duty) who have any medical expenditures paid for by the "Veterans Administration/ CHAMPVA, excluding TRICARE (VA)" as "VA patients." For more details on the expenditure data in MEPS, see Medical Expenditure Panel Survey, 2014, Section 2.5.11.

8 MEPS began including service era in 2011; however, the Veteran and VA patient samples sizes for 2011 forward were not large enough for this analysis.
} 
Differences in demographic characteristics may explain some proportion of the unadjusted differences in health care needs between Veterans and non-Veterans. Following our earlier analyses comparing Veterans and non-Veterans, we adjusted prevalence rates for VA patients and non-VA patient Veterans to account for these demographic differences.

There are also differences in the socioeconomic characteristics of VA patients and non-VA patients that may be related to diagnosed disease prevalence (Table 5-2). VA patients are older, have lower incomes, are less likely to be employed, and are less likely to be married than Veterans who do not use VA health care.

Figure 5-8

Age Distribution of Veterans, by VA Patient Status

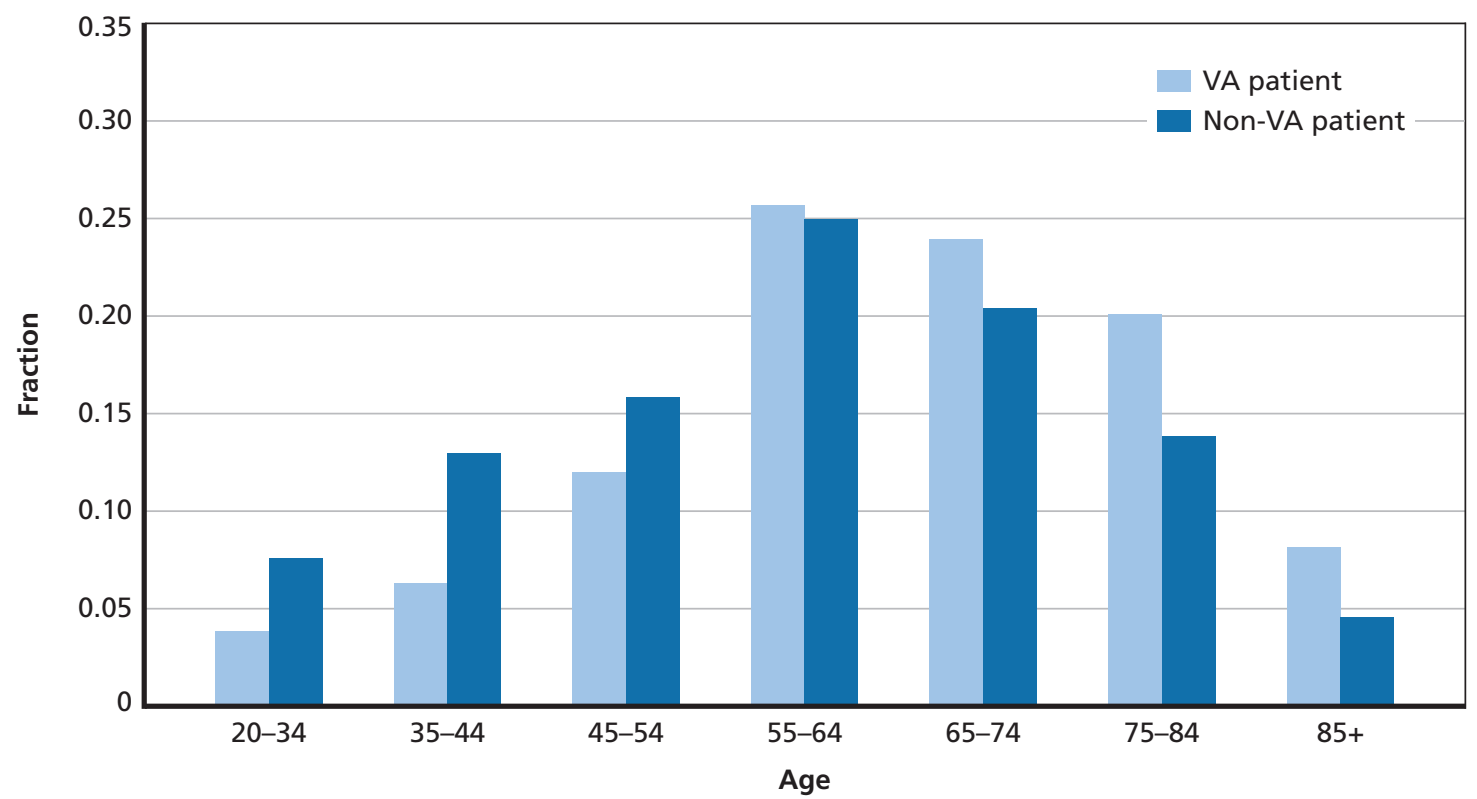

SOURCE: RAND analysis of MEPS, 2006-2012.

NOTES: Sample size, VA patients $=4,871$, and sample size, non-VA patients $=7,442$. Sample sizes may be smaller for some conditions due to missing values.

RAND RR1165z1-5.8

Table 5-2

Socioeconomic Characteristics of Veterans, by VA Patient Status, 2006-2012

\begin{tabular}{lcc}
\hline \multicolumn{1}{c}{ Characteristic } & Veterans, VA Patients & Veterans, Non-VA Patients \\
\hline Over age 65 & $52.2 \%$ & $38.7 \%$ \\
Married & $62.6 \%$ & $68.0 \%$ \\
Less than high school education & $9.1 \%$ & $5.8 \%$ \\
Employed* & $41.3 \%$ & $62.8 \%$ \\
Average household income & $\$ 35,981$ & $\$ 45,278$ \\
\hline
\end{tabular}

SOURCE: RAND analysis of MEPS, 2006-2012.

NOTES: Veterans, VA patients and Veterans, non-VA patients are mutually exclusive categories. Sample size, VA patients $=4,871$, and sample size, non-VA patients $=7,442$.

* Non-employed individuals include both people who are unemployed and people who are out of the labor force, such as retirees. 


\subsubsection{Prevalence of Health Conditions of Veterans, by Use of VA Health Care Services}

The results from the previous section showed that the prevalence of most health conditions is higher for Veterans than for non-Veterans, even after adjusting for demographic composition of these two populations. The analysis also showed that there is variation within Veterans across some demographic characteristics, such as age. In this section, we compared diagnosed disease prevalence rates between VA patients (as defined above) and Veterans who have not had any medical expenditures paid for by VA or CHAMPVA during the past year ("non-VA patients").

In Figure 5-9 and Figure 5-10, we present the results for selected high- and low-prevalence conditions among VA patients and non-VA patients. Conditions are grouped for scaling (see Appendix C.2 for a more comprehensive list of health conditions). The unadjusted prevalence rates of diagnosed diseases are consistently statistically greater for VA patients than for non-VA patients, both among high- and low-prevalence conditions. Among the high-prevalence health conditions, differences in unadjusted diagnosed disease prevalence rates range from 7.7 to 19.5 percentage points (for cancer and hypertension, respectively). Among low-prevalence conditions, differences in unadjusted diagnosed disease prevalence rates range from 2.8 to 8.0 percentage points (for asthma and GERD, respectively).

In the next sections, we examine the extent to which differences in the demographic characteristics of VA and non-VA patients are driving these results.

Figure 5-9

Unadjusted Prevalence of Diagnosed High-Prevalence Health Conditions Among Veterans, by VA Patient Status

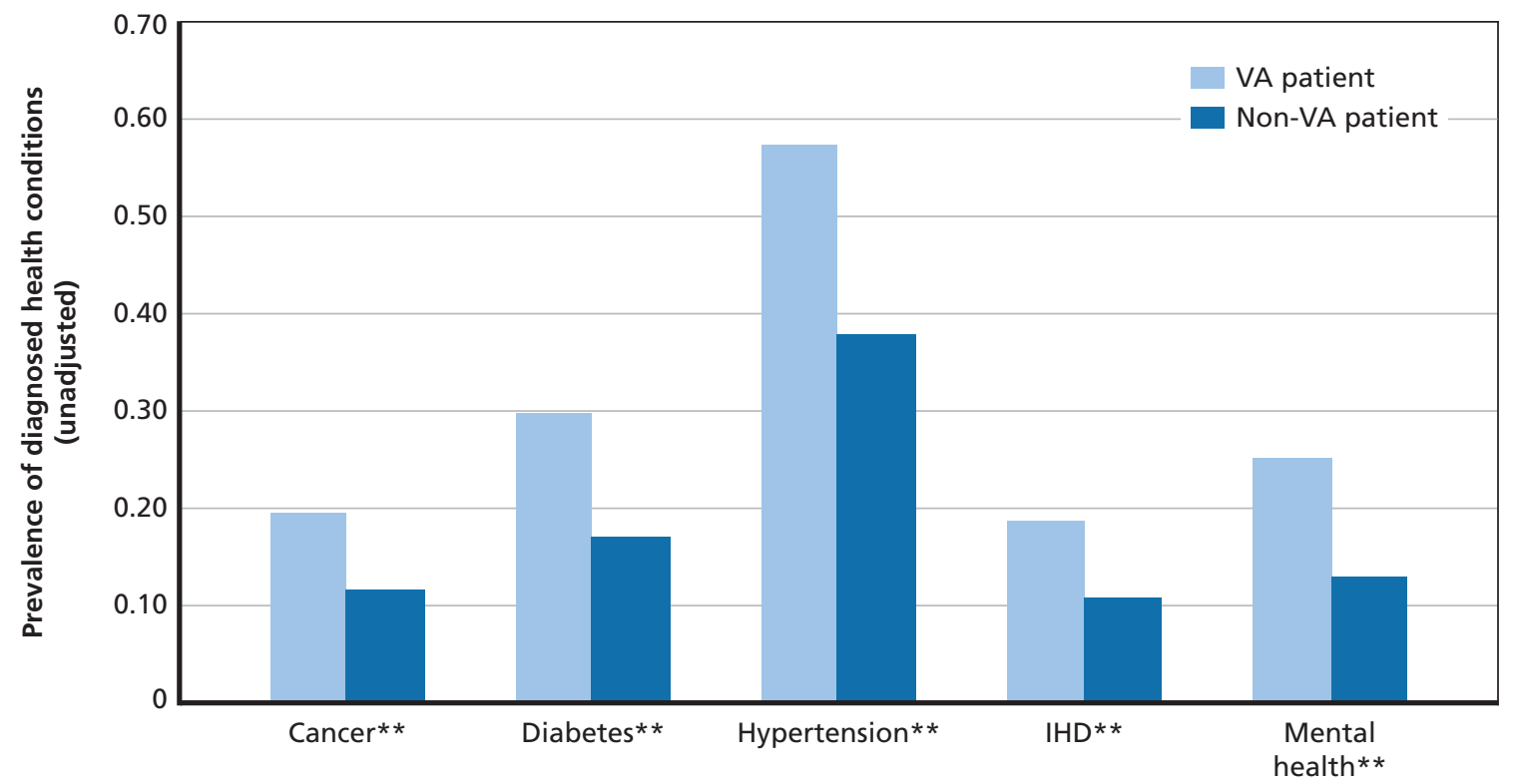

SOURCE: RAND analysis of MEPS, 2006-2012.

NOTES: ** indicates a statistically significant difference between VA patients and Veterans who are not VA patients at $p$-value $<0.05$. Sample size, VA patients $=4,871$, and sample size, non-VA patients $=7,442$. Sample sizes may be smaller for some conditions due to missing values. Cancer includes any malignancy, and Mental Health includes any mental health condition.

RAND RR1165z1-5.9 
Figure 5-10

Unadjusted Prevalence of Diagnosed Lower-Prevalence Health Conditions Among Veterans, by VA Patient Status

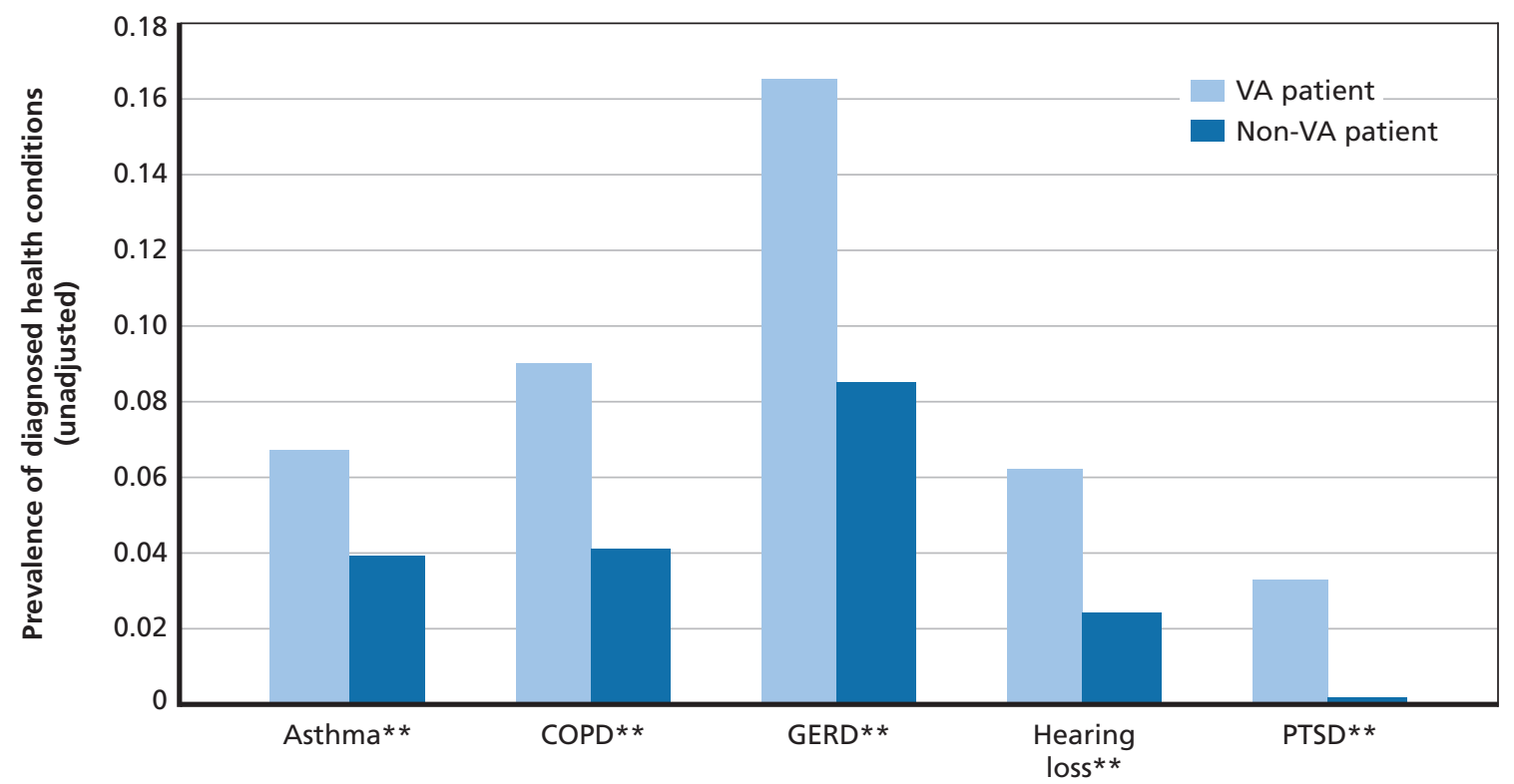

SOURCE: RAND analysis of MEPS, 2006-2012.

NOTES: ** indicates a statistically significant difference between VA patients and Veterans who are not VA patients at $p$-value $<0.05$. Sample size, VA patients $=4,871$, and sample size, non-VA patients $=7,442$. Sample sizes may be smaller for some conditions due to missing values.

RAND RR116521-5.10

\subsubsection{Prevalence of Health Conditions, by VA Patient Status, Adjusted for Demographic Differences}

As noted in Section 5.4.1, VA patients tend to have greater unadjusted rates of diagnosed diseases relative to Veterans who are not VA patients. Because use of VA health care services may be correlated with key demographic characteristics, we estimated disease prevalence for Veterans who are VA patients and for those who do not use VA services, adjusting for age, sex, racel ethnicity, geographic residence, and time trends (as described for comparisons of Veterans and non-Veterans in Section 5.3.2). Again, we refer to this estimation specification as Model 1.

We present predicted prevalence rates for several key conditions in Figures 5-11 and 5-12, grouped by prevalence level for scale (see Table C-13 for full results). In general, adjusting for demographic characteristics reduced the differences in prevalence rates between VA and non-VA patients, but VA patients still appear to have significantly greater rates of diagnosed diseases. Among high-prevalence conditions, the differences in prevalence rates among VA and non-VA patients range from 5.0 to 16.6 percentage points (for cancer and any mental health condition, respectively). Among low-prevalence conditions, the differences in prevalence rates range from 3.8 to 8.2 percentage points (for hearing loss and PTSD, respectively). The predicted prevalence of chronic conditions that are potentially related to military service for Veterans exposed to Agent Orange is higher for VA patients (U.S. Department of Veterans Affairs, 2015f).

There are several potential factors that may drive the differences in prevalence rates between VA patients and Veterans who do not use VA health care. First, Veterans who use VA 
Figure 5-11

Adjusted Prevalence of Diagnosed High-Prevalence Health Conditions Among Veterans, by VA Patient Status

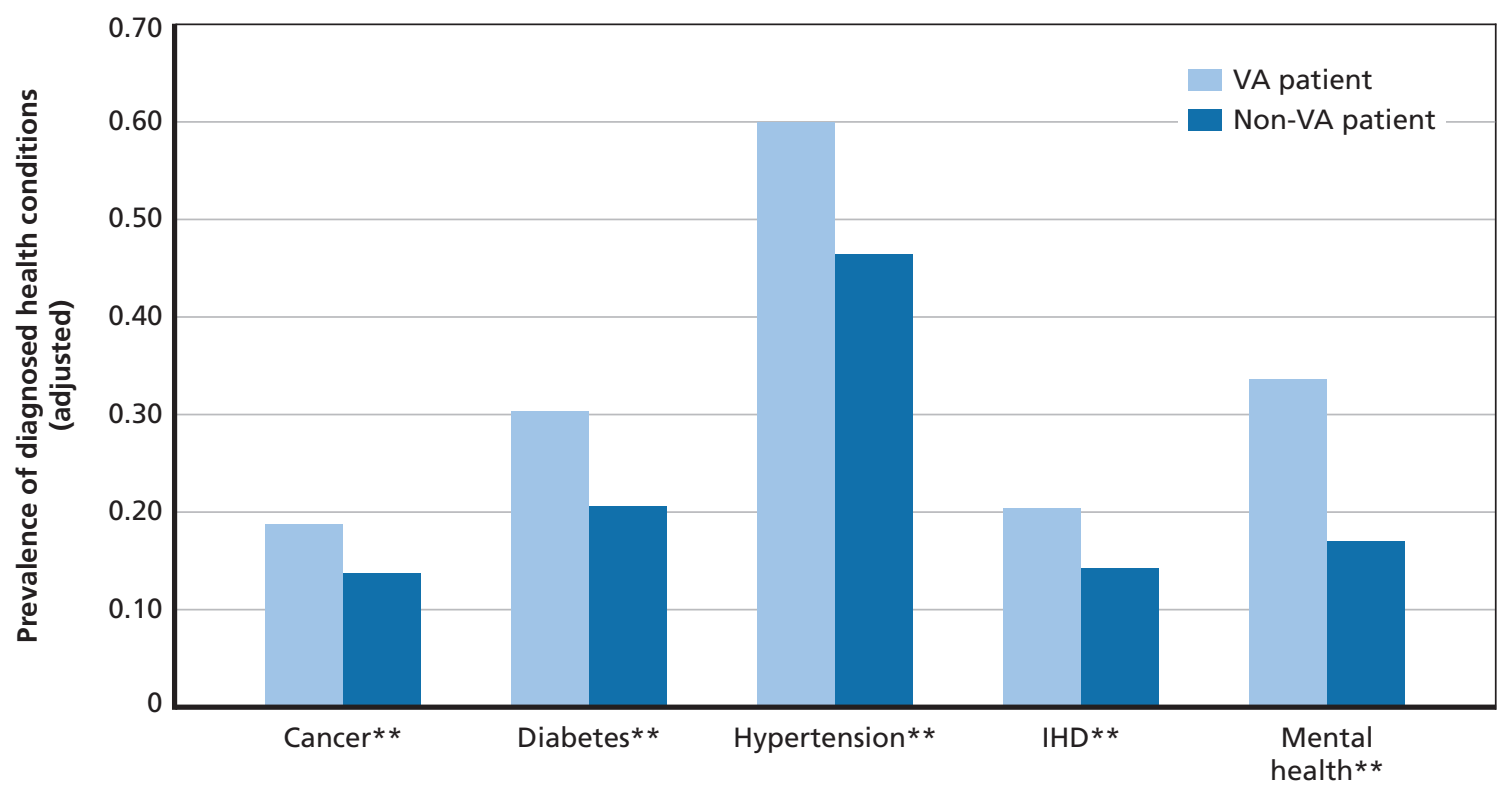

SOURCE: RAND analysis of MEPS, 2006-2012.

NOTES: ** indicates a statistically significant difference between VA patients and Veterans who are not VA patients at $p$-value $<0.05$. Sample size, VA patients $=4,871$, and sample size, non-VA patients $=7,442$. Sample sizes may be smaller for some conditions due to missing values. The adjusted prevalence rates are the predicted prevalence rates from a logit estimation that included indicators for sex, five race/ethnicity categories, 14 age categories, four Census regions, residential location in an MSA, and a nonlinear time trend. VA patient status is defined as having any expenditures paid by VA at the person, not condition, level. Cancer includes any malignancy, and Mental Health includes any mental health condition.

RAND RR1165z1-5.11

health care services may be sicker than Veterans who do not use VA health care. Second, Veterans may be more likely to seek out and use VA health care services if they have health conditions that they believe VA is better equipped to treat, such as PTSD. Finally, VA providers may diagnose some conditions, particularly those that are service-connected, at a higher rate than other health care providers.

We examined the sensitivity of our Model 1 findings by including additional individuallevel characteristics, including marital status, educational attainment (four categories), current student status, current employment status, health insurance coverage, and interactions of sex and race/ethnicity (full results are available in Table C-27). Again, we refer to this more detailed estimation specification as Model 2. The gap between VA patients and non-VA patients closes for most conditions using Model 2, except for cerebrovascular disease and lipid disorder, for which the gap widens slightly, and for cancer, hearing loss, and hypertension, for which the 
Figure 5-12

Adjusted Prevalence of Diagnosed Lower-Prevalence Health Conditions Among Veterans, by VA Patient Status

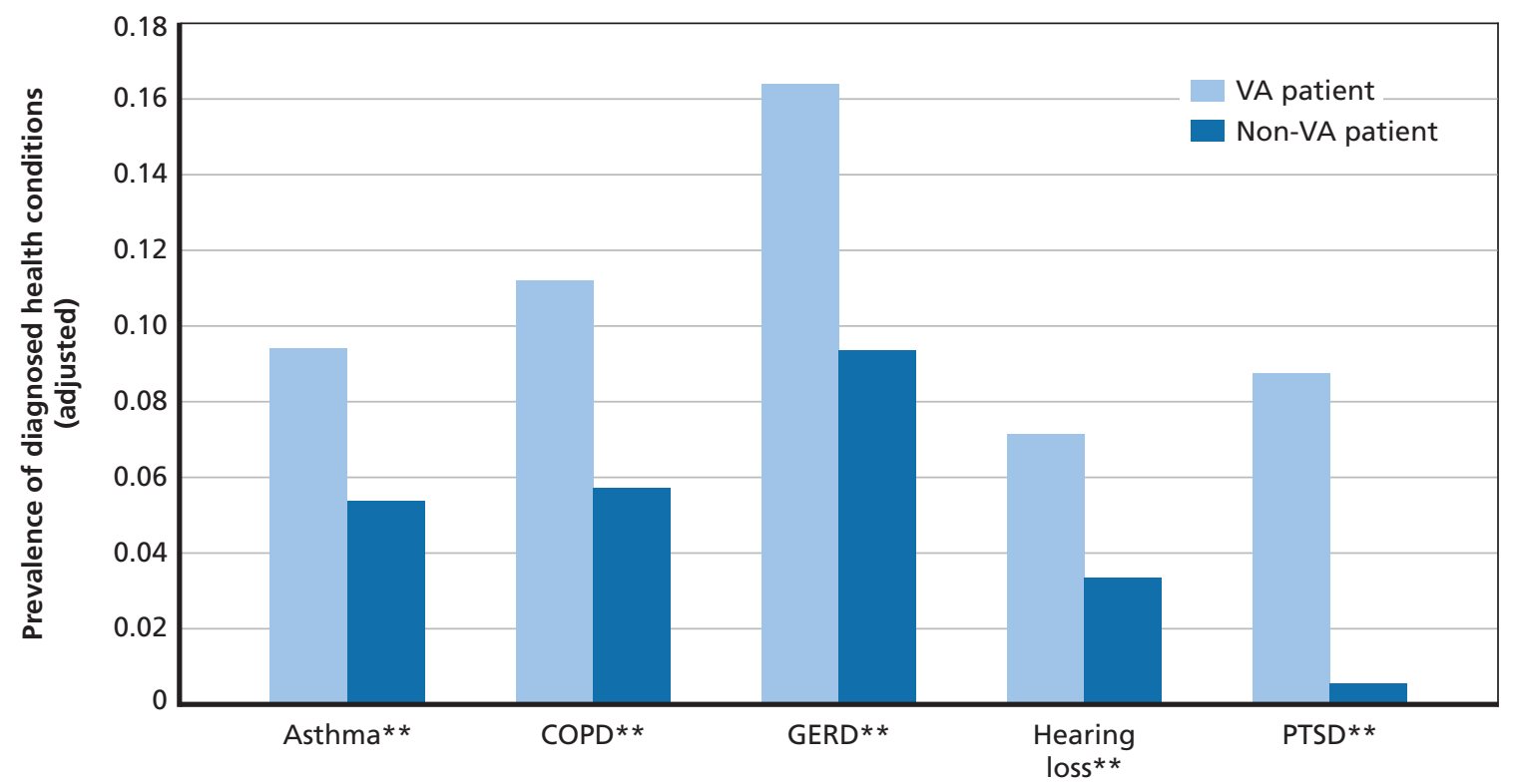

SOURCE: RAND analysis of MEPS, 2006-2012.

NOTES: ** indicates a statistically significant difference between VA patients and Veterans who are not VA patients at $p$-value $<0.05$. Sample size, VA patients $=4,871$, and sample size, non-VA patients $=7,442$. Sample sizes may be smaller for some conditions due to missing values. The adjusted prevalence rates are the predicted prevalence rates from a logit estimation that included indicators for sex, five race/ethnicity categories, 14 age categories, four Census regions, residential location in an MSA, and a nonlinear time trend. VA patient status is defined as having any expenditures paid by VA at the person, not condition, level.

RAND RR1165z1-5.12

prevalence gap remains the same. The largest decrease in gap is observed in the prevalence of any mental health condition, followed by PTSD. Health insurance coverage was associated with a higher prevalence of most conditions, though many of the differences were not statistically different from zero. Having a college degree was associated with lower prevalence rates, particularly for COPD, hypertension, and IHD.

We examined the extent to which VA patients have greater disease burden, as measured by the Charlson Comorbidity Index and the presence of a limitation in IADLs (Figure 5-13). Due to sample size limitations, we were unable to estimate differences in the presence of ADLs across VA and non-VA patients. VA patients were more likely to have at least one Charlson condition and one IADL limitation relative to non-VA patients. Full results are available in Appendix C.2. We also found that VA patients are more likely to have a comorbid mental health condition (Figure 5-14). 
Figure 5-13

Adjusted Means of Disease Burden Measures for Veterans, by VA Patient Status

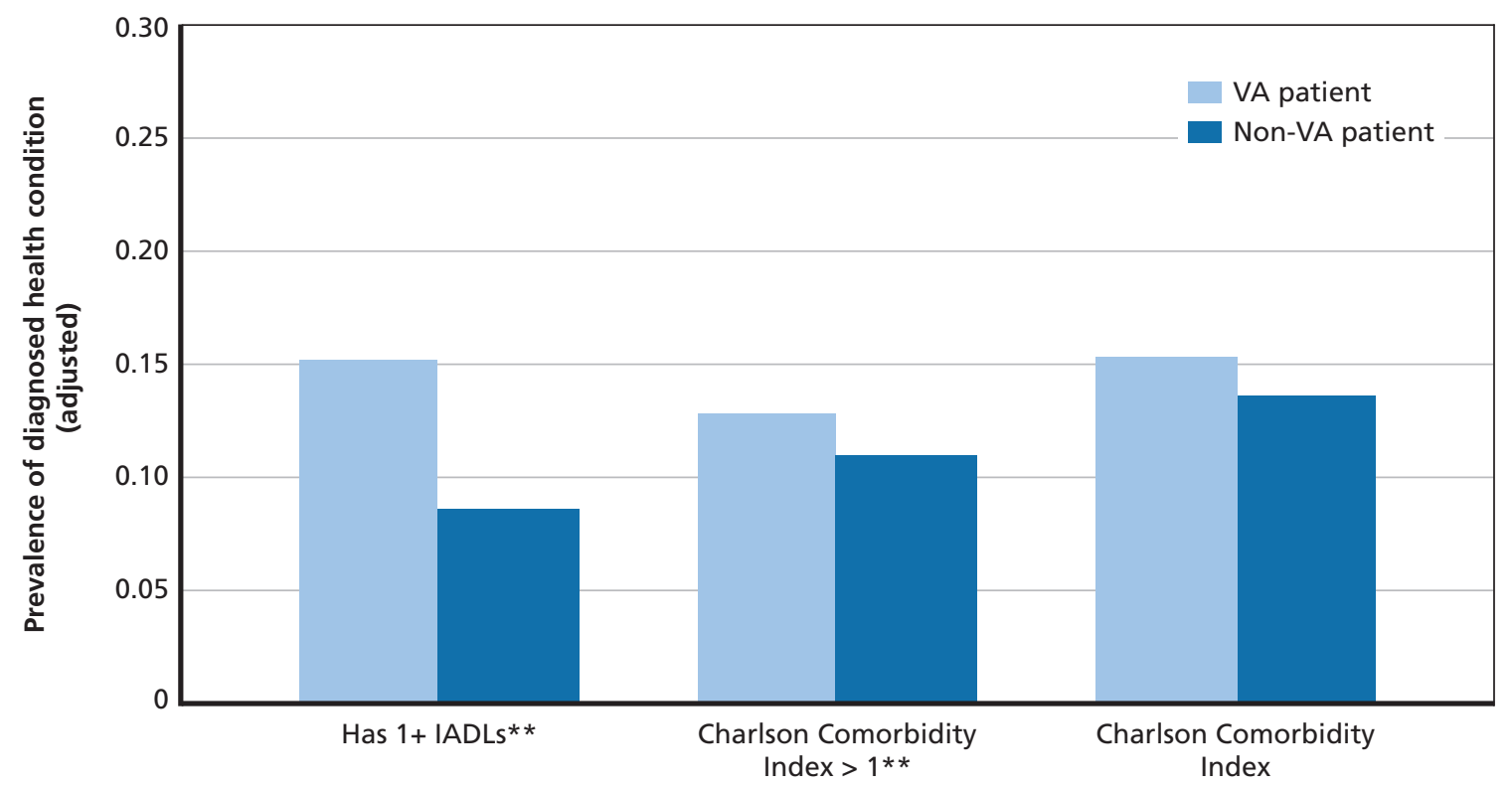

SOURCE: RAND analysis of MEPS, 2006-2012.

NOTES: ** indicates a statistically significant difference between VA patients and Veterans who are not VA patients at $p$-value $<0.05$. Sample size, VA patients $=4,871$, and sample size, non-VA patients $=7,442$. Sample sizes may be smaller for some conditions due to missing values. The adjusted prevalence rates are the predicted prevalence rates from a logit estimation that included indicators for sex, five race/ethnicity categories, 14 age categories, four Census regions, residential location in an MSA, and a nonlinear time trend. VA patient status is defined as having any expenditures paid by VA at the person, not condition, level.

RAND RR1165z1-5.13

\subsubsection{Heterogeneity by Demographic Characteristics}

As noted, Veterans who use VA health care services have many health care needs that are distinct from those of Veterans who do not use VA health care services. This section explores how VA patients' health conditions vary by demographic characteristic and how the differences vary by demographic characteristic, including age, sex, and race/ethnicity.

In Figure 5-15, we present adjusted diagnosed disease prevalence for VA patients and for non-VA patient Veterans by age category for diabetes, hypertension, hearing loss, and PTSD (full results are in Tables C-16 and C-17, respectively). The changes in diagnosed disease preva- 
Figure 5-14

Adjusted Means of Comorbid Mental Health Condition (Mental Health Condition + Another Condition/Limitation) for Veterans, by VA Patient Status

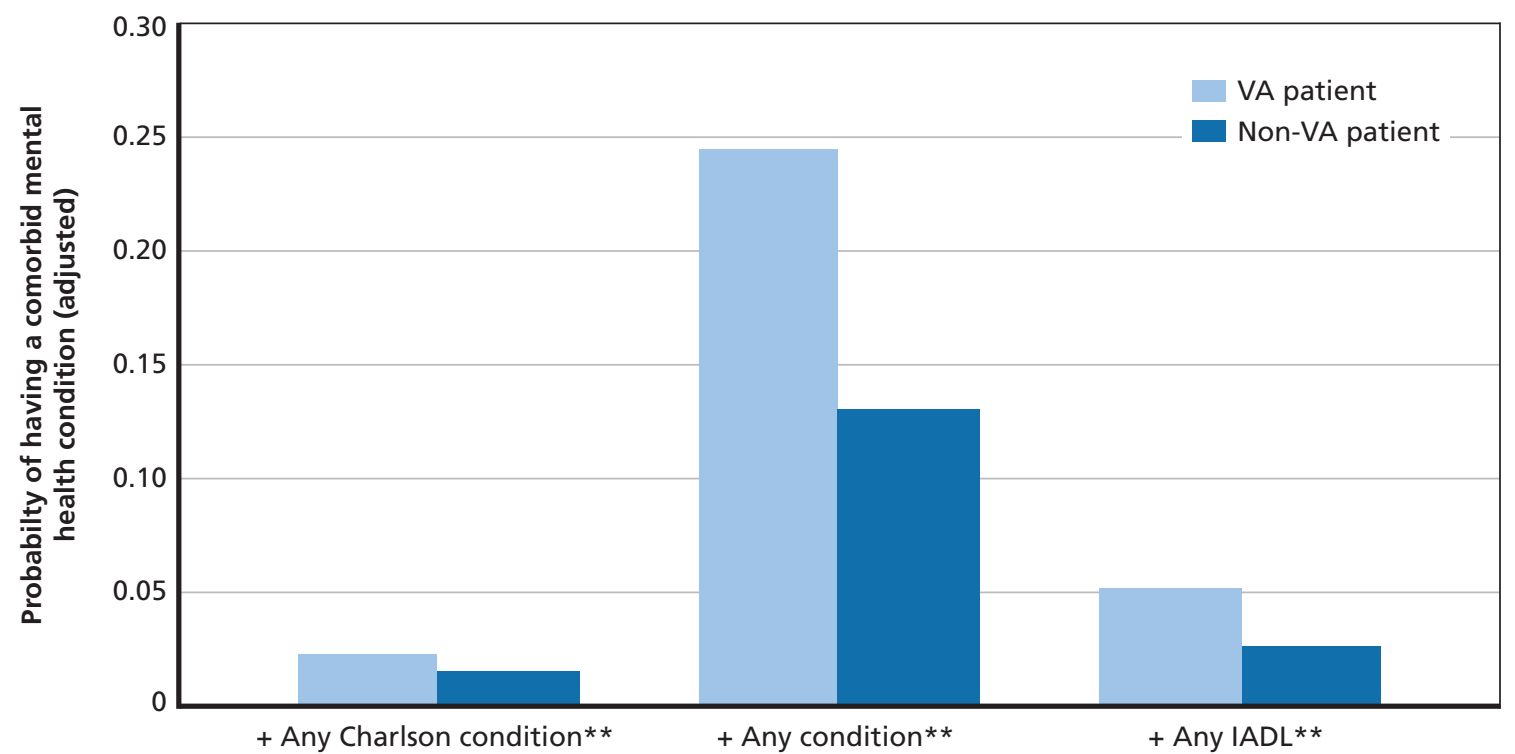

SOURCE: RAND analysis of MEPS, 2006-2012.

NOTES: ** indicates a statistically significant difference between VA patients and Veterans who are not VA patients at $p$-value $<0.05$. Sample size, VA patients $=4,871$, and sample size, non-VA patients $=7,442$. Sample sizes may be smaller for some conditions due to missing values. The adjusted prevalence rates are the predicted prevalence rates from a logit estimation that included indicators for sex, five race/ethnicity categories, 14 age categories, four Census regions, residential location in an MSA, and a nonlinear time trend. VA patient status is defined as having any expenditures paid by VA at the person, not condition, level.

RAND RR1165z1-5.14

lence of diabetes, hypertension, and hearing loss across the age distribution are similar for both VA and non-VA patients. Veterans using VA services, however, tend to be diagnosed with PTSD at significantly greater rates than non-VA patients, particularly at younger ages and between ages 55 and 64. Given that MEPS data cover 2006 to 2012, these ages align with service in the post-9/11 and Vietnam eras.

In Appendix C.2.2, Tables C-16 and C-17 show the prevalence of differences by age, sex, and race/ethnicity. Among both women and men, VA patients consistently have higher prevalence of health conditions than non-VA patients. 
Figure 5-15

Prevalence of Selected Diagnosed Conditions Among Veterans, by VA Patient Status and Age
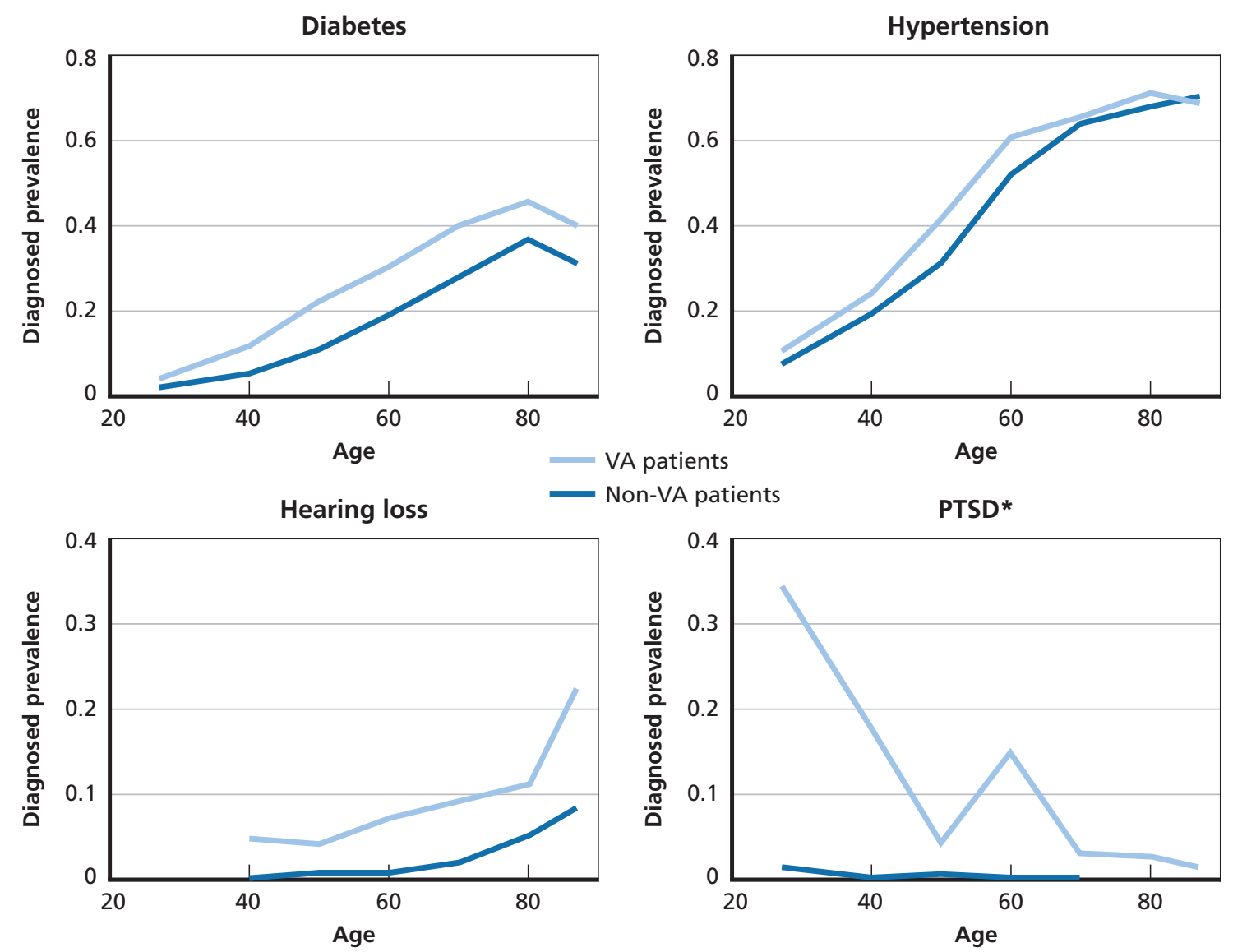

Non-VA patients $\quad$ PTSD*

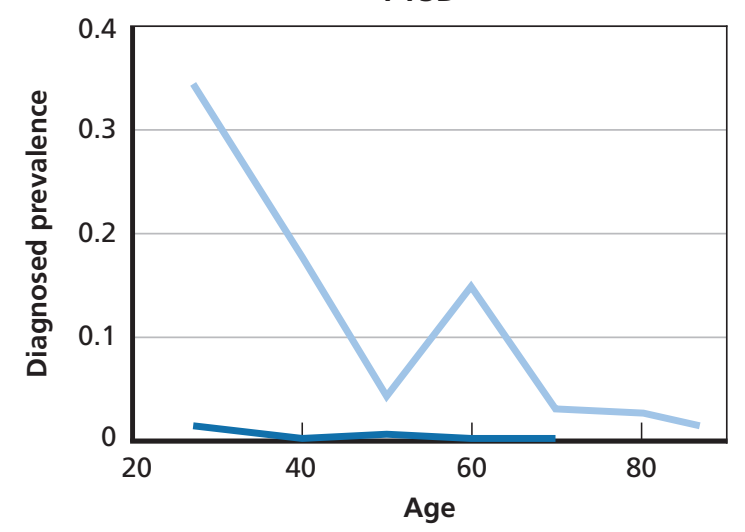

SOURCE: RAND analysis of MEPS, 2006-2012.

NOTES: Sample size, VA patients $=4,871$, and sample size, non-VA patients $=7,442$. Sample sizes may be smaller for some conditions due to missing values. The adjusted prevalence rates are the predicted prevalence rates from a logit estimation that included indicators for sex, five race/ethnicity categories, 14 age categories, four Census regions, residential location in an MSA, and a nonlinear time trend. VA patient status is defined as having any expenditures paid by VA at the person, not condition, level.

* Due to sample size, prevalence of PTSD among older Veterans who are not VA patients could not be estimated. RAND RR1165z1-5.15 


\subsubsection{Prevalence of Rare Health Conditions Among VA Patients}

Due to the small sample size of VA patients in MEPS, we could not include rare conditions in our analysis. To fill this gap in the preceding analysis, we utilized VA encounter data to provide a more complete picture of the unique health care needs of VA patients. An important caveat is that prevalence estimates using VA encounter data only capture conditions treated at VA. However, as noted in Chapter Two, most Veterans with service-connected conditions use VA health care services. The unadjusted prevalence estimates using 2014 VA encounter data are presented in Figure 5-16.

Figure 5-16

VA Encounter-Based Prevalence of Diagnosed Health Conditions Among VA Patients

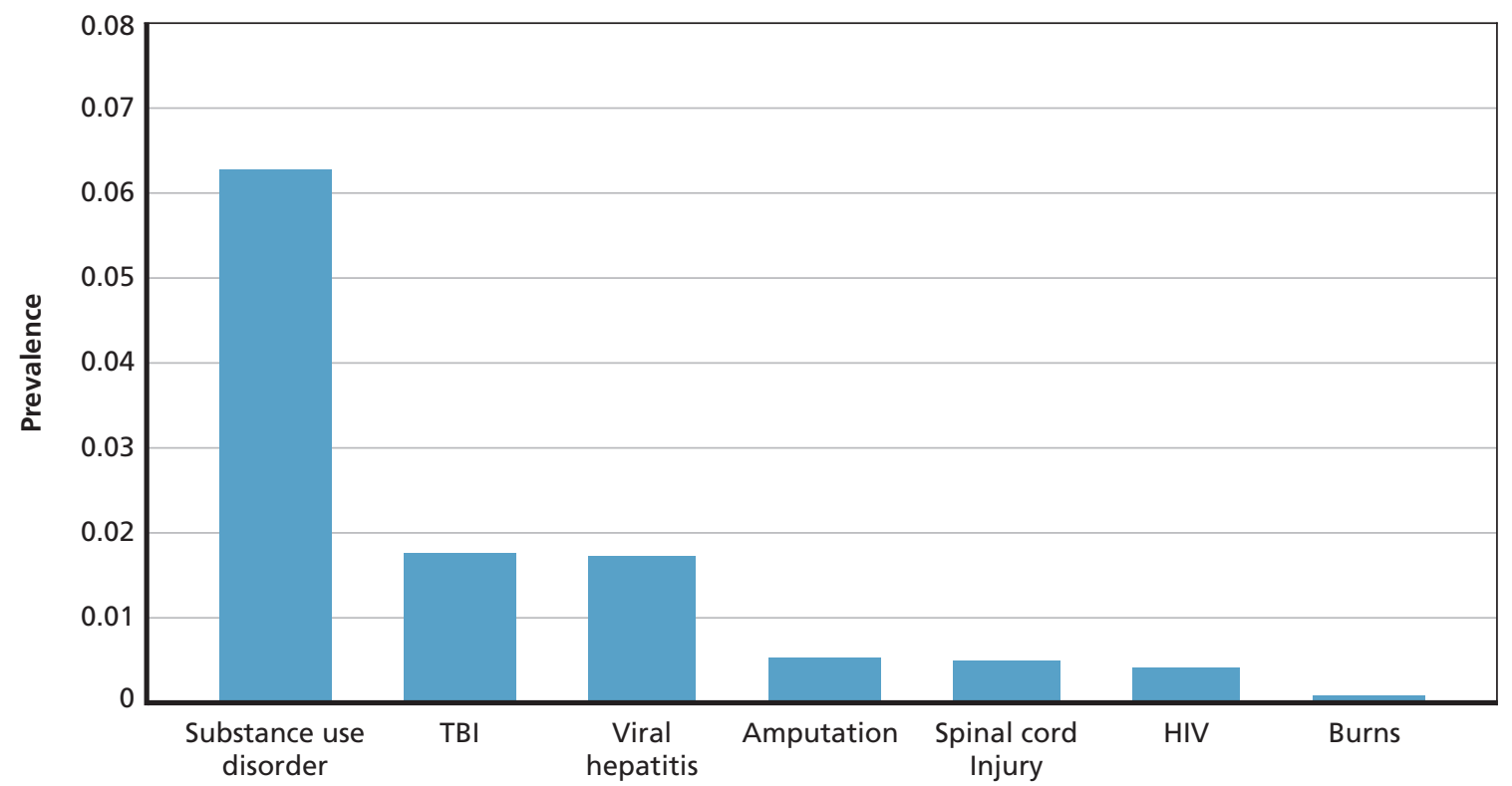

SOURCE: RAND analysis of VA encounter data (2014).

NOTES: VA encounter sample size, VA patients $=5,871,766$.

RAND RR1165z1-5.16 


\subsubsection{Prevalence of Health Conditions among VA Patients, by VA Priority Group and Reliance}

In this section, we explore the associations between the prevalence of health conditions among VA patients and the patients' interactions with VA. Specifically, we consider the prevalence of health conditions by priority group and one measure of VA reliance.

It may also be informative to understand how the health conditions of VA patients vary by priority group. Figure 5-17 shows the prevalence of selected health conditions among VA patients in 2014, by priority group (PG), and Table C-20 shows the full results. These prevalence estimates are based on VA encounter data, so they capture only the VA-diagnosed prevalence rates, which may be different from the total prevalence rates based on diagnosis independent of provider. The estimates show that prevalence for hypertension, diabetes, and cancer are highest among VA patients in priority group 4. This could be because these patients have higher rates of disease or because they are more reliant on VA health care services. We cannot disentangle the two. Not surprisingly, PTSD and TBI, which are highly connected to service, have the highest prevalence among VA patients in priority group 1.

In Table 5-3, we use MEPS data to explore how VA patients whose medical expenditures are paid only by VA differ from VA patients who have some health expenditures paid by other sources of health coverage (e.g., Medicare). We define VA patients as "VA reliant" if all medical expenditures, independent of provider, were VA paid or VA and self or family paid. According to our estimates using MEPS, approximately 13 percent of all VA patients can be classified as reliant. VA-reliant patients are younger than those who also use other sources of health coverage (Appendix C-21).

Due to demographic differences between the two groups, we present adjusted prevalence rates in Table 5-3. The results suggest that even after adjusting for differences in characteristics that may be related to disease prevalence, the prevalence rates for some health conditions, including diabetes, GERD, and cancer, are lower for VA-reliant patients than other VA patients. However, it is difficult to draw any inferences from these estimates because they are imprecise due to the small sample size of VA reliant patients.

\subsubsection{Health Care Needs of Recently Separated Veterans and VA Patients}

The preceding analysis indicates that Veterans who become VA patients have greater rates of diagnosed disease prevalence than Veterans who do not use VA health care, even after controlling for key demographic characteristics. A combination of Veteran characteristics and VA policies may lead Veterans with greater health care needs or specific health conditions to use VA health care services at higher rates than healthier Veterans.

We explored the association of specific health conditions with VA use by comparing the prevalence of health conditions among recently separated Veterans, estimated with MHS encounter data, to the prevalence of the same health conditions among VA patients, estimated with VA encounter data. We assumed that nearly all health care for active military is provided by MHS and, thus, health condition prevalence estimates based on MHS encounter data reflect the true prevalence of diagnosed health conditions in this population. We used these estimates as a benchmark to determine whether some health conditions are over- or underrepresented in VA encounters. We did not have the permissions necessary to match MHS encounter data to VA encounter data at the individual level; therefore, we compared national prevalence rates (or rates in comparable large demographic groups). To match samples from MHS and VA as closely as possible, we focused on Veterans under age 35. We further restricted 
Figure 5-17

VA Encounter-Based Prevalence of Diagnosed Health Conditions, by Priority Group
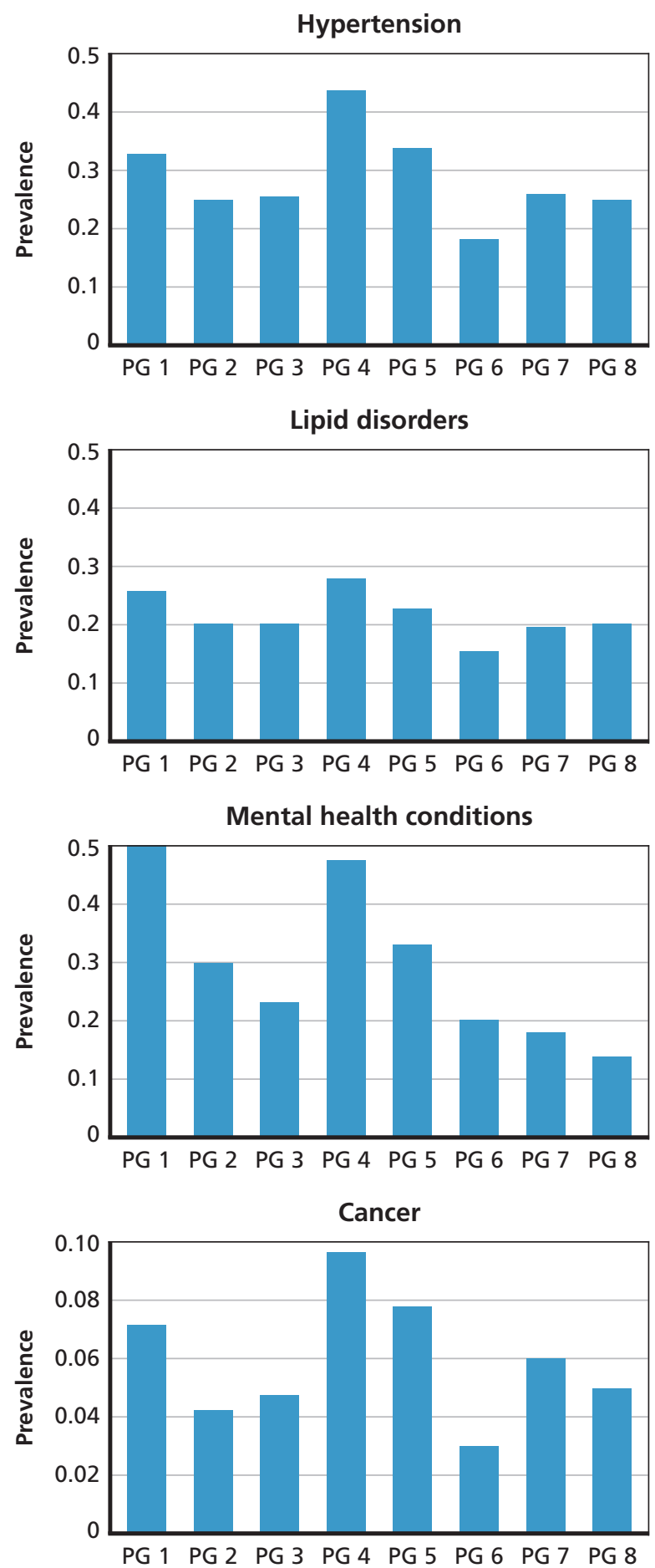

SOURCE: RAND analysis of VA encounter data (2014).

NOTES: VA encounter sample size, VA patients $=5,871,766$.

RAND RR1165z1-5.17

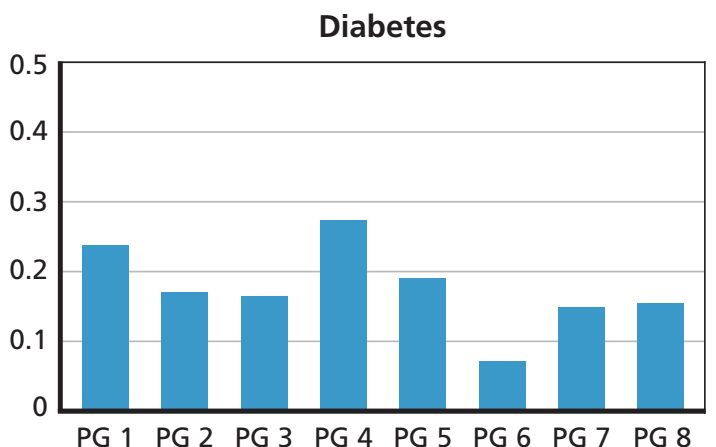

$\begin{array}{llllllllll}\text { PG } 1 & \text { PG } 2 & \text { PG } 3 & \text { PG } 4 & \text { PG } 5 & \text { PG } 6 & \text { PG } 7 & \text { PG } 8\end{array}$

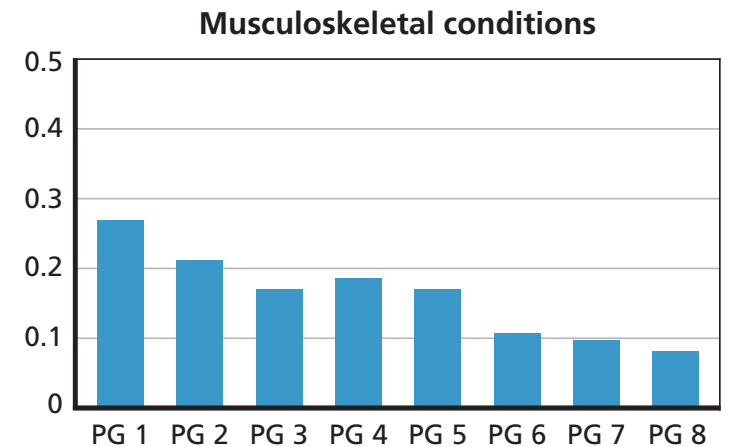

PG 1 PG 2 PG 3 PG 4 PG 5 PG 6 PG 7 PG 8

PTSD

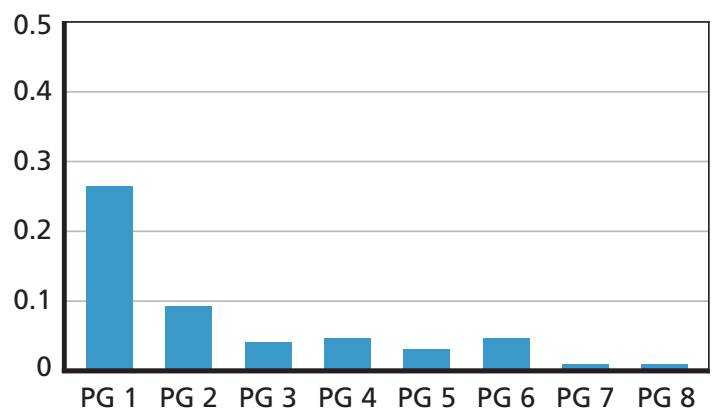

Traumatic brain injury

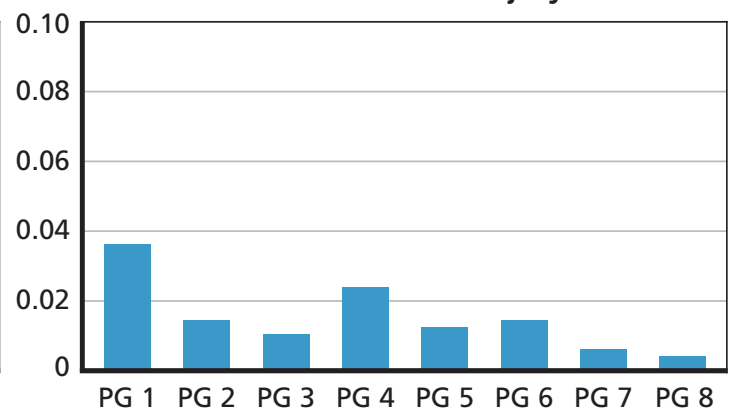


Table 5-3

Prevalence of Diagnosed Health Conditions among VA Patients, by VA Reliance

\begin{tabular}{|c|c|c|c|c|c|c|}
\hline \multirow[b]{2}{*}{ Health Condition } & \multicolumn{2}{|c|}{ All Ages } & \multirow{2}{*}{$\begin{array}{c}\begin{array}{c}\text { P-Value } \\
\text { of } \\
\text { Difference }\end{array}\end{array}$} & \multicolumn{2}{|c|}{ Age $<65$} & \multirow{2}{*}{$\begin{array}{c}\begin{array}{c}\text { P-Value } \\
\text { of } \\
\text { Difference }\end{array}\end{array}$} \\
\hline & $\begin{array}{l}\text { VA Reliant } \\
\text { (13\%) }\end{array}$ & $\begin{array}{c}\text { VA Non-Reliant } \\
(87 \%)\end{array}$ & & $\begin{array}{l}\text { VA Reliant } \\
\text { (20\%) }\end{array}$ & $\begin{array}{c}\text { VA Non-Reliant } \\
(\mathbf{8 0} \%)\end{array}$ & \\
\hline \multirow[t]{2}{*}{ Cancer } & 0.115 & 0.210 & $<0.000$ & 0.062 & 0.113 & 0.064 \\
\hline & $(0.026)$ & $(0.030)$ & & $(0.022)$ & $(0.039)$ & \\
\hline \multirow[t]{2}{*}{ COPD } & 0.120 & 0.105 & 0.423 & 0.098 & 0.075 & 0.239 \\
\hline & $(0.029)$ & $(0.024)$ & & $(0.037)$ & $(0.030)$ & \\
\hline \multirow[t]{2}{*}{ Diabetes } & 0.267 & 0.350 & 0.001 & 0.223 & 0.288 & 0.035 \\
\hline & $(0.037)$ & $(0.036)$ & & $(0.047)$ & $(0.051)$ & \\
\hline \multirow[t]{2}{*}{ GERD } & 0.116 & 0.180 & 0.003 & 0.110 & 0.158 & 0.072 \\
\hline & $(0.025)$ & $(0.032)$ & & $(0.033)$ & $(0.044)$ & \\
\hline \multirow[t]{2}{*}{ Hearing loss } & 0.093 & 0.092 & 0.971 & 0.071 & 0.085 & 0.566 \\
\hline & $(0.031)$ & $(0.024)$ & & $(0.045)$ & $(0.037)$ & \\
\hline \multirow{2}{*}{$\begin{array}{l}\text { Mental health } \\
\text { conditions }\end{array}$} & 0.273 & 0.321 & 0.050 & 0.358 & 0.411 & 0.107 \\
\hline & $(0.037)$ & $(0.036)$ & & $(0.055)$ & $(0.055)$ & \\
\hline \multirow[t]{2}{*}{ PTSD } & 0.081 & 0.078 & 0.821 & 0.119 & 0.118 & 0.973 \\
\hline & $(0.025)$ & $(0.022)$ & & $(0.039)$ & $(0.038)$ & \\
\hline
\end{tabular}

SOURCE: RAND analysis of MEPS, 2006-2012.

NOTES: ** indicates a statistically significant difference between VA-reliant patients and VA non-reliant patients at $p$-value $<0.05$. Sample size, VA patients $=4,871$; VA-reliant patients $=740 ;$ VA non-reliant patients $=4,131$. A VA patient is considered reliant if all medical expenses in the year were VA and family/ self-paid. A VA patient is considered non-reliant if he or she has some medical expenses paid by a non-VA health insurance source. The adjusted prevalence rates are the predicted prevalence rates from a logit estimation that included indicators for sex, five race/ethnicity categories, 14 age categories, four Census regions, residential location in an MSA, and a nonlinear time trend. VA patient status is defined as having any expenditures paid by VA at the person, not condition, level.

the MHS sample to Veterans who separated from service in 2012 or 2013. Separation dates and VA enrollment dates are not available in the VA encounter data. A limitation of this approach is that the prevalence of age-related chronic conditions is very low for this age group, so it is difficult to discern whether Veterans with these conditions disproportionately choose to use VA health care.

Figure 5-18 shows the unadjusted prevalence of health conditions estimated separately using MHS and VA encounter data. We report results on health conditions for which there are differences in prevalence rates between MHS and VA patients and on common chronic conditions with non-zero prevalence estimates. A table with the full set of conditions is available in Appendix C.2.

The results suggest that young Veterans' use of VA may be affected by health status. Specifically, although the prevalence of diagnosed mental health conditions overall is slightly higher among VA patients than newly separated Veterans, the prevalence of diagnosed PTSD among VA patients in particular is disproportionately larger among VA patients than among 
Figure 5-18

Health Condition Prevalence in MHS and VA Encounter Data for Young Veterans

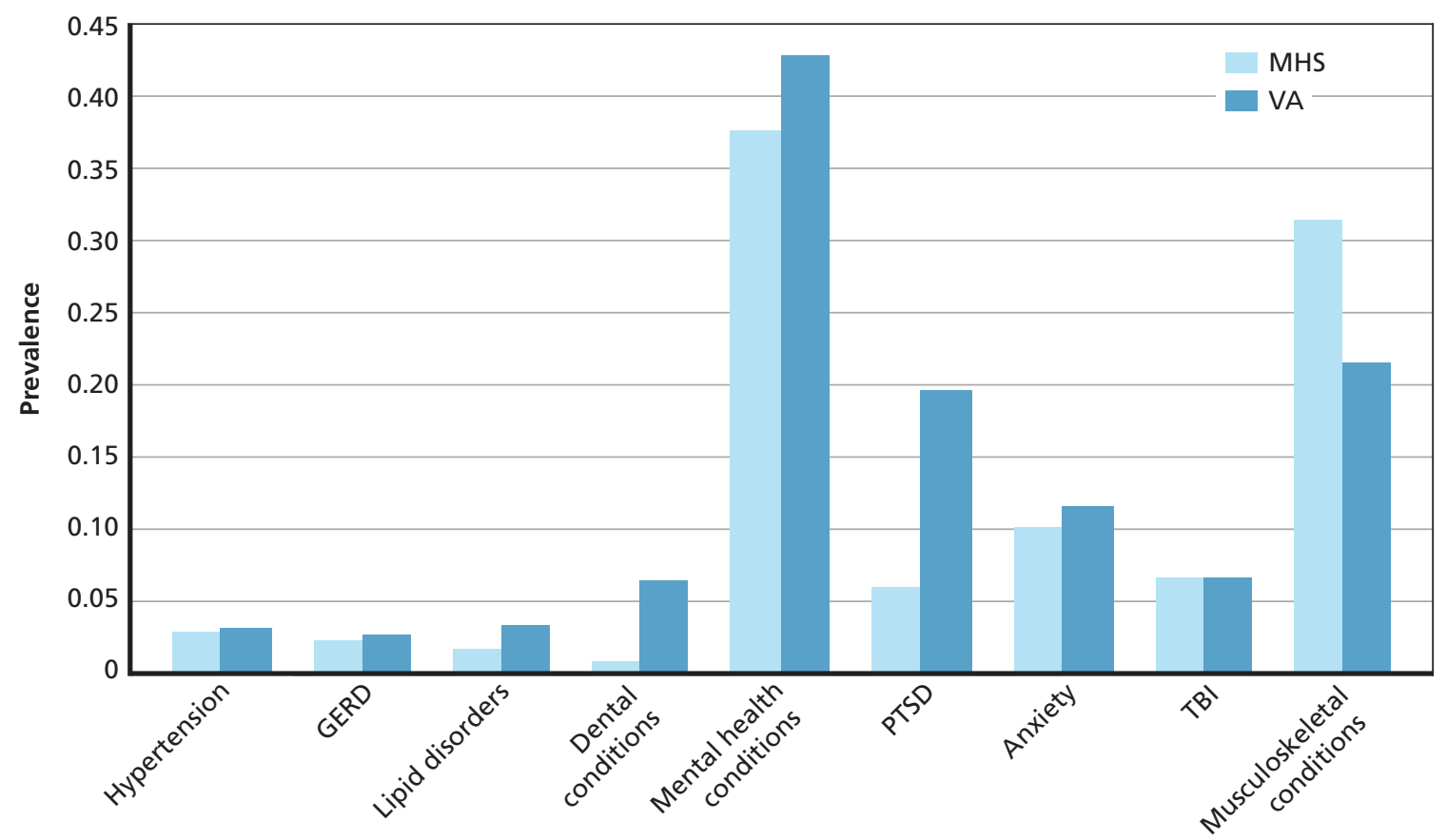

SOURCE: RAND analysis of MHS encounter data (2012-2013) and VA encounter data (2014).

NOTES: Sample size, separating military personnel aged less than 35 in MHS = 325,849, and sample size,

VA patients in the VA encounter data aged less than $35=503,205$. Mental Health includes any mental

health condition. Musculoskeletal conditions are those associated with chronic pain.

RAND RR1165z1-5.18

the full population of service members separating from DoD. This finding is consistent with differences in PTSD prevalence by VA patient status in the MEPS analysis. This may in part reflect disincentives for active duty military personnel to seek mental health services, including stigma and fear of negative career repercussions. Veterans with musculoskeletal conditions appear to be underrepresented in the VA patient population. However, some musculoskeletal conditions are not permanent or require less treatment over time, which could explain the difference in prevalence rates. Given the data limitations, it is not clear whether these differences are driven by individual choice or VA policy, or whether diagnosis of some conditions is more likely in particular health care systems.

\subsection{Future Health Care Needs of Veterans}

\subsubsection{Factors Driving Veteran Health Care Needs}

Changes in the demographic composition of the Veteran population are likely to play a prominent role in determining future health care needs. For example, the prevalence rate of many chronic health conditions, such as diabetes, IHD, and many types of cancer, increases with age (Figures 5-6 and 5-15), and we predict that the average age of Veterans will increase over the next 10 years, especially for women (Figure 3-6). The number of female Veterans is also predicted to increase (Chapter Three), which could cause an increase in the overall prevalence 
of health conditions that disproportionately affect women. However, the number of female Veterans as a proportion of the total Veteran population will remain small, suggesting that this change in demographic composition is not likely to cause major shifts in Veterans' health conditions over the next 10 years.

Another plausible predictor of Veterans' future health conditions is their experience while in active military service. In particular, deployment and exposure to combat may increase the risk of injury and chronic health conditions and exacerbate existing conditions, although the evidence is mixed (Buckman et al., 2009; Kline et al., 2010; Hoge, Auchterlonie, \& Milliken, 2006; Dobkin and Shabani, 2009). This is particularly important for considering demand for VA health services, because Veterans with service-connected disabilities are placed in a higher priority group for enrollment in VA than those without such conditions. The results in Section 5.3 indicate that the prevalence of mental health conditions, especially PTSD, is much higher among Veterans than among similar non-Veterans. The prevalence of these conditions is even higher among Veterans who use VA (Figure 5-12), making this an important factor to consider for future VA demand. Other conditions, such as TBI and musculoskeletal conditions, have also been tied to military service.

Deployment rates have been consistently high among recently separated Veterans, as seen in Figure 5-19. If the scope and intensity of U.S. military operations continue to decline, we expect fewer new Veterans in the future to have been deployed while on active duty. The projected separations under the assumption of no conflicts over the next 10 years show a steep decline in the fraction of new Veterans that deployed during service. As a result, we expect an associated decline in service-connected injuries and chronic health conditions among newly separated Veterans in the future. We also expect that those who deployed but did not separate from service soon after deployment and will be observed separating over the next 10 years are likely to have fewer health problems than those who did separate immediately, compounding this effect.

Figure 5-19

Fraction of Recently Separated Veterans Under Age 35 Who Deployed on Active Duty

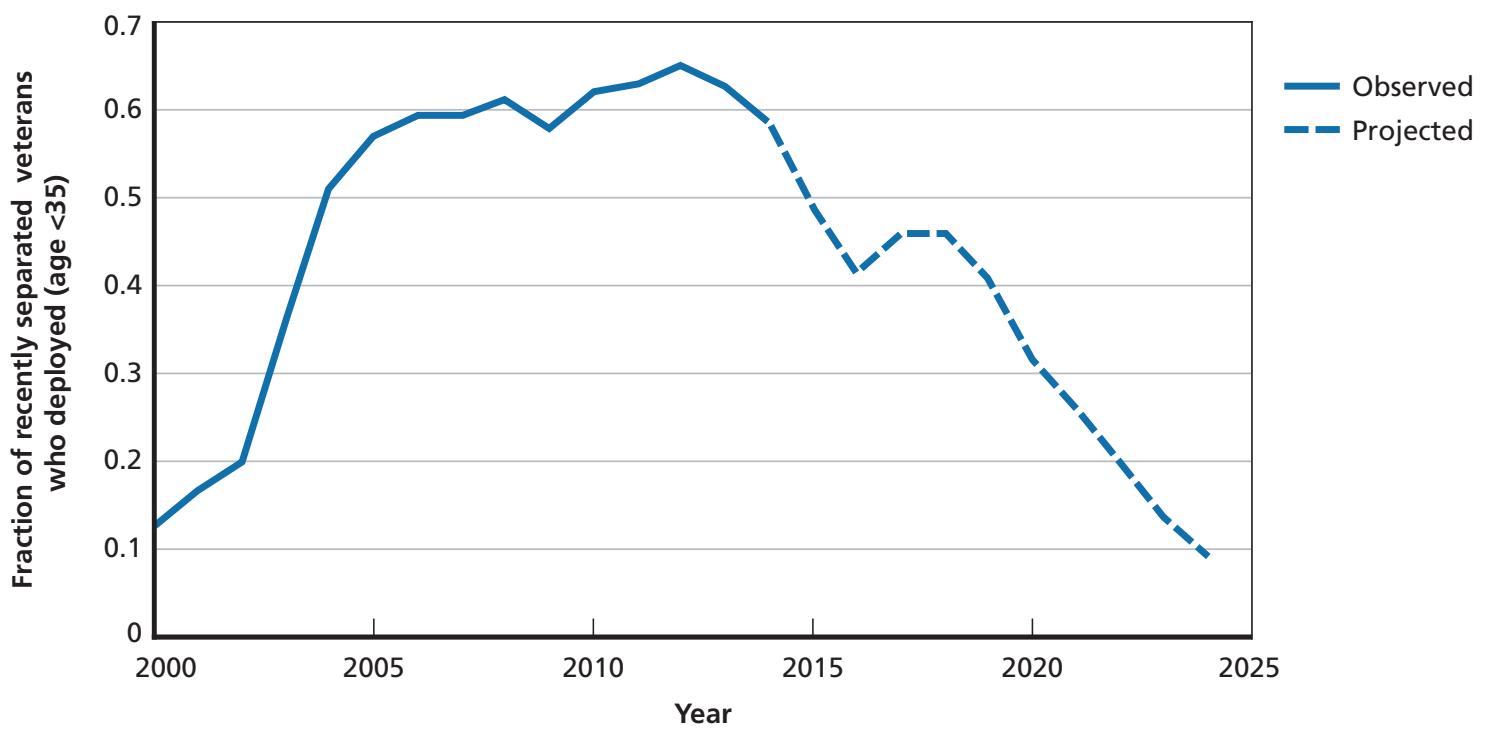

SOURCE: RAND analysis of DoD data.

RAND RR1165z1-5.19 


\subsubsection{Projected Health Care Needs of Veterans}

The projections of health condition prevalence among Veterans incorporate a time trend of the prevalence of each health condition and account for changes in the demographic composition of the Veteran population. Because health conditions may be affected by service experience, the projections of conditions that are likely to have a substantial service connection-PTSD and mental health conditions, which include PTSD_are adjusted to account for this. This adjustment assumes that the prevalence of the condition remains constant within five-year birth cohorts rather than within five-year age bands and assumes a prevalence rate for future newly separated Veterans. For example, the prevalence of PTSD is higher among Veterans in their 60s than it is among Veterans just younger and older, probably because this cohort was more likely to have served in Vietnam. As a result, we do not expect that current 50-year-olds will suddenly have a higher prevalence of PTSD in 10 years when they turn 60. We used estimates of the health conditions of current military service members and among Veterans who have recently separated from service to inform the prevalence of health conditions among those who will separate from service and become Veterans during 2015-2024. The details of this empirical methodology are in Appendix C.1.5, and the sensitivity of the results to our assumptions is explored in Appendix C.4.3.

Figure 5-20 shows the projected trend in prevalence for selected health conditions among Veterans. The dashed lines around the projection line are conservative bounds (described in detail in Appendix C.1.5.6). Based on our projections, chronic health conditions that afflict both Veterans and non-Veterans are expected to increase moderately over the next 10 years. The projected increases in the prevalence of hypertension, diabetes, and cancer are centered around 10 percent, while the prevalence of lipid disorders remains relatively stable. Given that these conditions are more common among older adults, the projections are consistent with the projected aging of the Veteran population over the next 10 years. However, while aging does tend to increase the prevalence of IHD, we estimated that prevalence rates for IHD will actually decline during 2015-2024. This finding is consistent with long-standing trends toward decreasing prevalence of acute coronary syndrome across all age groups in the U.S. population (Krumholz, Normand, \& Wang, 2014; Talbott et al., 2013). However, this decline largely represents an extrapolation of recent declines in the prevalence of IHD noted in MEPS; this finding assumes that the previous trend toward reduced prevalence of IHD will continue. The relatively large confidence bands suggest that the trend is uncertain and actual prevalence may not decline as sharply (Appendix C.4.5).

PTSD has been connected to military service experiences, particularly deployment-related combat exposure (Ramchand, Rudavsky, Grant, Tanielian, \& Jaycox, 2015). Given that this condition is caused by specific trauma more generally, it is likely that this condition is predominantly determined by military experience rather than age among the Veteran population. We adjusted the projection of the prevalence of PTSD by linking estimated prevalence to birth cohorts rather than age, as discussed above. In order to make this adjustment, we assumed a prevalence rate of 5.4 percent for newly separated Veterans from 2015 to 2024 . This prevalence rate, estimated using MHS data, is the average of the annual prevalence of PTSD among service members separating between 2009 and 2014. We also assume 35 percent of Veterans with PTSD remit in the first year post-service. The adjusted results are in Figure 5-21, and we project that the prevalence of PTSD will remain about the same from 2015 to $2024 .{ }^{9}$

9 Without the cohort adjustment, PTSD is projected to be 9.2 percent among Veterans in 2024, a 6-percentage-point increase. 
Figure 5-20

Projected Prevalence of Selected Health Conditions Among Veterans (2015-2024)
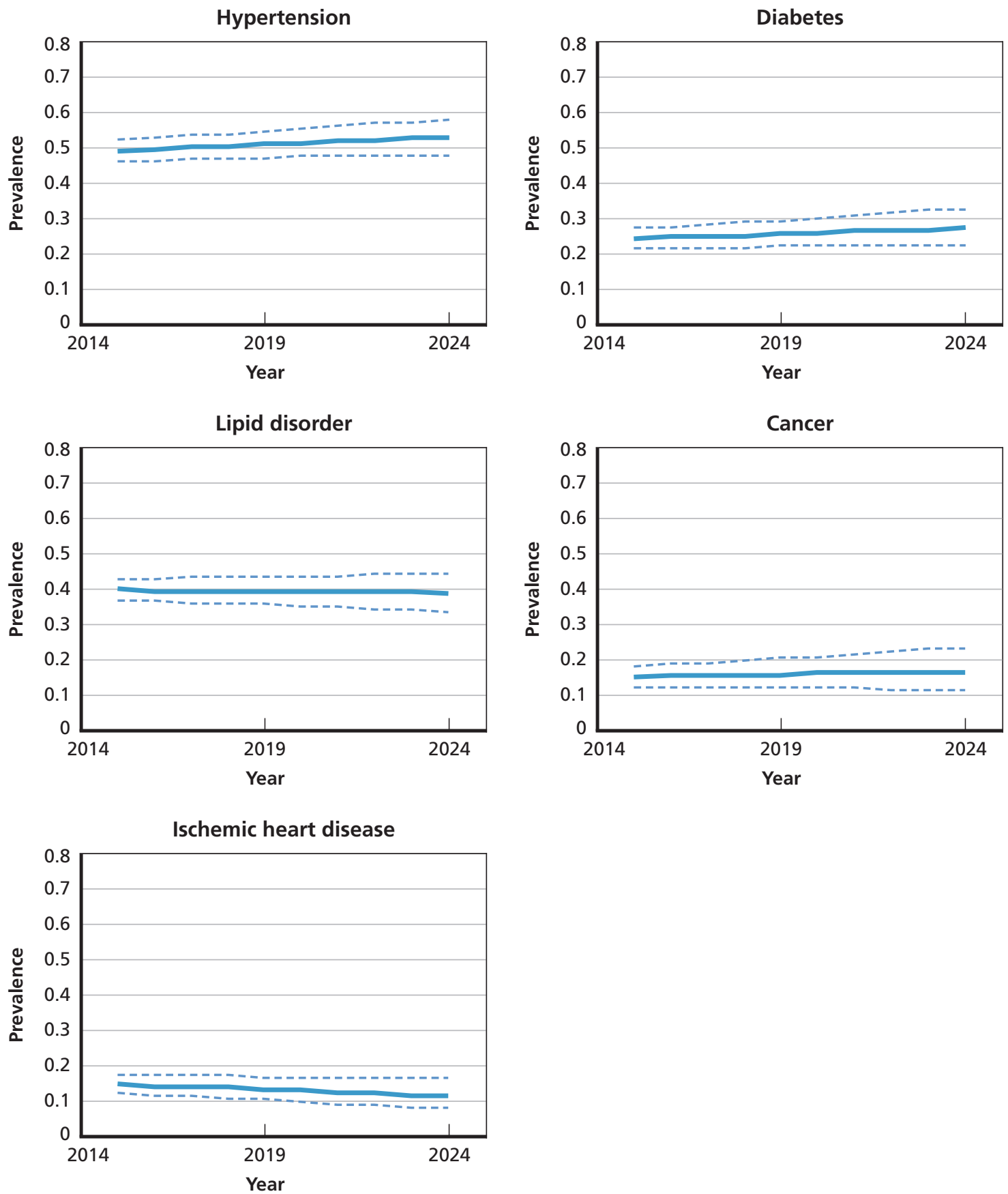

SOURCE: RAND analysis of VA, DoD, Census, and MEPS data.

NOTES: MEPS sample size, Veterans $=12,313$. Solid lines indicate the projected prevalence for each health condition, which accounts for the changes in the composition of the Veteran population by age, sex, race/ethnicity, Census region, and MSA. The dashed lines indicate conservative bounds for the projected prevalence rates. (See Appendix C.1.5 for methodological details.)

RAND RR1165z1-5.20 
Figure 5-21

Projected Prevalence of Service-Connected Conditions Among Veterans (2015-2024)
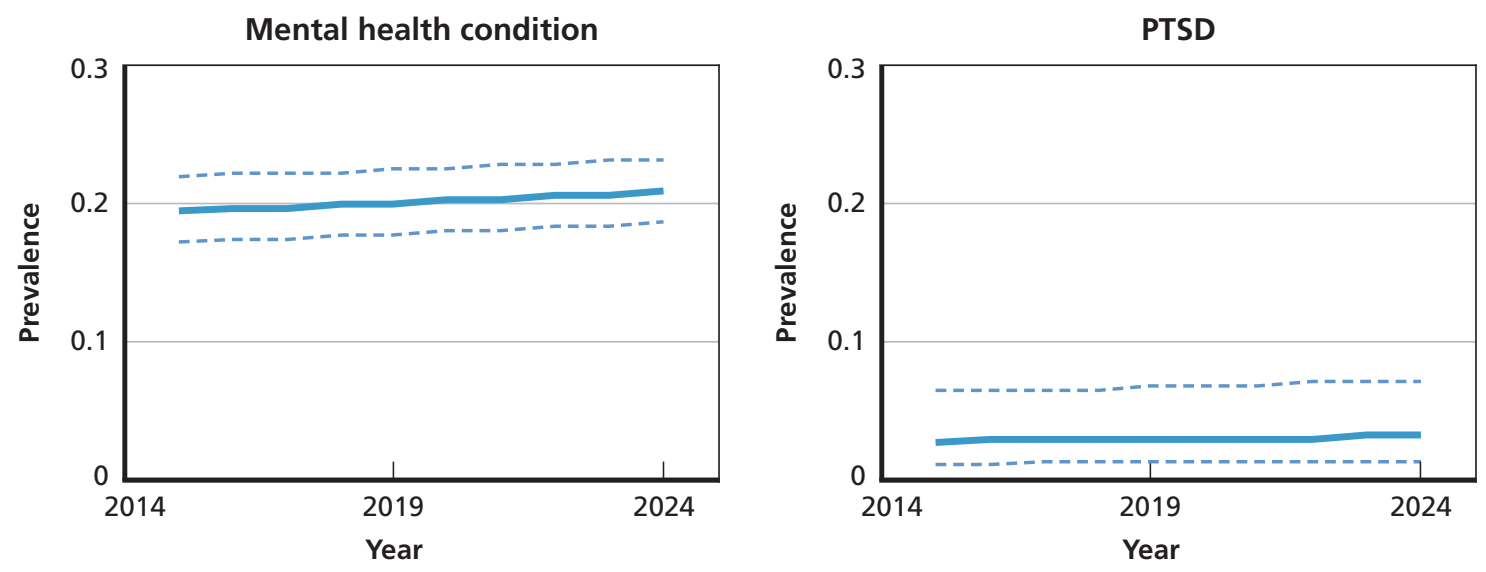

SOURCE: RAND analysis of VA, DoD, Census, and MEPS data.

NOTES: MEPS sample size, Veterans $=12,313$. Solid lines indicate the projected prevalence for each health condition, which accounts for the changes in the composition of the Veteran population by age, sex, race/ ethnicity, Census region, and MSA. The dashed lines indicate conservative bounds for the projected prevalence rates. (See Appendix C.1.5 for methodological details.)

RAND RR1165z1-5.21

PTSD is also included in the umbrella of any mental health condition, and for this population, PTSD is likely to have a strong effect on the prevalence of mental health conditions. Therefore, we also present adjusted results for the prevalence of any mental health condition in Figure 5-21. We used the same adjustment approach as for PTSD and assumed that the prevalence of any mental health condition among new Veterans will be 32.5 percent (also estimated with MHS). For mental health conditions, we assume a remission rate of 6.3 percent in the first year post-service, and zero thereafter. The remission rate is based on the fraction of Veterans with mental health conditions that have PTSD and the PTSD remission rate. With the adjustment, the prevalence of mental health conditions among Veterans is projected to increase to 20.7 percent by 2024. However, the umbrella of mental health conditions includes conditions that may not be related to service or that may have higher or lower remission rates than PTSD. If we treat mental health conditions like other predominantly age-related chronic conditions, such as hypertension, we project that the prevalence of mental health conditions among Veterans will be 26.1 percent by 2024. The details of these projection results are in Appendix C.4.3.

One particular limitation of our projections of health conditions relates to the timing of when a condition presents or when the individual seeks treatment from VA for the condition. For instance, if a Veteran experiences a hidden wound of war, such as PTSD or TBI, and either does not experience symptoms right away (e.g., delayed onset), does not enroll in VA and therefore does not receive treatment for some time after the injury, or does not file a claim for the condition soon after separating from the military, there may be a gap between injury or separation and when VA will treat the condition. Our projections are unable to account for this pattern. The data used to project health care needs (MEPS, MHS encounter, and VA encounter) lack information on when an injury occurred, when the Veteran separated from service, when the Veteran filed a disability claim, and when the Veteran enrolled in VA. Therefore, we are unable to determine whether receipt of treatment for these hidden wounds of war occurred 
shortly after the injury or experience or if there was a delay. Using historical data on the time between separation and either when a disability claim was filed or when the condition was first treated by VA would allow for an adjustment to our projections that accounts for future cases of hidden wounds of war for which VA does not yet have visibility.

The projection results for the full set of conditions that could be estimated using MEPS are included in Appendix C.4. Many service-connected injuries and chronic conditions of interest have low prevalence in the general population and cannot be reliably estimated using MEPS, so they are excluded from the projections.

\subsection{Future Health Care Needs of VA Patients}

\subsubsection{Factors Driving VA Patient Health Care Needs}

VA patients are a subset of the Veteran population; thus, the same factors and trends that affect the health care needs of Veterans overall will also affect the needs of VA patients. To project VA patient health care needs, we used the same approach and the same set of assumptions that we used for the Veteran population.

In addition to the factors and trends that affect the health care needs of the Veteran population overall, the needs in the VA patient population are determined in part by who uses VA health care services. Changes in VA policies - such as new priority group cutoffs for enrollment eligibility, additions of new presumptive service-connected conditions, or changes to the enhanced eligibility benefits for new combat Veterans - will directly affect the number and composition of Veterans who are eligible to receive VA health care services. External factors that affect access to affordable health care, such as fluctuations in the economic climate or changes in the eligibility rules for other public health programs (e.g., Medicare), have the potential to affect VA use rates and the composition of the VA patient population. We assumed that these factors remain constant over the next 10 years in these baseline projections. The effects of policy changes on future Veteran, enrollee, and patient counts are considered in the scenario analyses in Chapter Six.

\subsubsection{Projected Health Care Needs of VA Patients}

We projected the health care needs of VA patients using MEPS and VA encounter data (data sources are detailed in Section 2.6). MEPS was used to estimate and project prevalence of more-common chronic conditions based on receipt of care from any provider. We used VA encounter data to estimate and project demand for VA health care services and to estimate and project the prevalence of predominantly service-connected conditions, which cannot be reliably estimated using MEPS because of their low prevalence.

Figure 5-22 shows projected VA patient health care needs. The prevalence rates of hypertension and diabetes are projected to increase, and the prevalence rates of lipid disorders and cancer are projected to remain constant. As for Veterans overall, the prevalence of IHD is projected to decline among VA patient (see Appendix C.4.5 for further discussion).

As noted, the prevalence of PTSD, which is also a component of mental health conditions, is more likely linked to military experience than age. We adjusted the projections of mental health conditions and PTSD among VA patients using the same approach we used 
Figure 5-22

Projected Prevalence of Selected Health Conditions Among VA Patients (2015-2024)
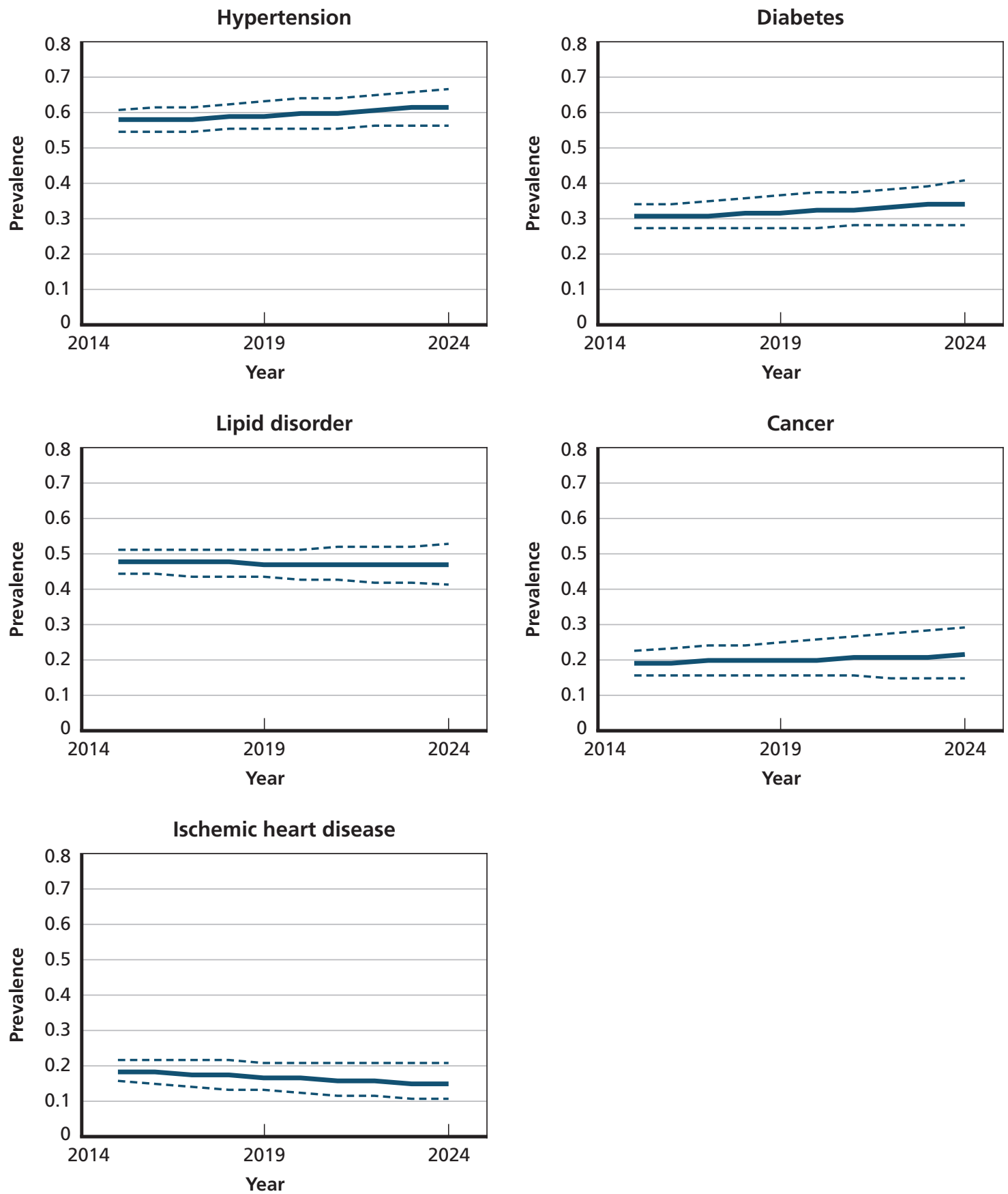

SOURCE: RAND analysis of VA, DoD, Census, and MEPS data. NOTES: MEPS sample size, VA patients $=4,871$. Solid lines indicate the projected prevalence for each health condition, which accounts for the changes in the composition of the VA patient populations by age, sex, race/ ethnicity, Census region, and MSA. The dashed lines indicate conservative bounds for the projected prevalence rates. (See Appendix C.1.5 for methodological details.)

RAND RR1165z1-5.22 
to adjust the projected prevalence of these conditions for all Veterans. However, we assumed a higher prevalence of mental health conditions and PTSD among newly separated Veterans who become VA patients than we did among newly separated Veterans overall. We assumed that the prevalence rates are 48.8 percent for any mental health condition and 17.3 percent for PTSD. These prevalence rates were derived from VA encounter data, MHS encounter data, and recently published work comparing prevalence rates between Veterans who use VA health care services and those who do not (Dursa, Reinhard, Barth, \& Schneiderman, 2014). We also assume a PTSD remission rate of 30 percent in the first year post-VA enrollment and a mental health condition remission rate of 10 percent in the first year post-VA enrollment. The approach is detailed in Appendix C.1.5. Despite their magnitude, the prevalence rates for newly separated Veterans who become VA patients will not have a large effect on the overall prevalence rates for the VA patient population, because new patients are a small portion of the total VA patient population.

The projected prevalence rates of mental health conditions and PTSD among VA patients are presented in Figure 5-23. The overall prevalence of any mental health condition among VA patients is projected to be 33 percent by 2024, and the prevalence of PTSD is projected to increase to be just over 10 percent in 2024. As with all Veterans, mental health conditions are not purely service-connected. If we treat mental health conditions like other predominantly age-related chronic conditions, such as hypertension, we project that the prevalence of mental health conditions among VA patients will be about 37 percent by 2024 . The details of these projection results are in Appendix C.4.

We used VA encounter data to estimate the prevalence of two conditions of particular interest to VA: TBI and musculoskeletal conditions. VA encounter data were used to estimate the prevalence of VA-diagnosed/treated health conditions among VA patients. ${ }^{10}$ MEPS prevalence estimates include diagnoses made by VA and non-VA providers, and because many VA patients use non-VA providers for some health care services, MEPS provides a more comprehensive estimate of diagnosed prevalence rates among VA patients. However, the VA encounter estimates provide a better picture of the condition-based demand for VA health care services.

Our projections are presented in Figure 5-24. We project increases in the number of VA patients with both conditions. The prevalence of TBI, like PTSD and mental health conditions, is more likely linked to military experience than age. We used the same approach to adjust the projected prevalence of TBI. The projected trend in the prevalence of musculoskeletal conditions reflects historical trends in the prevalence of these conditions, as measured with VA encounter data and projected changes to the age and sex composition of the VA patient population. The fit of the model is discussed in Appendix C.5.

It is important to note that the trends may be partially driven by increases in VA use for these conditions, rather than trends in true diagnosed prevalence. In addition, the rates of diagnosis of these conditions may have increased due to heightened awareness and improved understanding of these conditions. We are not able to disentangle these effects.

10 Additionally, the prevalence of TBI is too low to be reliably estimated with MEPS. 
Figure 5-23

Projected Prevalence of Service-Connected Conditions of VA Patients (2015-2024)
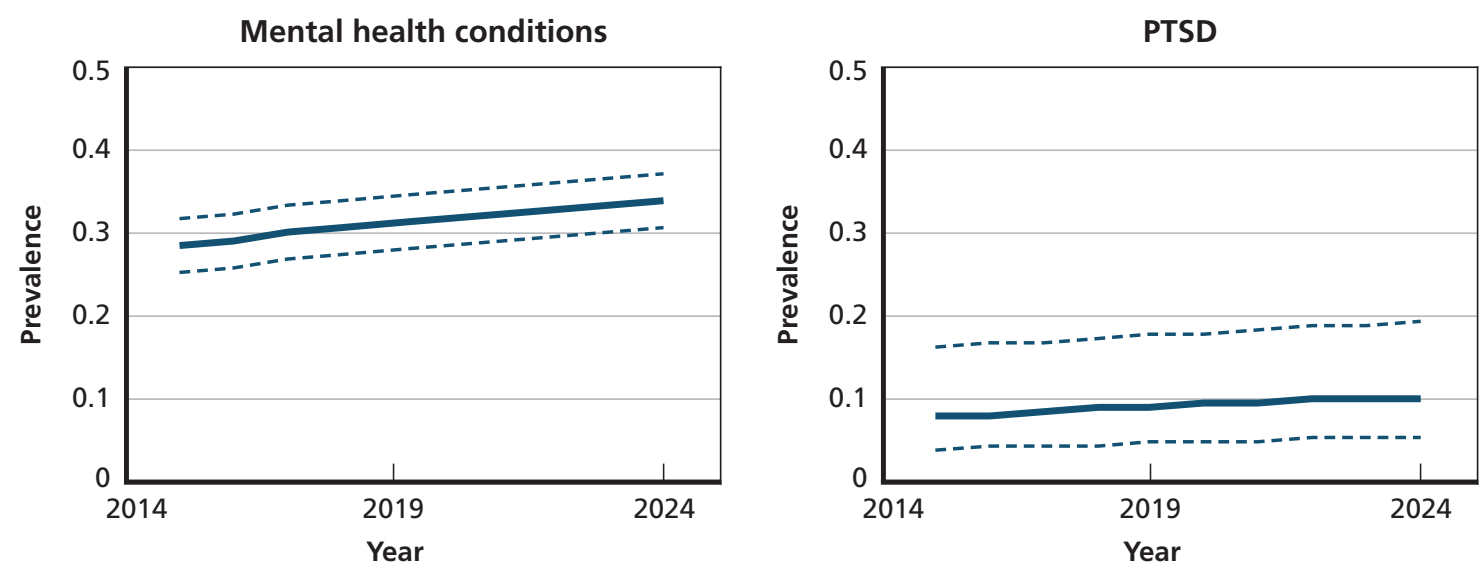

SOURCE: RAND analysis of VA, DoD, Census, and MEPS data.

NOTES: MEPS sample size, Veterans $=12,313$. Solid lines indicate the projected prevalence for each health condition, which accounts for the changes in the composition of the Veteran population by age, sex, race/ethnicity, Census region, and MSA. The dashed lines indicate conservative bounds for the projected prevalence rates. (See Appendix C.1.5 for methodological details.)

RAND RR1165z1-5.23

Figure 5-24

VA Encounter-Based Projections of Health Conditions Among VA Patients (2015-2024)

TBI

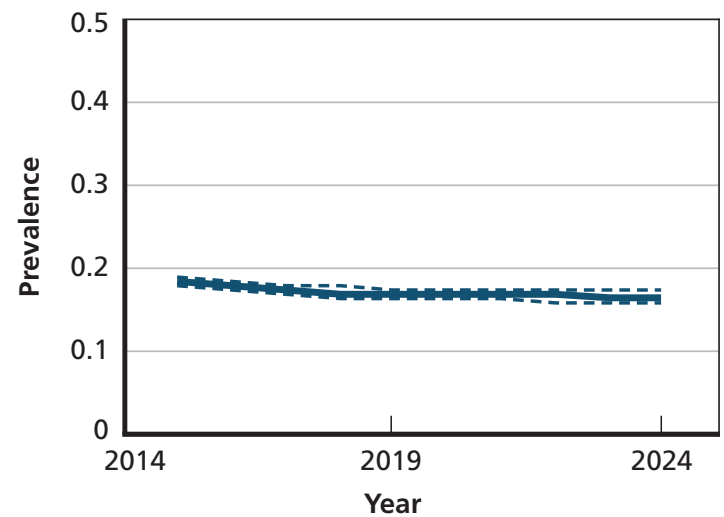

Musculoskeletal conditions

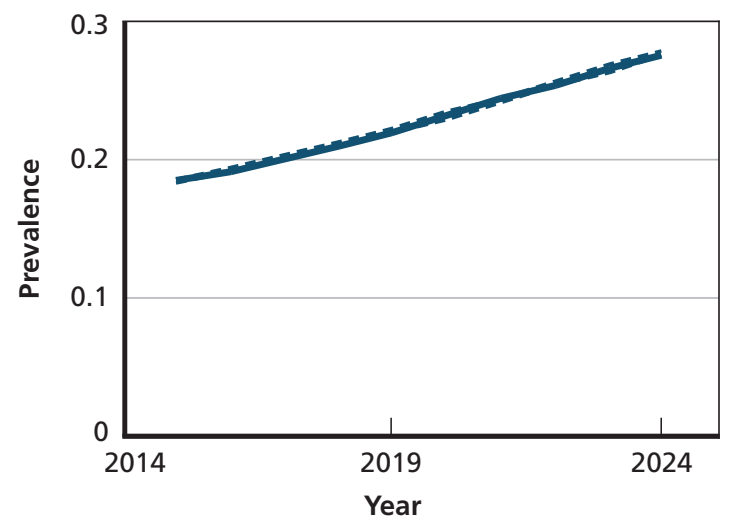

SOURCE: RAND analysis of VA, DoD, Census, and MEPS data.

NOTES: VA encounter sample size, VA patients $=5,871,766$. Solid lines indicate the projected prevalence for each health condition, which accounts for the changes in the composition of the VA patient populations by age, sex, race/ethnicity, Census region, and MSA. The dashed lines indicate conservative bounds for the projected prevalence rates. (See Appendix C.1.5 for methodological details.) Musculoskeletal conditions are those associated with chronic pain.

RAND RR1165z1-5.24 


\subsection{Summary of Key Findings}

Veterans have a higher prevalence of many chronic physical health conditions than nonVeterans, in part because they are older. The prevalence of many chronic conditions, including cancer, diabetes, and GERD, is higher among Veterans than non-Veterans. This difference diminishes after adjusting for the differences in the demographic composition (particularly, age and sex) of the Veteran and non-Veteran populations. The remaining differences are small for most physical health conditions, including the prevalence rates of hypertension, heart disease, and lipid disorders, which are nearly identical across the two populations.

Veterans have a higher prevalence of mental health conditions than demographically matched non-Veterans. In contrast to the physical health conditions, the relative mental health status of Veterans worsens when they are compared with demographically matched non-Veterans. PTSD stands out as an important unique health need; the prevalence among Veterans is 13.5 times that of non-Veterans.

VA patients have a higher prevalence of chronic health conditions than Veterans who do not use VA health care services. Among Veterans, the prevalence rates (adjusted for demographic differences) of most chronic conditions we examined, including cancer, heart-related conditions, diabetes, and hypertension, are higher for VA patients than for Veterans who do not use VA health care services. This is at least partially the result of VA prioritizing enrollment by Veterans with higher prevalence of chronic health conditions (including those with serviceconnected conditions, disabilities, and lower incomes). The prevalence of any mental health condition among VA patients is nearly twice the prevalence among non-VA patients, and the prevalence of PTSD is 17.4 times larger. PTSD is associated with combat experience, which is an important predictor of Veterans' use of VA health care services.

The prevalence of diabetes, hypertension, and cancer among Veterans is projected to increase moderately over the next 10 years. For Veterans, we project moderate increases in the prevalence of diabetes ( 3 percentage points) and hypertension ( 4 percentage points), but a moderate decline in the prevalence of IHD (3 percentage points). These projections follow the recent trends in these conditions among the U.S. population.

The prevalence of mental health conditions among Veterans and VA patients is projected to increase. The prevalence of any mental health conditions and PTSD among Veterans is projected to increase by about 0.5 percentage points. Among VA patients, the prevalence of mental health conditions is projected to increase by 5.3 percentage points, while the prevalence of PTSD will increase by approximately by 2.4 percentage points. The expected declines in deployment and combat exposure among the newest Veterans and VA patients over the next 10 years will contribute to a slowing of the rate of increase of mental health conditions and somewhat smaller increases for PTSD in particular.

The gap in the prevalence of many health conditions between VA patients and Veterans who do not use VA health care services is projected to widen over the next $\mathbf{1 0}$ years. The projected increases in the prevalence of mental health conditions and PTSD among VA patients is larger than that among all Veterans, as cited above. The prevalence of diabetes, GERD, and cancer are also projected to increase more among VA patients than all Veterans, while the prevalence of other chronic conditions are projected to change by the same amount for the two populations. 


\subsection{Introduction}

Estimates of changes in populations and health care needs over time rely on a set of assumptions about how the policy environment and world will change over time. The demographic projections in this report are driven by historical information on military separation, Veteran mortality, and migration. For the health care needs analyses in Chapter Five, we inferred the future prevalence of health conditions from historical prevalence rates. These analyses assume that the future will resemble the recent past in many important dimensions, including the general size and function of the armed forces, migration patterns, the health care options and coverage available to Veterans, and the mission and function of VA in terms of health care delivery.

What if these dimensions change? The purpose of this chapter is to describe how changes to the status quo could affect Veteran, enrollee, and patient populations and more broadly influence demand for VA care. To do this, we analyzed a range of policy scenarios. Policy scenarios are a tool that can help decisionmakers understand the effects of "what ifs" as they plan for the future, especially when considered in conjunction with robust, validated baseline projections. We first identified a set of plausible scenarios, as described below, and then used modeling techniques to estimate the effect of each scenario on the size of the future VA patient population. As part of our evaluation of each scenario, we assessed how the scenario and impacts could be integrated into current VA modeling efforts, and, if it would not be possible to incorporate these changes into existing models, we propose a range of policy or modeling options to integrate and mitigate scenario impacts as appropriate.

Despite the usefulness of policy scenarios, there are two important caveats that apply to this chapter. First, each individual scenario is speculative and is based on assumptions that may diverge from actual conditions in the future. Second, due to time and data constraints, we were able to describe and evaluate only a limited set of policy scenarios. We chose these scenarios to be illustrative of important "what ifs" that VA could face in the future. However, specific details, such as the nature of a potential future conflict, cannot be reliably predicted. 


\subsection{Scenario Methodology Overview}

\section{Overview of Scenario Analysis Data and Methods}

- To select scenarios for analysis, we generated candidate scenarios by reviewing scenarios that had been previously explored in VA analyses, conducting a targeted literature review, and consulting with other assessment teams and RAND subject-matter experts.

- We categorized scenarios by whether they would result in a change in (1) the population of eligible or enrolled Veterans or (2) the proportion of enrollees who use VA health care services.

- We selected analysis scenarios that were most likely to have substantive impacts on the size of Veteran or patient populations or Veterans' health care needs. These are:

- Changes to VA eligibility by priority group

- Changes in presumptive VA eligibility

- Impact of future conflict on VA use

- Improving access to VA Care

- The ACA's coverage expansion.

- We used scenario-specific methods to develop and evaluate each scenario.

- These scenario-specific methods are described below and in more detail in Appendix D.

The research described in this chapter reflects two methodological steps. First, we developed five specific scenarios. Next, we developed scenario-specific evaluation methodologies to estimate the impact of each scenario on populations and demand for VA health care. The scenariospecific methodologies are described in detail in the following sections and in Appendix D. The last section in this chapter discusses key results and conclusions from our scenario analyses. See Chapter Two for a summary of VA's current use of scenarios in modeling and planning processes, focusing in particular on EHCPM.

This paragraph describes our methodology for choosing and defining specific scenarios for analysis. As a first step, we generated a list of candidate scenarios by reviewing scenarios that had been previously explored in VA analyses, ${ }^{1}$ conducting a targeted literature review, and consulting with other assessment teams and RAND subject-matter experts. Candidate scenarios involved either a change in VA policy, a change in policy elsewhere in the government, or an external trend. We explored candidate scenarios that would have plausible impact on either the size of Veteran and patient populations or Veterans' health care needs. Through this process, we identified 12 candidate scenarios. Next, we developed a framework to help organize and guide our selection of five scenarios for analysis. The framework is described below. We selected for analysis those scenarios that were most likely to have substantive impacts on the size of Veteran or patient populations or Veterans' health care needs. Some of the candidate scenarios that were not selected for inclusion are discussed at the end of this chapter.

\footnotetext{
1 The VA EHCPM analysts and the VA OACT have conducted scenario analysis; we reviewed documentation on these analyses and notes from our meetings with these offices.
} 


\subsection{Scenario Typology and Framework}

We first categorized scenarios by whether they would result primarily in a change in (1) the population of eligible or enrolled Veterans or (2) the proportion of enrollees that are VA patients (i.e., that use VA health care services). These potential changes align with the analyses presented earlier in this report, including projections of the demographics of the Veteran population (Chapter Three) and projections of VA health care enrollees and patients (Chapter Four).

The primary outcome for all scenarios was the estimated change in the number of VA patients by calendar year. Following the framework laid out in Chapter Two, the number of VA patients is the product of the number of eligible Veterans, the enrollment rate, and the use rate, or, with the y subscript denoting year:

$$
\text { Patients }_{y}=\text { EligibleVeterans }_{y} * \text { EnrollmentRate }_{y} * \text { UseRate }_{y}
$$

Changes in the Veteran population-either in the number of Veterans or shifts in the composition of the Veteran population-can have important implications for the demand for VA health care. Even if the number of Veterans remains constant, shifts in the age, sex, priority group, or health status of Veterans can change demand for VA services to the extent that Veteran subpopulations utilize VA services differently.

Changes that directly affect how many VA enrollees choose to be VA patients would also influence the number of VA patients. As described in Chapter Four, demographic and other characteristics of Veterans, including whether Veterans have access to other sources of health coverage, are significant predictors of whether they use VA health care.

We also categorized scenarios into one of two types of policies or trends: (1) VA policies or legislation focusing on VA, or (2) broader policy changes outside of VA's control and external trends. We differentiated between these two policy or trend types to help describe whether VA has direct, indirect, or no control over the scenario.

The five specific scenarios are listed in Figure 6-1, a two-by-two framework incorporating the scenario types and change types introduced above. As shown in Quadrant A, we selected two scenarios that would change Veteran eligibility for health benefits. Other VA-focused policies or trends that directly influence the number of Veterans eligible for health benefits would likely share many characteristics and aspects of these two scenarios. As shown in Quadrant $\mathrm{B}$, the hypothetical future conflict scenario describes how an influx of service members in response to a new conflict could translate into a flow of Veterans and ultimately VA patients. The future conflict analyses assess a range of plausible military manpower scenarios and their effect on projections of VA patients. In Quadrant C, the scenario analyzes policy options that could influence Veterans' decisions to use VA health care by changing actual or perceived access and quality of care in the VA system. Finally, the ACA coverage expansion scenario in Quadrant D describes the potential impacts of major shifts in the non-VA coverage available to Veterans on their decision to use or not use VA care. A range of other policies external to VAsuch as changes in state Medicaid policy and the generosity and cost of employer-sponsored insurance-share characteristics with this fifth scenario. 
Figure 6-1

Assessment A Scenario Framework

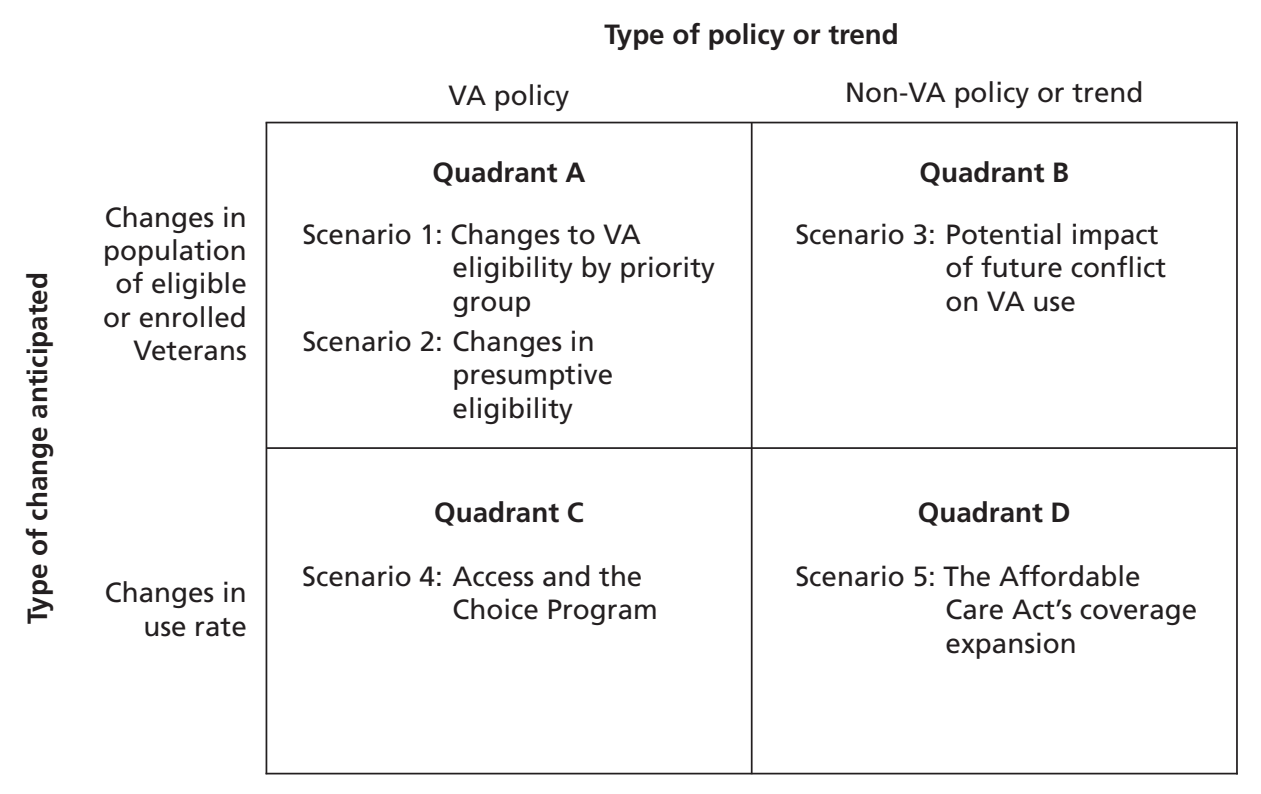

RAND RR1165z1-6.1

The following sections describe each scenario in more detail. We included contextual factors and details that could be important in determining the impact of these scenarios on demand for VA health care.

\subsection{Changes to VA Eligibility, by Priority Group}

\subsubsection{Description}

Under current VA policy, Veterans in priority groups $8 \mathrm{e}$ and $8 \mathrm{~g}$ are not eligible to en roll for VA health care services. These Veterans satisfy the length-of-service requirement and the discharge criteria but do not have a service-connected disability rating, do not have a disability rendering them housebound, and do not qualify for Medicaid, and they have household income exceeding 110 percent of their GMT income limit. We modeled changes to VA eligibility by priority group by estimating the effects of a change in which these lower priority groups gained eligibility for health care services. In the first subscenario, we examined how many Veterans would become eligible for services if eligibility was extended to priority group $8 \mathrm{e}$ and the expected number of VA patients that would result. Second, we further examined how many Veterans would become eligible for services if eligibility was extended to the next-lowest priority group, $8 \mathrm{~g}$, and estimated the expected number of VA patients that would result. 


\subsubsection{Approach}

Data and Methods for Scenario One, Changes to VA Eligibility by Priority Group

- We sorted all Veterans eligible for priority group assignments into priority groups in order to both estimate the size of the $8 \mathrm{e}$ and $8 \mathrm{~g}$ Veteran populations and to test use rates for each eligible priority group..

- We used the 2013 ACS Public Use Microdata Sample (PUMS), which contains self-reported information on service-connected disability rating, VA health service use, family and household income, family and household size, individual income from a variety of sources, area of residence and Medicaid use.

- We employed an algorithm that assigns a priority group to each Veteran contained in the 2013 ACS, based on available data and adjustment factors drawn from administrative records.

In order to sort all Veterans into priority groups, this analysis had two essential components:

1. The identification of all U.S. Veterans in a nationally representative data set. To do this, we used the 2013 ACS PUMS, or 2013 ACS, which contains self-reported information on service-connected disability rating, VA health service use, family and household income, family and household size, individual income from a variety of sources, area of residence, and Medicaid use. The 2013 ACS, with demographic adjustments from VA administrative records, identifies approximately 21.9 million Veterans in the United States, with slightly more than 6 million Veterans using VA health services.

2. A priority group classification algorithm that assigns a priority group to each Veteran contained in the 2013 ACS, based on the available data described above and several adjustment factors drawn from administrative records (see Appendix D.1 for a thorough description of this algorithm).

Testing subscenarios in which eligibility for VA health services is expanded to new priority groups amounts to taking the algorithm's estimates of these ineligible priority groups and applying a use rate, estimated from the most similar eligible priority group, to arrive at an estimate of new VA patients.

Table 6-1 shows the baseline ACS estimates of Veterans using VA health services - that is, VA patients, by priority group, in comparison with VA administrative records of this population, along with a brief description of each priority group's eligibility requirements (for a thorough description of the eligibility requirements for each priority group, see U.S. Department of Veterans Affairs, 2015c). This comparison provides a validity test: The ACS estimates of VA patients should match administrative records. Indeed, these baseline ACS estimates are very close to the administrative records, with much of the deviation attributable to sampling error and comparisons across two distinct years.

However, the advantage of this approach is not in replicating administrative records, but in estimating the number of potentially eligible Veterans by priority group in the general Veteran population, a task for which administrative records are ill-suited. Although the administrative data have records of Veterans in these priority groups who are eligible to use VA health services (e.g., 8e Veterans who are eligible for services for their 0 -percent service-connected disability care), there are otherwise no estimates of the size or composition of this $8 \mathrm{e}$ and $8 \mathrm{~g}$ population. 
Table 6-1

VA Patients According to 2014 VA Administrative Data and 2013 ACS Estimates, by Priority Group

\begin{tabular}{|c|c|c|}
\hline Priority Group & VA Patients, 2014 & VA Patients in ACS, 2013 \\
\hline 1 - Service Connected $50 \%+$ & $1,599,076$ & $1,641,000$ \\
\hline 2 - Service Connected $30 \%-40 \%$ & 489,192 & 502,000 \\
\hline 3 - Service Connected 20\%/POW/Special & 741,713 & 761,000 \\
\hline $4-\mathrm{AA} /$ Housebound or Catastrophic & 191,342 & 117,000 \\
\hline 5 - Non-Service Connected Below Income & $1,315,317$ & $1,350,000$ \\
\hline 6 - All Other Not Required to Make Co-Pay & 276,275 & 304,000 \\
\hline 7 - Non-Compensable Non-Service Connected Below GMT & 174,810 & 192,000 \\
\hline $\begin{array}{l}8 a, 8 b, 8 c, 8 d-\text { Non-Compensable Non-Service Connected } \\
\text { Below GMT }+10 \% \text {, or previously VA eligible }\end{array}$ & $1,055,685$ & $1,124,000$ \\
\hline $8 e, 8 g-$ Users & 26,115 & 32,000 \\
\hline Priority Group Not Assigned & 2,241 & \\
\hline Total & $5,871,766$ & $6,022,000$ \\
\hline
\end{tabular}

SOURCE: RAND calculations using 2013 ACS PUMS.

We tested two subscenarios for Scenario 1. The first estimated the number of new VA patients under the condition that Veterans in priority group 8e were eligible for these services; they currently are not eligible. The 8 e subscenario uses the estimates of the number of Veterans classified as $8 \mathrm{e}$ and average priority group 8 use rates to estimate how extending eligibility to $8 \mathrm{e}$ would affect the number of eligible Veterans and the number of expected VA patients. Veterans in priority group $8 \mathrm{~g}$ are also currently ineligible for VA health care services, so the $8 \mathrm{e}$ and $8 \mathrm{~g}$ subscenario estimates the effects of expanding enrollment eligibility to both these priority groups.

\subsubsection{Results}

Table 6-2 shows estimates of all 21.9 million Veterans in the 2013 ACS by priority group, ${ }^{2}$ compared with the corresponding number of ACS-estimated VA patients by priority group. These two numbers allow us to calculate priority group-specific use rates, which are the ratios of VA patients to eligible Veterans in each priority group.

When the subscenarios below result in shifts in the number of eligible Veterans by priority group, these use rates will then translate the change in eligible Veterans into a change in expected VA patients in that priority group.

Priority group 8e subscenario. Table 6-2 shows that there are 78,000 Veterans who, whether enrolled or not, would be categorized into priority group $8 \mathrm{e}$ if they applied for VA health care benefits. Thus, if eligibility to enroll in VA health care were extended to priority group $8 \mathrm{e}$, there would be 78,000 newly eligible Veterans. Given that Veterans in priority group 8 overall have an average use rate of 0.44 , there would be an expected 34,000 new

\footnotetext{
2 In Chapter Three, we report that there are 21.6 million Veterans. This estimate was calculated using the 2014 ACS,
} whereas the 21.9 million reported here uses the 2013 ACS. 
Table 6-2

2013 ACS Estimates and Use Rates, by Priority Group and Eligibility Status

\begin{tabular}{|c|c|c|c|}
\hline Priority Group and Eligibility Status & $\begin{array}{l}\text { All Veterans in ACS, } \\
2013\end{array}$ & $\begin{array}{l}\text { VA Patients in ACS, } \\
2013\end{array}$ & $\begin{array}{l}\text { Average Use Rate in } \\
\text { ACS (Patients/Eligible) }\end{array}$ \\
\hline 1 & $1,961,000$ & $1,641,000$ & 0.84 \\
\hline 2 & 745,000 & 502,000 & 0.67 \\
\hline 3 & $1,310,000$ & 761,000 & 0.58 \\
\hline 4 & 377,000 & 117,000 & 0.31 \\
\hline 5 & $4,151,000$ & $1,350,000$ & 0.33 \\
\hline 6 & $1,073,000$ & 304,000 & 0.28 \\
\hline 7 & 749,000 & 192,000 & 0.26 \\
\hline $8 a, 8 b, 8 c, 8 d$ & $2,572,000$ & $1,124,000$ & 0.44 \\
\hline $8 \mathrm{e}, 8 \mathrm{~g}$ users & 32,000 & 32,000 & 1.00 \\
\hline $8 \mathrm{e}$ nonusers & 78,000 & & \\
\hline $8 \mathrm{~g}$ nonusers & $4,727,000$ & & \\
\hline Ineligible due to discharge status & $1,246,000$ & & \\
\hline Ineligible due to length of service & $2,903,000$ & & \\
\hline Total eligible for services & $12,970,000$ & & \\
\hline Total eligible to apply for enrollment ${ }^{a}$ & $17,775,000$ & & \\
\hline Total Veterans & $21,924,000$ & $6,022,000$ & \\
\hline
\end{tabular}

SOURCE: RAND calculations using 2013 ACS PUMS.

a Not all Veterans who are eligible to apply for VA health care services are eligible to enroll and receive VA health care services under current policy.

VA patients as a result $(78,000 \times 0.44)$, increasing the overall number of VA patients by 0.6 percent. $^{3}$

Priority group $\mathbf{8 e}$ and $\mathbf{8 g}$ subscenario. Table 6-2 shows that there are 4,727,000 Veterans who would be categorized into priority group $8 \mathrm{~g}$ if they applied for VA health care benefits. ${ }^{4}$ Thus, when combined with the 78,000 Veterans in $8 \mathrm{e}$, there would be 4,805,000 newly eligible Veterans if eligibility to enroll in VA health care were extended to both $8 \mathrm{e}$ and $8 \mathrm{~g}$. As above, given the average priority group 8 use rate of 0.44 , there would be an expected $2,114,000$ new $8 \mathrm{e}$ and $8 \mathrm{~g}$ VA patients as a result $(4,805,000 \times 0.44)$, an increase of 35.1 percent over the current VA patient population.

\footnotetext{
3 It is possible that higher-income Veterans who are not currently eligible to enroll would have lower use rates than Veterans currently enrolled in priority group 8 . If this were the case, we may overestimate that number of Veterans that would be likely to use care if the VA expanded eligibility to all Veterans in priority group 8.

4 This estimate of the size of potential priority group $8 \mathrm{~g}$ Veterans is determined by sorting Veterans into higher priority groups. The next step is to subtract those who do not satisfy length-of-service or discharge requirements from the remaining unsorted Veteran population. See Appendix D for a description of determining how many Veterans do not satisfy these criteria. However, it should be noted that the length-of-service and discharge requirements are treated independently and thus represent an overestimate of the ineligible Veteran population.
} 


\subsubsection{Discussion}

We estimate that expanding enrollment eligibility for VA health care to include priority group 8e would bring in a modest number of new Veteran users, increasing the number of patients by 0.6 percent. However, we estimate that there are a substantial number of Veterans in priority group 8g. An expansion to include this group would markedly increase the current number of eligible Veterans and users. Expanding eligibility to enroll in VA health care to include both groups of Veterans (priority groups $8 \mathrm{e}$ and $8 \mathrm{~g}$ ) would bring in a large number of new Veteran users, increasing the number of patients by 35.1 percent. However, such an estimate is based on current priority group 8 use rates, and this estimate may be different if the population of Veterans in this priority group changes.

The subscenarios tested here correspond to expansions of eligibility or generosity of the VA program. We did not test any contractions in eligibility or generosity for two reasons. First, historically, such contractions have been accompanied by grandfathering in currently eligible Veterans, and because this analysis is static in its analysis of 2013 Veterans, these future dynamics are outside the scope of this analysis's capabilities. Second, such a contraction-based analysis does not require estimates of eligible Veterans, just current and projected enrollees and users, for which administrative records are well-suited. Nevertheless, our baseline counts of all eligible Veterans by priority group give estimates of the overall size of each of these priority groups, which may be useful for other scenario-based models.

\subsection{Changes in Presumptive Eligibility}

\subsubsection{Description}

When a Veteran's military service has resulted in physical or mental impairment, that Veteran is granted such benefits as compensation and health care depending on the extent of impairment. However, not all impairments can be factually linked by evidence to military service. Since 1921, Congress has authorized VA to establish criteria for impairments that are likely (but unproven) to be service-connected. Since that time, more than 150 conditions have been categorized as service-connected. In the Agent Orange Act of 1991, Congress directed VA to periodically review the quality of the evidence for presuming service-connection for impairments experienced by Veterans (Samet \& Bodurow, 2008). If VA finds that there has been a systematic impact on Veteran health, VA then revises its eligibility criteria. The revised eligibility standard typically specifies that when a Veteran served in a particular location during a particular time frame and has a particular condition - that condition is presumed to have been caused by military service and the Veteran is now eligible to enroll in VA health care.

VA contracts with IOM to review every two years the medical literature on Vietnam-era Veterans. Table 6-3 shows IOM's latest evidentiary findings for Vietnam Veterans for a variety of health conditions. In 2006, IOM moved hypertension from "Inadequate or Insufficient Evidence to Determine an Association" to "Limited or Suggestive Evidence of an Association." As of the writing of this report, VA had not added hypertension to the list of presumptive conditions for Veterans who served in Vietnam. This scenario examines the potential impact of adding hypertension to the list of conditions that would allow VA to presume that a Vietnamera Veteran with hypertension is service-connected. 
Table 6-3

IOM's Assessment of the Evidence Connecting Certain Health Conditions to Military Service in Vietnam

Sufficient Evidence of an Association

Soft-tissue sarcoma (including heart); non-Hodgkin lymphoma; chronic lymphocytic leukemia (including hairy cell leukemia and other chronic B-cell leukemias); Hodgkin lymphoma; chloracne

Limited or Suggestive Evidence of an Association

Laryngeal cancer; cancer of the lung, bronchus, or trachea; prostate cancer; multiple myeloma; $A L$ amyloidosis (category change in 2006); early-onset peripheral neuropathy (category change in 2010); Parkinson disease (category change in 2006); porphyria cutanea tarda; hypertension (category change in 2006); ischemic heart disease (category change in 2008); stroke (category change in 2012); Type 2 diabetes (mellitus); spina bifida in offspring of exposed people

Inadequate or Insufficient Evidence to Determine an Association

Cancers of the oral cavity (including lips and tongue), pharynx (including tonsils), or nasal cavity (including ears and sinuses); cancers of the pleura, mediastinum, and other unspecified sites in the respiratory system and intrathoracic organs; esophageal cancer (category change in 2006); stomach cancer (category change in 2006); colorectal cancer (including small intestine and anus) (category change in 2006); hepatobiliary cancers (liver, gallbladder, and bile ducts); pancreatic cancer (category change in 2006); bone and joint cancer; melanoma; nonmelanoma skin cancer (basal-cell and squamous-cell); breast cancer; cancers of reproductive organs (cervix, uterus, ovary, testes, and penis; excluding prostate); urinary bladder cancer; renal cancer (kidney and renal pelvis); cancers of brain and nervous system (including eye); endocrine cancers (thyroid, thymus, and other endocrine organs); leukemia (other than chronic B-cell leukemias, including chronic lymphocytic leukemia and hairy cell leukemia); cancers at other and unspecified sites; infertility; spontaneous abortion (other than after paternal exposure to TCDD [tetrachlorodibenzodio], which appears not to be associated); neonatal or infant death and stillbirth in offspring of exposed people; low birth weight in offspring of exposed people; birth defects (other than spina bifida) in offspring of exposed people; childhood cancer (including acute myeloid leukemia) in offspring of exposed people; neurobehavioral disorders (cognitive and neuropsychiatric); neurodegenerative diseases, excluding Parkinson disease; chronic peripheral nervous system disorders; hearing loss (added in 2010); respiratory disorders (wheeze or asthma, chronic obstructive pulmonary disease, and farmer's lung); gastrointestinal, metabolic, and digestive disorders (changes in hepatic enzymes, lipid abnormalities, and ulcers); immune system disorders (immune suppression, allergy, and autoimmunity); circulatory disorders (other than hypertension, ischemic heart disease, and stroke); endometriosis; disruption of thyroid homeostasis; eye problems (added in 2010); bone conditions (added in 2010)

Limited or Suggestive Evidence of No Association

Spontaneous abortion after paternal exposure to TCDD [tetrachlorodibenzodio]

SOURCE: Institute of Medicine of the National Academies, 2013. Underlined modifications from earlier editions published in 2005, 2007, 2009, and 2011.

NOTES: Italics: Category change in response to additional evidence. Bold: Additional conditions added to the conditions being monitored. 


\subsubsection{Approach}

Data and Methods for Scenario Two, Changes in Presumptive Eligibility

- We used data from MEPS to estimate the maximum number of Veterans who would newly use VA health care if VA included hypertension presumptively as a serviceconnected condition for Veterans who served in Vietnam.

- We employed an algorithm that assigns a priority group to each Veteran contained in the 2013 ACS, based on available data and adjustment factors drawn from administrative records.

The purpose of this scenario was to estimate the maximum number of Veterans who would newly utilize VA health care if VA decided that hypertension could be presumptively included as a service-connected condition for Veterans who served in Vietnam. We relied on estimates from NSV for information about the number of Veterans who served in Vietnam. We used VA business intelligence data to determine the proportion of these Veterans who are currently VA patients (we use age as a proxy for service in the Vietnam era). For the prevalence of hypertension in Veterans and current VA patients, we relied on MEPS. (See Chapter Five and Appendix $\mathrm{C}$ for more information.) We applied the ratios of service in theater and hypertension to the Veteran populations who were eligible to receive VA health care but were not enrolled in VA health care, or were enrolled in the higher priority groups (7 and 8). Our detailed methods are described in Appendix D.

\subsubsection{Results}

The progression from Vietnam-era Veteran to likely enrollee is illustrated in Figure 6-2. The total height of the first bar represents the 7 million living Veterans who served during the Vietnam era, and the lower portion represents the approximately 3 million who served in theater. Of the 3 million, 61 percent are not enrolled or are in priority group 7 or 8 (where care is not free) (second bar). Among those not enrolled (but eligible for enrollment with otherthan-dishonorable discharges) or in priority group 7 or 8 , we estimate that 65 percent have hypertension (third bar). This hypertension prevalence rate is an average of the prevalence rate for enrolled Veterans in priority groups 7 and 8 (70 percent) and the rate for the non-enrolled Vietnam-era Veterans (62 percent). The height of the fourth bar is equivalent to those who could enroll as a result of a presumptive service connection for hypertension. Of course, not all of those who are eligible will enroll, so we therefore estimate that of the non-enrollees who have hypertension, 31 percent are likely to enroll and become patients (approximately 363,000 new patients).

\subsubsection{Discussion}

An increase of 363,000 new patients would represent an increase of 6.4 percent in VA's total patient population (5.7 million in 2014 as reported in EHCPM output). The key driver of our estimate is the use rate for Veterans with hypertension: While we calculated use rates for the relevant Veteran population in MEPS, actual enrollment and use rates by the newly eligible may be higher or lower than our assumptions. 
Figure 6-2

Progression from Vietnam-Era Veterans to Likely New VA Patients If Hypertension Is Adopted as a Presumptively Service-Connected Condition

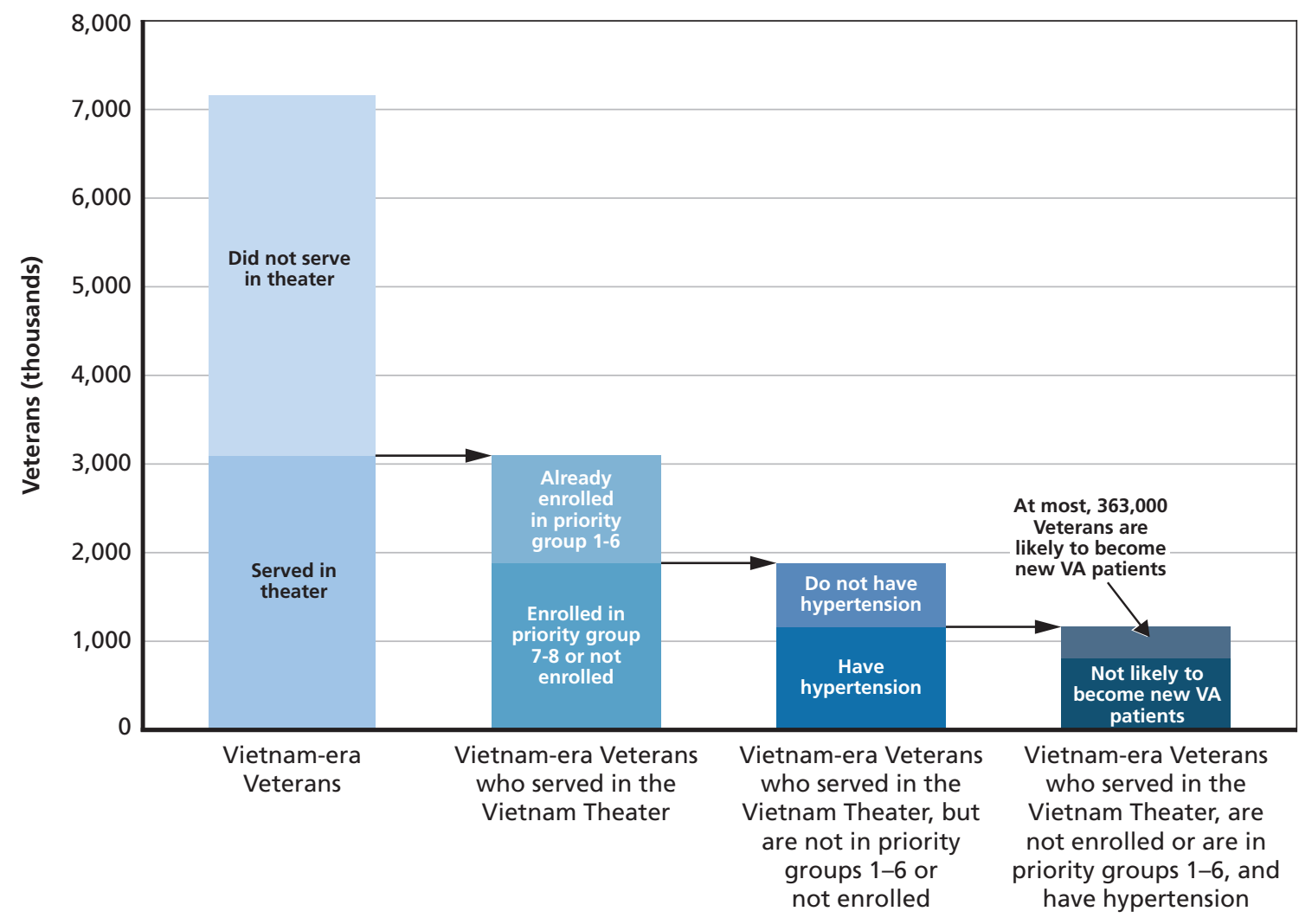

Veteran status

SOURCE: RAND analysis of NSV, MEPS, ACS, and VA Business Intelligence (Enrollment) data.

RAND RR1165z1-6.2

\subsection{Potential Impact of Future Military Conflict on VA Use}

\subsubsection{Description}

The United States has spent roughly 25-35 percent of the past 115 years engaged in some form of armed conflict. If past experience is any guide, the chances of future conflict are high (Kavanagh, 2013). Conflict affects the size of the VA patient population in three significant ways. First, the authorized end-strength of the military may grow larger, which translates into larger cohorts of Veterans when those additional personnel eventually separate. Second, conflict may increase the number of service members who will qualify for VA health benefits, through a wide range of mechanisms, including activation of reserve/National Guard units, deployment into a theater of combat, increased risk of service-connected disability, and automatic placement into higher VA priority groups. Third, exposure to the conflict environment may carry unique health risks that are rare among the non-Veteran population. Consequently, Veterans with conflict-related health conditions are likely to rely on VA for a larger portion of their care. Taken together, this suggests that future conflict is both likely to occur and likely to affect the demand for VA health care. 
In the future conflict scenario, we examined the potential consequences of future conflict on demand for VA health care over the next 10 years. These analyses are not models of war itself. Rather, they are models of how changes in both the size of the military and the average exposure to conflict among service members have consequences for the number of new patients using VA health care services. We examined 36 future conflict scenarios based on different assumptions about how end-strength would be affected, how widely service members would be exposed to conflict, and how rates of VA health care enrollment or reliance on VA for health care would vary. We then observed the commonalities across scenario assumptions-which patterns tended to hold, regardless of the specific assumptions made.

In projecting the implications of conflict for VA health care, we are not breaking new ground. VA, DoD, and Congressional Budget Office analysts are among those who have built models to examine this contingency. However, conflict consequences are difficult to model, and transparency on current modeling approaches is relatively scarce, ${ }^{5}$ so there is significant room for contributions to this type of modeling. In addition, we examined how different combinations of assumptions interact to produce different consequences, which may aid future model consumers in understanding how models differ, and when those differences matter. We also built on previous RAND research on force planning and personnel patterns, which imbues our model with unique insights into service member separation choices and how these would be shaped by the near-term policy environment. This includes research into the poor performance of stop-loss (Brady, 2014), positive impact of deployment on retention rate (Hosek \& Martorell, 2009), difficulties maintaining deployment readiness among reserve components (Brauner, Jackson, \& Gayton, 2012; Pint et al., 2015), and the effectiveness of incentive-based retention strategies (Asch et al., 2010).

\subsubsection{Approach}

\section{Data and Methods for Scenario Three, Impact of Future Conflict on Demand} for VA Care

- We used data from DMDC and the U.S. Census Bureau, DoD planning documents, the NSV, and the SoE to examine 36 potential future conflicts and their impact on demand for VA health care between 2015 and 2024.

- We employed a conversion process that begins with a starting population (current Veterans in VA's model) and winnows this down to the segment that will eventually rely on VA for at least part of its health care. Our projections involved two conversions: how endstrengths of the U.S. military translate into separations from the military and how separations from the military translate into VA patients.

As in VA's own models, ours modeled a conversion process by which a starting population (service members in these projections; Veterans in VA's model) is winnowed down to just the segment that will eventually rely on VA health care for at least part of its care. Many of these conversions are difficult to predict (e.g., when the next war will break out, and how widespread exposure to the conflict environment will be), so we calculate conversion rates under different assumptions, and then examine the resulting range of outcomes. Our projections involved two conversions: (1) how the end-strength of the U.S. military translates into separations from the

\footnotetext{
5 Some of this is by necessity. Much of the defense modeling on this topic is understandably classified.
} 
military, and (2) how separations from the military translate into VA patients. In our models, the first conversion and final projection figures were calibrated and validated against the VA population projections in Chapter Three. The second conversion was validated against the enrollment and reliance rates in Chapter Four.

Military end-strength scenarios. The total size of the U.S. military is difficult to forecast, because it can change rapidly to accommodate the current security needs of the United States. Therefore, we created six potential scenarios of end-strength for the 2015-2024 period. These projections included the active, reserve, and National Guard components of the Army, Navy, Air Force, and Marine Corps_-about 2.2 million service members in 2014. Each scenario extrapolated from manpower statistics and planning documents.

Three scenarios ("buildup") started from historical data (Defense Manpower Data Center, 2014; U.S. Census Bureau, 2012b) for the post-2000 period to estimate how high end-strength could rise. They projected the total end-strength that would result if each component of each service rose to its highest historical level for the post-2000 period, peaked, and then steadily declined afterward. This rise-peak-decline pattern is typical for a wartime surge. For the Army and Marine Corps, maximum end-strength occurred between 2009 and 2011, to better meet the challenges of the wars in Afghanistan and Iraq. For the Navy and Air Force, this occurred between 2002 and 2004, before a new wave of technological improvements allowed both services to reduce the quantity of personnel necessary to operate their respective fleets. While reduction from those levels was driven by technical change, it is conceivable that new roles and technologies could lead to these staffing levels being re-attained in future conflicts. In fact, an air and sea war, perhaps fought over the Pacific, could exceed these force size projections. ${ }^{6}$

The other three end-strength scenarios ("drawdown") estimated how low end-strength could fall. They follow a similar strategy as the "buildup" scenarios, but project the end strength that could result from the sequestration cuts scheduled to occur by 2019, as described in DoD planning documents (Office of the Under Secretary of Defense (Comptroller), 2015), the Quadrennial Defense Review (U.S. Department of Defense, 2014), and the Congressional Budgeting Office's analysis of them (Congressional Budget Office, 2014a). The planned cuts formed the backbone of these projections because, with few exceptions, they are lower than any historical force level in the 2000-2014 period. In the cases where they are not (active duty Marine Corps and Army Reserve/National Guard in 2000), we used historical lows instead of the sequestration cuts. Figure 6-3 reports the total end-strength for each scenario over time. ${ }^{7}$ Appendix D reports all historical/planned maximums and minimums for each segment of the U.S. military.

For all scenarios, the drawdown to minimum or buildup to maximum was implemented over the course of three years. Given the minimum, maximum, and current end-strengths observed, this approximates the end-strength rate of change observed during the 2007-2010 surge.

Combat exposure scenarios. While end-strength could have consequences on the demand for VA care, the total size of the U.S. military is not necessarily related to how much combat exposure service members will receive. To capture those potential effects, we created

\footnotetext{
6 Because the United States has not fought an air and sea war since World War II (and that was fought concurrently with a large land war), there is not enough historical performance data to project an end-strength scenario.

7 The equivalent 2024 figures for the active duty Army are as follows: 622,000 (increase $\times 110$ percent), 566,000 (increase, starting 2016), 538,000 (ramp up, starting 2018), 510,000 (temporary decrease), 420,000 (permanent decrease), and 378,000 (decrease $\times 110$ percent).
} 
Figure 6-3

End-Strength Scenarios (total non-civilian U.S. military personnel)

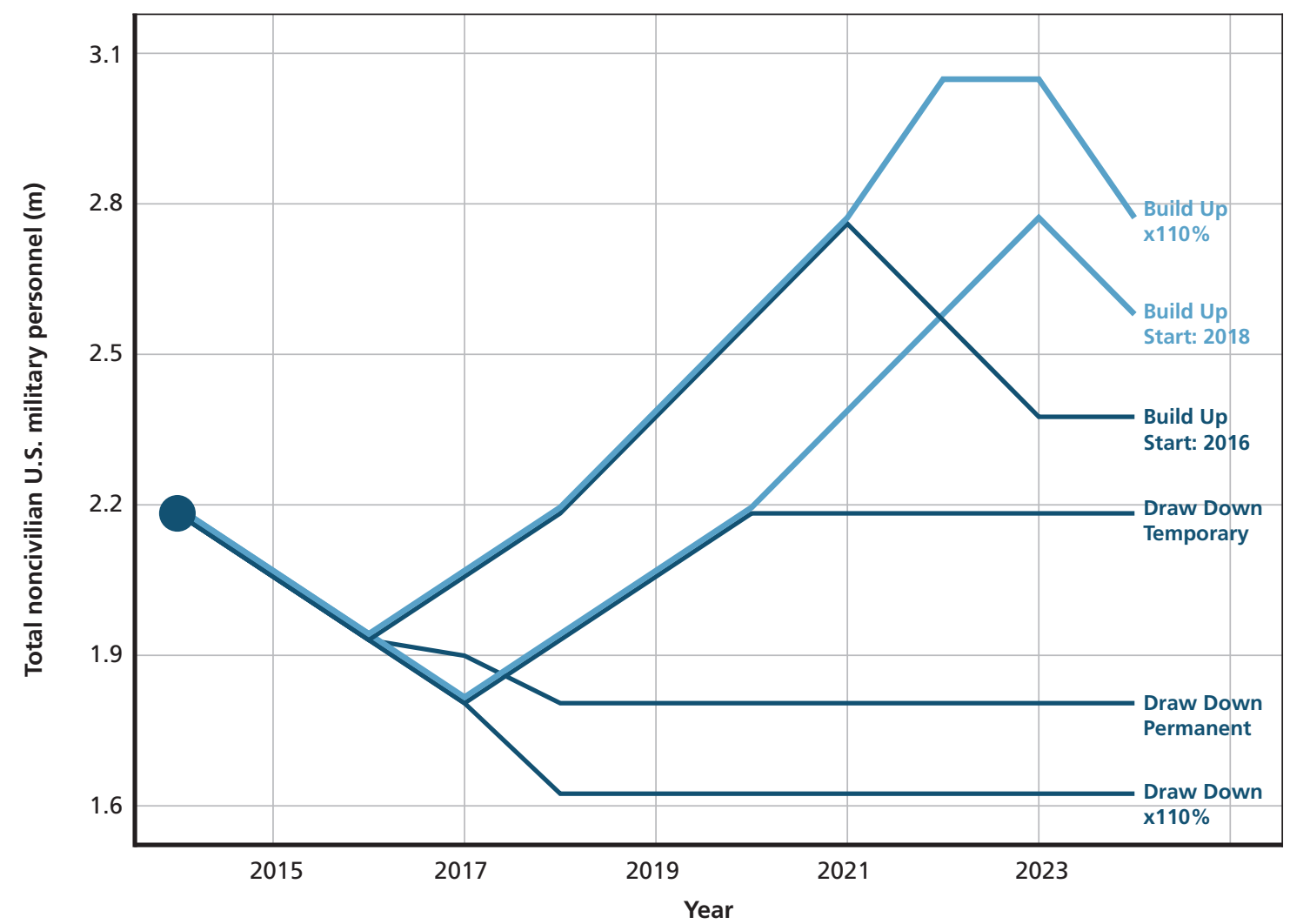

SOURCE: RAND calculations based on DoD administrative data (2015), Census Bureau data (2012), Congressional Budget Office analysis (2014), and U.S. Army posture statements (2014).

RAND RR1165z1-6.3

three potential scenarios of possible combat exposure. Each scenario projected the total number of combat exposures that would result if each component of each service experienced the maximum, minimum, and average rate of hostile deployment that it witnessed between 2000 and 2014 for each year of the 2015-2024 period. ${ }^{8}$ Appendix D reports all historical ranges for each segment of the U.S. military.

Separating service members. A great deal of research has examined service member separation patterns. This research suggests a complex relationship between personal expectations, life situation, deployment, and the chances of re-enlisting. While extended hostile deployments tend to decrease the chances of reenlistment, moderate deployments actually increase those chances (Hosek \& Martorell, 2009). Moreover, the current system of targeted incentives has proven an effective tool for shaping these chances to meet current personnel needs (Asch et al., 2010). Consequently, rates of separation tend to reflect DoD personnel needs, much more than individual factors. Therefore, we modeled separation patterns from the perspective of DoD. Under average conditions, what rates of separation are typical among the various ranks,

8 In these scenarios, the combat exposure rates do not vary with surges in end-strength, because they do not correspond strongly in the historical data for the 2000-2014 period. While surges in end-strength may correspond with a more intense war effort, they also lower each service member's chance of being deployed. 
components, and services? When end-strength needs change, how are these cuts or increases typically realized among the various ranks, components, and services? We formulated a regression model to examine the relationship between total end-strength, changes in end-strength, and separation rates for each service, component, and officer/enlisted segment. This model contained 48 separate coefficients to characterize all of these features, and the interactions between them. However, the basic patterns they revealed can be summarized in just four points:

- Enlisted service members average less time on active service than officers before separating, and account for most of the extra separation changes when required end-strength declines. To be precise, DMDC separation records suggest that, in 2013, 75 percent of enlisted service members separated within eight years, while 75 percent of officers separated over the course of 16 years. Among active component service members, those figures were 11 years and 22 years, respectively.

- Active component and reserve/guard component personnel levels experience some countervailing movement over time. It is not unusual to see the active component swell when the reserve/guard component shrinks, and vice versa. This is especially common at the beginning and end of conflicts, as service members from one are converted into service members in the other. However, it is also common during cutbacks.

- Reserve/guard component personnel spend less time on active duty than active component personnel, and tend to experience proportionally larger swings in total size. DMDC separation records suggest that, in 2013, 75 percent of active component service members separated within 12 years of active service, while 75 percent of reserve component service members separated with less than a year of active duty service.

- Soldiers and Marines had fewer years of active service at the time of separation than did airmen and sailors, on average. DMDC separation records suggest that, in 2013, 75 percent of airmen/sailors separated within 12 years, but 75 percent of soldiers/Marines separated within seven years. Among active component service members, those figures were 15 years and 10 years, respectively.

Model predictions for the cumulative number of separating service members ${ }^{9}$ were calibrated against the demographic models discussed in Chapter Three. Our minimal conflict, permanent drawdown scenario assumptions produced the same predicted increase in the size of the post-9/11 Veteran cohort by 2024. Appendix D reports the typical years of service at time of separation for active and enlisted personnel over this time period.

Enrolled VA health care users. Combining these projections with various survey sources, we then calculated how many of these separating service members might go on to rely on VA for at least some of their health care needs. This analysis involved using VA's NSV to calculate the probability that Veterans with different characteristics would enroll in VA health care, and also VA's SoE to ascertain the probability that Veterans with different characteristics would rely on VA for at least some portion of their health care needs. Implicitly, these probabilities are modeling both the chances that a Veteran would be eligible for VA health benefits, and that he or she would choose to enroll and rely upon VA care.

9 Minus those with less than two years of active duty services, and no hostile deployments. For the reserves and National Guard, we made some allowances for those who served a full term for which they were called up, qualifying for VA without serving a full 24 months. 
However, implicit in this conversion rate is the assumption that future conflicts will affect the chances of enrollment and reliance in the same way that they did during the 2000-2014 period. For example, one of the reasons why conversion rates are higher for deployed Veterans is that deployed Veterans are more likely to suffer a service connected disability. However, differences in the weapons technology, battlefield medicine, and geographic location of futures conflicts can alter the chances of acquiring such a disability.

To examine the consequences of such variation, we crafted two scenarios of the chances that separating service members would become VA users. One scenario assumed a 10 percent increase in the conversion rate, while the other assumed a 10 percent decrease in those rates. Our baseline rates were validated against the rates calculated in Chapter Four. See Chapter Four for a more detailed treatment of reliance and enrollment rates.

\subsubsection{Results}

Figure 6-4 reports the results of 18 projections (the doughnut plots) of separating service members for each of six end-strength scenarios (the rows), and three conflict exposure scenarios (the columns). The size of each doughnut is proportional to the total number of separations projected to take place between 2014 and 2024. The three colors divide the separations into three broad categories: (1) ineligible - those with less than 24 months of active duty service and no combat exposure (light gray); (2) served two or more years - those who separated with at least 24 months of active duty service, but no combat exposure (medium blue); ${ }^{10}$ and (3) deployed - those who experienced at least one deployment into a conflict zone before separating (dark blue). These categories are based on the historical maximum, average, and minimum observed deployment rates for each segment of the military during the post-9/11 period. The precise historical figures are reported in Appendix D.

Across scenarios, the number of projected service members who will separate between 2015 and 2024 varies from 2.7 to 3.5 million. However, a substantial portion, mostly reservists, will not have been on active duty long enough to qualify for VA benefits. That leaves between 1.6 and 2.6 million that will be on active duty for at least two years before separation and/or have experienced a deployment to a hostile area. ${ }^{11}$

Looking across scenarios, the largest increase in end-strength did not necessarily translate into the sharpest increase in separations, because many of those new recruits will remain in service for a number of years afterward. According to DMDC records, 50 percent will still be in service six years later, and 25 percent will still be in service 12 years later. Because the impact of a recruiting surge is diffused over a number of years, the number of separations rises gradually, rather than abruptly. In contrast, drawdowns have a much more immediate effect. When end-strength decreases, additional separations are encouraged through changes in incentives, and these excess separations fully enter the Veteran pool within the first couple of years. Consequently, a recruitment surge would need to be more than three times as large as a drawdown to have the same near-term consequences for VA.

Conflict can have a major impact on how separation translates into potential users of VA services. One major mechanism is activation. Reservists who would not otherwise qualify for

\footnotetext{
10 Reservists and National Guard personnel who were called up and served for their full term are counted as having served $2+$ years.

11 For reservists and National Guard officers, we account for some basic exceptions to the threshold of 24 months of active service.
} 
Figure 6-4

Cumulative Separations (2015-2024)

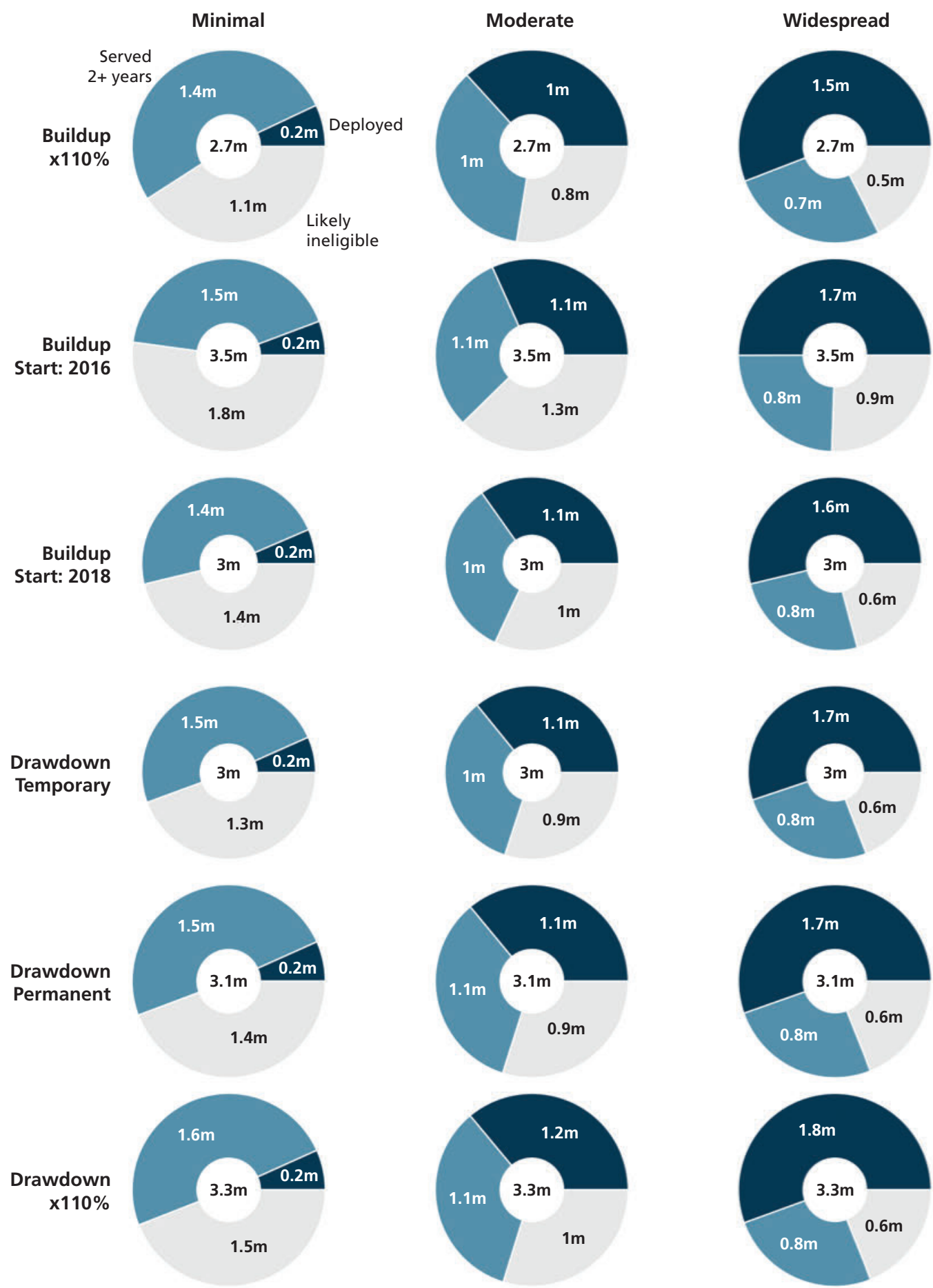

SOURCE: RAND calculations based on DoD administrative data (2015), Census Bureau data (2012), Congressional Budget Office analysis (2014), and U.S. Army posture statements (2014). NOTE: $m=$ million 
VA benefits would become eligible through deployment and active duty service. Conflict exposure itself also carries consequences for eligibility and the chances of relying on VA to provide care. The net result is that conflict can allow even a relatively small cohort of new separating service members to translate into a larger impact for VA, because proportionally more are likely to become users of VA health care. The "Buildup Start: 2016" row examines the volume of separations that might result if the United States began surging toward its historical maximum size in 2016, and then slowly started drawing back down after reaching that maximum. Between 2014 and 2024, 3.5 million service members were projected to separate. In a minimal conflict scenario, historical rates of deployment (the lowest observed in the post-9/11 period) and reenlistment suggest that approximately 200,000 of them might be deployed, and 1.5 million might spend enough time on active duty to meet VA requirements. In a widespread conflict scenario, historical rates of deployment (the highest observed in the post-9/11 period), 1.7 million might be deployed, and an additional 800,000 would be projected to serve sufficient time on active duty. This would amount to nearly a 50 percent increase in the number of potentially eligible Veterans, and an 850 percent increase in the number of likely deployed Veterans.

Figure 6-5 estimates how many of these separating service members would likely become VA patients under various scenario assumptions. The 36 lines are cumulative, charting how many new users would be likely to have used VA health care by that year under a given set of assumptions. The lines are colored to reflect the end-strength scenario. The black bars on the right identify which subset of lines reflects different sets of conflict exposure and enrollment

Figure 6-5

Cumulative New VA Patients Under Various Scenario Assumptions

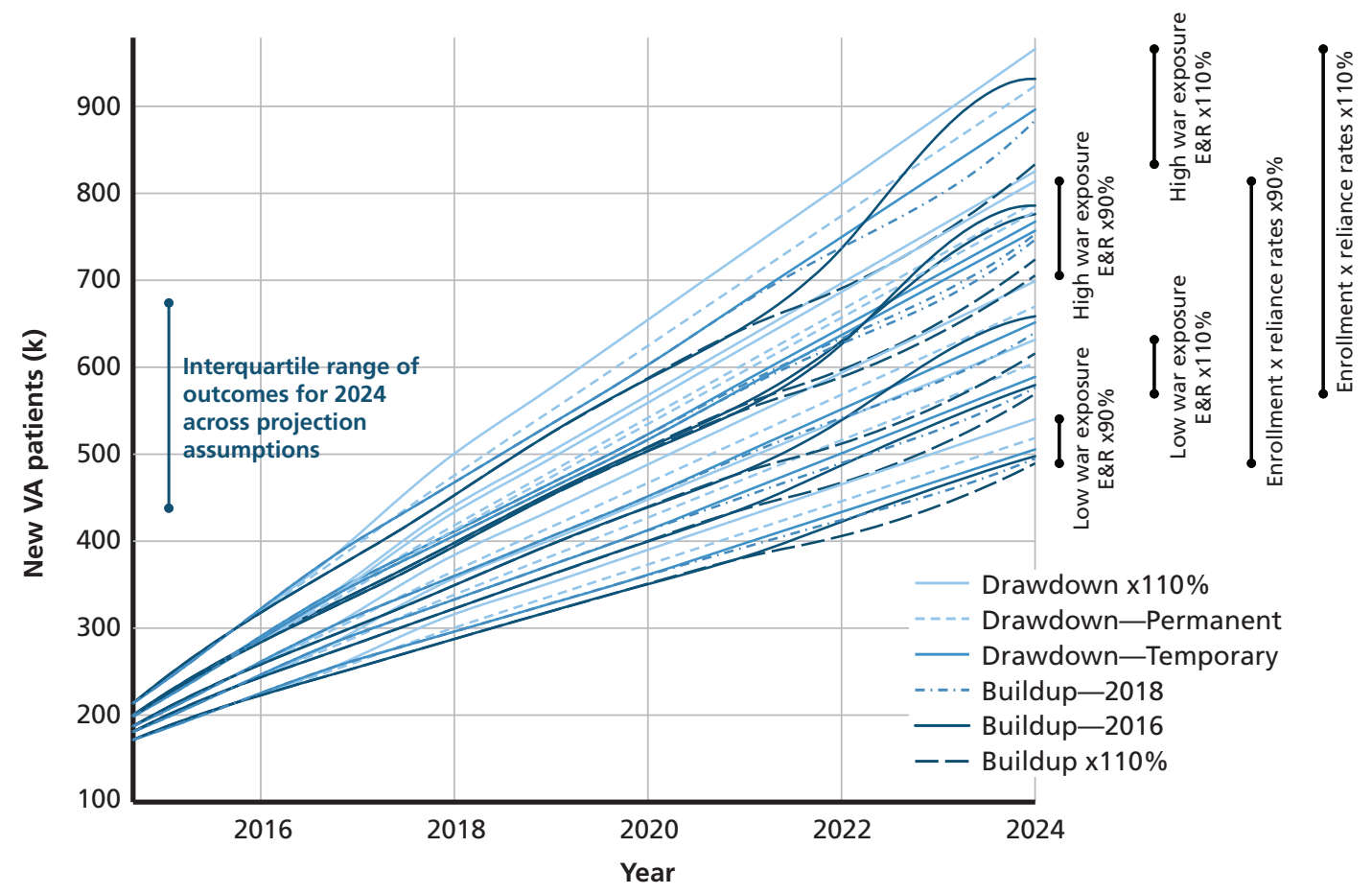

SOURCE: RAND calculations based on DoD administrative data (2015), Census Bureau data (2012), Congressional Budget Office analysis (2014), U.S. Army posture statements (2014), NSV survey responses, and SoE survey responses.

RAND RR1165z1-6.5 
and reliance assumptions (marked on the chart as E\&R). For example, the bar labeled "High War Exposure, E\&R x 90\%" denotes the assumption that a larger percentage of separating service members will have experienced at least one hostile deployment, but that the conversion rate of separating services members to VA patients will be lower than it was during the 20002014 period. It spans from 700,000 VA patients to a little more than 800,000 VA patients, because all of the scenarios that make this assumption project between 700,000 and 830,000 new VA patients by 2024.

In terms of end-strength, the biggest impacts on the number of additional VA patients occurred in scenarios that involved either a massive drawdown in the size of the military or a surge happening within the next couple of years, followed by ample time to draw back down. However, the total impact of the end-strength scenarios is relatively small compared with the other assumptions_-at most generating a difference of about 100,000 patients by 2024 .

Our two enrollment and reliance scenarios considered a 20 -percent difference in the assumed rate at which eligible separating service members would become VA patients. The projected effect varied greatly, depending on the other scenario assumptions, but may generate up to 150,000 additional patients by 2024 .

However, all of these pale in comparison with the effects of conflict exposure as a driver of demand for VA health care. Military conflict affects these projections at multiple stagesraising the number of eligible reservists and National Guard officers, increasing the rate of conversion to VA patients, and so on. Cumulatively, these effects could raise the number of VA patients by 200,000-300,000 over the next decade.

\subsubsection{Discussion}

Our analysis reveals three major findings. First, while a military buildup would produce more Veterans in the long term, drawdowns create a much steeper annual rise in the size of the Veteran population, because the population impact of a buildup is spread over a larger number of years. Drawdowns are much more likely to require VA to quickly and significantly increase its capacity. A sharp drawdown after a large buildup affects VA more than any other end-strength scenario. Second, conflict exposure increases demand for VA health care more than any other projection assumption. Looking across projections, the smallest wartime cohort of separating Veterans generates more VA usage than the largest peacetime cohort. Third, only some service members will become VA patients. The conversion process - from service member to eligible Veteran to VA patient-plays a significant role in determining how much demand VA would need to meet.

If we reflect on the Afghanistan and Iraq experiences, the past 15 years have witnessed the sequence of events most likely to generate a rise in demand for VA services. The combination of war and sequestration increases future eligibility and demand for VA health care among service members, and then quickly moves those service members into the Veteran pool.

Looking forward to the near future, the majority of our scenarios predicted 500,000 to 925,000 new VA patients by 2024 . However, most high-conflict projections estimated 750,000-925,000, while most low-conflict projections estimated just 500,000-600,000 new patients. This suggests that even moderate levels of hostile deployment can have big repercussions for the size of the incoming cohort of VA patients.

However, previous cohorts, especially the Vietnam cohort, were much larger than recent cohorts, so the difference would be small relative to the entire VA user population. To be precise, of the 21.6 million Veterans in 2014, only 2.6 million served during the post-9/11 period. 
Projecting forward, 1.7 million new post-9/11 Veterans are expected to join that cohort by 2024, while the overall size of the Veteran population declines to 17.5 million. These projections suggest that, depending on end-strength and conflict intensity, the number of new Veterans may vary between 1.6 million and 2.6 million. That amounts to approximately a 50 percent difference in the projected size of the post-9/11 cohort, but only a 5 percent difference in the size of the 2024 Veteran population.

\subsection{Improving Access to VA Care}

\subsubsection{Description}

VA has a range of policy tools at its disposal to address actual and perceived barriers to access. The Choice Program, which enables Veterans meeting certain criteria to receive VA-paid care from community providers, is one example. However, other, more-expansive policies are also possible. For example, the Choice Program, which is a temporary three-year program that applies to previously enrolled Veterans only, could be made permanent or expanded to all Veterans (VA, 2015e). In this scenario, we modeled the potential impact on VA (in terms of new patients) of eliminating all actual and perceived barriers to access, regardless of the policy tool or approach used. This analysis is a useful bounding exercise for the potential impacts of the policies to improve access. As in other scenarios, we assumed that the underlying VA health care delivery system and access to health care through non-VA channels remain constant over time.

\subsubsection{Approach}

\section{Data and Methods for Scenario Four, Improving Access to VA Care}

- We analyzed Veterans' responses to the 2010 NSV to calculate (1) the proportion of Veterans who selected an access-based reason for never using VA health care and (2) the proportion of Veterans who were VA patients.

- We calculated the number of Veterans who would use VA health care if those who reported access-related reasons for not using VA health care started using VA health care at the same rate as their peers. We did this by multiplying the number not using VA for access-related reasons by the use rate among Veterans who were patients or did not report access-related reasons for not using VA health care.

- We conducted a similar analysis using data from the SoE to determine the number of enrolled non-users who might become VA patients if access barriers were lifted.

We analyzed Veterans' responses to the 2010 NSV to calculate the proportion of Veterans who are not enrolled to receive VA care and who selected an access-related reason for not using VA health care. We also used NSV data to calculate VA use rates for each Veteran subgroup, excluding from the denominator those who reported an access-related reason. In a separate analysis, we used ACS data to estimate the proportion of Veterans in each health insurance coverage subgroup who are eligible to receive VA services. Our approach for this last analysis is outlined in detail in Section 6.4 and Appendix D.

We combined these inputs to calculate the number of Veterans who might enroll in and use VA health care if access improved. More specifically, we calculated the number of potential new VA patients if Veterans who we estimate to be eligible for VA care and who reported access issues started using VA services at the same rates as Veterans who did not report access problems. 
We repeated these steps for Veterans who are enrolled to receive VA care but are not current VA patients using the 2013 SoE. While NSV and SoE both describe Veterans' reported access-related reasons for not using VA health care, the two surveys sample different populations and offer complementary insight into how use of VA health care might change if access improved. Unlike SoE, NSV surveys Veterans who are not currently enrolled to receive VA health care services. This population of Veterans is important in our analyses because if access to VA care improved, we would expect additional Veterans to enroll to receive VA health care services. While SoE has a larger sample size and is more recent than NSV (2013 versus 2010), it is limited to enrolled Veterans only. Both surveys have information on enrolled Veterans who choose not use VA health care services. We present results from both surveys in an effort to present a range of plausible impacts if VA were to improve access to its health care.

In addition to the analyses described above, we fitted multivariate logit models using NSV data to describe how various characteristics of Veterans predicted the likelihood of selfreporting one or more access-related reasons for non-use.

Using this approach, we acknowledge that some Veterans would choose not to use VA health care even if there were no actual or perceived access, quality, or other differences between VA care and community-based care. The number of Veterans who choose to become new VA patients is affected by many factors, including access to other sources of health coverage and other features of the conceptual model described in Figure 2-1.

\subsubsection{Results}

\subsubsection{NSV Analyses}

We found that a significant share of Veterans reported an access-related reason for not using VA health. Of the 14.8 million Veterans who self-reported that they never used VA health care benefits, 1.8 million (or 12.4 percent of non-users) reported not using VA care because either "VA care is difficult to access (parking, distance, appointment availability)" or there was "too much trouble or red tape" (Figure 6-6). ${ }^{12}$ Many of the choices available to respondents-for example, "never considered getting any health care from VA" or "don't think VA health care would be as good as that available elsewhere"-are broad responses that could reflect access, convenience, amenity, and quality of care reasons for not receiving care. The actual number of Veterans not using VA care who experienced access problems may be higher than the 12.4 percent noted above, to the extent that these Veterans selected more-general responses in lieu of specific responses related to access.

Next, we considered whether certain Veteran characteristics were associated with selfreport of an actual or perceived access issue. The results from multivariate models suggested that some Veteran characteristics increased the odds of a Veteran reporting either "VA care is difficult to access" or "Too much trouble or red tape" as the reason why he or she has not used VA health care in the past (Figure 6-7). Non-patient Veterans with incomes greater than 133 percent of the FPL were less likely to report one of these two reasons than non-patient Veterans with incomes under 133 percent of the FPL. Non-patient Veterans with poorer selfreported health status had increasingly higher odds of reporting one of these two reasons compared with non-patient Veterans self-reporting that they were in excellent health. Odds ratios for age, sex, service branch, and residence Census region were not statistically distinguishable from 1 and were not included in Figure 6-7.

\footnotetext{
12 Note that some respondents selected both reasons, and therefore the combined rate is not the sum of the two rates
} reported in Figure 6-6. 
Figure 6-6

Selected Reasons for Not Utilizing VA Care (percentage of non-user Veterans)

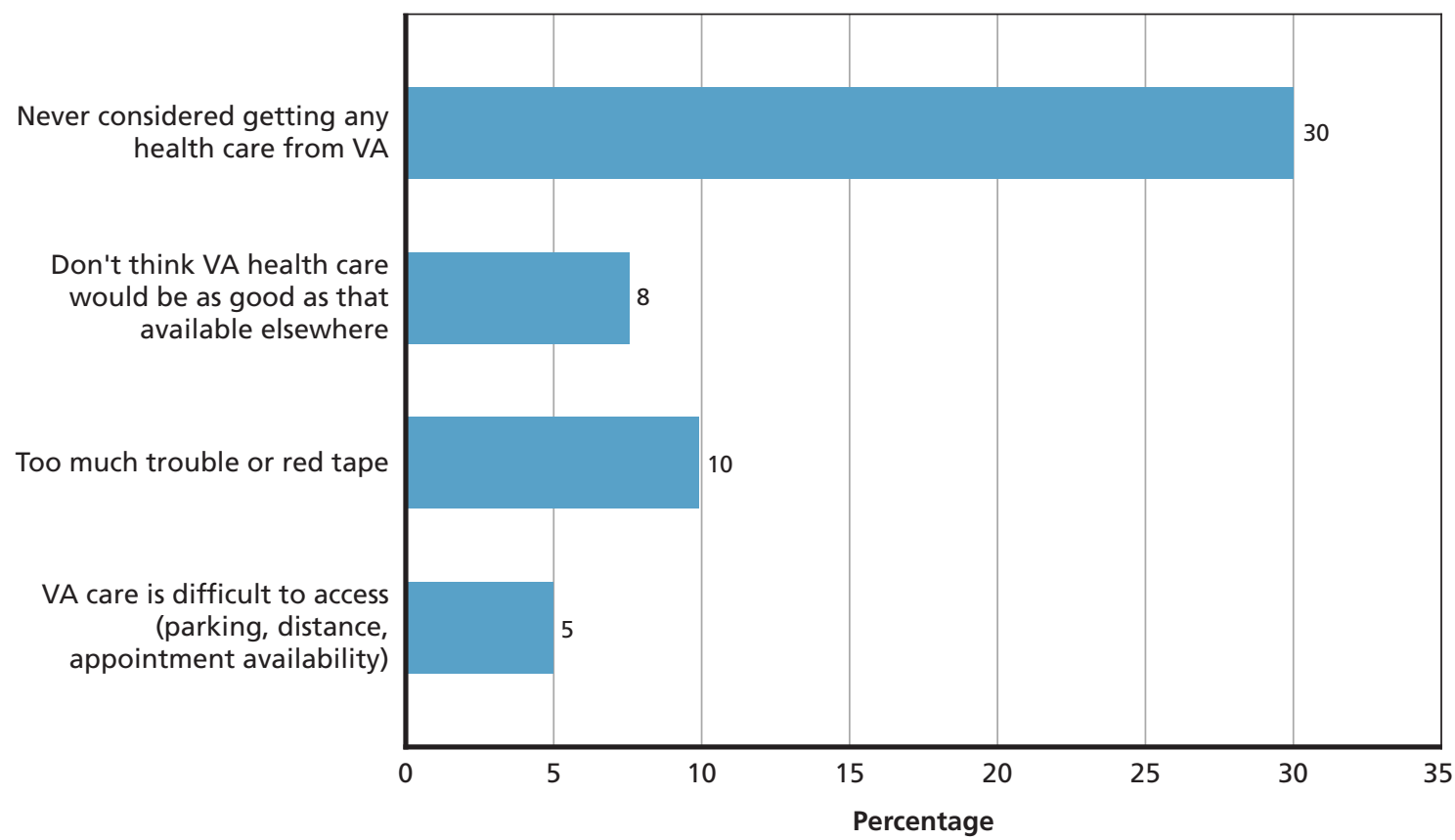

SOURCE: RAND analysis of 2010 NSV data (Item E2).

RAND RR1165z1-6-6

Figure 6-7

Relationship Between Veteran Characteristics and Self-Reported Access Barriers

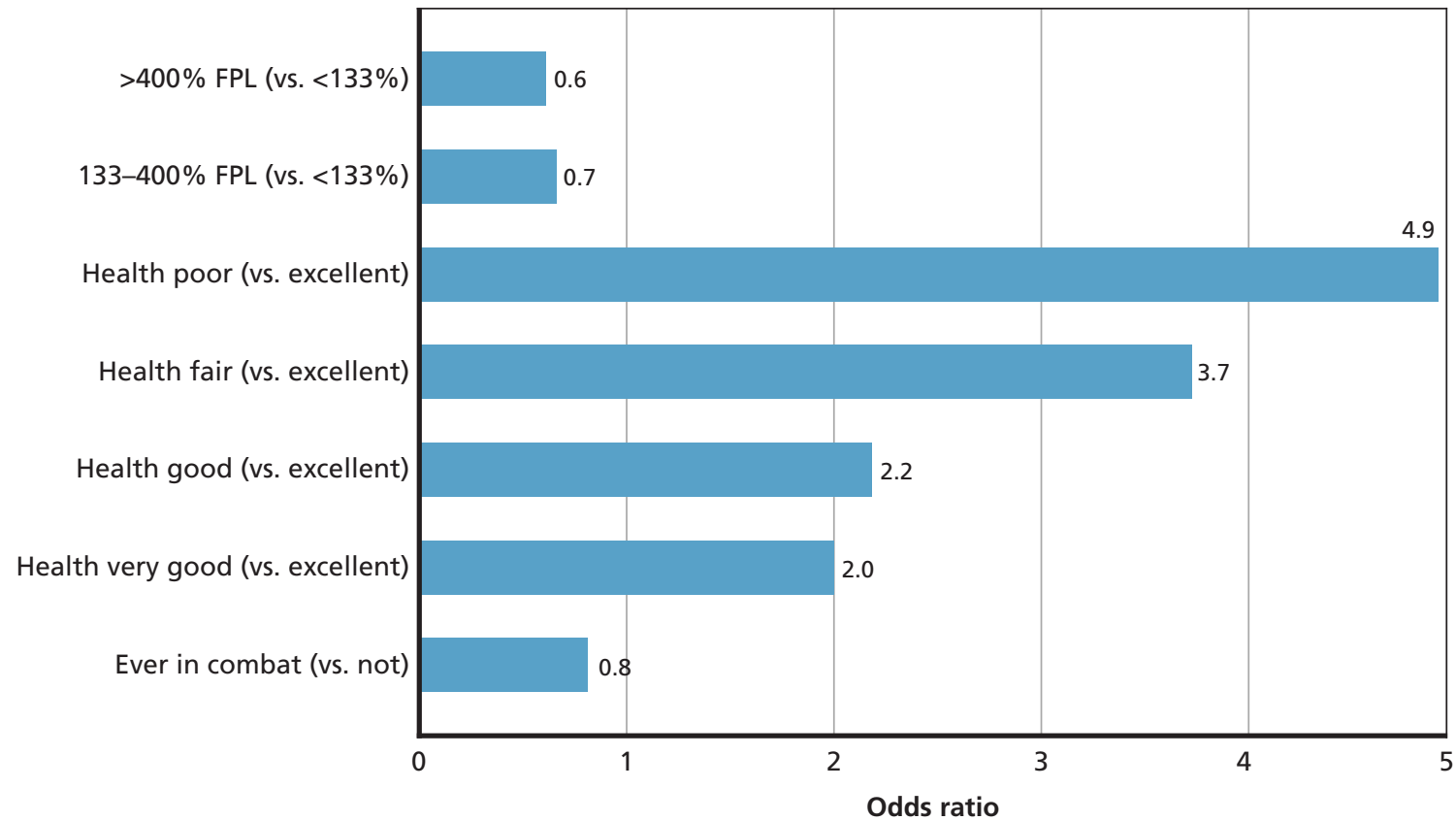

SOURCE: RAND analysis of 2010 NSV data.

NOTES: Odds ratios less than 1 imply that the Veteran subgroup has a lower likelihood of reporting an access-related reason for non-use than the reference group noted in parentheses. For example, Veterans at or about 400 percent of the FPL are 60 percent as likely to report an access-related reason than Veterans below 133 percent of the FPL. Likewise, odds ratios greater than 1 indicate that the group has a higher likelihood of reporting an access-related reason for non-use than the reference group.

RAND RR1165z1-6-7 
Table 6-4 reports estimates of the number of Veterans who are not VA patients and who report an access-related reason for their non-use in NSV; the proportion of Veterans in each coverage category that we estimate are eligible to receive care; the utilization rate for Veterans in subgroups by other health insurance coverage who did not report an access issue; and an upper bound estimate of new patients that VA should expect if all non-patients reporting accessrelated reasons for their non-use became VA patients at the same rates as their peers. The bulk of new patients would have private health insurance, Medicare coverage, or no coverage other than VA. We estimated that an upper bound of 235,000 Veterans who were formerly not VA patients might start using VA care if there were no access barriers in the VA health care system.

\subsubsection{SoE Analyses}

In order to explore how the elimination of access-related barriers would influence patient counts among current enrollees, we identified five SoE items that are most closely aligned with Veteran access to care:

1. "Veterans like me can get in and out of an appointment at VA in a reasonable time."

2. "When Veterans like me go to VA for an appointment, they do not wait a long time to see the doctor."

3. "It is easy to get to my local VA facility."

4. "There is a VA provider in my area that offers all of the health care services that Veterans like me need."

5. "It is easy for Veterans like me to get around in the VA health care facility."

Overall, 13 percent of Veterans who are not patients reported that they "completely disagreed" or "disagreed" with at least one of the five items listed above.

Table 6-4

Upper Bound of New Patients Responding to Improved Access

\begin{tabular}{lcccc}
\hline & $\begin{array}{c}\text { Non-Patients } \\
\text { Reporting an } \\
\text { Access Reason } \\
\text { (thousands) }\end{array}$ & $\begin{array}{c}\text { Proportion of } \\
\text { Veterans Eligible } \\
\text { to Receive Care } \\
\text { Po) }\end{array}$ & $\begin{array}{c}\text { Use Rate } \\
\text { (\%) }\end{array}$ & $\begin{array}{c}\text { Upper Bound } \\
\text { of New Patients } \\
\text { (thousands) }\end{array}$ \\
\hline No Coverage or VA only & 122 & 60 & 52 & 38 \\
Private only & 811 & 28 & 19 & 43 \\
Medicare only & 172 & 53 & 42 & 39 \\
Medicaid only & 44 & 98 & 48 & 21 \\
TRICARE only & 89 & 47 & 39 & 16 \\
Other (single) coverage only & 11 & 62 & 32 & $\mathbf{2}$ \\
Private and Medicare only & 390 & 39 & 17 & $\mathbf{2 5}$ \\
Medicare and TRICARE only & 49 & 47 & 51 & 12 \\
All other combinations & 151 & 71 & 34 & 37 \\
Total & $\mathbf{1 , 8 3 7}$ & 44 & 29 & $\mathbf{2 3 5}$ \\
\hline
\end{tabular}

SOURCE: RAND analysis of 2010 NSV data. 
We multiplied SoE estimates of the number of enrolled Veterans who were not 2013 VA patients by the proportion of non-patient Veterans in each age category reporting a potential access problem (columns $A$ through $\mathrm{C}$ in Table 6-5). We then assumed that, at most, these Veterans would begin using VA health care at the same rates as Veterans in the same age band, excluding all Veterans who reported potential access problems (column D). ${ }^{13}$ The resulting estimate is 212,000 new VA patients if all access barriers were eliminated.

\subsubsection{Discussion}

Even with nearly 2 million Veterans reporting an access-related reason for not using VA care in NSV, low use rates overall imply relatively small changes in new VA patients if access barriers were lifted. If non-patient Veterans reporting access issues started using VA care at the same rates as their peers who do not report access problems, we estimate that 235,000 Veterans will become new VA patients. Our complementary analysis of SoE data suggests that improving access to VA care could lead to an additional 212,000 currently enrolled Veterans opting to use VA care. The estimates from SoE are slightly lower than the estimates from NSV, in part because NSV includes all Veterans, while SoE focuses on enrolled Veterans only.

Our analysis made two important assumptions. First, we assumed that NSV and SoE accurately identified the proportion of non-VA-patient Veterans with an actual or perceived barrier to access. Neither survey is explicitly designed for this purpose, and neither directly asks Veterans to report or describe access barriers. VA would benefit from future surveys focusing specifically on the relationship between Veterans' perceptions of VA care and their decision of whether to receive care from VA. Second, we assumed that Veterans reporting access issues would use VA services at the same rates as their peers if actual or perceived access issues were to disappear. The actual use rate for these Veterans might be higher or lower than what we observed in survey data.

Despite these limitations, our analysis suggests that policies to improve access will have a modest impact on number of new VA patients. The relatively small effect reflects that many Veterans choose to receive some or all of their care from non-VA sources for reasons that do not appear to be entirely related to access. Additional data collection and analysis are needed to

Table 6-5

Upper Bound of New Patients Among Enrolled Veterans Responding to Improved Access

\begin{tabular}{lccccc}
\hline & $\begin{array}{c}\text { A. Total Enrollees } \\
\text { Who Were Not } \\
\text { Patients in 2013 } \\
\text { (thousands) }\end{array}$ & $\begin{array}{c}\text { B. Proportion of Veterans } \\
\text { Responding “Disagree" or } \\
\text { "Completely Disagree" to an } \\
\text { Access Item (\%) }\end{array}$ & $\begin{array}{c}\text { C. Non-Patients } \\
\text { Reporting } \\
\text { Access Reason } \\
\text { (thousands) }\end{array}$ & $\begin{array}{c}\text { D. Use Rate of New Patients } \\
\text { (\%) }\end{array}$ & $\begin{array}{c}\text { E. Upper Bound } \\
\text { (thousands) }\end{array}$ \\
\hline Age 18-44 & 399 & 11.8 & 47 & 70.6 & 33 \\
Age 45-64 & 735 & 11.5 & 85 & 78.8 & 67 \\
Age 65+ & 1,047 & 14.0 & 148 & 75.4 & 111 \\
Total & 2,181 & 12.8 & 279 & 76.0 & 212 \\
\hline
\end{tabular}

SOURCE: RAND analysis of 2013 SoE data.

13 We calculated this hypothetical use rate by dividing the number of VA patients in each age band by the sum of the number of patients and the number of non-patients who did not report an access issue in each age band. 
better understand the drivers of Veterans' decisions to use VA care rather than their other coverage. These inputs could be used to better predict how Veterans would respond-in terms of enrollment and utilization-to specific improvements in access to VA-provided care or wider availability of VA-purchased care.

\subsection{The Affordable Care Act's Coverage Expansion}

\subsubsection{Description}

The ACA introduced dramatic changes to the U.S. health insurance and health care delivery landscape. The ACA aimed to increase health insurance rates through several channels. First, the ACA as originally implemented would have expanded Medicaid eligibility to 133 percent of the FPL across the United States. ${ }^{14}$ A 2012 Supreme Court decision (National Federation of Independent Business v. Sebelius, 2012) left the decision to expand or not expand Medicaid to the states. As of April 2015, 33 states have expanded or are considering to expand Medicaid. Second, the ACA created insurance Marketplaces operated by the states or federal government, paired with subsidies for low-income individuals and penalties for individuals who remain uninsured (Kaiser Family Foundation, 2015). The ACA also instituted an individual mandate requiring most people to obtain insurance, an employer mandate requiring that businesses with 50 or more workers offer coverage, and numerous other reforms.

The specific scenario that we evaluate focuses on the ACA's coverage expansion provisions. These include:

- Medicaid expansion enabling all individuals in participating states with family incomes at or below 133 percent of the FPL to enroll in the Medicaid program.

- Subsidies for Marketplace insurance, which are available to individuals with incomes between 100 and 400 percent of the FPL who are not eligible for Medicaid and who do not have an affordable offer of coverage from an employer. The ACA's Marketplaces are online portals for buying and selling individual market (that is, non-employer) coverage. Individuals with incomes between 100 and 133 percent of the FPL are eligible for Medicaid in expansion states and Marketplace subsidies in non-expansion states.

- The individual mandate, which requires most Americans to obtain health insurance or pay a tax penalty.

- The employer mandate, which requires businesses with 50 or more workers to offer health insurance coverage or face penalties. In practice, the employer mandate has little effect on our analysis, because most employers with 50 or more workers offered insurance before the ACA was enacted and will continue to do so in the future.

The ACA includes a wide range of other provisions, including payment and delivery demonstrations, prohibitions on co-payments for preventive care, and taxes on expansive employerprovided health insurance plans. While these dimensions of the ACA are not explicitly considered in our coverage expansion scenario, we touch on many of these issues in this section.

14 Due to a 5-percent income "disregard" that is part of the modified adjusted gross income threshold used to determine Medicaid eligibility, the effective income threshold is 138 percent of the FPL. We use 133 percent of the FPL throughout this report for consistency. 
By February 2015, 11.7 million people were enrolled in Marketplace plans (Office of the Assistant Secretary for Planning and Evaluation, 2015b) and an additional 11.7 million people were newly enrolled in Medicaid and the Children's Health Insurance Program (Centers for Medicare \& Medicaid Services, 2015a). Recent survey-based research confirms significant gains in coverage (Carman \& Eibner, 2014; Long et al., 2013; Sommers et al., 2014), although the net decrease in uninsured rates reflects the fact that some people gaining Medicaid or Marketplace coverage previously had other sources of coverage. One group that is of particular interest to policymakers and researchers is the set of people in the so-called "coverage gap" - that is, low-income individuals who lack affordable coverage options because they may not qualify for Marketplace subsidies and are in a state that opted not to expand Medicaid (Garfield, Damico, Stephens, \& Rouhani, 2014).

While the ACA did not directly affect Veterans' eligibility to enroll in or receive VA care, there are important spillover effects of the ACA coverage expansion on Veterans and, ultimately, VA. Some uninsured Veterans may choose to enroll in VA health care to obtain qualifying coverage and avoid individual mandate penalties (U.S. Department of Veterans Affairs, 2015d). Other uninsured low-income Veterans, including some who were previously enrolled to receive VA health care or expect to use VA health care in the future, ${ }^{15}$ may qualify for subsidies to purchase coverage in insurance Marketplaces, or they may live in states that opted to expand Medicaid. These individuals may transition out of the VA health care system and into the community setting to receive some or all of their care.

The net impact of the coverage expansion on VA depends on the number of Veterans who face new incentives to enroll in VA health care compared with the number of current VA patients who obtain new private or Medicaid coverage and opt to shift to non-VA, communitybased providers. Analysis of Veterans' incomes in the 2013 ACS suggests that more than half of Veterans report family income of less than 400 percent of the FPL, which is the threshold for eligibility for Marketplace subsidies (Table 6-6). Approximately 13 percent of Veterans fall below 133 percent of the FPL threshold for Medicaid eligibility in expanding states. These proportions are similar in states that are and are not expanding Medicaid. Based on self-reported income alone, it appears that the health care decisions for a significant share of Veterans could be influenced by Marketplace subsidies or Medicaid expansion. Because our analysis considered states' decisions to expand Medicaid, it also took into account the geographic distribution of Veterans. Veterans who live in non-expansion states are therefore less likely to gain a new source of insurance under the ACA than Veterans who live in expansion states.

The net impact also hinges on the proportion of Veterans in different populations who use VA services at all each year, or the use rate. Many Veterans have at least one other source of health insurance coverage other than VA health care, and Veterans with other coverage have markedly different use rates than Veterans without other sources of coverage. Slightly more than half of Veterans reporting no non-VA sources of coverage have used VA health care services in the past, and 43 percent report using VA health care services in the past six months (Figure 6-8). Only 8 percent of Veterans reporting private coverage alone report using VA health care in the past six months.

15 Individuals who have an offer for "qualifying coverage" are not eligible to receive ACA subsidies. Enrollment to receive VA health care is considered qualifying coverage (U.S. Department of Veterans Affairs, 2015d). However, it is not clear whether there are processes in place to prevent a Veteran currently enrolled in VA health care from receiving subsidies to purchase care through an insurance Marketplace. Veterans may need to dis-enroll from VA health care in order to qualify for ACA subsidies. 
Table 6-6

Distribution of Veterans, by Income and State Medicaid Expansion Decisions

\begin{tabular}{lcc}
\hline $\begin{array}{l}\text { Income Relative to the } \\
\text { Federal Poverty Limit }\end{array}$ & $\begin{array}{c}\text { States Expanding } \\
\text { Medicaid, } \\
\text { of Vercentage }\end{array}$ & $\begin{array}{c}\text { States Not Expanding } \\
\text { Medicaid, } \\
\text { of Vercentage }\end{array}$ \\
\hline$<100 \% \mathrm{FPL}$ & $4.8 \%$ & $4.1 \%$ \\
$100-133 \% \mathrm{FPL}$ & $2.5 \%$ & $2.2 \%$ \\
$134-400 \% \mathrm{FPL}$ & $23.3 \%$ & $19.4 \%$ \\
$>400 \% \mathrm{FPL}$ & $24.8 \%$ & $18.8 \%$ \\
Subtotal & $55.4 \%$ & $44.6 \%$ \\
Total & & \\
\hline
\end{tabular}

SOURCE: RAND calculations using 2013 ACS PUMS.

a States announcing expansion by April 2015 are included in the expanding category (Kaiser Family Foundation, 2015). Four states (Alaska, Florida, Missouri, and Utah) that are considering expansion are included in the not expanding category.

Figure 6-8

VA Health Care Use Rates, by Veteran Health Insurance Coverage

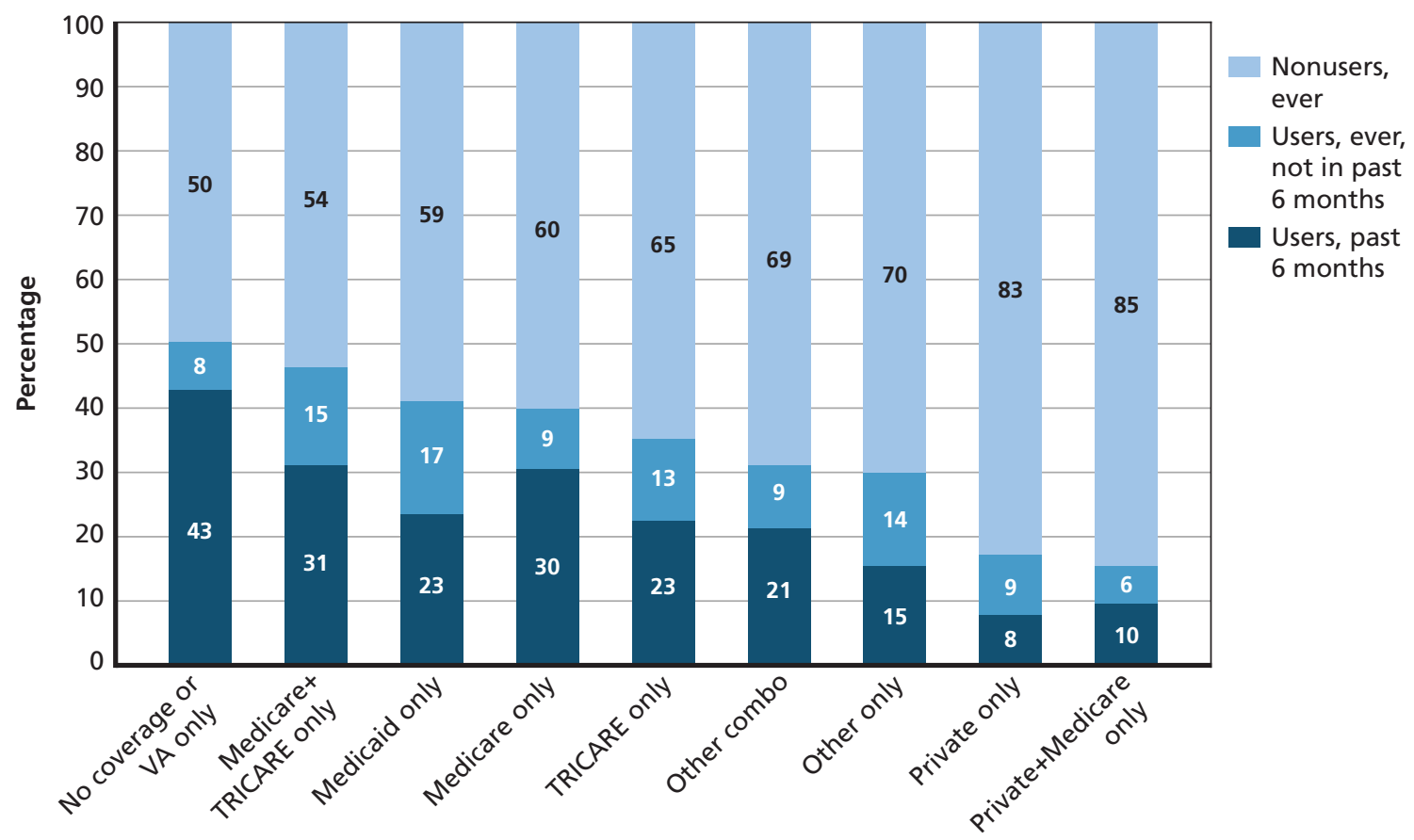

SOURCE: RAND analysis of 2010 NSV, items E2, E3, and F1, weighted by sampling weights. RAND RR1165z1-6-8 
The key assumptions underlying the ACA coverage expansion scenario center on whether and how Veterans who change their coverage status will change the way that they use VA health care. For example, VA health care enrollees who gain Marketplace or Medicaid coverage could continue to use VA health care as before the ACA, or they could shift to community providers and reduce or stop using VA health care. Many of the ideal inputs for evaluating the impact of the coverage expansion on VA are not yet available for analysis. We propose a set of "base case" coverage changes, enrollment rate, and use rate assumptions to illustrate key analytic steps and impacts. We also describe the implications of different assumptions in Appendix D.

\subsubsection{Approach}

\section{Data and Methods for Scenario Five, Impact of the Affordable Care Act}

- We estimated the impact of two effects of the ACA's coverage expansion: (1) enrollment of previously uninsured Veterans in response to the individual mandate and (2) uptake of new private and Medicaid coverage by Veterans currently enrolled in and using VA health care. We used NSV data to estimate both the number of uninsured, under-65 Veterans (those not enrolled in VA and with no other source of coverage) and the number of under-65 Veterans enrolled in VA care but with no other source of coverage.

- We then analyzed these estimates to determine how many Veterans would obtain coverage through the ACA and assessed whether and how Veterans who change their coverage status because of the ACA will change the way they use VA health care services.

We separately estimated the impact of two countervailing effects of the coverage expansion: (1) enrollment of previously uninsured Veterans to gain qualifying health coverage and avoid individual mandate penalties, some of whom will actually use VA services and some of whom will enroll only to avoid penalties, and (2) uptake of new private and Medicaid coverage by Veterans currently enrolled in and using VA health care. Figure 6-9 illustrates these effects. If the outcome of interest is the number of VA patients, then the two key flows are those to and from VA patients. The net impact of the coverage expansion is the flow of new, previously uninsured patients minus the flow of current patients who transition entirely to other sources of care.

The net change in terms of patient counts nationally may be small when considering both impacts. However, individual shifts within demographic and priority group categories may be significant and important for VA to incorporate into its modeling activities. For example, lowincome Veterans in states that chose to expand Medicaid eligibility may be less likely to use VA health services than their peers in states that opted not to expand Medicaid. Based on our review of the 2014 EHCPM model documentation, we do not believe that the most-recent VA projections of demand reflect any ACA-related changes in the enrolled population or use rates.

We used NSV data to estimate the number of uninsured Veterans - that is, Veterans who are not enrolled in VA health care and have no other source of coverage. We also used NSV data to estimate the number of Veterans who are enrolled in VA health care but have no other source of coverage. We then estimated how many of these Veterans would enroll in health insurance coverage in 2016 as a result of the ACA, ignoring their eligibility for VA health care. This analysis allowed us to separate the uninsured, non-enrolled Veteran population into two groups: (1) those who without VA eligibility would gain health coverage through the ACA and (2) those who would remain uninsured. We limited our analysis to Veterans under age 65, because most Veterans over age 65 are enrolled in Medicare, and because those age 65 and over are ineligible for the ACA's Medicaid expansion and Marketplace subsidies. 
Figure 6-9

Coverage Expansion Patient Flows

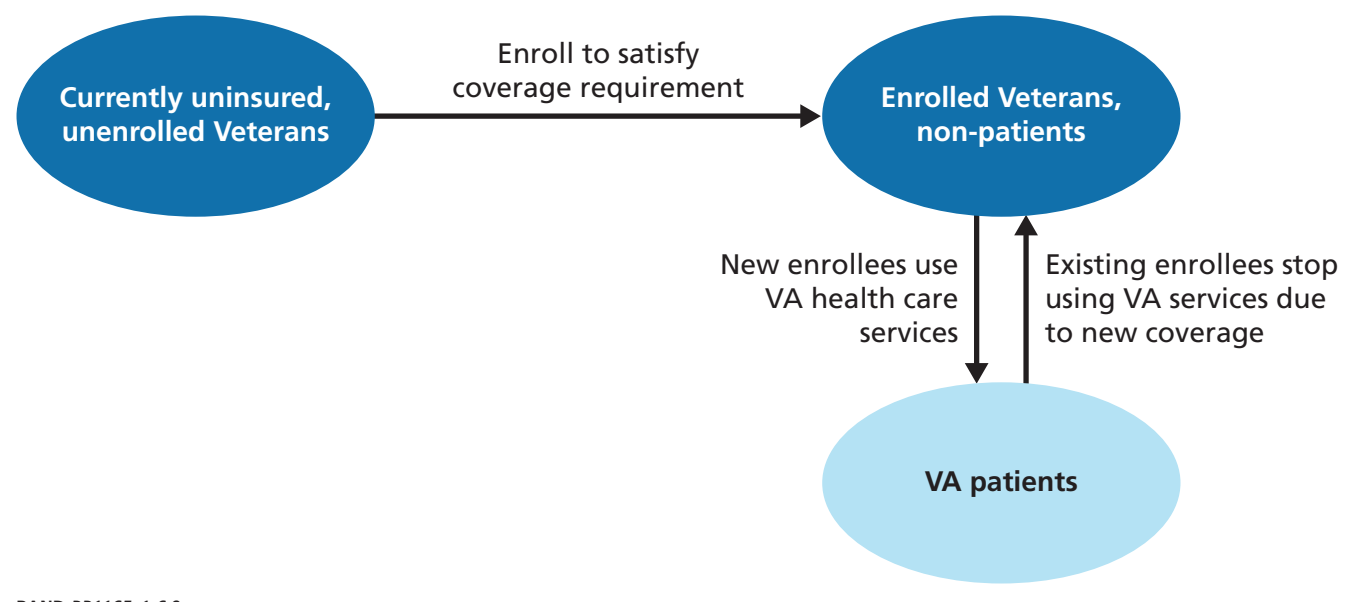

RAND RR1165z1-6.9

The estimate was based on results from the RAND COMPARE (COMPrehensive Assessment of Reform Efforts) microsimulation model, which is a model built to estimate the effects of the ACA (Cordova, Girosi, Nowak, Eibner, \& Finegold, 2013). The model takes into account the four coverage expansion provisions described above, as well as changes to insurance regulations that will affect health insurance premiums and, consequently, enrollment decisions. The model also accounts for the ACA's effects on employers, although, in net, we estimate little impact on employer health insurance provision. We focused on individuals in the general population with the same age distribution as uninsured, non-enrolled Veterans. We calculated the number of Veterans who would be expected to enroll in Medicaid, Marketplace plans, or other private coverage by combining the estimates of Veteran populations with the coverage change estimates from COMPARE.

While we could estimate with some accuracy the number of Veterans who would be eligible for Medicaid and for subsidized health coverage through the Marketplaces, predicting their choice to take up this coverage, enroll for VA coverage, or remain uninsured is much more difficult. This is true for both those Veterans currently enrolled at VA and those not enrolled. While we begin with a set of baseline enrollment and use rates with some grounding in observed rates in the Veteran population, it is important to recognize that actual enrollment and use rates are not known and may deviate from the enrollment and use patterns observed in current Veteran and patient data.

We expect that Veterans with different health coverage options will enroll in VA health care at different rates; we assume that Veterans who are eligible for Marketplace subsidies will be less likely to enroll in VA than Veterans who are not eligible for subsidies, and Veterans who gain access to Medicaid will be less likely to enroll in VA than Veterans who gain Marketplace subsidies. We began by setting a base rate for the percentage of uninsured, non-enrolled Veterans who we predict will not gain coverage under the ACA. We applied this same base rate to Veterans over 400 percent of the FPL who we predict will gain coverage. These Veterans will not have access to subsidized coverage under the ACA. We set the enrollment rate for Veterans who we predict will gain coverage and can receive Marketplace subsidies at half of the base rate, to reflect that subsidies may make Marketplace coverage an attractive option, particularly 
for Veterans who had not enrolled previously in VA care. We assumed that Veterans who will gain coverage through Medicaid will enroll in VA health care at one-quarter of the base rate, reflecting Medicaid's minimal cost-sharing requirements.

We assumed that Veterans induced by ACA to enroll in VA health care use VA services at the rate observed for the entire under-65 Veteran population with private insurance only. While this is lower than the use rate for enrolled Veterans overall, it reflects the fact that some Veterans will en roll solely for the purpose of obtaining qualifying coverage. As we note below, the use rate for these Veterans may increase over time. We report results for a range of enrollment and use rates in Appendix D.

Finally, we did not explicitly account for changes in use rates due to ACA-driven changes in VA priority group assignment. For example, in theory, Veterans could move from priority group 7 to priority group 5 if they live in a state with Medicaid expansion and now qualify under the higher-income threshold. The move to priority group 5 would face no cost-sharing for VA health care. However, the income threshold for priority group 5 is considerably higher than the higher Medicaid threshold, so we do not believe Medicaid expansion will affect the number of Veterans in priority group 5. More generally, the larger change for these Veterans is that they are gaining a new source of coverage from Medicaid, and we expect this change rather than a reduction in VA cost-sharing to be the more important driver of whether the Veteran chooses to become a VA patient.

\subsubsection{Results}

The following two sections report separate results for the two flows of patients in Figure 6-9first, a flow of new enrollees and patients from previously uninsured Veterans, and second, a flow of patients away from VA health care as they gain other coverage. A third section reports net results after combining these two components.

\subsubsection{New Enrollees and Patients from the Previously Uninsured}

If the post-ACA coverage transitions of Veterans were to resemble those for the general population, we estimate that half of the 1.1 million uninsured, non-enrolled Veterans in 2013 would enroll in another source of coverage-for example, Medicaid coverage or coverage through a Marketplace plan-by 2016. This rate would be lower for uninsured Veterans under 133 percent of the FPL, because not all states have expanded Medicaid. The remaining half of uninsured Veterans would be expected to remain uninsured through 2016. Table 6-7 reports the number of non-enrolled, uninsured Veterans by income category, the proportion of Veterans in each category to gain coverage, and the resulting number of Veterans predicted to gain other coverage. As a reminder, these results predict what uninsured Veterans would do if they did not have the option to enroll at VA. They are useful for identifying how many uninsured Veterans would choose to gain coverage and face a decision between ACA options and VA coverage, and how many would only consider whether to enroll at VA to avoid paying the penalty for being uninsured.

Not all uninsured Veterans are eligible to receive VA health care. We used eligibility rates calculated from ACS data and for specific income bands (as described in more detail in the changes to VA eligibility scenario) to estimate the proportion of Veterans eligible to enroll in VA care (Table 6-8).

The next steps estimate what proportion of Veterans will (1) enroll in VA health care and (2) become VA patients. Table 6-9 (column A) tracks these steps, starting with estimates of VA 
Table 6-7

Coverage Changes Under ACA for Previously Uninsured Veterans, by Income Category

\begin{tabular}{lccc}
\hline Income Category & $\begin{array}{c}\text { Non-Enrolled, } \\
\text { Previously Uninsured } \\
\text { Veterans (thousands) }\end{array}$ & $\begin{array}{c}\text { Proportion } \\
\text { Predicted to Gain } \\
\text { Other Coverage (\%) }\end{array}$ & $\begin{array}{c}\text { Non-Enrolled, Previously Uninsured } \\
\text { Veterans Predicted to Gain Other } \\
\text { Coverage (thousands) }\end{array}$ \\
\hline$\leq 133 \% \mathrm{FPL}$ & 588 & 34.4 & 202 \\
$134-400 \% \mathrm{FPL}$ & 390 & 68.8 & 268 \\
$>400 \% \mathrm{FPL}$ & 132 & 62.7 & 83 \\
Total & 1,109 & 49.9 & 553 \\
\hline
\end{tabular}

SOURCE: Authors' analysis based on 2010 NSV data and the RAND COMPARE microsimulation model.

Table 6-8

Eligibility for Previously Uninsured Veterans, by Income Category

\begin{tabular}{|c|c|c|c|}
\hline Income Category & $\begin{array}{l}\text { Non-Enrolled, Previously } \\
\text { Uninsured Veterans } \\
\text { (thousands) }\end{array}$ & $\begin{array}{c}\text { Proportion Eligible to } \\
\text { Receive VA Health Care } \\
\text { (\%) }\end{array}$ & $\begin{array}{c}\text { Eligible, Non-Enrolled, } \\
\text { Previously Uninsured } \\
\text { Veterans } \\
\text { (thousands) }\end{array}$ \\
\hline \multicolumn{4}{|c|}{ Veterans Not Predicted to Gain Coverage Under ACA } \\
\hline$\leq 133 \% \mathrm{FPL}$ & 386 & 100.0 & 386 \\
\hline $134-400 \% \mathrm{FPL}$ & 122 & 57.6 & 70 \\
\hline$>400 \% \mathrm{FPL}$ & 49 & 13.1 & 6 \\
\hline Subtotal & 556 & 83.0 & 462 \\
\hline \multicolumn{4}{|c|}{ Veterans Predicted to Gain Coverage Under ACA } \\
\hline$\leq 133 \% \mathrm{FPL}$ & 202 & 100.0 & 202 \\
\hline $134-400 \% \mathrm{FPL}$ & 268 & 57.6 & 154 \\
\hline$>400 \% \mathrm{FPL}$ & 83 & 13.1 & 11 \\
\hline Subtotal & 553 & 66.4 & 367 \\
\hline Total & 1,109 & 74.8 & 829 \\
\hline
\end{tabular}

SOURCE: Authors' analysis based on 2010 NSV data, 2013 ACS PUMS data, and the RAND COMPARE microsimulation model.

eligible, non-enrolled, previously uninsured Veterans from Table 6-8. Approximately one in four Veterans is currently enrolled to receive health care services, although the enrollment rate is significantly higher ( 47 percent) for Veterans without other sources of coverage. We apply a 50 -percent enrollment rate for Veterans not predicted to enroll in another source of coverage as a result of the ACA. For Veterans who we predict will enroll in other coverage, we apply the same 50-percent enrollment rate for Veterans in the highest income category and lower enrollment rates for Veterans in lower income categories (because these individuals are ineligible for the ACA's subsidies), as discussed above: 25 percent for those who are eligible for Marketplace subsidies and 12.5 percent for those who enroll in Medicaid. With these enrollment assump- 
Table 6-9

Predicted VA Enrollees and Patients

\begin{tabular}{|c|c|c|c|c|c|}
\hline Income Category & $\begin{array}{c}\text { A. Non-Enrolled, } \\
\text { Previously Uninsured } \\
\text { Veterans Gaining } \\
\text { Coverage Under ACA } \\
\text { (thousands) }\end{array}$ & $\begin{array}{l}\text { B. Enrollment } \\
\text { Rate (\%) }\end{array}$ & $\begin{array}{l}\text { C. Predicted } \\
\text { Enrollees } \\
\text { (thousands) }\end{array}$ & $\begin{array}{l}\text { D. Use } \\
\text { Rate (\%) }\end{array}$ & $\begin{array}{l}\text { E. Predicted } \\
\text { Patients } \\
\text { (thousands) }\end{array}$ \\
\hline \multicolumn{6}{|c|}{ Veterans Not Predicted to Gain Coverage Under ACA } \\
\hline$\leq 133 \% \mathrm{FPL}$ & 385 & 50.0 & 192 & 24.2 & 47 \\
\hline $134-400 \% \mathrm{FPL}$ & 70 & 50.0 & 35 & 21.2 & 7 \\
\hline$>400 \% \mathrm{FPL}$ & 6 & 50.0 & 3 & 14.4 & $<1$ \\
\hline Subtotal & 462 & 50.0 & 231 & 23.6 & 54 \\
\hline \multicolumn{6}{|c|}{ Veterans Predicted to Gain Coverage Under ACA } \\
\hline$\leq 133 \% \mathrm{FPL}$ & 202 & 12.5 & 25 & 24.2 & 6 \\
\hline $134-400 \% \mathrm{FPL}$ & 154 & 25.0 & 39 & 21.2 & 8 \\
\hline$>400 \% \mathrm{FPL}$ & 11 & 50.0 & 5 & 14.4 & 1 \\
\hline Subtotal & 367 & 18.9 & 69 & 21.8 & 15 \\
\hline Total & 829 & 36.2 & 300 & 23.2 & 70 \\
\hline
\end{tabular}

SOURCE: Authors' analysis based on 2010 NSV data, 2013 ACS PUMS data, and the RAND COMPARE microsimulation model.

tions, we estimate an increase of 300,000 Veterans enrolled in VA health care (column C in Table 6-9).

Our next step was to convert these estimates of new enrollees to estimates of new patients. We used a real-world use rate-calculated across enrolled and non-enrolled Veterans with private insurance-for this analysis (column D in Table 6-9). ${ }^{16}$ In the short term, Veterans enrolling only to satisfy the ACA's individual mandate may have relatively low use rates compared with patients with private coverage. At a 50-percent base enrollment rate and the use rates described above, we estimated that ACA coverage expansion will result in 70,000 new VA patients who were previously uninsured and not enrolled (column E in Table 6-9).

A limitation of this analysis is that we had to make relatively strong assumptions about which Veterans would enroll and use care due to the ACA's individual mandate requirements. In Appendix D, we conduct sensitivity analyses to estimate how the results might change under a variety of alternative assumptions about enrollment and use rates.

\subsubsection{Transitions Away from VA Health Care Due to New Coverage Options}

Using NSV data, we estimated that there were 1.4 million Veterans enrolled in VA health care without another source of health coverage (column A in Table 6-10). Of these Veterans, approximately half would gain another source of coverage as a result of the ACA (column A in Table 6-10). How many would take up this other coverage is unknown.

\footnotetext{
16 We used the private use rate rather than a combination of private and Medicaid use rates because there are very few Veterans with Medicaid coverage only in the NSV data.
} 
Table 6-10

Predicted Coverage Changes for Veterans Enrolled in VA Health Care Only, by Income Category

\begin{tabular}{lcccccc}
\hline & $\begin{array}{c}\text { A. Veterans } \\
\text { Enrolled in } \\
\text { VA Health } \\
\text { Care Only }\end{array}$ & $\begin{array}{c}\text { B. Proportion } \\
\text { Gaining } \\
\text { Coverage } \\
\text { (\%) (RAND } \\
\text { COMPARE) }\end{array}$ & $\begin{array}{c}\text { C. Veterans } \\
\text { Enrolled in VA } \\
\text { Health Care Only } \\
\text { and Gaining } \\
\text { Other Coverage } \\
\text { (thousands) }\end{array}$ & $\begin{array}{c}\text { D. Use } \\
\text { Rates, VA } \\
\text { Health Care } \\
\text { Only (\%) }\end{array}$ & $\begin{array}{c}\text { E. Change } \\
\text { in Use Rate } \\
\text { for Veterans } \\
\text { Gaining Other } \\
\text { Coverage (\%) }\end{array}$ & $\begin{array}{c}\text { F. Estimated } \\
\text { Reduction in } \\
\text { Vatients } \\
\text { (thousands) }\end{array}$ \\
\hline$\leq 133 \% \mathrm{FPL}$ & 728 & 34.4 & 251 & 97.9 & -25.0 & 61 \\
$134-400 \% \mathrm{FPL}$ & 462 & 68.8 & 318 & 98.3 & -25.0 & 78 \\
$>400 \% \mathrm{FPL}$ & 191 & 62.7 & 119 & 80.6 & -25.0 & 24 \\
Total & 1,381 & 49.8 & 688 & 95.1 & -25.0 & 164 \\
\hline
\end{tabular}

SOURCE: Authors' analysis based on ACS and the RAND COMPARE microsimulation model.

The current use rates for this subpopulation of Veterans - that is, Veterans who are enrolled in VA health care and have no other source of coverage-are high (column D in Table 6-10). We assumed that use rates would fall by 25 percent for Veterans who gain other coverage (column E in Table 6-10). If all VA-enrolled Veterans who could enroll in ACA coverage do so and immediately shift to this lower use rate, we would expect 164,000 fewer VA patients each year (column F in Table 6-10). The magnitude of this impact hinges on the ACA take-up rate and the use rate assumption. We discuss the impacts of different use rate assumptions in Appendix D.

\subsubsection{Net Impacts and Medicaid Expansion in All States}

The net overall change in VA patients hinges on assumptions for enrollment and use rates. Using the base rates described above, we estimated a net decrease of 94,000 VA patients (70,000 new users, minus 164,000 current users who stop using VA care). However, other rates can lead to increases in the number of VA patients.

We looked separately at the impact of Medicaid expansion in every state. Some states that have yet to expand Medicaid are considering doing so, and it is possible that others will follow suit. If we apply the change in Medicaid enrollment that we have observed to date in expanding states to non-expanding states, Medicaid expansion in all states results in 28,000 fewer VA patients. ${ }^{17}$ The net impact on VA from broader Medicaid expansion-including the decrease from the base case analysis-is a decrease of 122,000 VA patients.

\subsubsection{Discussion}

Our analysis suggests that the net impact of the ACA coverage expansion in terms of VA patients is modest, with a net decrease of about 94,000 VA patients per year. However, this net result combines separate flows of Veterans in and out of VA health care, and different assumptions can result in a range of net impacts, including increases in the number of VA patients. We expect that Veterans enrolling in Medicaid to gain qualifying coverage will use VA care at rates different from current enrollees without other sources of coverage. Specifically, we pre-

17 Our estimate of 30,000 fewer patients includes 8,000 fewer new patients, as more Veterans opt for Medicaid to gain qualifying coverage, as well as 22,000 fewer patients among the already enrolled, as more Veterans access care through Medicaid. 
dict that they will use VA care at rates that more closely resemble current enrollees with access to private care or lower. A large proportion of these Veterans may not need or want to receive VA care, but rather are enrolling to satisfy the ACA individual mandate. Over time, these Veterans may start using VA care at higher rates. Finally, whether current VA patients take up offers for coverage through Medicaid or Marketplace plans hinges in part on their satisfaction with VA health care as their primary source for care, on Marketplace subsidies, and on family composition. For example, Veterans seeking coverage for themselves and dependents may be better served by enrolling in a Marketplace or other commercial family plan rather than en rolling in VA.

\subsection{Summary of Scenario Impacts}

Table 6-11 and Figure 6-10 summarize impacts of all scenarios on projected patient counts. The table shows only mid-range estimates for each scenario. However, there is considerable uncertainty about the factors behind these estimates. More-sophisticated analysis of Veteran

Table 6-11

Scenario Impacts on Projected VA Patient Counts

\begin{tabular}{|c|c|c|c|c|c|c|c|c|c|c|c|}
\hline \multirow[b]{2}{*}{ Scenario } & \multicolumn{11}{|c|}{ Millions of VA Patients, 2014-2024 } \\
\hline & 2014 & 2015 & 2016 & 2017 & 2018 & 2019 & 2020 & 2021 & 2022 & 2023 & 2024 \\
\hline Baseline (from Chapter Four) & 5.87 & 5.99 & 6.06 & 6.11 & 6.14 & 6.16 & 6.15 & 6.14 & 6.12 & 6.09 & 6.07 \\
\hline \multicolumn{12}{|c|}{ Scenario 1: Changes to VHA Eligibility by Priority Group } \\
\hline Priority Group 8e Eligibility & 5.87 & 6.02 & 6.09 & 6.14 & 6.17 & 6.19 & 6.18 & 6.17 & 6.15 & 6.13 & 6.10 \\
\hline $\begin{array}{l}\text { Priority Group } 8 \mathrm{e} \text { and } 8 \mathrm{~g} \\
\text { Eligibility }\end{array}$ & 5.87 & 8.10 & 8.20 & 8.26 & 8.30 & 8.33 & 8.32 & 8.30 & 8.28 & 8.24 & 8.21 \\
\hline $\begin{array}{l}\text { Scenario 2: Changes in } \\
\text { Presumptive Eligibility }\end{array}$ & 5.87 & 6.35 & 6.42 & 6.48 & 6.51 & 6.53 & 6.52 & 6.51 & 6.49 & 6.46 & 6.44 \\
\hline \multicolumn{12}{|c|}{ Scenario 3: Impact of Future Conflict on VA Use } \\
\hline Minimal Conflict & 5.87 & 5.99 & 6.03 & 6.07 & 6.10 & 6.13 & 6.17 & 6.20 & 6.24 & 6.27 & 6.32 \\
\hline Median Conflict & 5.87 & 6.01 & 6.08 & 6.14 & 6.20 & 6.26 & 6.31 & 6.36 & 6.41 & 6.47 & 6.53 \\
\hline Widespread Conflict & 5.87 & 6.03 & 6.12 & 6.20 & 6.28 & 6.36 & 6.43 & 6.51 & 6.59 & 6.68 & 6.75 \\
\hline $\begin{array}{l}\text { Scenario 4: Improving Access to } \\
\text { VA Care }\end{array}$ & 5.87 & 6.23 & 6.30 & 6.35 & 6.38 & 6.40 & 6.39 & 6.38 & 6.36 & 6.33 & 6.31 \\
\hline \multicolumn{12}{|c|}{ Scenario 5: ACA Coverage Expansion } \\
\hline ACA Overall & 5.87 & 5.99 & 5.96 & 6.01 & 6.04 & 6.06 & 6.05 & 6.04 & 6.02 & 6.00 & 5.98 \\
\hline ACA Medicaid Expansion & 5.87 & 5.99 & 5.94 & 5.98 & 6.01 & 6.03 & 6.03 & 6.01 & 5.99 & 5.97 & 5.95 \\
\hline
\end{tabular}


Figure 6-10

Scenario Impacts on Projected VA Patient Counts

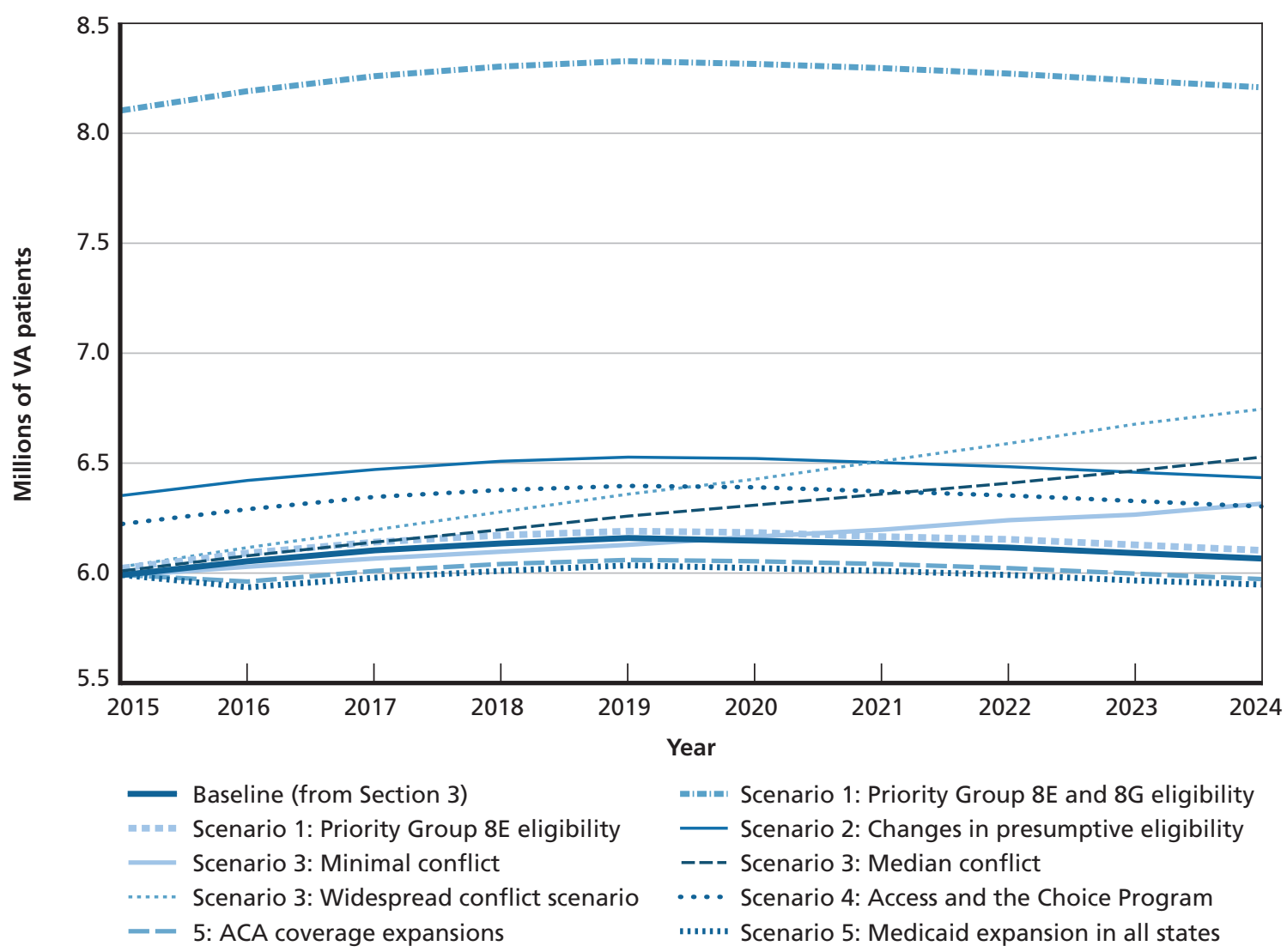

RAND RR1165z1-6.10

health coverage decisions given the characteristics of the choices available to Veterans was not possible in the limited time for this study, but such analysis could help VA anticipate future changes in its patient population under these and other possible scenarios.

\subsection{Discussion}

The five specific scenarios help quantify the impacts of "what if" policy and trend questions on baseline projections. The impacts of some scenarios, like the priority groups $8 \mathrm{e}$ and $8 \mathrm{~g}$ scenario and, to a lesser extent, the access and presumptive eligibility scenarios, suggest significant increases in the VA patient population. We estimate that increasing eligibility to receive VA health care services to include priority groups $8 \mathrm{e}$ and $8 \mathrm{~g}$ would increase the number of VA patients by nearly 40 percent. Not coincidentally, the scenarios with the largest potential impact on VA in terms of patient counts are those that address eligibility, access, and patient choices. These scenarios could come about as the result of VA policy or congressional action rather than external policies or trends. Other scenarios, such as the ACA coverage expansion scenario, suggest more-modest effects, with changes in projections of VA patients over time 
on the order of tens or hundreds of thousands of patients per year, in the context of a base of approximately 6 million patients. Still, the impacts that we describe are potentially important for VA's resource allocation and planning, and for improving access and quality of care.

We considered a range of other potential drivers of change in VA patient projections that were not evaluated as formal scenarios.

Changes to the cost of employer-sponsored insurance: For example, the so-called "Cadillac tax" on high-cost employer-sponsored health plans introduced in ACA will go into effect in 2018 and could affect the non-VA coverage options available to some Veterans. More generally, ACA's employer mandate could drive some small and large firms to change the way that they provide coverage to their employees, or whether they provide coverage at all. Veterans could increasingly rely on VA for care if employers drop coverage or reduce the generosity of coverage over time, or if premiums for employer-sponsored insurance increase significantly over time.

Changes in the generosity of specialty drug coverage: Other trends in commercial insurance could drive Veterans to access more of their care through VA than through other sources. Commercial insurers increasingly place expensive biologic and other specialty prescription drugs on formulary specialty tiers, which are then often tied to significant costsharing, including co-insurance rates. Eligible Veterans can avoid paying hundreds or thousands of dollars in cost-sharing for specialty drugs by accessing these drugs through VA. This kind of "wraparound" use of VA increases enrollment and patient counts (because we define a VA patient as a Veteran using any VA health care services, even if it is only prescription drugs), and results in a considerable cost to VA in terms of the purchase and administration cost of the drug, but in the short term, it introduces few new demands on the VA health care system. However, for Veterans enrolling in VA to receive a single "gateway" service, such as expensive specialty drugs, their proportion of care may expand over time. Current VA projections on the impact of specific specialty drugs (for example, expensive new hepatitis $C$ treatments) focus on direct drug costs only and not on the impact of changes in specialty drug coverage outside of VA on projections of demand for VA care overall.

Changes in access to mental health services: The evolving mental health landscape is an example of a change that could lower VA patient projections over time. Recent legislative changes have increased non-Veterans' access to and coverage for mental health care. The implementation of the Mental Health Parity and Addiction Equity Act in 2010, combined with the inclusion of mental health coverage as an essential benefit in 2014, has greatly increased the mental health coverage available through private insurance plans. The Office of the Assistant Secretary for Planning and Evaluation in the U.S. Department of Health and Human Services has estimated that this 2010 law, combined with the ACA, will lead to greater mental health and substance abuse treatment coverage for more than 62 million Americans (Beronio, Po, Skopec, \& Glied, 2013). Additionally, mental health coverage has now been fully phased in to parity in Medicare (wherein cost-sharing for mental health care must be on par with medical and surgical care) in 2014, increasing coverage of these services for 48 million Medicare beneficiaries (Medicare Improvements for Patients and Providers Act, 2008). Given traditionally high levels of reliance on VA mental health services amid the limited availability of civilian coverage, these large changes may manifest in markedly different trends. In our analyses of MEPS data through 2012, Veterans who have access to employer-provided insurance or Medicare had modest increases in VA mental health spending over time compared with considerable increases for Veterans without other sources of coverage, suggesting that Veterans may be 
increasingly accessing mental health services outside VA. Given this expansion in the civilian sector and this early evidence of differential responsiveness depending on Veterans' alternative health service coverage statuses, careful attention should be paid to the projected utilization of VA mental health services going forward.

Changes in assistance programs and Veteran income: The Social Security Disability Insurance (SSDI) program provides Medicare coverage and monthly cash benefits to more than 13 million Americans and, according to counts from the 2013 ACS, more than one-third of 1 million Veterans or their households. However, this program is facing impending insolvency, and in the exhaustion of the SSDI Trust Fund, projected by the Congressional Budget Office to occur in the last quarter of 2016, all benefits currently being paid out would be immediately cut by 20 percent in the current-law framework. Because this income is included in household income when determining VA priority groups, such a decline could cause Veterans otherwise ineligible for enrollment to now fall under priority group $8 \mathrm{~b}$ or $8 \mathrm{~d}$ income thresholds, as well as reassign currently eligible Veterans to higher priority groups with lower or no co-pays, both of which would directly affect program costs. In our analysis of 2013 ACS data in combination with the priority group classification algorithm described in this chapter, we found that of the 335,000 Veterans in the 2013 ACS either receiving SSDI benefits themselves or residing in a household with an SSDI beneficiary, 260,000 were eligible for VA health services (among whom slightly more than half $[132,000]$ were VA patients), while 75,000 Veterans had household incomes too high to qualify for enrollment. Although a preliminary analysis suggests that there would be a limited number of newly eligible Veterans given a 20 percent SSDI benefit cut, there is the potential for substantial reshuffling from priority groups 7 and $8 \mathrm{a}-8 \mathrm{~d}$ to priority group 5. Additionally, SSDI benefits will continue to fall in every year after 2016 if there is no policy action going forward, increasing the extent to which Veterans are affected. Because of the size of this population and the wide range of shifts that can occur in both the presence and the absence of policy changes, careful attention should be paid to how these shifts will affect Veterans receiving SSDI benefits in the years to come. 



\section{Conclusions and Recommendations}

In this assessment, we estimated the current and future demographic characteristics of Veterans, estimated the current health care needs of Veterans and VA patients, projected future health care needs of the Veteran population, estimated the extent to which Veterans rely on VA for their health care, and considered how future policy changes or trends may affect the population of eligible or enrolled Veterans or the rate at which Veterans rely on VA for health care. In this chapter, we summarize the findings of these analyses and offer recommendations for consideration as VA plans to meet future needs for health care services.

\subsection{Summary of Assessment A Findings}

\subsubsection{Current and Future Demographic Characteristics}

We estimated the total number of Veterans in 2014 to be approximately 21.6 million. Over the 10-year projection horizon, we estimate that this number will decrease by 19 percent, to 17.5 million, by 2024. The most significant population decline will occur in the Ohio River Valley region, with some marginal increases in population size in parts of the Southeast. The share of female Veterans is expected to grow, and the average age of Veterans will also increase over this period. The largest change in the relative share of service era cohorts is a decrease in pre-Vietnam- and Vietnam-era Veterans and a large increase in the number of Gulf War and, especially, post-9/11 Veterans.

Between 2015 and 2024, the Veteran population is expected to become more geographically concentrated in urban areas, a result that is consistent with expectations about migration of the U.S. population more generally. The proportion of Veterans in both the youngest and oldest age groups is expected to increase over the same period, which will result in more variation in geographic age patterns. Trends in geographic distribution by age are expected to reflect cohort changes where Veterans reside rather than patterns of migration. Overall, the average age of the population is expected to rise slightly. In areas where the proportion of older Veterans is projected to decline over the 2015-2024 period, the cause is likely that insufficient numbers of younger Veterans are moving to these regions over time. On the other hand, areas that will see a larger proportion of older Veterans over time are those where middle-age Veterans currently live and are expected to stay as they age. While Veterans are mobile, our estimates suggest that migration is relatively small and not likely to be a major factor in Veteran demographics.

As mentioned in Chapter Three, Veterans are half as likely as non-Veterans to live below the poverty line, and they also enjoy higher median incomes. In addition, Veterans are less 
likely than non-Veterans to be unemployed. Disability status is a strong predictor of unemployment, and the rate of unemployment within the Veteran population varies widely by service era and age. Veterans are also more likely than non-Veterans to have health insurance, in addition to VA benefits, through both the public and private health insurance sectors. In fact, the majority of Veterans enrolled in VA health care have other health insurance options.

Although approximately one in 10 U.S. adults is a Veteran, Veterans account for 16 percent of homeless adults and 13 percent of sheltered homeless adults. Female Veterans are three to four times more likely than non-Veteran women to become homeless. Health conditions are more prevalent in homeless Veterans than they are in homeless non-Veterans, and these rates increase as time spent in homelessness increases. However, despite these concerns, the overall rate of homelessness among Veterans is low and has been declining over time. The U.S. Department of Housing and Urban Development reports that there were approximately 49,933 homeless Veterans in 2014 (less than 0.25 percent of the Veteran population), representing a 33-percent decline in homelessness among Veterans since 2010.

\subsubsection{Enrollment in and Reliance on VA Health Care Services 7.1.2.1. Enrollee and Patient Projections}

To project the number of VA enrollees and patients over the 2015-2024 period, we used a sensitivity analysis approach. We considered three different sets of assumptions, each of which accounted for factors that likely affect Veterans' enrollment decisions and VA use rates. In 2014, there were 9.09 million VA enrollees and 5.87 million VA patients, and, by 2024, we project between 9.33 and 10.00 million VA enrollees and between 5.81 and 6.18 million VA patients. The differences in our projections are driven primarily by enrollment and use rates among Veterans who served in Iraq and Afghanistan. In 2009, 6 percent of VA patients deployed in Iraq or Afghanistan, but, by 2014, this percentage increased to 12 percent. Assuming this pattern continues, we project that, by 2024, approximately 20 percent of VA patients will be Veterans of deployment in Iraq or Afghanistan.

Our estimates suggest that the Veteran population will shrink by 19 percent between 2015 and 2024. Over the same period, we project that the VA patient population will peak in 2019 and begin to taper off or decline through 2024. The less-pronounced decline in the size of the patient population relative to the Veteran population is related to recent increased enrollment and use of VA health care, particularly among younger Veterans. The rate of use among Veterans under age 35 has increased threefold since 2005. The growth in the younger VA patient population may be related to outreach efforts on the part of VA, streamlined enrollment processes, and enhanced eligibility rules for Veterans of Afghanistan and Iraq. These trends contributed to significant growth in the total VA patient population between 2000 and 2014, despite a decline in the overall Veteran population. While we estimate that these growth rates will taper off in the near future, the projected declines in the size of the patient population are small relative to the projected declines in the total number of Veterans.

\subsubsection{Reliance on VA}

The goals of our health care needs analysis were to describe and project numbers of people (Veterans overall and VA patients in particular) and prevalence rates of health conditionsbut not to estimate demand for or utilization of services. However, because MEPS captures all health care that is received by its respondents - from VA and from other sources - the data provide an opportunity to estimate Veteran reliance on VA. Reliance represents the share of 
health care services that patients receive from VA compared with other sources, and is important to VA planners because it can have an effect on demand for VA services. For example, if reliance increases due to rising health care costs in the civilian sector, VA may need to provide more care even if the number of VA patients is unchanged. Because VA's EHCPM also estimates reliance, we were able to generate estimates of reliance on a subset of health care services, which can then be compared with the results of VA's model.

We computed two reliance rates: (1) the share of health care services that VA patients receive from VA and (2) the share of health care services that all Veterans receive from VA, where, by definition, the second rate will be smaller than the first because some Veterans receive none of their health care from VA. Reliance rates for both populations were calculated for prescribed medications, all office visits, inpatient surgery, and specific office visit categories.

Among both VA patients and all Veterans, the highest rate of reliance on VA services is for office-based laboratory services, at 34 percent and 22 percent, respectively. Office-based radiology services rank second, at 32 percent for patients and 20 percent for all Veterans, in terms of reliance. Younger Veterans (and patients) rely on VA for more of their prescription drug, office visit, and inpatient surgical care than do older Veterans (and patients), a result that can likely be explained by differences in other health insurance status. Veterans and patients with lower incomes also rely on VA for both of these services. Living outside of a metropolitan area, being uninsured, and being in relatively poorer health are characteristics that are associated with greater reliance on VA for prescription drug services and office visits.

For many service categories, EHCPM yields different reliance estimates from those calculated in MEPS. For example, EHCPM predicts that 66 percent of prescription drugs are delivered to enrollees through VA, whereas MEPS results suggest lower rates: 30 percent for patients and 16 percent for all Veterans. It is unclear what drives these differences between EHCPM and MEPS, although they may be partially related to differences in methodologies, including (1) the population for whom reliance is estimated (e.g., enrollees or patients), (2) the data used to produce these estimates (e.g., survey, administrative, or commercial), and (3) the extent to which all health care encounters are captured in the data (e.g., based on self-reports or payer information). While we know MEPS has limitations, we have only partial visibility into the strengths and weaknesses of the EHCPM approach. For these reasons, we cannot fully determine why the approaches differ, or speculate about which approach is preferable.

\subsubsection{Current and Projected Health Care Needs}

Our health care needs analysis compared diagnosed conditions of Veterans and non-Veterans, as well as those of VA patients and Veterans who do not use VA health care.

Without adjusting for demographic differences between Veterans and non-Veterans (for example, Veterans are older and predominately male), we found that the prevalence rates of many diagnosed health conditions are higher in the Veteran population. Veterans are more likely than non-Veterans to be diagnosed with cancer, diabetes, hearing loss, and PTSD. A limitation of our analysis is that we observed only diagnosed conditions rather than true underlying health status. Because Veterans are more likely to be insured than non-Veterans, they may be more likely to receive a diagnosis than their non-Veteran counterparts.

Veterans are also on average older than non-Veterans, and many conditions that we explored in our analysis are more likely to be diagnosed as individuals age. In addition, Veterans are predominantly male (greater than 90 percent), whereas only 40 percent of U.S. non- 
Veterans are male. ${ }^{1}$ Therefore, conditions that are more likely to affect men will naturally have higher unadjusted prevalence rates in the Veteran population. Other characteristics that differ between the two populations may have similar effects on unadjusted rates. To compare health care needs for Veterans with their non-Veteran counterparts, we adjusted our estimates to account for age, sex, race/ethnicity, region of residence, and whether the individual resides in a metropolitan area.

After adjusting for these characteristics, we found that Veterans continue to have higher prevalence rates of most conditions, including mental health conditions. This result is particularly salient for PTSD. Veterans are 13.5 times more likely than non-Veterans to be diagnosed with the condition. Even though fewer than 5 percent of Veterans are diagnosed with PTSD, this condition is even rarer in the non-Veteran population. PTSD and mental health conditions more generally are negatively correlated with income and employment status; higher income and being employed are correlated with lower rates of PTSD and mental health problems. Yet, these Veteran to non-Veteran patterns hold even if we adjust for income and employment status, with Veterans still more likely than non-Veterans to be diagnosed with PTSD and mental health conditions. Unemployed Veterans have particularly high rates of PTSD, both overall and in comparison with unemployed non-Veterans.

Our adjusted estimates showed that Veterans are also more likely than non-Veterans to be diagnosed with chronic conditions, such as diabetes and hypertension. In general, differences between Veterans and non-Veterans become more pronounced with age, although, for mental health conditions, we found the greatest differences at younger ages.

Despite these higher rates of diagnosis among the Veteran population, an analysis of disease burden on daily living suggests that Veterans are less likely than non-Veterans to need help with ADLs, such as bathing and dressing, as well as IADLs, including such chores as housework, buying groceries, and using the phone. Veterans are no more likely than non-Veterans to have multiple comorbid or co-occurring conditions. We offer a couple of explanations for why Veterans may be more likely to have chronic and other conditions, but less likely to experience difficulties with daily functions. One possibility is that the types of health conditions faced by Veterans are not the ones that create daily living challenges, such as memory, personal care, mobility, or work-disabling conditions. Alternatively, because we are unable to control for combat experience using survey data, our ADLs results may reflect better functioning among non-combat Veterans. We also examined adjusted differences in comorbid mental health and estimated that Veterans were approximately three percentage points more likely than nonVeterans to have a diagnosed mental health conditions and any of the 29 other conditions we investigated in our health care needs analysis. Veterans were statistically neither more nor less likely than non-Veterans to experience comorbid mental health with any of the 16 Charlson conditions, any ADL limitation, or any IADL limitation.

Next, we examined how the diagnosed health care needs of VA patients differ from Veterans who do not use VA. Unadjusted prevalence rates of most conditions are higher among VA patients than they are among Veterans who do not use VA, with the largest differences exhibited in hypertension, diabetes, GERD, cancer, COPD, and PTSD. Even after controlling for a

\footnotetext{
1 The entire U.S. population is nearly half male (49 percent), but that includes Veterans, most of whom are male. When the population is divided into Veterans and non-Veterans, data indicate that only 40 percent of non-Veterans are male. In addition, survey data used to generate these percentages exclude individuals in correctional, juvenile, and other institutions, who are most likely male and therefore drive the percentage of non-Veteran males down.
} 
basic set of demographic variables, VA patients have higher rates of these conditions, although the differences between the two groups' prevalence rates are smaller. PTSD once again stands out as a condition that is much more common in VA patients than non-patients

We investigated whether disease burden was more prominent in VA patients than in Veterans who do not use VA. VA patients were more likely to have a Charlson comorbidity index greater than one and were more likely to have at least one IADL limitation, relative to Veterans who do not use VA. Similarly, VA patients with a mental health diagnosis are more likely than Veterans who do not use VA to also be diagnosed with one of the other health conditions examined in Chapter Five.

We used VA encounter data to examine rare, service-connected conditions that may uniquely affect Veterans. Among the conditions we considered, the prevalence rate-measured as the percentage of Veterans who receive treatment from VA for a particular condition-is highest for substance use disorder at 6.3 percent. Slightly less than 2 percent of Veterans who receive treatment at VA were diagnosed with TBI. Amputation and spinal cord injury were each diagnosed in 0.5 percent of VA patients, and the prevalence rate for burns was 0.1 percent.

We also projected the prevalence of health conditions over the 10-year time horizon, 2015-2024, using MEPS data covering health care received in all sectors, not just from VA. We predict an increase in the prevalence of chronic conditions that affect both Veterans and non-Veterans, such as hypertension, diabetes, and cancer, all of which become more common as an individual ages. Mental health conditions are expected to become slightly more common among Veterans, rising from 19.4 percent in 2015 to 20.7 percent in 2024 . The prevalence of PTSD, however, is expected to remain relatively constant at around 3 percent. Like Veterans more generally, the prevalence of diagnosed hypertension, diabetes, and cancer is expected to rise among VA patients. The patterns of mental health and PTSD are also similar for Veterans and VA patients, though the levels and projected increases are higher among patients. The prevalence of diagnosed mental health conditions is expected to increase from 28 percent to 34 percent, and the rate of diagnosed PTSD is projected to grow from 8 percent to 10 percent.

\subsubsection{Sensitivity of Projections to Policy Changes}

Our estimates of the demographic characteristics of the current and future Veteran population, as well as our analysis of the health care needs of Veterans and VA patients, assumed, implicitly, that the policy arena and other characteristics of the current environment would continue into the future, with the exception of specific data inputs and trends. For example, our analysis accounted for DoD's planned reduction in the size of the active duty military. Our baseline projections, however, did not account for future policy scenarios that might affect demand for VA health services. In order to test alternative assumptions about the future, we examined five scenarios that represent ways that policy changes may affect the number of eligible or enrolled Veterans or the rate at which Veterans rely on VA for health care.

Three scenarios involved VA policy changes - making priority groups $8 \mathrm{e}$ and $8 \mathrm{~g}$ eligible to enroll in VA, changing presumptive eligibility that would make Veterans who served in Vietnam and who now have hypertension eligible for benefits, and making VA health care services more accessible by removing barriers. In all of these cases, the number of VA enrollees and VA patients would increase. Allowing groups $8 \mathrm{e}$ and $8 \mathrm{~g}$ to enroll could have an enormous effect on the number of VA patients. Opening eligibility to these two groups could result in 4.8 million new enrollees, and more than 2.1 million new VA patients (a 35.1-percent increase 
in the patient population). Adding hypertension to the list of presumptive eligibility conditions would results in 363,000 new patients, or an increase of 6.4 percent.

Much attention has been paid in the past few years to access problems Veterans face in getting care from VA. Among respondents of NSV who report not using VA services, 12.4 percent $(1.8$ million) report that the barriers to access are a reason for non-use. If these obstacles are addressed, we estimate that up to 235,000 new patients might use VA for some of their health care needs.

The remaining two scenarios considered changes external to VA's control—a hypothetical future conflict and coverage expansion through the ACA.

We considered how the number of total Veterans and VA patients will increase if the U.S. military becomes engaged in a conflict in the next 10 years. Across different levels of combat exposure and policy environments, our results suggest that the number of new eligible Veterans may range from 1.6 to 2.6 million between 2015 and $2024-200,000$ to 1.8 million of whom will have combat exposure. In terms of new patients, depending on the assumptions of the conflict scenario, most of our analyses predicts between 500,000 and 925,000 new patients between 2015 and 2024.

The ACA may have opposite effects on enrollment and patient status for VA. On the one hand, some eligible, unenrolled Veterans may enroll with VA to satisfy the ACA's individual mandate, and some of these new enrollees may then choose to use VA for their health care needs. On the other hand, some VA patients may secure other insurance through either ACA's Medicaid expansion or the law's new subsidies for private coverage. These Veterans might then rely on that new insurance option, thereby reducing or eliminating their reliance on VA. We estimate that approximately 307,000 Veterans could enroll with VA as a result of the ACA mandate, and, of those, approximately 74,000 could become patients. Simultaneously, we estimate that 172,000 fewer current patients will rely on VA for health care needs. We estimate a net decrease of 98,000 VA patients in 2016 under the ACA coverage expansion scenario. We also estimate that this decrease will be larger if more states opt to expand Medicaid to cover all low-income adults.

\subsection{Limitations}

Our analysis has several important limitations. One critical constraint is that the data sets available to conduct the analysis are themselves limited. There has not been a full accounting of the Veteran population since the 2000 Census, and we had to cobble together data from several sources to estimate the total number of U.S. Veterans and their demographic characteristics. Compounding this issue is that available data sources often define Veterans slightly differently. For example, ACS asks about whether the individual ever served on active duty in the U.S. armed forces, reserves, or National Guard. MEPS, in contrast, asks whether the respondent was honorably discharged from active duty in the U.S. Army, Navy, Air Force, Marine Corps, or Coast Guard. The lack of alignment in definitions makes it difficult to directly compare the data, and could in some cases cause us to overestimate the number of eligible Veterans (for example, because Veterans who were other than honorably discharged are not eligible for VA care). 
In addition to the data challenges associated with defining the Veteran population, there are data challenges in understanding Veterans' health care needs. First, VA administrative data do not capture health care utilization across all sectors, including VA-provided and civilianprovided care. While MEPS captures all types of care, the sample of Veterans is small and may not be fully representative of the population. Second, the data sets we analyzed did not address respondents' underlying health status, and could not shed light on undiagnosed conditions or health care needs. Collecting information on undiagnosed health conditions is difficult but not impossible-for example, the National Health and Nutrition Examination Survey combines interviews and physical examination. While access to such data for Veterans could be useful, it would also require a costly and complex data collection process in which respondents participated in health examinations. Additional analyses would be necessary to determine whether collecting this type of data for Veterans would be worth the cost.

While a strength of our analysis was that we were able to combine both DoD and VA data to understand the diagnosed health conditions of new Veterans, we were unable to do a personspecific transition analysis between service member and Veteran. That is, the data available to use were not granular enough to allow us to observe individuals' transitions; all comparisons are at an aggregate level. More-granular information would allow for additional refinements, such as better understanding demographic differences in how active duty personnel transition into VA care.

Our analysis focused on projections of Veterans, their health and demographic characteristics, and the number of VA patients that can be expected over time. However, we did not address Veterans' demand for specific types of health care services. Understanding how demand for specific services will evolve in the future would be useful for VA planning purposes. Projecting demand, however, requires even more-detailed data and introduces additional uncertainty because changes in technologies, treatments, and scientific knowledge may influence the type of health care services that Veterans will require.

An additional limitation is that, in some cases, it was very difficult for us to distinguish between age and cohort effects. For example, recent Veterans are more likely to use VA health care than other Veterans. In part, this reflects a general pattern that young Veterans are more reliant on VA than older Veterans, who are more likely to have access to outside health insurance through an employer or Medicare. However, higher utilization among newer Veterans may also reflect generational changes in utilization patterns that may persist over time. Even with sophisticated statistical methods, it is not always possible to differentiate these two possibilities. To some degree, only time will tell exactly how utilization of VA services will evolve among Afghanistan and Iraq Veterans as they age.

While not a limitation per se, the scenarios that we evaluated in this section are speculative and based on assumptions that may diverge from actual conditions in the future.

Finally, we were limited in our ability to compare our analyses with analyses conducted by VA due to limited documentation of existing VA models. 


\subsection{Recommendations for Consideration}

\section{Plan for a Changing Veteran Landscape}

For decades, the number of VA enrollees and patients has been increasing, despite a declining Veteran population. Beginning in 2019, we project that the number of VA patients will begin to taper off (Figure 7-1). This is a change that VA has not experienced in several decades, and it suggests that planning for the future may require a new approach. If VA responds to increasing enrollee and patient counts, and to the health care demands that this population requires, by expanding facilities, infrastructure, staffing, and other resources, the result may be a largerthan-needed footprint after 2019, when the population begins to taper off. Rather, VA should begin to plan for a shrinking population now by considering alternative approaches to meeting the needs of its population, such as purchasing care from the civilian sector even while the patient population continues to grow.

\section{Improve Tracking of Some Veteran Populations}

The U.S. Census is the most comprehensive source of information on Veteran status, but the most recent time these data were collected was 2000. Since then, detailed information on Veterans has been gathered only in ACS, which is known to underrepresent the Veteran population. Therefore, analyses that require full-scale accounting of the U.S. Veteran population rely on data that are 15 years old. While DoD data provide insight into the number of service members who become Veterans when they leave the armed forces, and a number of household surveys capture respondents' Veteran status and include socioeconomic, demographic, and health questions, current efforts to study the Veteran population require analysts to cobble together multiple data sources to augment the 2000 Census. Considering the duration of the post-9/11 conflicts and the number of service members and Veterans who have been exposed to combat during this period, as well as the policies that have been established to address the needs of the Veteran population, the nation is overdue for an update to the Veteran accounting that was

Figure 7-1

Trends in the Veteran Population, Enrollment, and Use of VA Care

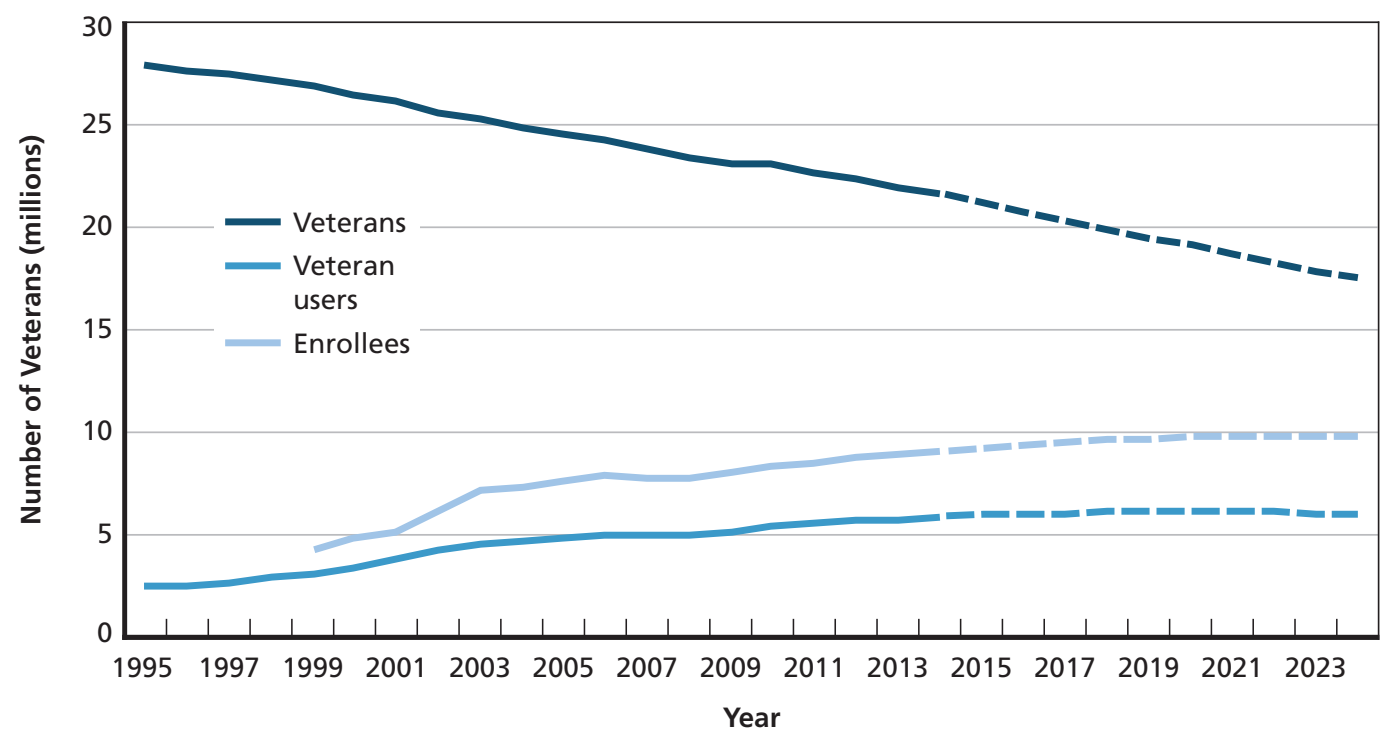


possible in the 2000 U.S. Census. Recapturing Veteran information in the U.S. Census will also allow sampling strategies used in smaller surveys to be refined to reflect the current population of Veterans. We recommend re-implementing data collection on the Veteran population in the 2020 Census.

Relatedly, efforts should be taken to closely monitor the nation's newest Veterans, those who served in the post-9/11 era, many of whom have experienced combat. It has been several decades since a generation of Veterans has been exposed to combat in such large proportions and over such a long period of time, and VA and the nation as a whole must prepare to respond to their evolving needs. Policies_ — such as the five-year enhanced eligibility for VA benefits — are already in place to provide extra coverage to combat Veterans immediately after they separate from the military. While Veterans tend to rely on VA less as they age and gain access to other sources of health care, post-9/11 Veterans may continue to use VA at higher rates because of increased rates of service-connected conditions often related to combat and because enhanced eligibility policies encouraged early use of VA. Closely monitoring this new generation of Veterans will ensure that VA is able to respond rapidly and appropriately to a young population whose needs may be different and evolving in a way that VA has not seen in several decades.

Because current VA encounter data only provide visibility of care that is delivered within VA, little is known about the health care needs of several other groups of Veterans. For instance, many Veterans enroll with VA at some point after separating from the military but then never use VA for care. They represent a population of potential consumers of VA health care under certain future circumstances, which we explore in our scenario analysis. Additional tracking of this non-using enrolled population could be achieved through surveys_including "MEPS for Vets" as described below_that address each Veteran's health care needs and where he or she is receiving treatment for these conditions.

VA policy currently prohibits Veterans in priority groups 8e and 8g, comprising higherincome Veterans without disabilities, from newly enrolling. There are no publicly available estimates of the number of Veterans in each of these priority groups. However, using the 2013 ACS, we estimated that there are 78,000 Veterans who meet the criteria for priority group 8e, and 4.7 million Veterans who meet the criteria for priority group 8g; none of these Veterans is currently eligible to enroll for VA health care services. We estimate that if VA were to allow priority group 8e Veterans to enroll, 34,000 new Veterans would become VA patients if they use $\mathrm{VA}$ at the same rate as other priority group 8 Veterans. Because $8 \mathrm{~g}$ is a much larger population (approximately 4.7 million Veterans), an extension of eligibility rules to include them would have a major impact on VA patient counts, by as many as 2.1 million new patients. Tracking these Veterans, especially for the purposes of being resourced to care for them if a policy change reopens enrollment privileges, is important.

Finally, requirements for eligibility for VA benefits are at least 24 months of military service, concluding with an honorable discharge, and for reservists, a period of activation. If future policies relax these requirements for Veterans who are not currently eligible for benefits, VA might experience an increase in the number of new enrollees and patients. For instance, service members who serve for fewer than 24 months are ineligible to receive VA benefits, but it is possible that someone may deploy to a combat zone before separating under these circumstances. If policy were changed such that the 24-month rule were relaxed if a Veteran had experienced combat, this may also result in an inflow of new VA patients. We did not have data on either of these groups for the analyses in this assessment, so we did not attempt to quantify how many Veterans may be affected by these hypothetical future policy changes. 


\section{Anticipate Potential Shifts in the Geographic Distribution of Veterans}

Between 2015 and 2024, the geographic distribution of Veterans will experience only moderate shifts, but there are opportunities to improve VA coverage in response to some of the movement, particularly among certain age groups. The Ohio River Valley and upper Midwest are expected to experience declines in the Veteran population, which suggests existing facilities may be consolidated. Regions such as Washington, D.C.; Charlotte, North Carolina; and San Antonio and Austin, Texas, are projected to see growth in the total number of Veterans, but VA has already positioned health services in those areas. Other growth areas in the West, including Montana, Wyoming, and Colorado, do not have VA coverage and may therefore benefit from the installation of telehealth and CBOC services.

Because the overall Veteran population will continue to age over the projection horizon, health services related to aging will be needed everywhere. However, younger Veterans under age 35 are expected to become more concentrated in a number of areas—such as Los Angeles; Dallas; Washington, D.C.; northern New Jersey; northern California; central Washington state; the Midwest; Wyoming; and Utah-thereby creating a need for health care services geared toward young adults in these regions.

Finally, the Veteran population in the Southwest has the most uneven alignment between Veterans and VA health care services. Population growth is expected, including among young Veterans, and the region is already characterized by widely spaced facilities with limited access to alternative VAMCs in close proximity to the patient population. As the demand for services expands, the Southwest region presents an opportunity for VA to grow to meet the health care needs of its growing population.

\section{Improve Data Collection Regarding Health Care Utilization}

The analyses in this report relied on a variety of data sources, including public use surveys that report whether a respondent is a Veteran, VA medical encounter data, other VA data sources, and DoD MHS claims data. While all of these data sources provided some of the information that was required for this independent assessment, and together they enabled an analysis of our key questions, none provided a comprehensive overview of Veteran reliance on VA, defined as the share of health care services that VA patients (or Veterans more generally) receive from VA versus other sources.

In Chapter Four, we used MEPS to estimate Veteran and VA patient reliance on VA. However, our analysis has limitations. First, the number of Veterans and VA patients surveyed in MEPS is small. Second, we were able to define several health care services in these data, but not the full range of services that are delivered or that EHCPM estimates. One way to allow for a more thorough, independent analysis of reliance is to oversample Veterans in MEPS and, if necessary, collect additional information on more-detailed services received through VA and other health care sources. This "MEPS for Vets" could build upon the existing MEPS at a much lower cost than it would require to develop a new survey.

\section{Incorporate Separation Patterns and Health Care Needs of Current Service Members into Projections}

In this assessment, we incorporated data on current service members, who will become Veterans in the future, in several of our analyses. Our demographic projection model incorporates counts of service member separations to augment Census 2000 data on Veterans. To estimate Veterans' future health care needs, we acquired data on diagnosed health conditions among 
service members separating from active duty and who received care through the MHS. Finally, in our scenario analyses, we estimated the number of service members who might separate from active duty and become Veterans in the case of a hypothetical future conflict.

Similar to our demographic projections approach, VetPop2014 uses separation data to account for new Veterans as they leave the military. VetPop2014 uses information on age, sex, and active/reserve status. Our approach uses that same information but also includes service branch and race/ethnicity, both of which may be important factors in accurately estimating Veteran counts and health care needs of the Veteran population.

Incorporating service branch allows the model to capture variation in sex across the services - which ranges from 7 percent female in the Marine Corps to 19 percent female in the Air Force - and, consequently, estimates of the sex composition of the Veteran population. Additionally, if the model accounts for an individual's branch of service, projections of future Veteran counts can better handle changes in end-strength and differences in separation patterns (years of service, retirement rates, etc.), which vary considerably by service.

Both the RAND approach to modeling Veteran demographic characteristics and VetPop2014 predict greater race/ethnicity heterogeneity in future Veteran populations, with VetPop2014 predicting greater minority growth than the RAND model. This difference may be a reflection of different approaches to controlling for minority mortality; the RAND model allows for race/ethnicity-specific mortality, and VetPop2014 does not. In addition, to the extent that there are differences in some health conditions by race/ethnicity, including this characteristic in models of health care projections will lead to more accuracy in predictions.

We estimate that future conflicts could lead to large increases in the number of Veterans who might seek care through VA, as well as large increases in the number of Veterans with combat exposure. For example, in some scenarios, we found that a conflict similar to those in Afghanistan and Iraq could lead to more than 2 million new Veterans and more than 500,000 new patients. Accounting for future conflicts is inherently uncertain. However, developing tools to estimate how conflicts could affect demand for VA services could make it easier for VA planners to respond in the event that a conflict scenario emerges. With an existing model in place, parameters and assumptions could be changed as policy details and information become available.

At present, VA does not have access to DoD MHS encounter data. For this study, we utilized MHS data from 2008 through 2014 to explore whether current service members (future Veterans) have different health care needs from current Veterans. We estimate that current service members are much more likely than current Veterans to have a diagnosed musculoskeletal condition at the time of separation from service. On the other hand, the rates of PTSD and mental health are higher in the existing Veteran population than they are among separating service members. This result may reflect a disincentive to present with mental health conditions while serving in the military. To the extent that individuals who separate from the military and become Veterans over our 2015-2024 projection window have different health care needs from the patients currently being served by VA, the addition of MHS data may be a crucial input to projecting the needs that VA must meet in the future.

Despite the important addition of MHS data to the analyses in this assessment, time constraints prevented us from conducting a longitudinal analysis of the transition from service member to Veteran. Ideally, the analysis would link medical records at the individual level to determine which health conditions that were diagnosed during an individual's time in service carry over to VA for follow-up and continued treatment. This analysis is feasible with the data 
used in this assessment, and we therefore recommend that it be undertaken to improve estimates of the types of health conditions and numbers of patients that VA should be prepared to treat as service members separate from the military.

\section{Develop Analytic Framework to Perform Scenario Testing}

Our analysis of five future scenarios highlights the importance of developing methods and models that can respond quickly and agilely to policy changes. While some of the policy changes we considered resulted in modest changes in the number of new Veterans and new VA patients, others estimated as many as hundreds of thousands of new Veterans and patients. VA OACT has a Veteran Healthcare Scenario Model that is able to estimate, for instance, how changes in demographic characteristics or economic conditions (such as employment or income) may affect demand for VA services and related costs. Expanding this model to include such events as future conflicts, changes in the civilian health sector, unanticipated changes in perceptions about health care quality, and groundbreaking new technologies, to name a few, will enable VA to address the types of uncertainties that current models may not address. Having methods in place to estimate the effects of these types of changes on Veteran demand for health care services will improve VA's efforts to meet the health care needs of its patient population.

\subsection{Overall Assessment A Conclusions}

Although the number of Veterans is expected to decline over the next decade, the number of Veterans seeking health care services through VA is expected to increase until its peak in 2019 and taper off or even decrease thereafter. These continued increases in VA utilization reflect changes in VA policies, such as outreach to enroll new patients and expansions to presumptive eligibility criteria, and may also reflect external trends, such as increasing costs in the private health insurance sector. We also estimate that VA patients will become somewhat older and sicker over time, a pattern that could increase demand for VA services despite the relative stability in the size of the patient population.

Reflecting VA patients' older age, the diagnosed prevalence of common chronic conditions (e.g., diabetes, cancer) is two to three times higher among Veterans than among nonVeterans. Thirty-three percent of all patients seen at VA have a mental health condition, and 8 percent have PTSD. When combined with the otherwise rare conditions related to combatamputation, TBI, blindness, and severe burns - and the vulnerable circumstances of some patients, VA handles a patient mix that is uniquely different from what community providers are used to.

However, demand for VA health care services is sensitive to the size and demographics of the VA population, Veterans' underlying health conditions, changes in eligibility and access, and shifts in Veterans' reliance on VA. Limitations with current VA data systems make it difficult to fully account for all of these issues when estimating the future needs of the patient population. There has not been a full accounting of all U.S. Veterans since the 2000 Census. As a result, it is difficult to be fully confident in estimates of even the size of the Veteran population, let alone their mix of demographic characteristics. Current VA surveys provide limited information on Veterans' total health care utilization and health care needs, particularly 
for Veterans who do not currently access health care at VA. Yet, understanding the needs of patients who do not use VA care is critical for projecting how the patient mix might shift due to policy changes, such as eligibility expansions.

Even among VA patients who regularly use health care, current VA data sources inadequately capture patients' total health care utilization. Both our analysis using MEPS and VA's own analysis using EHCPM suggest that many VA patients rely only partially on VA for their health care services. Yet reliance is sensitive to factors that are both inside and outside of VA's control, including perceptions about the quality of care available at VA facilities, wait times, and the cost of private-sector care. Better data to understand the full profile of Veterans' health care needs could help VA plan for changes in demand that could arise due to these internal and external factors. 

APPENDIX A

Current and Future Demographic Trends in the Veteran Population

\section{A.1. Data and Analytic Approach}

\section{A.1.1. Definition}

\section{A.1.1.1. Veterans}

For the population projection exercise, the team defines Veterans consistently with ACS, which defines Veterans as having "ever served on active duty in the U.S. Armed Forces, military Reserves or National Guard. Active duty does not include training for the Reserves or National Guard, but does include activation, for example, for the Persian Gulf War." Once an individual has ceased to serve on active duty in any of these capacities, he or she is considered a Veteran for the purposes of the projections. Note that the team does not have information on length of service (only eras of active duty), or on status of discharge. Thus, not all Veterans in the projection exercise may qualify for VA services. For example, Veterans who served less than two years, or Veterans with "bad paper" discharges (dishonorable, other-than-honorable, and bad conduct discharges) are all ineligible for VA services, but are included in the projection exercise. No federal agency publishes the numbers of "bad paper" discharges, but a range of sources suggests that dishonorable discharges represent 1 percent and other bad paper discharges are an additional 3 percent of all separations (Carter, 2013; Philipps, 2013; Wicker, 1991). Chapter Four derives VA users from the overall population projection estimates.

\section{A.1.1.2. Definition of Demographic and Geographic Characteristics}

See Table A-1 for the demographic and geographic characteristics used in our analysis.

Race/ethnicity is coded as non-Hispanic white, non-Hispanic black, Hispanic, non-Hispanic Asian, or non-Hispanic other. In cases where individuals report multiple race categories, they are coded as "non-Hispanic other" as limited by the data.

The analysis defines seven service eras: pre-1950, Korean War (July 1950-January 1955), pre-Vietnam peace era (February 1955-July 1964), Vietnam era (August 1964-April 1975), post-Vietnam peace era (May 1975-July 1990), Gulf War era (August 1990-August 2001), and post-9/11 (September 2001 or later). Individuals are grouped into the most recent active duty wartime era they served in (if they report multiple periods of service), or, if they only served during peace time, they are grouped into their most recent peacetime era.

For units of geography the team uses PUMA: Public Use Microdata Area. Since 2005, the ACS has collected information based on PUMAs. PUMAs are geographic units used by the U.S. Census Bureau. The state governments drew PUMA boundaries for the 2000 Census to allow reporting of detailed data for all areas. There were a total of 2,071 PUMAs in the 2000 Census and 2,351 in the 2013 ACS. Because PUMA boundaries changed over time, the team 
Table A-1

Demographic and Geographic Characteristics

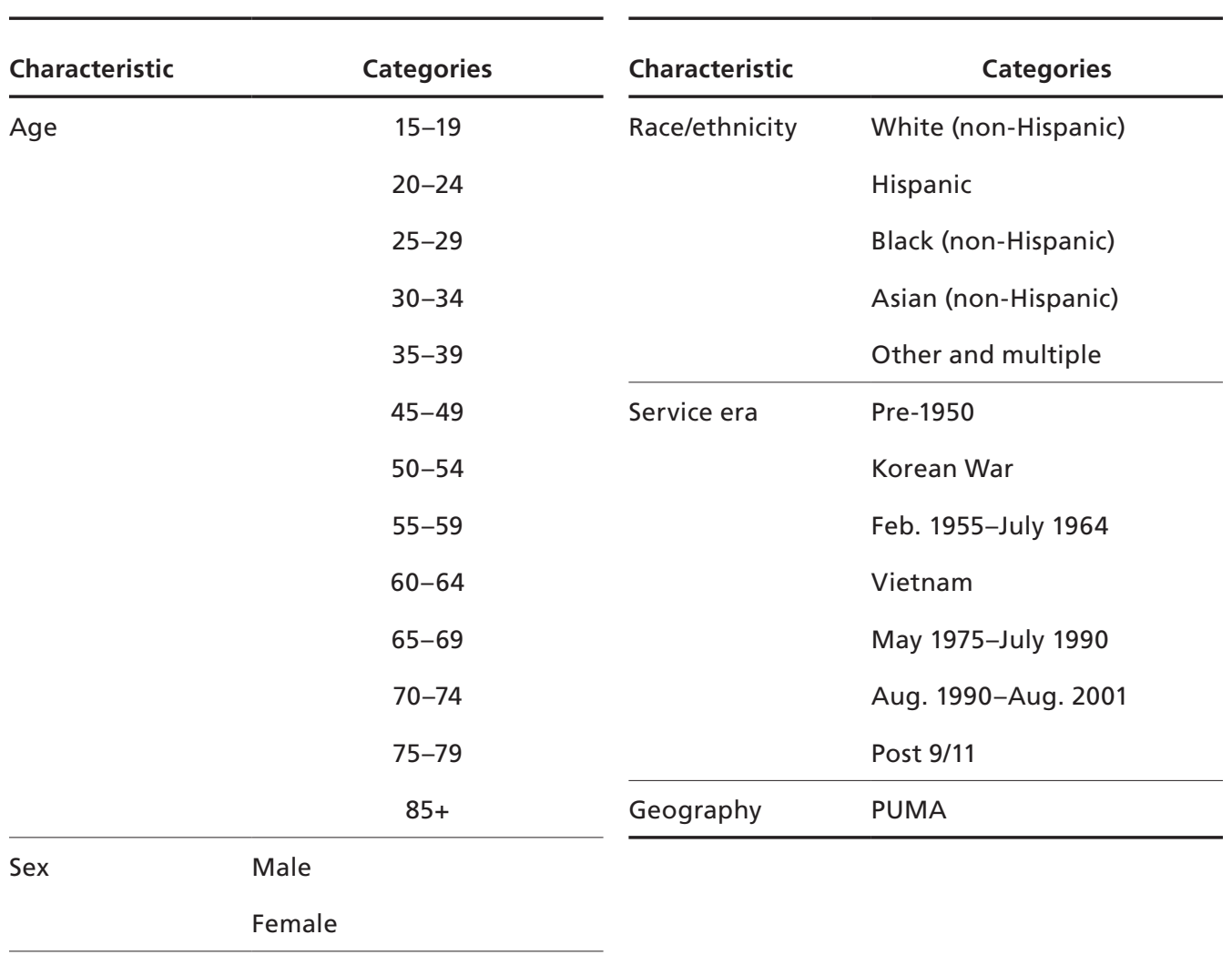

generated geographical areas compatible across the surveys, taking the 2013 ACS boundaries as the baseline. These comparable areas are used throughout the population projection exercise. Note that rural and urban areas may be simultaneously contained within a single PUMA (they are not necessarily 100 percent rural or 100 percent urban). Our use of PUMAs is partly driven by necessity (they are the smallest geographic unit available in the ACS data we rely on), but PUMAs also provide some benefits to our modeling approach. PUMAs are designed to contain populations of 100,000 , which ensures that each PUMA contains population sizes amenable to even relatively small cell sizes (older Asian female Veterans, for example). Large cities, such as Los Angeles, contain several PUMAs. In areas with sparser populations, a single PUMA may contain multiple counties. In contrast, geographic units, such as county, are not based on population size; as of 2013 there were 3,144 counties and county equivalents in the United States, and it is likely that many counties would have Veteran populations too sparse to model reliably. However, the use of PUMA does present some special challenges for mapping purposes. Since PUMAs are groups of 100,000 people (in practice, they can sometimes reach upward of 150,000), they can be geographically small in dense urban areas. In fact, 25 percent of all PUMAs fall within a 40-mile radius of just 10 cities. For example, Figure A-1 depicts the PUMAs that contain the population of Los Angeles, California. There are 54 PUMAs within 20 miles of downtown Los Angeles - most of which would be near invisible on a page-size map of the United States. As such, rather than shading these maps by the population inside each PUMA, we shaded them according to the total population near each PUMA. Specifically, we shaded each PUMA based on the total population of the PUMA within 40 miles of each PUMA center. 
Figure A-1

PUMAs in Los Angeles, California

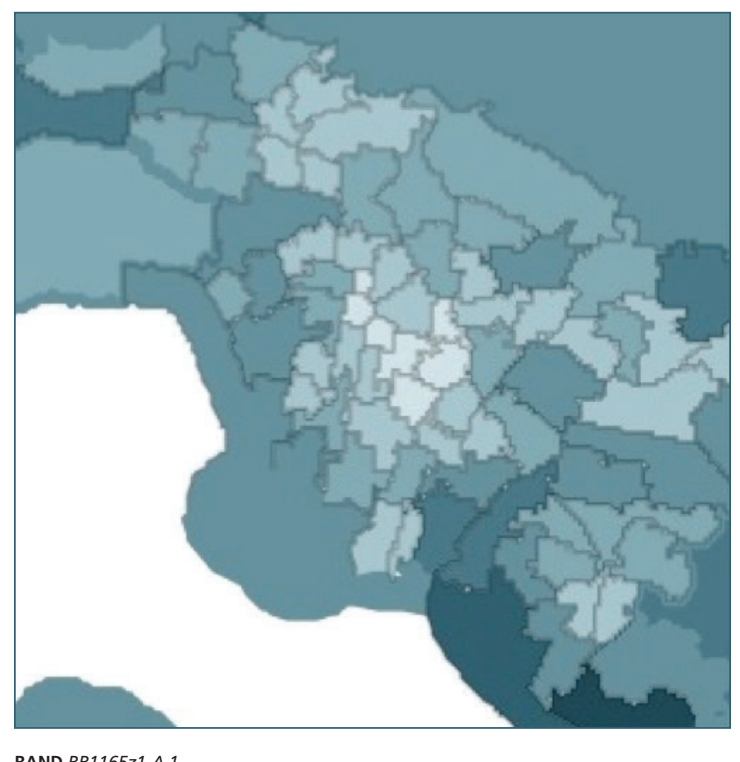

\section{A.1.2. Data Sources}

2000 Census: The 2000 Census collected information about the 115.9 million housing units and 281.4 million people in the United States on April 1, 2000. A 5-percent sample of people and housing units received a more detailed long form survey that contained questions including Veteran status and periods of service. As a starting point, the team used the 5-percent sample 2000 Census data to assess the baseline Veteran population in 2000. The 2010 Census did not include a long form, and did not collect information on Veteran status. The 2010 Census short form included only basic demographic questions (e.g., name, relationship with head of household, sex, age, Hispanic origin, race) and household information (e.g., number of people in the household, whether the home is owned or rented). The 2000 Census long form asked detailed demographic and household questions, including Veteran status and time that person served on active duty in the U.S. Armed Forces. As of the 2010 Census, detailed sociodemographic and other information is collected in the ACS, rather than the Census.

Active Duty Master and Loss files 2000-2014: The Active Duty Master file provides an inventory of all individuals on active duty (excluding reservists on active duty for training) for the Army, Navy, Marine Corps, Air Force, Coast Guard, Public Health Service, and National Oceanic and Atmospheric Administration Commissioned Corps at a point in time. Relevant personal data elements include date of birth, sex, race, and ethnic group. Relevant military data elements include months of service and basic active service date, as well as anticipated service contract end date. The Active Duty Military Personnel Transaction File contains a transaction record for every individual entrance, separation, or reenlistment in the Army, Navy, Air Force, Marine Corps, and Coast Guard within a specific time frame. The active duty loss files are subsets of the Master/Transaction file. The team used these data to supplement the 2000 Census counts for April 2000-December 2014. Each separation or "loss" indicates an incoming Veteran to the civilian population. 
Work Experience (WEX) and Contingency Tracking System (CTS) files 20002014: The WEX contains a longitudinal record for each individual who has served in the active or reserve forces since September 1990. For those individuals, the WEX includes information on service back to 1975. The file is organized by "transactions"; in other words, a new record is generated whenever there is a change in the key variables—service/component/reserve category, pay grade, occupation (primary, secondary, or duty), and unit identification code. The WEX is built from information in DMDC's Active Duty Master Personnel Edit File, equivalent reserve files, and the underlying service files. Information on actual deployment can be found in a sequence of "contingency files." The most recent of them is the CTS file. It contains one record for every activation or deployment in support of the conflicts in Iraq and Afghanistan. Using the WEX and CTS files enabled the team to identify Veterans who have served in the reserves or National Guard and who were activated at some point and add them to the incoming Veteran population each year. Note that the CTS file will not identify reserve or National Guard personnel who were activated for other conflicts, such as Bosnia, and the team acknowledges that the analysis will slightly underestimate the reserve/National Guard Veteran population.

2005-2009 and 2009-2013 ACS five-year estimates: ACS is an ongoing mandatory survey conducted by the U.S. Census Bureau that collects data each year to bridge intercensal periods and provide detailed information about the population, including Veteran status. The ACS also includes information on current location and location in the previous year. The analysis uses the ACS to determine Veteran geographic distribution and migration patterns. It was not possible to use the ACS to accurately measure the number of Veterans in the population; the ACS is generally acknowledged to undercount Veterans, though it is assumed to accurately capture the distribution of Veteran characteristics (age, sex, race/ethnicity, service cohort, location). For example, the 2013 ACS estimates of total Veteran population are roughly equivalent to our own estimates of the number of Veterans observed in the 2000 Census who are estimated to still be living in 2013 - that is, the ACS estimates effectively undercount by the number of new Veterans who entered the population from 2000 through 2013. For this reason, the team produced a set of population projections using a combination of Census 2000 data, DoD data, and ACS data. More specifically, the team used the 2005-2009 and 20092013 ACS five-year estimates available through the American FactFinder website (U.S. Census Bureau, undated a). ACSs prior to 2005 do not have information about residence in previous year, which is necessary for migration estimates.

Since 2005, the ACS has collected information on PUMAs of residence one year before the survey. PUMAs are geographic units used by the U.S. Census Bureau. The state governments drew PUMA boundaries for the 2000 Census to allow reporting of detailed data for all areas. There were a total of 2,071 PUMAs in the 2000 Census and 2,351 in the 2013 ACS. Because PUMA boundaries changed over time, the team generated geographical areas compatible across the surveys, taking the 2013 ACS boundaries as the baseline. These comparable areas are used throughout the population projection exercise. Note that rural and urban areas may be simultaneously contained within a single PUMA (they are not necessarily 100 -percent rural or 100-percent urban). 


\section{A.1.3. Population Projection}

The projection is estimated using a cohort component approach, a standard demographic method of projecting populations based on births and deaths over time (Preston, Heuveline, \& Guillot, 2001). "Births" in this application of the model are new Veterans. Once the overall national projection has been estimated, the analysis then considers Veteran location and migration through the period. Broadly, the team begins with a comprehensive count of Veterans from the 2000 Demographic Census. The next step is to add observed new Veterans each year through 2014 using Department of Defense data, and apply annual age, sex, and race/ethnicity specific mortality rates to everyone from 2000 through 2024. Once the total 2014 population is calculated, the analysis distributes the Veteran population geographically according to observed Veteran data (along a variety of characteristics). The team estimated annual Veteran migration, based on observed Veteran migratory movements, and applied those migration rates to the estimated 2014 population to derive the final 2014 population and distribution. The team then applied the derived 2014 geographic distribution to the 2015 population estimates, and applied migration adjustments to derive the 2015 population distribution. This process continues through 2024. Projections are calculated using the U.S. Census Bureau's Rural and Urban Projection software (See U.S. Census Bureau, undated b).

\section{A.1.3.1. National Projection}

The national population projection consists of two main components: baseline Veteran population (at 2000) and projected new Veterans (through 2024).

1. The analysis begins with a well-measured historical baseline Veteran population and adds the number of new Veterans entering the civilian population each year afterward.

2. From the initial year that the Veteran population is assessed, the baseline and incoming Veteran population is progressed through a cohort component projection model in which age, sex, and race/ethnicity groups are subjected to age, sex, and race/ethnicity specific mortality throughout the projection period (until 2024). ${ }^{1}$

3. Once the total Veteran population is projected through 2024, the team estimates location and migration of Veterans each year throughout the period based on observed and projected trends.

Projections are produced separately for each service era, and combined for national totals.

\section{A.1.3.2. New Veterans}

"Births" in the population projection are assessed using data containing a census of observed transitions to Veteran status, and are extrapolated for future periods. The majority of new Veterans 2000-2014 are measured using the Active Duty Master and Loss Files. We supplement this with the WEX and CTS files to identify reserve and National Guard personnel who have been activated at some point (2000-2014). For the 2015-2024 period, we estimate the number of new Veterans each year using transition probabilities based on age, sex, race/ethnicity, and branch of service based on total force size derived from WEX. We also assume downsizing of

1 Note that projections begin at 17; individuals may join the Armed Forces with parental consent at age 17. 
the Armed Forces following announcements by the Army (Tan, 2015) and other information, ${ }^{2}$ with a total active duty force of 1.25 million by 2018 (89 percent of 2010). Specifically, by 2018 we assume an Army of 445,000 (79 percent of 2010), Air Force of 311,000 (96 percent of 2010), Navy of 311,000 (97 percent of 2010), and Marine Corps of 186,000 (93 percent of 2010). We further assume that there will be no significant future conflicts during the projection period, which also impacts the number of reserve and National Guard personnel who will be activated during the period.

\section{A.1.3.3. Mortality Rates}

Mortality rates are based on a combination of mortality rates published by CDC and mortality rates obtained from VA OACT. OACT estimates 2014 Veteran population mortality using a variety of administrative data, IRS data, and Social Security Administration data. The Veteran-specific rates are available by sex, but not race/ethnicity. Our analysis used the most recent (2011) CDC rates to derive race/ethnicity specific mortality rates that reflect OACT rates overall, proportionately distributing mortality rates across sex-race/ethnicity groups proportionate to differences observed in the national population. This approach is summarized below:

1. Calculate the proportion of Veterans in each age group for each race/ethnicity, e.g., for ages 20-24.

2. Multiply the proportion of Veterans in each age-race/ethnicity cell by the national mortality rate (deaths per 100,000) for that cell. This would be the death rates of Veterans if they had the same rates as civilians in each age-race/ethnicity cell.

3. Calculate the ratio of overall Veteran mortality rate to the rate if Veterans had the same rates as civilians to get the proportional difference in rates.

4. Multiply the civilian race/ethnicity rates by this difference in rates at each age. This gives us the same mortality rates as the VetPop2014 model, but spread proportionately through the race/ethnicity groups based on civilian rates. It does assume that the inflation/deflation factor at each age is the same for each race/ethnicity.

\section{A.1.4. Baseline Geographic Distribution, 2005-2024}

While the ACS undercounts Veterans, the analysis assumes that it accurately captures Veterans' geographic distribution. The analysis applies Veteran geographic distribution (by fiveyear age group, sex, race/ethnicity, service cohort, and PUMA) from the 2005-2009 and 2009-2013 ACS five-year estimates to the national Veteran populations for 2005-2024 in order to assess initial geographic distribution. 2005-2009 ACS five-year estimates were used to distribute 2005-2008 national population estimates. There are no multi-year ACS estimates for 2004-2008 or 2005-2008 periods. 2009-2013 ACS five-year estimates were used to distribute 2009-2013 national population estimates and 2014-2024 national population projections.

\footnotetext{
2 Personal communication from Air Force Enterprise Readiness Analysis Division (HQ USAF/A1PF) via email, Septem-
} ber 9, 2014 . 
A.1.5. Migration, 2014-2024

Next the team applies migration to the initial 2014-2024 distributions, as outlined below:

- Number of migrants (numerator of rates): Calculate number of migrants by five-year age group, sex, race/ethnicity, service cohort, PUMA, and PUMA in previous year using 2009-2013 ACS.

- Population of PUMA of origin at the beginning of the time interval (denominator of out-migration rates): Calculate number of Veterans by five-year age group, sex, race/ ethnicity, service cohort, and PUMA in previous year using 2009-2013 ACS. These data are merged to the number of migrants file.

- Population of PUMA of destination at the end of the time interval (denominator of in-migration rates): Calculate number of Veterans by five-year age group, sex, race/ ethnicity, service cohort, and PUMA using 2009-2013 ACS. These data are merged to the number of migrants file.

- Convert groups of PUMA in previous year to the PUMA level: Some PUMAs in previous year are combined in groups of PUMAs in ACS for confidentiality reasons. The team converts groups of PUMAs back to the PUMA level by disaggregating the number of migrants and population at the beginning of the period. This distribution is performed based on the population at the end of the period as a weight for each combination of fiveyear age group, sex, race/ethnicity, service cohort, PUMA, and group of PUMA in previous year. The files with relationship between PUMAs of migration and PUMAs are available in the Integrated Public Use Microdata Series (IPUMS-USA) website (Integrated Public Use Microdata Series, undated).

- Convert 2000 PUMAs into 2010 PUMAs: The 2009-2011 PUMA codes are based on the 2000 Demographic Census classification. We convert these codes into the 2010 Demographic Census classification, based on a geographic correspondence engine developed by the Missouri Census Data Center (2012). This conversion is applied to both the PUMA of current residence and the PUMA in previous year (after the conversion procedure from the topic above). The 2012-2013 PUMA codes are already available in the 2010 Demographic Census classification.

- Append 2009-2011 and 2012-2013 data and add distance: The team appends back 2009-2011 and 2012-2013 data, after the 2000-2010 PUMA conversion. Information on distance between PUMAs is estimated based on shapefiles available in the Census Bureau website (U.S. Census Bureau, undated c, undated d). Distance is merged to the 2009-2013 data with migration information.

- In-Migration rates: Calculate in-migration rates of Veterans by five-year age group, sex, race/ethnicity, service cohort, PUMA, and PUMA in previous year using 2009-2013 ACS. The denominator of these rates is the population of PUMA of destination at the end of the time interval. The team divided the estimated rates by five to originate annual migration rates, because data relates to 2009-2013.

- Out-Migration rates: Calculate out-migration rates of Veterans by five-year age group, sex, race/ethnicity, service cohort, PUMA, and PUMA in previous year using 2009-2013 ACS. The denominator of these rates is the population of PUMA of origin at the beginning of the time interval. The team divided the estimated rates by five to originate annual migration rates, because data relates to 2009-2013. 
- Future in- and out-migration rates (gravity models): The team estimates migration rates with zero-inflated Poisson regression models, based on 2009-2013 ACS, and applies these rates to the 2014-2024 period. These models are appropriate when the count dependent variable has a high incidence of zeros. For instance, out of 2,909,616 cells in the inmigration model, 2,133,534 have zero migrants (73 percent) and 776,082 have non-zero observations (27 percent). This approach predicts in- and out-migrants as a function of age, sex, race/ethnicity, service cohort, and squared distance, using population at risk as exposure. For in-migration, population at risk is located in the PUMA of destination at the end of the time interval, while population in the PUMA of origin at the beginning of the time interval is used as a control variable. For out-migration, population at risk is located in the PUMA of origin at the beginning of the time interval, while population in the PUMA of destination at the end of the time interval is used as a control variable. Regression coefficients from this model are used to predict in- and out-migration rates for 2014-2024 by applying coefficients to projected Veteran populations. In effect, the analysis assumes that age, sex, race/ethnicity, and service cohort migration patterns remain constant over the next 10 years. Regression models do not include year. Models that included year as a predictor (to capture time trends in migration) indicated that there were not significant time trends: The year effect was orders of magnitude smaller than other predictors and did not contribute meaningfully to predicted migration trends. More details about these gravity models are presented below.

- Number of in- and out-migrants: The analysis applies the predicted rates from the zeroinflated Poisson regression models to the initial 2014 population projection to obtain the number of in- and out-migrants in 2014 for each five-year age group, sex, race/ethnicity, service cohort, PUMA, and PUMA in previous year. Then the team collapses information on PUMA in previous year, in order to get the number of in- and out-migrants for each five-year age group, sex, race/ethnicity, service cohort, and PUMA.

- Adjustment of the number of in-migrants: The team adjusts the number of in-migrants in each cell based on the overall count of in-migrants and out-migrants in a specific year. More specifically, the adjusted number of in-migrants equals the original number of inmigrants, multiplied by the overall sum of out-migrants in 2014, divided by the overall sum of in-migrants in 2014. This procedure assures that overall net migration in 2014 equals to zero. The assumption behind this adjustment is that out-migration counts are more accurate than in-migration counts. Out-migration cells were estimated based on residence in a previous year, which is a group of PUMAs. The team allocates both the counts of migrants and the population of origin at the beginning of the time interval into the PUMAs within the group of PUMAs. This approach gives a higher chance of all cells having migrants, because the distribution is based on the population of Veterans in the area of destination at the end of the time interval, as described above. In-migration rates were estimated with information already at the PUMA level, which might generate more cells with small counts and affect the overall number of in-migrants.

- Net migration: The team subtracts adjusted in-migrants by out-migrants for each fiveyear age group, sex, race/ethnicity, service cohort, and PUMA cell and applies this net migration to the initial 2014 population, in order to get the final 2014 population.

- Weight calibration of counts of Veterans: The team performs a final adjustment of the counts of Veterans in all cells with a weight calibration procedure known as iterative proportional fitting (raking) of complex survey weights, through the ipfraking package 
in Stata. This procedure ensures that marginal counts of Veterans by five-year age group, sex, race/ethnicity, and service cohort at the PUMA level equal the national population projection in each year.

The analysis iterates through this process for subsequent years; i.e., use the final 2014 distribution (population after migration) as the baseline for the 2015 national population projection. Table A-2 summarizes the overall counts of Veterans by projected year, number of inmigrants, adjusted in-migrants, out-migrants, and net migration. The final three columns give an idea of the migration rates. As discussed above, the team utilized out-migration rates as the standard, which decrease from 2.97 percent in 2014 to 1.61 percent in 2024 and is consistent with lower mobility through time within the national territory.

\section{A.1.5.1. Gravity Models}

As described above, gravity models were estimated with zero-inflated Poisson regression models. Gravity models estimate migration rates based on population in the area of origin (at the beginning of the period), population in the area of destination (at the end of the period), distance between areas, and other control variables (age, sex, race/ethnicity, and service cohort). The implementation of gravity models is consistent with the study of internal migration determinants, which dates back to classical economic development theory, where migration is considered to be a mechanism that establishes regional spatial-economic equilibrium (Ravenstein, 1885, 1889). Migrants move from low-income to high-income areas and from densely to sparsely populated areas. Population streams are expected to occur between the poorest and wealthiest places and countries. Migration decisions are determined by "push" and "pull" factors in areas of origin and destination. Intervening obstacles (such as distance, physical barriers, immigration laws), as well as personal factors also influence migration flows (de Haas,

Table A-2

Counts of Veterans and Migration Variables, 2014-2024

\begin{tabular}{ccccccccc}
\hline Year & Veterans & In-Migrants & $\begin{array}{c}\text { Adjusted } \\
\text { In-Migrants }\end{array}$ & $\begin{array}{c}\text { Out- } \\
\text { Migrants }\end{array}$ & $\begin{array}{c}\text { Net } \\
\text { Migration }\end{array}$ & $\begin{array}{c}\text { In-Migration } \\
\text { Rate (\%) }\end{array}$ & $\begin{array}{c}\text { Adjusted } \\
\text { In-Migration } \\
\text { Rate (\%) }\end{array}$ & $\begin{array}{c}\text { Out-Migration } \\
\text { Rate (\%) }\end{array}$ \\
\hline 2014 & $21,579,290$ & 553,963 & 641,122 & 641,122 & 0.0 & 2.57 & 2.97 & 2.97 \\
\hline 2015 & $21,179,305$ & 553,188 & 612,482 & 612,482 & 0.0 & 2.61 & 2.89 & 2.89 \\
\hline 2016 & $20,763,195$ & 545,726 & 576,489 & 576,489 & 0.0 & 2.63 & 2.78 & 2.78 \\
\hline 2017 & $20,346,285$ & 533,323 & 537,150 & 537,150 & 0.0 & 2.62 & 2.64 & 2.64 \\
\hline 2018 & $19,928,403$ & 518,292 & 495,146 & 495,146 & 0.0 & 2.60 & 2.48 & 2.48 \\
\hline 2019 & $19,511,393$ & 503,313 & 452,512 & 452,512 & 0.0 & 2.58 & 2.32 & 2.32 \\
\hline 2020 & $19,097,747$ & 488,767 & 412,335 & 412,335 & 0.0 & 2.56 & 2.16 & 2.16 \\
\hline 2021 & $18,689,523$ & 476,259 & 374,713 & 374,713 & 0.0 & 2.55 & 2.00 & 2.00 \\
\hline 2022 & $18,287,262$ & 464,319 & 341,120 & 341,120 & 0.0 & 2.54 & 1.87 & 1.87 \\
\hline 2023 & $17,888,878$ & 453,210 & 310,196 & 310,196 & 0.0 & 2.53 & 1.73 & 1.73 \\
\hline 2024 & $17,494,154$ & 444,004 & 281,887 & 281,887 & 0.0 & 2.54 & 1.61 & 1.61 \\
\hline
\end{tabular}

SOURCE: National projections and 2009-2013 ACS five-year estimates. 
2007, 2009; Greenwood, Hunt, Rickman, \& Treyz, 1991; Lee, 1966; McDowell \& de Haan, 1997; Passaris, 1989). Economic, environmental, and demographic factors are assumed to drive migrants away from their places of origin and attract them to new places of destination.

Based on the regional equilibrium framework, distance is expected to play an intervening role on the levels of population flows. Previous studies took distances between areas into account by utilizing gravity models to estimate migration (Head, 2000; Lowry, 1966; Pöyhönen, 1963; Tinbergen, 1962). Gravity models address the distance between areas, as well as the changing population in the areas over time. The idea behind these models is to use distance between areas and population trends as instrumental variables to estimate the level of migration, before analyzing the migration rates. Gravity models consider the population in the area of origin (at the beginning of the period), the population in the area of destination (at the end of the period), distance between areas, and the proportion of migrants already living in a specific area (dependent variable). Distance is constant over time, but the population at the beginning and end of the period in each area has varying out- and in-migration trends over time.

Zero-inflated Poisson statistical regressions can generate gravity models for inter-regional migration flows, with a dependent variable measured in discrete units (integer counts of migrants) and a discrete probability distribution (Stillwell, 2009). These models are appropriate for this analysis, because they do not maintain error variances as constant for the different sizes of estimated flows, as is the case of "log-normal" models. In the case of migration flows between PUMAs, the model is also recommended because there are a significant number of smaller flows among the areas, as well as a small number of larger migration flows. The Poisson regression equation is

$$
\mathrm{M}_{i j}=\mathrm{b}_{0}+\mathrm{b}_{1} \times \mathrm{P}_{i}+\mathrm{b}_{2} \times \mathrm{P}_{j}+\mathrm{b}_{3} \times \mathrm{d}_{i j}+\varepsilon_{i j}
$$

where $M_{i j}$ represents migrants at the end of the period between areas of origin (i) and destination $(j) ; b_{0}$ is the constant; $b_{1}$ is the regression coefficient associated with the population in the area of origin at the beginning of the period $\left(\mathrm{P}_{\mathrm{i}}\right) ; \mathrm{b}_{2}$ is the coefficient associated with the population in the area of destination at the end of the period $\left(\mathrm{P}_{\mathrm{j}}\right) ; \mathrm{b}_{3}$ is related to the distance between PUMAs $\left(\mathrm{d}_{\mathrm{ij}}\right)$; and $\varepsilon_{\mathrm{ij}}$ is the random error term associated with all pairs of PUMAs.

In order to generate these flows, it is necessary to use migration information that indicates the location of residence at a specific previous moment. Information about the PUMA of previous residence (where the person was living one year before the survey) is included in the ACS since 2005. This migration information allows the estimation of (1) the population at the beginning of the period by age group, sex, race/ethnicity, service cohort, and PUMA $\left(\mathrm{P}_{\mathrm{i}}\right)$; (2) the population at the end of the period by age group, sex, race/ethnicity, service cohort, and PUMA $\left(\mathrm{P}_{\mathrm{j}}\right)$; and (3) migrants at the end of the period by age group, sex, race/ethnicity, service cohort, and PUMA of both origin and destination $\left(\mathrm{M}_{\mathrm{ij}}\right)$. This study utilized a matrix of distances between all PUMA centroids, in order to estimate the $\mathrm{d}_{\mathrm{ij}}$ component of the Poisson regression equation. The cells with no migration flows or no population are replaced by zero in the regression. As a way to control for the high prevalence of cells with zero counts of migrants (dependent variable), a dummy variable indicates whether the cell has zero migrants within the "inflate" option in the zero-inflated Poisson model.

The results from zero-inflated Poisson regression models are illustrated in Table A-3. The first model deals with in-migration, in which population in PUMA of destination at the end 
of the period is taken as the exposure variable. The model about out-migration utilizes population in PUMA of origin at the beginning of the period as the exposure variable. The general trends of variables are similar between these models and can be better visualized in the panels in Figure A-2. Men have lower chances to migrate, compared to women. On one hand, Veterans in older service cohorts are less likely to migrate, compared with those in 9/11 era. On the other hand, older Veterans are more likely to migrate, compared with younger Veterans (25-29 age group), specially starting at the 70-74 age group. These two results (service cohort and age) counterbalance each other, since Veterans in older service cohorts (less likely to migrate) are older in age (more likely to migrate). All race/ethnicity groups are more likely to migrate than whites. As expected, longer distances between PUMAs have negative impacts on the likelihood of migrating. This information was included as squared distance in order to make the variable more spread throughout the national territory, as well as to be consistent with gravity models. For the in-migration model, population in origin at the beginning of the period has a positive effect on migration, as we would expect of more populated areas sending more migrants. For the out-migration model, population in destination at the end of the period has a negative effect on migration, which is contrary to the original hypothesis, but this coefficient is not statistically significant.

Table A-3

Estimates from Zero-Inflated Poisson Regression Models for Number of Migrants (Dependent Variable)

\begin{tabular}{|c|c|c|}
\hline Independent Variables & In-Migration Model & Out-Migration Model \\
\hline Constant & $\begin{array}{l}-2.254 * * * \\
(0.0148)\end{array}$ & $\begin{array}{l}-1.803 * * * \\
(0.0138)\end{array}$ \\
\hline Female & ref. & ref. \\
\hline Male & $\begin{array}{l}-1.168 * * * \\
(0.0117)\end{array}$ & $\begin{array}{l}-1.321 * * * \\
(0.0108)\end{array}$ \\
\hline 9/11 era & ref. & ref. \\
\hline Gulf War era & $\begin{array}{l}-0.313^{* * *} \\
(0.0154)\end{array}$ & $\begin{array}{l}-0.264 * * * \\
(0.0153)\end{array}$ \\
\hline Peacetime post-Vietnam & $\begin{array}{c}-0.930 * * * \\
(0.0193)\end{array}$ & $\begin{array}{l}-0.874 * * * \\
(0.0190)\end{array}$ \\
\hline Vietnam era & $\begin{array}{l}-2.014 * * * \\
(0.0304)\end{array}$ & $\begin{array}{l}-1.836 * * * \\
(0.0290)\end{array}$ \\
\hline Peacetime pre-Vietnam & $\begin{array}{l}-2.237 * * * \\
(0.0419)\end{array}$ & $\begin{array}{l}-2.031 * * * \\
(0.0391)\end{array}$ \\
\hline Korean conflict & $\begin{array}{l}-3.241 * * * \\
(0.0470)\end{array}$ & $\begin{array}{l}-2.838^{* * *} \\
(0.0446)\end{array}$ \\
\hline
\end{tabular}


Table A-3-Continued

\begin{tabular}{|c|c|c|}
\hline Independent Variables & In-Migration Model & Out-Migration Model \\
\hline \multirow[t]{2}{*}{ Pre-1950 } & $-3.746 * * *$ & $-3.356 * * *$ \\
\hline & $(0.0594)$ & $(0.0563)$ \\
\hline \multirow[t]{2}{*}{$17-19$ years } & $1.059 * * *$ & $1.891 * * *$ \\
\hline & $(0.0745)$ & $(0.0813)$ \\
\hline \multirow[t]{2}{*}{ 20-24 years } & $0.574 * * *$ & $0.464 * * *$ \\
\hline & $(0.0189)$ & $(0.0189)$ \\
\hline $25-29$ years & ref. & ref. \\
\hline \multirow[t]{2}{*}{$30-34$ years } & $0.166 * * *$ & $0.222 * * *$ \\
\hline & $(0.0158)$ & $(0.0154)$ \\
\hline \multirow[t]{2}{*}{$35-39$ years } & $0.240 * * *$ & $0.257 * * *$ \\
\hline & $(0.0198)$ & $(0.0196)$ \\
\hline \multirow[t]{2}{*}{$40-44$ years } & $0.240 * * *$ & $0.295^{* * *}$ \\
\hline & $(0.0203)$ & $(0.0200)$ \\
\hline \multirow[t]{2}{*}{ 45-49 years } & $0.317 * * *$ & $0.327 * * *$ \\
\hline & $(0.0213)$ & $(0.0210)$ \\
\hline \multirow[t]{2}{*}{$50-54$ years } & $0.254 * * *$ & $0.265^{* * *}$ \\
\hline & $(0.0236)$ & $(0.0231)$ \\
\hline \multirow[t]{2}{*}{$55-59$ years } & $0.759 * * *$ & $0.762 * * *$ \\
\hline & $(0.0261)$ & $(0.0255)$ \\
\hline \multirow[t]{2}{*}{$60-64$ years } & $-0.180 * * *$ & $0.136 * * *$ \\
\hline & $(0.0352)$ & $(0.0333)$ \\
\hline \multirow[t]{2}{*}{$65-69$ years } & $0.0651^{*}$ & $0.277 * * *$ \\
\hline & $(0.0349)$ & $(0.0337)$ \\
\hline \multirow[t]{2}{*}{$70-74$ years } & $0.766 * * *$ & $0.777 * * *$ \\
\hline & $(0.0415)$ & $(0.0392)$ \\
\hline \multirow[t]{2}{*}{$75-79$ years } & $1.406 * * *$ & $1.306^{* * *}$ \\
\hline & $(0.0449)$ & $(0.0423)$ \\
\hline \multirow[t]{2}{*}{$80-84$ years } & $2.051 * * *$ & $1.813 * * *$ \\
\hline & $(0.0492)$ & $(0.0470)$ \\
\hline \multirow[t]{2}{*}{$85+$ years } & $2.091 * * *$ & $1.982 * * *$ \\
\hline & $(0.0619)$ & $(0.0582)$ \\
\hline White & ref. & ref. \\
\hline
\end{tabular}


Table A-3-Continued

\begin{tabular}{|c|c|c|}
\hline Independent Variables & In-Migration Model & Out-Migration Model \\
\hline Black & $\begin{array}{l}0.688 * * * \\
(0.01000)\end{array}$ & $\begin{array}{l}0.752 * * * \\
(0.00942)\end{array}$ \\
\hline Hispanic & $\begin{array}{l}0.285^{* * *} \\
(0.0126)\end{array}$ & $\begin{array}{l}0.339 * * * \\
(0.0120)\end{array}$ \\
\hline Asian & $\begin{array}{l}0.815^{* * *} \\
(0.0250)\end{array}$ & $\begin{array}{l}1.187^{* * *} \\
(0.0218)\end{array}$ \\
\hline Other & $\begin{array}{l}1.718 * * * \\
(0.0218)\end{array}$ & $\begin{array}{l}1.968 * * * \\
(0.0206)\end{array}$ \\
\hline Squared distance & $\begin{array}{l}-0.0000000222 * * * \\
(0.00000000233)\end{array}$ & $\begin{array}{l}-0.000000012 * * * \\
(0.00000000191)\end{array}$ \\
\hline $\begin{array}{l}\text { Population in origin at the } \\
\text { beginning of period }\end{array}$ & $\begin{array}{l}0.000963 * * * \\
(0.0000236)\end{array}$ & \\
\hline $\begin{array}{l}\text { Population in destination at } \\
\text { the end of period }\end{array}$ & & $\begin{array}{r}-0.0000036 \\
(0.000022)\end{array}$ \\
\hline Exposure variable & $\begin{array}{l}\text { Pop. in destination } \\
\text { at the end of period }\end{array}$ & $\begin{array}{l}\text { Pop. in origin } \\
\text { at the beginning of period }\end{array}$ \\
\hline Inflate model & & \\
\hline Constant & $\begin{array}{r}-31.38 * * * \\
(0.00114)\end{array}$ & $\begin{array}{r}-33.88 * * * \\
(0.00114)\end{array}$ \\
\hline Indicator of cells & $62.55^{* * *}$ & $67.48 * * *$ \\
\hline without migrants & $(0.00305)$ & $(0.00270)$ \\
\hline Non-zero observations & 776,082 & 776,082 \\
\hline Zero observations & $2,133,534$ & $1,132,194$ \\
\hline Total observations & $2,909,616$ & $1,908,276$ \\
\hline
\end{tabular}

SOURCE: 2009-2013 ACS five-year estimates.

NOTES: Robust standard errors in parentheses. *** $p<0.01,{ }^{* *} p<0.05,{ }^{*} p<0.1$. 
Figure A-2

Coefficients from Zero-Inflated Poisson Regression Models for Number of Migrants (Dependent Variable)

Coefficients from in-migration model
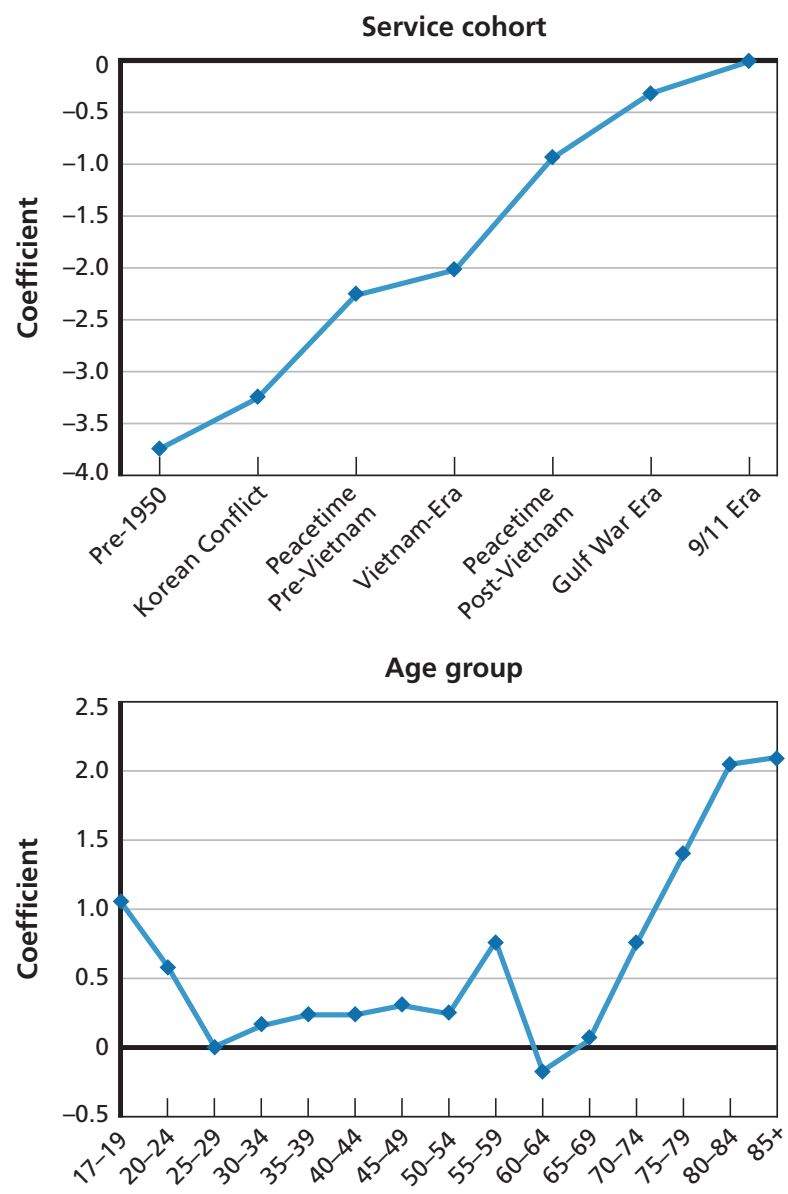

Race/ethnicity

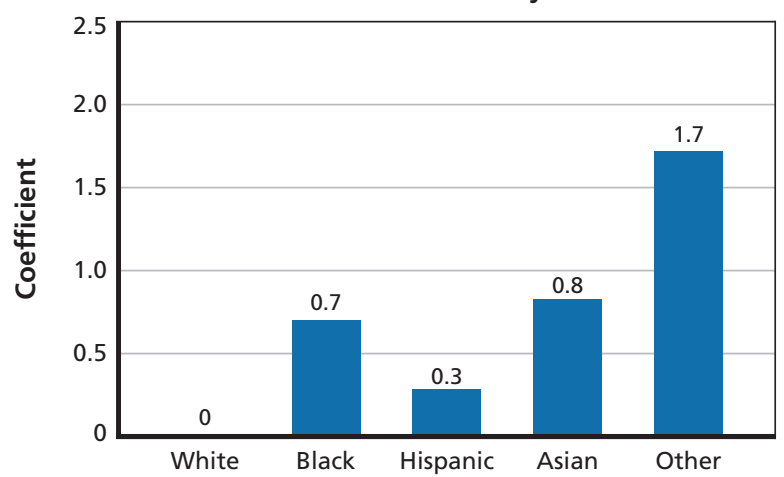

Coefficients from out-migration model

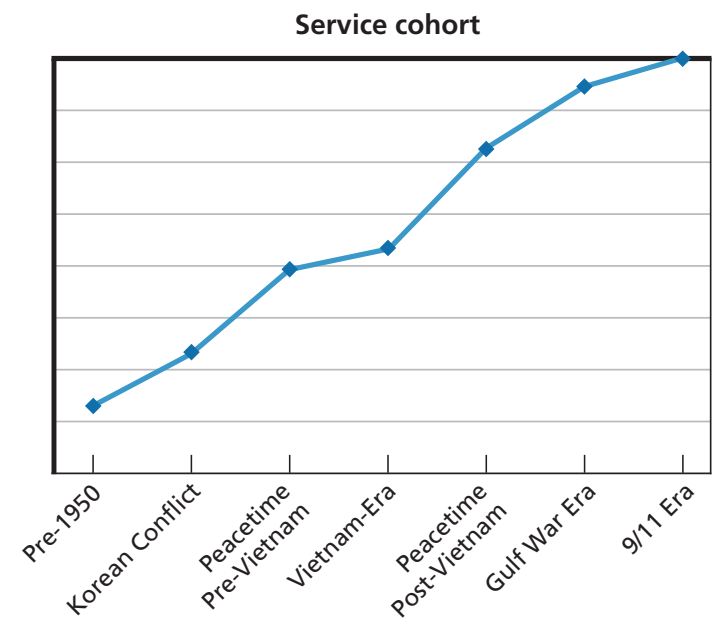

Age group

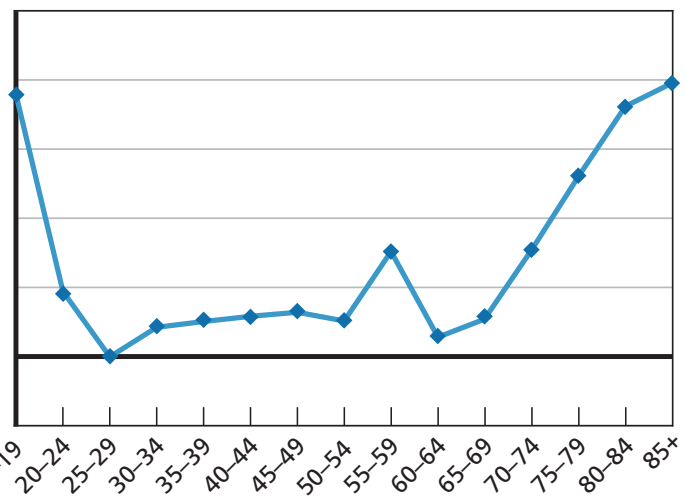

SOURCE: 2009-2013 ACS 5-year estimates.

RAND RR1165z1-A.2

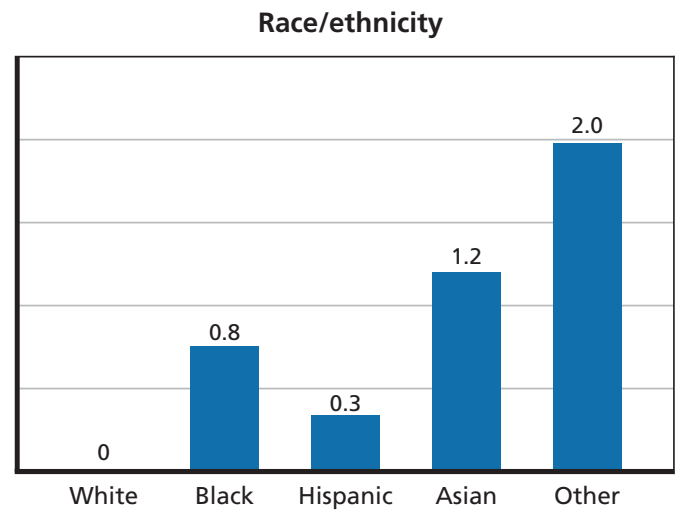




\section{A.1.6. Spatial Regression Models}

The team used spatial regression models to examine how growth and movement in the Veteran population is related to the location of VA facilities. These models follow the standard linear modeling format except for two cases.

First, observations are weighted so that all observations of approximately the same location have weights that sum to one. For example, there are 19 PUMAs within a 10-mile radius of downtown Chicago. Each PUMA could be given a weight of 1/19th to indicate that all cases are observed Veterans located near the same set of VA facilities. The weighting procedure used here follows this same principle, but without using a hard cut-off. Instead, each observation receives a weight of

$$
\frac{\sum_{i} 2^{-D_{i j} / k}}{\sum_{j} \sum_{i} 2^{-D_{i j} / k}}
$$

where $\mathrm{D}$ is a matrix of distances between observations $\mathrm{i}$ and $\mathrm{j} . \mathrm{K}$ is a scaling constant that indicates roughly what distance $i$ and $j$ are far enough away that the location of $j$ only counts as 50 percent the same as the location of $i$. This weighting allows observations to be scored along a continuum from "same location" to "different location" instead of using a hard cutoff.

Second, the population/net migration of each PUMA is predicted using the distance between its center and the nearest VA facility or other geographic feature. When the resulting coefficients are negative, it indicates that distance from a facility corresponds to smaller populations, i.e., more people tend to locate close to facilities. For estimation purposes, these distances are logged, because the effects of distance on behavior are known to be nonlinear. For example, the difference between 10 miles and 20 miles makes a significant impact on population behavior, but the difference between 210 miles and 220 miles has relatively little impactboth are about equally far away. 


\section{A.1.6.1. Spatial Regression Models of Migration in Relation to VA Facilities}

Table A-4 shows Veteran migration in 2014 as a function of VA facilities.

Table A-4

Migration in 2014 as a Function of VA Facilities

\begin{tabular}{|c|c|c|c|c|c|}
\hline \multirow[b]{2}{*}{ Covariate } & \multicolumn{5}{|c|}{$\begin{array}{l}\text { Adjusted Prevalence } \\
\text { (Std. Errors) }\end{array}$} \\
\hline & Model 1 & Model 2 & Model 3 & Model 4 & Model 5 \\
\hline \multirow[t]{2}{*}{ VAMC } & & & 59.28 & 53.71 & 52.59 \\
\hline & & & $(5.75)$ & $(5.94)$ & $(6.74)$ \\
\hline \multirow[t]{2}{*}{ CBOC } & & 64.22 & & 39.33 & 37.85 \\
\hline & & (10.56) & & (10.74) & (11.17) \\
\hline \multirow[t]{2}{*}{ City 2 million + } & & & & & 2.29 \\
\hline & & & & & $(6.11)$ \\
\hline \multirow[t]{2}{*}{ Southwest } & & & & & 3.72 \\
\hline & & & & & (4.99) \\
\hline \multirow[t]{2}{*}{ Intercept } & 79.78 & -132.59 & -156.40 & -264.25 & -289.74 \\
\hline & $(4.55)$ & $(64.22)$ & $(23.35)$ & (37.54) & $(48.47)$ \\
\hline
\end{tabular}

SOURCE: RAND analysis of VA, DoD, and Census data.

NOTES: Results in Table A-4 indicate that overall, Veterans tend to move farther away from VAMC and CBOC VA facilities-not closer. Distances to VAMC and CBOC have significant positive associations with net migration, indicating that Veterans are moving to PUMAs that are farther from facilities.

\section{A.1.7. Comparison to the VetPop2014 Model}

Two primary public sources of information regarding the complete current and projected Veteran population are the ACS, conducted by the U.S. Census, and the VetPop2014 model, produced by VA. According to the 2013 ACS there were 19.6 million Veterans ${ }^{3}$ - which is somewhat below RAND's estimate of 21.9 million for the same time point. In contrast, the 2014 estimates from the VetPop2014 and RAND models are quite similar: 21.9 million and 21.6 million, respectively. The VetPop2014 model was developed by OACT for Veteran population projections from 2014 to 2043. The model provides Veteran counts by age, sex, service era, and race/ethnicity at the county level.

VetPop2014 is the culmination of years of work and refinement by OACT. VetPop2014 represents the seventh iteration of the OACT Veteran Population Projection Model (the previous iteration was developed in 2011). Here we highlight ways in which our approach differs methodologically from VetPop2014 and how our projection results differ. Documentation for the VetPop2014 model is scarce, and we rely on information contained in an online two-page abstract (Office of the Actuary, 2014) and discussions with the VetPop2014 team at OACT. As a result, we do not assess the quality of the VetPop2014 model here.

3 Based on the 2013 ACS. 
Broadly speaking, VetPop2014 shares a similar projection approach to our model: Mortality rates are applied to a baseline population, new Veterans are added to the baseline population over time, and Veterans migrate throughout the projection period. The original baseline population for the VetPop model was Census 2000, as with our projections. (Recall that Census 2010 did not include Veteran status.) Key differences between the models are in data inputs and in migration modeling.

VetPop2014 applies age- and sex-specific mortality rates derived from mortality data that include (a) Veteran-specific information from VA administrative data and (b) U.S. population data from the Social Security Administration and the IRS. VetPop2014 assumes a slight mortality improvement for older Veterans due to longevity improvement, although by 2024 this is negligible. The VetPop2014 model does not incorporate race/ethnic-specific mortality rates. Our model differs here in that we apply race/ethnic-specific (in addition to age- and sexspecific) rates. As described in Section A.1.3.3, the mortality rates we use are a modification of the VetPop2014 rates we originally obtained from OACT that also incorporates the national race/ethnic differences in mortality reported by CDC. We do not assume any improvement in mortality through the projection period.

VetPop2014 projects annual separations (new Veterans) by age and sex, and by active and reserve component, using DoD data. The abstract states that "[b]ased on DoD's annual military separation data from FY1980 to FY2013 . . . VetPop2014 . . . developed a set of Time Series Models to project annual separations for various age and gender groups. . . . VetPop2014 Model then used historical county separation data based on VA administrative records along with migration information from the IRS to project the county level separation from FY2014 to FY2044 using predictive modeling techniques." VetPop2014 assumes that conflicts in the Gulf end by 2018, and that there are no other major conflicts in the next 30 years. Our model uses individual-level DoD administrative data to derive separation rates by age, sex, race/ethnicity, branch of service, and active and reserve status. Our projected separations reflect branchspecific trends in sex composition and separation rates, which is not reflected in VetPop2014 to our knowledge. ${ }^{4}$ Our projected separations also assume no future major conflicts, but we also assume downsizing of the various branches by 2018 following announcements by the Army and our internal estimates for the other branches. It is not clear whether VetPop2014 similarly assumes downsizing. Our model also excludes separations from the reserves and National Guard if they have not served any active duty time (either in the other branches, or while in the reserves/National Guard). This exclusion is based on the need for consistency with the way ACS measures Veterans as well as the requirement for active duty in order to qualify for VA access. We do not know whether VetPop2014 similarly excludes unactivated reserve and National Guard personnel. Unlike the VetPop2014 model, the RAND model does not derive geography-based separation rates, but rather geographically distributes new Veterans according to the Veteran age, sex, race/ethnicity, and service era distribution observed in the country at the time.

Finally, VetPop2014 models migration at the county level using historical data from VA, IRS, and the ACS. Predictive migration models are developed for various age (five groups)

4 The sex composition of the various branches varied in 2014: Women composed 12 percent of the Army, 19 percent of the Air Force, 16 percent of the Navy, and 7 percent of the Marine Corps according to our data. Similarly, separation rates varied in 2014: 14 percent for the Army, 13 percent for the Navy, 11 percent for the Air Force, and 16 percent for the Marine Corps. 
and sex cohorts. While we do not know more about the VetPop2014 migration models, this is where our models likely differ most. Our migration models are based only on data from the ACS, but the gravity models we use to predict migration from 2014 through 2024 reflect a wider range of Veteran characteristics: sex, service era, age (15 groups), and race/ethnicity-as well as distance and origin/destination population sizes. All of these characteristics were significant predictors of migration in our models. However, as we noted previously, migration plays a relatively minor role in overall population distribution in our models. We do not know how significant the role of migration is in the VetPop2014 model.

Figure A-3 compares RAND's projections of the number of Veterans with those from VetPop2014. Despite the differences between VetPop2014 and our model, the projected 2024 Veteran populations are relatively similar in size (19 and 17.5 million, respectively) and sex composition (11 percent female in both models). However, there are differences in terms of race/ethnic composition of the Veteran population, as shown in Table A-5. VetPop2014 predicts slightly higher percentages of black and Hispanic Veterans, while we predict higher per-

Figure A-3

Comparison of RAND and VetPop2014 Projections

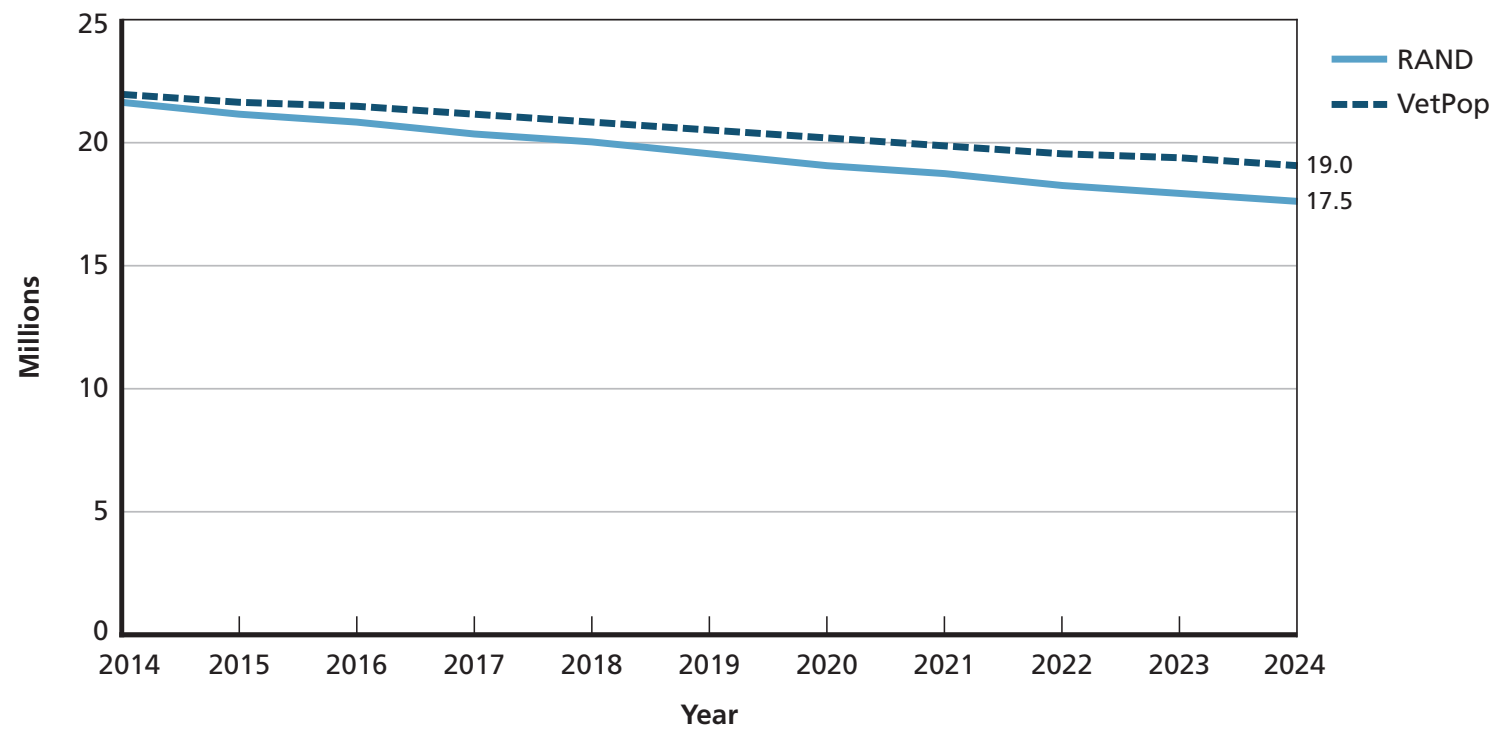

SOURCE: RAND analysis of VA, DoD, and Census data, and VetPop2014.

Table A-5

Projected Race/Ethnicity of Veteran Population in 2024, RAND and VetPop2014

\begin{tabular}{lcc}
\hline Race/Ethnicity & RAND & VetPop2014 \\
\hline White & 75.8 & 73.4 \\
Black & 12.7 & 13.9 \\
Hispanic & 7.3 & 8.6 \\
Asian & 2.3 & 1.8 \\
Other & 1.9 & 2.3 \\
\hline
\end{tabular}


centages of white and Asian Veterans. This is most likely a result of our differing mortality rates (white and Asian Veterans have lower mortality rates than black and Hispanic Veterans in our model, consistent with national mortality rates).

As the Veteran population is projected to grow more diverse over time, we suggest that the VetPop2014 model consider race/ethnicity differences in mortality rates. We also encourage detailed methodological documentation of the VetPop2014 model to provide transparency regarding the assumptions and methodology used for VA's projections.

\section{A.2. Detailed Results}

To ease tabular presentation of data, we refer to Census divisions (see Tables A-6 through A-11). There are nine Census divisions:

1. Pacific: Alaska, Washington, Oregon, California, and Hawaii

2. Mountain: Montana, Idaho, Wyoming, Nevada, Utah, Colorado, Arizona, and New Mexico

3. West North Central: North Dakota, South Dakota, Nebraska, Kansas, Minnesota, Iowa, and Missouri

4. West South Central: Oklahoma, Texas, Arkansas, and Louisiana

5. East North Central: Wisconsin, Illinois, Indiana, Ohio and Michigan

6. East South Central: Kentucky, Tennessee, Mississippi, and Alabama

7. Middle Atlantic: New York, Pennsylvania, and New Jersey

8. South Atlantic: Maryland, Delaware, West Virginia, Virginia, Washington DC, North Carolina, South Carolina, Georgia, and Florida

9. New England: Vermont, New Hampshire, Maine, Massachusetts, Connecticut and Rhode Island.

Over time, the East North Central and Middle Atlantic areas lose proportionately greater population (from 25 percent to 19 percent), while the South Atlantic area grows from 23 percent to 28 percent of all Veterans. There is slight movement of Asians from the Pacific to South Atlantic region (10-percent reduction/increase).

Younger Veterans are slightly less visible in the Pacific region over time. Older Veterans (45+) increase representation in the South Atlantic and West South Central regions, and decrease in the East North Central region.

Gulf War and later eras increase from 27 percent to 42 percent of the Veteran population by 2024 . There is no substantial geographic redistribution of cohorts. 
Table A-6

Veteran Demographics, by Census Division, 2014

\begin{tabular}{lcccccccc}
\hline Division & Total & Male & Female & Non-Hispanic Non-Hispanic & White & Black & Hispanic & $\begin{array}{c}\text { Non-Hispanic Non-Hispanic } \\
\text { Asian }\end{array}$ \\
& & & & Projected Populations & & \\
\hline & & & & & & \\
Pacific & $3,022,664$ & $2,768,670$ & 253,994 & $2,208,026$ & 216,651 & 311,222 & 221,788 & 64,977 \\
\hline Mountain & $1,774,653$ & $1,613,843$ & 160,810 & $1,443,094$ & 79,193 & 187,154 & 29,381 & 35,831 \\
\hline West North Central & 1,546617 & $1,434,679$ & 111,938 & $1,412,166$ & 75,838 & 30,530 & 9,123 & 18,960 \\
\hline West South Central & $2,498,912$ & $2,265,665$ & 233,247 & $1,798,409$ & 339,004 & 296,039 & 23,904 & 41,556 \\
\hline East North Central & $3,053,885$ & $2,852,543$ & 201,342 & $2,633,206$ & 307,780 & 69,691 & 16,749 & 26,459 \\
\hline East South Central & $1,422,091$ & $1,300,567$ & 121,524 & $1,146,055$ & 236,226 & 21,943 & 5,351 & 12,516 \\
\hline Middle Atlantic & $2,269,477$ & $2,121,759$ & 147,718 & $1,894,593$ & 227,448 & 108,235 & 22,759 & 16,442 \\
\hline South Atlantic & $5,033,535$ & $4,519,420$ & 514,115 & $3,751,124$ & 959,885 & 212,504 & 52,451 & 57,571 \\
\hline New England & 957,457 & 893,680 & 63,777 & 887,186 & 32,867 & 23,093 & 6,259 & 8,052 \\
\hline National & $21,579,294$ & $19,770,826$ & $1,808,465$ & $17,173,859$ & $2,474,892$ & $1,260,411$ & 387,765 & 282,364 \\
\hline
\end{tabular}

SOURCE: RAND analysis of VA, DoD, and Census data. 
Table A-7

Demographics, by Census Division, 2024

\begin{tabular}{lcccccccc}
\hline Division & Total & Male & Female & Non-Hispanic Non-Hispanic & White & Black & Hispanic & $\begin{array}{c}\text { Non-Hispanic Non-Hispanic } \\
\text { Asian }\end{array}$ \\
\hline & & & & Projected Populations & & \\
Pacific & $2,423,732$ & $2,169,126$ & 254,606 & $1,684,270$ & 193,428 & 260,508 & 205,130 & 80,396 \\
\hline Mountain & $1,588,614$ & $1,413,787$ & 174,827 & $1,247,369$ & 90,259 & 177,953 & 34,305 & 38,728 \\
\hline West North Central & $1,096,108$ & 984,939 & 111,169 & 965,072 & 67,238 & 36,084 & 7,640 & 20,074 \\
\hline West South Central & $2,328,168$ & $2,075,496$ & 252,672 & $1,598,352$ & 331,036 & 316,979 & 30,299 & 51,502 \\
\hline East North Central & $1,869,574$ & $1,707,224$ & 162,350 & $1,566,658$ & 203,306 & 57,218 & 16,548 & 25,844 \\
\hline East South Central & $1,267,367$ & $1,133,034$ & 134,333 & $1,007,906$ & 211,958 & 26,432 & 5,171 & 15,900 \\
\hline Middle Atlantic & $1,424,543$ & $1,292,532$ & 132,011 & $1,127,761$ & 163,605 & 94,635 & 19,752 & 18,790 \\
\hline South Atlantic & $4,847,982$ & $4,274,631$ & 573,351 & $3,481,203$ & 930,933 & 282,458 & 72,950 & 80,438 \\
\hline New England & 648,066 & 589,296 & 58,770 & 586,147 & 24,994 & 24,084 & 5,012 & 7,829 \\
\hline National & $17,494,154$ & $15,640,065$ & $1,854,089$ & $13,264,738$ & $2,216,757$ & $1,276,351$ & 396,807 & 393,501 \\
\hline
\end{tabular}

SOURCE: RAND analysis of VA, DoD, and Census data. 
Table A-8

Age, by Census Division, 2014

\begin{tabular}{lrrrr}
\hline Division & Age 17-34 & Age 35-44 & Age 45-64 & Age 65+ \\
\hline Pacific & 237,359 & 296,578 & 977,513 & $1,511,214$ \\
\hline Mountain & 148,263 & 190,906 & 594,758 & 840,726 \\
\hline West North Central & 113,518 & 147,140 & 514,242 & 771,717 \\
\hline West South Central & 236,840 & 313,560 & 853,026 & $1,095,487$ \\
\hline East North Central & 191,896 & 292,587 & $1,025,119$ & $1,544,282$ \\
\hline East South Central & 109,384 & 152,917 & 525,176 & 634,614 \\
\hline Middle Atlantic & 134,452 & 176,282 & 691,621 & $1,267,121$ \\
\hline South Atlantic & 386,518 & 547,519 & $1,801,087$ & $2,298,400$ \\
\hline New England & 51,308 & 74,032 & 300,832 & 531,284 \\
\hline National & $1,609,538$ & $2,191,522$ & $7,283,374$ & $10,494,845$ \\
\hline
\end{tabular}

SOURCE: RAND analysis of VA, DoD, and Census data.

Table A-9

Age, by Census Division, 2024

\begin{tabular}{lrrrr}
\hline Division & Age 17-34 & Age 35-44 & Age 45-64 & Age 65+ \\
\hline Pacific & 143,061 & 233,260 & 686,937 & $1,360,474$ \\
\hline Mountain & 95,288 & 190,341 & 475,298 & 827,687 \\
\hline West North Central & 84,965 & 128,026 & 343,202 & 539,914 \\
\hline West South Central & 188,528 & 287,286 & 719,945 & $1,132,409$ \\
\hline East North Central & 135,095 & 177,667 & 571,508 & 985,304 \\
\hline East South Central & 86,280 & 129,061 & 441,327 & 610,699 \\
\hline Middle Atlantic & 102,763 & 134,692 & 406,439 & 780,649 \\
\hline South Atlantic & 273,948 & 547,098 & $1,626,073$ & $2,400,863$ \\
\hline New England & 36,323 & 61,103 & 183,118 & 367,522 \\
\hline National & $1,146,251$ & $1,888,534$ & $5,453,847$ & $9,005,521$ \\
\hline SOURCE: & & &
\end{tabular}

SOURCE: RAND analysis of VA, DoD, and Census data. 
Table A-10

Veteran Population, by Region and Service Era, 2014

\begin{tabular}{llcccccc}
\hline \multirow{2}{*}{ Division } & \multicolumn{1}{c}{ Pre-1950 } & Conflict & $\begin{array}{c}\text { Pre-Vietnam } \\
\text { Peace }\end{array}$ & Vietnam Era & $\begin{array}{c}\text { Post-Vietnam } \\
\text { Peace }\end{array}$ & Gulf War Era & Post-9/11 Era \\
\hline & & & \multicolumn{5}{c}{ Veteran Populations } \\
\hline Pacific & 237,994 & 288,125 & 282,978 & 978,656 & 448,312 & 418,357 & 368,241 \\
\hline Mountain & 111,358 & 147,670 & 162,582 & 576,263 & 256,420 & 281,299 & 239,063 \\
\hline West North Central & 124,535 & 166,723 & 160,403 & 485,852 & 222,501 & 213,994 & 172,608 \\
\hline West South Central & 145,768 & 189,533 & 206,470 & 774,216 & 368,648 & 444,496 & 369,782 \\
\hline East North Central & 267,087 & 315,125 & 338,911 & 966,829 & 483,594 & 408,375 & 273,963 \\
\hline East South Central & 83,315 & 111,873 & 127,778 & 444,545 & 227,404 & 245,606 & 181,570 \\
\hline Middle Atlantic & 249,624 & 263,947 & 283,845 & 694,561 & 335,307 & 242,456 & 199,737 \\
\hline South Atlantic & 321,136 & 407,702 & 445,405 & $1,509,535$ & 777,054 & 883,420 & 689,284 \\
\hline New England & 96,428 & 109,704 & 111,387 & 305,282 & 148,266 & 105,352 & 81,038 \\
\hline National & $1,637,245$ & $2,000,402$ & $2,119,759$ & $6,735,739$ & $3,267,506$ & $3,243,355$ & $2,575,286$ \\
\hline
\end{tabular}

SOURCE: RAND analysis of VA, DoD, and Census data. 
Table A-11

Veteran Population, by Region and Service Era, 2024

\begin{tabular}{lccccccc}
\hline \multicolumn{1}{c}{ Division } & Pre-1950 & $\begin{array}{c}\text { Korean } \\
\text { Conflict }\end{array}$ & $\begin{array}{c}\text { Pre-Vietnam } \\
\text { Peace }\end{array}$ & Vietnam Era & $\begin{array}{c}\text { Post-Vietnam } \\
\text { Peace }\end{array}$ & Gulf War Era & Post-9/11 Era \\
\hline Pacific & & \multicolumn{7}{c}{ Veteran Populations } & & \\
\hline Mountain & 63,111 & 120,164 & 156,151 & 781,661 & 414,727 & 362,065 & 525,852 \\
\hline West North Central & 29,881 & 44,809 & 93,440 & 491,604 & 250,756 & 289,636 & 388,487 \\
\hline West South Central & 31,898 & 55,375 & 70,366 & 291,892 & 171,083 & 192,528 & 282,967 \\
\hline East North Central & 67,875 & 85,954 & 145,300 & 499,117 & 350,815 & 304,070 & 416,443 \\
\hline East South Central & 21,945 & 30,958 & 55,939 & 365,751 & 234,455 & 238,821 & 319,499 \\
\hline Middle Atlantic & 62,030 & 68,430 & 149,546 & 356,930 & 269,366 & 195,004 & 323,238 \\
\hline South Atlantic & 81,330 & 135,021 & 321,510 & $1,382,221$ & 754,834 & 948,648 & $1,224,419$ \\
\hline New England & 24,894 & 35,250 & 53,301 & 194,089 & 115,156 & 89,760 & 135,616 \\
\hline National & 422,578 & 639,629 & $1,140,866$ & $5,071,347$ & $2,950,231$ & $3,018,207$ & $4,251,297$ \\
\hline
\end{tabular}

SOURCE: RAND analysis of VA, DoD, and Census data. 
While the number of male Veterans is projected to decline steadily, the number of female Veterans is projected to increase very slightly at the same time (Figure A-4). The relative share of female Veterans will increase by 25 percent, from 8 percent to 10 percent of the Veteran population by 2024.

Veterans' mean age will increase slowly throughout the period (Figure A-5). Male Veterans' mean age will rise much more slowly than female Veterans' mean age, although female Veterans are substantially younger overall.

Figure A-4

Total Population, by Sex, 2014-2024

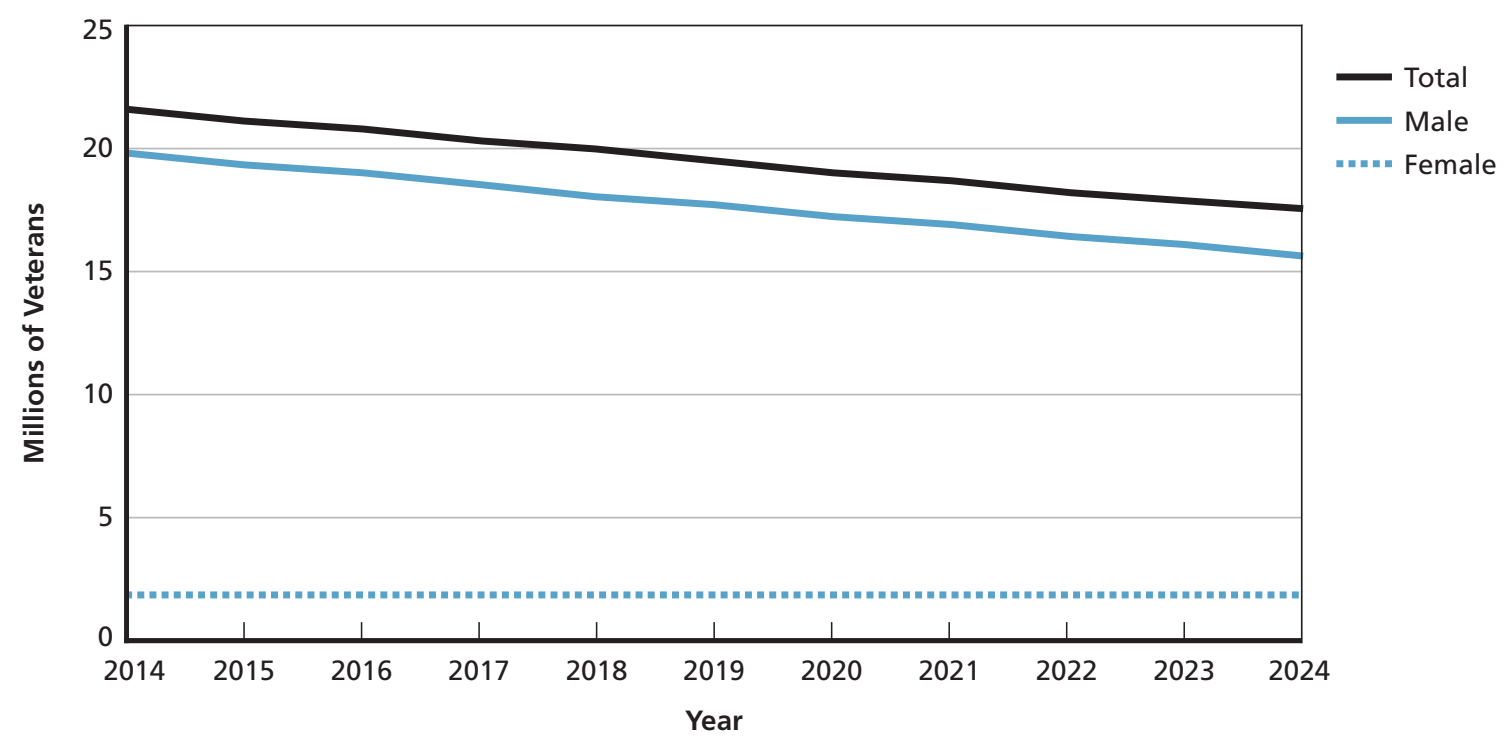

SOURCE: RAND analysis of VA, DoD, and Census data.

RAND RR1165z1-A.4 
Figure A-5

Average Age, by Sex, 2014-2024

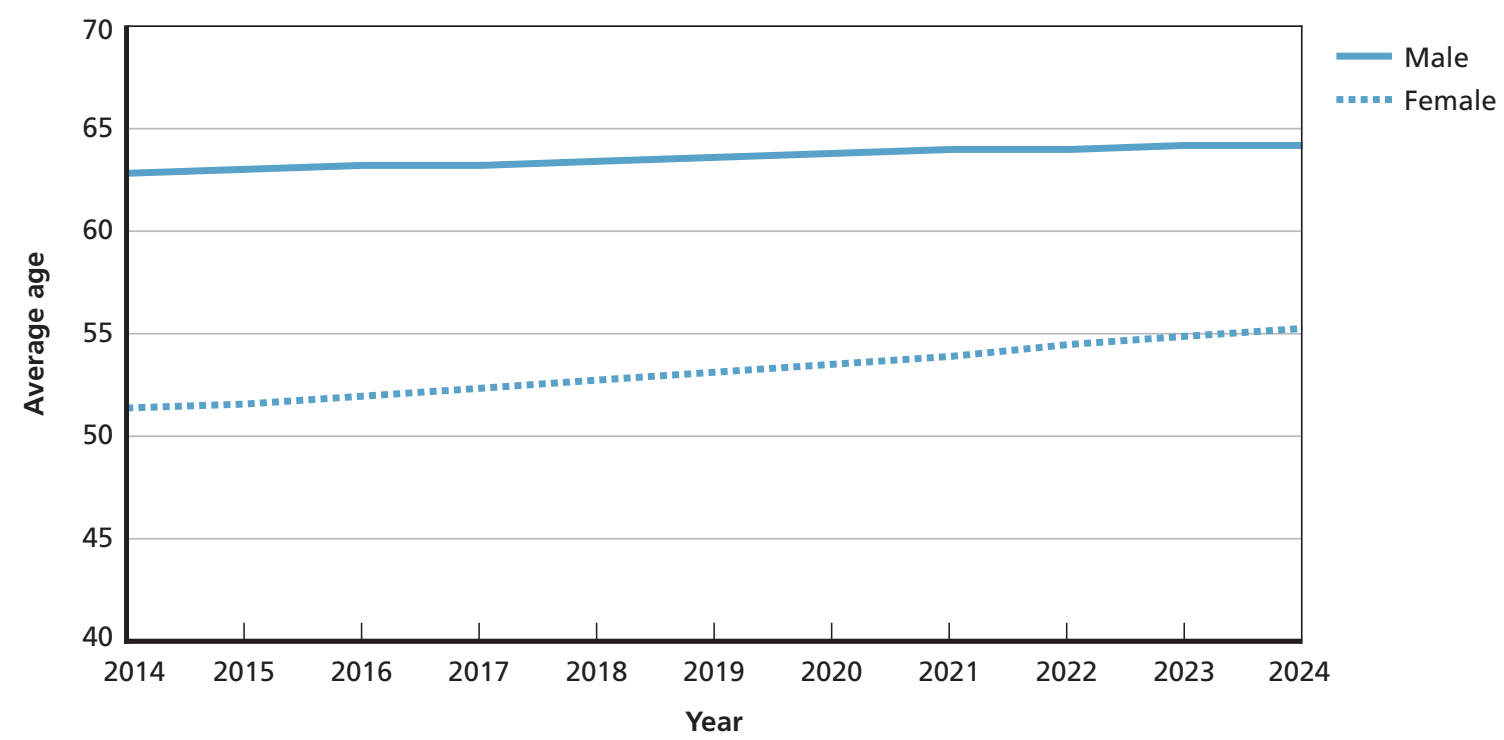

SOURCE: RAND analysis of VA, DoD, and Census data.

RAND RR1165z1-A.5

Figures A-6 through A-16 present snapshots of the geographic distribution of various Veteran demographic characteristics in 2014 and in 2024 in order to provide an overview of key changes over time. Total Veteran population counts and average ages by sex are presented for 2014-2024, followed by the density of the Veteran population and percentages of Veterans by specific race/ethnicity and age categories. The number of Veteran net migrants in 2014 and 2024 is also presented, as well as the change in net migration rates between 2014 and 2024 . 
Figure A-6

Total Veteran Population Counts: 2014 and 2024
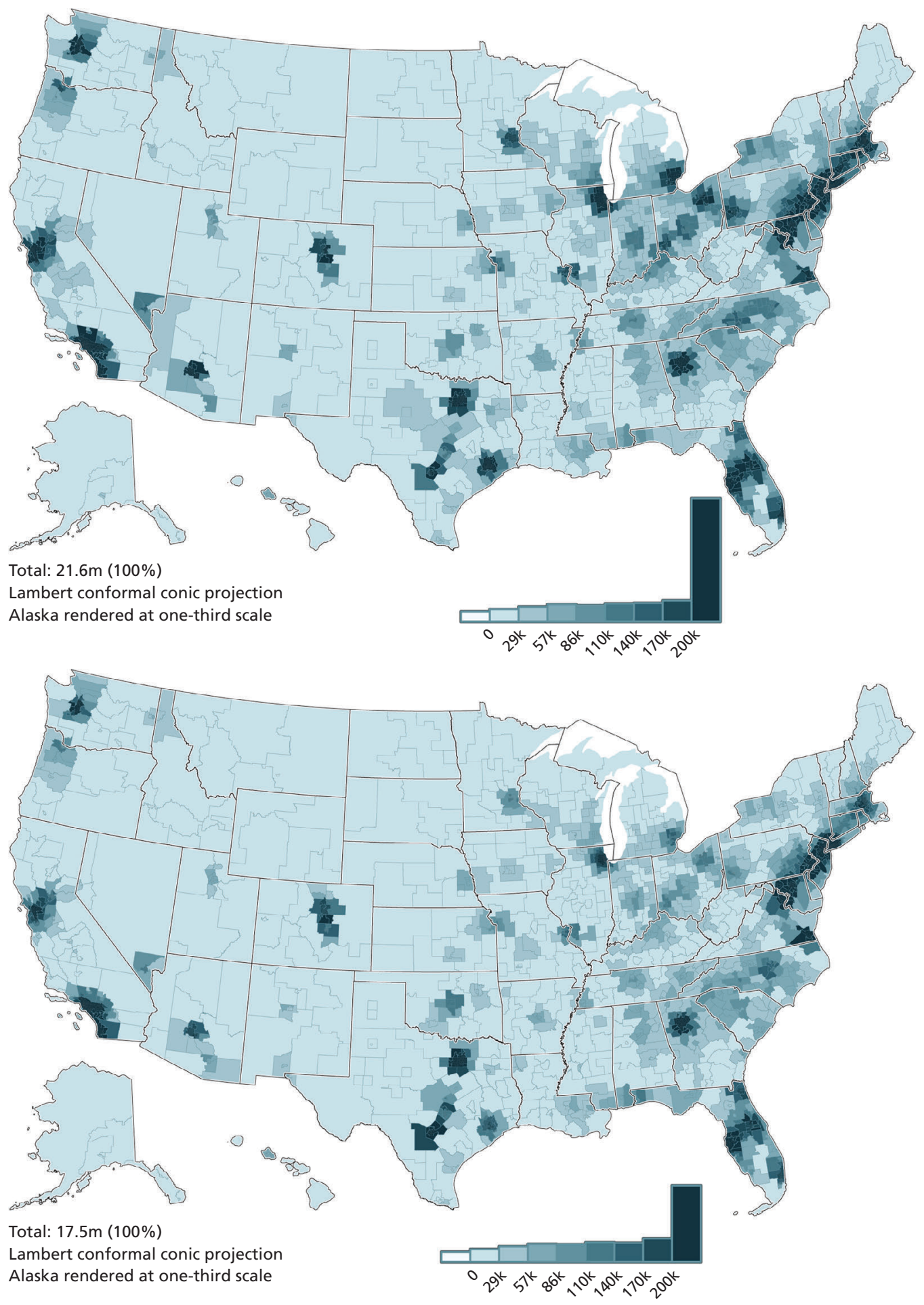

SOURCE: RAND analysis of VA, DoD, and Census data.

RAND RR1165z1-A.6 
Figure A-7

Geographic Density of Veterans in 2014, Change by 2024
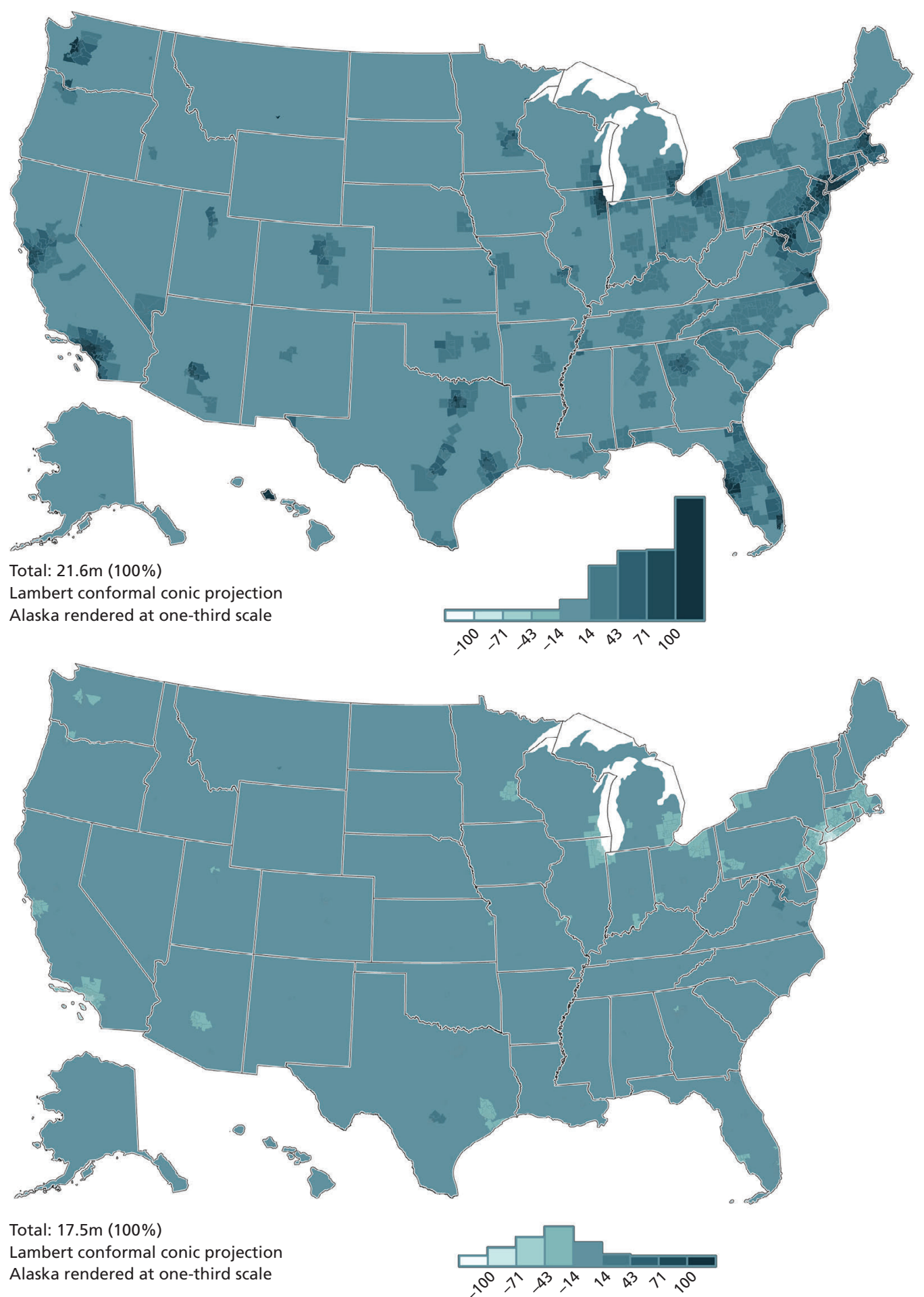

SOURCE: RAND analysis of VA, DoD, and Census data.

RAND RR1165z1-A.7 
Figure A-8

Percentage of Non-Hispanic White Veterans: 2014 and 2024
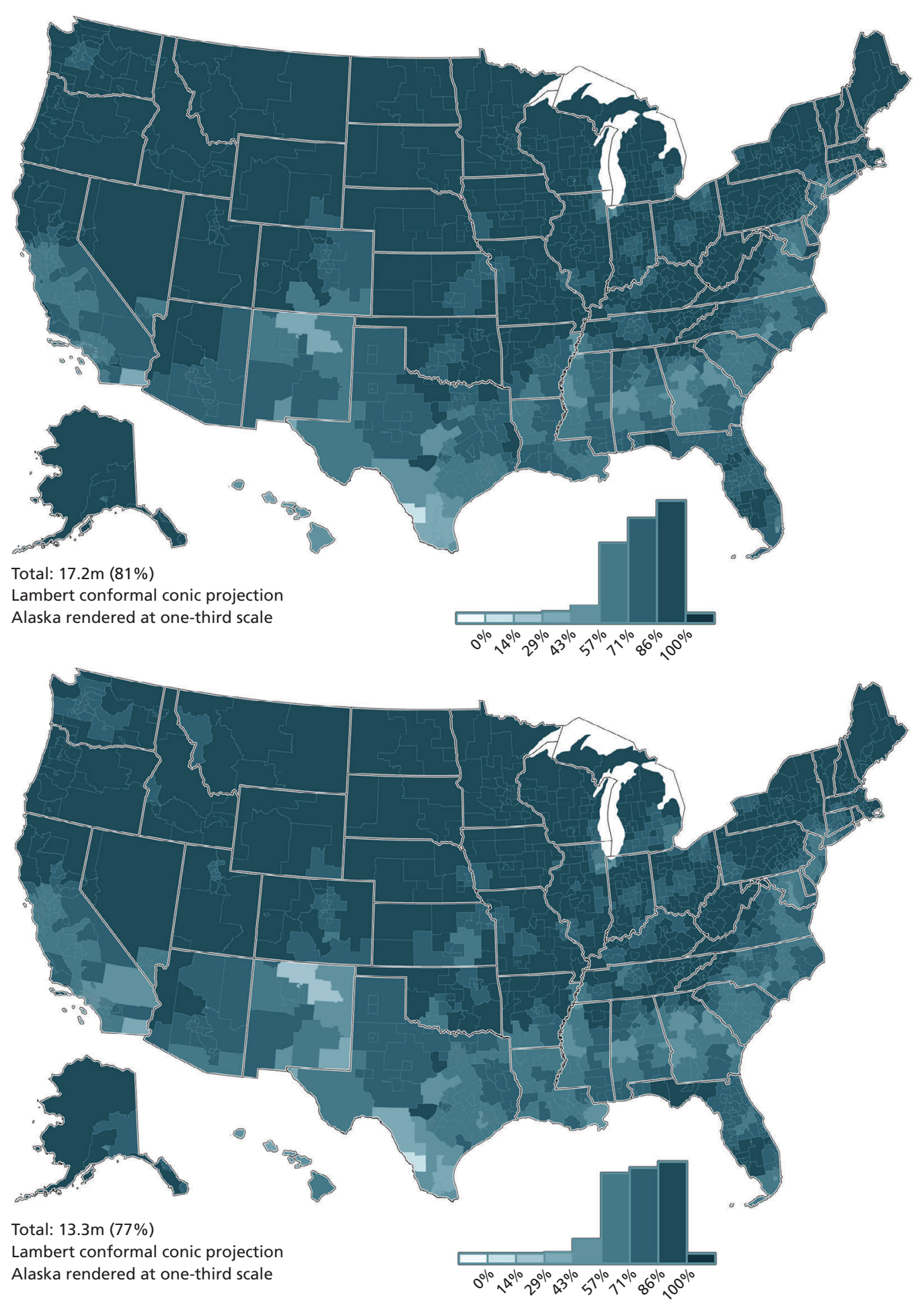

SOURCE: RAND analysis of VA, DoD, and Census data.

RAND RR1165z1-A.8 
Figure A-9

Percentage of Black Veterans: 2014 and 2024
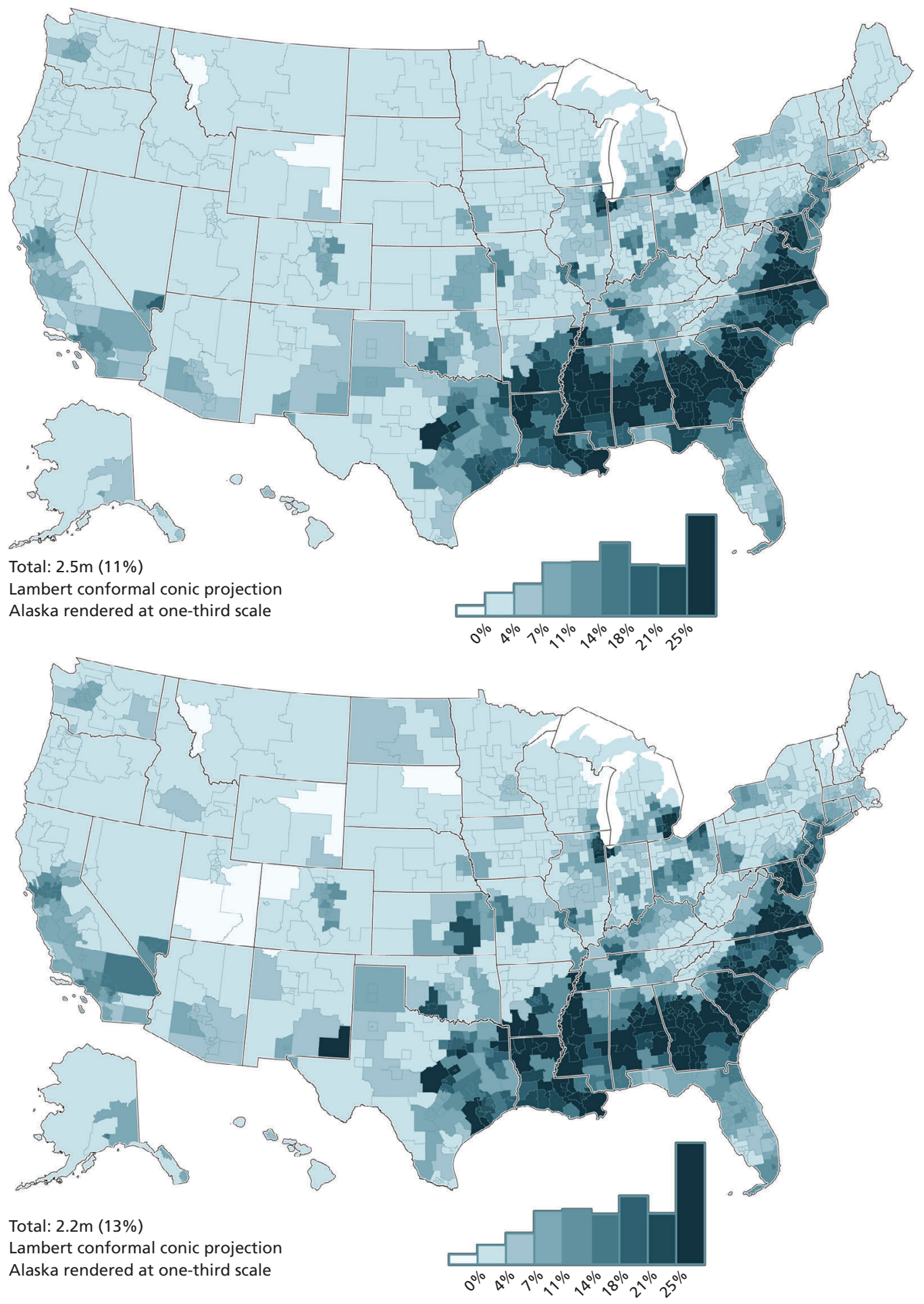

SOURCE: RAND analysis of VA, DoD, and Census data.

RAND RR1165z1-A.9 
Figure A-10

Percentage of Black Veterans: 2014 and 2024
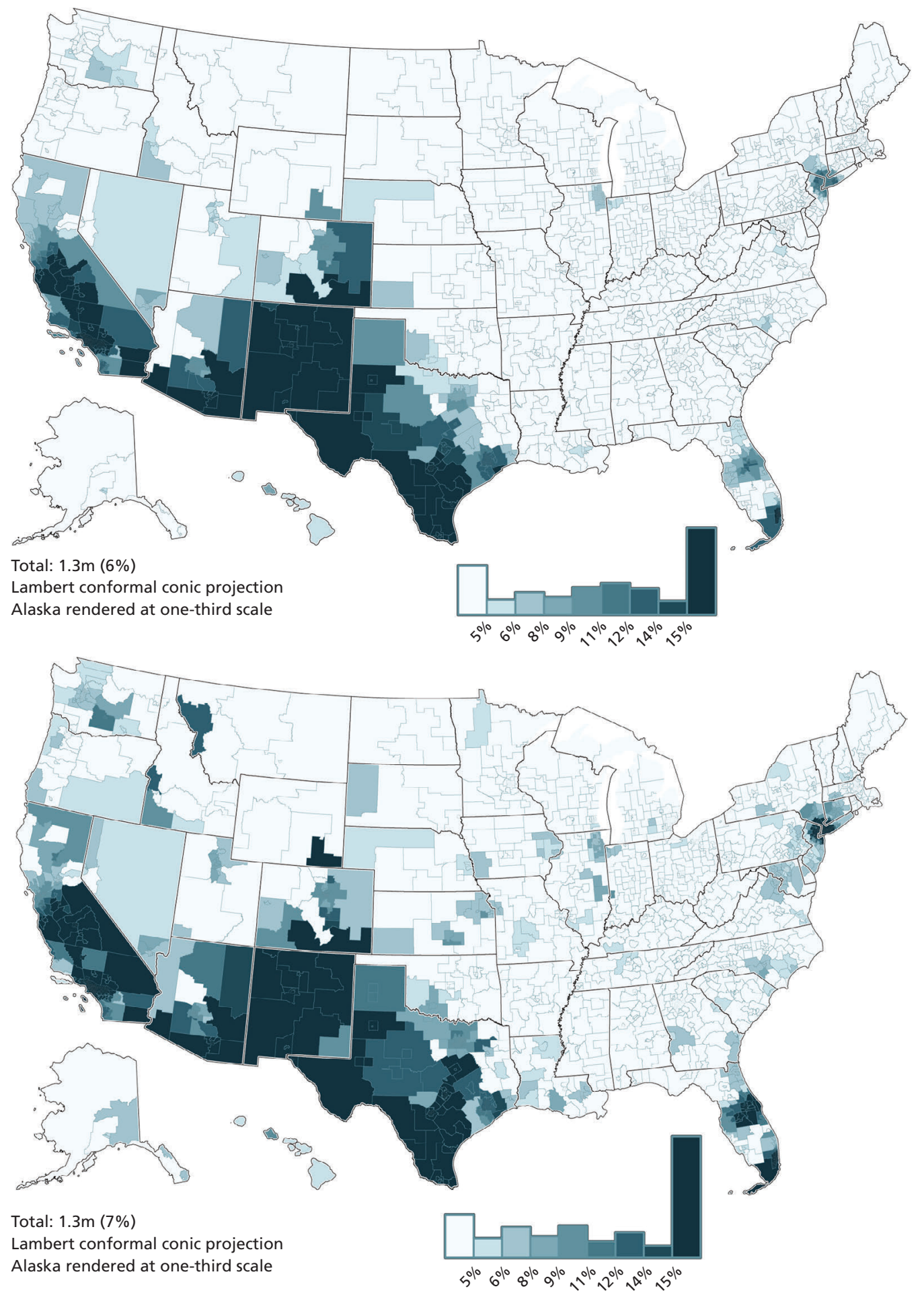

SOURCE: RAND analysis of VA, DoD, and Census data.

RAND RR1165z1-A.10 
Figure A-11

Percentage of Veterans Under Age 35: 2014 and 2024
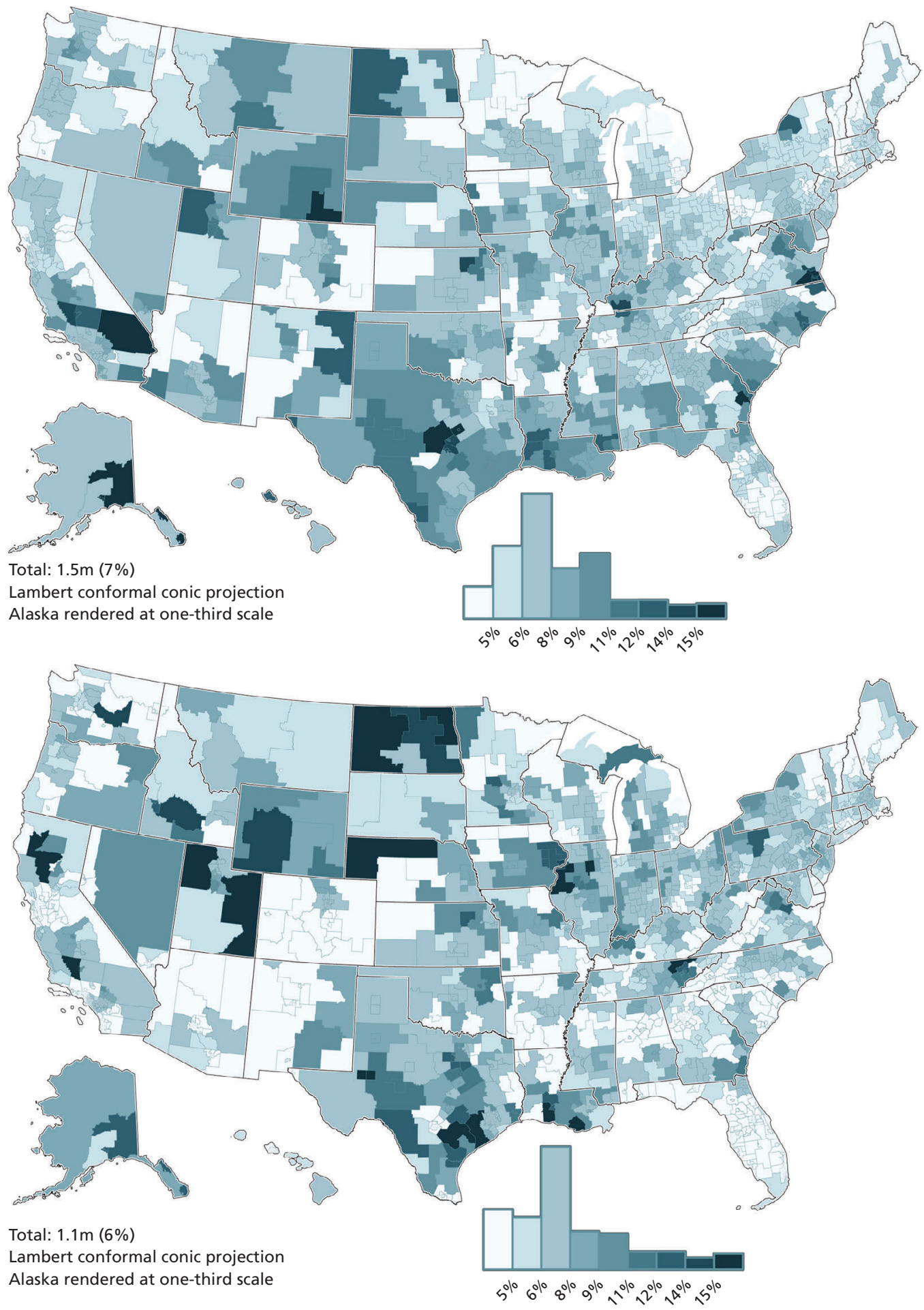

SOURCE: RAND analysis of VA, DoD, and Census data.

RAND RR1165z1-A.11 
Figure A-12

Percentage of Veterans Under Age 35 in 2014, Change by 2024
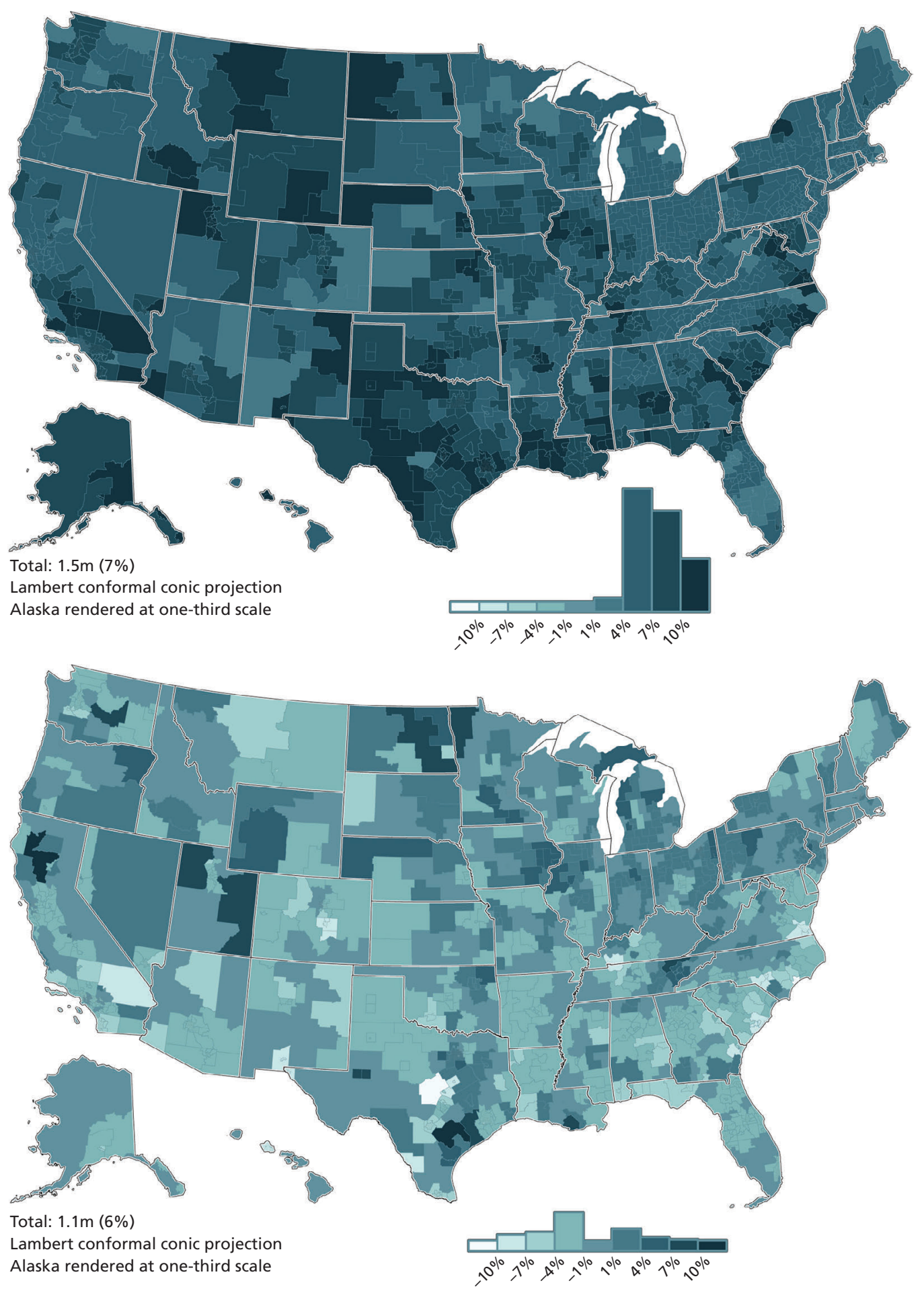

SOURCE: RAND analysis of VA, DoD, and Census data.

RAND RR1165z1-A.12 
Figure A-13

Percentage of Veterans Over Age 65: 2014 and 2024
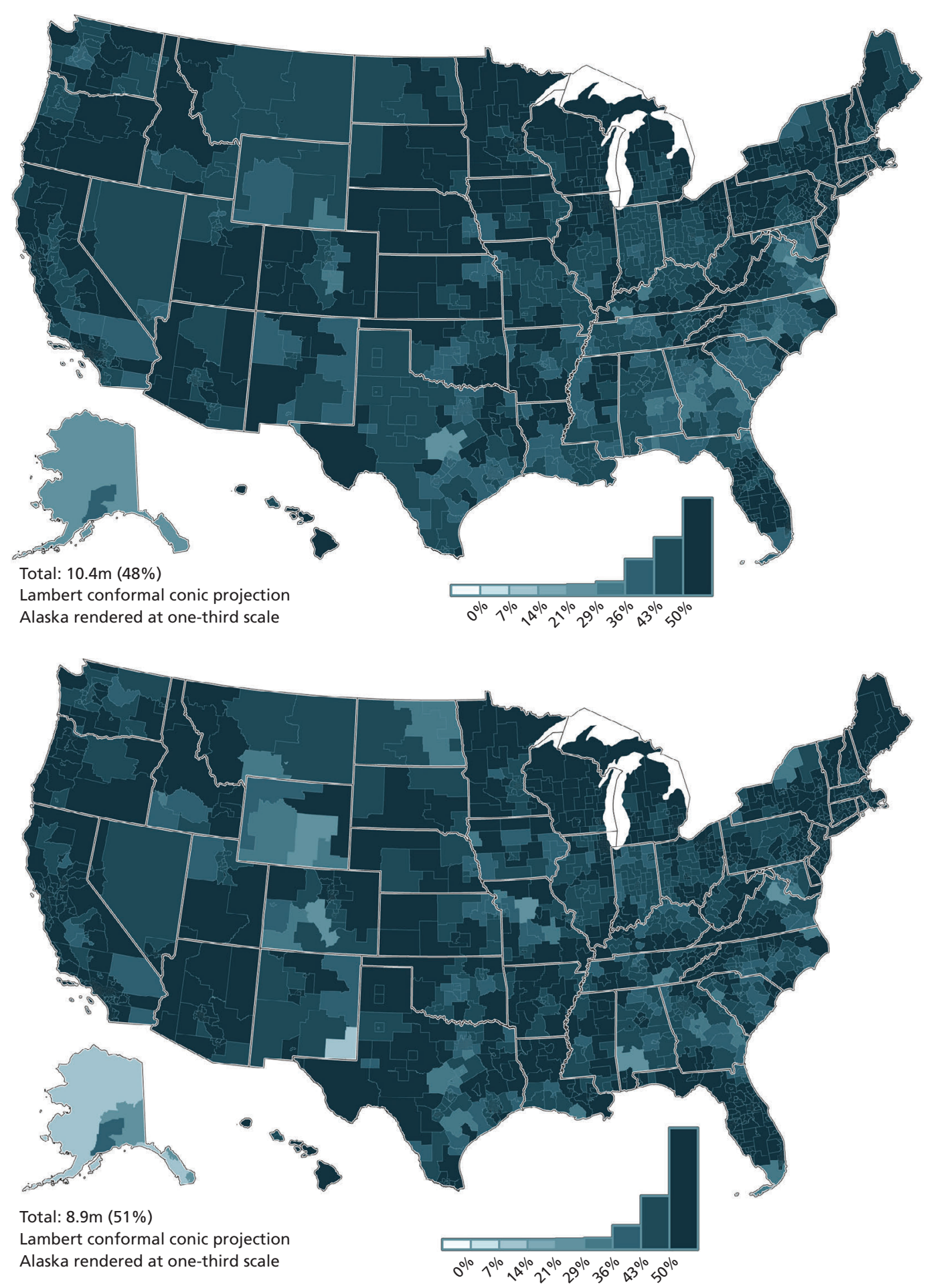

SOURCE: RAND analysis of VA, DoD, and Census data. 
Figure A-14

Percentage of Veterans Over Age 65 in 2014, Change by 2024

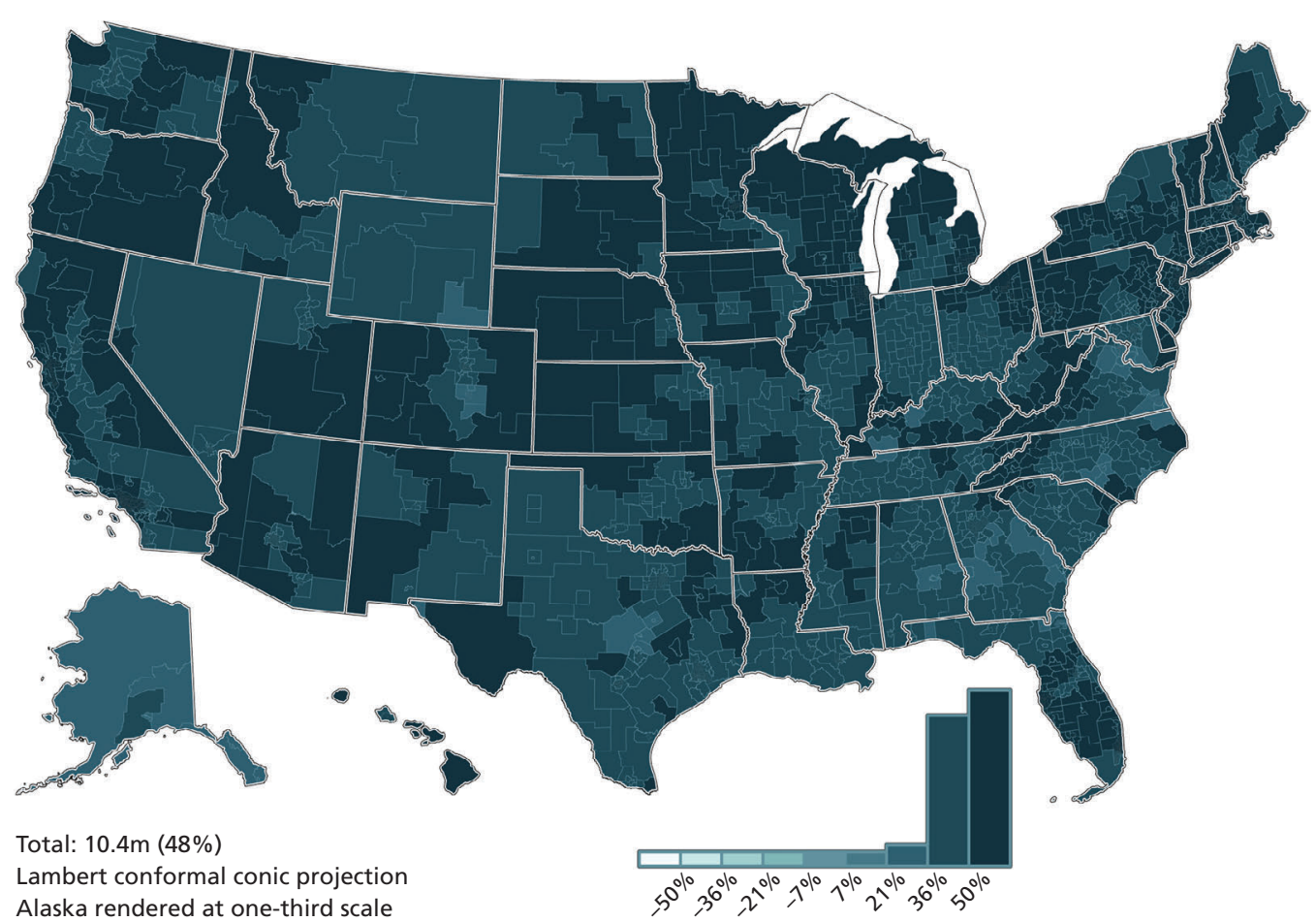

Alaska rendered at one-third scale

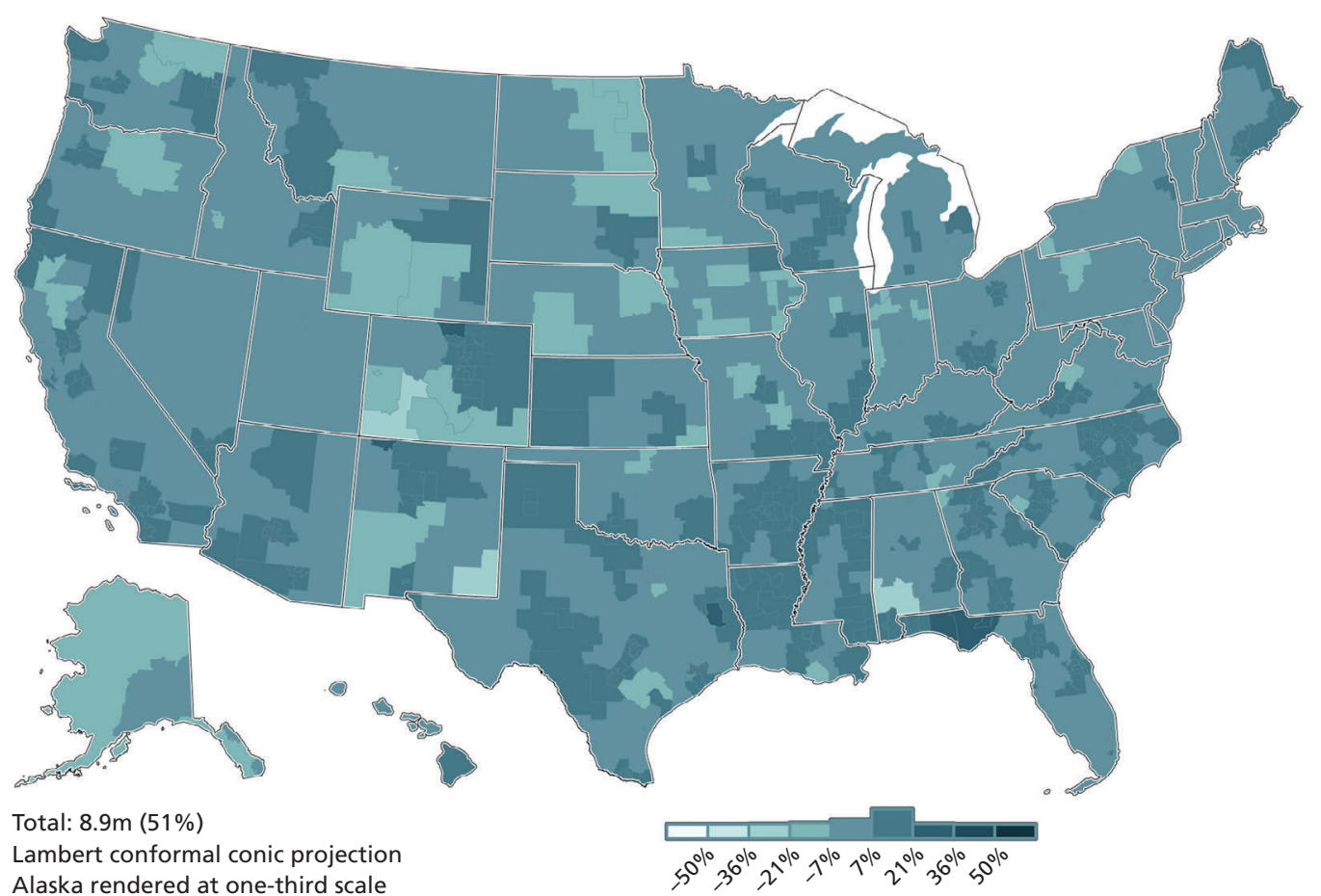

SOURCE: RAND analysis of VA, DoD, and Census data.

RAND RR1165z1-A.14 
Figure A-15

Number of Net Migrants: 2014 and 2024
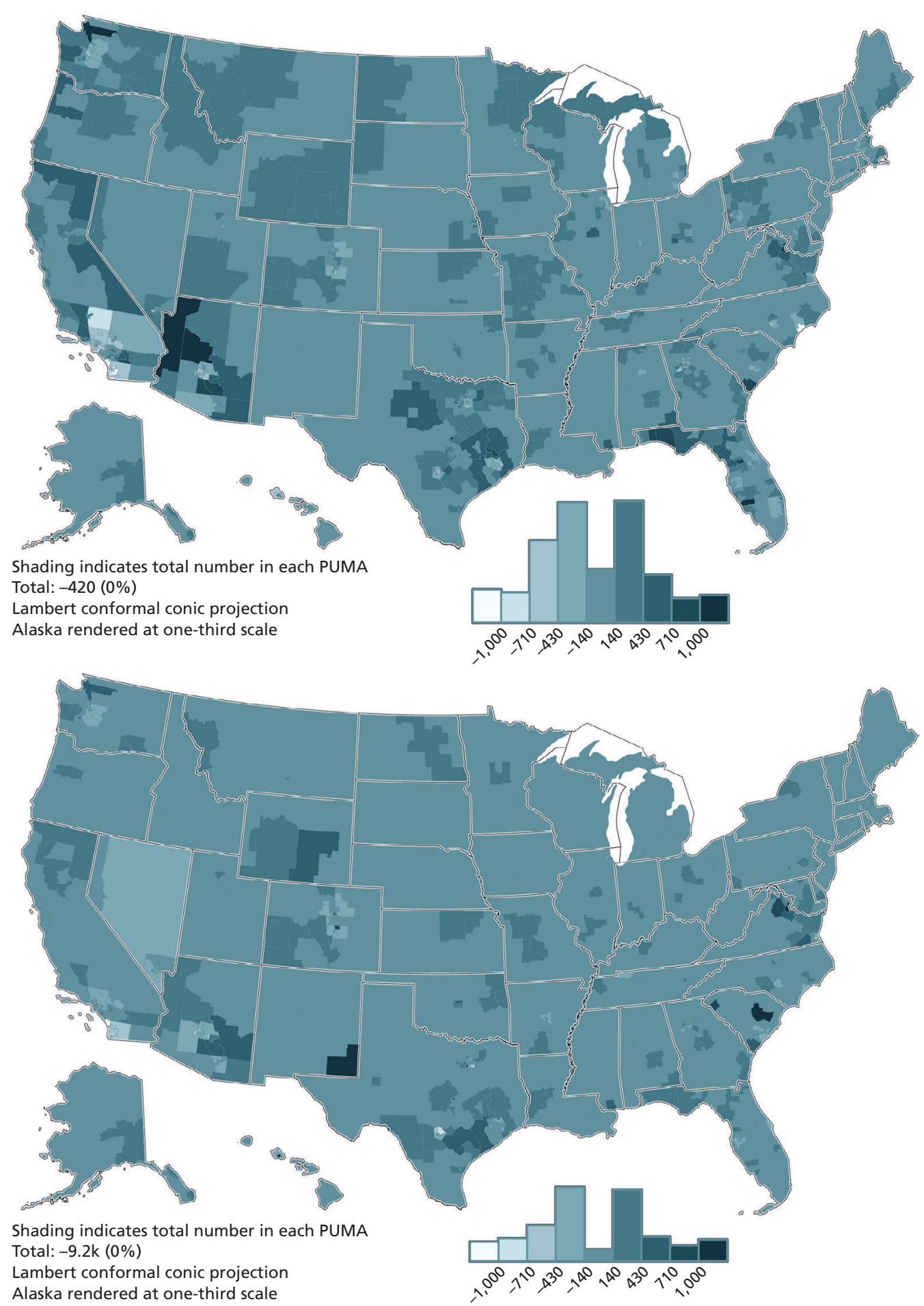

SOURCE: RAND analysis of VA, DoD, and Census data.

RAND RR116521-A.15 
Figure A-16

Change in Net Migration Rates: 2014 to 2024

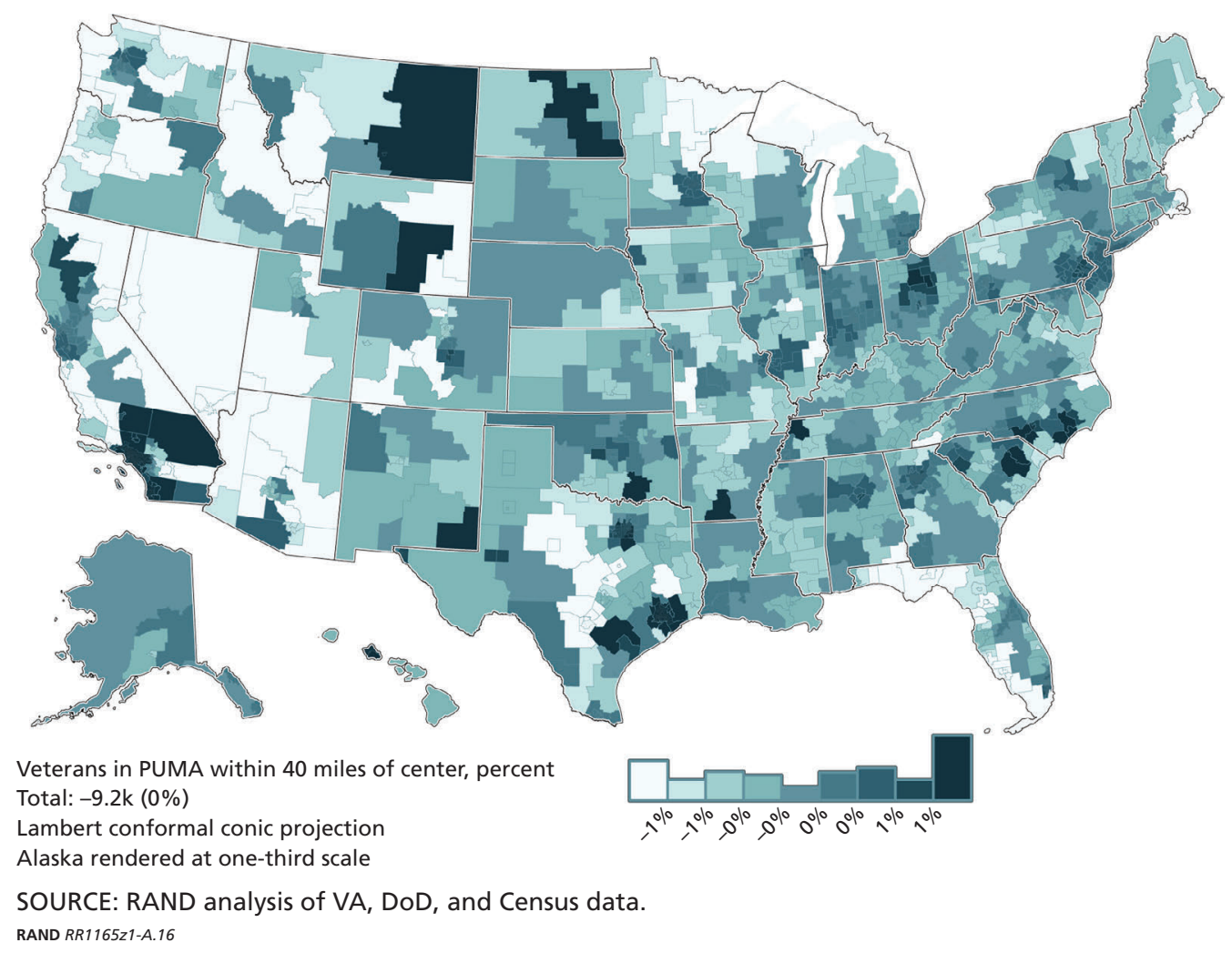

\section{A.3. Population Projection Sensitivity Analysis}

\section{A.3.1. Alternative Models}

In this section, we examine how the population projections vary when using different mortality rates. Recall that the rates used in the RAND Veteran projection are based on a combination of the VetPop2014 mortality rates and national-level race/ethnic-specific mortality rates from the CDC.

Here we apply mortality rates from (1) the VetPop2014 model (no race/ethnic differences in rates), (2) the observed national-level mortality rates from the CDC, and (3) a significantly elevated risk of mortality linked to poverty-area residence identified in other literature.

Also recall that our population projections begin from 2000 and continue through 2024. Figure A-17 presents the 2014-2024 period under the four mortality schedules. The RAND projection and VetPop2014 model are virtually equivalent, which is to be expected given that the overall mortality rate was simply distributed across race/ethnic groups. As discussed in Section A.1.7, although total Veteran population estimates across the RAND and VetPop2014 projections are similar, there are differences in the race/ethnic composition of the two populations: White and Asian Veterans are slightly more prominent in the RAND projection due to their slightly lower mortality rates than other groups.

Contrasting the RAND projection with the national mortality rates obtained from the CDC indicates that the mortality rates for Veterans are slightly lower than for the nation as a 
Figure A-17

Model Comparison

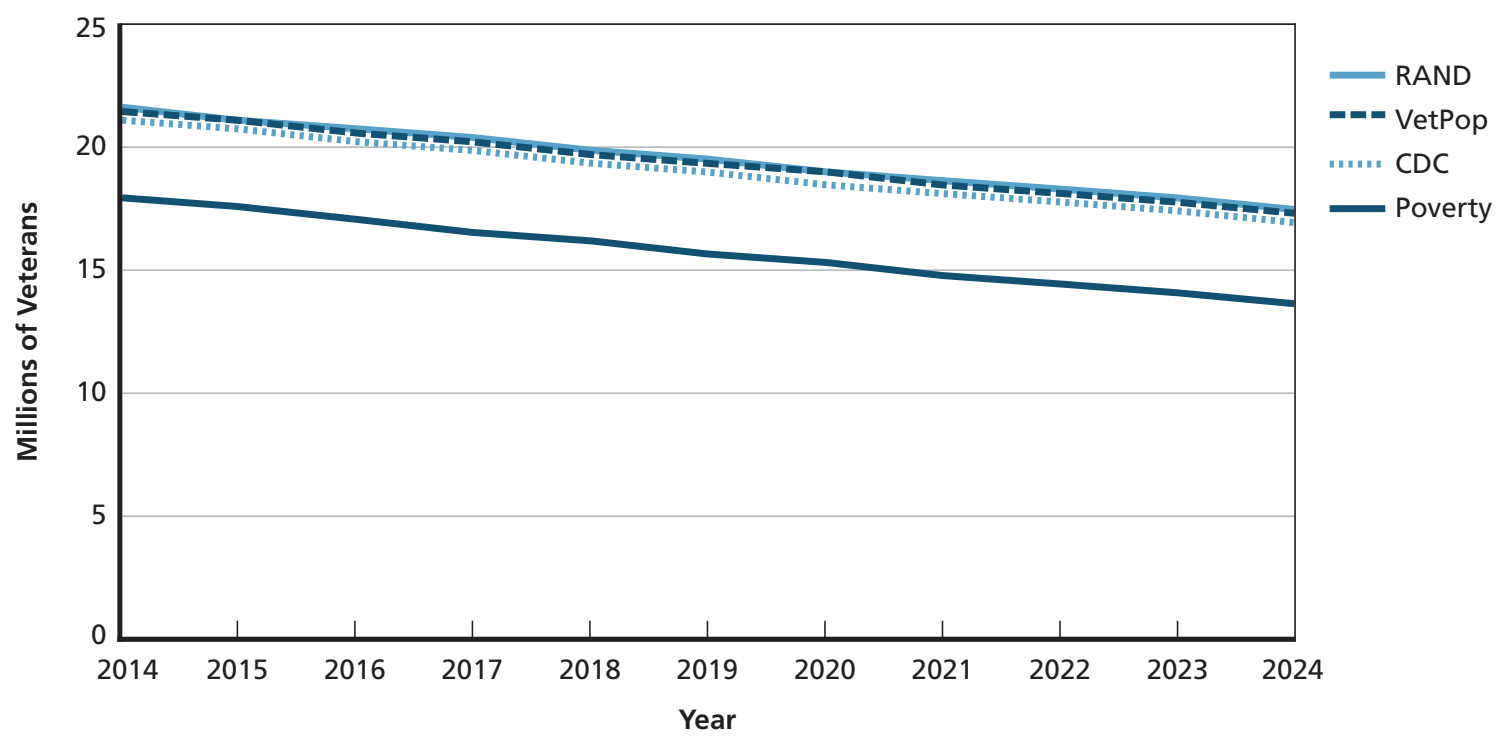

RAND RR1165z1-A.17

whole. By 2024, the RAND estimate projects 17.5 million Veterans, while using CDC mortality rates on the same population leads to a 2024 population of 17.0 million Veterans. While Veterans may have more health care needs than other civilians (see Chapter Four), they may also have access to VA care; whereas many civilians may lack health care. Further, Veterans have higher median incomes and are less likely to live below the poverty line than other civilians (National Center for Veterans Analysis and Statistics, 2014b).

Finally, to examine how highly elevated mortality risk impacts our population projections, we apply the same elevated risk of all-cause mortality associated with poverty-area residence (rate ratio 1.78) that was identified in a longitudinal study of over 10,000 individuals ages 25-74, from 1971 to 1987 (Waitzman \& Smith, 1998). There are many studies linking poverty to mortality; this specific one was chosen based on the large sample size and long follow-up period. As it is for illustrative purposes only, it is sufficient for the present needs. Applying nearly doubled mortality rates to the population projection does have a substantial impact, as anticipated: by 2024 the projection estimates a Veteran population of 13.7 million (22 percent lower than the RAND estimate). Again, this estimate assumes all Veterans live in federally designated poverty areas, and is for illustrative purpose only.

Overall, the comparison suggests that the projection is relatively robust to different mortality schedules, assuming the mortality schedules are not extreme. 


\section{A.3.1.1. Comparison of Observed Versus Gravity Model Net Migration in 2014-2024}

Net migration estimated with observed in-migration and out-migration rates from 2009 through 2013 ACS is compared with net migration estimated with predicted in-migration and out-migration rates from gravity models using the same survey (see Figure A-18). In 2014, there are less PUMAs with stronger negative or positive observed net migration, compared with predicted net migration. In other words, predicted net migration is more evenly distributed across the different categories of migration level than observed net migration. This result might be an indication that gravity models estimate rates that smooth the rates across the country, even though these rates did generate some areas with highly positive net migration in northwest Arizona. By 2024, net migration estimated with predicted rates from gravity models are even more equally distributed across the categories of migration level, compared to observed net migration. As a result, there are fewer areas with strong positive net migration originated with predicted rates than observed rates. Besides these differences, the same general patterns are observed across the country: (1) positive net migration to southeastern New Mexico, northwestern Florida, Colorado, southern Wyoming, western South Carolina, and northern Virginia; and (2) negative net migration from Nevada, Los Angeles, Phoenix, and San Antonio.

Even with some differences between net migration estimated with observed and predicted rates, the overall distribution of Veterans by PUMAs is similar throughout these two approaches (see Figure A-19). This similarity is probably due to the fact the overall in-migration and out-migration rates are small among the Veteran population. Observed rates varied from 3.03 percent in 2014 to 1.71 percent in 2024, while predicted rates varied from 2.97 percent in 2014 to 1.61 percent in 2024 . The team utilizes predicted results originated from gravity models to both net migration and population of Veterans, because they smooth the trends across the small geographical areas of PUMAs and the final results are consistent with observed trends. 
Figure A-18

Observed and Predicted Net Migration, 2014 and 2024

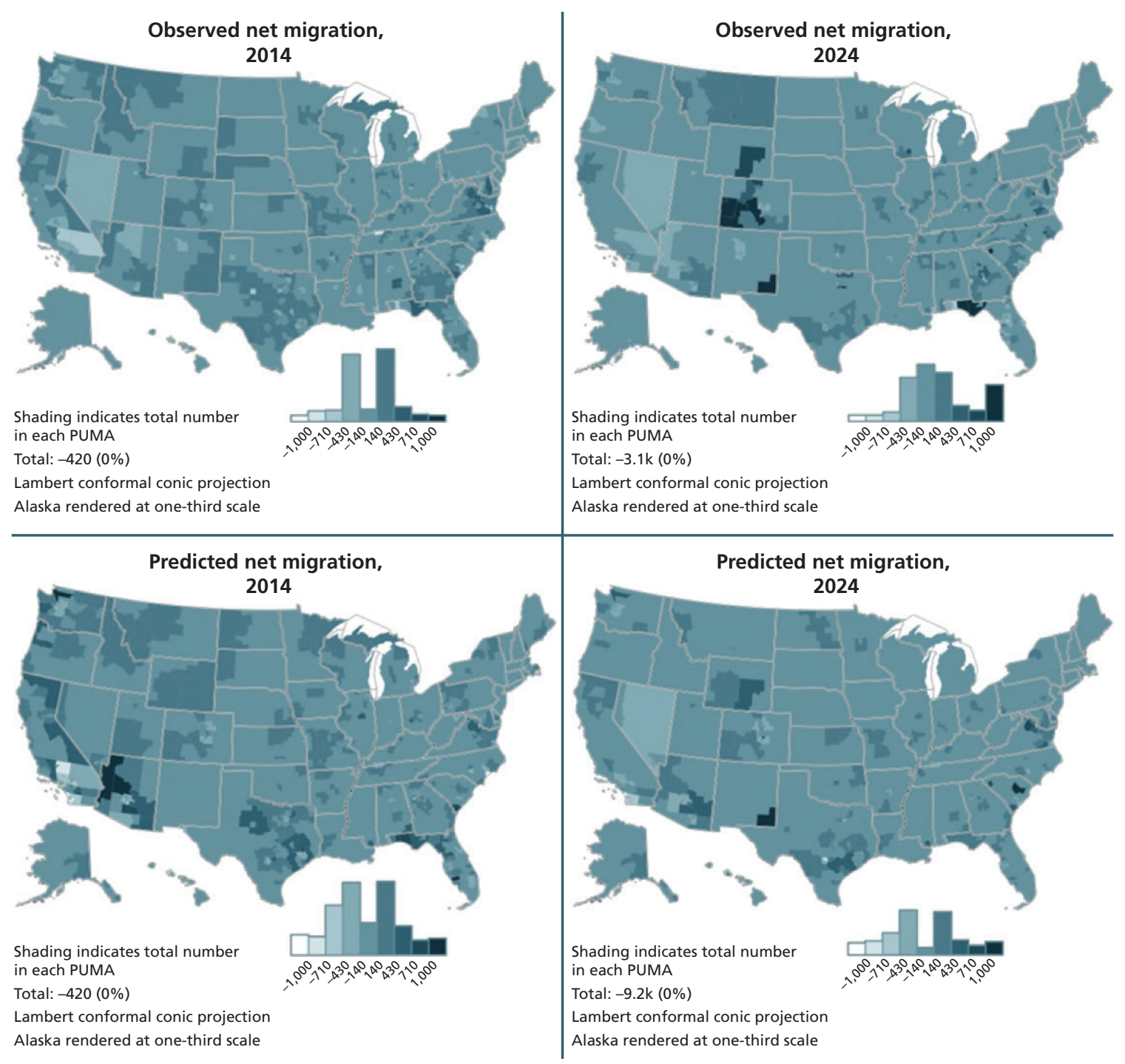

RAND RR1165z1-A.18 
Figure A-19

Observed and Predicted Veteran Population, 2014 and 2024

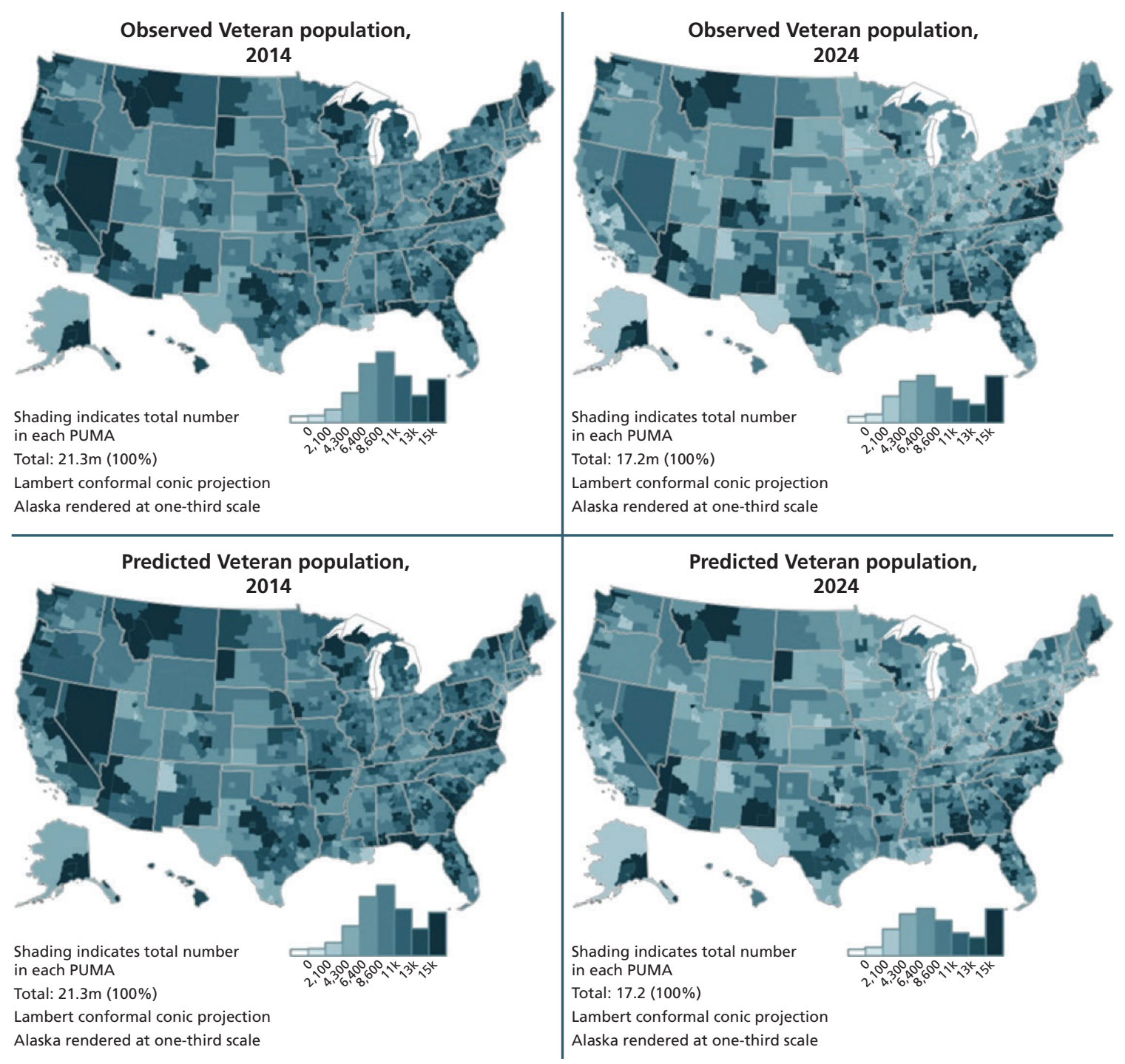

RAND RR1165z1-A.19 



\section{Enrollment in and Reliance on the VA Health Care System}

\section{B.1. Statistical Model for the Number of New Enrollees}

We used VA administrative data, the Veteran population projections from Chapter Three, and DoD data to estimate the probability that a yet-to-enroll Veteran enrolls in the VA health care system. Estimates are based on a generalized linear model indexed by age $(a)$, sex $(g)$, and CTS deployment status $(d)$, and include a linear effect of time to capture secular trends in enrollment by year $(t)$ :

$$
\mathrm{NE}_{\text {agdt }} \sim \operatorname{Binomial}\left(\mathrm{U}_{\text {agdt }}, \mathrm{p}_{\text {agdt }}\right), \text { with } \mathrm{p}_{a g d t}=\operatorname{expit}\left(\delta_{a, g}+\theta_{d}+\eta C_{a, g}+\beta \mathrm{t}\right),
$$

where $\mathrm{NE}_{\text {agdt }}$ is the number of new enrollees, $\mathrm{U}_{\text {agdt }}$ is the number of yet-to-enroll Veterans, $\mathrm{p}_{\text {agdt }}$ is the probability that a yet-to-enroll Veteran enrolls, $\delta_{a, g}$ is an age-and-sex specific effect, $\theta_{d}$ is the effect of Iraq-Afghanistan deployment on the probability of enrollment, and $\mathrm{C}_{\mathrm{a}, \mathrm{g}}$ is the percentage of Veterans in age group $a$ and sex $g$ who served in Vietnam. The percent of Vietnam Veterans, $\mathrm{C}_{a, g}$, is included in the model to disentangle the effect of age and the Vietnam service era on enrollment. This is important because, for example, a 2001 change in VA policy related to presumptive conditions associated with Agent Orange exposure led to an increase in the enrollment rates for Vietnam Veterans when they were in their 50s. Explicitly modeling this effect ensures that we do not predict a sharp increase in enrollment for all service era cohorts as they enter their 50s. We will explore including similar terms for other service cohorts.

Due to data limitations, this model does not distinguish between enrollment rates for Veterans deployed in Iraq and Afghanistan who are within the five-year window of enhanced eligibility and those who are outside of this window. The VA administrative data that we received do not contain information on separation date; therefore, we were unable to distinguish new enrollees who had deployed as part of the conflicts in Iraq and Afghanistan and separated within the past five years from those who had separated more than five years ago. With proper approvals, DoD data on separations and deployment could be merged with VA enrollment data. However, we did not have such approvals. 


\section{B.2. Algorithm for Projecting VA Enrollment}

The following algorithm was used to project VA enrollment:

1. Start with the current number of enrollees in an age group $a, \operatorname{sex} g$, and Iraq-Afghanistan deployment status $\mathrm{d}$ in year $\mathrm{t}, \mathrm{E}_{a}^{t}$, suppressing the notation indicating sex and IraqAfghanistan deployment status for ease of notation. We observe $\mathrm{E}_{a}^{2014}$, but this number is projected in all subsequent years.

2. Estimate the number of currently enrolled Veterans who are alive and enrolled in the next year by applying age-specific mortality rates to the current enrollees and increasing their age by one year: $\widetilde{\mathrm{E}}_{a+1}^{t+1}$

3. Obtain Vet $\mathrm{t}_{a+1}^{t+1}$ as described in Chapter Three.

4. Estimate the number of yet-to-enroll Veterans by subtracting the projected number of enrolled Veterans from the total number of Veterans in that year ${ }^{1}$ :

$$
\mathrm{U}_{a+1}^{t+1}=\mathrm{Vet}_{a+1}^{t+1}-\widetilde{\mathrm{E}}_{a+1}^{t+1}
$$

5. Estimate the number of new enrollees by applying estimated enrollment rates for year $t+1$, based on equation (1), to the number of yet-to-enroll Veterans:

$$
\mathrm{NE}_{a+1}^{t+1}=\mathrm{U}_{a+1}^{t+1} \widehat{\operatorname{Pr}}(\text { Enrollment })
$$

(Recall that the probability of enrollment depends on Iraq-Afghanistan Global War on Terror deployment status.)

6. Add the number of new enrollees to the existing population of enrollees:

$$
\mathrm{E}_{a+1}^{t+1}=\widetilde{\mathrm{E}}_{a+1}^{t+1}+\mathrm{NE}_{a+1}^{t+1}
$$

7. Repeat to predict through 2024.

Our algorithm assumes that enrollment rates for Iraq- or Afghanistan-deployed Veterans, based on equation (1), differ systematically from other Veterans, but otherwise depends only on age, sex, and a linear time trend. The effect of Iraq-Afghanistan deployment on enrollment $\left(\theta_{\mathrm{d}}\right)$ is constant over time, so therefore this model does not capture expected decreases in enrollment rates among Veterans who were deployed (see Chapter Four for discussion).

We projected the number of enrollees based on three different sets of assumptions on the future probability of new enrollment. First, we assumed that the probability of enrollment will continue to follow the recent pattern of enrollment by directly using equation (1). Second, we assumed that the probability of enrollment among Veterans who were deployed will follow the recent pattern of enrollment among Veterans who never deployed by dropping the $\theta_{\mathrm{d}}$ term in equation (1). Third, we assumed that the probability of enrollment among Veterans who

\footnotetext{
1 Note that mortality rates were used in Chapter Three to estimate $\operatorname{Vet}_{a+1}^{t+1}$. The number of yet-to-enroll Veterans $\mathrm{U}_{a+1}^{t+1}$ incorporates mortality rates indirectly because it is a function of $\mathrm{Vet}_{a+1}^{t+1}$ and $\widetilde{\mathrm{E}}_{a+1}^{t+1}$, both of which incorporate mortality rates.
} 
deployed will decrease over time by allowing the effect of deployment on future enrollment, $\theta_{\mathrm{d}}$, to decrease by 5 percent each year.

\section{B.3. Projecting the Number of VA Patients}

We projected the number of VA patients from 2014 through 2024 by multiplying the number of enrollees by the probability of use among enrollees. We estimated the probability that, in a given year ( $t$ ), a VA enrollee is a VA patient using a generalized linear model applied to individual-level data from the SoE:

$$
\operatorname{logit}\left(\operatorname{Pr}\left[\mathrm{VA}_{i}=1\right]\right)=\alpha+\gamma_{1} \text { age }_{i}+\gamma_{2} \text { gender }_{i}+\gamma_{3} \text { cohort }_{i}+\gamma_{5} \mathrm{GWOT}_{i}+\beta t
$$

In equation (2), $V A_{i}$ is equal to 1 if $i$ th enrollee is a VA patient, age $\mathrm{e}_{\mathrm{i}}$ is a vector of five-year age categories, gender ${ }_{i}$ is indicator for male, cohort ${ }_{i}$ is a vector of indicators for seven service eras, and $\mathrm{GWOT}_{\mathrm{i}}$ is an indicator that the enrollee was deployed as part of Iraq and Afghanistan, and $t$ is a linear effect of calendar year.

\section{B.4. Probability of VA Use, by VA Enrollee Characteristics}

Tables B-1 through B-5 show results from our analysis.

Table B-1

Probability That a VA Enrollee Uses VA Health Care: Baseline Model

\begin{tabular}{|c|c|c|}
\hline \multirow[b]{2}{*}{ Demographic Group } & \multicolumn{2}{|c|}{ Estimates for VA Patient Population Projections } \\
\hline & Probability of Using VA & Standard Errors \\
\hline \multicolumn{3}{|l|}{ Age } \\
\hline$<25$ & 0.741 & $(0.014)$ \\
\hline $25-29$ & 0.694 & $(0.010)$ \\
\hline $30-34$ & 0.670 & $(0.010)$ \\
\hline $35-39$ & 0.663 & $(0.010)$ \\
\hline $40-44$ & 0.655 & $(0.009)$ \\
\hline $45-49$ & 0.632 & $(0.009)$ \\
\hline $50-54$ & 0.657 & $(0.008)$ \\
\hline $55-60$ & 0.681 & $(0.006)$ \\
\hline $60-64$ & 0.700 & $(0.005)$ \\
\hline $65-69$ & 0.686 & $(0.005)$ \\
\hline $70-74$ & 0.633 & $(0.007)$ \\
\hline $75-79$ & 0.584 & $(0.007)$ \\
\hline $80-84$ & 0.573 & $(0.009)$ \\
\hline $85+$ & 0.558 & $(0.012)$ \\
\hline
\end{tabular}


Table B-1-Continued

\begin{tabular}{lll}
\hline & \multicolumn{2}{c}{ Estimates for VA Patient Population Projections } \\
\cline { 2 - 3 } Demographic Group & Probability of Using VA & Standard Errors \\
\hline Sex & 0.686 & $(0.007)$ \\
\hline Male & 0.651 & $(0.002)$ \\
Service era & & \\
Post 9/11 & 0.506 & $(0.009)$ \\
\hline Gulf War & 0.516 & $(0.007)$ \\
\hline Post-Vietnam peace & 0.668 & $(0.009)$ \\
\hline Vietnam & 0.676 & $(0.004)$ \\
\hline Pre-Vietnam peace & 0.719 & $(0.007)$ \\
\hline Korean conflict & 0.762 & $(0.006)$ \\
\hline Pre-1950s & 0.764 & $(0.009)$ \\
\hline Deployed to Iraq or Afghanistan & & $(0.008)$ \\
\hline No & 0.651 & \\
\hline Yes & 0.676 & $(0.02)$ \\
\hline SolvcF & & \\
\hline
\end{tabular}

SOURCE: SoE (2010-2014).

NOTES: Sample size $=209,979$. The adjusted probabilities of using VA health care services conditional on enrollment are shown in the first column. The standard errors are in column (2). These estimates show the differences in the probability of VA health service use within each demographic dimension (e.g., age), while controlling for other demographic differences. For example, any differences in the adjusted probabilities of use by age are devoid of the impact of Afghanistan and Iraq deployment on the probabilitiy that younger Veterans use VA health services.

Table B-2

Probability That a VA Enrollee Uses VA Health Care, by Demographic Characteristics

\begin{tabular}{lllllll}
\hline & \multicolumn{2}{c}{ Model 1} & & \multicolumn{2}{c}{ Model 2} \\
\cline { 2 - 3 } \cline { 5 - 7 } & $\begin{array}{c}\text { Probability of } \\
\text { Using VA }\end{array}$ & $\begin{array}{c}\text { Standard } \\
\text { Errors }\end{array}$ & & $\begin{array}{c}\text { Probability of } \\
\text { Using VA }\end{array}$ & $\begin{array}{c}\text { Standard } \\
\text { Errors }\end{array}$ \\
\hline Age & & & & & \\
\hline 25 & 0.490 & $(0.056)$ & & 0.571 & $(0.061)$ \\
\hline $25-29$ & 0.525 & $(0.026)$ & & 0.584 & $(0.025)$ \\
\hline $30-34$ & 0.583 & $(0.022)$ & & 0.635 & $(0.021)$ \\
\hline $35-39$ & 0.608 & $(0.024)$ & & 0.631 & $(0.022)$ \\
\hline $40-44$ & 0.614 & $(0.020)$ & & 0.623 & $(0.020)$ \\
\hline $45-49$ & 0.613 & $(0.018)$ & & 0.613 & $(0.018)$ \\
\hline
\end{tabular}


Table B-2-Continued

\begin{tabular}{|c|c|c|c|c|}
\hline & \multicolumn{2}{|c|}{ Model 1} & \multicolumn{2}{|c|}{ Model 2} \\
\hline & $\begin{array}{l}\text { Probability of } \\
\text { Using VA }\end{array}$ & $\begin{array}{l}\text { Standard } \\
\text { Errors }\end{array}$ & $\begin{array}{c}\text { Probability of } \\
\text { Using VA }\end{array}$ & $\begin{array}{c}\text { Standard } \\
\text { Errors }\end{array}$ \\
\hline $50-54$ & 0.601 & $(0.016)$ & 0.597 & $(0.016)$ \\
\hline $55-60$ & 0.654 & $(0.013)$ & 0.640 & $(0.013)$ \\
\hline $60-64$ & 0.649 & $(0.010)$ & 0.640 & $(0.010)$ \\
\hline $65-69$ & 0.686 & $(0.010)$ & 0.680 & $(0.010)$ \\
\hline $70-74$ & 0.641 & $(0.013)$ & 0.632 & $(0.013)$ \\
\hline $75-79$ & 0.617 & $(0.016)$ & 0.600 & $(0.015)$ \\
\hline $80-84$ & 0.604 & $(0.020)$ & 0.585 & $(0.019)$ \\
\hline $85+$ & 0.604 & $(0.030)$ & 0.593 & $(0.029)$ \\
\hline \multicolumn{5}{|l|}{ Sex } \\
\hline Female & 0.677 & $(0.009)$ & 0.661 & $(0.009)$ \\
\hline Male & 0.622 & $(0.004)$ & 0.623 & $(0.004)$ \\
\hline \multicolumn{5}{|l|}{ Service era } \\
\hline Post 9/11 & 0.625 & $(0.016)$ & 0.518 & $(0.017)$ \\
\hline Gulf War & 0.585 & $(0.012)$ & 0.548 & $(0.012)$ \\
\hline Post-Vietnam peace & 0.628 & $(0.016)$ & 0.635 & $(0.015)$ \\
\hline Vietnam & 0.619 & $(0.009)$ & 0.642 & $(0.008)$ \\
\hline Pre-Vietnam peace & 0.657 & $(0.016)$ & 0.726 & $(0.014)$ \\
\hline Korean conflict & 0.676 & $(0.018)$ & 0.746 & $(0.015)$ \\
\hline Pre-1950s & 0.652 & $(0.030)$ & 0.724 & $(0.026)$ \\
\hline \multicolumn{5}{|c|}{ Deployed to Iraq or Afghanistan } \\
\hline No & 0.625 & $(0.005)$ & 0.619 & $(0.005)$ \\
\hline Yes & 0.631 & $(0.016)$ & 0.665 & $(0.017)$ \\
\hline \multicolumn{5}{|l|}{ Employment status } \\
\hline Employed & 0.561 & $(0.007)$ & 0.590 & $(0.006)$ \\
\hline Not employed & 0.632 & $(0.017)$ & 0.634 & $(0.016)$ \\
\hline Not in the labor force & 0.672 & $(0.005)$ & 0.652 & $(0.005)$ \\
\hline \multicolumn{5}{|l|}{ Income (\$) } \\
\hline Less than 11,000 & 0.645 & $(0.010)$ & 0.654 & $(0.010)$ \\
\hline $11,000-15,999$ & 0.737 & $(0.014)$ & 0.753 & $(0.013)$ \\
\hline $16,000-20,999$ & 0.691 & $(0.014)$ & 0.698 & $(0.013)$ \\
\hline $21,000-25,999$ & 0.703 & $(0.013)$ & 0.712 & $(0.012)$ \\
\hline
\end{tabular}


Table B-2-Continued

\begin{tabular}{|c|c|c|c|c|}
\hline & \multicolumn{2}{|c|}{ Model 1} & \multicolumn{2}{|c|}{ Model 2} \\
\hline & $\begin{array}{l}\text { Probability of } \\
\text { Using VA }\end{array}$ & $\begin{array}{l}\text { Standard } \\
\text { Errors }\end{array}$ & $\begin{array}{c}\text { Probability of } \\
\text { Using VA }\end{array}$ & $\begin{array}{c}\text { Standard } \\
\text { Errors }\end{array}$ \\
\hline $26,000-30,999$ & 0.657 & $(0.012)$ & 0.666 & $(0.012)$ \\
\hline $31,000-35,999$ & 0.666 & $(0.014)$ & 0.664 & $(0.014)$ \\
\hline $36,000-40,999$ & 0.648 & $(0.013)$ & 0.638 & $(0.013)$ \\
\hline $41,000-45,999$ & 0.615 & $(0.017)$ & 0.608 & $(0.017)$ \\
\hline $46,000-50,999$ & 0.627 & $(0.014)$ & 0.614 & $(0.014)$ \\
\hline $51,000-55,999$ & 0.606 & $(0.022)$ & 0.597 & $(0.021)$ \\
\hline $56,000+$ & 0.534 & $(0.007)$ & 0.518 & $(0.007)$ \\
\hline \multicolumn{5}{|c|}{ Health status is excellent or very good } \\
\hline No & 0.658 & $(0.004)$ & 0.643 & $(0.004)$ \\
\hline Yes & 0.574 & $(0.006)$ & 0.600 & $(0.006)$ \\
\hline \multicolumn{5}{|c|}{ Non-VA health insurance coverage } \\
\hline No & 0.619 & $(0.015)$ & 0.631 & $(0.014)$ \\
\hline Yes & 0.628 & $(0.004)$ & 0.625 & $(0.004)$ \\
\hline \multicolumn{5}{|c|}{ Non-VA prescription insurance coverage } \\
\hline No & 0.780 & $(0.008)$ & 0.769 & $(0.008)$ \\
\hline Yes & 0.556 & $(0.006)$ & 0.561 & $(0.006)$ \\
\hline \multicolumn{5}{|l|}{ Priority group } \\
\hline 1 & & & 0.832 & $(0.007)$ \\
\hline 2 & & & 0.702 & $(0.008)$ \\
\hline 3 & & & 0.634 & $(0.008)$ \\
\hline 4 & & & 0.740 & $(0.016)$ \\
\hline 5 & & & 0.557 & $(0.010)$ \\
\hline 6 & & & 0.530 & $(0.012)$ \\
\hline 7 & & & 0.672 & $(0.018)$ \\
\hline 8 & & & 0.508 & $(0.007)$ \\
\hline \multicolumn{5}{|c|}{ Enhanced eligibility for Iraq or Afghanistan } \\
\hline No & & & 0.626 & $(0.004)$ \\
\hline Yes & & & 0.628 & $(0.020)$ \\
\hline
\end{tabular}

SOURCE: SOE (2014).

NOTES: Sample size $=32,285$. The adjusted probabilities of using VA health care services conditional on enrollment are shown in columns (1) and (3). The associated standard errors are in columns (2) and (4). These estimates show the differences in the probability of VA health service use within each demographic dimension (e.g., age), while controlling for other demographic differences. For example, any differences in the adjusted probabilities of use by age are devoid of the impact of Afghanistan and Iraq deployment on the probability that younger Veterans use VA health services. 
Table B-3

Characteristics of Non-Veterans, Veterans, and VA Patients in MEPS

\begin{tabular}{|c|c|c|c|c|}
\hline \multirow[b]{2}{*}{ Demographic Group } & \multicolumn{4}{|c|}{$\begin{array}{c}\text { Distribution by Demographic Characteristics } \\
\text { (Standard Errors) }\end{array}$} \\
\hline & Non-Veterans & Veterans & $\begin{array}{l}\text { Veterans, } \\
\text { VA Patients }\end{array}$ & $\begin{array}{c}\text { Veterans, } \\
\text { Non-VA } \\
\text { Patients }\end{array}$ \\
\hline \multicolumn{5}{|l|}{ Age } \\
\hline \multirow[t]{2}{*}{$20-34$} & 0.300 & 0.062 & 0.038 & 0.076 \\
\hline & $(0.003)$ & $(0.004)$ & $(0.004)$ & $(0.005)$ \\
\hline \multirow[t]{2}{*}{$35-44$} & 0.192 & 0.104 & 0.063 & 0.129 \\
\hline & $(0.002)$ & $(0.004)$ & $(0.005)$ & $(0.006)$ \\
\hline \multirow[t]{2}{*}{$45-54$} & 0.202 & 0.144 & 0.120 & 0.158 \\
\hline & $(0.002)$ & $(0.005)$ & $(0.007)$ & $(0.006)$ \\
\hline \multirow[t]{2}{*}{$55-64$} & 0.154 & 0.252 & 0.257 & 0.249 \\
\hline & $(0.002)$ & $(0.007)$ & $(0.010)$ & $(0.008)$ \\
\hline \multirow[t]{2}{*}{$65-74$} & 0.084 & 0.218 & 0.240 & 0.204 \\
\hline & $(0.002)$ & $(0.006)$ & $(0.010)$ & $(0.007)$ \\
\hline \multirow[t]{2}{*}{$75-84$} & 0.049 & 0.162 & 0.201 & 0.138 \\
\hline & $(0.001)$ & $(0.006)$ & $(0.009)$ & $(0.006)$ \\
\hline \multirow[t]{2}{*}{$85+$} & 0.019 & 0.059 & 0.081 & 0.045 \\
\hline & $(0.001)$ & $(0.004)$ & $(0.007)$ & $(0.004)$ \\
\hline \multicolumn{5}{|l|}{ Sex } \\
\hline \multirow[t]{2}{*}{ Male } & 0.438 & 0.932 & 0.937 & 0.929 \\
\hline & $(0.002)$ & $(0.004)$ & $(0.005)$ & $(0.005)$ \\
\hline \multirow[t]{2}{*}{ Female } & 0.562 & 0.068 & 0.063 & 0.071 \\
\hline & $(0.002)$ & $(0.004)$ & $(0.005)$ & $(0.005)$ \\
\hline \multicolumn{5}{|l|}{ Race/ethnicity } \\
\hline \multirow[t]{2}{*}{ White (non-Hispanic) } & 0.662 & 0.823 & 0.824 & 0.822 \\
\hline & $(0.008)$ & $(0.007)$ & $(0.009)$ & $(0.009)$ \\
\hline \multirow[t]{2}{*}{ Hispanic } & 0.150 & 0.043 & 0.040 & 0.045 \\
\hline & $(0.007)$ & $(0.003)$ & $(0.004)$ & $(0.004)$ \\
\hline \multirow[t]{2}{*}{ Black (non-Hispanic) } & 0.116 & 0.100 & 0.106 & 0.096 \\
\hline & $(0.005)$ & $(0.005)$ & $(0.006)$ & $(0.006)$ \\
\hline \multirow[t]{2}{*}{ Asian } & 0.051 & 0.012 & 0.007 & 0.016 \\
\hline & $(0.003)$ & $(0.003)$ & $(0.002)$ & $(0.003)$ \\
\hline \multirow[t]{2}{*}{ Other and multiple } & 0.021 & 0.022 & 0.024 & 0.021 \\
\hline & $(0.002)$ & $(0.002)$ & $(0.003)$ & $(0.003)$ \\
\hline
\end{tabular}


Table B-3-Continued

\begin{tabular}{|c|c|c|c|c|}
\hline \multirow[b]{2}{*}{ Demographic Group } & \multicolumn{4}{|c|}{$\begin{array}{l}\text { Distribution by Demographic Characteristics } \\
\text { (Standard Errors) }\end{array}$} \\
\hline & Non-Veterans & Veterans & $\begin{array}{l}\text { Veterans, } \\
\text { VA Patients }\end{array}$ & $\begin{array}{c}\text { Veterans, } \\
\text { Non-VA } \\
\text { Patients }\end{array}$ \\
\hline \multicolumn{5}{|l|}{ Lives in an MSA } \\
\hline \multirow[t]{2}{*}{ No } & 0.154 & 0.187 & 0.213 & 0.170 \\
\hline & $(0.010)$ & $(0.013)$ & $(0.018)$ & $(0.012)$ \\
\hline \multirow[t]{2}{*}{ Yes } & 0.846 & 0.813 & 0.787 & 0.830 \\
\hline & $(0.010)$ & $(0.013)$ & $(0.018)$ & $(0.012)$ \\
\hline \multicolumn{5}{|l|}{ Married } \\
\hline \multirow[t]{2}{*}{ No } & 0.477 & 0.341 & 0.374 & 0.320 \\
\hline & $(0.004)$ & $(0.009)$ & $(0.011)$ & $(0.010)$ \\
\hline \multirow[t]{2}{*}{ Yes } & 0.523 & 0.659 & 0.626 & 0.680 \\
\hline & $(0.004)$ & $(0.009)$ & $(0.011)$ & $(0.010)$ \\
\hline \multicolumn{5}{|l|}{ Student or currently in school } \\
\hline \multirow[t]{2}{*}{ No } & 0.906 & 0.992 & 0.992 & 0.992 \\
\hline & $(0.001)$ & $(0.001)$ & $(0.001)$ & $(0.001)$ \\
\hline \multirow[t]{2}{*}{ Yes } & 0.094 & 0.008 & 0.008 & 0.008 \\
\hline & $(0.001)$ & $(0.001)$ & $(0.001)$ & $(0.001)$ \\
\hline \multicolumn{5}{|l|}{ Educational attainment } \\
\hline \multirow[t]{2}{*}{$<$ High school } & 0.176 & 0.071 & 0.091 & 0.058 \\
\hline & $(0.003)$ & $(0.004)$ & $(0.006)$ & $(0.004)$ \\
\hline \multirow[t]{2}{*}{ High school diploma or GED } & 0.308 & 0.340 & 0.350 & 0.333 \\
\hline & $(0.004)$ & $(0.009)$ & $(0.011)$ & $(0.010)$ \\
\hline \multirow[t]{2}{*}{ Some college } & 0.180 & 0.210 & 0.203 & 0.214 \\
\hline & $(0.002)$ & $(0.006)$ & $(0.009)$ & $(0.007)$ \\
\hline \multirow[t]{2}{*}{ College } & 0.336 & 0.380 & 0.356 & 0.396 \\
\hline & $(0.005)$ & $(0.008)$ & $(0.010)$ & $(0.010)$ \\
\hline \multicolumn{5}{|c|}{ Currently employed (not on active duty) } \\
\hline \multirow[t]{2}{*}{ No } & 0.293 & 0.454 & 0.587 & 0.372 \\
\hline & $(0.003)$ & $(0.009)$ & $(0.012)$ & $(0.010)$ \\
\hline \multirow[t]{2}{*}{ Yes } & 0.707 & 0.546 & 0.413 & 0.628 \\
\hline & $(0.003)$ & $(0.009)$ & $(0.012)$ & $(0.010)$ \\
\hline
\end{tabular}


Table B-3-Continued

\begin{tabular}{|c|c|c|c|c|}
\hline \multirow[b]{2}{*}{ Demographic Group } & \multicolumn{4}{|c|}{$\begin{array}{c}\text { Distribution by Demographic Characteristics } \\
\text { (Standard Errors) }\end{array}$} \\
\hline & Non-Veterans & Veterans & $\begin{array}{l}\text { Veterans, } \\
\text { VA Patients }\end{array}$ & $\begin{array}{c}\text { Veterans, } \\
\text { Non-VA } \\
\text { Patients }\end{array}$ \\
\hline \multicolumn{5}{|l|}{ Income categories } \\
\hline \multirow[t]{2}{*}{ Poor } & 0.124 & 0.063 & 0.081 & 0.052 \\
\hline & $(0.003)$ & $(0.003)$ & $(0.004)$ & $(0.003)$ \\
\hline \multirow[t]{2}{*}{ Near poor } & 0.044 & 0.035 & 0.048 & 0.026 \\
\hline & $(0.001)$ & $(0.002)$ & $(0.004)$ & $(0.002)$ \\
\hline \multirow[t]{2}{*}{ Low income } & 0.135 & 0.114 & 0.139 & 0.099 \\
\hline & $(0.002)$ & $(0.004)$ & $(0.007)$ & $(0.004)$ \\
\hline \multirow[t]{2}{*}{ Middle income } & 0.304 & 0.307 & 0.326 & 0.294 \\
\hline & $(0.003)$ & $(0.005)$ & $(0.008)$ & $(0.006)$ \\
\hline \multirow[t]{2}{*}{ High income } & 0.393 & 0.481 & 0.406 & 0.528 \\
\hline & $(0.005)$ & $(0.007)$ & $(0.011)$ & $(0.008)$ \\
\hline \multicolumn{5}{|l|}{ Income } \\
\hline \multirow[t]{2}{*}{ Total household income } & $33,546.49$ & $41,708.24$ & $35,980.76$ & $45,278.40$ \\
\hline & (304.781) & $(541.289)$ & (753.759) & $(646.528)$ \\
\hline
\end{tabular}

SOURCE: MEPS, 2006-2012.

NOTES: Columns (3) (Veterans, VA patients) and (4) (Veterans, non-VA patients) are two mutually exclusive categories of column (2) (Veterans). Sample size, Veterans $=12,313$; sample size, nonVeterans $=150,225$; Sample size, VA patients $=4,871$; sample size, non-VA patients $=7,442$. 


\section{B.4.1. Projections of VA Enrollees and Patients}

Table B-4

Projected VA Enrollment Counts (in millions), 2015-2024

\begin{tabular}{|c|c|c|c|c|c|c|c|}
\hline \multirow[b]{2}{*}{ Year } & \multirow[b]{2}{*}{$\begin{array}{l}\text { Number of } \\
\text { Veterans }\end{array}$} & \multicolumn{2}{|c|}{$\begin{array}{l}\text { "Status Quo" } \\
\text { Assumption }\end{array}$} & \multicolumn{2}{|c|}{$\begin{array}{l}\text { "Never Deployed" } \\
\text { Assumption }\end{array}$} & \multicolumn{2}{|c|}{$\begin{array}{c}\text { "Decreasing Enrollment" } \\
\text { Assumption }\end{array}$} \\
\hline & & $\begin{array}{l}\text { Number of } \\
\text { VA Enrollees }\end{array}$ & $\begin{array}{l}\text { Percentage of } \\
\text { Veterans }\end{array}$ & $\begin{array}{l}\text { Number of } \\
\text { VA Enrollees }\end{array}$ & $\begin{array}{l}\text { Percentage of } \\
\text { Veterans }\end{array}$ & $\begin{array}{l}\text { Number of } \\
\text { VA Enrollees }\end{array}$ & $\begin{array}{l}\text { Percentage of } \\
\text { Veterans }\end{array}$ \\
\hline 2015 & 21.18 & 9.27 & $44 \%$ & 9.16 & $43 \%$ & 9.27 & $44 \%$ \\
\hline 2016 & 20.76 & 9.43 & $45 \%$ & 9.23 & $44 \%$ & 9.42 & $45 \%$ \\
\hline 2017 & 20.35 & 9.57 & $47 \%$ & 9.29 & $46 \%$ & 9.55 & $47 \%$ \\
\hline 2018 & 19.93 & 9.69 & $49 \%$ & 9.32 & $47 \%$ & 9.65 & $48 \%$ \\
\hline 2019 & 19.51 & 9.80 & $50 \%$ & 9.36 & $48 \%$ & 9.73 & $50 \%$ \\
\hline 2020 & 19.10 & 9.85 & $52 \%$ & 9.35 & $49 \%$ & 9.76 & $51 \%$ \\
\hline 2021 & 18.69 & 9.90 & $53 \%$ & 9.35 & $50 \%$ & 9.78 & $52 \%$ \\
\hline 2022 & 18.29 & 9.95 & $54 \%$ & 9.35 & $51 \%$ & 9.80 & $54 \%$ \\
\hline 2023 & 17.89 & 9.98 & $56 \%$ & 9.35 & $52 \%$ & 9.80 & $55 \%$ \\
\hline 2024 & 17.49 & 10.0 & $57 \%$ & 9.33 & $53 \%$ & 9.80 & $56 \%$ \\
\hline
\end{tabular}

SOURCE: RAND analysis of VA, DoD, and Census data.

Table B-5

Projected VA Patient Counts (in millions), 2015-2024

\begin{tabular}{|c|c|c|c|c|c|c|c|}
\hline \multirow[b]{2}{*}{ Year } & \multirow[b]{2}{*}{$\begin{array}{l}\text { Number of } \\
\text { Veterans }\end{array}$} & \multicolumn{2}{|c|}{$\begin{array}{l}\text { "Status Quo" } \\
\text { Assumption }\end{array}$} & \multicolumn{2}{|c|}{$\begin{array}{l}\text { "Never Deployed" } \\
\text { Assumption }\end{array}$} & \multicolumn{2}{|c|}{$\begin{array}{c}\text { "Decreasing Enrollment" } \\
\text { Assumption }\end{array}$} \\
\hline & & $\begin{array}{l}\text { Number of } \\
\text { VA Patients }\end{array}$ & $\begin{array}{l}\text { Percentage of } \\
\text { Veterans }\end{array}$ & $\begin{array}{l}\text { Number of } \\
\text { VA Patients }\end{array}$ & $\begin{array}{l}\text { Percentage of } \\
\text { Veterans }\end{array}$ & $\begin{array}{l}\text { Number of } \\
\text { VA Patients }\end{array}$ & $\begin{array}{l}\text { Percentage of } \\
\text { Veterans }\end{array}$ \\
\hline 2015 & 21.18 & 5.99 & $28 \%$ & 5.93 & $28 \%$ & 5.99 & $28 \%$ \\
\hline 2016 & 20.76 & 6.06 & $29 \%$ & 5.95 & $29 \%$ & 6.06 & $29 \%$ \\
\hline 2017 & 20.35 & 6.12 & $30 \%$ & 5.96 & $29 \%$ & 6.11 & $30 \%$ \\
\hline 2018 & 19.93 & 6.16 & $31 \%$ & 5.96 & $30 \%$ & 6.14 & $31 \%$ \\
\hline 2019 & 19.51 & 6.20 & $32 \%$ & 5.95 & $30 \%$ & 6.16 & $32 \%$ \\
\hline 2020 & 19.10 & 6.20 & $32 \%$ & 5.92 & $31 \%$ & 6.15 & $32 \%$ \\
\hline 2021 & 18.69 & 6.21 & $33 \%$ & 5.89 & $32 \%$ & 6.14 & $33 \%$ \\
\hline 2022 & 18.29 & 6.20 & $34 \%$ & 5.87 & $32 \%$ & 6.12 & $33 \%$ \\
\hline 2023 & 17.89 & 6.19 & $35 \%$ & 5.84 & $33 \%$ & 6.09 & $34 \%$ \\
\hline 2024 & 17.49 & 6.18 & $35 \%$ & 5.81 & $33 \%$ & 6.07 & $35 \%$ \\
\hline
\end{tabular}

SOURCE: RAND analysis of VA, DoD, and Census data. 


\section{Health Care Needs of the Veteran Population}

\section{C.1. Data and Analytic Approach}

\section{C.1.1. Definitions}

\section{C.1.1.1. Veterans}

The two main populations of interest are (1) Veterans meeting VA eligibility requirements (Veterans) and (2) Veterans using VA services (VA patients). There is also an intermediate population of Veterans enrolled in VA, of which VA patients are a subset, but we did not consider this group in our analyses. The criteria used to identify these two subgroups varied according to the information available in each data source.

Veterans: When using nationally representative surveys, we defined Veterans based on self-reported Veteran status. We used honorable discharge as a proxy of VA eligibility and limited the sample to this population when possible. We also considered separated military personnel in DoD administrative records Veterans.

VA patients: When using nationally representative surveys, we classified Veterans as VA patients if they met any of the following criteria: They reported using VA services, they reported using non-VA services that were paid for by VA, or if there was some indication that VA paid for at least some of the health care services received by the respondent when possible. If the survey did not identify source of payment, we defined VA patients based on self-reported health insurance coverage. In this case, the respondent would likely be misclassified if he or she was enrolled in VA but did not use services. VA patients were identified in VA administrative data as those Veterans who were enrolled in VA and received some health service from VA in the previous year.

\section{C.1.1.2. Definition of Demographic and Geographic Characteristics}

Demographic and geographic groups were used throughout the analyses in this section and will be referred to simply as demographics. Standardizing the definition of these groups was imperative for many reasons; most importantly, the population projections of Chapter Three were combined with the analyses of this section to produce projections of the health care needs of Veterans and VA patients. By using a standardized definition of demographic characteristics, we ensured seamless integration of the results.

Our analyses attempt to generate results for demographic groups defined by age, sex, race, ethnicity, and geographic area. Where appropriate, additional demographic groups included service cohort (e.g., Vietnam, Afghanistan, Iraq), Veteran versus non-Veteran, and VA patient versus non-VA patient. Table C-1 summarizes the main demographic and geographic characteristics used throughout this section. 


\begin{tabular}{|c|c|c|c|}
\hline Characteristic & Group Description & Characteristic & Group Description \\
\hline \multirow[t]{8}{*}{ Age } & $15-24$ & & Black (non-Hispanic) \\
\hline & $25-34$ & & Asian \\
\hline & $35-44$ & & Other and multiple \\
\hline & $45-54$ & Service cohort & Pre-1950 \\
\hline & $55-64$ & & Korean War \\
\hline & $65-74$ & & Feb. 1955-July 1964 \\
\hline & $75-84$ & & Vietnam \\
\hline & $85+$ & & May 1975-July 1990 \\
\hline \multirow[t]{2}{*}{ Sex } & Male & & Aug. 1990-Aug. 2001 \\
\hline & Female & & Post 9/11 \\
\hline Race/ethnicity & White (non-Hispanic) & Geography & $\begin{array}{l}\text { Public Use Microdata } \\
\text { Area (PUMA) }\end{array}$ \\
\hline
\end{tabular}

Demographic characteristics were not available in all data sources and in some cases were not available at the same resolution. For example, geographic location was not always available at the PUMA level, and when this occurred we used available geographic information, such as state or Census region (Northeast, Midwest, South, and West).

\section{C.1.1.3. Health Conditions and Health Behaviors}

We considered a common set of key conditions when carrying out analysis of patient-level data (derived from survey, claims, or encounter data) to inform this subtask. We used several sources to identify important conditions. We began with 39 diagnostic groups identified by the VA Healthcare Analysis and Information Group (HAIG); these were chosen on the basis of prevalence, cost, and morbidity. To avoid missing conditions useful for this analysis but of less relevance to the HAIG, we added several conditions based on four different sources. First, we used the CDC Morbidity and Mortality Weekly Report, including indicators of chronic disease (Holt et al., 2015), leading causes of morbidity and mortality (Johnson et al., 2014), 10 conditions associated with the greatest total expenditures (Soni, 2011), common causes of disability among adults (Centers for Disease Control and Prevention, 2009), and from the National Vital Statistics Report “Deaths: Final Data for 2011” (Hoyert \& Xu, 2012). Second, we used the Healthcare Cost and Utilization Project's HCUPnet (Agency for Healthcare Research and Quality, undated) and the National Ambulatory Medical Care Survey/National Hospital Ambulatory Medical Care Survey (Centers for Disease Control and Prevention, undated) to identify additional conditions associated with large numbers of discharges in U.S. community hospitals or large numbers of outpatient visits. Third, we used conditions identified in Assessment B (RAND Health, 2015) as critical for understanding the workforce and infrastructure needs of VA. Fourth, we identified conditions that disproportionately affect Veterans relative to non-Veterans using a review of research studies and analyses of national survey data. After combining and de-duplicating these lists, we identified codes to be used with administrative 
data (including ICD-9 or ICD-9 code groupings produced by AHRQ's Clinical Classifications Software [CCS]). (See Section C.1.2 below.)

For the final report, we selected health conditions that were either associated with high prevalence rates among Veterans or those that disproportionately affected Veterans in comparison to non-Veterans. Analyses of individual-level survey data used additional self-reported health conditions when available, as well as self-reported difficulty with ADLs and health behaviors, including alcohol use, tobacco use, and exercise.

\section{C.1.2. Identification of Clinical Conditions in Survey and Encounter Data}

As discussed below in greater detail, the primary data sources for identifying health conditions among Veterans and non-Veterans were MEPS and encounter data from VA and MHS (the military insurance system for active military service members and dependents). All three data sources included person-level information on conditions based on ICD-9 codes. In the public use files of MEPS, the ICD-9 codes were truncated at the three-digit level or were converted into corresponding CCS codes, which are published by AHRQ (see Healthcare Cost and Utilization Project, 2015). VA and MHS encounter data included full five-digit ICD-9 codes. We used 29 coding algorithms (i.e., combinations of ICD-9 and/or CCS codes) to identify relevant clinical populations within these data sources (see Table C-2).

We identified the algorithms themselves by first searching in PubMed for studies that validated the use of particular ICD-9 codes for specified conditions with good sensitivity and positive predictive value (PPV). We searched PubMed using the following terms: (ICD-9[TIAB] OR international classification of diseases[TIAB] OR international classification of diseases[MH]) AND (validation OR sensitivity OR positive predictive value OR specificity OR accuracy OR ((claims OR administrative) AND data)). If no studies for particular conditions could be identified, we instead used relevant AHRQ CCS categories, which were designed to group conditions into categories suitable for use by health service researchers. If we were unable to identify a relevant CCS category, we identified ICD-9 codes whose descriptions were relevant for that condition.

We used these coding algorithms to directly identify conditions in VA and MHS encounter data. We attempted to identify conditions with greater accuracy by requiring that one inpatient encounter be labeled with the associated ICD-9 codes or that two outpatient encounters are labeled with the associated ICD-9 codes; this is a common approach for identifying conditions within VA encounter data (Bedimo et al., 2009; Justice et al., 2006; Park et al., 2014). We make exceptions for TBI, acute coronary syndrome, and AMI. We identify TBI cases even if there is only a single outpatient or inpatient diagnosis flagged because acute causes of TBI (concussion, skull fracture, etc.) may only be described at the initial visit, per guidance from DoD (U.S. Department of Defense, 2010). In contrast, we identify acute coronary syndrome and AMI if one or more inpatient stays had a principal diagnosis code associated with those conditions (Petersen et al., 1999). In MEPS data (which lack five-digit ICD-9 codes), we used three-digit ICD-9 codes, CCS codes, or combinations of the two to identify conditions. For example, PTSD has a single ICD-9 code (309.81); this code cannot be uniquely identified using three-digit ICD-9 codes or CCS codes alone, but the combination of the ICD-9 code 309 and the CCS code 651 uniquely identifies PTSD. 
Table C-2

Identification of Health Conditions

\begin{tabular}{|c|c|c|}
\hline Condition & ICD-9 & Comment \\
\hline $\begin{array}{l}\text { Acute coronary } \\
\text { syndrome }\end{array}$ & $\begin{array}{l}410.00 ; 410.01 ; 410.10 ; 410.11 ; 410.20 ; \\
410.21 ; 410.30 ; 410.31 ; 410.40 ; 410.41 ; \\
410.50 ; 410.51 ; 410.60 ; 410.61 ; 410.70 ; \\
410.71 ; 410.80 ; 410.81 ; 410.90 ; 410.91 ; \\
411.1\end{array}$ & $\begin{array}{l}\text { Per Varas-Lorenzo et al. (2008): "PPVs of the codes } \\
410 \text { and } 411 \text { for acute coronary syndrome were } 0.96 \\
(95 \% \mathrm{Cl}: 0.92-0.98) \text { and } 0.86(95 \% \mathrm{Cl}: 0.83-0.88) \text {, } \\
\text { respectively." We did not estimate the incidence of } \\
\text { acute coronary syndrome using MEPS data because } \\
\text { it is unlikely that survey respondents distinguished } \\
\text { between recent and remote episodes of acute } \\
\text { coronary syndrome. }\end{array}$ \\
\hline AMI & 410.xx & $\begin{array}{l}\text { Per Petersen et al. (1999), PPV of principal diagnosis } \\
\text { of } 410 . x x \text { for AMI is } 97 \% \text {. We did not estimate the } \\
\text { incidence of AMI using MEPS data because it is unlikely } \\
\text { that survey respondents distinguished between recent } \\
\text { and remote episodes of AMI. }\end{array}$ \\
\hline
\end{tabular}

All cerebrovascular 430.xx; 431.xx; 432.xx; 433.xx; 434.xx; CCS category

$\begin{array}{ll}\text { diseases } & \text { 435.xx; 436.xx; 437.xx; 438.xx }\end{array}$

All malignant 140.xx-209.xx ICD-9 codes selected had 79\% sensitivity, $80 \%$ PPV cancer using hospital discharge records (Brackley, Penning, \& Lesperance, 2006).

All mental health 290.xx; 291.xx; 292.xx; 293.xx; 294.xx; No validation study was identified, but a similar range conditions 295.xx; 296.xx; 297.xx; 298.xx; 299.xx; of ICD-9 codes has been used to identify mental health 300.xx; 301.xx; 302.xx; 303.xx; 304.xx; conditions in administrative data (Garvey Wilson, 305.xx; 306.xx; 307.xx; 308.xx; 309.xx; Messer, \& Hoge, 2009). Of note, this list excludes 311.xx; V11.xx disorders of development and childhood.

Amputation $\quad$ V49.74; V49.75; V49.76; V49.77; 897.0; We were unable to identify a validation study for $897.1 ; 897.2 ; 897.3 ; 897.4 ; 897.5 ; 897.6 ;$ ICD-9 codes identifying patients with a history of 897.7; V49.70; V49.71; V49.72; V49.73; amputation. ICD-9 codes were identified by searching V49.60; V49.61; V49.62; V49.63; a database of ICD-9 code descriptions for descriptions $997.60 ; 997.61 ; 997.62 ; 997.69 ; 885.0 ; \quad$ related to amputations, or history thereof. $885.1 ; 886.0 ; 886.1 ; 895.0 ; 895.1$; 896.0; 896.1; 896.2; 896.3; 905.9; 353.6; V49.66; V49.67; V49.64; V49.65; 887.0; 887.1; 887.2; 887.3; 887.4; 887.5; $887.6 ; 887.7$

Arthritis 274.xx; 712.xx; 713.xx; 715.xx; 716.xx; We aimed to types of arthritis that are more likely 717.xx; 718.xx; 719.xx to be due to injury (and possibly related to military service). There was no predefined, validated set of diagnoses for doing this, so we chose broad groups of ICD-9 codes related to non-immunologically mediated arthritis; we excluded diagnoses such as rheumatoid arthritis.

\begin{tabular}{|c|c|c|}
\hline Asthma & $\begin{array}{l}493.00 ; 493.01 ; 493.02 ; 493.10 ; 493.11 ; \\
493.12 ; 493.20 ; 493.21 ; 493.22 ; 493.81 ; \\
493.82 ; 493.90 ; 493.91 ; 493.92\end{array}$ & $\begin{array}{l}\text { ICD-9 codes derived from accepted quality measures } \\
\text { related to COPD and asthma (Centers for Medicare \& } \\
\text { Medicaid Services, 2015b) }\end{array}$ \\
\hline Burns & $\begin{array}{l}\text { 906.5; 906.6; 906.7; 906.8; 906.9; } \\
\text { 940.xx; 941.xx; 942.xx; 943.xx; 944.xx; } \\
\text { 945.xx; 946.xx; 947.xx; 948.xx; 949.xx }\end{array}$ & CCS category \\
\hline $\begin{array}{l}\text { Chronic renal } \\
\text { failure }\end{array}$ & $\begin{array}{l}\text { 403.xx; 404.xx; 581.xx; 582.xx; 583.xx; } \\
\text { 585.xx; 586.xx; 587.xx; V56.xx }\end{array}$ & $\begin{array}{l}\text { ICD-9 coding algorithms had poor sensitivity for } \\
\text { identifying chronic renal failure in Medicare claims } \\
\text { (3-26\%), but } 90 \% \text { PPV (Winkelmayer et al., 2005). This } \\
\text { version was adapted from Winkelmayer et al. (2005) } \\
\text { for use with MEPS. }\end{array}$ \\
\hline
\end{tabular}


Table C-2-Continued

\begin{tabular}{|c|c|c|}
\hline Condition & ICD-9 & Comment \\
\hline Colon cancer & $\begin{array}{l}\text { 153.0; 153.1; } 153.2 ; 153.3 ; 153.4 ; 153.5 ; \\
153.6 ; 153.7 ; 153.8 ; 153.9 ; 154.0 ; 154.1 ; \\
154.8 ; 230.3 ; \text { V10.05; V10.06 }\end{array}$ & $\begin{array}{l}\text { With exception of carcinoid tumors (209.xx), } \\
\text { colon cancer otherwise defined as in CCS category } \\
\text { (Setoguchi et al., 2007). Use of two ICD-9 codes was } \\
80.4 \% \text { sensitive, } 99.5 \% \text { specific for colorectal cancer, } \\
\text { and codes had a } 69.4 \% \text { PPV. }\end{array}$ \\
\hline COPD & $\begin{array}{l}\text { 491.0; 491.1; 491.20; 491.21; 491.8; } \\
\text { 491.9; 492.0; 492.8; 494.xx; 494.0; } \\
\text { 494.1; 496.xx }\end{array}$ & $\begin{array}{l}\text { ICD-9 codes derived from accepted quality measures } \\
\text { related to COPD and asthma (Centers for Medicare \& } \\
\text { Medicaid Services, undated). }\end{array}$ \\
\hline Diabetes & $\begin{array}{l}250.00 ; 250.01 ; 250.02 ; 250.10 ; 250.12 ; \\
250.20 ; 250.22 ; 250.30 ; 250.32 ; \\
250.40 ; 250.42 ; 250.50 ; 250.52 ; \\
250.60 ; 250.62 ; 250.70 ; 250.72 ; 250.80 ; \\
250.82 ; 250.90 ; 250.92 ; 250.93 ; 271.4 ; \\
357.2 ; 648.00 ; 648.01 ; 648.02 ; 648.03 \\
648.04 ; 962.3\end{array}$ & $\begin{array}{l}\text { ICD-9 codes derived from accepted quality measures } \\
\text { related to diabetes Types I and II, and are similar } \\
\text { to those of CCS category (Centers for Medicare \& } \\
\text { Medicaid Services, 2015b; Chao et al., 2013; Hirsch \& } \\
\text { Scheck McAlearney, 2013). Most studies incorporated } \\
\text { prescription medication fills, so ICD-9 alone may } \\
\text { overstate prevalence; however requiring medication } \\
\text { fills would exclude diet-treated diabetes. }\end{array}$ \\
\hline GERD & $530.11 ; 530.81$ & $\begin{array}{l}\text { We were unable to identify a validation study for ICD- } \\
9 \text { codes identifying patients with a history of GERD. } \\
\text { ICD-9 codes were identified by searching a database } \\
\text { of ICD- } 9 \text { code descriptions for descriptions related to } \\
\text { reflux, or history thereof. }\end{array}$ \\
\hline Hearing loss & 389.xx & $\begin{array}{l}\text { No validation study identified, but 389.xx encompasses } \\
\text { hearing loss ICD-9 codes. }\end{array}$ \\
\hline Heart failure & $\begin{array}{l}398.91 ; 428.0 ; 428.1 ; 428.20 ; 428.21 ; \\
428.22 ; 428.23 ; 428.30 ; 428.31 ; \\
428.32 ; 428.33 ; 428.40 ; 428.41 ; \\
428.42 ; 428.43 ; 428.9 ; 425.0 ; 425.1 ; \\
425.11 ; 425.18 ; 425.2 ; 425.3 ; 425.4 ; \\
425.7 ; 425.8 ; 425.9\end{array}$ & $\begin{array}{l}\text { ICD-9 codes derived from accepted quality measures } \\
\text { related to heart failure (Centers for Medicare \& } \\
\text { Medicaid Services, 2015b). }\end{array}$ \\
\hline Hypertension & 401.xx; 402.xx; 403.xx; 404.xx; 405.xx & $\begin{array}{l}\text { Per Quan et al. (2009): "The administrative data } \\
\text { hypertension definition of ' } 2 \text { claims within } 2 \text { years or } 1 \\
\text { hospitalization' had the highest validity relative to the } \\
\text { other definitions evaluated (sensitivity } 75 \% \text {, specificity } \\
94 \%, \text { PPV } 81 \% \text {, negative predictive value } 92 \% \text {, and K } \\
\text { 0.71)." Study used ICD-9 401.xx, 402.xx, 403.xx, 404.xx, } \\
\text { 405.xx. }\end{array}$ \\
\hline IHD & $\begin{array}{l}\text { 410.xx; 411.0; 411.1; 411.8; 411.81; } \\
\text { 411.89; 412.xx; 413.0; 413.1; 413.9; } \\
\text { 414.0; 414.00; 414.01; 414.06; 414.2; } \\
\text { 414.3; 414.4; 414.8; 414.9; V45.81; } \\
\text { V458.2 }\end{array}$ & CCS category \\
\hline Lipid disorders & $272.0 ; 272.1 ; 272.2 ; 272.3 ; 272.4$ & CCS category \\
\hline Major depression & $296.2 x ; 296.3 x$ & $\begin{array}{l}\text { Derived from Fiest et al. (2014) but definition not } \\
\text { useful in MEPS public-use data, and Fiest et al. (2014) } \\
\text { found that depression coding was not reliable in VA } \\
\text { ( } 30 \% \text { sensitivity). }\end{array}$ \\
\hline
\end{tabular}


Table C-2-Continued

\begin{tabular}{|c|c|c|}
\hline Condition & ICD-9 & Comment \\
\hline $\begin{array}{l}\text { Musculoskeletal } \\
\text { conditions } \\
\text { associated with } \\
\text { chronic pain }\end{array}$ & $\begin{array}{l}\text { 715.95; 715.96; 717.6; 717.7; 717.9; } \\
\text { 718.46; 719.40; 719.45; 719.46; } \\
\text { 720.07201; 720.2; 721.3; 721.42; } \\
\text { 721.7; 721.90; 721.91; 722.10; 722.52; } \\
\text { 722.6; 722.70; 722.73; 722.80; 722.82; } \\
\text { 722.83; 724.00; 724.02; 724.2; 724.3; } \\
\text { 724.5; 724.6; 724.79; 724.8; 724.9; } \\
\text { 726.5; 726.64; 729.1; 729.90; 733.92; } \\
\text { 733.98; 736.6; 738.4; 756.11; 756.12; } \\
\text { 805.4; 836.0; 836.1; 843.9; 844.; 844.0; } \\
\text { 844.1; 844.2; 846.0; 846.1; 846.8; } \\
\text { 846.9; 847.2; 847.3; 847.4; 847.9; 905.1; } \\
\text { 907.2; 907.3; 953.2;953.3; 953.4 }\end{array}$ & $\begin{array}{l}\text { We did not identify a study that validated an } \\
\text { algorithm for case identification of musculoskeletal } \\
\text { conditions against a gold standard. Instead, we } \\
\text { derived this algorithm from Beehler et al. (2013), which } \\
\text { aimed to identify patients with chronic pain due to } \\
\text { musculoskeletal conditions within the VA ambulatory } \\
\text { population. Of note, the definition from Beehler et } \\
\text { al. excludes many acute musculoskeletal conditions, } \\
\text { such as fractures, in order to identify musculoskeletal } \\
\text { conditions more similar to other categories used } \\
\text { in the assessment, which primarily concern chronic } \\
\text { conditions. This definition also excludes non- } \\
\text { musculoskeletal conditions associated with chronic } \\
\text { pain. }\end{array}$ \\
\hline PTSD & 309.81 & $\begin{array}{l}\text { McCarron et al. (2014) noted that ICD-9 code } 309.81 \\
\text { has PPV > } 89 \% \text { in VA data; Holowka et al. (2014) noted } \\
\text { accuracy for one-year prevalence of PTSD is about } 70 \% \\
\text { (sensitivity } 91.4 \% \text {, specificity } 80.9 \text { ) with ICD-9 code } \\
309.81 \text {. }\end{array}$ \\
\hline Prostate cancer & 185.xx; 233.4; V10.46 & $\begin{array}{l}\text { ICD-9 codes selected had } 73 \% \text { sensitivity, } 79 \% \text { PPV } \\
\text { (Brackley et al., 2006). }\end{array}$ \\
\hline Schizophrenia & 295.xx & $\begin{array}{l}\text { Algorithm not validated but used in Hsu et al. (2008) } \\
\text { study of VA mental health. We excluded ICD-9 codes } \\
\text { for non-specific psychosis to avoid using episodes of } \\
\text { psychosis not associated with schizophrenia, such as } \\
289.9 \text { (pyschosis not otherwise specified). }\end{array}$ \\
\hline Spinal cord injury & $\begin{array}{l}\text { 344.9; 344.8; 907.5; 907.9; 344.2; } \\
\text { 344.3; 344.30; 344.31; 344.32; 344.4; } \\
\text { 344.40; 344.41; 344.42; 344.5; 344.60; } \\
\text { 344.61; 344.81; 344.89; 907.1;907.3; } \\
\text { 907.4; 952.00; 952.01; 952.02; 952.03; } \\
\text { 952.04; 952.05; 952.06; 952.07; } \\
\text { 952.08; 952.09; 952.10; 952.11; 952.12; } \\
\text { 952.13; 952.14; 952.15; 952.16; 952.17; } \\
\text { 952.18; 952.19; 952.2; 952.3; 952.4; } \\
\text { 952.8; 952.9; 806.38; 806.39; 806.4; } \\
\text { 806.5; 806.60; 806.61; 806.62; 806.69; } \\
\text { 806.70; 806.71; 806.72; 806.79; 806.8; } \\
\text { 806.9;907.2; 806.00; 806.01; 806.02; } \\
\text { 806.03; 806.04; 806.05; 806.06; } \\
\text { 806.07; 806.08; 806.09; 806.10; } \\
\text { 806.11; 806.12; 806.13; 806.14; 806.15; } \\
\text { 806.16; 806.17; 806.18; 806.19; 806.20; } \\
\text { 806.21; 806.22; 806.23; 806.24; } \\
\text { 806.25; 806.26; 806.27; 806.28; } \\
\text { 806.29; 806.30; 806.31; 806.32; } \\
\text { 806.33; 806.34; 806.35; 806.36; } \\
\text { 806.37; 344.0; 344.00; 344.01; 344.02; } \\
\text { 344.03; 344.04; 344.09; 344.1 }\end{array}$ & $\begin{array}{l}\text { CCS category combined with non-validated ICD-9 } \\
\text { algorithm used in Netherlands study (Hagen et al., } \\
\text { 2009; van Asbeck, Post, \& Pangalila, 2000); Hagen et al. } \\
\text { (2009) used combinations of 344.x, 907.2, 806.x, 952.x; } \\
\text { study showed the codes had good specificity (>90\%) } \\
\text { but poor sensitivity, but this combination of all codes } \\
\text { was not tested. }\end{array}$ \\
\hline
\end{tabular}


Table C-2-Continued

\begin{tabular}{|c|c|c|}
\hline Condition & ICD-9 & Comment \\
\hline Substance abuse & $\begin{array}{l}\text { 303.90; 305.00; 305.90; 303.93; } \\
\text { 305.20; 305.03; 304.00; 303.91; } \\
\text { 304.20; 304.30; 305.60; 304.80; } \\
\text { 304.90; 305.01; 304.23; 291.81; } \\
\text { 292.84; 305.02; 304.01; 305.63; } \\
\text { 304.31; 305.50; 304.21; 303.00; } \\
\text { 305.23; 304.03; 304.40; 304.33; } \\
\text { 303.92; 292.0; 305.22; 305.21; 305.70; } \\
\text { 291.89; 304.10; 304.83; 303.01; 305.62; } \\
\text { 305.93; 304.43; 304.22; 305.40; } \\
\text { 305.53; 304.70; 305.61; 291.0; 304.32; } \\
\text { 304.60; 292.89; 305.73; 305.51; } \\
\text { 304.41; 304.73; 305.91; 304.71; 292.81; } \\
\text { 304.11; 304.81; 304.02; 291.9; 303.02; } \\
\text { 292.12; 304.93; 304.91; 305.52; 303.03; } \\
\text { 305.71; 304.63; 305.72; 304.13; 305.43; } \\
\text { 305.92; 304.92; 292.11; 304.42; 291.1; } \\
\text { 291.3; 305.41; 305.30; 304.61; 304.82; } \\
\text { 305.42; 304.72; 305.33; 292.2; 304.12; } \\
\text { 304.50; 304.62; 304.53; 292.82; 291.5; } \\
\text { 304.51; 305.31; 305.80; 305.32; 291.4; } \\
\text { 304.52; 292.83; 305.81; 305.83; 305.82 }\end{array}$ & $\begin{array}{l}\text { Definitions drawn from Watkins et al. (2011) and } \\
\text { derived using expert assessment, although it was not } \\
\text { validated. }\end{array}$ \\
\hline TBI & $\begin{array}{l}\text { 800.xx; 801.xx; 803.xx; 804.xx; 850.xx; } \\
\text { 851.xx; 852.xx; 853.xx; 854.xx; 310.2; } \\
\text { 950.1; 950.2; 950.3; V15.52; 959.01; } \\
\text { 905.0; 907.0 }\end{array}$ & $\begin{array}{l}\text { Used VA list of ICD-9 codes corresponding to TBI } \\
\text { (Taylor, 2012). Carlson et al. (2013) found } 70 \% \\
\text { sensitivity, } 85 \% \text { PPV in population that screened } \\
\text { positive for potential TBI, but validation study for } \\
\text { entire VA population not found. }\end{array}$ \\
\hline $\begin{array}{l}\text { Viral Hepatitis and } \\
\text { Hepatitis C }\end{array}$ & $070.4 ; 070.5 ; 070.6 ; 070.7$ & $\begin{array}{l}\text { CCS category; in MEPS this includes all viral hepatitis } \\
\text { because it is not possible to separate hepatitis C and } \\
\text { hepatitis at } 3 \text { digit level OR via CCS. Kramer et al. } \\
\text { (2008) showed } 93 \% \text { PPV, } 92 \% \text { NPV for hepatitis C in } \\
\text { VA patients with HIV. Niu, Forde, \& Goldberg (2015) } \\
\text { showed that } 1 \text { inpatient or } 2 \text { outpatient ICD-9 codes } \\
\text { for Hepatitis C had } 88 \% \text { PPV. }\end{array}$ \\
\hline Vision loss & $\begin{array}{l}\text { 367.0; 367.1; 367.20; 367.21; 367.22; } \\
\text { 367.31; 367.32; 367.4; 367.51; 367.52; } \\
\text { 367.53; 367.81; 367.89; 367.9; 368.00; } \\
\text { 368.01; 368.02; 368.03; 368.10; 368.11; } \\
\text { 368.12; 368.13; 368.14; 368.15; 368.16; } \\
\text { 368.2; 368.30; 368.31; 368.32; 368.33; } \\
\text { 368.34; 368.40; 368.41; 368.42; } \\
\text { 368.43; 368.44; 368.45; 368.46; } \\
\text { 368.47; 368.51; 368.52; 368.53; } \\
\text { 368.54; 368.55; 368.59; 368.60; } \\
\text { 368.61; 368.62; 368.63; 368.69; 368.8; } \\
\text { 368.9; 369.00; 369.01; 369.02; 369.03; } \\
\text { 369.04; 369.05; 369.06; 369.07; 369.08; } \\
\text { 369.10; 369.11; 369.12; 369.13; 369.14; } \\
\text { 369.15; 369.16; 369.17; 369.18; 369.20; } \\
\text { 369.21; 369.22; 369.23; 369.24; 369.25; } \\
\text { 369.3; 369.4; 369.60; 369.61; 369.62; } \\
\text { 369.63; 369.64; 369.65; 369.66; 369.67; } \\
\text { 369.68; 369.69; 369.70; 369.71; 369.72; } \\
\text { 369.73; 369.74; 369.75; 369.76; 369.8; } \\
\text { 369.9; V41.0 }\end{array}$ & CCS category, which includes mild vision loss. \\
\hline
\end{tabular}


Table C-2-Continued

\begin{tabular}{|c|c|c|}
\hline Condition & ICD-9 & Comment \\
\hline Women's health & 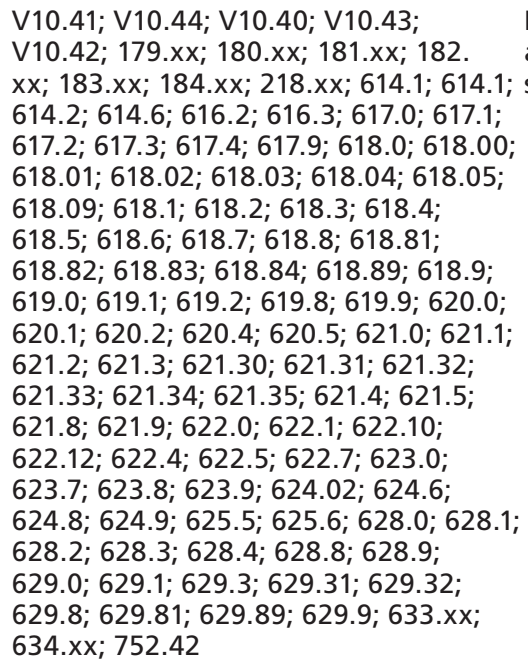 & $\begin{array}{l}\text { Represents conditions for which surgery is indicated, } \\
\text { and uses ICD-9 codes provided by consultation from } \\
\text { subject matter experts at VA. }\end{array}$ \\
\hline
\end{tabular}

\section{C.1.3. Data Sources}

The analysis relied on several data sources, including Veteran survey data and nationally representative survey data (that include some measure of Veteran status) and administrative data provided by VA. The passages following Table C-3 briefly discuss the main advantages and limitations of each available data source.

The Medical Expenditure Panel Survey (MEPS) is a recurring, nationally representative longitudinal survey of the U.S. civilian noninstitutionalized population drawn from the NHIS sampling frame. The individual-level data contain information on Veteran status (but does not include information on service era), age, sex, race/ethnicity, marital status, education, and income. Health information is obtained using open-ended questions about medical conditions present during the past year. These open-ended responses are then collapsed into ICD-9 codes.

MEPS is the only publicly available data source from which we can estimate diagnosed prevalence rates for all Veterans (who are defined based on self-report of being honorably discharged by the military). ${ }^{1}$ We infer whether an individual in the MEPS is a VA patient based on the respondents' source of payment for health care. Specifically, we defined active VA patients as those respondents who had any payment by VA for services used. Unfortunately, the MEPS data do not enable us to identify all Veterans who are eligible for VA services; we can identify only those eligible Veterans who use VA services. There are also some specific conditions for which MEPS is incapable of providing reliable estimates due to sample size limitations; these primarily consist of relatively rare conditions, such as polytrauma, TBI, and medically unexplained illness. The analyses use data from 2006 to 2012.

The National Health Interview Survey (NHIS), sponsored by the CDC, is an individual-level annual survey that includes information on demographics, Census region, general health, cancer screening, and self-reported medical conditions (including asthma, arthritis, cancer, diabetes), health behaviors (including alcohol use, smoking, exercise), physical

\footnotetext{
1 Prior to 2006, the survey instrument asked whether anyone in the household had ever served on active duty in the Armed Forces of the United States [emphasis added].
} 
Table C-3

Summary of Data Sources

\begin{tabular}{|c|c|c|c|c|c|c|}
\hline Data File & Population & Health & $\begin{array}{l}\text { Geographic } \\
\text { Location }\end{array}$ & $\begin{array}{l}\text { Veteran } \\
\text { Status }\end{array}$ & VA Enrolled & VA Patient \\
\hline \multicolumn{7}{|c|}{ Nationally representative surveys } \\
\hline MEPS $^{a}$ & $\begin{array}{l}\text { Civilians, Veterans, VA } \\
\text { patients }\end{array}$ & Yes & Census region & Yes & Yes & Yes \\
\hline $\mathrm{NHIS}^{\mathrm{b}}$ & $\begin{array}{l}\text { Civilians, Veterans, VA } \\
\text { enrollees }\end{array}$ & Yes & Census region & Yes & Yes & Yes \\
\hline BRFSSC & $\begin{array}{l}\text { National Veterans, } \\
\text { civilians }\end{array}$ & Yes & State & Yes & Yes & Yes \\
\hline U.S. Census & Civilians, Veterans & No & Yes & No & No & No \\
\hline $\operatorname{ACS}^{d}$ & $\begin{array}{l}\text { Civilians, Veterans, } \\
\text { eligible Veterans, } \\
\text { enrollees }\end{array}$ & Limited & Yes & Yes & Yes & No \\
\hline \multicolumn{7}{|c|}{ Veteran/VA surveys } \\
\hline NSV & Veterans & Limited & No & Yes & Yes & Yes \\
\hline SoE & VA enrollees & Limited & Yes & Yes & Yes & Yes \\
\hline \multicolumn{7}{|c|}{ Administrative data } \\
\hline VA Encounter & VA patients & Yes & Yes & Yes & Yes & Yes \\
\hline VA Enrollment & $\begin{array}{l}\text { VA patients, VA } \\
\text { enrollees }\end{array}$ & No & Yes & Yes & Yes & Yes \\
\hline MHS & Active duty military & Yes & Yes & N/A & $\mathrm{N} / \mathrm{A}$ & N/A \\
\hline $\begin{array}{l}\text { Active Duty } \\
\text { Master and Loss }\end{array}$ & Active duty military & No & No & N/A & $\mathrm{N} / \mathrm{A}$ & N/A \\
\hline Work Experience & Service members & No & No & $\mathrm{N} / \mathrm{A}$ & $\mathrm{N} / \mathrm{A}$ & $\mathrm{N} / \mathrm{A}$ \\
\hline $\begin{array}{l}\text { Contingency } \\
\text { Tracking System }\end{array}$ & Service members & No & Yes & $\mathrm{N} / \mathrm{A}$ & $\mathrm{N} / \mathrm{A}$ & $\mathrm{N} / \mathrm{A}$ \\
\hline
\end{tabular}

NOTE: For the survey data sources, details vary regarding how Veteran status, enrollee states, and patient status is assessed. Enrollee status is typically based on a self-report of enrollment or use of VA insurance. Patient status may be inferred in some cases based on utilization of VA services, or payment for services by the VA.

a See Medical Expenditure Panel Survey, undated.

b See Centers for Disease Control and Prevention, 2012.

' See Centers for Disease Control and Prevention, 2014a.

d See U.S. Census Bureau, 2015.

or functional limitations, and mental health (adult mental health, stress). We defined eligible Veterans to be those who self-reported having served in active military duty. It is not possible to identify VA patients in this data. We analyzed data from 2011 to 2013, which cover the period when military service information is available. We used NHIS to estimate the prevalence of the selected health conditions among Veterans and non-Veterans. Where possible, we compared these estimates from those derived using MEPS which were based on ICD-9-based health conditions. 
The Behavioral Risk Factor Surveillance System (BRFSS), sponsored by the CDC, is a nationally representative survey fielded monthly by state health departments that collects individual-level information on health behaviors and risk factors associated with the leading causes of premature mortality and morbidity among adults in the United States. We used data covering 2013. The survey allows for periodic experimental modules and for states to ask additional questions beyond the core instrument. The survey asks whether respondents have ever served in active military duty in the core instruments, and we used this response to identify Veterans. There are also several experimental modules (for certain states) that have asked more detailed questions about deployment, service, and a few specific Veterans' health issues (e.g., PTSD, traumatic brain injury), but we did not use this information because the sample sizes were small and the populations were not nationally representative. We used BRFSS to estimate the prevalence of the self-reported health conditions among Veterans and non-Veterans. BRFSS was also used to examine differences in health behaviors (e.g., smoking) and other health indicators (e.g., obesity) between Veterans and non-Veterans.

The VA Survey of Veteran Enrollees Health and Reliance Upon VA (SoE) is a recurring nationwide survey of more than 40,000 Veterans enrolled in the VA system. The survey includes each enrollee's demographic and socioeconomic characteristics, general health status, period of military service, and priority group (from administrative record). It also includes information relevant to enrollees' relationships with the VA system, including when and why individuals enrolled in VA, health insurance coverage, long-term care insurance, use of VA and non-VA services, and payer information. We used this survey to determine the characteristics of Veteran VA enrollees predicting use (or non-use) of VA services and, in combination with demographic projections, future rates of VA use.

VA encounter data include individual-level information on diagnoses, demographic characteristics and geographic location (state). VA encounter data was used to estimate current and prior condition prevalence patterns among active VA patients. This data set has larger sample sizes which allow us to estimate the prevalence of service-connected health conditions that exhibit very low prevalence in the national population. A limitation of these data is that it does not capture Veterans not enrolled in VA. Moreover, the database includes only health conditions of VA patients that were treated at VA. This may represent only a subset of total health conditions if VA patients also seek care from non-VA providers. Most Veterans with service-connected disabilities use VA (RAND analysis of FY14 VHA Support Service Center Current Enrollment Cube Data), so the prevalence estimates for these conditions are expected to be more representative of the overall prevalence in the Veteran population.

The VA Enrollment data are derived from the Health Eligibility Center enrollment files via the Assistant Deputy Under Secretary for Health enrollment file. The data include the counts of VA patients and VA enrollees by state of residence, sex, age group, and IraqAfghanistan deployment status. We used data covering the period 2005-2014.

MHS encounter data include information on diagnoses and demographic characteristics of active military personnel. We used MHS encounter data to estimate the prevalence of health conditions among separating personnel, which may be useful in predicting health conditions among future VA service users and in determining variation in demand for VA services by health condition.

The DMDC Loss files are administrative data used to identify recently separated military personnel. The DMDC Loss files were linked to the MHS encounter data using a scrambled identifier. 
The Work Experience (WEX) file contains a longitudinal record for each individual who has served in the active or reserve forces since September 1990. The file contains information on service/component/reserve category, pay grade, occupation (primary, secondary, or duty), and unit identification code. The WEX is built from information in DMDC's Active Duty Master Personnel Edit file, equivalent reserve files, and the underlying service files.

The Contingency Tracking System (CTS) data are administrative data that indicate whether active military personnel were ever deployed. These data were linked to the DMDC Loss Files to track the fraction of separating soldiers that were every deployed over time.

\section{C.1.4. Prevalence Estimated from Surveys Versus Administrative Health Records}

Our findings are based on data from both the MEPS survey and VA administrative health records, which can lead to differences in estimated prevalence of disease. We reviewed the literature to assess the sensitivity and robustness of our estimates.

We identified four studies of North American populations that compared self-reported health conditions to findings from administrative health records that in these studies are considered the reference standard or "gold" standard (Martin, Leff, Calonge, Garrett, \& Nelson, 2000; Muggah, Graves, Bennett, \& Manuel, 2013; Okura, Urban, Mahoney, Jacobsen, \& Rodeheffer, 2004; Singh, 2009). Table C-4 summarizes these four studies, describing the survey or administrative data used as well as the population covered and the reference standard data used.

These four studies reported the sensitivity and specificity across conditions (see Table C-5). The high specificity across conditions reflects the low chance that respondents report a condition that is not found in the medical record. Sensitivity varied across conditions and tended to be highest for diabetes and hypertension. Sensitivity may be higher for chronic conditions that require ongoing care than episodic or acute conditions. Differences in sensitivity and specificity across studies could arise because of differences in populations studied, differences in the reference standard, and differences in how completely administrative health records captured health care.

Direction of bias in self-report data: A review by Bhandari and Wagner (2006) concluded that respondents tend to under-report health care utilization, especially for recall periods longer than six months (they reported on recall of up to 12 months). This is consistent with

Table C-4

Summary of Published Studies Comparing Self-Reported Versus Administrative Health Data

\begin{tabular}{|c|c|c|c|c|}
\hline Description & Muggah et al., 2013 & Singh, 2009 & Okura et al., 2004 & Martin et al., 2000 \\
\hline $\begin{array}{l}\text { Survey } \\
\text { instrument }\end{array}$ & $\begin{array}{l}\text { Canadian Community } \\
\text { Health Survey }\end{array}$ & $\begin{array}{l}\text { Veteran's Quality of } \\
\text { Life Study }\end{array}$ & $\begin{array}{l}\text { "Has a medical provider } \\
\text { ever told you that you } \\
\text { had any of following } \\
\text { conditions?" }\end{array}$ & $\begin{array}{l}\text { Behavioral Risk Factor } \\
\text { Survey }\end{array}$ \\
\hline $\begin{array}{l}\text { Reference } \\
\text { standard }\end{array}$ & $\begin{array}{l}\text { Diagnostic codes in } \\
\text { Annual Health } \\
\text { Review (AHR) }\end{array}$ & $\begin{array}{l}\text { VA medical records: } \\
\text { ICD-9 codes and } \\
\text { pharmacy records }\end{array}$ & Medical Record Review & $\begin{array}{l}\text { Diagnostic codes in } \\
\text { AHR }\end{array}$ \\
\hline Population & Ontario & $\begin{array}{l}\text { Veterans who received } \\
\text { care at VA Veterans } \\
\text { Integrated Service } \\
\text { Network-13 facilities }\end{array}$ & Minnesota & Colorado HMO \\
\hline $\mathrm{N}$ & 85,459 & 40,508 & 2,037 & 599 \\
\hline
\end{tabular}


Table C-5

Summary of Published Comparisons of Health Conditions Based on Self-Report Versus Administrative Health Data

\begin{tabular}{|c|c|c|c|c|c|c|c|c|}
\hline $\begin{array}{l}\text { Health } \\
\text { Condition }\end{array}$ & Sensitivity & Specificity & Sensitivity ${ }^{a}$ & ${ }^{a}$ Specificity ${ }^{a}$ & Sensitivity & Specificity & Sensitivity & Specificity \\
\hline Diabetes & 0.73 & 0.99 & 0.91 & 0.95 & 0.66 & 0.99 & 0.73 & 0.99 \\
\hline Hypertension & 0.65 & 0.96 & & & 0.82 & 0.92 & 0.83 & 0.83 \\
\hline $\mathrm{CHF}$ & 0.26 & 0.99 & & & & & & \\
\hline Stroke & 0.49 & 0.99 & & & 0.78 & 0.99 & & \\
\hline Asthma & 0.55 & 0.96 & & & & & & \\
\hline COPD & 0.26 & 0.97 & & & & & & \\
\hline Depression & & & 0.72 & 0.82 & & & & \\
\hline AMI & & & & & 0.89 & 0.98 & & \\
\hline $\begin{array}{l}\text { Hyper- } \\
\text { cholesterolemia }\end{array}$ & & & & & & & 0.59 & 0.94 \\
\hline
\end{tabular}

findings by Muggah et al. (2013), who found that estimated prevalence of health care conditions based on self-report tended to be lower than estimates based on administrative health records. In contrast, a study by Zuvekas and Olin (2009) found close agreement between survey-reported utilization from MEPS and Medicare claims data among Medicare-enrolled respondents. Inconsistencies between self-report and medical records were more likely for men and patients with less education (Okura et al., 2004; Singh, 2009).

Consistency across surveys: $\mathrm{Li}$ et al. (2012) estimated prevalence of a range of health conditions using NHIS, BRFSS, and the National Health and Nutrition Examination Survey, and found that these surveys produced similar estimated prevalence of diabetes, hypertension, and stroke. An earlier comparison by Fahimi, Link, Mokdad, Schwartz, and Levy (2008) produced similarly reassuring findings. Many of the issues related to BRFSS are attributable to the use of landline phones; it is not clear whether inclusion of cell phones in the sampling frame (since 2011) has ameliorated bias problems. Earlier studies have found that compared to BRFSS responders, non-responders tend to be younger, non-white, less educated, non-English speakers, and unmarried (Voigt, Koepsell, \& Daling, 2003).

\section{C.1.5. Analytic Approach}

All analyses of survey data were weighted using survey sampling weights to ensure the representativeness of the results. Weights were distributed with each survey dataset and typically accounted for the sampling design and nonresponse. We adjusted standard errors for clustering at the person-level in estimations where panel data were treated as repeated cross-sections (e.g., MEPS data, which generally includes two years of data for each survey respondent). 


\section{C.1.5.1. Current Differences in the Prevalence of Health Conditions Between Veterans and Non-Veterans}

Differences in the prevalence of health conditions between Veterans and non-Veterans can be summarized by comparing observed prevalence rates between the two groups. Any observed differences in prevalence provide insight that the two populations are different. However, this approach alone cannot separate prevalence differences due to the varying demographic composition of Veterans and non-Veterans from prevalence differences due to other factors. In order to identify the "unique" health care needs of Veterans, it is important that estimated differences between Veterans and non-Veterans do not capture differences in the demographic characteristics between the two groups. For example, we expect Veterans to have a higher prevalence of hypertension than non-Veterans because Veterans are known to be older when compared with non-Veterans, and the prevalence of hypertension increases with age. For a complete summary of the demographic differences between Veterans and non-Veterans, see Table C-18.

To account for any demographic differences between Veterans and non-Veterans, we calculated adjusted prevalence rates. An adjusted prevalence uses a model to predict the prevalence of a health condition for a reference population. This is achieved by predicting the prevalence of a health condition for a set of observations that has the same demographic composition as the population of interest. In our case, the population of interest is the Veteran population. Thus, the non-Veteran adjusted prevalence estimates the prevalence among non-Veterans if their demographic composition matched the demographic composition of Veterans, and the adjusted Veteran prevalence estimates the prevalence among Veterans using the demographic composition of Veterans.

All comparisons made between Veterans and non-Veterans using adjusted prevalence rates account for the different demographic composition of the two populations. Therefore, any remaining differences after adjustment can be attributed to factors other than demographics (e.g., military service or deployment). This provides insight into health conditions that are unique to Veterans - those that are attributable to factors other than the demographic composition of the Veteran population.

As discussed above, calculating adjusted prevalence rates for Veterans and non-Veterans requires a model that predicts the prevalence of health conditions by Veteran status and other demographics. Below, we discuss the logistic regression model we used and the associated notation:

1. $i$ represents an individual captured in the survey or administrative data of interest.

2. For each individual, $\mathrm{H}_{\mathrm{i}}=1$ denotes the presence of a health condition or behavior of interest as identified by relevant ICD-9 or by self-reported measures, or $\mathrm{H}_{\mathrm{i}}=0$ if the condition is absent.

3. Vet indicates whether the individual is a Veteran $\left(V e t_{\mathrm{i}}=1\right.$ for Veterans and $V e t_{\mathrm{i}}=0$ for non-Veterans).

4. $Z_{\mathrm{i}}$ is a vector of mutually exclusive demographic and geographic indicator variables defined by individual is geographic location (either Census Region [Northeast, Midwest, South, and Midwest] or state), age category (5- or 10-year intervals), sex, and race/ ethnicity. Service cohort is not available in MEPS, which is the principal data source for this analysis; the effect of particular service cohorts can only be indirectly approximated by determining how differences between Veterans and non-Veterans vary with age. 
5. $X_{\mathrm{i}}$ is a vector of individual characteristics that are associated with a given health condition, and that may be associated with differences in disease prevalence, including whether the individual lives in an urban location, marital status, education, employment/student status, smoking, and health insurance coverage.

We chose to use logistic regression models at the individual level of the form

$$
\operatorname{logit}\left(\mathrm{PR}\left[\mathrm{H}_{i}=1\right)=\alpha+\gamma_{1} \mathrm{Vet}_{i}+\gamma_{2} \mathrm{Z}_{i}+\gamma_{3} \mathrm{Vet}_{i} \times \mathrm{Z}_{i}+\mathrm{X}_{i} \beta+\mathrm{f}\left(\tau_{i}\right)\right. \text {, }
$$

where $\tau_{\mathrm{i}}$ is the calendar year of observation $i$, and $\mathrm{f}\left(\tau_{\mathrm{i}}\right)$ is some function of time (e.g., a spline function or a set of year indicators). The function $\mathrm{f}\left(\tau_{\mathrm{i}}\right)$ accounts for time trends in the prevalence of health conditions. The interaction term $\left(\gamma_{3} \mathrm{Vet}_{\mathrm{i}} \times \mathrm{Z}_{\mathrm{i}}\right)$ allows differences in prevalence between Veterans and non-Veterans to vary by demographic characteristics.

Let $\hat{\mathrm{h}}_{1}\left(\mathrm{Vet}_{i}, \mathrm{Z}_{i}, \mathrm{X}_{i}, \tau_{i}\right)=\operatorname{expit}\left(\hat{\alpha}+\hat{\gamma}_{1} \mathrm{Vet}_{i}+\hat{\gamma}_{2} \mathrm{Z}_{i}+\hat{\gamma}_{3} \mathrm{Vet}_{i} \times \mathrm{Z}_{i}+\mathrm{X}_{i} \hat{\beta}+\mathrm{f}\left(\tau_{i}\right)\right)$ be the estimated prevalence for an observation in year $\tau_{\mathrm{i}}$ with demographics $Z_{\mathrm{i}}$, Veteran status $V_{\mathrm{i}}$, and covariates $\mathrm{X}_{\mathrm{i}}$. The non-Veteran adjusted prevalence for year $\tau$ in demographic group $\mathrm{Z}$ is given by

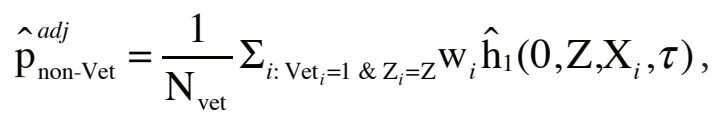

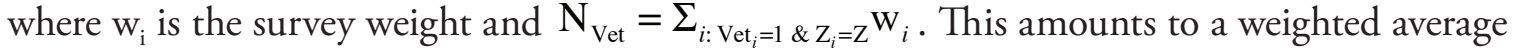
of estimated prevalences among Veterans with demographics $Z$, where the estimated prevalence is evaluated for Vet $=0$ in year $\tau$. In other words, this estimates the prevalence of a health condition among Veterans with specific demographics as if (contrary to fact) they had been nonVeterans. The adjusted prevalence for Veterans in year $\tau$ and demographic group $Z$ is given by

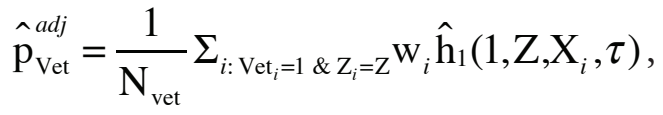

where everything is as before. Note that the only difference between the Veteran and nonVeteran adjusted prevalence is that the Veteran adjusted prevalence sets the Veteran status to 1 for all observations, and the non-Veteran adjusted prevalence sets the Veteran status to 0 for all observations.

We considered 14 age groups, four census regions, two sexes, and five races/ethnicities. All of the possible combinations among these characteristics represent 560 different demographic subgroups. If estimates for the prevalence of a health condition in any one subgroup were calculated using only data from that subgroup (a stratified estimation approach), each subgroup would need a sufficient sample size to provide a stable prevalence estimate. However, MEPS has only about 4,000 total VA patients, so many of the 560 possible subgroups do not have a sufficient sample size to obtain precise estimates of prevalence using this stratified approach. For this reason, we instead used a regression approach that uses all available data to estimate the prevalence. This is achieved by breaking the prevalence down into five factors: (1) a baseline prevalence, (2) the association with age, (3) the association with Census region, (4) the association with sex, and (5) the association with race. Each of the factors is estimated 
using data pooled across all other demographic characteristics. For example, the association of being an older patient with disease prevalence is estimated using the entire population, and this association is applied to all of the subgroups that include older patients. Put another way, the regression approach implies that the association of age with a heath condition is the same across all other demographic characteristics — that is, the effect of age is similar for males and females, blacks and whites, and so on.

Specifying a model with an extremely rich set of covariates $\mathrm{X}_{\mathrm{i}}$ increases the risk of including factors endogenous to the Veteran experience; in other words, experience in the military may lead to differences in education, marital status, and other characteristics. Including these variables in the analyses might minimize differences between Veterans and non-Veterans. The baseline analysis did not include all possible elements of $\mathrm{X}_{\mathrm{i}}$, due to their potential endogeneity with Veteran status (the baseline analysis included an indicator of urban residential location [residence in a metropolitan statistical area]), but we considered an extended model that included these additional characteristics as sensitivity analyses in Section C.3.1.

This general approach was applied to MEPS, NHIS, and BRFSS to assess the robustness of the findings. NHIS and BRFSS have larger sample sizes but measure fewer health conditions than MEPS. BRFSS was also used to examine differences in health behaviors (e.g., smoking) and other health indicators (e.g., obesity) between Veterans and non-Veterans.

\section{C.1.5.2. Current Differences in the Prevalence of Health Conditions Between VA Patients and Non-VA Patients}

The analyses comparing VA patients to non-VA patients followed the same structure as the analyses comparing Veterans to non-Veterans (C.1.5.1).

Differences in the prevalence of health conditions between VA patients and non-VA patients can be summarized by comparing observed prevalence rates between the two groups. Any observed differences in prevalence provide insight that the two populations are different. However, the demographic composition of VA patients differs from that of non-VA patients, and any observed differences in prevalence may be attributable to these demographic differences. For a complete summary of the demographic differences between VA patients and non-VA patients, see Table C-18.

In order to adjust for the different demographic compositions of VA patients and non-VA patients, we calculated adjusted prevalence rates. An adjusted prevalence uses a model to predict the prevalence of a health condition for a reference population. This is achieved by predicting the prevalence of a health condition for a set of observations that has the same demographic composition as the population of interest. For the analyses of this section, the population of interest is the Veteran population. Thus, the adjusted prevalence for VA patients estimates the prevalence among VA patients if their demographic composition matched the demographic composition of all Veterans, and the adjusted prevalence for non-VA patients estimates the prevalence among non-VA patients if their demographic composition matched the demographic composition of all Veterans.

All comparisons made between VA patients and non-VA patients using adjusted prevalence rates account for the different demographic composition of the two populations. Therefore, any remaining differences after adjustment can be attributed to factors other than demographics. If large differences remain, this suggests that the VA patient population differs from that of the general Veteran population. 
Calculating adjusted prevalence rates for VA patients and non-VA patients requires a model that predicts the prevalence of health conditions by VA patient status and other demographics. We used the following notation:

1. $i$ represents an individual captured in the survey or administrative data of interest.

2. $\mathrm{H}_{\mathrm{i}}=1$ denotes the presence of a health condition or behavior of interest as identified by relevant ICD-9 or by self-report, and $\mathrm{H}_{\mathrm{i}}=0$ if the condition is absent.

3. $\mathrm{VA}_{\mathrm{i}}$ indicates whether the individual is a VA patient $\left(\mathrm{VA}_{\mathrm{i}}=1\right.$ for $\mathrm{VA}$ patients and $\mathrm{VA}_{\mathrm{i}}=$ 0 otherwise).

4. $\mathrm{Z}_{\mathrm{i}}$ is a vector of mutually exclusive demographic and geographic indicator variables defined by individual geographic location (either Census Region [Northeast, Midwest, South and Midwest] or state), age category (5 or 10 year intervals), sex, and race/ethnicity. Service cohort is not available in MEPS, which is the principal data source for this analysis; the effect of particular service cohorts can only be indirectly approximated by determining how differences between Veterans and non-Veterans vary with age.

5. $X_{\mathrm{i}}$ is a vector of individual characteristics that are associated with health conditions that may be associated with differences in disease, including whether the individual lives in an urban location, marital status, education, employment/student status, smoking, and health insurance coverage.

We chose to use logistic regression models at the individual level of the form

$$
\operatorname{logit}\left(\operatorname{Pr}\left[\mathrm{H}_{i}=1\right]\right)=\alpha+\gamma_{1} \mathrm{VA}_{i}+\gamma_{2} \mathrm{Z}_{i}+\gamma_{3} \mathrm{VA}_{i} \times \mathrm{Z}_{i}+\mathrm{X}_{i} \beta+\mathrm{f}\left(\tau_{i}\right)
$$

where $\tau_{\mathrm{i}}$ is the calendar year of observation $i$, and $\mathrm{f}\left(\tau_{\mathrm{i}}\right)$ is some function of time (e.g., a spline function or a set of year indicators). The function $f\left(\tau_{i}\right)$ accounts for any time trends in the prevalence of health conditions across the entire Veteran population. The interaction term $\left(\gamma_{3} \mathrm{VA}_{\mathrm{i}} \times \mathrm{Z}_{\mathrm{i}}\right)$ allows differences in prevalence between VA patients and non-VA patients to vary by demographic characteristics.

Let $\hat{\mathrm{h}}_{2}\left(\mathrm{VA}_{i}, \mathrm{Z}_{i}, \mathrm{X}_{i}, \tau_{i}\right)=\operatorname{expit}\left(\hat{\alpha}+\hat{\gamma}_{1} \mathrm{VA}_{i}+\hat{\gamma}_{2} \mathrm{Z}_{i}+\hat{\gamma}_{3} \mathrm{VA}_{i} \times \mathrm{Z}_{i}+\mathrm{X}_{i} \hat{\beta}+\hat{\mathrm{f}}\left(\tau_{i}\right)\right)$ be the estimated prevalence for an observation in year $\tau_{\mathrm{i}}$ with demographics $Z_{\mathrm{i}}$, VA patient status $\mathrm{VA}_{\mathrm{i}}$, and covariates $\mathrm{X}_{\mathrm{i}}$. The VA patient adjusted prevalence for year $\tau$ in demographic group $\mathrm{Z}$ is given by

$$
\hat{\mathrm{p}}_{\mathrm{vA}}^{\text {adj }}=\frac{1}{\mathrm{~N}_{\text {vet }}} \sum_{i: \mathrm{Z}_{i}=\mathrm{Z}} \mathrm{w}_{i} \hat{\mathrm{h}}_{2}\left(1, \mathrm{Z}, \mathrm{X}_{i}, \tau\right),
$$

where $\mathrm{w}_{\mathrm{i}}$ is the survey weight and $\mathrm{N}_{\mathrm{vet}}=\Sigma_{i: \mathrm{Z}_{i}=\mathrm{Z}} \mathrm{W}_{i}$. This amounts to a weighted average of estimated prevalences among all Veterans with demographics $Z$, where the estimated prevalence is evaluated for $\mathrm{VA}=1$ in year $\tau$. In other words, we estimate the prevalence of a health condition among all Veterans with specific demographics as if they all were VA patients. The adjusted prevalence for non-VA patients in year $\tau$ and demographic group $\mathrm{Z}$ is given by

$$
\hat{\mathrm{p}}_{\text {non-VA }}^{\text {adj }}=\frac{1}{\mathrm{~N}_{\text {vet }}} \sum_{i: Z_{i}=\mathrm{Z}} \mathrm{w}_{i} \hat{\mathrm{h}}_{2}\left(0, \mathrm{Z}, \mathrm{X}_{i}, \tau\right) \text {, }
$$


where everything is as before. Note that the only difference between the VA patient and non-VA patient adjusted prevalence is that the VA patient adjusted prevalence sets the VA patient status to 1 for all observations, and the non-VA patient adjusted prevalence sets the VA patient status to 0 for all observations.

We used MEPS as the primary data source for this analysis because it identifies a broad set of diagnosed health conditions and the use of VA health care services, which is used to identify VA patients.

\section{C.1.5.3. Projecting Future Prevalence Among Veterans}

Projections of the future prevalence (and counts) of key health conditions among Veterans were undertaken in three main steps. First, the Veteran population was projected forward by demographic groups in Chapter Three. Second, the prevalence of key health conditions were projected forward by demographic groups using the models of Section C.1.5.1 (details below). Finally, the number of Veterans in each demographic group was multiplied by the corresponding prevalence to get the projected number of Veterans with a particular health condition from 2015 through 2024.

Section C.1.5.1 focuses on the current prevalence of health conditions among Veterans. Assuming that the prevalence of key health conditions is static across the next decade within demographic groups (a strong assumption), the number of Veterans with specific health conditions can be estimated by multiplying these static prevalence rates by the population projections from Chapter Three (all within demographic groups). Any changes in the overall prevalence of a health condition using this approach can be attributed to the changing demographic composition of Veterans because the demographic-specific prevalence rates are assumed to remain constant.

Assuming constant demographic-specific prevalence rates is a strong assumption and not expected to hold. The model given by equation (1) accounts for any time trends in the prevalence of health conditions through the inclusion of $\mathrm{f}\left(\tau_{\mathrm{i}}\right)$. Therefore, these models can be used to project forward the demographic-specific prevalence rates while accounting for trends over time. Recall, $\tau_{\mathrm{i}}$ is the calendar year of observation. By setting $\tau_{\mathrm{i}}$ to a future year and estimating the adjusted prevalence given in equation (3), the projected demographic-specific prevalence is obtained. For example, suppose we want to estimate the prevalence in 2020. We simply set $\tau=2020$ in equation (3):

$$
\hat{\mathrm{p}}_{\mathrm{vet}}^{\text {adj }}(\mathrm{Z}, 2020)=\frac{1}{\mathrm{~N}_{\text {vet }}} \sum_{\mathrm{Vet}_{i}=1 \& \mathrm{Z}_{i}=\mathrm{Z}} \mathrm{W}_{i} \hat{\mathrm{h}}_{1}\left(1, \mathrm{Z}, \mathrm{X}_{i}, 2020\right) .
$$

These demographic-specific prevalence projections are multiplied by the Veteran population projections of Chapter Three to obtain the projected number of Veterans with specific health conditions.

These baseline results were adjusted for likely service-connected conditions. First, the estimated prevalence rates for 2014 were fixed for each birth-year based cohort and carried forward over the projection period (2015-2024). This assumes that the prevalence of PTSD is not related to age, but instead, related to birth cohort. This allows us to use age to indirectly control for service during times of war, such as Vietnam. Specifically, the cohort that was born in 1980 has the same prevalence of PTSD in 2014 at age 34 as they would in 2024 at age 44. 
Second, prevalence estimates of newly separated Veterans were estimated using MHS encounter data. Finally, we allow for recovery from these conditions. This approach captures the more relevant trends in service-connected health conditions. The details of this adjustment are in Section C.1.5.5.

\section{C.1.5.4. Projecting Future Prevalence Among VA Patients}

Projections of the future prevalence (and counts) of key health conditions among VA patients were undertaken in four main steps. First, the Veteran population was projected forward by demographic groups in Chapter Three. Second, the number of VA patients were projected forward by demographic groups in Chapter Four. Third, the prevalence of key health conditions were projected forward by demographic groups using a modification of the models of Section C.1.5.2 (details below). Finally, the number of VA patients in each demographic group was multiplied by the corresponding prevalence to get the projected number of VA patients with a particular health condition from 2015 through 2024.

Section C.1.5.2 focuses on the current prevalence of health conditions among VA patients. Assuming that the prevalence of key health conditions is static across the next decade within demographic groups (a strong assumption), the number of VA patients with specific health conditions can be estimated by multiplying these static prevalence rates by the VA patient population projections from above (all within demographic groups). Any changes in the overall prevalence of a health condition using this approach can be attributed to the changing demographic composition of the VA patient population because the demographic-specific prevalence rates are assumed to remain constant.

Assuming constant demographic-specific prevalence rates is a strong assumption and not expected to hold. The model given by equation (4) accounts for any time trends in the prevalence of health conditions through the inclusion of $\mathrm{f}\left(\tau_{\mathrm{i}}\right)$. Therefore, these models can be used to project forward the demographic-specific prevalence rates of health conditions among VA patients while accounting for trends over time. However, in order to estimate a time trend that is consistent with our estimates for the Veteran population as a whole and to improve the precision of the trend estimate, we modified equation (4) to include an indicator for being a Veteran and to include non-Veterans in the prevalence estimation. Analogous to the Veteran case, we projected the demographic-specific prevalence among VA patients by the calendar year of observation, $\tau_{\mathrm{i}}$, to a future year and estimating the prevalence given by the modified equation (4). These demographic-specific prevalence projections were multiplied by the VA patient population projections to obtain the projected number of VA patients with specific health conditions. We generate projections using prevalence estimates from MEPS and from VA encounter data. In each case, the approach is the same. ${ }^{2}$ However, the interpretation is different. The MEPS results predict the prevalence of health conditions among VA patients, regardless of whether treatment for the specific condition is received at VA, whereas the VA encounter analysis predicts the prevalence of health conditions treated by VA among VA patients.

We also adjust these baseline projections for service-connected conditions using the same approach described in Section C.1.5.5

\footnotetext{
2 For the projections using VA encounter data, equation (4) was estimated without modification because these data include only Veterans.
} 


\section{C.1.5.5. Projecting Future Prevalence of Service-Connected Conditions}

TBI, PTSD, and other mental health conditions are connected to deployment and combat exposure (Ramchand et al., 2015). Given that TBI and PTSD are caused by specific trauma and have relatively low prevalence rates in the non-Veteran population, it is likely that these conditions are predominantly determined by military experience, rather than age, among the Veteran population. The umbrella measure of "any mental health condition" includes PTSD and other conditions that may be linked to deployment to a combat zone, so we consider it to be linked to military service rather than age for the projections.

We account for this relationship by using a birthyear-based cohort approach to project the prevalence of prevalence of TBI, PTSD, and mental health conditions for Veterans and VA patients. The future prevalence of each condition is projected separately for Veterans who separated from service before 2015 ("Pre-2015 Veterans") and for future Veterans who will separate from service in 2015-2024 ("Post-2014 Veterans").

Pre-2015 Veterans (VA patients). The estimated prevalence of each condition among Veterans in 2014 is held constant within cohorts, defined by birthyear, as the population ages. For example, the prevalence of PTSD among Veterans who were born in 1994 was 11 percent when they turned 20 years old in 2014. We assumed that the prevalence of PTSD will remain constant in these Veterans as they age, so that when they turn 30 years old in 2024, the prevalence of PTSD is 11 percent. PTSD may improve with treatment, in which case this assumption of constant prevalence within cohort would cause us to overestimate the prevalence of PTSD among Veterans and VA patients. However, research findings suggest that the highest rate of remission is within the first year post-diagnosis, ${ }^{3}$ and Veterans who separated from service before 2015 are one or more years post-service in the projection years (2015-2024). Further, the overall profile of remission is not well documented, and our data do not include information about when Veterans experienced the traumatic events, so we do not adjust for remission among this group. The projected prevalence is demographic-specific, so the overall prevalence for a birthyear cohort will change over time if the demographic composition of the cohort changes through mortality or VA use along non-age dimensions (e.g. sex).

Post-2014 Veterans (VA patients). The implicit assumption in the projections of nonservice-connected chronic conditions, such as hypertension and diabetes, is that the prevalence rate among new Veterans is the same as the prevalence rate among existing Veterans with the same demographic characteristics (e.g., age and sex). We cannot make this assumption in the case of service-connected conditions; therefore, we must assume a future prevalence rate for each health condition among new Veterans. Our underlying assumption is that there will be no future conflicts during the ten-year projection period, so new Veterans will have less exposure to combat and thus lower prevalence of TBI, PTSD, and mental health conditions. The

\footnotetext{
3 Based on a literature review, Tanielian and Jaycox (2008) suggest that the three-month remission rate of PTSD is 30-40 percent depending on type of treatment, but the probability of relapse is unknown. A review of longitudinal PTSD studies by Santiago et al. (2013) indicates that approximately 40 percent of PTSD cases remit after one year. A recent literature review and meta-analysis by Morina et al. (2014) found that 44 percent of PTSD cases were non-cases at follow-up (mean 40 months). A report by the Congressional Budget Office (2012) found that of VA patients initially diagnosed with PTSD, 75-80 percent of those who continue to use VA health services receive treatment for PTSD in the four years following diagnosis. This estimate conflates the PTSD remission rate and non-random exit from the VA health system, i.e., those who no longer need services for PTSD may drop out of the VA patient sample, but is likely a better representation of the prevalence among VA patients. Frieden and Collins (2013) find that approximately 77 percent of new TBI cases do not require treatment for more than one year post-injury.
} 
prevalence rates we assume for the projections are derived from our analysis of MHS encounter data and from the existing research on the relationship between these health conditions and deployment. We also allow for recovery or remission of the health conditions in the first year post-separation for Veterans and post-enrollment for VA patients. The assumed prevalence and remission rates are discussed in detail below.

Estimation. The projections of the prevalence of PTSD (and mental health conditions) among Veterans and VA patients are based on the 2014 demographic-specific prevalence estimates from MEPS. The projections of the prevalence of TBI among VA patients are based on the 2014 demographic-specific prevalence estimates from VA encounter data. VA encounter data reflect the health conditions of VA patients only, so we project the prevalence of this condition for VA patients only. All projections are made using demographic-group level data, but we abstract from this in the following discussion for simplicity.

The projected prevalence of health condition $H C$ among Veterans in year $t$ is the weighted average of the prevalence among the pre-2015 Veterans in each birthyear cohort and the prevalence among the post-2014 Veterans in each birthyear cohort. This is given by

$$
\mathrm{p}_{\mathrm{c}, \mathrm{t}}^{\mathrm{HC}}=\left(1-\mathrm{f}_{\mathrm{c}, \mathrm{t}}^{\text {post sep }}\right) \times \mathrm{p}_{\mathrm{c}, 2014}^{\mathrm{HC}}+\mathrm{f}_{\mathrm{c}, \mathrm{t}}^{\text {new sep }} \times \mathrm{p}_{\text {assumed }}^{\mathrm{HC} \text { initial }}+\mathrm{f}_{\mathrm{c}, \mathrm{t}}^{\text {(post sep - new sep) }} \times \mathrm{p}_{\text {assumed }}^{\mathrm{HC} \text { chronic }},
$$

where $\mathrm{f}_{\mathrm{c}, \mathrm{t}}^{\text {post sep }}$ is the fraction Veterans in year $t$ who were born in year $c$ and who separated from service after 2014, $\mathrm{p}_{\mathrm{c}, 2014}^{\mathrm{HC}}$ is the prevalence of the health condition $H C$ among Veterans who separated before 2015, and $\mathrm{p}_{\text {assumed }}^{\mathrm{HC}}$ is the prevalence rate that is assumed for Veterans who separated after 2014.

The first term is the prevalence of $H C$ among Veterans who separated from service before 2015, $\mathrm{p}_{\mathrm{c}, 2014}^{\mathrm{HC}}$, weighted by the fraction of Veterans separated before $2015,\left(1-\mathrm{f}_{\mathrm{c}, \mathrm{t}}^{\text {post sep }}\right)$. The second term is the initial prevalence of $H C$ among those who separated after 2014, $\mathrm{p}_{\text {assumed }}^{\mathrm{HC} \text { intial }}$, weighted by the fraction of Veterans who separated in year $t, \mathrm{f}_{\mathrm{c}, \mathrm{t}}^{\text {new sep }}$. The third term is the prevalence of $H C$ requiring more than one year of treatment, $\mathrm{p}_{\text {assumed }}^{\mathrm{HC} \text { chronic }}$, weighted by the fraction of Veterans who separated from service after 2014 but before year $t, \mathrm{f}_{\mathrm{c}, t}^{\text {(post sep - new sep) }}$ where $\mathrm{p}_{\text {assumed }}^{\mathrm{HC} \text { chronic }}$ is equal to $\mathrm{p}_{\text {assumed }}^{\mathrm{HC} \text { initial }}$ times the fraction of $H C$ cases who require ongoing treatment.

We follow the same approach for VA patients, with one exception. The prevalence rate and remission probability of the health conditions for future VA patients is determined by the year in which the Veteran enrolled in VA rather than by year of separation from service. That is, we split future Veterans into two groups: Veterans who were VA patients in 2014 and those who were not (post-2014 VA patients). The group of post-2014 VA patients includes all Veterans who separated post-2015 and also includes new VA patients who separated before 2015. This date is more relevant than the separation date for VA patients under the assumption that new VA patients who did not immediately enroll in VA post-separation are less likely to have a service-connected $H C$ than those who began using VA health care services right away. We make the following assumptions regarding prevalence and remission rates for the estimation:

- The prevalence of PTSD among new Veterans in 2015-2024 is 5.4 percent. This prevalence rate, estimated using MHS data, is the average of the annual prevalence rates of PTSD among service members separating 2009-2014. It is a little lower than the estimated prevalence of 7.9 percent among Iraq and Afghanistan Veterans who did not deploy 
and do not use VA in Dursa et al. (2014). The MHS estimate may overstate the prevalence of PTSD among new Veterans in 2015-2024, because only two-thirds as many of them will have deployed to Iraq or Afghanistan. However, the MHS estimate may be lower than the actual rate of PTSD if service members are reluctant to seek diagnosis and treatment for PTSD before separation, but seek care after. In addition, as discussed above, due to data limitations and lack on consensus in the literature regarding the profile of remission and relapse of these conditions, we do not formally adjust the prevalence to account for this, which may lead to projections that are too high.

- The prevalence of PTSD among new VA patients in 2015-2024 is 17.3 percent. Veterans with service-connected conditions are more likely to use VA. We estimated the prevalence ratio of PTSD among VA patients relative to all Veterans as the ratio between the prevalence of PTSD among VA patients under age 35 in 2014 (VA encounter data) and the prevalence of PTSD among separating service members under age 35 in 20122013 (MHS encounter data). We then inflate the assumed prevalence of 5.4 percent for all Veterans by this ratio (3.2). This resulting PTSD prevalence estimate for new VA patients is very close to the 17.5 percent prevalence rate of PTSD among VA patients who served post-2001 and who did not deploy to Iraq and Afghanistan estimated by Dursa et al. (2014).

- The one-year remission rate of PTSD is 35 percent for all Veterans and 30 percent for VA patients. A review of the literature suggests that the rate of remission in one year is 30-45 percent (Tanielian and Jaycox, 2008; Santiago et al., 2013; Morina et al., 2014), so we choose a middle value for the projections with all Veterans. However, a report by the Congressional Budget Office (2012) found that, of VA patients initially diagnosed with PTSD, 75-80 percent of those that continue to use VA health services receive treatment for PTSD in the four years following diagnosis. This suggests that PTSD among VA patients may be more difficult to treat, motivating us to choose a lower rate of remission for this population.

- The prevalence of mental health conditions among new Veterans in 2015-2024 is 32.5 percent. This is estimated using MHS encounter data following the approach we use for PTSD.

- The prevalence of mental health conditions among new VA patients in 2015-2024 is 48.8 percent. This was estimated from MHS and VA encounter data following the approach for PTSD.

- The one-year remission rate for mental health conditions is 6.3 percent for all Veterans and 10 percent for VA patients. The umbrella of mental health conditions includes a host of conditions, making it difficult to choose an appropriate overall remission rate for the condition, as it depends on how well each condition responds to treatment and the fraction of Veterans with each condition. However, mental health conditions include PTSD, so to be consistent with the remission rate assumed for PTSD, we apply the same rate to mental health, scaled by the fraction of Veterans with mental health conditions that have PTSD. Using 2014 MHS data, we find that 18 percent of newly separated Veterans with mental health conditions have PTSD. Therefore, we assume a 6.3 percent $(=0.18 * 0.35)$ one-year remission rate for mental health conditions among all Veterans. Similarly, we assume a scaled PTSD remission rate for mental health conditions among VA patients. Using VA encounter data, we find that about one-third of VA patients with any mental health condition have PTSD, so we assume a 10 percent rate $(=1 / 3 * 30 \%)$ for mental 
health conditions among VA patients. As Veterans can also recover from other mental health conditions, this is likely an underestimate of the recovery rate.

- The prevalence of TBI among new VA patients in each year 2015-2024 is 0.5 percent. This assumed prevalence of TBI among new VA patients reflects our assumption of no future conflict during the next decade and is derived from a CDC report of the number of TBI-related emergency department visits among adults in the United States (Centers for Disease Control and Prevention, 2014c). ${ }^{4}$ The prevalence of TBI among separating service members under the age of 35 in MHS encounters and the prevalence among VA patients under the age of 35 in VA encounter data are very similar, so no adjustments were made to the CDC rate to account for selective use of VA health care services.

- We assume that 20 percent of TBI cases among VA patients who are predicted to enroll in each year 2015-2024 require ongoing treatment. This is based on estimates from Frieden and Collins (2013).

- For all conditions-TBI, PTSD, and other mental health conditions - the probability of remission is assumed to be zero one or more years post-separation(enrollment). This assumption implies that the prevalence of health conditions among Veterans who separated before 2015 (and VA patients who enrolled in VA before 2015) is fixed for each birthyear cohort because these populations are already one year post-separation(enrollment) in the first projection year.

Limitations. The data we are using for the baseline estimates of prevalence (MEPS, VA encounter, and MHS) capture only conditions that are treated in each year. If Veterans do not require doctor visits each year treat an ongoing condition, we will underestimate the fraction of Veterans who require treatment for each condition.

The research on the prevalence of PTSD among Veterans who have not deployed is thin, making it challenging to estimate prevalence rates under the assumption of no future conflicts over the projection period. Dursa et al. (2014) and Hoge et al. (2004) are exceptions in a literature that focuses on service-connected conditions among Veterans who deployed. These two studies find an unexpectedly high rate of PTSD among Iraq and Afghanistan-era Veterans who did not deploy and who are pre-deployment, respectively. If these prevalence rates are too large, then our projections overstate the prevalence of PTSD among future Veterans.

The research on the course of remission for those with PTSD is also sparse. We aggregated the results of several literature review for our assumptions, but did not find a strong source documenting PTSD over the life course to use for this analysis. We are not able to sign the direction of the bias from our estimation assumptions because some Veterans will take longer than one year to remit (biasing our estimates upward) and some Veterans will relapse (biasing our estimates downward.

\section{C.1.5.6. Upper and Lower Bounds for Projections}

Computational limitations prevented us from calculating 95-percent prediction intervals for our projections. However, crude upper and lower bounds were estimated by calculating the 95-percent confidence intervals for the demographic-specific prevalence rates, and by performing the projections with the demographic-specific prevalence rates set to both the upper and

4 This report indicates that there were 470 TBI-related emergency department visits per 100,000 adults age 25-44 years old in 2009-2010. 
lower bounds. Specifically, we multiplied the demographic-specific prevalence upper bounds (from the 95-percent confidence intervals) by the population projections, and used the resulting projections as an upper bound. Similarly, we multiplied the demographic-specific prevalence lower bounds (from the 95-percent confidence intervals) by the population projections, and used the resulting projections as a lower bound. These bounds are expected to be more conservative (wider) than actual 95-percent prediction intervals under this modeling framework because they ignore the correlation between the predicted prevalence rates across demographic groups. Other modeling strategies would have yielded different projections and different uncertainties associated with the projections. An alternative approach would have been to model the prevalence rates within demographic groups as a time series, which accounts for the correlation between prevalence within demographic groups across time.

\section{C.2. Current Health Care Needs Prevalence Results}

\section{C.2.1. Prevalence for Veterans and Non-Veterans}

Table C-6

Prevalence of Diagnosed Health Conditions for Veterans and Non-Veterans

\begin{tabular}{|c|c|c|c|c|c|}
\hline \multirow[b]{2}{*}{ Health Conditions } & \multicolumn{2}{|c|}{$\begin{array}{l}\text { Unadjusted Mean } \\
\text { (Standard Deviation) }\end{array}$} & \multicolumn{2}{|c|}{$\begin{array}{l}\text { Adjusted Mean } \\
\text { (Standard Error) }\end{array}$} & \multirow{2}{*}{$\begin{array}{c}\text { Difference in } \\
\text { Adjusted Means } \\
\text { (Veteran - } \\
\text { Non-Veteran) }\end{array}$} \\
\hline & Veteran & Non-Veterans & Veteran & Non-Veterans & \\
\hline \multirow[t]{2}{*}{ Asthma } & 0.05 & 0.057 & 0.058 & 0.056 & 0.002 \\
\hline & $(0.003)$ & $(0.001)$ & $(0.004)$ & $(0.003)$ & \\
\hline \multirow[t]{2}{*}{ Cancer } & 0.141 & 0.047 & 0.155 & 0.112 & $0.043^{*}$ \\
\hline & $(0.005)$ & $(0.001)$ & $(0.009)$ & $(0.007)$ & \\
\hline \multirow[t]{2}{*}{$\mathrm{CHF}$} & 0.016 & 0.007 & 0.01 & 0.011 & -0.001 \\
\hline & $(0.002)$ & 0.000 & $(0.002)$ & $(0.002)$ & \\
\hline \multirow[t]{2}{*}{ COPD } & 0.058 & 0.028 & 0.063 & 0.046 & $0.017^{*}$ \\
\hline & $(0.003)$ & $(0.001)$ & $(0.006)$ & $(0.004)$ & \\
\hline \multirow[t]{2}{*}{ Diabetes } & 0.218 & 0.107 & 0.228 & 0.202 & 0.026 \\
\hline & $(0.006)$ & $(0.001)$ & $(0.008)$ & $(0.008)$ & \\
\hline \multirow[t]{2}{*}{ GERD } & 0.116 & 0.066 & 0.122 & 0.100 & $0.022^{*}$ \\
\hline & $(0.004)$ & $(0.001)$ & $(0.008)$ & $(0.006)$ & \\
\hline \multirow[t]{2}{*}{ Hearing loss } & 0.037 & 0.01 & 0.043 & 0.031 & $0.012^{*}$ \\
\hline & $(0.002)$ & 0.000 & $(0.005)$ & $(0.005)$ & \\
\hline \multirow[t]{2}{*}{ Hypertension } & 0.451 & 0.244 & 0.475 & 0.466 & 0.009 \\
\hline & $(0.007)$ & $(0.002)$ & $(0.010)$ & $(0.008)$ & \\
\hline \multirow[t]{2}{*}{ IHD } & 0.138 & 0.043 & 0.142 & 0.138 & 0.004 \\
\hline & $(0.005)$ & $(0.001)$ & $(0.008)$ & $(0.007)$ & \\
\hline
\end{tabular}


Table C-6-Continued

\begin{tabular}{|c|c|c|c|c|c|}
\hline \multirow[b]{2}{*}{ Health Conditions } & \multicolumn{2}{|c|}{$\begin{array}{l}\text { Unadjusted Mean } \\
\text { (Standard Deviation) }\end{array}$} & \multicolumn{2}{|c|}{$\begin{array}{l}\text { Adjusted Mean } \\
\text { (Standard Error) }\end{array}$} & \multirow{2}{*}{$\begin{array}{l}\text { Difference in } \\
\text { Adjusted Means } \\
\text { (Veteran - } \\
\text { Non-Veteran) }\end{array}$} \\
\hline & Veteran & Non-Veterans & Veteran & Non-Veterans & \\
\hline \multirow[t]{2}{*}{ Lipid disorder } & 0.393 & 0.193 & 0.409 & 0.392 & $0.017^{*}$ \\
\hline & $(0.007)$ & $(0.002)$ & $(0.010)$ & $(0.008)$ & \\
\hline \multirow[t]{2}{*}{ Low back pain } & 0.133 & 0.113 & 0.138 & 0.133 & 0.005 \\
\hline & $(0.004)$ & $(0.001)$ & $(0.007)$ & $(0.006)$ & \\
\hline \multirow[t]{2}{*}{ Mental health condition } & 0.175 & 0.180 & 0.200 & 0.174 & $0.026^{*}$ \\
\hline & $(0.005)$ & $(0.002)$ & $(0.009)$ & $(0.006)$ & \\
\hline \multirow[t]{2}{*}{ PTSD } & 0.014 & 0.002 & 0.027 & 0.002 & $0.025^{*}$ \\
\hline & $(0.002)$ & 0.000 & $(0.006)$ & 0.000 & \\
\hline
\end{tabular}

SOURCE: RAND analysis of MEPS, 2006-2012.

NOTES: Sample size, non-Veterans $=150,225$ and sample size, Veterans $=12,313$. Sample sizes may be smaller for some conditions due to missing values. The unadjusted prevalence in results columns (1) and (2) are equivalent to the fraction of Veterans and non-Veterans who have been diagnosed with each condition. The adjusted prevalence in results columns (3) and (4) are the predicted prevalence from a logit estimation that included indicators for sex, five race/ethnicity categories, 14 age categories, four census regions, residential location in an MSA, and a nonlinear time trend. These estimates show the differences in prevalence of health conditions for Veterans and for non-Veterans with the same demographic profile as Veterans. Cancer includes any malignancy and mental health condition includes any mental health condition.

* The difference in adjusted means is statistically different from zero at the 5 percent level ( $p$-value $<0.05)$.

Table C-7

Predicted Means of Disease Burden Measures, by Veteran Status

\begin{tabular}{|c|c|c|c|}
\hline & \multicolumn{2}{|c|}{$\begin{array}{l}\text { Adjusted Mean } \\
\text { (Standard Error) }\end{array}$} & \multirow{2}{*}{$\begin{array}{c}\text { P-Value for } \\
\text { Difference in } \\
\text { Adjusted Means }\end{array}$} \\
\hline & Veteran & Non-Veteran & \\
\hline \multirow[t]{2}{*}{ Has 1+ IADLs } & 0.072 & 0.086 & $<0.000$ \\
\hline & $(0.005)$ & $(0.004)$ & \\
\hline \multirow[t]{2}{*}{ Has 1+ ADLs } & 0.044 & 0.054 & 0.004 \\
\hline & $(0.004)$ & $(0.004)$ & \\
\hline \multirow[t]{2}{*}{ Charlson Comorbidity Index $>1$} & 0.098 & 0.094 & 0.415 \\
\hline & $(0.005)$ & $(0.005)$ & \\
\hline \multirow[t]{2}{*}{ Charlson Comorbidity Index } & 0.119 & 0.119 & 0.959 \\
\hline & $(0.008)$ & $(0.007)$ & \\
\hline
\end{tabular}

SOURCE: RAND analysis of MEPS, 2006-2012.

NOTES: Sample size, non-Veterans $=150,225$ and sample size, Veterans $=12,313$. Sample sizes may be smaller for some conditions due to missing values. Predicted or adjusted means were obtained from estimating logistic regressions with the following additional covariates included: sex (male is the omitted category), five race/ethnicity categories, 14 age categories, four Census regions, an MSA indicator, and year fixed effects using the margins command in Stata treating Veterans as though they had similar observable characteristics as civilians. The Charlson Comorbidity Index model was estimated using a Poisson regression. 
Table C-8

Adjusted Means of Comorbid Mental Health Condition (Mental Health Condition + Another Condition/Limitation) for Veterans and Non-Veterans

\begin{tabular}{lccc}
\hline & \multicolumn{2}{c}{$\begin{array}{c}\text { Adjusted Mean } \\
\text { (Standard Error) }\end{array}$} & $\begin{array}{c}\text { P-Value for } \\
\text { Difference in } \\
\text { Adjusted Means }\end{array}$ \\
\cline { 2 - 4 } & Veteran & Non-Veteran & 0.420 \\
\hline $\begin{array}{l}\text { Has any IADL + any mental health } \\
\text { condition }\end{array}$ & 0.027 & 0.025 & 0.519 \\
\hline $\begin{array}{l}\text { Has any ADL + any mental health } \\
\text { condition }\end{array}$ & $0.004)$ & $(0.003)$ & $<0.000$ \\
\hline $\begin{array}{l}\text { Has any condition + any mental health } \\
\text { condition }\end{array}$ & 0.184 & 0.014 & \\
\hline $\begin{array}{l}\text { Has any Charlson condition + any mental } \\
\text { health condition }\end{array}$ & 0.016 & 0.015 & 0.014 \\
\hline
\end{tabular}

SOURCE: RAND analysis of MEPS, 2006-2012.

NOTES: Sample size, non-Veterans $=150,225$ and sample size, Veterans $=12,313$. Sample sizes may be smaller for some conditions due to missing values. Predicted or adjusted means were obtained from estimating logistic regressions with the following additional covariates included: sex (male is the omitted category), five race/ethnicity categories, 14 age categories, four Census regions, an MSA indicator, and year fixed effects using the margins command in Stata treating Veterans as though they had similar observable characteristics as civilians. The Charlson Comorbidity Index model was estimated using a Poisson regression.

Table C-9

Prevalence of Diagnosed Health Conditions for Veterans, by Demographic Characteristics

\begin{tabular}{lccccccc}
\hline & \multicolumn{7}{c}{$\begin{array}{c}\text { Adjusted Prevalence } \\
\text { (Standard Error) }\end{array}$} \\
\cline { 2 - 8 } Demographic Group & Cancer & COPD & Diabetes & HBP & $\begin{array}{c}\text { Hearing } \\
\text { Loss }\end{array}$ & IHD & PTSD \\
\hline Age & & & & & \\
20-34 & 0.005 & 0.005 & 0.023 & 0.075 & IS & 0.006 & 0.095 \\
\hline $35-44$ & $(0.003)$ & $(0.004)$ & $(0.007)$ & $(0.016)$ & & $(0.004)$ & $(0.037)$ \\
\hline $45-54$ & 0.016 & 0.017 & 0.065 & 0.191 & 0.010 & 0.016 & 0.044 \\
& $(0.006)$ & $(0.007)$ & $(0.012)$ & $(0.023)$ & $(0.006)$ & $(0.006)$ & $(0.015)$ \\
\hline $55-64$ & 0.041 & 0.035 & 0.137 & 0.337 & 0.018 & 0.063 & 0.016 \\
& $(0.010)$ & $(0.010)$ & $(0.018)$ & $(0.027)$ & $(0.007)$ & $(0.012)$ & $(0.006)$ \\
\hline $65-74$ & 0.097 & 0.083 & 0.223 & 0.542 & 0.027 & 0.127 & 0.053 \\
& $(0.014)$ & $(0.015)$ & $(0.022)$ & $(0.028)$ & $(0.008)$ & $(0.018)$ & $(0.016)$ \\
\hline & 0.210 & 0.091 & 0.317 & 0.644 & 0.045 & 0.193 & 0.013 \\
& $(0.024)$ & $(0.017)$ & $(0.027)$ & $(0.025)$ & $(0.011)$ & $(0.023)$ & $(0.006)$ \\
\hline
\end{tabular}


Table C-9-Continued

\begin{tabular}{|c|c|c|c|c|c|c|c|}
\hline \multirow[b]{2}{*}{ Demographic Group } & \multicolumn{7}{|c|}{$\begin{array}{l}\text { Adjusted Prevalence } \\
\text { (Standard Error) }\end{array}$} \\
\hline & Cancer & COPD & Diabetes & HBP & $\begin{array}{l}\text { Hearing } \\
\text { Loss }\end{array}$ & IHD & PTSD \\
\hline \multirow[t]{2}{*}{$75-84$} & 0.298 & 0.099 & 0.398 & 0.693 & 0.074 & 0.317 & 0.011 \\
\hline & $(0.030)$ & $(0.018)$ & $(0.030)$ & $(0.025)$ & $(0.017)$ & $(0.031)$ & $(0.006)$ \\
\hline \multirow[t]{2}{*}{$85+$} & 0.317 & 0.113 & 0.342 & 0.702 & 0.141 & 0.333 & 0.006 \\
\hline & $(0.034)$ & $(0.025)$ & $(0.034)$ & $(0.030)$ & $(0.033)$ & $(0.036)$ & $(0.006)$ \\
\hline \multicolumn{8}{|l|}{ Sex } \\
\hline \multirow[t]{2}{*}{ Male } & 0.149 & 0.070 & 0.238 & 0.508 & 0.045 & 0.164 & 0.032 \\
\hline & $(0.015)$ & $(0.012)$ & $(0.019)$ & $(0.021)$ & $(0.010)$ & $(0.017)$ & $(0.009)$ \\
\hline \multirow[t]{2}{*}{ Female } & 0.133 & 0.096 & 0.225 & 0.442 & 0.016 & 0.072 & 0.022 \\
\hline & $(0.025)$ & $(0.025)$ & $(0.030)$ & $(0.032)$ & $(0.009)$ & $(0.023)$ & $(0.010)$ \\
\hline \multicolumn{8}{|l|}{ Race/ethnicity } \\
\hline \multirow[t]{2}{*}{ White (non-Hispanic) } & 0.158 & 0.074 & 0.230 & 0.494 & 0.046 & 0.167 & 0.027 \\
\hline & $(0.016)$ & $(0.012)$ & $(0.019)$ & $(0.022)$ & $(0.011)$ & $(0.017)$ & $(0.009)$ \\
\hline \multirow[t]{2}{*}{ Hispanic } & 0.057 & 0.034 & 0.302 & 0.500 & 0.031 & 0.110 & 0.043 \\
\hline & $(0.017)$ & $(0.014)$ & $(0.034)$ & $(0.033)$ & $(0.015)$ & $(0.022)$ & $(0.018)$ \\
\hline \multirow[t]{2}{*}{ Black (non-Hispanic) } & 0.100 & 0.051 & 0.265 & 0.603 & 0.024 & 0.122 & 0.048 \\
\hline & $(0.015)$ & $(0.013)$ & $(0.025)$ & $(0.023)$ & $(0.008)$ & $(0.017)$ & $(0.015)$ \\
\hline \multirow[t]{2}{*}{ Asian } & 0.088 & 0.036 & 0.217 & 0.443 & 0.034 & 0.022 & 0.008 \\
\hline & $(0.036)$ & $(0.017)$ & $(0.047)$ & $(0.049)$ & $(0.015)$ & $(0.011)$ & $(0.007)$ \\
\hline \multirow[t]{2}{*}{ Other and multiple } & 0.074 & 0.089 & 0.319 & 0.491 & 0.055 & 0.179 & 0.077 \\
\hline & $(0.024)$ & $(0.029)$ & $(0.048)$ & $(0.046)$ & $(0.027)$ & $(0.040)$ & $(0.034)$ \\
\hline \multicolumn{8}{|l|}{ Residence in an MSA } \\
\hline \multirow[t]{2}{*}{ No } & 0.132 & 0.083 & 0.248 & 0.506 & 0.043 & 0.165 & 0.052 \\
\hline & $(0.017)$ & $(0.016)$ & $(0.024)$ & $(0.025)$ & $(0.012)$ & $(0.020)$ & $(0.017)$ \\
\hline \multirow[t]{2}{*}{ Yes } & 0.153 & 0.068 & 0.234 & 0.504 & 0.044 & 0.160 & 0.027 \\
\hline & $(0.016)$ & $(0.012)$ & $(0.019)$ & $(0.021)$ & $(0.010)$ & $(0.016)$ & $(0.008)$ \\
\hline
\end{tabular}

SOURCE: RAND analysis of MEPS, 2006-2012.

NOTES: Sample size, Veterans $=12,313$. Cells labeled IS indicate that the prevalence model for the specific health condition could not be estimated due to insufficient sample size. The adjusted prevalence estimates are the predicted prevalence from a logit estimation that included an indicators for sex, five race/ethnicity categories, 14 age categories, four census regions, residential location in an MSA, and a nonlinear time trend. These estimated differences control for the demographic differences within demographic group. HBP is hypertension. 
Table C-10

Difference in the Prevalence of Diagnosed Health Conditions for Veterans and Non-Veterans, by Demographic Characteristics

Difference in Adjusted Prevalence (Standard Error)

\begin{tabular}{|c|c|c|c|c|c|c|c|c|}
\hline Demographic Group & Asthma & Cancer & COPD & Diabetes & GERD & $\begin{array}{l}\text { Hearing } \\
\text { Loss }\end{array}$ & $\begin{array}{l}\text { Mental } \\
\text { Health } \\
\text { Condition }\end{array}$ & PTSD \\
\hline \multicolumn{9}{|l|}{ Age } \\
\hline $20-34$ & $\begin{array}{r}-0.023 \\
(0.011)\end{array}$ & $\begin{array}{r}-0.003 \\
(0.002)\end{array}$ & $\begin{array}{r}-0.003 \\
(0.004)\end{array}$ & $\begin{array}{c}0.004 \\
(0.007)\end{array}$ & $\begin{array}{l}0.018 \\
(0.012)\end{array}$ & IS & $\begin{array}{c}0.107 \\
(0.027)\end{array}$ & $\begin{array}{l}0.069 \\
(0.022)\end{array}$ \\
\hline $35-44$ & $\begin{array}{r}-0.018 \\
(0.015)\end{array}$ & $\begin{array}{r}-0.005 \\
(0.005)\end{array}$ & $\begin{array}{l}0.002 \\
(0.006)\end{array}$ & $\begin{array}{c}0.010 \\
(0.011)\end{array}$ & $\begin{array}{l}0.022 \\
(0.012)\end{array}$ & $\begin{array}{c}0.003 \\
(0.004)\end{array}$ & $\begin{array}{l}0.016 \\
(0.019)\end{array}$ & $\begin{array}{l}0.027 \\
(0.010)\end{array}$ \\
\hline $45-54$ & $\begin{array}{c}0.024 \\
(0.014)\end{array}$ & $\begin{array}{r}-0.001 \\
(0.008)\end{array}$ & $\begin{array}{c}0.006 \\
(0.008)\end{array}$ & $\begin{array}{c}0.034 \\
(0.015)\end{array}$ & $\begin{array}{l}0.026 \\
(0.013)\end{array}$ & $\begin{array}{l}0.006 \\
(0.004)\end{array}$ & $\begin{array}{l}0.058 \\
(0.022)\end{array}$ & $\begin{array}{l}0.007 \\
(0.004)\end{array}$ \\
\hline $55-64$ & $\begin{array}{c}0.031 \\
(0.014)\end{array}$ & $\begin{array}{c}0.005 \\
(0.011)\end{array}$ & $\begin{array}{l}0.035 \\
(0.013)\end{array}$ & $\begin{array}{c}0.024 \\
(0.017)\end{array}$ & $\begin{array}{l}0.003 \\
(0.015)\end{array}$ & $\begin{array}{c}0.004 \\
(0.004)\end{array}$ & $\begin{array}{c}0.050 \\
(0.020)\end{array}$ & $\begin{array}{l}0.037 \\
(0.011)\end{array}$ \\
\hline $65-74$ & $\begin{array}{c}0.033 \\
(0.020)\end{array}$ & $\begin{array}{c}0.046 \\
(0.019)\end{array}$ & $\begin{array}{l}0.018 \\
(0.014)\end{array}$ & $\begin{array}{c}0.003 \\
(0.022)\end{array}$ & $\begin{array}{l}0.001 \\
(0.017)\end{array}$ & $\begin{array}{c}0.003 \\
(0.006)\end{array}$ & $\begin{array}{c}0.003 \\
(0.017)\end{array}$ & $\begin{array}{l}0.009 \\
(0.005)\end{array}$ \\
\hline $75-84$ & $\begin{array}{l}0.028 \\
(0.018)\end{array}$ & $\begin{array}{c}0.095 \\
(0.026)\end{array}$ & $\begin{array}{l}0.014 \\
(0.016)\end{array}$ & $\begin{array}{c}0.024 \\
(0.025)\end{array}$ & $\begin{array}{l}0.032 \\
(0.020)\end{array}$ & $\begin{array}{r}-0.005 \\
(0.011)\end{array}$ & $\begin{array}{c}0.022 \\
(0.021)\end{array}$ & $\begin{array}{l}0.008 \\
(0.004)\end{array}$ \\
\hline $85+$ & $\begin{array}{c}0.038 \\
(0.024)\end{array}$ & $\begin{array}{c}0.127 \\
(0.033)\end{array}$ & $\begin{array}{l}0.040 \\
(0.023)\end{array}$ & $\begin{array}{c}0.024 \\
(0.035)\end{array}$ & $\begin{array}{c}0.073 \\
(0.024)\end{array}$ & $\begin{array}{c}0.014 \\
(0.022)\end{array}$ & $\begin{array}{c}0.017 \\
(0.028)\end{array}$ & $\begin{array}{l}0.004 \\
(0.004)\end{array}$ \\
\hline Sex & & & & & & & & \\
\hline Male & $\begin{array}{r}-0.006 \\
(0.004)\end{array}$ & $\begin{array}{c}0.019 \\
(0.004)\end{array}$ & $\begin{array}{l}0.009 \\
(0.003)\end{array}$ & $\begin{array}{c}0.023 \\
(0.005)\end{array}$ & $\begin{array}{l}0.023 \\
(0.005)\end{array}$ & IS & $\begin{array}{c}0.052 \\
(0.009)\end{array}$ & $\begin{array}{l}0.043 \\
(0.010)\end{array}$ \\
\hline Female & $\begin{array}{c}0.030 \\
(0.016)\end{array}$ & $\begin{array}{c}0.007 \\
(0.011)\end{array}$ & $\begin{array}{l}0.012 \\
(0.011)\end{array}$ & $\begin{array}{c}0.010 \\
(0.016)\end{array}$ & $\begin{array}{c}0.013 \\
(0.015)\end{array}$ & IS & $\begin{array}{c}0.055 \\
(0.025)\end{array}$ & $\begin{array}{l}0.025 \\
(0.011)\end{array}$ \\
\hline Race/ethnicity & & & & & & & & \\
\hline White (non-Hispanic) & $\begin{array}{c}0.012 \\
(0.009)\end{array}$ & $\begin{array}{c}0.013 \\
(0.007)\end{array}$ & $\begin{array}{c}0.012 \\
(0.007)\end{array}$ & $\begin{array}{c}0.020 \\
(0.009)\end{array}$ & $\begin{array}{l}0.018 \\
(0.010)\end{array}$ & IS & $\begin{array}{c}0.028 \\
(0.015)\end{array}$ & $\begin{array}{c}0.028 \\
(0.008)\end{array}$ \\
\hline Hispanic & $\begin{array}{c}0.021 \\
(0.017)\end{array}$ & $\begin{array}{r}-0.001 \\
(0.008)\end{array}$ & $\begin{array}{l}0.001 \\
(0.008)\end{array}$ & $\begin{array}{c}0.019 \\
(0.019)\end{array}$ & $\begin{array}{l}0.031 \\
(0.015)\end{array}$ & IS & $\begin{array}{c}0.134 \\
(0.028)\end{array}$ & $\begin{array}{l}0.048 \\
(0.018)\end{array}$ \\
\hline Black (non-Hispanic) & $\begin{array}{c}0.005 \\
(0.012)\end{array}$ & $\begin{array}{c}0.016 \\
(0.008)\end{array}$ & $\begin{array}{c}0.006 \\
(0.008)\end{array}$ & $\begin{array}{r}-0.011 \\
(0.015)\end{array}$ & $\begin{array}{l}0.006 \\
(0.010)\end{array}$ & IS & $\begin{array}{c}0.063 \\
(0.016)\end{array}$ & $\begin{array}{l}0.052 \\
(0.015)\end{array}$ \\
\hline Asian & $\begin{array}{c}0.027 \\
(0.022)\end{array}$ & $\begin{array}{l}0.019 \\
(0.017)\end{array}$ & $\begin{array}{l}0.014 \\
(0.010)\end{array}$ & $\begin{array}{c}0.002 \\
(0.024)\end{array}$ & $\begin{array}{l}0.018 \\
(0.019)\end{array}$ & IS & $\begin{array}{c}0.159 \\
(0.063)\end{array}$ & $\begin{array}{l}0.009 \\
(0.007)\end{array}$ \\
\hline
\end{tabular}


Table C-10-Continued

Difference in Adjusted Prevalence

(Standard Error)

\begin{tabular}{|c|c|c|c|c|c|c|c|c|}
\hline Demographic Group & Asthma & Cancer & COPD & Diabetes & GERD & $\begin{array}{l}\text { Hearing } \\
\text { Loss }\end{array}$ & $\begin{array}{l}\text { Mental } \\
\text { Health } \\
\text { Condition }\end{array}$ & PTSD \\
\hline Other and multiple & $\begin{array}{c}0.011 \\
(0.029)\end{array}$ & $\begin{array}{r}-0.006 \\
(0.015)\end{array}$ & $\begin{array}{c}0.007 \\
(0.019)\end{array}$ & $\begin{array}{c}0.008 \\
(0.033)\end{array}$ & $\begin{array}{c}0.033 \\
(0.027)\end{array}$ & IS & $\begin{array}{c}0.091 \\
(0.047)\end{array}$ & $\begin{array}{l}0.075 \\
(0.038)\end{array}$ \\
\hline \multicolumn{9}{|l|}{ Residence in an MSA } \\
\hline No & $\begin{array}{r}-0.003 \\
(0.011)\end{array}$ & $\begin{array}{c}0.010 \\
(0.008)\end{array}$ & $\begin{array}{c}0.009 \\
(0.009)\end{array}$ & $\begin{array}{c}0.011 \\
(0.014)\end{array}$ & $\begin{array}{c}0.028 \\
(0.014)\end{array}$ & IS & $\begin{array}{c}0.065 \\
(0.022)\end{array}$ & $\begin{array}{l}0.059 \\
(0.020)\end{array}$ \\
\hline Yes & $\begin{array}{c}0.016 \\
(0.009)\end{array}$ & $\begin{array}{c}0.013 \\
(0.006)\end{array}$ & $\begin{array}{c}0.011 \\
(0.006)\end{array}$ & $\begin{array}{c}0.017 \\
(0.009)\end{array}$ & $\begin{array}{c}0.016 \\
(0.008)\end{array}$ & IS & $\begin{array}{c}0.051 \\
(0.014)\end{array}$ & $\begin{array}{l}0.030 \\
(0.008)\end{array}$ \\
\hline
\end{tabular}

SOURCE: RAND analysis of MEPS, 2006-2012.

NOTES: Sample size, non-Veterans $=150,225$ and sample size, Veterans $=12,313$. Sample sizes may be smaller for some conditions due to missing values. Cells labeled IS indicate that the prevalence model for the specific health condition could not be estimated due to insufficient sample size. The adjusted prevalence estimates are the predicted prevalence from a logit estimation that included an indicators for sex, five race/ethnicity categories, 14 age categories, four census regions, residential location in an MSA, and a nonlinear time trend. These estimated differences control for the demographic differences between Veterans and non-Veterans and within demographic subgroups. Cancer includes any malignancy, and Mental Health includes any mental health condition.

Table C-11

Difference in the Prevalence of Diagnosed Health Conditions for Veterans and Non-Veterans, by Poverty/Income and Employment Status

\begin{tabular}{|c|c|c|c|c|c|c|c|c|}
\hline & \multicolumn{8}{|c|}{$\begin{array}{l}\text { Adjusted Mean } \\
\text { (Standard Error) }\end{array}$} \\
\hline & Asthma & Cancer & COPD & Diabetes & GERD & $\begin{array}{l}\text { Hearing } \\
\text { Loss }\end{array}$ & $\begin{array}{c}\text { Mental } \\
\text { Health } \\
\text { Condition }\end{array}$ & PTSD \\
\hline \multicolumn{9}{|l|}{ Poverty/income category } \\
\hline $\begin{array}{l}\text { Poor/neg income non- } \\
\text { Veterans }\end{array}$ & $\begin{array}{c}0.079 \\
(0.002)\end{array}$ & $\begin{array}{l}0.043 \\
(0.002)\end{array}$ & $\begin{array}{c}0.053 \\
(0.002)\end{array}$ & $\begin{array}{c}0.152 \\
(0.003)\end{array}$ & $\begin{array}{c}0.076 \\
(0.002)\end{array}$ & $\begin{array}{c}0.010 \\
(0.001)\end{array}$ & $\begin{array}{c}0.235 \\
(0.003)\end{array}$ & $\begin{array}{l}0.005 \\
(0.001)\end{array}$ \\
\hline $\begin{array}{l}\text { Poor/neg income } \\
\text { Veterans }\end{array}$ & $\begin{array}{c}0.064 \\
(0.009)\end{array}$ & $\begin{array}{l}0.055 \\
(0.005)\end{array}$ & $\begin{array}{c}0.063 \\
(0.007)\end{array}$ & $\begin{array}{c}0.144 \\
(0.009)\end{array}$ & $\begin{array}{c}0.075 \\
(0.008)\end{array}$ & $\begin{array}{c}0.011 \\
(0.002)\end{array}$ & $\begin{array}{c}0.282 \\
(0.015)\end{array}$ & $\begin{array}{l}0.043 \\
(0.009)\end{array}$ \\
\hline Near poor non-Veterans & $\begin{array}{c}0.068 \\
(0.003)\end{array}$ & $\begin{array}{l}0.045 \\
(0.002)\end{array}$ & $\begin{array}{c}0.044 \\
(0.002)\end{array}$ & $\begin{array}{c}0.146 \\
(0.004)\end{array}$ & $\begin{array}{c}0.067 \\
(0.003)\end{array}$ & $\begin{array}{c}0.010 \\
(0.001)\end{array}$ & $\begin{array}{c}0.193 \\
(0.005)\end{array}$ & $\begin{array}{l}0.003 \\
(0.001)\end{array}$ \\
\hline Near poor Veterans & $\begin{array}{c}0.058 \\
(0.011)\end{array}$ & $\begin{array}{c}0.072 \\
(0.008)\end{array}$ & $\begin{array}{c}0.041 \\
(0.007)\end{array}$ & $\begin{array}{c}0.141 \\
(0.012)\end{array}$ & $\begin{array}{l}0.087 \\
(0.010)\end{array}$ & $\begin{array}{c}0.013 \\
(0.003)\end{array}$ & $\begin{array}{c}0.245 \\
(0.020)\end{array}$ & $\begin{array}{r}0.052 \\
(0.014)\end{array}$ \\
\hline $\begin{array}{l}\text { Low income non- } \\
\text { Veterans }\end{array}$ & $\begin{array}{c}0.057 \\
(0.002)\end{array}$ & $\begin{array}{l}0.038 \\
(0.001)\end{array}$ & $\begin{array}{c}0.034 \\
(0.001)\end{array}$ & $\begin{array}{c}0.133 \\
(0.002)\end{array}$ & $\begin{array}{c}0.060 \\
(0.002)\end{array}$ & $\begin{array}{c}0.008 \\
(0.001)\end{array}$ & $\begin{array}{c}0.171 \\
(0.003)\end{array}$ & $\begin{array}{l}0.002 \\
(0.000)\end{array}$ \\
\hline
\end{tabular}


Table C-11-Continued

\begin{tabular}{|c|c|c|c|c|c|c|c|c|}
\hline & \multicolumn{8}{|c|}{$\begin{array}{l}\text { Adjusted Mean } \\
\text { (Standard Error) }\end{array}$} \\
\hline & Asthma & Cancer & COPD & Diabetes & GERD & $\begin{array}{l}\text { Hearing } \\
\text { Loss }\end{array}$ & $\begin{array}{l}\text { Mental } \\
\text { Health } \\
\text { Condition }\end{array}$ & PTSD \\
\hline \multirow[t]{2}{*}{ Low income Veterans } & 0.064 & 0.069 & 0.043 & 0.143 & 0.083 & 0.011 & 0.198 & 0.031 \\
\hline & $(0.007)$ & $(0.005)$ & $(0.005)$ & $(0.008)$ & $(0.007)$ & $(0.002)$ & $(0.011)$ & $(0.006)$ \\
\hline \multirow{2}{*}{$\begin{array}{l}\text { Middle income non- } \\
\text { Veterans }\end{array}$} & 0.050 & 0.040 & 0.025 & 0.120 & 0.054 & 0.008 & 0.150 & 0.001 \\
\hline & $(0.001)$ & $(0.001)$ & $(0.001)$ & $(0.002)$ & $(0.001)$ & $(0.001)$ & $(0.002)$ & $(0.000)$ \\
\hline \multirow[t]{2}{*}{ Middle income Veterans } & 0.052 & 0.056 & 0.039 & 0.143 & 0.070 & 0.013 & 0.195 & 0.027 \\
\hline & $(0.004)$ & $(0.003)$ & $(0.003)$ & $(0.005)$ & $(0.004)$ & $(0.001)$ & $(0.008)$ & $(0.004)$ \\
\hline \multirow{2}{*}{$\begin{array}{l}\text { High income non- } \\
\text { Veterans }\end{array}$} & 0.044 & 0.045 & 0.015 & 0.098 & 0.058 & 0.009 & 0.125 & 0.001 \\
\hline & $(0.001)$ & $(0.001)$ & $(0.001)$ & $(0.002)$ & $(0.001)$ & $(0.001)$ & $(0.002)$ & $(0.000)$ \\
\hline \multirow[t]{2}{*}{ High income Veterans } & 0.047 & 0.063 & 0.021 & 0.125 & 0.073 & 0.014 & 0.148 & 0.014 \\
\hline & $(0.004)$ & $(0.003)$ & $(0.002)$ & $(0.005)$ & $(0.004)$ & $(0.001)$ & $(0.006)$ & $(0.003)$ \\
\hline \multicolumn{9}{|l|}{ Employment status } \\
\hline \multirow{2}{*}{$\begin{array}{l}\text { Unemployed non- } \\
\text { Veterans }\end{array}$} & 0.080 & 0.048 & 0.048 & 0.159 & 0.081 & 0.010 & 0.236 & 0.004 \\
\hline & $(0.002)$ & $(0.001)$ & $(0.001)$ & $(0.002)$ & $(0.002)$ & $(0.001)$ & $(0.003)$ & $(0.000)$ \\
\hline \multirow[t]{2}{*}{ Unemployed Veterans } & 0.073 & 0.076 & 0.056 & 0.170 & 0.096 & 0.014 & 0.277 & 0.080 \\
\hline & $(0.005)$ & $(0.003)$ & $(0.004)$ & $(0.005)$ & $(0.005)$ & $(0.001)$ & $(0.009)$ & $(0.010)$ \\
\hline \multirow[t]{2}{*}{ Employed non-Veterans } & 0.042 & 0.036 & 0.014 & 0.093 & 0.047 & 0.007 & 0.126 & 0.001 \\
\hline & $(0.001)$ & $(0.001)$ & $(0.001)$ & $(0.001)$ & $(0.001)$ & $(0.001)$ & $(0.001)$ & $(0.000)$ \\
\hline \multirow[t]{2}{*}{ Employed Veterans } & 0.045 & 0.042 & 0.019 & 0.111 & 0.059 & 0.013 & 0.145 & 0.011 \\
\hline & $(0.004)$ & $(0.003)$ & $(0.002)$ & $(0.005)$ & $(0.003)$ & $(0.002)$ & $(0.006)$ & $(0.002)$ \\
\hline
\end{tabular}

SOURCE: RAND analysis of MEPS, 2006-2012.

NOTES: Sample size, non-Veterans $=150,225$ and sample size, Veterans $=12,313$. Sample sizes may be smaller for some conditions due to missing values. Model controls for age, sex, race/ethnicity, Census region, and MSA. 
Table C-12

Financial Insecurities, Predicted Means for Veterans and Non-Veterans

\begin{tabular}{|c|c|c|c|}
\hline Outcome & $\begin{array}{c}\text { Veterans } \\
\text { (Standard Error) }\end{array}$ & $\begin{array}{l}\text { Non-Veterans } \\
\text { (Standard Error) }\end{array}$ & $\begin{array}{c}\text { P-Value of } \\
\text { Difference in Means }\end{array}$ \\
\hline Panel A. All NHIS respondents $(\mathrm{N}=34,540)$ & $\mathrm{N}=3,309$ & $\mathrm{~N}=31,231$ & \\
\hline Moderately to severely worried about paying bills & $\begin{array}{c}0.270 \\
(0.012)\end{array}$ & $\begin{array}{c}0.326 \\
(0.004)\end{array}$ & $0.000 *$ \\
\hline $\begin{array}{l}\text { Moderately to severely worried about health care } \\
\text { costs }\end{array}$ & $\begin{array}{c}0.257 \\
(0.013)\end{array}$ & $\begin{array}{c}0.340 \\
(0.004)\end{array}$ & $0.000 *$ \\
\hline Moderately to severely worried about housing costs & $\begin{array}{c}0.204 \\
(0.011)\end{array}$ & $\begin{array}{c}0.260 \\
(0.003)\end{array}$ & $0.000 *$ \\
\hline Participate in TANF & $\begin{array}{c}0.011 \\
(0.003)\end{array}$ & $\begin{array}{c}0.011 \\
(0.001)\end{array}$ & 0.893 \\
\hline $\begin{array}{l}\text { Panel B. NHIS respondents reporting any Chronic } \\
\text { Condition^ }(N=18,645)\end{array}$ & $\mathrm{N}=2,398$ & $N=16,247$ & \\
\hline Moderately to severely worried about paying bills & $\begin{array}{c}0.317 \\
(0.016)\end{array}$ & $\begin{array}{c}0.368 \\
(0.006)\end{array}$ & $0.024^{*}$ \\
\hline $\begin{array}{l}\text { Moderately to severely worried about health care } \\
\text { Costs }\end{array}$ & $\begin{array}{l}0.290 \\
(0.017)\end{array}$ & $\begin{array}{c}0.369 \\
(0.006)\end{array}$ & $0.014^{*}$ \\
\hline Moderately to severely worried about housing cost & $\begin{array}{c}0.241 \\
(0.015)\end{array}$ & $\begin{array}{c}0.290 \\
(0.006)\end{array}$ & $0.025^{*}$ \\
\hline Participate in TANF & $\begin{array}{c}0.012 \\
(0.004)\end{array}$ & $\begin{array}{c}0.014 \\
(0.001)\end{array}$ & $0.003^{*}$ \\
\hline $\begin{array}{l}\text { Panel C. NHIS respondents reporting a Health } \\
\text { Problem Requiring Special Equipment }(\mathrm{N}=2,982)\end{array}$ & $N=396$ & $\mathrm{~N}=2,486$ & \\
\hline Moderately to severely worried about paying bills & $\begin{array}{c}0.379 \\
(0.039)\end{array}$ & $\begin{array}{c}0.467 \\
(0.022)\end{array}$ & $0.024^{*}$ \\
\hline $\begin{array}{l}\text { Moderately to severely worried about health care } \\
\text { costs }\end{array}$ & $\begin{array}{c}0.348 \\
(0.038)\end{array}$ & $\begin{array}{c}0.444 \\
(0.021)\end{array}$ & $0.014^{*}$ \\
\hline Moderately to severely worried about housing costs & $\begin{array}{c}0.295 \\
(0.036)\end{array}$ & $\begin{array}{c}0.376 \\
(0.021)\end{array}$ & $0.025^{*}$ \\
\hline Participate in TANF & $\begin{array}{c}0.004 \\
(0.004)\end{array}$ & $\begin{array}{c}0.026 \\
(0.006)\end{array}$ & $0.003^{*}$ \\
\hline
\end{tabular}

SOURCE: RAND analysis of National Health Interview Survey (NHIS) (2013).

NOTES: Models control for age, sex, race/ethnicity, and Census regions. TANF = Temporary Assistance for Needy Families.

* The difference in adjusted means is statistically different from zero at the 5 percent level ( $p$-value $<0.05)$.

^ Chronic Conditions: Hypertension, CHD, Angina, heart condition/disease, emphysema, asthma, cancer, weak/ failing kidneys, liver disease, arthritis, hepatitis. 


\section{C.2.2. Prevalence for Veterans by VA Patient Status}

Table C-13

Prevalence of Diagnosed Health Conditions, by VA Patient Status

\begin{tabular}{|c|c|c|c|c|c|}
\hline \multirow[b]{2}{*}{ Health Conditions } & \multicolumn{2}{|c|}{$\begin{array}{l}\text { Unadjusted Mean } \\
\text { (Standard Deviation) }\end{array}$} & \multicolumn{2}{|c|}{$\begin{array}{l}\text { Adjusted Mean } \\
\text { (Standard Error) }\end{array}$} & \multirow{2}{*}{$\begin{array}{l}\text { Difference in } \\
\text { Adjusted Means } \\
\text { (VA - Non-VA) }\end{array}$} \\
\hline & VA Patients & $\begin{array}{c}\text { Veterans Not } \\
\text { Using VA }\end{array}$ & VA Patients & $\begin{array}{l}\text { Veterans Not } \\
\text { Using VA }\end{array}$ & \\
\hline \multirow[t]{2}{*}{ Asthma } & 0.067 & 0.039 & 0.094 & 0.053 & $0.041 *$ \\
\hline & $(0.005)$ & $(0.003)$ & $(0.016)$ & $(0.010)$ & \\
\hline \multirow[t]{2}{*}{ Cancer } & 0.193 & 0.116 & 0.188 & 0.138 & $0.050 *$ \\
\hline & $(0.008)$ & $(0.002)$ & $(0.019)$ & $(0.015)$ & \\
\hline \multirow[t]{2}{*}{ Cerebrovascular disease } & 0.055 & 0.025 & 0.039 & 0.022 & $0.017^{*}$ \\
\hline & $(0.005)$ & $(0.002)$ & $(0.009)$ & $(0.005)$ & \\
\hline \multirow[t]{2}{*}{$\mathrm{CHF}$} & 0.03 & 0.009 & 0.011 & 0.005 & $0.006 *$ \\
\hline & $(0.003)$ & $(0.001)$ & $(0.004)$ & $(0.002)$ & \\
\hline \multirow[t]{2}{*}{ COPD } & 0.090 & 0.041 & 0.112 & 0.057 & $0.055^{*}$ \\
\hline & $(0.006)$ & $(0.003)$ & $(0.019)$ & $(0.010)$ & \\
\hline \multirow[t]{2}{*}{ Diabetes } & 0.296 & 0.169 & 0.304 & 0.207 & $0.097^{*}$ \\
\hline & $(0.009)$ & $(0.006)$ & $(0.023)$ & $(0.018)$ & \\
\hline \multirow[t]{2}{*}{ GERD } & 0.165 & 0.085 & 0.164 & 0.093 & $0.071^{*}$ \\
\hline & $(0.008)$ & $(0.005)$ & $(0.021)$ & $(0.013)$ & \\
\hline \multirow[t]{2}{*}{ Hearing loss } & 0.062 & 0.024 & 0.071 & 0.033 & $0.038^{*}$ \\
\hline & $(0.005)$ & $(0.003)$ & $(0.017)$ & $(0.008)$ & \\
\hline \multirow[t]{2}{*}{ Hypertension } & 0.573 & 0.378 & 0.600 & 0.466 & $0.134^{*}$ \\
\hline & $(0.008)$ & $(0.005)$ & $(0.022)$ & $(0.022)$ & \\
\hline \multirow[t]{2}{*}{ IHD } & 0.187 & 0.108 & 0.204 & 0.142 & $0.062 *$ \\
\hline & $(0.008)$ & $(0.005)$ & $(0.020)$ & $(0.015)$ & \\
\hline \multirow[t]{2}{*}{ Lipid disorder } & 0.498 & 0.33 & 0.513 & 0.385 & $0.128^{*}$ \\
\hline & $(0.010)$ & $(0.008)$ & $(0.023)$ & $(0.021)$ & \\
\hline \multirow[t]{2}{*}{ Low back pain } & 0.178 & 0.105 & 0.170 & 0.098 & $0.072^{*}$ \\
\hline & $(0.007)$ & $(0.005)$ & $(0.020)$ & $(0.013)$ & \\
\hline
\end{tabular}


Table C-13-Continued

\begin{tabular}{|c|c|c|c|c|c|}
\hline \multirow[b]{2}{*}{ Health Conditions } & \multicolumn{2}{|c|}{$\begin{array}{l}\text { Unadjusted Mean } \\
\text { (Standard Deviation) }\end{array}$} & \multicolumn{2}{|c|}{$\begin{array}{l}\text { Adjusted Mean } \\
\text { (Standard Error) }\end{array}$} & \multirow{2}{*}{$\begin{array}{c}\text { Difference in } \\
\text { Adjusted Means } \\
\text { (VA - Non-VA) }\end{array}$} \\
\hline & VA Patients & $\begin{array}{l}\text { Veterans Not } \\
\text { Using VA }\end{array}$ & VA Patients & $\begin{array}{l}\text { Veterans Not } \\
\text { Using VA }\end{array}$ & \\
\hline \multirow[t]{2}{*}{ Mental health condition } & 0.251 & 0.128 & 0.337 & 0.171 & $0.166^{*}$ \\
\hline & $(0.009)$ & $(0.005)$ & $(0.027)$ & $(0.017)$ & \\
\hline \multirow[t]{2}{*}{ PTSD } & 0.033 & 0.002 & 0.087 & 0.005 & $0.082 *$ \\
\hline & $(0.004)$ & $(0.001)$ & $(0.023)$ & $(0.002)$ & \\
\hline
\end{tabular}

SOURCE: RAND analysis of MEPS, 2006-2012.

NOTES: Sample size, non-Veterans $=150,225$ and sample size, Veterans $=12,313$. Sample sizes may be smaller for some conditions due to missing values. The unadjusted prevalence in results columns (1) and (2) are equivalent to the fraction of VA patients and Veterans not using VA health services that have been diagnosed with each condition. The adjusted prevalence in results columns (3) and (4) are the predicted prevalence rates from a logit estimation that included indicators for sex, five race/ethnicity categories, 14 age categories, four census regions, residential location in an MSA, and a nonlinear time trend. These estimates show the differences in the prevalence of health conditions for VA patients and Veterans not using VA health services, both with the same demographic profile of the overall Veteran population.

${ }^{*}$ The difference in adjusted means is statistically different from zero at the 5 percent level ( $p$-value $\left.<0.05\right)$.

Table C-14

Predicted Means of Disease Burden Measures Among, by VA Patient Status

\begin{tabular}{lccc}
\hline & \multicolumn{2}{c}{$\begin{array}{c}\text { Adjusted Mean } \\
\text { (Standard Error) }\end{array}$} & $\begin{array}{c}\text { P-Value for } \\
\text { Difference in }\end{array}$ \\
\cline { 2 - 3 } & VA Patients & $\begin{array}{c}\text { Non-VA } \\
\text { Patients }\end{array}$ & $\begin{array}{c}\text { Adjusted Means } \\
\text { Adjed }\end{array}$ \\
\hline Has 1+ IADLs & 0.152 & 0.086 & $<0.000$ \\
\hline Charlson Comorbidity Index $>1$ & 0.128 & 0.110 & 0.049 \\
\hline Charlson Comorbidity Index & 0.153 & $(0.012)$ & 0.132 \\
\hline
\end{tabular}

SOURCE: RAND analysis of MEPS, 2006-2012.

NOTES: $N=11,251$ for the total Veteran sample. Predicted or adjusted means were obtained from estimating logistic regressions with the following additional covariates included: sex (male is the omitted category), five race/ethnicity categories, 14 age categories, four Census regions, an MSA indicator, and year fixed effects using the margins command in Stata treating Veterans as though they had similar observable characteristics as civilians. The Charlson Comorbidity Index model was estimated using a Poisson regression. 
Table C-15

Adjusted Means of Comorbid Mental Health Condition (Mental Health Condition + Another Condition/Limitation) for Veterans, by VA Patient Status

\begin{tabular}{|c|c|c|c|}
\hline & \multicolumn{2}{|c|}{$\begin{array}{l}\text { Adjusted Mean } \\
\text { (Standard Error) }\end{array}$} & \multirow{2}{*}{$\begin{array}{l}\text { P-Value for } \\
\text { Difference in } \\
\text { Adjusted Means }\end{array}$} \\
\hline & VA Patient & Non-VA Patient & \\
\hline \multirow[t]{2}{*}{ Has any IADL + any mental health condition } & 0.052 & 0.026 & $<0.000$ \\
\hline & $(0.004)$ & $(0.003)$ & \\
\hline \multirow[t]{2}{*}{ Has any condition + any mental health condition } & 0.244 & 0.130 & $<0.000$ \\
\hline & $(0.009)$ & $(0.006)$ & \\
\hline \multirow[t]{2}{*}{ Has any Charlson condition + any mental health condition } & 0.022 & 0.015 & 0.048 \\
\hline & $(0.003)$ & $(0.002)$ & \\
\hline
\end{tabular}

SOURCE: RAND analysis of MEPS, 2006-2012.

NOTES: $\mathrm{N}=12,313$ for the total Veteran sample. Predicted or adjusted means were obtained from estimating logistic regressions with the following additional covariates included: sex (male is the omitted category), five race/ethnicity categories, 14 age categories, four Census regions, an MSA indicator, and year fixed effects using the margins command in Stata treating Veterans as though they had similar observable characteristics as civilians. The Charlson Comorbidity Index model was estimated using a Poisson regression.

Table C-16

Prevalence of Diagnosed Health Conditions for VA Patients, by Demographic Characteristics

\begin{tabular}{|c|c|c|c|c|c|c|c|}
\hline \multirow[b]{2}{*}{ Demographic Group } & \multicolumn{7}{|c|}{$\begin{array}{l}\text { Adjusted Prevalence } \\
\text { (Standard Error) }\end{array}$} \\
\hline & Cancer & COPD & Diabetes & HBP & $\begin{array}{l}\text { Hearing } \\
\text { Loss }\end{array}$ & IHD & PTSD \\
\hline \multicolumn{8}{|l|}{ Age } \\
\hline $20-34$ & $\begin{array}{c}0.013 \\
(0.009)\end{array}$ & IS & $\begin{array}{l}0.041 * \\
(0.019)\end{array}$ & $\begin{array}{l}0.103 * * \\
(0.035)\end{array}$ & IS & $\begin{array}{c}0.004 \\
(0.005)\end{array}$ & $\begin{array}{l}0.346 * * \\
(0.105)\end{array}$ \\
\hline $35-44$ & $\begin{array}{c}0.018 \\
(0.009)\end{array}$ & $\begin{array}{c}0.032 \\
(0.022)\end{array}$ & $\begin{array}{l}0.114 * * \\
(0.029)\end{array}$ & $\begin{array}{l}0.243 * * \\
(0.044)\end{array}$ & $\begin{array}{c}0.050 \\
(0.035)\end{array}$ & $\begin{array}{l}0.028 * \\
(0.013)\end{array}$ & $\begin{array}{l}0.178 * * \\
(0.055)\end{array}$ \\
\hline $45-54$ & $\begin{array}{l}0.057 * * \\
(0.016)\end{array}$ & $\begin{array}{l}0.073 * * \\
(0.024)\end{array}$ & $\begin{array}{l}0.223 * * \\
(0.037)\end{array}$ & $\begin{array}{l}0.417 * * \\
(0.046)\end{array}$ & $\begin{array}{l}0.042 * \\
(0.020)\end{array}$ & $\begin{array}{l}0.108 \text { ** } \\
(0.027)\end{array}$ & $\begin{array}{l}0.041 * \\
(0.017)\end{array}$ \\
\hline $55-64$ & $\begin{array}{l}0.117 * * \\
(0.024)\end{array}$ & $\begin{array}{l}0.150 * * \\
(0.033)\end{array}$ & $\begin{array}{l}0.305^{* *} \\
(0.040)\end{array}$ & $\begin{array}{l}0.606 * * \\
(0.041)\end{array}$ & $\begin{array}{l}0.072 * * \\
(0.023)\end{array}$ & $\begin{array}{l}0.169 * * \\
(0.030)\end{array}$ & $\begin{array}{l}0.147 * * \\
(0.043)\end{array}$ \\
\hline $65-74$ & $\begin{array}{l}0.254 * * \\
(0.039)\end{array}$ & $\begin{array}{l}0.115^{* *} \\
(0.028)\end{array}$ & $\begin{array}{l}0.400 * * \\
(0.044)\end{array}$ & $\begin{array}{l}0.661 * * \\
(0.039)\end{array}$ & $\begin{array}{l}0.093 * * \\
(0.027)\end{array}$ & $\begin{array}{l}0.208 * * \\
(0.033)\end{array}$ & $\begin{array}{l}0.032 * \\
(0.016)\end{array}$ \\
\hline $75-84$ & $\begin{array}{l}0.313 * * \\
(0.044)\end{array}$ & $\begin{array}{l}0.091 * * \\
(0.024)\end{array}$ & $\begin{array}{l}0.459 * * \\
(0.045)\end{array}$ & $\begin{array}{l}0.712 * * \\
(0.037)\end{array}$ & $\begin{array}{l}0.114 * * \\
(0.030)\end{array}$ & $\begin{array}{l}0.356 \text { ** } \\
(0.045)\end{array}$ & $\begin{array}{c}0.027 \\
(0.016)\end{array}$ \\
\hline $85+$ & $\begin{array}{l}0.312 * * \\
(0.047)\end{array}$ & $\begin{array}{l}0.108^{* *} \\
(0.033)\end{array}$ & $\begin{array}{l}0.396 * * \\
(0.051)\end{array}$ & $\begin{array}{l}0.692 * * \\
(0.046)\end{array}$ & $\begin{array}{l}0.223 * * \\
(0.059)\end{array}$ & $\begin{array}{l}0.380 * * \\
(0.053)\end{array}$ & $\begin{array}{c}0.013 \\
(0.013)\end{array}$ \\
\hline
\end{tabular}


Table C-16-Continued

\begin{tabular}{|c|c|c|c|c|c|c|c|}
\hline \multirow[b]{2}{*}{ Demographic Group } & \multicolumn{7}{|c|}{$\begin{array}{l}\text { Adjusted Prevalence } \\
\text { (Standard Error) }\end{array}$} \\
\hline & Cancer & COPD & Diabetes & HBP & $\begin{array}{l}\text { Hearing } \\
\text { Loss }\end{array}$ & IHD & PTSD \\
\hline \multicolumn{8}{|l|}{ Sex } \\
\hline Male & $\begin{array}{l}0.189 * * \\
(0.027)\end{array}$ & $\begin{array}{l}0.108 * * \\
(0.024)\end{array}$ & $\begin{array}{l}0.338 * * \\
(0.035)\end{array}$ & $\begin{array}{l}0.589 * * \\
(0.035)\end{array}$ & $\begin{array}{l}0.097 * * \\
(0.026)\end{array}$ & $\begin{array}{l}0.218^{* *} \\
(0.029)\end{array}$ & $\begin{array}{l}0.084 * * \\
(0.023)\end{array}$ \\
\hline Female & $\begin{array}{l}0.224 * * \\
(0.050)\end{array}$ & $\begin{array}{l}0.113 * * \\
(0.039)\end{array}$ & $\begin{array}{l}0.263 * * \\
(0.052)\end{array}$ & $\begin{array}{l}0.492 * * \\
(0.053)\end{array}$ & $\begin{array}{c}0.048 \\
(0.029)\end{array}$ & $\begin{array}{l}0.137 * * \\
(0.049)\end{array}$ & $\begin{array}{l}0.052 * \\
(0.023)\end{array}$ \\
\hline \multicolumn{8}{|l|}{ Race/ethnicity } \\
\hline White (non-Hispanic) & $\begin{array}{l}0.203 * * \\
(0.029)\end{array}$ & $\begin{array}{l}0.116 * * \\
(0.026)\end{array}$ & $\begin{array}{l}0.327 * * \\
(0.035)\end{array}$ & $\begin{array}{l}0.570 * * \\
(0.035)\end{array}$ & $\begin{array}{l}0.100 * * \\
(0.027)\end{array}$ & $\begin{array}{l}0.224 * * \\
(0.030)\end{array}$ & $\begin{array}{l}0.074 * * \\
(0.022)\end{array}$ \\
\hline Hispanic & $\begin{array}{l}0.069 * * \\
(0.025)\end{array}$ & $\begin{array}{l}0.035^{\star} \\
(0.015)\end{array}$ & $\begin{array}{l}0.419 * * \\
(0.058)\end{array}$ & $\begin{array}{l}0.547 * * \\
(0.054)\end{array}$ & $\begin{array}{l}0.084^{*} \\
(0.042)\end{array}$ & $\begin{array}{l}0.130 * * \\
(0.036)\end{array}$ & $\begin{array}{l}0.088 * \\
(0.036)\end{array}$ \\
\hline Black (non-Hispanic) & $\begin{array}{l}0.119 * * \\
(0.024)\end{array}$ & $\begin{array}{l}0.067 * * \\
(0.022)\end{array}$ & $\begin{array}{l}0.339 * * \\
(0.040)\end{array}$ & $\begin{array}{l}0.691 * * \\
(0.033)\end{array}$ & $\begin{array}{l}0.051 * * \\
(0.018)\end{array}$ & $\begin{array}{l}0.160 * * \\
(0.030)\end{array}$ & $\begin{array}{l}0.100 * * \\
(0.031)\end{array}$ \\
\hline Asian & $\begin{array}{c}0.193 \\
(0.122)\end{array}$ & $\begin{array}{c}0.053 \\
(0.040)\end{array}$ & $\begin{array}{l}0.354^{* *} \\
(0.109)\end{array}$ & $\begin{array}{l}0.491 * * \\
(0.085)\end{array}$ & $\begin{array}{c}0.028 \\
(0.024)\end{array}$ & $\begin{array}{c}0.009 \\
(0.009)\end{array}$ & $\begin{array}{c}0.046 \\
(0.043)\end{array}$ \\
\hline Other and multiple & $\begin{array}{l}0.126 * * \\
(0.046)\end{array}$ & $\begin{array}{l}0.123^{*} \\
(0.054)\end{array}$ & $\begin{array}{l}0.459 * * \\
(0.078)\end{array}$ & $\begin{array}{l}0.649 * * \\
(0.056)\end{array}$ & $\begin{array}{l}0.116 * \\
(0.053)\end{array}$ & $\begin{array}{l}0.236 * * \\
(0.063)\end{array}$ & $\begin{array}{l}0.165^{\star} \\
(0.064)\end{array}$ \\
\hline Residence in an MSA & & & & & & & \\
\hline No & $\begin{array}{l}0.162 * * \\
(0.027)\end{array}$ & $\begin{array}{l}0.125^{* *} \\
(0.030)\end{array}$ & $\begin{array}{l}0.342 * * \\
(0.041)\end{array}$ & $\begin{array}{l}0.615^{* *} \\
(0.038)\end{array}$ & $\begin{array}{l}0.086 * * \\
(0.028)\end{array}$ & $\begin{array}{l}0.219 * * \\
(0.033)\end{array}$ & $\begin{array}{l}0.113 * * \\
(0.035)\end{array}$ \\
\hline Yes & $\begin{array}{l}0.198 * * \\
(0.028)\end{array}$ & $\begin{array}{l}0.104 * * \\
(0.024)\end{array}$ & $\begin{array}{l}0.333^{* *} \\
(0.035)\end{array}$ & $\begin{array}{l}0.575^{* *} \\
(0.035)\end{array}$ & $\begin{array}{l}0.097 * * \\
(0.025)\end{array}$ & $\begin{array}{l}0.213^{* *} \\
(0.029)\end{array}$ & $\begin{array}{l}0.072^{* *} \\
(0.021)\end{array}$ \\
\hline
\end{tabular}

SOURCE: RAND analysis of MEPS, 2006-2012.

NOTES: Sample size, VA patients $=4,871$. Cells labeled IS indicate that the prevalence model for the specific health condition could not be estimated due to insufficient sample size. The adjusted prevalence estimates are the predicted prevalence from a logit estimation that included an indicators for sex, five race/ethnicity categories, 14 age categories, four census regions, residential location in an MSA, and a nonlinear time trend. These estimated differences control for the demographic differences within demographic group. HBP is hypertension. * $\mathrm{p}<0.05 ; * * \mathrm{p}<0.01$. 
Table C-17

Prevalence of Diagnosed Health Conditions for Non-VA Patient Veterans, by Demographic Characteristics

\begin{tabular}{|c|c|c|c|c|c|c|c|}
\hline \multirow[b]{2}{*}{ Demographic Group } & \multicolumn{7}{|c|}{$\begin{array}{l}\text { Adjusted Prevalence } \\
\text { (Standard Error) }\end{array}$} \\
\hline & Cancer & COPD & Diabetes & HBP & $\begin{array}{l}\text { Hearing } \\
\text { Loss }\end{array}$ & IHD & PTSD \\
\hline \multicolumn{8}{|l|}{ Age } \\
\hline \multirow[t]{2}{*}{$20-34$} & 0.002 & 0.008 & $0.019 *$ & $0.074 * *$ & IS & 0.007 & 0.016 \\
\hline & $(0.002)$ & $(0.006)$ & $(0.008)$ & $(0.019)$ & & $(0.006)$ & $(0.013)$ \\
\hline \multirow[t]{2}{*}{$35-44$} & $0.017^{*}$ & $0.014^{*}$ & $0.055^{* *}$ & $0.191 * *$ & 0.002 & $0.014^{*}$ & 0.004 \\
\hline & $(0.008)$ & $(0.006)$ & $(0.014)$ & $(0.027)$ & $(0.001)$ & $(0.005)$ & $(0.005)$ \\
\hline \multirow[t]{2}{*}{$45-54$} & $0.037 * *$ & $0.020 *$ & $0.108^{* *}$ & $0.316 * *$ & 0.009 & $0.047 * *$ & 0.005 \\
\hline & $(0.014)$ & $(0.009)$ & $(0.020)$ & $(0.033)$ & $(0.005)$ & $(0.013)$ & $(0.004)$ \\
\hline \multirow[t]{2}{*}{$55-64$} & $0.091 * *$ & $0.043 * *$ & $0.190 * *$ & $0.521 * *$ & 0.008 & $0.109 * *$ & 0.003 \\
\hline & $(0.018)$ & $(0.014)$ & $(0.025)$ & $(0.034)$ & $(0.004)$ & $(0.021)$ & $(0.003)$ \\
\hline \multirow[t]{2}{*}{$65-74$} & $0.191 * *$ & $0.080 * *$ & $0.279 * *$ & $0.644 * *$ & $0.022 *$ & $0.198 * *$ & 0.002 \\
\hline & $(0.031)$ & $(0.021)$ & $(0.032)$ & $(0.031)$ & $(0.009)$ & $(0.030)$ & $(0.003)$ \\
\hline \multirow[t]{2}{*}{$75-84$} & $0.300 * *$ & $0.119 * *$ & $0.371 * *$ & $0.685^{* *}$ & $0.051 *$ & $0.303 * *$ & IS \\
\hline & $(0.040)$ & $(0.028)$ & $(0.038)$ & $(0.032)$ & $(0.021)$ & $(0.040)$ & \\
\hline \multirow[t]{2}{*}{$85+$} & $0.336 * *$ & $0.125 * *$ & $0.307 * *$ & $0.704^{\star *}$ & $0.086 * *$ & $0.296 * *$ & IS \\
\hline & $(0.048)$ & $(0.037)$ & $(0.044)$ & $(0.039)$ & $(0.032)$ & $(0.046)$ & \\
\hline \multicolumn{8}{|l|}{ Sex } \\
\hline \multirow[t]{2}{*}{ Male } & $0.133^{* *}$ & $0.052 * *$ & $0.193 * *$ & $0.472 * *$ & $0.023 * *$ & $0.142 * *$ & 0.005 \\
\hline & $(0.019)$ & $(0.013)$ & $(0.021)$ & $(0.026)$ & $(0.008)$ & $(0.019)$ & $(0.004)$ \\
\hline \multirow[t]{2}{*}{ Female } & $0.082 * *$ & $0.095 * *$ & $0.213 * *$ & $0.415 * *$ & 0.002 & $0.035^{*}$ & 0.002 \\
\hline & $(0.025)$ & $(0.034)$ & $(0.036)$ & $(0.038)$ & $(0.002)$ & $(0.017)$ & $(0.002)$ \\
\hline \multicolumn{8}{|l|}{ Race/ethnicity } \\
\hline \multirow[t]{2}{*}{ White (non-Hispanic) } & $0.140 * *$ & $0.055^{* *}$ & $0.188 * *$ & $0.461 * *$ & $0.023 * *$ & $0.143 * *$ & 0.003 \\
\hline & $(0.020)$ & $(0.013)$ & $(0.020)$ & $(0.026)$ & $(0.008)$ & $(0.019)$ & $(0.003)$ \\
\hline \multirow[t]{2}{*}{ Hispanic } & $0.051 * *$ & 0.036 & $0.239 * *$ & $0.479 * *$ & 0.006 & $0.103 * *$ & 0.009 \\
\hline & $(0.019)$ & $(0.023)$ & $(0.038)$ & $(0.039)$ & $(0.004)$ & $(0.027)$ & $(0.010)$ \\
\hline \multirow[t]{2}{*}{ Black (non-Hispanic) } & $0.091 * *$ & $0.045^{*}$ & $0.231 * *$ & $0.553 * *$ & 0.01 & $0.102 * *$ & 0.012 \\
\hline & $(0.019)$ & $(0.018)$ & $(0.030)$ & $(0.030)$ & $(0.007)$ & $(0.020)$ & $(0.012)$ \\
\hline \multirow[t]{2}{*}{ Asian } & $0.053^{*}$ & 0.033 & $0.177 * *$ & $0.439 * *$ & 0.028 & 0.029 & IS \\
\hline & $(0.022)$ & $(0.019)$ & $(0.043)$ & $(0.053)$ & $(0.015)$ & $(0.015)$ & \\
\hline \multirow[t]{2}{*}{ Other and multiple } & 0.038 & $0.072^{*}$ & $0.241 * *$ & $0.370 * *$ & 0.023 & $0.152 * *$ & 0.015 \\
\hline & $(0.021)$ & $(0.034)$ & $(0.055)$ & $(0.058)$ & $(0.018)$ & $(0.047)$ & $(0.016)$ \\
\hline
\end{tabular}


Table C-17-Continued

\begin{tabular}{|c|c|c|c|c|c|c|c|}
\hline \multirow[b]{2}{*}{ Demographic Group } & \multicolumn{7}{|c|}{$\begin{array}{l}\text { Adjusted Prevalence } \\
\text { (Standard Error) }\end{array}$} \\
\hline & Cancer & COPD & Diabetes & HBP & $\begin{array}{l}\text { Hearing } \\
\text { Loss }\end{array}$ & IHD & PTSD \\
\hline \multicolumn{8}{|l|}{ Residence in an MSA } \\
\hline No & $\begin{array}{l}0.120 * * \\
(0.022)\end{array}$ & $\begin{array}{l}0.054^{* *} \\
(0.016)\end{array}$ & $\begin{array}{l}0.236 * * \\
(0.028)\end{array}$ & $\begin{array}{l}0.485^{* *} \\
(0.032)\end{array}$ & $\begin{array}{l}0.030 * \\
(0.012)\end{array}$ & $\begin{array}{l}0.154 * * \\
(0.025)\end{array}$ & $\begin{array}{l}0.004 \\
(0.003)\end{array}$ \\
\hline Yes & $\begin{array}{l}0.134 * * \\
(0.019)\end{array}$ & $\begin{array}{l}0.061 * * \\
(0.016)\end{array}$ & $\begin{array}{l}0.195^{* *} \\
(0.023)\end{array}$ & $\begin{array}{l}0.459 * * \\
(0.028)\end{array}$ & $\begin{array}{l}0.022 * \\
(0.010)\end{array}$ & $\begin{array}{l}0.141 * * \\
(0.022)\end{array}$ & $\begin{array}{l}0.005 \\
(0.004)\end{array}$ \\
\hline
\end{tabular}

SOURCE: RAND analysis of MEPS, 2006-2012.

NOTES: Sample size, non-VA patient Veterans $=7,442$. Cells labeled IS indicate that the prevalence model for the specific health condition could not be estimated due to insufficient sample size. The adjusted prevalence estimates are the predicted prevalence from a logit estimation that included an indicators for sex, five race/ ethnicity categories, 14 age categories, four census regions, residential location in an MSA, and a nonlinear time trend. These estimated differences control for the demographic differences within demographic group. HBP is hypertension. * $\mathrm{p}<0.05 ; * * \mathrm{p}<0.01$.

\section{C.2.3. Differences in Characteristics by Veteran and VA Patient Status}

Table C-18

Characteristics of Non-Veterans, Veterans, and VA Patients in MEPS

\begin{tabular}{|c|c|c|c|c|}
\hline \multirow[b]{2}{*}{ Demographic Group } & \multicolumn{4}{|c|}{$\begin{array}{l}\text { Distribution by Demographic Characteristics } \\
\text { (Standard Error) }\end{array}$} \\
\hline & Veterans & Non-Veterans & $\begin{array}{c}\text { Veterans, VA } \\
\text { Patients }\end{array}$ & $\begin{array}{l}\text { Veterans, Non- } \\
\text { VA Patients }\end{array}$ \\
\hline \multicolumn{5}{|l|}{ Age } \\
\hline \multirow[t]{2}{*}{$20-34$} & 0.062 & 0.300 & 0.038 & 0.076 \\
\hline & $(0.004)$ & $(0.003)$ & $(0.004)$ & $(0.005)$ \\
\hline \multirow[t]{2}{*}{$35-44$} & 0.104 & 0.192 & 0.063 & 0.129 \\
\hline & $(0.004)$ & $(0.002)$ & $(0.005)$ & $(0.006)$ \\
\hline \multirow[t]{2}{*}{$45-54$} & 0.144 & 0.202 & 0.120 & 0.158 \\
\hline & $(0.005)$ & $(0.002)$ & $(0.007)$ & $(0.006)$ \\
\hline \multirow[t]{2}{*}{$55-64$} & 0.252 & 0.154 & 0.257 & 0.249 \\
\hline & $(0.007)$ & $(0.002)$ & $(0.010)$ & $(0.008)$ \\
\hline \multirow[t]{2}{*}{$65-74$} & 0.218 & 0.084 & 0.240 & 0.204 \\
\hline & $(0.006)$ & $(0.002)$ & $(0.010)$ & $(0.007)$ \\
\hline \multirow[t]{2}{*}{$75-84$} & 0.162 & 0.049 & 0.201 & 0.138 \\
\hline & $(0.006)$ & $(0.001)$ & $(0.009)$ & $(0.006)$ \\
\hline \multirow[t]{2}{*}{$85+$} & 0.059 & 0.019 & 0.081 & 0.045 \\
\hline & $(0.004)$ & $(0.001)$ & $(0.007)$ & $(0.004)$ \\
\hline
\end{tabular}


Table C-18-Continued

\begin{tabular}{|c|c|c|c|c|}
\hline \multirow[b]{2}{*}{ Demographic Group } & \multicolumn{4}{|c|}{$\begin{array}{c}\text { Distribution by Demographic Characteristics } \\
\text { (Standard Error) }\end{array}$} \\
\hline & Veterans & Non-Veterans & $\begin{array}{l}\text { Veterans, VA } \\
\text { Patients }\end{array}$ & $\begin{array}{l}\text { Veterans, Non- } \\
\text { VA Patients }\end{array}$ \\
\hline \multicolumn{5}{|l|}{ Sex } \\
\hline \multirow[t]{2}{*}{ Male } & 0.932 & 0.438 & 0.937 & 0.929 \\
\hline & $(0.004)$ & $(0.002)$ & $(0.005)$ & $(0.005)$ \\
\hline \multirow[t]{2}{*}{ Female } & 0.068 & 0.562 & 0.063 & 0.071 \\
\hline & $(0.004)$ & $(0.002)$ & $(0.005)$ & $(0.005)$ \\
\hline \multicolumn{5}{|l|}{ Race/ethnicity } \\
\hline \multirow[t]{2}{*}{ White (non-Hispanic) } & 0.823 & 0.662 & 0.824 & 0.822 \\
\hline & $(0.007)$ & $(0.008)$ & $(0.009)$ & $(0.009)$ \\
\hline \multirow[t]{2}{*}{ Hispanic } & 0.043 & 0.150 & 0.040 & 0.045 \\
\hline & $(0.003)$ & $(0.007)$ & $(0.004)$ & $(0.004)$ \\
\hline \multirow[t]{2}{*}{ Black (non-Hispanic) } & 0.100 & 0.116 & 0.106 & 0.096 \\
\hline & $(0.005)$ & $(0.005)$ & $(0.006)$ & $(0.006)$ \\
\hline \multirow[t]{2}{*}{ Asian } & 0.012 & 0.051 & 0.007 & 0.016 \\
\hline & $(0.003)$ & $(0.003)$ & $(0.002)$ & $(0.003)$ \\
\hline \multirow[t]{2}{*}{ Other and multiple } & 0.022 & 0.021 & 0.024 & 0.021 \\
\hline & $(0.002)$ & $(0.002)$ & $(0.003)$ & $(0.003)$ \\
\hline \multicolumn{5}{|l|}{ Lives in an MSA } \\
\hline \multirow[t]{2}{*}{ No } & 0.187 & 0.154 & 0.213 & 0.170 \\
\hline & $(0.013)$ & $(0.010)$ & $(0.018)$ & $(0.012)$ \\
\hline \multirow[t]{2}{*}{ Yes } & 0.813 & 0.846 & 0.787 & 0.830 \\
\hline & $(0.013)$ & $(0.010)$ & $(0.018)$ & $(0.012)$ \\
\hline \multicolumn{5}{|l|}{ Married } \\
\hline \multirow[t]{2}{*}{ No } & 0.341 & 0.477 & 0.374 & 0.320 \\
\hline & $(0.009)$ & $(0.004)$ & $(0.011)$ & $(0.010)$ \\
\hline \multirow[t]{2}{*}{ Yes } & 0.659 & 0.523 & 0.626 & 0.680 \\
\hline & $(0.009)$ & $(0.004)$ & $(0.011)$ & $(0.010)$ \\
\hline \multicolumn{5}{|c|}{ Student or currently in school } \\
\hline \multirow[t]{2}{*}{ No } & 0.992 & 0.906 & 0.992 & 0.992 \\
\hline & $(0.001)$ & $(0.001)$ & $(0.001)$ & $(0.001)$ \\
\hline \multirow[t]{2}{*}{ Yes } & 0.008 & 0.094 & 0.008 & 0.008 \\
\hline & $(0.001)$ & $(0.001)$ & $(0.001)$ & $(0.001)$ \\
\hline
\end{tabular}


Table C-18-Continued

\begin{tabular}{|c|c|c|c|c|}
\hline \multirow[b]{2}{*}{ Demographic Group } & \multicolumn{4}{|c|}{$\begin{array}{c}\text { Distribution by Demographic Characteristics } \\
\text { (Standard Error) }\end{array}$} \\
\hline & Veterans & Non-Veterans & $\begin{array}{l}\text { Veterans, VA } \\
\text { Patients }\end{array}$ & $\begin{array}{l}\text { Veterans, Non- } \\
\text { VA Patients }\end{array}$ \\
\hline \multicolumn{5}{|l|}{ Educational attainment } \\
\hline \multirow[t]{2}{*}{$<$ High school } & 0.071 & 0.176 & 0.091 & 0.058 \\
\hline & $(0.004)$ & $(0.003)$ & $(0.006)$ & $(0.004)$ \\
\hline \multirow[t]{2}{*}{ High school diploma or GED } & 0.340 & 0.308 & 0.350 & 0.333 \\
\hline & $(0.009)$ & $(0.004)$ & $(0.011)$ & $(0.010)$ \\
\hline \multirow[t]{2}{*}{ Some college } & 0.210 & 0.180 & 0.203 & 0.214 \\
\hline & $(0.006)$ & $(0.002)$ & $(0.009)$ & $(0.007)$ \\
\hline \multirow[t]{2}{*}{ College } & 0.380 & 0.336 & 0.356 & 0.396 \\
\hline & $(0.008)$ & $(0.005)$ & $(0.010)$ & $(0.010)$ \\
\hline \multicolumn{5}{|c|}{ Currently employed (not on active duty) } \\
\hline \multirow[t]{2}{*}{ No } & 0.454 & 0.293 & 0.587 & 0.372 \\
\hline & $(0.009)$ & $(0.003)$ & $(0.012)$ & $(0.010)$ \\
\hline \multirow[t]{2}{*}{ Yes } & 0.546 & 0.707 & 0.413 & 0.628 \\
\hline & $(0.009)$ & $(0.003)$ & $(0.012)$ & $(0.010)$ \\
\hline \multicolumn{5}{|l|}{ Income categories } \\
\hline \multirow[t]{2}{*}{ Poor } & 0.063 & 0.124 & 0.081 & 0.052 \\
\hline & $(0.003)$ & $(0.003)$ & $(0.004)$ & $(0.003)$ \\
\hline \multirow[t]{2}{*}{ Near poor } & 0.035 & 0.044 & 0.048 & 0.026 \\
\hline & $(0.002)$ & $(0.001)$ & $(0.004)$ & $(0.002)$ \\
\hline \multirow[t]{2}{*}{ Low income } & 0.114 & 0.135 & 0.139 & 0.099 \\
\hline & $(0.004)$ & $(0.002)$ & $(0.007)$ & $(0.004)$ \\
\hline \multirow[t]{2}{*}{ Middle income } & 0.307 & 0.304 & 0.326 & 0.294 \\
\hline & $(0.005)$ & $(0.003)$ & $(0.008)$ & $(0.006)$ \\
\hline \multirow[t]{2}{*}{ High income } & 0.481 & 0.393 & 0.406 & 0.528 \\
\hline & $(0.007)$ & $(0.005)$ & $(0.011)$ & $(0.008)$ \\
\hline \multicolumn{5}{|l|}{ Income } \\
\hline \multirow[t]{2}{*}{ Total household income } & $41,708.24$ & $33,546.49$ & $35,980.76$ & $45,278.40$ \\
\hline & $(541.289)$ & $(304.781)$ & (753.759) & $(646.528)$ \\
\hline
\end{tabular}

SOURCE: RAND analysis of MEPS, 2006-2012.

NOTES: Columns (3) (Veterans, VA patients) and (4) (Veterans, non-VA patients) are two mutually exclusive categories of column (2) (Veterans). Sample size, Veterans $=12,313$; sample size, nonVeterans $=150,225$; sample size, VA patients $=4,871$; sample size, non-VA patients $=7,442$. 


\section{C.2.4. Prevalence Estimates with MHS and VA Encounter Data and Reliance}

Table C-19

Prevalence of Health Conditions in VA Encounter Data

\begin{tabular}{|c|c|}
\hline Health Condition & Prevalence \\
\hline Mental health condition & $32.1 \%$ \\
\hline Hypertension & $29.2 \%$ \\
\hline Lipid disorders & $22.2 \%$ \\
\hline Diabetes & $18.8 \%$ \\
\hline Arthritis & $18.2 \%$ \\
\hline Musculoskeletal conditions & $18.0 \%$ \\
\hline Mood disorder & $14.3 \%$ \\
\hline IHD & $12.3 \%$ \\
\hline Benign prostate hypertrophy & $11.0 \%$ \\
\hline Dermatologic conditions & $10.6 \%$ \\
\hline PTSD & $9.8 \%$ \\
\hline Hearing loss & $8.3 \%$ \\
\hline Dental conditions & $8.0 \%$ \\
\hline GERD & $6.6 \%$ \\
\hline Obesity & $6.3 \%$ \\
\hline Anxiety disorders & $6.3 \%$ \\
\hline Substance use disorder & $6.3 \%$ \\
\hline Malignant cancer & $6.2 \%$ \\
\hline COPD & $5.9 \%$ \\
\hline Cardiac dysrhythmias & $5.7 \%$ \\
\hline Major depression & $4.7 \%$ \\
\hline Chronic renal failure & $4.4 \%$ \\
\hline Anemia & $4.2 \%$ \\
\hline Thyroid disorders & $3.8 \%$ \\
\hline Heart failure & $3.1 \%$ \\
\hline Cerebrovascular disease & $2.9 \%$ \\
\hline Peripheral vascular disease & $2.9 \%$ \\
\hline Benign cancers & $2.7 \%$ \\
\hline Prostate cancer & $2.6 \%$ \\
\hline Conduction disorders & $2.3 \%$ \\
\hline Bipolar disorder & $1.9 \%$ \\
\hline
\end{tabular}


262 Characteristics and Unique Health Care Needs of the Patient Population Served by VA

Table C-19-Continued

\begin{tabular}{|c|c|}
\hline Health Condition & Prevalence \\
\hline TBI & $1.8 \%$ \\
\hline Viral hepatitis & $1.7 \%$ \\
\hline Alular disorders & $1.7 \%$ \\
\hline Dementia & $1.6 \%$ \\
\hline Asthma & $1.6 \%$ \\
\hline Schizophrenia & $1.4 \%$ \\
\hline Movement disorders & $1.3 \%$ \\
\hline Headache & $1.2 \%$ \\
\hline Chronic liver disease & $1.1 \%$ \\
\hline Skin cancer & $0.9 \%$ \\
\hline Kidney stones & $0.9 \%$ \\
\hline Rheumatologic disease & $0.7 \%$ \\
\hline Personality disorders & $0.7 \%$ \\
\hline Lung cancer & $0.6 \%$ \\
\hline Epilepsy & $0.5 \%$ \\
\hline Osteoporosis & $0.5 \%$ \\
\hline Amputation & $0.5 \%$ \\
\hline Colon cancer & $0.5 \%$ \\
\hline Spinal cord injury & $0.5 \%$ \\
\hline Acute coronary syndrome & $0.5 \%$ \\
\hline HIV & $0.4 \%$ \\
\hline Women's health & $0.4 \%$ \\
\hline AMI & $0.3 \%$ \\
\hline Multiple sclerosis & $0.2 \%$ \\
\hline Breast cancer & $0.1 \%$ \\
\hline Burns & $0.1 \%$ \\
\hline
\end{tabular}

SOURCE: RAND analysis of VA encounter data (2014).

NOTE: Sample size, Veterans in VA encounter data $=5,871,766$. 
Table C-20

Prevalence of Health Conditions Among VA Patients, by Priority Group

\begin{tabular}{|c|c|c|c|c|c|c|c|c|}
\hline \multirow[b]{2}{*}{ Health Condition } & \multicolumn{8}{|c|}{ Priority Group } \\
\hline & 1 & 2 & 3 & 4 & 5 & 6 & 7 & $8^{*}$ \\
\hline $\begin{array}{l}\text { Acute coronary } \\
\text { syndrome }\end{array}$ & $0.61 \%$ & $0.31 \%$ & $0.30 \%$ & $0.94 \%$ & $0.63 \%$ & $0.13 \%$ & $0.27 \%$ & $0.22 \%$ \\
\hline AMI & $0.46 \%$ & $0.23 \%$ & $0.23 \%$ & $0.75 \%$ & $0.48 \%$ & $0.10 \%$ & $0.21 \%$ & $0.17 \%$ \\
\hline Amputation & $0.87 \%$ & $0.27 \%$ & $0.28 \%$ & $6.42 \%$ & $0.08 \%$ & $0.02 \%$ & $0.31 \%$ & $0.03 \%$ \\
\hline Anemia & $4.93 \%$ & $3.04 \%$ & $3.06 \%$ & $11.38 \%$ & $5.21 \%$ & $1.43 \%$ & $3.18 \%$ & $2.77 \%$ \\
\hline Anxiety disorders & $9.61 \%$ & $6.68 \%$ & $5.09 \%$ & $8.55 \%$ & $6.48 \%$ & $4.25 \%$ & $3.34 \%$ & $2.54 \%$ \\
\hline Arthritis & $24.84 \%$ & $20.72 \%$ & $17.99 \%$ & $20.88 \%$ & $17.59 \%$ & $11.63 \%$ & $11.81 \%$ & $10.60 \%$ \\
\hline Asthma & $2.36 \%$ & $1.87 \%$ & $1.26 \%$ & $1.68 \%$ & $1.45 \%$ & $0.80 \%$ & $1.01 \%$ & $0.86 \%$ \\
\hline Benign cancers & $3.14 \%$ & $2.41 \%$ & $2.47 \%$ & $2.82 \%$ & $3.13 \%$ & $2.04 \%$ & $2.31 \%$ & $1.84 \%$ \\
\hline Bipolar disorder & $3.09 \%$ & $1.52 \%$ & $1.32 \%$ & $5.13 \%$ & $2.02 \%$ & $0.45 \%$ & $0.87 \%$ & $0.60 \%$ \\
\hline $\mathrm{BPH}$ & $9.83 \%$ & $8.26 \%$ & $9.82 \%$ & $15.67 \%$ & $11.26 \%$ & $8.83 \%$ & $12.48 \%$ & $14.14 \%$ \\
\hline Breast cancer & $0.12 \%$ & $0.08 \%$ & $0.06 \%$ & $0.12 \%$ & $0.12 \%$ & $0.02 \%$ & $0.07 \%$ & $0.06 \%$ \\
\hline Burns & $0.11 \%$ & $0.06 \%$ & $0.06 \%$ & $0.23 \%$ & $0.10 \%$ & $0.03 \%$ & $0.05 \%$ & $0.03 \%$ \\
\hline Cardiac dysrhythmias & $6.11 \%$ & $4.03 \%$ & $4.38 \%$ & $11.79 \%$ & $6.96 \%$ & $2.62 \%$ & $5.45 \%$ & $4.89 \%$ \\
\hline Cerebrovascular diseases & $3.27 \%$ & $1.90 \%$ & $1.99 \%$ & $10.02 \%$ & $3.62 \%$ & $1.03 \%$ & $2.33 \%$ & $1.87 \%$ \\
\hline Chronic liver disease & $1.37 \%$ & $0.87 \%$ & $0.87 \%$ & $2.20 \%$ & $1.54 \%$ & $0.52 \%$ & $0.81 \%$ & $0.61 \%$ \\
\hline Chronic renal failure & $4.9 \%$ & $2.7 \%$ & $3.0 \%$ & $11.3 \%$ & $5.1 \%$ & $1.3 \%$ & $4.0 \%$ & $4.1 \%$ \\
\hline Colon cancer & $0.46 \%$ & $0.37 \%$ & $0.41 \%$ & $1.00 \%$ & $0.78 \%$ & $0.30 \%$ & $0.50 \%$ & $0.40 \%$ \\
\hline Conduction disorders & $2.49 \%$ & $1.41 \%$ & $1.66 \%$ & $5.00 \%$ & $2.77 \%$ & $0.79 \%$ & $2.32 \%$ & $2.34 \%$ \\
\hline COPD & $6.68 \%$ & $3.86 \%$ & $4.14 \%$ & $13.26 \%$ & $8.39 \%$ & $2.38 \%$ & $4.27 \%$ & $3.67 \%$ \\
\hline Dementia & $1.71 \%$ & $0.95 \%$ & $1.09 \%$ & $10.18 \%$ & $1.51 \%$ & $0.33 \%$ & $2.00 \%$ & $1.18 \%$ \\
\hline Dental conditions & $22.67 \%$ & $3.06 \%$ & $2.48 \%$ & $5.65 \%$ & $3.48 \%$ & $2.43 \%$ & $1.30 \%$ & $0.83 \%$ \\
\hline Diabetes & $24.02 \%$ & $17.14 \%$ & $16.37 \%$ & $27.58 \%$ & $19.10 \%$ & $7.08 \%$ & $15.14 \%$ & $15.68 \%$ \\
\hline Epilepsy & $0.85 \%$ & $0.41 \%$ & $0.39 \%$ & $1.78 \%$ & $0.58 \%$ & $0.14 \%$ & $0.25 \%$ & $0.19 \%$ \\
\hline GERD & $8.87 \%$ & $5.77 \%$ & $5.42 \%$ & $10.81 \%$ & $6.84 \%$ & $3.51 \%$ & $4.23 \%$ & $4.40 \%$ \\
\hline Headache & $2.76 \%$ & $1.51 \%$ & $0.81 \%$ & $0.60 \%$ & $0.65 \%$ & $0.56 \%$ & $0.35 \%$ & $0.29 \%$ \\
\hline Hearing loss & $8.33 \%$ & $9.30 \%$ & $11.55 \%$ & $8.61 \%$ & $6.25 \%$ & $6.60 \%$ & $9.99 \%$ & $8.15 \%$ \\
\hline Heart failure & $3.84 \%$ & $1.78 \%$ & $2.00 \%$ & $8.97 \%$ & $4.26 \%$ & $0.76 \%$ & $2.47 \%$ & $1.88 \%$ \\
\hline HIV & $0.35 \%$ & $0.34 \%$ & $0.32 \%$ & $0.89 \%$ & $0.72 \%$ & $0.13 \%$ & $0.45 \%$ & $0.27 \%$ \\
\hline Hypertension & $32.81 \%$ & $24.91 \%$ & $25.23 \%$ & $43.40 \%$ & $33.39 \%$ & $18.07 \%$ & $25.84 \%$ & $24.90 \%$ \\
\hline IHD & $12.96 \%$ & $8.89 \%$ & $9.22 \%$ & $19.18 \%$ & $13.01 \%$ & $5.69 \%$ & $13.28 \%$ & $15.02 \%$ \\
\hline Kidney stones & $1.07 \%$ & $0.85 \%$ & $0.79 \%$ & $1.08 \%$ & $0.95 \%$ & $0.62 \%$ & $0.72 \%$ & $0.57 \%$ \\
\hline
\end{tabular}


Table C-20-Continued

\begin{tabular}{|c|c|c|c|c|c|c|c|c|}
\hline \multirow[b]{2}{*}{ Health Condition } & \multicolumn{8}{|c|}{ Priority Group } \\
\hline & 1 & 2 & 3 & 4 & 5 & 6 & 7 & $8^{*}$ \\
\hline Lipid disorders & $25.78 \%$ & $20.10 \%$ & $20.15 \%$ & $27.71 \%$ & $22.79 \%$ & $15.63 \%$ & $19.38 \%$ & $20.09 \%$ \\
\hline Lung cancer & $0.68 \%$ & $0.31 \%$ & $0.36 \%$ & $1.08 \%$ & $0.86 \%$ & $0.18 \%$ & $0.45 \%$ & $0.31 \%$ \\
\hline Major depression & $8.90 \%$ & $4.27 \%$ & $3.06 \%$ & $7.01 \%$ & $4.03 \%$ & $1.85 \%$ & $1.92 \%$ & $1.32 \%$ \\
\hline Malignant cancer & $7.18 \%$ & $4.26 \%$ & $4.71 \%$ & $9.62 \%$ & $7.80 \%$ & $3.00 \%$ & $5.98 \%$ & $4.92 \%$ \\
\hline $\begin{array}{l}\text { Mental health } \\
\text { conditions }\end{array}$ & $50.15 \%$ & $29.97 \%$ & $23.45 \%$ & $47.82 \%$ & $33.22 \%$ & $20.18 \%$ & $18.11 \%$ & $13.90 \%$ \\
\hline Mood disorder & $24.17 \%$ & $13.42 \%$ & $10.30 \%$ & $22.90 \%$ & $13.76 \%$ & $6.76 \%$ & $6.63 \%$ & $4.97 \%$ \\
\hline Movement disorders & $1.90 \%$ & $0.94 \%$ & $0.91 \%$ & $3.56 \%$ & $1.14 \%$ & $0.52 \%$ & $1.20 \%$ & $0.98 \%$ \\
\hline Multiple sclerosis & $0.49 \%$ & $0.19 \%$ & $0.12 \%$ & $0.71 \%$ & $0.12 \%$ & $0.08 \%$ & $0.11 \%$ & $0.09 \%$ \\
\hline $\begin{array}{l}\text { Musculoskeletal } \\
\text { conditions }\end{array}$ & $26.99 \%$ & $21.15 \%$ & $16.96 \%$ & $18.72 \%$ & $16.95 \%$ & $10.62 \%$ & $9.79 \%$ & $8.45 \%$ \\
\hline Obesity & $8.76 \%$ & $6.28 \%$ & $5.75 \%$ & $6.41 \%$ & $6.47 \%$ & $4.01 \%$ & $4.92 \%$ & $3.96 \%$ \\
\hline Osteoporosis & $0.62 \%$ & $0.37 \%$ & $0.38 \%$ & $1.60 \%$ & $0.64 \%$ & $0.16 \%$ & $0.51 \%$ & $0.39 \%$ \\
\hline $\begin{array}{l}\text { Peripheral vascular } \\
\text { disease }\end{array}$ & $3.27 \%$ & $1.92 \%$ & $2.05 \%$ & $8.64 \%$ & $3.63 \%$ & $1.18 \%$ & $2.34 \%$ & $1.89 \%$ \\
\hline Personality disorders & $1.16 \%$ & $0.56 \%$ & $0.52 \%$ & $2.36 \%$ & $0.77 \%$ & $0.14 \%$ & $0.18 \%$ & $0.12 \%$ \\
\hline Prostate cancer & $3.29 \%$ & $1.73 \%$ & $1.88 \%$ & $3.86 \%$ & $2.87 \%$ & $1.00 \%$ & $2.54 \%$ & $2.29 \%$ \\
\hline PTSD & $26.46 \%$ & $9.45 \%$ & $3.89 \%$ & $4.86 \%$ & $3.30 \%$ & $4.52 \%$ & $1.22 \%$ & $0.96 \%$ \\
\hline Rheumatologic disease & $0.95 \%$ & $0.60 \%$ & $0.61 \%$ & $0.96 \%$ & $0.79 \%$ & $0.49 \%$ & $0.65 \%$ & $0.59 \%$ \\
\hline Schizophrenia & $2.75 \%$ & $0.55 \%$ & $0.60 \%$ & $6.20 \%$ & $1.13 \%$ & $0.07 \%$ & $0.29 \%$ & $0.15 \%$ \\
\hline Skin cancer & $0.90 \%$ & $0.72 \%$ & $0.84 \%$ & $1.38 \%$ & $1.15 \%$ & $0.66 \%$ & $0.99 \%$ & $0.83 \%$ \\
\hline Sleep disorders & $5.37 \%$ & $3.16 \%$ & $2.70 \%$ & $3.28 \%$ & $2.52 \%$ & $1.77 \%$ & $1.86 \%$ & $1.58 \%$ \\
\hline Spinal cord injury & $0.85 \%$ & $0.24 \%$ & $0.25 \%$ & $4.49 \%$ & $0.15 \%$ & $0.05 \%$ & $0.13 \%$ & $0.06 \%$ \\
\hline Substance abuse & $7.89 \%$ & $5.16 \%$ & $4.82 \%$ & $14.20 \%$ & $9.04 \%$ & $3.16 \%$ & $3.03 \%$ & $2.07 \%$ \\
\hline TBI & $3.59 \%$ & $1.49 \%$ & $1.07 \%$ & $2.40 \%$ & $1.24 \%$ & $1.48 \%$ & $0.57 \%$ & $0.40 \%$ \\
\hline Thyroid disorders & $4.48 \%$ & $3.23 \%$ & $3.27 \%$ & $6.40 \%$ & $3.99 \%$ & $2.20 \%$ & $3.13 \%$ & $3.13 \%$ \\
\hline Alular disorders & $1.75 \%$ & $1.23 \%$ & $1.29 \%$ & $3.19 \%$ & $2.07 \%$ & $0.80 \%$ & $1.64 \%$ & $1.45 \%$ \\
\hline Viral hepatitis & $1.72 \%$ & $1.10 \%$ & $1.25 \%$ & $4.96 \%$ & $2.91 \%$ & $0.44 \%$ & $1.00 \%$ & $0.71 \%$ \\
\hline Vision loss & $13.37 \%$ & $10.16 \%$ & $10.11 \%$ & $21.14 \%$ & $11.13 \%$ & $5.80 \%$ & $8.31 \%$ & $6.65 \%$ \\
\hline Women's health & $0.61 \%$ & $0.57 \%$ & $0.38 \%$ & $0.22 \%$ & $0.38 \%$ & $0.20 \%$ & $0.24 \%$ & $0.17 \%$ \\
\hline
\end{tabular}

SOURCE: RAND analysis of VA encounter data (2014).

NOTES: Sample size, Veterans in VA encounter data $=5,871,766$.

* Priority group 8 includes $8 \mathrm{a}-8 \mathrm{~d}$. 
Table C-21

Prevalence of Diagnosed Health Conditions Among VA Patients, by VA Reliance

Panel A. Demographic characteristics

\begin{tabular}{|c|c|c|c|c|}
\hline & \multicolumn{2}{|c|}{ All Ages } & \multicolumn{2}{|c|}{ Age $<65$ Only } \\
\hline & VA Reliant & VA Non-Reliant & VA Reliant & VA Non-Reliant \\
\hline \multicolumn{5}{|l|}{ Age } \\
\hline \multirow[t]{2}{*}{$\%$ Age $17-29$} & 0.063 & 0.014 & 0.089 & 0.030 \\
\hline & $(0.014)$ & $(0.003)$ & $(0.019)$ & $(0.006)$ \\
\hline \multirow[t]{2}{*}{$\%$ Age $30-39$} & 0.072 & 0.042 & 0.102 & 0.094 \\
\hline & $(0.014)$ & $(0.004)$ & $(0.019)$ & $(0.010)$ \\
\hline \multirow[t]{2}{*}{$\%$ Age $40-49$} & 0.082 & 0.087 & 0.116 & 0.196 \\
\hline & $(0.013)$ & $(0.007)$ & $(0.018)$ & $(0.014)$ \\
\hline \multirow[t]{2}{*}{$\%$ Age $50-64$} & 0.490 & 0.301 & 0.694 & 0.679 \\
\hline & $(0.024)$ & $(0.011)$ & $(0.028)$ & $(0.015)$ \\
\hline \multirow[t]{2}{*}{$\% 65+$} & 0.293 & 0.556 & & \\
\hline & $(0.023)$ & $(0.015)$ & & \\
\hline \multicolumn{5}{|l|}{ Race/ethnicity } \\
\hline \multirow[t]{2}{*}{$\%$ Hispanic } & 0.062 & 0.037 & 0.071 & 0.051 \\
\hline & $(0.012)$ & $(0.004)$ & $(0.015)$ & $(0.007)$ \\
\hline \multirow[t]{2}{*}{$\%$ White, non-Hispanic } & 0.729 & 0.839 & 0.723 & 0.766 \\
\hline & $(0.023)$ & $(0.009)$ & $(0.025)$ & $(0.013)$ \\
\hline \multirow[t]{2}{*}{$\%$ Black, non-Hispanic } & 0.179 & 0.094 & 0.179 & 0.138 \\
\hline & $(0.018)$ & $(0.006)$ & $(0.020)$ & $(0.010)$ \\
\hline \multirow[t]{2}{*}{$\%$ Asian, non-Hispanic } & 0.005 & 0.007 & 0.002 & 0.008 \\
\hline & $(0.003)$ & $(0.003)$ & $(0.002)$ & $(0.003)$ \\
\hline \multirow[t]{2}{*}{$\%$ Other, non-Hispanic, multiple } & 0.025 & 0.023 & 0.024 & 0.037 \\
\hline & $(0.008)$ & $(0.003)$ & $(0.009)$ & $(0.007)$ \\
\hline \multicolumn{5}{|l|}{ Sex and marital status } \\
\hline \multirow[t]{2}{*}{$\%$ Male } & 0.117 & 0.087 & 0.083 & 0.042 \\
\hline & $(0.016)$ & $(0.006)$ & $(0.017)$ & $(0.006)$ \\
\hline \multirow[t]{2}{*}{$\%$ Married } & 0.395 & 0.344 & 0.385 & 0.343 \\
\hline & $(0.029)$ & $(0.012)$ & $(0.032)$ & $(0.017)$ \\
\hline \multicolumn{5}{|l|}{ Education and employment } \\
\hline \multirow[t]{2}{*}{$\%$ Less than high school } & 0.117 & 0.087 & 0.083 & 0.042 \\
\hline & $(0.016)$ & $(0.006)$ & $(0.017)$ & $(0.006)$ \\
\hline
\end{tabular}


Table C-21-Continued

\begin{tabular}{|c|c|c|c|c|}
\hline & \multicolumn{2}{|c|}{ All Ages } & \multicolumn{2}{|c|}{ Age $<65$ Only } \\
\hline & VA Reliant & VA Non-Reliant & VA Reliant & VA Non-Reliant \\
\hline \multirow[t]{2}{*}{$\%$ High school graduate or GED } & 0.395 & 0.344 & 0.385 & 0.343 \\
\hline & $(0.029)$ & $(0.012)$ & $(0.032)$ & $(0.017)$ \\
\hline \multirow[t]{2}{*}{$\%$ Some college } & 0.282 & 0.193 & 0.303 & 0.219 \\
\hline & $(0.030)$ & $(0.009)$ & $(0.035)$ & $(0.012)$ \\
\hline \multirow[t]{2}{*}{$\%$ College+ } & 0.206 & 0.376 & 0.229 & 0.396 \\
\hline & $(0.022)$ & $(0.011)$ & $(0.029)$ & $(0.015)$ \\
\hline \multirow[t]{2}{*}{$\%$ Not employed } & 0.527 & 0.596 & 0.419 & 0.313 \\
\hline & $(0.028)$ & $(0.013)$ & $(0.031)$ & $(0.015)$ \\
\hline \multirow[t]{2}{*}{$\%$ Current student } & 0.021 & 0.006 & 0.030 & 0.013 \\
\hline & $(0.006)$ & $(0.001)$ & $(0.009)$ & $(0.003)$ \\
\hline \multicolumn{5}{|l|}{ Residential location } \\
\hline \multirow[t]{2}{*}{ Lives in MSA } & 0.758 & 0.792 & 0.769 & 0.803 \\
\hline & $(0.030)$ & $(0.018)$ & $(0.029)$ & $(0.017)$ \\
\hline \multirow[t]{2}{*}{ Northwest } & 0.111 & 0.166 & 0.099 & 0.148 \\
\hline & $(0.015)$ & $(0.011)$ & $(0.016)$ & $(0.015)$ \\
\hline \multirow[t]{2}{*}{ Midwest } & 0.233 & 0.226 & 0.231 & 0.229 \\
\hline & $(0.026)$ & $(0.011)$ & $(0.028)$ & $(0.015)$ \\
\hline \multirow[t]{2}{*}{ South } & 0.461 & 0.416 & 0.473 & 0.438 \\
\hline & $(0.029)$ & $(0.015)$ & $(0.032)$ & $(0.019)$ \\
\hline \multirow[t]{2}{*}{ West } & 0.195 & 0.192 & 0.196 & 0.185 \\
\hline & $(0.024)$ & $(0.013)$ & $(0.025)$ & $(0.016)$ \\
\hline
\end{tabular}

Panel B. Prevalence of selected health conditions

\begin{tabular}{|c|c|c|c|c|c|c|}
\hline & \multicolumn{3}{|c|}{ All Ages } & \multicolumn{3}{|c|}{ Age $<65$} \\
\hline & VA Reliant & $\begin{array}{c}\text { Non-VA } \\
\text { Reliant }\end{array}$ & $\begin{array}{l}\text { P-Value of } \\
\text { Difference }\end{array}$ & VA Reliant & $\begin{array}{l}\text { Non-VA } \\
\text { Reliant }\end{array}$ & $\begin{array}{l}\text { P-Value of } \\
\text { Difference }\end{array}$ \\
\hline \multirow[t]{2}{*}{ Asthma } & 0.076 & 0.08 & 0.810 & 0.064 & 0.076 & 0.476 \\
\hline & $(0.022)$ & $(0.019)$ & & $(0.023)$ & $(0.026)$ & \\
\hline \multirow[t]{2}{*}{ Acute coronary syndrome } & 0.094 & 0.119 & 0.267 & 0.073 & 0.095 & 0.434 \\
\hline & $(0.026)$ & $(0.024)$ & & $(0.030)$ & $(0.035)$ & \\
\hline \multirow[t]{2}{*}{ Cerebrovascular disease } & 0.025 & 0.032 & 0.384 & 0.01 & 0.013 & 0.474 \\
\hline & $(0.009)$ & $(0.010)$ & & $(0.006)$ & $(0.008)$ & \\
\hline
\end{tabular}


Table C-21-Continued

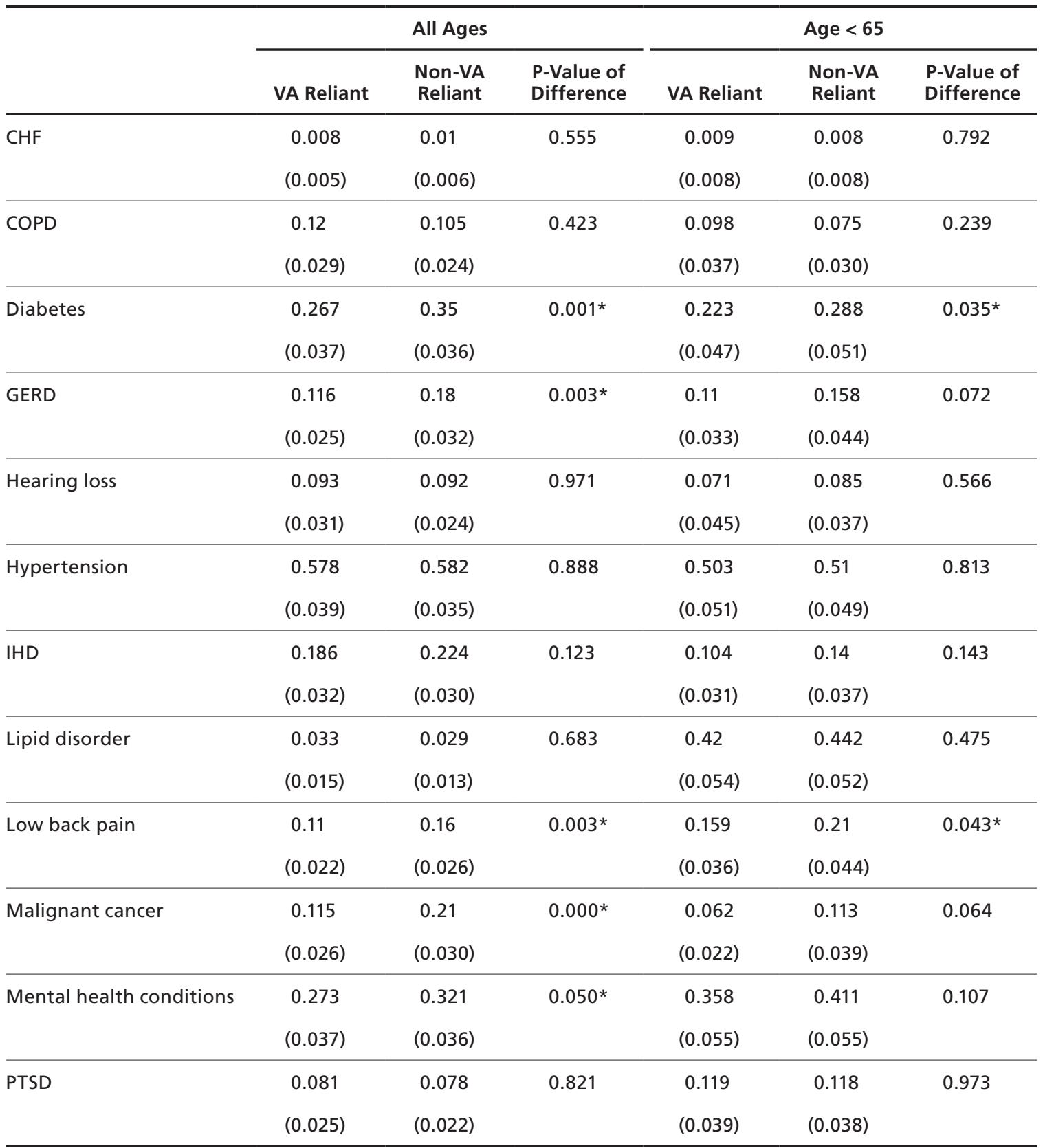

SOURCE: RAND analysis of MEPS, 2006-2012.

NOTES: Sample size, VA patients $=4,871$. A VA patient is considered reliant if all medical expenses in the year were VA and family/self-paid. A VA patient is considered non-reliant if he or she has some medical expenses paid by a non-VA health insurance source. The adjusted prevalence rates are the predicted prevalence rates from a logit estimation that included indicators for sex, five race/ethnicity categories, 14 age categories, four Census regions, residential location in an MSA, and a nonlinear time trend. VA patient status is defined as having any expenditures paid by VA at the person, not condition, level.

* indicates $p$-value $<0.05$ for the difference between VA reliant VA patients and VA patients who are not reliant on VA. 
Table C-22

Prevalence of Health Conditions in MHS and VA Encounter Data for Veterans Younger Than 35 Years Old

\begin{tabular}{|c|c|c|}
\hline Health Condition & $\begin{array}{c}\text { MHS } \\
\text { Encounter }\end{array}$ & $\begin{array}{c}\text { VA } \\
\text { Encounter }\end{array}$ \\
\hline Mental health conditions & $37.67 \%$ & $42.84 \%$ \\
\hline Musculoskeletal conditions & $31.59 \%$ & $21.55 \%$ \\
\hline PTSD & $5.95 \%$ & $19.65 \%$ \\
\hline Mood disorder & $11.96 \%$ & $19.02 \%$ \\
\hline Arthritis & $26.83 \%$ & $14.85 \%$ \\
\hline Anxiety disorders & $10.19 \%$ & $11.67 \%$ \\
\hline Substance use disorder & $9.22 \%$ & $9.49 \%$ \\
\hline TBI & $6.64 \%$ & $6.63 \%$ \\
\hline Dental conditions & $0.83 \%$ & $6.42 \%$ \\
\hline Major depression & $4.79 \%$ & $6.41 \%$ \\
\hline Headache & $5.26 \%$ & $3.36 \%$ \\
\hline Lipid disorders & $1.66 \%$ & $3.30 \%$ \\
\hline Obesity & $3.73 \%$ & $3.25 \%$ \\
\hline Hypertension & $2.97 \%$ & $3.17 \%$ \\
\hline GERD & $2.38 \%$ & $2.68 \%$ \\
\hline Bipolar disorder & $0.99 \%$ & $2.61 \%$ \\
\hline Asthma & $2.10 \%$ & $1.44 \%$ \\
\hline Personality disorders & $1.83 \%$ & $1.19 \%$ \\
\hline Hearing loss & $1.79 \%$ & $1.19 \%$ \\
\hline Thyroid disorders & $0.96 \%$ & $1.12 \%$ \\
\hline Women's health & $1.66 \%$ & $1.09 \%$ \\
\hline Cardiac dysrhythmias & $1.81 \%$ & $0.99 \%$ \\
\hline Schizophrenia & $0.23 \%$ & $0.94 \%$ \\
\hline Diabetes & $0.29 \%$ & $0.92 \%$ \\
\hline Anemia & $1.65 \%$ & $0.81 \%$ \\
\hline Benign cancers & $1.50 \%$ & $0.79 \%$ \\
\hline Kidney stones & $0.73 \%$ & $0.52 \%$ \\
\hline Epilepsy & $0.43 \%$ & $0.48 \%$ \\
\hline Cancer & $0.26 \%$ & $0.32 \%$ \\
\hline HIV & $0.09 \%$ & $0.26 \%$ \\
\hline Viral hepatitis & $0.07 \%$ & $0.26 \%$ \\
\hline
\end{tabular}


Table C-22-Continued

\begin{tabular}{lcc}
\hline Health Condition & $\begin{array}{c}\text { MHS } \\
\text { Encounter }\end{array}$ & $\begin{array}{c}\text { VA } \\
\text { Encounter }\end{array}$ \\
\hline Chronic liver disease & $0.24 \%$ & $0.25 \%$ \\
\hline Amputation & $0.19 \%$ & $0.22 \%$ \\
\hline Movement disorders & $0.42 \%$ & $0.22 \%$ \\
\hline Spinal cord injury & $0.17 \%$ & $0.22 \%$ \\
\hline Valvular disorders & $0.40 \%$ & $0.17 \%$ \\
\hline Chronic renal failure & $0.12 \%$ & $0.17 \%$ \\
\hline Rheumatologic disease & $0.16 \%$ & $0.17 \%$ \\
\hline IHD & $0.05 \%$ & $0.15 \%$ \\
\hline Cerebrovascular diseases & $0.17 \%$ & $0.14 \%$ \\
\hline Multiple sclerosis & $0.06 \%$ & $0.13 \%$ \\
\hline Conduction disorders & $0.17 \%$ & $0.13 \%$ \\
\hline COPD & $0.10 \%$ & $0.12 \%$ \\
\hline Heart failure & $0.06 \%$ & $0.09 \%$ \\
\hline Burns & $0.33 \%$ & $0.08 \%$ \\
\hline Peripheral vascular disease & $0.14 \%$ & $0.08 \%$ \\
\hline Benign prostate hypertrophy & $0.02 \%$ & $0.07 \%$ \\
\hline SO & & \\
\hline
\end{tabular}

SOURCE: RAND analysis of MHS encounter data (2012-2013) and VA encounter data (2014).

NOTES: Sample size, the number of separating military personnel aged less than 35 in MHS encounter data is 325,849, and the number of Veterans in VA encounter data aged less than 35 is 503,205.

\section{C.3. Current Health Care Needs Sensitivity Analysis}

\section{C.3.1. Alternative Prevalence Models for Veterans and Non-Veterans}

In this appendix, we describe our baseline specification (Model 1) and differences between the baseline specification and two alternative specifications (Models 2 and 3) that were used to assess the sensitivity of our findings to these baseline specifications.

- Model 1 adjusts for a respondent's sex, age (14 age categories: 20-24, 25-30, . . , 80-85, and 85+), race/ethnicity (five racial/ethnic categories: Hispanic, non-Hispanic white, nonHispanic black, non-Hispanic Asian, or other/multiple), four Census regions of residence (Northeast, Midwest, South, and West), an indicator for residence in an MSA, and a nonlinear time trend that adjusts for the year surveyed. The nonlinear time trend includes the following measures: (1) an indicator if the survey year was 2006, 2007, or after 2007; (2) a linear time trend for years after 2008 (e.g., equals 0 for 2006-2008, 1 for 2009, 2 for 2010, etc.). 
- Model 2 adds the following covariates to Model 1: education (four categories), interactions between sex and race/ethnicity, and indicators of health insurance coverage, marital status, student status, and employment status.

- Model 3 replaces the nonlinear time trend in Model 1 with indicators for each calendar year.

In Table C-23, we present estimated prevalence of health conditions for Veterans and nonVeterans using these three model specifications. The results are quantitatively similar across all three models.

Table C-23

The Prevalence of Diagnosed Health Conditions for Veterans and Non-Veterans: Alternative Specifications

\begin{tabular}{|c|c|c|c|c|c|c|}
\hline \multirow[b]{2}{*}{ Health Conditions } & \multicolumn{2}{|c|}{$\begin{array}{c}\text { Adjusted Mean } \\
\text { (Standard Error) } \\
\text { Model } 1\end{array}$} & \multicolumn{2}{|c|}{$\begin{array}{c}\text { Adjusted Mean } \\
\text { (Standard Error) } \\
\text { Model } 2\end{array}$} & \multicolumn{2}{|c|}{$\begin{array}{c}\text { Adjusted Mean } \\
\text { (Standard Error) } \\
\text { Model } 3\end{array}$} \\
\hline & Veteran & Non-Veterans & Veteran & Non-Veterans & Veteran & Non-Veterans \\
\hline \multirow[t]{2}{*}{ Asthma } & 0.058 & 0.056 & 0.054 & 0.055 & 0.054 & 0.053 \\
\hline & $(0.004)$ & $(0.003)$ & $(0.006)$ & $(0.004)$ & $(0.004)$ & $(0.002)$ \\
\hline \multirow[t]{2}{*}{ Cancer } & 0.155 & 0.112 & 0.141 & 0.105 & 0.154 & 0.111 \\
\hline & $(0.009)$ & $(0.007)$ & $(0.010)$ & $(0.008)$ & $(0.007)$ & $(0.005)$ \\
\hline \multirow[t]{2}{*}{$\mathrm{CHF}$} & 0.01 & 0.011 & 0.009 & 0.010 & 0.012 & 0.014 \\
\hline & $(0.002)$ & $(0.002)$ & $(0.002)$ & $(0.002)$ & $(0.002)$ & $(0.002)$ \\
\hline \multirow[t]{2}{*}{ COPD } & 0.063 & 0.046 & 0.055 & 0.036 & 0.064 & 0.046 \\
\hline & $(0.006)$ & $(0.004)$ & $(0.006)$ & $(0.004)$ & $(0.004)$ & $(0.003)$ \\
\hline \multirow[t]{2}{*}{ Diabetes } & 0.228 & 0.202 & 0.214 & 0.187 & 0.223 & 0.197 \\
\hline & $(0.008)$ & $(0.008)$ & $(0.009)$ & $(0.008)$ & $(0.007)$ & $(0.006)$ \\
\hline \multirow[t]{2}{*}{ GERD } & 0.122 & 0.100 & 0.105 & 0.085 & 0.118 & 0.097 \\
\hline & $(0.008)$ & $(0.006)$ & $(0.008)$ & $(0.006)$ & $(0.006)$ & $(0.004)$ \\
\hline \multirow[t]{2}{*}{ Hearing loss } & 0.043 & 0.031 & 0.040 & 0.029 & 0.041 & 0.030 \\
\hline & $(0.005)$ & $(0.005)$ & $(0.006)$ & $(0.005)$ & $(0.004)$ & $(0.003)$ \\
\hline \multirow[t]{2}{*}{ Hypertension } & 0.475 & 0.466 & 0.463 & 0.456 & 0.466 & 0.458 \\
\hline & $(0.010)$ & $(0.008)$ & $(0.012)$ & $(0.011)$ & $(0.008)$ & $(0.006)$ \\
\hline \multirow[t]{2}{*}{ IHD } & 0.142 & 0.138 & 0.128 & 0.123 & 0.152 & 0.148 \\
\hline & $(0.008)$ & $(0.007)$ & $(0.009)$ & $(0.008)$ & $(0.006)$ & $(0.006)$ \\
\hline \multirow[t]{2}{*}{ Lipid disorder } & 0.409 & 0.392 & 0.399 & 0.380 & 0.412 & 0.395 \\
\hline & $(0.010)$ & $(0.008)$ & $(0.011)$ & $(0.010)$ & $(0.008)$ & $(0.006)$ \\
\hline \multirow[t]{2}{*}{ Low back pain } & 0.138 & 0.133 & 0.136 & 0.124 & 0.130 & 0.126 \\
\hline & $(0.007)$ & $(0.006)$ & $(0.008)$ & $(0.007)$ & $(0.005)$ & $(0.004)$ \\
\hline
\end{tabular}


Table C-23-Continued

\begin{tabular}{|c|c|c|c|c|c|c|}
\hline \multirow[b]{2}{*}{ Health Conditions } & \multicolumn{2}{|c|}{$\begin{array}{c}\text { Adjusted Mean } \\
\text { (Standard Error) } \\
\text { Model } 1\end{array}$} & \multicolumn{2}{|c|}{$\begin{array}{c}\text { Adjusted Mean } \\
\text { (Standard Error) } \\
\text { Model } 2\end{array}$} & \multicolumn{2}{|c|}{$\begin{array}{c}\text { Adjusted Mean } \\
\text { (Standard Error) } \\
\text { Model } 3\end{array}$} \\
\hline & Veteran & Non-Veterans & Veteran & Non-Veterans & Veteran & Non-Veterans \\
\hline \multirow{2}{*}{$\begin{array}{l}\text { Mental health } \\
\text { condition }\end{array}$} & 0.200 & 0.174 & 0.185 & 0.158 & 0.190 & 0.165 \\
\hline & $(0.009)$ & $(0.006)$ & $(0.010)$ & $(0.007)$ & $(0.007)$ & $(0.004)$ \\
\hline \multirow[t]{2}{*}{ PTSD } & 0.027 & 0.002 & 0.016 & 0.001 & 0.020 & 0.002 \\
\hline & $(0.006)$ & 0.000 & $(0.005)$ & $(0.000)$ & $(0.003)$ & $(0.000)$ \\
\hline
\end{tabular}

SOURCE: RAND analysis of MEPS, 2006-2012.

NOTES: Sample size, non-Veterans $=150,225$ and sample size, Veterans $=12,313$. Sample sizes may be smaller for some conditions due to missing values. The predicted prevalence rates are from a logit estimation that included indicators for sex, five race/ethnicity categories, 14 age categories, four census regions, and residential location in an MSA. Models 1 and 2 include a nonlinear time trend, while Model 3 includes indicators for each calendar year. Model 3 also includes indicators for 4 education categories, health insurance coverage, being married, being a student or in school, being employed, and interactions of sex and race/ethnicity. These estimates show the differences in prevalence of health conditions for Veterans and for non-Veterans with the same demographic profile as Veterans. Cancer includes any malignancy and mental health condition includes any mental health condition.

In Tables C-24, C-25, and C-26, we report the estimated odds ratios for the Model 2 control variables. These tables describe the association between each control variable and disease prevalence, while adjusting for the remaining Model 2 control variables. In general, these additional controls explain some of the variation in prevalence rates, but, as shown in Table C-21, overall predicted prevalence rates based on Model 2 are similar to baseline estimates from Model 1. 
Table C-24

Odds Ratios of Each Disease Condition for Control Variables Included in Model 2: Sample: Veterans and Non-Veterans

\begin{tabular}{|c|c|c|c|c|}
\hline \multirow[b]{2}{*}{ Demographic Characteristics } & \multicolumn{4}{|c|}{$\begin{array}{l}\text { Model 2: Estimated Odds Ratio for each Disease Condition } \\
\text { (Standard Error) }\end{array}$} \\
\hline & Asthma & Cancer & COPD & $\mathrm{CHF}$ \\
\hline \multirow[t]{2}{*}{ Married } & $0.740 * * *$ & $1.122 * *$ & $0.642 * * *$ & $0.610 * * *$ \\
\hline & $(0.0328)$ & $(0.0511)$ & $(0.0340)$ & $(0.065)$ \\
\hline \multirow[t]{2}{*}{ Student status } & 0.900 & 0.830 & $0.294 * * *$ & $0.107 * * *$ \\
\hline & $(0.0930)$ & $(0.217)$ & $(0.0801)$ & $(0.091)$ \\
\hline \multirow[t]{2}{*}{ Have public or private health insurance } & $1.710 * * *$ & $2.055^{* * *}$ & $1.447 * * *$ & 1.085 \\
\hline & $(0.118)$ & $(0.212)$ & $(0.133)$ & $(0.199)$ \\
\hline \multirow[t]{2}{*}{ High school diploma or GED } & $0.834 * * *$ & 1.059 & $0.596 * * *$ & 0.952 \\
\hline & $(0.0480)$ & $(0.0750)$ & $(0.0457)$ & $(0.129)$ \\
\hline \multirow[t]{2}{*}{ Some college or associate degree } & 0.926 & 1.099 & $0.536 * * *$ & 0.786 \\
\hline & $(0.0594)$ & $(0.0829)$ & $(0.0479)$ & $(0.128)$ \\
\hline \multirow[t]{2}{*}{ College degree or higher } & $0.869 * *$ & $1.390 * * *$ & $0.348 * * *$ & $0.745^{*}$ \\
\hline & $(0.0535)$ & $(0.0956)$ & $(0.0338)$ & $(0.116)$ \\
\hline \multirow[t]{2}{*}{ Employed } & $0.522 * * *$ & $0.621 * * *$ & $0.312 * * *$ & $0.272 * * *$ \\
\hline & $(0.0242)$ & $(0.0347)$ & $(0.0238)$ & $(0.039)$ \\
\hline \multirow[t]{2}{*}{ White (non-Hispanic), } & $1.438 * * *$ & $2.465 * * *$ & $3.045^{* * *}$ & 0.816 \\
\hline & $(0.157)$ & $(0.287)$ & $(0.504)$ & $(0.331)$ \\
\hline \multirow[t]{2}{*}{ Black (non-Hispanic), male } & $1.402 * *$ & $1.406 * * *$ & $1.500 * *$ & 0.936 \\
\hline & $(0.184)$ & $(0.183)$ & $(0.264)$ & $(0.396)$ \\
\hline \multirow[t]{2}{*}{ Asian, male } & 1.014 & 1.024 & 1.024 & 1.885 \\
\hline & $(0.177)$ & $(0.211)$ & $(0.306)$ & $(1.305)$ \\
\hline \multirow[t]{2}{*}{ Other and multiple, male } & $1.847 * * *$ & 1.248 & $3.381 * * *$ & 0.486 \\
\hline & $(0.398)$ & $(0.384)$ & $(0.919)$ & $(0.328)$ \\
\hline
\end{tabular}

SOURCE: RAND analysis of MEPS, 2006-2012.

NOTES: Model 2 included indicators for sex, five race/ethnicity categories, 14 age categories, four census regions, residential location in an MSA, a nonlinear time trend, four education categories, health insurance coverage, being married, being a student or in school, being employed, and interactions of sex and race/ethnicity. These estimates show the relative change in odds of having been diagnosed or treated for the given condition under a given demographic characteristic. An odds ratio greater than (less than) 1 means that the probability increases (decreases). *** $p<0.01,{ }^{* *} p<0.05,{ }^{*} p<0.1$ 
Table C-25

Odds Ratios of Each Disease Condition for Control Variables Included in Model 2: Sample: Veterans and Non-Veterans (cont.)

\begin{tabular}{|c|c|c|c|c|c|}
\hline \multirow[b]{2}{*}{ Demographic Characteristics } & \multicolumn{5}{|c|}{$\begin{array}{l}\text { Model 2: Estimated Odds Ratio for each Disease Condition } \\
\text { (Standard Error) }\end{array}$} \\
\hline & Diabetes & GERD & Hearing Loss & Hypertension & IHD \\
\hline \multirow[t]{2}{*}{ Married } & 0.978 & $0.931 *$ & 1.130 & $0.887 * * *$ & $0.887 * * *$ \\
\hline & $(0.0297)$ & $(0.0367)$ & $(0.0996)$ & $(0.0260)$ & $(0.0260)$ \\
\hline \multirow[t]{2}{*}{ Student status } & 0.781 & 1.089 & 2.001 & $0.778 * *$ & $0.778 * *$ \\
\hline & $(0.147)$ & $(0.216)$ & $(1.129)$ & $(0.0926)$ & $(0.0926)$ \\
\hline \multirow[t]{2}{*}{ Have public or private health insurance } & $1.683 * * *$ & $2.517 * * *$ & $1.469 * *$ & $1.786 * * *$ & $1.786 * * *$ \\
\hline & $(0.0847)$ & $(0.181)$ & $(0.275)$ & $(0.0670)$ & $(0.0670)$ \\
\hline \multirow[t]{2}{*}{ High school diploma or GED } & $0.861 * * *$ & 0.943 & 1.062 & $0.922 * *$ & $0.922 * *$ \\
\hline & $(0.0351)$ & $(0.0559)$ & $(0.135)$ & $(0.0320)$ & $(0.0320)$ \\
\hline \multirow[t]{2}{*}{ Some college or associate degree } & $0.816 * * *$ & 0.984 & 1.215 & $0.877 * * *$ & $0.877 * * *$ \\
\hline & $(0.0380)$ & $(0.0600)$ & $(0.172)$ & $(0.0367)$ & $(0.0367)$ \\
\hline \multirow[t]{2}{*}{ College degree or higher } & $0.667 * * *$ & $0.853 * *$ & $1.368 * *$ & $0.710 * * *$ & $0.710 * * *$ \\
\hline & $(0.0330)$ & $(0.0530)$ & $(0.181)$ & $(0.0265)$ & $(0.0265)$ \\
\hline \multirow[t]{2}{*}{ Employed } & $0.542 * * *$ & $0.596 * * *$ & 0.831 & $0.658 * * *$ & $0.658 * * *$ \\
\hline & $(0.0201)$ & $(0.0243)$ & $(0.0971)$ & $(0.0185)$ & $(0.0185)$ \\
\hline \multirow[t]{2}{*}{ White (non-Hispanic), male } & $0.718 * * *$ & $2.118 * * *$ & $1.574 * *$ & $1.121 * *$ & $1.121 * *$ \\
\hline & $(0.0440)$ & $(0.246)$ & $(0.301)$ & $(0.0564)$ & $(0.0564)$ \\
\hline \multirow[t]{2}{*}{ Black (non-Hispanic), male } & 0.939 & $1.265^{*}$ & 0.776 & $1.645^{* * *}$ & $1.645^{* * *}$ \\
\hline & $(0.0719)$ & $(0.169)$ & $(0.180)$ & $(0.0955)$ & $(0.0955)$ \\
\hline \multirow[t]{2}{*}{ Asian, male } & 0.915 & 0.861 & 0.899 & 0.997 & 0.997 \\
\hline & $(0.0993)$ & $(0.176)$ & $(0.322)$ & $(0.0855)$ & $(0.0855)$ \\
\hline \multirow[t]{2}{*}{ Other and multiple, male } & 1.218 & $1.793 * *$ & 1.299 & $1.467 * *$ & $1.467 * *$ \\
\hline & $(0.201)$ & $(0.442)$ & $(0.546)$ & $(0.238)$ & $(0.238)$ \\
\hline
\end{tabular}

SOURCE: RAND analysis of MEPS, 2006-2012.

NOTES: Model 2 included indicators for sex, five race/ethnicity categories, 14 age categories, four census regions, residential location in an MSA, a nonlinear time trend, four education categories, health insurance coverage, being married, being a student or in school, being employed, and interactions of sex and race/ethnicity. These estimates show the relative change in odds of having been diagnosed or treated for the given condition under a given demographic characteristic. An odds ratio greater than (less than) 1 means that the probability increases (decreases). ${ }^{* *} p<0.01,{ }^{* *} p<0.05,{ }^{*} p<0.1$ 
Table C-26

Odds Ratios of Each Disease Condition for Control Variables Included in Model 2: Sample: Veterans and Non-Veterans (cont.)

\begin{tabular}{|c|c|c|c|c|}
\hline \multirow[b]{2}{*}{ Demographic Characteristics } & \multicolumn{4}{|c|}{$\begin{array}{l}\text { Model 2: Estimated Odds Ratio for each Disease Condition } \\
\text { (Standard Error) }\end{array}$} \\
\hline & Lipid Disorder & Low Back Pain & $\begin{array}{l}\text { Mental Health } \\
\text { Condition }\end{array}$ & PTSD \\
\hline \multirow[t]{2}{*}{ Married } & $1.047 *$ & $0.853 * * *$ & $0.563 * * *$ & $0.383 * * *$ \\
\hline & $(0.0273)$ & $(0.0271)$ & $(0.0145)$ & $(0.0717)$ \\
\hline \multirow[t]{2}{*}{ Student status } & 0.898 & $0.746 * * *$ & $0.841 * *$ & 0.954 \\
\hline & $(0.131)$ & $(0.0746)$ & $(0.0624)$ & $(0.356)$ \\
\hline \multirow[t]{2}{*}{ Have public or private health insurance } & $2.472 * * *$ & $1.315^{* * *}$ & $1.551 * * *$ & $1.574^{*}$ \\
\hline & $(0.110)$ & $(0.0575)$ & $(0.0648)$ & $(0.371)$ \\
\hline \multirow[t]{2}{*}{ High school diploma or GED } & 0.984 & 1.001 & 0.963 & 1.105 \\
\hline & $(0.0377)$ & $(0.0411)$ & $(0.0381)$ & $(0.269)$ \\
\hline \multirow[t]{2}{*}{ Some college or associate degree } & 0.950 & 0.994 & 0.949 & 1.300 \\
\hline & $(0.0430)$ & $(0.0551)$ & $(0.0452)$ & $(0.362)$ \\
\hline \multirow[t]{2}{*}{ College degree or higher } & $0.852 * * *$ & 0.992 & $0.864 * * *$ & 0.902 \\
\hline & $(0.0350)$ & $(0.0462)$ & $(0.0357)$ & $(0.269)$ \\
\hline \multirow[t]{2}{*}{ Employed } & $0.640 * * *$ & $0.690 * * *$ & $0.459 * * *$ & $0.161 * * *$ \\
\hline & $(0.0199)$ & $(0.0228)$ & $(0.0136)$ & $(0.0342)$ \\
\hline \multirow[t]{2}{*}{ White (non-Hispanic), male } & $1.214^{* * *}$ & $1.523 * * *$ & $1.707 * * *$ & 1.687 \\
\hline & $(0.0731)$ & $(0.102)$ & $(0.0983)$ & $(0.618)$ \\
\hline \multirow[t]{2}{*}{ Black (non-Hispanic), male } & $0.820 * * *$ & 0.918 & $0.798 * * *$ & 1.540 \\
\hline & $(0.0624)$ & $(0.0755)$ & $(0.0651)$ & $(0.628)$ \\
\hline \multirow[t]{2}{*}{ Asian, male } & 1.140 & $0.750 * *$ & $0.677^{* * *}$ & 0.470 \\
\hline & $(0.100)$ & $(0.0898)$ & $(0.0778)$ & $(0.385)$ \\
\hline \multirow[t]{2}{*}{ Other and multiple, male } & $1.435^{* * *}$ & 1.188 & $1.588^{* * *}$ & $3.631 * *$ \\
\hline & $(0.193)$ & $(0.155)$ & $(0.195)$ & $(2.048)$ \\
\hline
\end{tabular}

SOURCE: RAND analysis of MEPS, 2006-2012.

NOTES: Model 2 included indicators for sex, five race/ethnicity categories, 14 age categories, four census regions, residential location in an MSA, a nonlinear time trend, four education categories, health insurance coverage, being married, being a student or in school, being employed, and interactions of sex and race/ethnicity. These estimates show the relative change in odds of having been diagnosed or treated for the given condition under a given demographic characteristic. An odds ratio greater than (less than) 1 means that the probability increases (decreases). ${ }^{* * *} p<0.01,{ }^{* *} p<0.05,{ }^{*} p<0.1$ 


\section{C.3.2. Alternative Prevalence Models for Veterans by VA Patient Status}

As reported in C-1, we conducted similar sensitivity tests for our analysis comparing Veterans by VA patient status. We again estimated Models 1-3 as described in C-1 for our analysis of Veterans only.

As shown in Table C-27, results are similar across the three models, though there is more variation in results across models in VA patient versus non-patient analyses than in the nonVeteran versus Veteran analysis. In Tables C-28, C-29, and C-30, we report the estimated odds ratios for the Model 2 control variables. These describe the association between each control variable and disease prevalence among Veterans, while adjusting for the remaining Model 2 control variables.

Table C-27

The Prevalence of Diagnosed Health Conditions by VA Patient Status: Alternative Specifications

\begin{tabular}{|c|c|c|c|c|c|c|}
\hline \multirow[b]{2}{*}{ Health Conditions } & \multicolumn{2}{|c|}{$\begin{array}{c}\text { Adjusted Mean } \\
\text { (Standard Error) } \\
\text { Model } 1\end{array}$} & \multicolumn{2}{|c|}{$\begin{array}{c}\text { Adjusted Mean } \\
\text { (Standard Error) } \\
\text { Model } 2\end{array}$} & \multicolumn{2}{|c|}{$\begin{array}{c}\text { Adjusted Mean } \\
\text { (Standard Error) } \\
\text { Model } 3\end{array}$} \\
\hline & VA Patients & $\begin{array}{c}\text { Veterans Not } \\
\text { Using VA }\end{array}$ & VA Patients & $\begin{array}{l}\text { Veterans Not } \\
\text { Using VA }\end{array}$ & VA Patients & $\begin{array}{c}\text { Veterans Not } \\
\text { Using VA }\end{array}$ \\
\hline \multirow[t]{2}{*}{ Asthma } & 0.094 & 0.053 & 0.063 & 0.033 & 0.074 & 0.042 \\
\hline & $(0.016)$ & $(0.010)$ & $(0.015)$ & $(0.009)$ & $(0.009)$ & $(0.006)$ \\
\hline \multirow[t]{2}{*}{ Cancer } & 0.188 & 0.138 & 0.172 & 0.120 & 0.190 & 0.139 \\
\hline & $(0.019)$ & $(0.015)$ & $(0.023)$ & $(0.018)$ & $(0.013)$ & $(0.010)$ \\
\hline \multirow[t]{2}{*}{ Cerebrovascular disease } & 0.039 & 0.022 & 0.047 & 0.025 & 0.048 & 0.027 \\
\hline & $(0.009)$ & $(0.005)$ & $(0.015)$ & $(0.009)$ & $(0.008)$ & $(0.005)$ \\
\hline \multirow[t]{2}{*}{$\mathrm{CHF}$} & 0.011 & 0.005 & IS & IS & 0.014 & 0.006 \\
\hline & $(0.004)$ & $(0.002)$ & & & $(0.004)$ & $(0.002)$ \\
\hline \multirow[t]{2}{*}{ COPD } & 0.112 & 0.057 & 0.084 & 0.047 & 0.106 & 0.053 \\
\hline & $(0.019)$ & $(0.010)$ & $(0.019)$ & $(0.011)$ & $(0.012)$ & $(0.006)$ \\
\hline \multirow[t]{2}{*}{ Diabetes } & 0.304 & 0.207 & 0.290 & 0.205 & 0.282 & 0.190 \\
\hline & $(0.023)$ & $(0.018)$ & $(0.029)$ & $(0.023)$ & $(0.015)$ & $(0.011)$ \\
\hline \multirow[t]{2}{*}{ GERD } & 0.164 & 0.093 & 0.159 & 0.092 & 0.156 & 0.088 \\
\hline & $(0.021)$ & $(0.013)$ & $(0.026)$ & $(0.016)$ & $(0.014)$ & $(0.008)$ \\
\hline \multirow[t]{2}{*}{ Hearing loss } & 0.071 & 0.033 & 0.066 & 0.031 & 0.071 & 0.032 \\
\hline & $(0.017)$ & $(0.008)$ & $(0.021)$ & $(0.010)$ & $(0.011)$ & $(0.005)$ \\
\hline \multirow[t]{2}{*}{ Hypertension } & 0.600 & 0.466 & 0.617 & 0.482 & 0.573 & 0.437 \\
\hline & $(0.022)$ & $(0.022)$ & $(0.028)$ & $(0.029)$ & $(0.016)$ & $(0.015)$ \\
\hline \multirow[t]{2}{*}{ IHD } & 0.204 & 0.142 & 0.199 & 0.139 & 0.204 & 0.143 \\
\hline & $(0.020)$ & $(0.015)$ & $(0.025)$ & $(0.019)$ & $(0.014)$ & $(0.011)$ \\
\hline \multirow[t]{2}{*}{ Lipid disorder } & 0.513 & 0.385 & 0.534 & 0.402 & 0.509 & 0.382 \\
\hline & $(0.023)$ & $(0.021)$ & $(0.029)$ & $(0.028)$ & $(0.016)$ & $(0.014)$ \\
\hline
\end{tabular}


Table C-27-Continued

\begin{tabular}{|c|c|c|c|c|c|c|}
\hline \multirow[b]{2}{*}{ Health Conditions } & \multicolumn{2}{|c|}{$\begin{array}{c}\text { Adjusted Mean } \\
\text { (Standard Error) } \\
\text { Model } 1\end{array}$} & \multicolumn{2}{|c|}{$\begin{array}{c}\text { Adjusted Mean } \\
\text { (Standard Error) } \\
\text { Model } 2\end{array}$} & \multicolumn{2}{|c|}{$\begin{array}{c}\text { Adjusted Mean } \\
\text { (Standard Error) } \\
\text { Model } 3\end{array}$} \\
\hline & VA Patients & $\begin{array}{c}\text { Veterans Not } \\
\text { Using VA }\end{array}$ & VA Patients & $\begin{array}{c}\text { Veterans Not } \\
\text { Using VA }\end{array}$ & VA Patients & $\begin{array}{c}\text { Veterans Not } \\
\text { Using VA }\end{array}$ \\
\hline \multirow[t]{2}{*}{ Low back pain } & 0.170 & 0.098 & 0.199 & 0.139 & 0.204 & 0.143 \\
\hline & $(0.020)$ & $(0.013)$ & $(0.025)$ & $(0.019)$ & $(0.014)$ & $(0.011)$ \\
\hline \multirow[t]{2}{*}{ Mental health condition } & 0.337 & 0.171 & 0.311 & 0.173 & 0.304 & 0.150 \\
\hline & $(0.027)$ & $(0.017)$ & $(0.034)$ & $(0.023)$ & $(0.017)$ & $(0.010)$ \\
\hline \multirow[t]{2}{*}{ PTSD } & 0.087 & 0.005 & 0.06 & 0.005 & 0.056 & 0.003 \\
\hline & $(0.023)$ & $(0.002)$ & $(0.024)$ & $(0.002)$ & $(0.010)$ & $(0.001)$ \\
\hline
\end{tabular}

SOURCE: RAND analysis of MEPS, 2006-2012.

NOTES: Sample size, Veterans $=12,313$. Cells labeled IS indicate that the prevalence model for the specific health condition could not be estimated due to insufficient sample size. The predicted prevalence rates are from a logit estimation that included indicators for sex, five race/ethnicity categories, 14 age categories, four census regions, and residential location in an MSA. Models 1 and 2 include a nonlinear time trend, while Model 3 includes indicators for each calendar year. Model 3 also includes indicators for four education categories, health insurance coverage, being married, being a student or in school, being employed, and interactions of sex and race/ ethnicity. These estimates show the differences in prevalence of health conditions for VA patients and Veterans not using VA health services, both with the same demographic profile of the overall Veteran population. Cancer includes any malignancy and mental health condition includes any mental health condition. 
Table C-28

Odds Ratios of Each Disease Condition for Control Variables Included in Model 2: Sample: VA Patients and Non-VA Patients (Veterans Only)

\begin{tabular}{|c|c|c|c|c|}
\hline \multirow[b]{2}{*}{ Demographic Characteristics } & \multicolumn{4}{|c|}{$\begin{array}{l}\text { Model 2: Estimated Odds Ratio for each Disease Condition } \\
\text { (Standard Error) }\end{array}$} \\
\hline & Asthma & Cancer & COPD & CHF \\
\hline \multirow[t]{2}{*}{ Married } & 0.899 & $1.373 * * *$ & 0.826 & IS \\
\hline & $(0.128)$ & $(0.149)$ & $(0.114)$ & \\
\hline \multirow[t]{2}{*}{ Student status } & 0.504 & $2.453^{*}$ & 0.443 & IS \\
\hline & $(0.357)$ & $(1.269)$ & $(0.495)$ & \\
\hline \multirow[t]{2}{*}{ Have public or private health insurance } & 1.506 & $2.045^{* *}$ & 0.820 & IS \\
\hline & $(0.406)$ & $(0.610)$ & $(0.208)$ & \\
\hline \multirow[t]{2}{*}{ High school diploma or GED } & 0.695 & 1.166 & $0.502 * * *$ & IS \\
\hline & $(0.168)$ & $(0.193)$ & $(0.103)$ & \\
\hline \multirow[t]{2}{*}{ Some college or associate degree } & 0.868 & 1.147 & $0.584 * *$ & IS \\
\hline & $(0.218)$ & $(0.207)$ & $(0.131)$ & \\
\hline \multirow[t]{2}{*}{ College degree or higher } & 0.710 & $1.598^{* * *}$ & $0.304 * * *$ & IS \\
\hline & $(0.172)$ & $(0.261)$ & $(0.0697)$ & \\
\hline \multirow[t]{2}{*}{ Employed } & $0.620 * * *$ & $0.601 * * *$ & $0.352 * * *$ & IS \\
\hline & $(0.109)$ & $(0.0705)$ & $(0.0656)$ & \\
\hline \multirow[t]{2}{*}{ White (non-Hispanic), male } & 1.640 & $2.386 * * *$ & $3.115 * * *$ & IS \\
\hline & $(0.592)$ & $(0.757)$ & $(1.321)$ & \\
\hline \multirow[t]{2}{*}{ Black (non-Hispanic), male } & 1.413 & 1.512 & 1.394 & IS \\
\hline & $(0.573)$ & $(0.526)$ & $(0.665)$ & \\
\hline \multirow[t]{2}{*}{ Asian, male } & 1.919 & 1.586 & 1.260 & IS \\
\hline & $(1.070)$ & $(0.985)$ & $(0.898)$ & \\
\hline \multirow[t]{2}{*}{ Other and multiple, male } & 1.343 & 1.663 & $3.544 * *$ & IS \\
\hline & $(0.791)$ & $(0.801)$ & $(2.081)$ & \\
\hline
\end{tabular}

SOURCE: RAND analysis of MEPS, 2006-2012.

NOTES: Cells labeled IS indicate that the prevalence model for the specific health condition could not be estimated due to insufficient sample size. The extended logit estimation included indicators for sex, five race/ ethnicity categories, 14 age categories, four census regions, residential location in an MSA, a nonlinear time trend, four education categories, health insurance coverage, being married, being a student or in school, being employed, and interactions of sex and race/ethnicity. These estimates show the relative change in odds of having been diagnosed or treated for the given condition under a given demographic characteristic. An odds ratio greater than (less than) 1 means that the probability increases (decreases). ${ }^{* *} p<0.01, * * p<0.05,{ }^{*} p<0.1$ 
Table C-29

Odds Ratios of Each Disease Condition for Control Variables Included in Model 2: Sample: VA Patients and Non-VA Patients (Veterans Only) (continued)

\begin{tabular}{|c|c|c|c|c|c|}
\hline \multirow[b]{2}{*}{ Demographic Characteristics } & \multicolumn{5}{|c|}{$\begin{array}{l}\text { Model 2: Estimated Odds Ratio for each Disease Condition } \\
\text { (Standard Error) }\end{array}$} \\
\hline & Diabetes & GERD & Hearing Loss & Hypertension & IHD \\
\hline \multirow[t]{2}{*}{ Married } & $1.187 * *$ & 1.146 & $1.487 * *$ & $1.154 * *$ & $1.358 * * *$ \\
\hline & $(0.100)$ & $(0.121)$ & $(0.278)$ & $(0.0827)$ & $(0.143)$ \\
\hline \multirow[t]{2}{*}{ Student status } & 1.960 & 0.363 & 2.111 & 0.705 & $0.163^{*}$ \\
\hline & $(1.381)$ & $(0.236)$ & $(2.384)$ & $(0.292)$ & $(0.175)$ \\
\hline \multirow[t]{2}{*}{ Have public or private health insurance } & 1.269 & $1.557 * *$ & 0.747 & $1.435^{* * *}$ & $1.864 * *$ \\
\hline & $(0.205)$ & $(0.335)$ & $(0.283)$ & $(0.181)$ & $(0.467)$ \\
\hline \multirow[t]{2}{*}{ High school diploma or GED } & 0.896 & 0.811 & 0.921 & $0.688 * * *$ & 0.799 \\
\hline & $(0.117)$ & $(0.139)$ & $(0.220)$ & $(0.0859)$ & $(0.122)$ \\
\hline \multirow[t]{2}{*}{ Some college or associate degree } & 0.970 & 0.876 & 1.032 & $0.689 * * *$ & 0.819 \\
\hline & $(0.137)$ & $(0.165)$ & $(0.284)$ & $(0.0920)$ & $(0.138)$ \\
\hline \multirow[t]{2}{*}{ College degree or higher } & 0.809 & 0.842 & 1.076 & $0.655^{* * *}$ & $0.694 * *$ \\
\hline & $(0.107)$ & $(0.147)$ & $(0.260)$ & $(0.0826)$ & $(0.110)$ \\
\hline \multirow[t]{2}{*}{ Employed } & $0.616 * * *$ & $0.637 * * *$ & 0.868 & $0.779 * * *$ & $0.552 * * *$ \\
\hline & $(0.0587)$ & $(0.0742)$ & $(0.190)$ & $(0.0613)$ & $(0.0701)$ \\
\hline \multirow[t]{2}{*}{ White (non-Hispanic), male } & $0.674 * *$ & $1.737 * *$ & 1.163 & 0.974 & $1.793 * *$ \\
\hline & $(0.114)$ & $(0.468)$ & $(0.558)$ & $(0.148)$ & $(0.431)$ \\
\hline \multirow[t]{2}{*}{ Black (non-Hispanic), male } & 0.749 & 1.083 & 0.640 & $1.656 * * *$ & 1.132 \\
\hline & $(0.144)$ & $(0.321)$ & $(0.356)$ & $(0.280)$ & $(0.307)$ \\
\hline \multirow[t]{2}{*}{ Asian, male } & $0.532^{*}$ & 0.932 & 1.205 & 0.801 & $0.177 * * *$ \\
\hline & $(0.193)$ & $(0.519)$ & $(0.749)$ & $(0.233)$ & $(0.114)$ \\
\hline \multirow[t]{2}{*}{ Other and multiple, male } & 1.217 & 1.631 & 1.302 & 0.936 & $2.393 * *$ \\
\hline & $(0.372)$ & $(0.716)$ & $(0.846)$ & $(0.255)$ & $(0.944)$ \\
\hline
\end{tabular}

SOURCE: RAND analysis of MEPS, 2006-2012.

NOTES: Cells labeled IS indicate that the prevalence model for the specific health condition could not be estimated due to insufficient sample size. The extended logit estimation included indicators for sex, five race/ ethnicity categories, 14 age categories, four census regions, residential location in an MSA, a nonlinear time trend, four education categories, health insurance coverage, being married, being a student or in school, being employed, and interactions of sex and race/ethnicity. These estimates show the relative change in odds of having been diagnosed or treated for the given condition under a given demographic characteristic. An odds ratio greater than (less than) 1 means that the probability increases (decreases). ${ }^{* *} p<0.01,{ }^{* *} p<0.05,{ }^{*} p<0.1$ 
Table C-30

Odds Ratios of Each Disease Condition for Control Variables Included in Model 2: Sample: VA Patients and Non-VA Patients (Veterans Only) (continued)

\begin{tabular}{|c|c|c|c|c|}
\hline \multirow[b]{2}{*}{ Demographic Characteristics } & \multicolumn{4}{|c|}{$\begin{array}{l}\text { Model 2: Estimated Odds Ratio for each Disease Condition } \\
\text { (Standard Error) }\end{array}$} \\
\hline & Lipid Disorder & Low Back Pain & $\begin{array}{l}\text { Mental Health } \\
\text { Condition }\end{array}$ & PTSD \\
\hline \multirow[t]{2}{*}{ Married } & $1.340 * * *$ & 1.059 & $0.778 * * *$ & 0.932 \\
\hline & $(0.0965)$ & $(0.0958)$ & $(0.0640)$ & $(0.235)$ \\
\hline \multirow[t]{2}{*}{ Student status } & 0.827 & 0.666 & 1.117 & 1.183 \\
\hline & $(0.597)$ & $(0.301)$ & $(0.408)$ & $(0.650)$ \\
\hline \multirow[t]{2}{*}{ Have public or private health insurance } & $1.775^{* * *}$ & 0.955 & $1.288^{*}$ & 0.975 \\
\hline & $(0.235)$ & $(0.151)$ & $(0.177)$ & $(0.361)$ \\
\hline \multirow[t]{2}{*}{ High school diploma or GED } & 1.034 & 0.843 & 1.015 & 1.116 \\
\hline & $(0.125)$ & $(0.128)$ & $(0.141)$ & $(0.550)$ \\
\hline \multirow[t]{2}{*}{ Some college or associate degree } & 1.103 & 0.874 & 0.868 & 0.938 \\
\hline & $(0.145)$ & $(0.145)$ & $(0.131)$ & $(0.510)$ \\
\hline \multirow[t]{2}{*}{ College degree or higher } & 1.126 & 0.951 & 0.998 & 1.185 \\
\hline & $(0.138)$ & $(0.146)$ & $(0.142)$ & $(0.601)$ \\
\hline \multirow[t]{2}{*}{ Employed } & $0.607 * * *$ & $0.714^{* * *}$ & $0.358 * * *$ & $0.150 * * *$ \\
\hline & $(0.0483)$ & $(0.0800)$ & $(0.0375)$ & $(0.0465)$ \\
\hline \multirow[t]{2}{*}{ White (non-Hispanic), male } & $1.330 *$ & 1.126 & 1.239 & 0.959 \\
\hline & $(0.209)$ & $(0.203)$ & $(0.218)$ & $(0.471)$ \\
\hline \multirow[t]{2}{*}{ Black (non-Hispanic), male } & 0.986 & 0.825 & 0.771 & 1.544 \\
\hline & $(0.174)$ & $(0.172)$ & $(0.157)$ & $(0.835)$ \\
\hline \multirow[t]{2}{*}{ Asian, male } & 1.197 & 0.717 & 1.643 & $0.106^{*}$ \\
\hline & $(0.361)$ & $(0.268)$ & $(0.651)$ & $(0.129)$ \\
\hline \multirow[t]{2}{*}{ Other and multiple, male } & $1.860 * *$ & 0.659 & 1.337 & 1.977 \\
\hline & $(0.540)$ & $(0.230)$ & $(0.377)$ & (1.374) \\
\hline
\end{tabular}

SOURCE: RAND analysis of MEPS, 2006-2012.

NOTES: Cells labeled IS indicate that the prevalence model for the specific health condition could not be estimated due to insufficient sample size. The extended logit estimation included indicators for sex, five race/ ethnicity categories, 14 age categories, four census regions, residential location in an MSA, a nonlinear time trend, four education categories, health insurance coverage, being married, being a student or in school, being employed, and interactions of sex and race/ethnicity. These estimates show the relative change in odds of having been diagnosed or treated for the given condition under a given demographic characteristic. An odds ratio greater than (less than) 1 means that the probability increases (decreases). ${ }^{* *} p<0.01, * * p<0.05,{ }^{*} p<0.1$ 


\section{C.3.3. Prevalence of Self-Reported Health Conditions}

\section{C.3.3.1. Veterans vs. Non-Veterans in NHIS}

Table C-31 provides estimates of the prevalence of self-reported health conditions for Veterans and non-Veterans, some of which overlap with the ICD-9-based health condition measures, using NHIS (2006-2013).

Table C-31

Prevalence of Self-Reported Health Conditions for Veterans and Non-Veterans in NHIS

\begin{tabular}{|c|c|c|c|c|c|}
\hline \multirow[b]{2}{*}{ Health Conditions } & \multicolumn{2}{|c|}{$\begin{array}{l}\text { Unadjusted Mean } \\
\text { (Standard Deviation) }\end{array}$} & \multicolumn{2}{|c|}{$\begin{array}{l}\text { Adjusted Mean } \\
\text { (Standard Error) }\end{array}$} & \multirow{2}{*}{$\begin{array}{c}\text { P-Value for } \\
\text { Difference in } \\
\text { Adjusted Means }\end{array}$} \\
\hline & Veteran & Non-Veterans & Veteran & Non-Veterans & \\
\hline \multirow[t]{2}{*}{ Angina } & 0.054 & 0.018 & 0.046 & 0.037 & $0.000 *$ \\
\hline & $(0.002)$ & $(0.000)$ & $(0.002)$ & $(0.002)$ & \\
\hline \multirow[t]{2}{*}{ Arthritis } & 0.354 & 0.209 & 0.349 & 0.302 & $0.000 *$ \\
\hline & $(0.005)$ & $(0.001)$ & $(0.005)$ & $(0.005)$ & \\
\hline \multirow[t]{2}{*}{ Asthma } & 0.095 & 0.125 & 0.095 & 0.103 & $0.006^{*}$ \\
\hline & $(0.003)$ & $(0.001)$ & $(0.003)$ & $(0.002)$ & \\
\hline \multirow[t]{2}{*}{ Cancer } & 0.171 & 0.071 & 0.178 & 0.126 & $0.000 *$ \\
\hline & $(0.004)$ & $(0.001)$ & $(0.004)$ & $(0.003)$ & \\
\hline \multirow[t]{2}{*}{ Chronic bronchitis } & 0.043 & 0.040 & 0.039 & 0.033 & $0.002 *$ \\
\hline & $(0.002)$ & $(0.001)$ & $(0.002)$ & $(0.001)$ & \\
\hline \multirow[t]{2}{*}{ Back pain } & 0.312 & 0.277 & 0.325 & 0.300 & $0.000 *$ \\
\hline & $(0.004)$ & $(0.002)$ & $(0.005)$ & $(0.004)$ & \\
\hline \multirow[t]{2}{*}{ Coronary heart disease } & 0.132 & 0.036 & 0.137 & 0.117 & $0.000 *$ \\
\hline & $(0.003)$ & $(0.001)$ & $(0.004)$ & $(0.003)$ & \\
\hline \multirow[t]{2}{*}{ Emphysema } & 0.045 & 0.015 & 0.041 & 0.032 & $0.000 *$ \\
\hline & $(0.002)$ & $(0.000)$ & $(0.002)$ & $(0.002)$ & \\
\hline \multirow[t]{2}{*}{ Myocardial infarction } & 0.099 & 0.026 & 0.088 & 0.074 & $0.000 *$ \\
\hline & $(0.003)$ & $(0.000)$ & $(0.003)$ & $(0.003)$ & \\
\hline \multirow[t]{2}{*}{ Hay fever } & 0.079 & 0.077 & 0.078 & 0.072 & $0.019 *$ \\
\hline & $(0.002)$ & $(0.001)$ & $(0.003)$ & $(0.002)$ & \\
\hline \multirow[t]{2}{*}{ Heart condition } & 0.137 & 0.067 & 0.127 & 0.103 & $0.000 *$ \\
\hline & $(0.003)$ & $(0.001)$ & $(0.004)$ & $(0.003)$ & \\
\hline \multirow[t]{2}{*}{ Hepatitis } & 0.046 & 0.028 & 0.041 & 0.032 & $0.000 *$ \\
\hline & $(0.002)$ & $(0.000)$ & $(0.002)$ & $(0.002)$ & \\
\hline \multirow[t]{2}{*}{ Hypertension } & 0.472 & 0.271 & 0.472 & 0.452 & $0.000 *$ \\
\hline & $(0.005)$ & $(0.002)$ & $(0.005)$ & $(0.005)$ & \\
\hline
\end{tabular}


Table C-31-Continued

\begin{tabular}{|c|c|c|c|c|c|}
\hline \multirow[b]{2}{*}{ Health Conditions } & \multicolumn{2}{|c|}{$\begin{array}{l}\text { Unadjusted Mean } \\
\text { (Standard Deviation) }\end{array}$} & \multicolumn{2}{|c|}{$\begin{array}{l}\text { Adjusted Mean } \\
\text { (Standard Error) }\end{array}$} & \multirow{2}{*}{$\begin{array}{c}\text { P-Value for } \\
\text { Difference in } \\
\text { Adjusted Means }\end{array}$} \\
\hline & Veteran & Non-Veterans & Veteran & Non-Veterans & \\
\hline \multirow[t]{2}{*}{ Jaw or front of ear pain } & 0.035 & 0.048 & 0.036 & 0.032 & $0.022^{*}$ \\
\hline & $(0.002)$ & $(0.001)$ & $(0.002)$ & $(0.001)$ & \\
\hline \multirow[t]{2}{*}{ Liver condition } & 0.020 & 0.013 & 0.019 & 0.014 & $0.000 *$ \\
\hline & $(0.001)$ & $(0.000)$ & $(0.001)$ & $(0.001)$ & \\
\hline \multirow[t]{2}{*}{ Migraine } & 0.092 & 0.155 & 0.099 & 0.085 & $0.000 *$ \\
\hline & $(0.003)$ & $(0.001)$ & $(0.003)$ & $(0.002)$ & \\
\hline \multirow[t]{2}{*}{ Neck pain } & 0.152 & 0.146 & 0.157 & 0.138 & $0.000 *$ \\
\hline & $(0.003)$ & $(0.001)$ & $(0.004)$ & $(0.003)$ & \\
\hline \multirow[t]{2}{*}{ Sinusitis } & 0.119 & 0.129 & 0.111 & 0.104 & $0.032^{*}$ \\
\hline & $(0.003)$ & $(0.001)$ & $(0.003)$ & $(0.002)$ & \\
\hline \multirow[t]{2}{*}{ Stroke } & 0.056 & 0.024 & 0.054 & 0.046 & $0.001^{*}$ \\
\hline & $(0.002)$ & $(0.000)$ & $(0.003)$ & $(0.002)$ & \\
\hline \multirow[t]{2}{*}{ Ulcer } & 0.104 & 0.066 & 0.086 & 0.071 & $0.000 *$ \\
\hline & $(0.002)$ & $(0.001)$ & $(0.003)$ & $(0.002)$ & \\
\hline
\end{tabular}

SOURCE: RAND analysis of National Health Interview Survey (NHIS) (2006-2013).

NOTES: The unadjusted prevalence in results columns (1) and (2) are equivalent to the fraction of Veterans and non-Veterans who self-reported each condition. The adjusted prevalence in results columns (3) and (4) are the predicted prevalence from a logit estimation that included indicators for sex, five race/ethnicity categories, five age categories, four census regions, and a nonlinear time trend. These estimates show the differences in prevalence of self-reported health conditions for Veterans and for non-Veterans with the same demographic profile as Veterans.

* The difference in adjusted means is statistically different from zero at the $5 \%$ level (p-value $<0.05)$. 


\section{C.3.3.2. Veterans vs. Non-Veterans in BRFSS}

In Table C-32, we provide estimates of the prevalence of self-reported health conditions and health behaviors for Veterans and non-Veterans, some of which overlap with the ICD-9-based health condition measures, using BRFSS (2013).

Table C-32

Prevalence of Self-Reported Health Outcomes for Veterans and Non-Veterans in BRFSS

\begin{tabular}{|c|c|c|c|c|c|}
\hline \multirow{2}{*}{$\begin{array}{l}\text { Health Conditions, } \\
\text { Indicators, and Behaviors }\end{array}$} & \multicolumn{2}{|c|}{$\begin{array}{l}\text { Unadjusted Mean } \\
\text { (Standard Deviation) }\end{array}$} & \multicolumn{2}{|c|}{$\begin{array}{l}\text { Adjusted Mean } \\
\text { (Standard Error) }\end{array}$} & \multirow{2}{*}{$\begin{array}{l}\text { Difference in } \\
\text { Adjusted Means } \\
\text { (V - C) }\end{array}$} \\
\hline & Non-Veterans & Veteran & Non-Veterans & Veteran & \\
\hline \multirow[t]{2}{*}{ Asthma^ } & 0.093 & 0.060 & 0.072 & 0.060 & $-0.011 *$ \\
\hline & $(0.001)$ & $(0.002)$ & $(0.001)$ & $(0.002)$ & \\
\hline \multirow[t]{2}{*}{ Cancer $^{\wedge}$} & 0.100 & 0.203 & 0.187 & 0.203 & $0.016^{*}$ \\
\hline & $(0.001)$ & $(0.003)$ & $(0.002)$ & $(0.003)$ & \\
\hline \multirow[t]{2}{*}{ COPD } & 0.061 & 0.095 & 0.077 & 0.095 & $0.019 *$ \\
\hline & $(0.001)$ & $(0.002)$ & $(0.002)$ & $(0.002)$ & \\
\hline \multirow[t]{2}{*}{ Diabetes } & 0.096 & 0.163 & 0.147 & 0.163 & $0.017 *$ \\
\hline & $(0.001)$ & $(0.003)$ & $(0.002)$ & $(0.003)$ & \\
\hline \multirow[t]{2}{*}{ High blood pressure } & 0.488 & 0.289 & 0.512 & 0.517 & $0.015^{*}$ \\
\hline & $(0.007)$ & $(0.002)$ & $(0.010)$ & $(0.009)$ & \\
\hline \multirow[t]{2}{*}{ Activity limitations } & 0.190 & 0.269 & 0.230 & 0.269 & $0.039 *$ \\
\hline & $(0.001)$ & $(0.003)$ & $(0.002)$ & $(0.003)$ & \\
\hline \multirow[t]{2}{*}{ Obese } & 0.282 & 0.293 & 0.289 & 0.293 & 0.004 \\
\hline & $(0.001)$ & $(0.004)$ & $(0.002)$ & $(0.004)$ & \\
\hline \multirow[t]{2}{*}{ Smoke now } & 0.181 & 0.188 & 0.164 & 0.188 & 0.024 \\
\hline & $(0.001)$ & $(0.003)$ & $(0.002)$ & $(0.003)$ & \\
\hline \multirow[t]{2}{*}{ Exercise in last 30 days } & 0.734 & 0.742 & 0.721 & 0.742 & $-0.021 *$ \\
\hline & $(0.001)$ & $(0.004)$ & $(0.003)$ & $(0.004)$ & \\
\hline \multirow[t]{2}{*}{ Insufficient sleep (< 7 hrs.) } & 0.354 & 0.372 & 0.310 & 0.372 & $0.063^{*}$ \\
\hline & $(0.002)$ & $(0.004)$ & $(0.002)$ & $(0.004)$ & \\
\hline
\end{tabular}

SOURCE: RAND analysis of Behavioral Risk Factor Surveillance Survey (BRFSS) (2013).

NOTES: The unadjusted prevalence in results columns (1) and (2) are equivalent to the fraction of Veterans and non-Veterans who self-reported each condition or behavior. The adjusted prevalence in results columns (3) and (4) are the predicted prevalence from a logit estimation that included indicators for sex, five race/ethnicity categories, five age categories, four census regions. These estimates show the differences in prevalence of selfreported health conditions for Veterans and for non-Veterans with the same demographic profile as Veterans.

* The difference in adjusted means is statistically different from zero at the $5 \%$ level ( $p$-value<0.05).

$\wedge$ These conditions do not directly map to the ICD-9 based definitions in MEPS. 


\section{C.4. Projections of Future Health Care Needs}

\section{C.4.1. Projections for Veterans}

C.4.1.1. Asthma

Table C-33

Projected Prevalence of Diagnosed Asthma Among Veterans, by Sex, 2014-2024

\begin{tabular}{|c|c|c|c|c|c|c|}
\hline \multirow[b]{2}{*}{ Year } & \multicolumn{2}{|c|}{ Male } & \multicolumn{2}{|c|}{ Female } & \multicolumn{2}{|c|}{ Total } \\
\hline & Count & Prevalence & Count & Prevalence & Count & Prevalence \\
\hline 2014 & $1,108,000$ & $5.6 \%$ & 166,000 & $9.2 \%$ & $1,274,000$ & $5.9 \%$ \\
\hline 2015 & $1,110,000$ & $5.7 \%$ & 171,000 & $9.4 \%$ & $1,281,000$ & $6.0 \%$ \\
\hline 2016 & $1,109,000$ & $5.9 \%$ & 176,000 & $9.7 \%$ & $1,285,000$ & $6.2 \%$ \\
\hline 2017 & $1,105,000$ & $6.0 \%$ & 181,000 & $9.9 \%$ & $1,286,000$ & $6.3 \%$ \\
\hline 2018 & $1,100,000$ & $6.1 \%$ & 186,000 & $10.2 \%$ & $1,286,000$ & $6.5 \%$ \\
\hline 2019 & $1,094,000$ & $6.2 \%$ & 192,000 & $10.4 \%$ & $1,286,000$ & $6.6 \%$ \\
\hline 2020 & $1,089,000$ & $6.3 \%$ & 197,000 & $10.7 \%$ & $1,286,000$ & $6.7 \%$ \\
\hline 2021 & $1,084,000$ & $6.4 \%$ & 202,000 & $11.0 \%$ & $1,286,000$ & $6.9 \%$ \\
\hline 2022 & $1,078,000$ & $6.6 \%$ & 208,000 & $11.2 \%$ & $1,286,000$ & $7.0 \%$ \\
\hline 2023 & $1,072,000$ & $6.7 \%$ & 213,000 & $11.5 \%$ & $1,285,000$ & $7.2 \%$ \\
\hline 2024 & $1,065,000$ & $6.8 \%$ & 219,000 & $11.8 \%$ & $1,284,000$ & $7.3 \%$ \\
\hline
\end{tabular}

SOURCE: RAND analysis of DoD, Census, VA, and MEPS data.

Table C-34

Projected Prevalence of Diagnosed Asthma Among Veterans, by Age, 2014-2024

\begin{tabular}{llllllll}
\hline & \multicolumn{2}{c}{$<35$} & \multicolumn{2}{c}{$35-64$} & \multicolumn{2}{c}{$65+$} \\
\cline { 2 - 5 } \cline { 7 - 8 } \cline { 7 - 8 } Year & Count & Prevalence & Count & Prevalence & Count & Prevalence \\
\hline 2014 & 70,000 & $4.4 \%$ & 557,000 & $5.9 \%$ & 647,000 & $6.2 \%$ \\
\hline 2015 & 70,000 & $4.5 \%$ & 548,000 & $6.0 \%$ & 663,000 & $6.3 \%$ \\
\hline 2016 & 69,000 & $4.5 \%$ & 544,000 & $6.2 \%$ & 672,000 & $6.4 \%$ \\
\hline 2017 & 68,000 & $4.6 \%$ & 543,000 & $6.3 \%$ & 675,000 & $6.6 \%$ \\
\hline 2018 & 66,000 & $4.7 \%$ & 543,000 & $6.5 \%$ & 677,000 & $6.7 \%$ \\
\hline 2019 & 65,000 & $4.8 \%$ & 544,000 & $6.6 \%$ & 676,000 & $6.8 \%$ \\
\hline 2020 & 65,000 & $4.9 \%$ & 545,000 & $6.8 \%$ & 676,000 & $6.9 \%$ \\
\hline 2021 & 64,000 & $5.1 \%$ & 545,000 & $6.9 \%$ & 678,000 & $7.1 \%$ \\
\hline 2022 & 63,000 & $5.2 \%$ & 544,000 & $7.1 \%$ & 679,000 & $7.2 \%$ \\
\hline 2023 & 63,000 & $5.3 \%$ & 542,000 & $7.2 \%$ & 680,000 & $7.4 \%$ \\
\hline 2024 & 62,000 & $5.4 \%$ & 542,000 & $7.4 \%$ & 680,000 & $7.6 \%$ \\
\hline
\end{tabular}

SOURCE: RAND analysis of DoD, Census, VA, and MEPS data. 
Table C-35

Projected Prevalence of Diagnosed Asthma Among Veterans, by Race/Ethnicity, 2014-2024

\begin{tabular}{|c|c|c|c|c|c|c|c|c|}
\hline \multirow[b]{2}{*}{ Year } & \multicolumn{2}{|c|}{ White, Non-Hispanic } & \multicolumn{2}{|c|}{ Black, Non-Hispanic } & \multicolumn{2}{|c|}{ Hispanic } & \multicolumn{2}{|c|}{ Asian/Other } \\
\hline & Count & Prevalence & Count & Prevalence & Count & Prevalence & Count & Prevalence \\
\hline 2014 & $1,033,000$ & $6.0 \%$ & 149,000 & $6.0 \%$ & 55,000 & $4.4 \%$ & 37,000 & $5.5 \%$ \\
\hline 2015 & $1,033,000$ & $6.2 \%$ & 152,000 & $6.2 \%$ & 57,000 & $4.5 \%$ & 39,000 & $5.7 \%$ \\
\hline 2016 & $1,031,000$ & $6.3 \%$ & 154,000 & $6.4 \%$ & 58,000 & $4.6 \%$ & 40,000 & $5.9 \%$ \\
\hline 2017 & $1,027,000$ & $6.4 \%$ & 157,000 & $6.5 \%$ & 60,000 & $4.7 \%$ & 42,000 & $6.0 \%$ \\
\hline 2018 & $1,022,000$ & $6.6 \%$ & 159,000 & $6.7 \%$ & 62,000 & $4.8 \%$ & 43,000 & $6.2 \%$ \\
\hline 2019 & $1,016,000$ & $6.7 \%$ & 162,000 & $6.9 \%$ & 63,000 & $4.9 \%$ & 45,000 & $6.3 \%$ \\
\hline 2020 & $1,010,000$ & $6.8 \%$ & 164,000 & $7.0 \%$ & 65,000 & $5.1 \%$ & 46,000 & $6.5 \%$ \\
\hline 2021 & $1,006,000$ & $7.0 \%$ & 167,000 & $7.2 \%$ & 66,000 & $5.2 \%$ & 48,000 & $6.7 \%$ \\
\hline 2022 & $1,000,000$ & $7.1 \%$ & 169,000 & $7.4 \%$ & 68,000 & $5.3 \%$ & 50,000 & $6.8 \%$ \\
\hline 2023 & 993,000 & $7.3 \%$ & 171,000 & $7.6 \%$ & 69,000 & $5.4 \%$ & 51,000 & $7.0 \%$ \\
\hline 2024 & 987,000 & $7.4 \%$ & 173,000 & $7.8 \%$ & 71,000 & $5.5 \%$ & 53,000 & $7.2 \%$ \\
\hline
\end{tabular}

SOURCE: RAND analysis of DoD, Census, VA, and MEPS data.

\section{C.4.1.2. Congestive Heart Failure Among Veterans}

See also sensitivity analysis in Section C.4.5.

Table C-36

Projected Prevalence of Diagnosed CHF Among Veterans, by Sex, 2014-2024

\begin{tabular}{|c|c|c|c|c|c|c|}
\hline \multirow[b]{2}{*}{ Year } & \multicolumn{2}{|c|}{ Male } & \multicolumn{2}{|c|}{ Female } & \multicolumn{2}{|c|}{ Total } \\
\hline & Count & Prevalence & Count & Prevalence & Count & Prevalence \\
\hline 2014 & 233,000 & $1.2 \%$ & 9,000 & $0.5 \%$ & 242,000 & $1.1 \%$ \\
\hline 2015 & 207,000 & $1.1 \%$ & 8,000 & $0.5 \%$ & 215,000 & $1.0 \%$ \\
\hline 2016 & 183,000 & $1.0 \%$ & 8,000 & $0.4 \%$ & 191,000 & $0.9 \%$ \\
\hline 2017 & 161,000 & $0.9 \%$ & 7,000 & $0.4 \%$ & 168,000 & $0.8 \%$ \\
\hline 2018 & 143,000 & $0.8 \%$ & 6,000 & $0.3 \%$ & 149,000 & $0.7 \%$ \\
\hline 2019 & 126,000 & $0.7 \%$ & 6,000 & $0.3 \%$ & 132,000 & $0.7 \%$ \\
\hline 2020 & 111,000 & $0.6 \%$ & 5,000 & $0.3 \%$ & 116,000 & $0.6 \%$ \\
\hline 2021 & 97,000 & $0.6 \%$ & 5,000 & $0.3 \%$ & 102,000 & $0.5 \%$ \\
\hline 2022 & 85,000 & $0.5 \%$ & 4,000 & $0.2 \%$ & 89,000 & $0.5 \%$ \\
\hline 2023 & 74,000 & $0.5 \%$ & 4,000 & $0.2 \%$ & 78,000 & $0.4 \%$ \\
\hline 2024 & 65,000 & $0.4 \%$ & 4,000 & $0.2 \%$ & 69,000 & $0.4 \%$ \\
\hline
\end{tabular}

SOURCE: RAND analysis of DoD, Census, VA, and MEPS data. 
Table C-37

Projected Prevalence of Diagnosed CHF Among Veterans, by Age, 2014-2024

\begin{tabular}{ccccccccc}
\hline & \multicolumn{2}{c}{$<$} & & \multicolumn{2}{c}{$35-64$} & & \multicolumn{2}{c}{$65+$} \\
\cline { 2 - 5 } \cline { 7 - 8 } \cline { 7 - 8 } Year & Count & Prevalence & & Count & Prevalence & & Count & Prevalence \\
\hline 2014 & $<1,000$ & $<0.1 \%$ & & 39,000 & $0.4 \%$ & & 203,000 & $1.9 \%$ \\
\hline 2015 & $<1,000$ & $<0.1 \%$ & & 33,000 & $0.4 \%$ & & 182,000 & $1.7 \%$ \\
\hline 2016 & $<1,000$ & $<0.1 \%$ & & 28,000 & $0.3 \%$ & & 162,000 & $1.6 \%$ \\
\hline 2017 & $<1,000$ & $<0.1 \%$ & & 24,000 & $0.3 \%$ & & 144,000 & $1.4 \%$ \\
\hline 2018 & $<1,000$ & $<0.1 \%$ & & 21,000 & $0.3 \%$ & 128,000 & $1.3 \%$ \\
\hline 2019 & $<1,000$ & $<0.1 \%$ & & 18,000 & $0.2 \%$ & & 113,000 & $1.1 \%$ \\
\hline 2021 & $<1,000$ & $<0.1 \%$ & & 16,000 & $0.2 \%$ & & 99,000 & $1.0 \%$ \\
\hline 2022 & 000 & $0.0 \%$ & & 14,000 & $0.2 \%$ & & 87,000 & $0.9 \%$ \\
\hline 2023 & 000 & $0.0 \%$ & & 12,000 & $0.2 \%$ & & 77,000 & $0.8 \%$ \\
\hline 2024 & 000 & $0.0 \%$ & & 11,000 & $0.1 \%$ & & 67,000 & $0.7 \%$ \\
\hline
\end{tabular}

SOURCE: RAND analysis of DoD, Census, VA, and MEPS data.

Table C-38

Projected Prevalence of Diagnosed CHF Among Veterans, by Race/Ethnicity, 2014-2024

\begin{tabular}{|c|c|c|c|c|c|c|c|c|}
\hline \multirow[b]{2}{*}{ Year } & \multicolumn{2}{|c|}{ White, Non-Hispanic } & \multicolumn{2}{|c|}{ Black, Non-Hispanic } & \multicolumn{2}{|c|}{ Hispanic } & \multicolumn{2}{|c|}{ Asian/Other } \\
\hline & Count & Prevalence & Count & Prevalence & Count & Prevalence & Count & Prevalence \\
\hline 2014 & 207,000 & $1.2 \%$ & 26,000 & $1.0 \%$ & 6,000 & $0.5 \%$ & 3,000 & $0.5 \%$ \\
\hline 2015 & 183,000 & $1.1 \%$ & 23,000 & $0.9 \%$ & 5,000 & $0.4 \%$ & 3,000 & $0.4 \%$ \\
\hline 2016 & 162,000 & $1.0 \%$ & 21,000 & $0.9 \%$ & 5,000 & $0.4 \%$ & 3,000 & $0.4 \%$ \\
\hline 2017 & 143,000 & $0.9 \%$ & 19,000 & $0.8 \%$ & 4,000 & $0.3 \%$ & 3,000 & $0.4 \%$ \\
\hline 2018 & 126,000 & $0.8 \%$ & 17,000 & $0.7 \%$ & 4,000 & $0.3 \%$ & 2,000 & $0.3 \%$ \\
\hline 2019 & 110,000 & $0.7 \%$ & 15,000 & $0.6 \%$ & 4,000 & $0.3 \%$ & 2,000 & $0.3 \%$ \\
\hline 2020 & 97,000 & $0.7 \%$ & 14,000 & $0.6 \%$ & 3,000 & $0.3 \%$ & 2,000 & $0.3 \%$ \\
\hline 2021 & 84,000 & $0.6 \%$ & 12,000 & $0.5 \%$ & 3,000 & $0.2 \%$ & 2,000 & $0.2 \%$ \\
\hline 2022 & 74,000 & $0.5 \%$ & 11,000 & $0.5 \%$ & 3,000 & $0.2 \%$ & 2,000 & $0.2 \%$ \\
\hline 2023 & 64,000 & $0.5 \%$ & 10,000 & $0.4 \%$ & 2,000 & $0.2 \%$ & 1,000 & $0.2 \%$ \\
\hline 2024 & 56,000 & $0.4 \%$ & 9,000 & $0.4 \%$ & 2,000 & $0.2 \%$ & 1,000 & $0.2 \%$ \\
\hline
\end{tabular}

SOURCE: RAND analysis of DoD, Census, VA, and MEPS data. 


\section{C.4.1.3. Chronic Obstructive Pulmonary Disease Among Veterans}

Table C-39

Projected Prevalence of Diagnosed COPD Among Veterans, by Sex, 2014-2024

\begin{tabular}{cccccccc}
\hline & \multicolumn{2}{c}{ Male } & & \multicolumn{2}{c}{ Female } & & \multicolumn{2}{c}{ Total } \\
\cline { 2 - 5 } \cline { 7 - 8 } \cline { 7 - 8 } Year & Count & Prevalence & Count & Prevalence & Count & Prevalence \\
\hline 2014 & $1,391,000$ & $7.0 \%$ & 115,000 & $6.3 \%$ & $1,506,000$ & $7.0 \%$ \\
\hline 2015 & $1,345,000$ & $6.9 \%$ & 115,000 & $6.3 \%$ & $1,460,000$ & $6.9 \%$ \\
\hline 2016 & $1,297,000$ & $6.8 \%$ & 115,000 & $6.3 \%$ & $1,412,000$ & $6.8 \%$ \\
\hline 2017 & $1,249,000$ & $6.7 \%$ & 115,000 & $6.3 \%$ & $1,364,000$ & $6.7 \%$ \\
\hline 2018 & $1,203,000$ & $6.6 \%$ & 116,000 & $6.3 \%$ & $1,319,000$ & $6.6 \%$ \\
\hline 2019 & $1,156,000$ & $6.5 \%$ & 116,000 & $6.3 \%$ & $1,272,000$ & $6.5 \%$ \\
\hline 2020 & $1,111,000$ & $6.4 \%$ & 116,000 & $6.3 \%$ & $1,227,000$ & $6.4 \%$ \\
\hline 2021 & $1,068,000$ & $6.3 \%$ & 117,000 & $6.3 \%$ & $1,185,000$ & $6.3 \%$ \\
\hline 2022 & $1,027,000$ & $6.2 \%$ & 117,000 & $6.3 \%$ & $1,144,000$ & $6.3 \%$ \\
\hline 2023 & 988,000 & $6.2 \%$ & 117,000 & $6.3 \%$ & $1,105,000$ & $6.2 \%$ \\
\hline 2024 & 949,000 & $6.1 \%$ & 117,000 & $6.3 \%$ & $1,066,000$ & $6.1 \%$ \\
\hline
\end{tabular}

SOURCE: RAND analysis of DoD, Census, VA, and MEPS data.

Table C-40

Projected Prevalence of Diagnosed COPD Among Veterans, by Age, 2014-2024

\begin{tabular}{|c|c|c|c|c|c|c|}
\hline \multirow[b]{2}{*}{ Year } & \multicolumn{2}{|c|}{$<35$} & \multicolumn{2}{|c|}{$35-64$} & \multicolumn{2}{|c|}{$65+$} \\
\hline & Count & Prevalence & Count & Prevalence & Count & Prevalence \\
\hline 2014 & 18,000 & $1.1 \%$ & 439,000 & $4.6 \%$ & $1,049,000$ & $10.0 \%$ \\
\hline 2015 & 17,000 & $1.1 \%$ & 410,000 & $4.5 \%$ & $1,033,000$ & $9.8 \%$ \\
\hline 2016 & 16,000 & $1.1 \%$ & 389,000 & $4.4 \%$ & $1,006,000$ & $9.6 \%$ \\
\hline 2017 & 16,000 & $1.1 \%$ & 374,000 & $4.3 \%$ & 976,000 & $9.5 \%$ \\
\hline 2018 & 15,000 & $1.1 \%$ & 358,000 & $4.3 \%$ & 946,000 & $9.3 \%$ \\
\hline 2019 & 14,000 & $1.0 \%$ & 346,000 & $4.2 \%$ & 912,000 & $9.2 \%$ \\
\hline 2020 & 13,000 & $1.0 \%$ & 334,000 & $4.1 \%$ & 880,000 & $9.0 \%$ \\
\hline 2021 & 13,000 & $1.0 \%$ & 320,000 & $4.1 \%$ & 851,000 & $8.9 \%$ \\
\hline 2022 & 12,000 & $1.0 \%$ & 307,000 & $4.0 \%$ & 825,000 & $8.8 \%$ \\
\hline 2023 & 11,000 & $1.0 \%$ & 294,000 & $3.9 \%$ & 799,000 & $8.7 \%$ \\
\hline 2024 & 11,000 & $0.9 \%$ & 283,000 & $3.9 \%$ & 772,000 & $8.6 \%$ \\
\hline
\end{tabular}

SOURCE: RAND analysis of DoD, Census, VA, and MEPS data. 
Table C-41

Projected Prevalence of Diagnosed COPD Among Veterans, by Race/Ethnicity, 2014-2024

\begin{tabular}{|c|c|c|c|c|c|c|c|c|}
\hline \multirow[b]{2}{*}{ Year } & \multicolumn{2}{|c|}{ White, Non-Hispanic } & \multicolumn{2}{|c|}{ Black, Non-Hispanic } & \multicolumn{2}{|c|}{ Hispanic } & \multicolumn{2}{|c|}{ Asian/Other } \\
\hline & Count & Prevalence & Count & Prevalence & Count & Prevalence & Count & Prevalence \\
\hline 2014 & $1,323,000$ & $7.7 \%$ & 113,000 & $4.6 \%$ & 43,000 & $3.4 \%$ & 27,000 & $4.1 \%$ \\
\hline 2015 & $1,278,000$ & $7.6 \%$ & 112,000 & $4.6 \%$ & 43,000 & $3.4 \%$ & 27,000 & $4.0 \%$ \\
\hline 2016 & $1,231,000$ & $7.5 \%$ & 110,000 & $4.5 \%$ & 43,000 & $3.4 \%$ & 27,000 & $4.0 \%$ \\
\hline 2017 & $1,186,000$ & $7.4 \%$ & 109,000 & $4.5 \%$ & 43,000 & $3.3 \%$ & 27,000 & $3.9 \%$ \\
\hline 2018 & $1,141,000$ & $7.3 \%$ & 108,000 & $4.5 \%$ & 42,000 & $3.3 \%$ & 27,000 & $3.9 \%$ \\
\hline 2019 & $1,097,000$ & $7.2 \%$ & 106,000 & $4.5 \%$ & 42,000 & $3.3 \%$ & 27,000 & $3.9 \%$ \\
\hline 2020 & $1,054,000$ & $7.1 \%$ & 105,000 & $4.5 \%$ & 42,000 & $3.3 \%$ & 27,000 & $3.8 \%$ \\
\hline 2021 & $1,012,000$ & $7.0 \%$ & 103,000 & $4.5 \%$ & 41,000 & $3.2 \%$ & 27,000 & $3.8 \%$ \\
\hline 2022 & 974,000 & $7.0 \%$ & 102,000 & $4.5 \%$ & 41,000 & $3.2 \%$ & 27,000 & $3.8 \%$ \\
\hline 2023 & 937,000 & $6.9 \%$ & 100,000 & $4.5 \%$ & 41,000 & $3.2 \%$ & 27,000 & $3.7 \%$ \\
\hline 2024 & 900,000 & $6.8 \%$ & 98,000 & $4.4 \%$ & 40,000 & $3.2 \%$ & 27,000 & $3.7 \%$ \\
\hline
\end{tabular}

SOURCE: RAND analysis of DoD, Census, VA, and MEPS data.

\section{C.4.1.4. Diabetes Among Veterans}

Table C-42

Projected Prevalence of Diagnosed Diabetes Among Veterans, by Sex, 2014-2024

\begin{tabular}{cccccccc}
\hline \multirow{2}{*}{ Year } & \multicolumn{2}{c}{ Male } & & \multicolumn{2}{c}{ Female } & & \multicolumn{2}{c}{ Total } \\
\cline { 2 - 5 } \cline { 7 - 8 } \cline { 7 - 8 } & Count & Prevalence & Count & Prevalence & Count & Prevalence \\
\hline 2014 & $4,880,000$ & $24.7 \%$ & 299,000 & $16.6 \%$ & $5,179,000$ & $24.0 \%$ \\
\hline 2015 & $4,845,000$ & $25.0 \%$ & 309,000 & $17.0 \%$ & $5,154,000$ & $24.3 \%$ \\
\hline 2016 & $4,800,000$ & $25.3 \%$ & 320,000 & $17.5 \%$ & $5,120,000$ & $24.7 \%$ \\
\hline 2017 & $4,757,000$ & $25.7 \%$ & 330,000 & $18.1 \%$ & $5,087,000$ & $25.0 \%$ \\
\hline 2018 & $4,712,000$ & $26.0 \%$ & 342,000 & $18.6 \%$ & $5,054,000$ & $25.4 \%$ \\
\hline 2020 & $4,605,000$ & $26.7 \%$ & 365,000 & $19.8 \%$ & $4,970,000$ & $26.0 \%$ \\
\hline 2021 & $4,549,000$ & $27.0 \%$ & 377,000 & $20.4 \%$ & $4,926,000$ & $26.4 \%$ \\
\hline 2022 & $4,498,000$ & $27.4 \%$ & 389,000 & $21.1 \%$ & $4,887,000$ & $26.7 \%$ \\
\hline 2024 & $4,445,000$ & $27.7 \%$ & 402,000 & $21.7 \%$ & $4,847,000$ & $27.1 \%$ \\
\hline
\end{tabular}

SOURCE: RAND analysis of DoD, Census, VA, and MEPS data. 
Table C-43

Projected Prevalence of Diagnosed Diabetes Among Veterans, by Age, 2014-2024

\begin{tabular}{cccccccc}
\hline \multirow{2}{*}{ Year } & \multicolumn{2}{c}{$<35$} & \multicolumn{2}{c}{$35-64$} & \multicolumn{2}{c}{$65+$} \\
\cline { 2 - 5 } \cline { 7 - 8 } \cline { 7 - 8 } & Count & Prevalence & Count & Prevalence & Count & Prevalence \\
\hline 2014 & 45,000 & $2.8 \%$ & $1,458,000$ & $15.4 \%$ & $3,676,000$ & $35.0 \%$ \\
\hline 2015 & 45,000 & $2.8 \%$ & $1,400,000$ & $15.4 \%$ & $3,709,000$ & $35.3 \%$ \\
\hline 2016 & 44,000 & $2.9 \%$ & $1,368,000$ & $15.5 \%$ & $3,708,000$ & $35.5 \%$ \\
\hline 2017 & 43,000 & $2.9 \%$ & $1,349,000$ & $15.7 \%$ & $3,696,000$ & $35.9 \%$ \\
\hline 2018 & 41,000 & $3.0 \%$ & $1,331,000$ & $15.8 \%$ & $3,682,000$ & $36.3 \%$ \\
\hline 2019 & 41,000 & $3.0 \%$ & $1,323,000$ & $16.1 \%$ & $3,649,000$ & $36.8 \%$ \\
\hline 2021 & 30,000 & $3.1 \%$ & $1,313,000$ & $16.3 \%$ & $3,617,000$ & $37.2 \%$ \\
\hline 2022 & 39,000 & $3.1 \%$ & $1,297,000$ & $16.5 \%$ & $3,590,000$ & $37.6 \%$ \\
\hline 2023 & 38,000 & $3.2 \%$ & $1,262,000$ & $16.8 \%$ & $3,547,000$ & $38.6 \%$ \\
\hline 2024 & 37,000 & $3.2 \%$ & $1,246,000$ & $17.0 \%$ & $3,514,000$ & $39.0 \%$ \\
\hline
\end{tabular}

SOURCE: RAND analysis of DoD, Census, VA, and MEPS data.

Table C-44

Projected Prevalence of Diagnosed Diabetes Among Veterans, by Race/Ethnicity, 2014-2024

\begin{tabular}{|c|c|c|c|c|c|c|c|c|}
\hline \multirow[b]{2}{*}{ Year } & \multicolumn{2}{|c|}{ White, Non-Hispanic } & \multicolumn{2}{|c|}{ Black, Non-Hispanic } & \multicolumn{2}{|c|}{ Hispanic } & \multicolumn{2}{|c|}{ Asian/Other } \\
\hline & Count & Prevalence & Count & Prevalence & Count & Prevalence & Count & Prevalence \\
\hline 2014 & $4,118,000$ & $24.0 \%$ & 619,000 & $25.0 \%$ & 298,000 & $23.7 \%$ & 143,000 & $21.3 \%$ \\
\hline 2015 & $4,074,000$ & $24.3 \%$ & 628,000 & $25.6 \%$ & 305,000 & $24.1 \%$ & 147,000 & $21.5 \%$ \\
\hline 2016 & $4,022,000$ & $24.6 \%$ & 636,000 & $26.2 \%$ & 311,000 & $24.5 \%$ & 150,000 & $21.7 \%$ \\
\hline 2017 & $3,973,000$ & $24.9 \%$ & 644,000 & $26.8 \%$ & 318,000 & $24.9 \%$ & 153,000 & $22.0 \%$ \\
\hline 2018 & $3,922,000$ & $25.2 \%$ & 651,000 & $27.3 \%$ & 324,000 & $25.4 \%$ & 156,000 & $22.2 \%$ \\
\hline 2019 & $3,865,000$ & $25.5 \%$ & 658,000 & $27.9 \%$ & 330,000 & $25.8 \%$ & 160,000 & $22.5 \%$ \\
\hline 2020 & $3,806,000$ & $25.8 \%$ & 665,000 & $28.5 \%$ & 336,000 & $26.3 \%$ & 163,000 & $22.7 \%$ \\
\hline 2021 & $3,747,000$ & $26.0 \%$ & 671,000 & $29.2 \%$ & 342,000 & $26.7 \%$ & 166,000 & $23.0 \%$ \\
\hline 2022 & $3,694,000$ & $26.4 \%$ & 677,000 & $29.8 \%$ & 348,000 & $27.2 \%$ & 169,000 & $23.3 \%$ \\
\hline 2023 & $3,638,000$ & $26.7 \%$ & 682,000 & $30.4 \%$ & 354,000 & $27.7 \%$ & 172,000 & $23.5 \%$ \\
\hline 2024 & $3,576,000$ & $27.0 \%$ & 686,000 & $31.0 \%$ & 359,000 & $28.1 \%$ & 175,000 & $23.8 \%$ \\
\hline
\end{tabular}

SOURCE: RAND analysis of DoD, Census, VA, and MEPS data. 


\section{C.4.1.5. Gastroesophageal Reflux Disease Among Veterans}

Table C-45

Projected Prevalence of Diagnosed GERD Among Veterans, by Sex, 2014-2024

\begin{tabular}{|c|c|c|c|c|c|c|}
\hline \multirow[b]{2}{*}{ Year } & \multicolumn{2}{|c|}{ Male } & \multicolumn{2}{|c|}{ Female } & \multicolumn{2}{|c|}{ Total } \\
\hline & Count & Prevalence & Count & Prevalence & Count & Prevalence \\
\hline 2014 & $2,495,000$ & $12.6 \%$ & 217,000 & $12.0 \%$ & $2,712,000$ & $12.6 \%$ \\
\hline 2015 & $2,465,000$ & $12.7 \%$ & 222,000 & $12.2 \%$ & $2,687,000$ & $12.7 \%$ \\
\hline 2016 & $2,435,000$ & $12.9 \%$ & 228,000 & $12.5 \%$ & $2,663,000$ & $12.8 \%$ \\
\hline 2017 & $2,406,000$ & $13.0 \%$ & 234,000 & $12.8 \%$ & $2,640,000$ & $13.0 \%$ \\
\hline 2018 & $2,376,000$ & $13.1 \%$ & 240,000 & $13.1 \%$ & $2,616,000$ & $13.1 \%$ \\
\hline 2019 & $2,342,000$ & $13.3 \%$ & 246,000 & $13.4 \%$ & $2,588,000$ & $13.3 \%$ \\
\hline 2020 & $2,309,000$ & $13.4 \%$ & 252,000 & $13.7 \%$ & $2,561,000$ & $13.4 \%$ \\
\hline 2021 & $2,275,000$ & $13.5 \%$ & 258,000 & $14.0 \%$ & $2,533,000$ & $13.6 \%$ \\
\hline 2022 & $2,241,000$ & $13.6 \%$ & 264,000 & $14.3 \%$ & $2,505,000$ & $13.7 \%$ \\
\hline 2023 & $2,204,000$ & $13.7 \%$ & 270,000 & $14.6 \%$ & $2,474,000$ & $13.8 \%$ \\
\hline 2024 & $2,165,000$ & $13.8 \%$ & 276,000 & $14.9 \%$ & $2,441,000$ & $14.0 \%$ \\
\hline
\end{tabular}

SOURCE: RAND analysis of DoD, Census, VA, and MEPS data.

Table C-46

Projected Prevalence of Diagnosed GERD Among Veterans, by Age, 2014-2024

\begin{tabular}{cccccccc}
\hline \multirow{2}{*}{ Year } & \multicolumn{2}{c}{$<35$} & \multicolumn{2}{c}{$35-64$} & \multicolumn{2}{c}{$65+$} \\
\cline { 2 - 5 } \cline { 7 - 8 } \cline { 7 - 8 } & Count & Prevalence & Count & Prevalence & Count & Prevalence \\
\hline 2014 & 43,000 & $2.6 \%$ & 979,000 & $10.3 \%$ & $1,689,000$ & $16.1 \%$ \\
\hline 2015 & 42,000 & $2.7 \%$ & 941,000 & $10.4 \%$ & $1,705,000$ & $16.2 \%$ \\
\hline 2016 & 41,000 & $2.7 \%$ & 918,000 & $10.4 \%$ & $1,705,000$ & $16.3 \%$ \\
\hline 2018 & 30,000 & $2.7 \%$ & 903,000 & $10.5 \%$ & $1,697,000$ & $16.5 \%$ \\
\hline 2019 & 39,000 & $2.8 \%$ & 888,000 & $10.6 \%$ & $1,689,000$ & $16.7 \%$ \\
\hline 2020 & 37,000 & $2.8 \%$ & 880,000 & $10.7 \%$ & $1,670,000$ & $16.8 \%$ \\
\hline 2021 & 37,000 & $2.9 \%$ & 860,000 & $10.9 \%$ & $1,637,000$ & $17.1 \%$ \\
\hline 2022 & 36,000 & $2.9 \%$ & 847,000 & $11.0 \%$ & $1,622,000$ & $17.3 \%$ \\
\hline 2023 & 35,000 & $2.9 \%$ & 834,000 & $11.1 \%$ & $1,606,000$ & $17.5 \%$ \\
\hline 2024 & 34,000 & $3.0 \%$ & 822,000 & $11.2 \%$ & $1,585,000$ & $17.6 \%$ \\
\hline
\end{tabular}

SOURCE: RAND analysis of DoD, Census, VA, and MEPS data. 
Table C-47

Projected Prevalence of Diagnosed GERD Among Veterans, by Race/Ethnicity, 2014-2024

\begin{tabular}{|c|c|c|c|c|c|c|c|c|}
\hline \multirow[b]{2}{*}{ Year } & \multicolumn{2}{|c|}{ White, Non-Hispanic } & \multicolumn{2}{|c|}{ Black, Non-Hispanic } & \multicolumn{2}{|c|}{ Hispanic } & \multicolumn{2}{|c|}{ Asian/Other } \\
\hline & Count & Prevalence & Count & Prevalence & Count & Prevalence & Count & Prevalence \\
\hline 2014 & $2,364,000$ & $13.8 \%$ & 236,000 & $9.6 \%$ & 69,000 & $5.5 \%$ & 42,000 & $6.2 \%$ \\
\hline 2015 & $2,335,000$ & $13.9 \%$ & 239,000 & $9.8 \%$ & 70,000 & $5.6 \%$ & 43,000 & $6.3 \%$ \\
\hline 2016 & $2,305,000$ & $14.1 \%$ & 242,000 & $10.0 \%$ & 72,000 & $5.7 \%$ & 44,000 & $6.4 \%$ \\
\hline 2017 & $2,276,000$ & $14.3 \%$ & 245,000 & $10.2 \%$ & 74,000 & $5.8 \%$ & 45,000 & $6.5 \%$ \\
\hline 2018 & $2,246,000$ & $14.4 \%$ & 248,000 & $10.4 \%$ & 75,000 & $5.9 \%$ & 47,000 & $6.6 \%$ \\
\hline 2019 & $2,214,000$ & $14.6 \%$ & 250,000 & $10.6 \%$ & 77,000 & $6.0 \%$ & 48,000 & $6.7 \%$ \\
\hline 2020 & $2,181,000$ & $14.8 \%$ & 252,000 & $10.8 \%$ & 78,000 & $6.1 \%$ & 49,000 & $6.8 \%$ \\
\hline 2021 & $2,149,000$ & $14.9 \%$ & 254,000 & $11.1 \%$ & 80,000 & $6.2 \%$ & 50,000 & $6.9 \%$ \\
\hline 2022 & $2,116,000$ & $15.1 \%$ & 256,000 & $11.3 \%$ & 81,000 & $6.4 \%$ & 51,000 & $7.1 \%$ \\
\hline 2023 & $2,081,000$ & $15.3 \%$ & 258,000 & $11.5 \%$ & 83,000 & $6.5 \%$ & 52,000 & $7.2 \%$ \\
\hline 2024 & $2,044,000$ & $15.4 \%$ & 259,000 & $11.7 \%$ & 84,000 & $6.6 \%$ & 54,000 & $7.3 \%$ \\
\hline
\end{tabular}

SOURCE: RAND analysis of DoD, Census, VA, and MEPS data.

\section{C.4.1.6. Hearing Loss Among Veterans}

Table C-48

Projected Prevalence of Diagnosed Hearing Loss Among Veterans, by Sex, 2014-2024

\begin{tabular}{cccccccc}
\hline & \multicolumn{2}{c}{ Male } & & \multicolumn{2}{c}{ Female } & & \multicolumn{2}{c}{ Total } \\
\cline { 2 - 5 } \cline { 7 - 8 } \cline { 7 - 8 } Year & Count & Prevalence & Count & Prevalence & Count & Prevalence \\
\hline 2014 & 910,000 & $4.6 \%$ & & 32,000 & $1.8 \%$ & 942,000 & $4.4 \%$ \\
\hline 2015 & 936,000 & $4.8 \%$ & & 33,000 & $1.8 \%$ & 969,000 & $4.6 \%$ \\
\hline 2016 & 958,000 & $5.1 \%$ & & 35,000 & $1.9 \%$ & 993,000 & $4.8 \%$ \\
\hline 2018 & 982,000 & $5.3 \%$ & & 36,000 & $2.0 \%$ & $1,018,000$ & $5.0 \%$ \\
\hline 2019 & $1,008,000$ & $5.6 \%$ & & 38,000 & $2.1 \%$ & $1,046,000$ & $5.2 \%$ \\
\hline 2020 & $1,030,000$ & $5.8 \%$ & & 41,000 & $2.2 \%$ & $1,071,000$ & $5.5 \%$ \\
\hline 2021 & $1,052,000$ & $6.1 \%$ & & 43,000 & $2.3 \%$ & $1,095,000$ & $5.7 \%$ \\
\hline 2022 & $1,069,000$ & $6.3 \%$ & & 45,000 & $2.5 \%$ & $1,114,000$ & $6.0 \%$ \\
\hline 2024 & $1,090,000$ & $6.6 \%$ & & 48,000 & $2.6 \%$ & $1,138,000$ & $6.2 \%$ \\
\hline 5023 & $1,112,000$ & $6.9 \%$ & 51,000 & $2.8 \%$ & $1,163,000$ & $6.5 \%$ \\
\hline
\end{tabular}

SOURCE: RAND analysis of DoD, Census, VA, and MEPS data. 
Table C-49

Projected Prevalence of Diagnosed Hearing Loss Among Veterans, by Age, 2014-2024

\begin{tabular}{cccccccc}
\hline & \multicolumn{2}{c}{$<35$} & \multicolumn{2}{c}{$35-64$} & \multicolumn{2}{c}{$65+$} \\
\cline { 2 - 4 } \cline { 7 - 8 } Year & Count & Prevalence & Count & Prevalence & Count & Prevalence \\
\hline 2014 & 5,000 & $0.3 \%$ & 138,000 & $1.5 \%$ & 799,000 & $7.6 \%$ \\
\hline 2015 & 5,000 & $0.3 \%$ & 134,000 & $1.5 \%$ & 830,000 & $7.9 \%$ \\
\hline 2016 & 5,000 & $0.3 \%$ & 133,000 & $1.5 \%$ & 854,000 & $8.2 \%$ \\
\hline 2017 & 5,000 & $0.3 \%$ & 134,000 & $1.6 \%$ & 879,000 & $8.6 \%$ \\
\hline 2018 & 5,000 & $0.4 \%$ & 136,000 & $1.6 \%$ & 905,000 & $8.9 \%$ \\
\hline 2020 & 5,000 & $0.4 \%$ & 142,000 & $1.8 \%$ & 948,000 & $9.7 \%$ \\
\hline 2021 & 5,000 & $0.4 \%$ & 144,000 & $1.8 \%$ & 965,000 & $10.1 \%$ \\
\hline 2022 & 5,000 & $0.4 \%$ & 147,000 & $1.9 \%$ & 986,000 & $10.5 \%$ \\
\hline 2024 & 5,000 & $0.4 \%$ & 139,000 & $1.7 \%$ & 927,000 & $9.3 \%$ \\
\hline
\end{tabular}

SOURCE: RAND analysis of DoD, Census, VA, and MEPS data.

Table C-50

Projected Prevalence of Diagnosed Hearing Loss Among Veterans, by Race/Ethnicity, 2014-2024

\begin{tabular}{|c|c|c|c|c|c|c|c|c|}
\hline \multirow[b]{2}{*}{ Year } & \multicolumn{2}{|c|}{ White, Non-Hispanic } & \multicolumn{2}{|c|}{ Black, Non-Hispanic } & \multicolumn{2}{|c|}{ Hispanic } & \multicolumn{2}{|c|}{ Asian/Other } \\
\hline & Count & Prevalence & Count & Prevalence & Count & Prevalence & Count & Prevalence \\
\hline 2014 & 873,000 & $5.1 \%$ & 35,000 & $1.4 \%$ & 19,000 & $1.5 \%$ & 14,000 & $2.1 \%$ \\
\hline 2015 & 896,000 & $5.3 \%$ & 37,000 & $1.5 \%$ & 20,000 & $1.6 \%$ & 15,000 & $2.2 \%$ \\
\hline 2016 & 916,000 & $5.6 \%$ & 39,000 & $1.6 \%$ & 22,000 & $1.7 \%$ & 16,000 & $2.3 \%$ \\
\hline 2017 & 938,000 & $5.9 \%$ & 41,000 & $1.7 \%$ & 23,000 & $1.8 \%$ & 17,000 & $2.4 \%$ \\
\hline 2018 & 961,000 & $6.2 \%$ & 43,000 & $1.8 \%$ & 24,000 & $1.9 \%$ & 18,000 & $2.6 \%$ \\
\hline 2019 & 981,000 & $6.5 \%$ & 45,000 & $1.9 \%$ & 26,000 & $2.0 \%$ & 19,000 & $2.7 \%$ \\
\hline 2020 & $1,000,000$ & $6.8 \%$ & 47,000 & $2.0 \%$ & 27,000 & $2.1 \%$ & 20,000 & $2.8 \%$ \\
\hline 2021 & $1,016,000$ & $7.1 \%$ & 49,000 & $2.1 \%$ & 29,000 & $2.2 \%$ & 21,000 & $2.9 \%$ \\
\hline 2022 & $1,034,000$ & $7.4 \%$ & 52,000 & $2.3 \%$ & 30,000 & $2.4 \%$ & 22,000 & $3.1 \%$ \\
\hline 2023 & $1,054,000$ & $7.7 \%$ & 54,000 & $2.4 \%$ & 32,000 & $2.5 \%$ & 24,000 & $3.2 \%$ \\
\hline 2024 & $1,071,000$ & $8.1 \%$ & 57,000 & $2.5 \%$ & 34,000 & $2.6 \%$ & 25,000 & $3.4 \%$ \\
\hline
\end{tabular}

SOURCE: RAND analysis of DoD, Census, VA, and MEPS data. 


\section{C.4.1.7. Hypertension Among Veterans}

Table C-51

Projected Prevalence of Diagnosed Hypertension Among Veterans, by Sex, 2014-2024

\begin{tabular}{|c|c|c|c|c|c|c|}
\hline \multirow[b]{2}{*}{ Year } & \multicolumn{2}{|c|}{ Male } & \multicolumn{2}{|c|}{ Female } & \multicolumn{2}{|c|}{ Total } \\
\hline & Count & Prevalence & Count & Prevalence & Count & Prevalence \\
\hline 2014 & $9,897,000$ & $50.1 \%$ & 620,000 & $34.3 \%$ & $10,517,000$ & $48.7 \%$ \\
\hline 2015 & $9,792,000$ & $50.6 \%$ & 637,000 & $35.0 \%$ & $10,429,000$ & $49.2 \%$ \\
\hline 2016 & $9,669,000$ & $51.0 \%$ & 654,000 & $35.9 \%$ & $10,323,000$ & $49.7 \%$ \\
\hline 2017 & $9,548,000$ & $51.6 \%$ & 672,000 & $36.8 \%$ & $10,220,000$ & $50.2 \%$ \\
\hline 2018 & $9,420,000$ & $52.1 \%$ & 691,000 & $37.7 \%$ & $10,111,000$ & $50.7 \%$ \\
\hline 2019 & $9,279,000$ & $52.5 \%$ & 709,000 & $38.6 \%$ & $9,988,000$ & $51.2 \%$ \\
\hline 2020 & $9,134,000$ & $52.9 \%$ & 728,000 & $39.5 \%$ & $9,862,000$ & $51.6 \%$ \\
\hline 2021 & $8,980,000$ & $53.3 \%$ & 747,000 & $40.5 \%$ & $9,727,000$ & $52.0 \%$ \\
\hline 2022 & $8,828,000$ & $53.7 \%$ & 765,000 & $41.4 \%$ & $9,593,000$ & $52.5 \%$ \\
\hline 2023 & $8,672,000$ & $54.1 \%$ & 784,000 & $42.3 \%$ & $9,456,000$ & $52.9 \%$ \\
\hline 2024 & $8,508,000$ & $54.4 \%$ & 801,000 & $43.2 \%$ & $9,309,000$ & $53.2 \%$ \\
\hline
\end{tabular}

SOURCE: RAND analysis of DoD, Census, VA, and MEPS data.

Table C-52

Projected Prevalence of Diagnosed Hypertension Among Veterans, by Age, 2014-2024

\begin{tabular}{lcccccc}
\hline \multirow{2}{*}{ Year } & \multicolumn{2}{c}{$<35$} & \multicolumn{2}{c}{$35-64$} & \multicolumn{2}{c}{$65+$} \\
\cline { 2 - 4 } \cline { 7 - 8 } \cline { 7 - 8 } & Count & Prevalence & Count & Prevalence & Count & Prevalence \\
\hline 2014 & 93,000 & $5.8 \%$ & $3,356,000$ & $35.4 \%$ & $7,068,000$ & $67.3 \%$ \\
\hline 2015 & 92,000 & $5.8 \%$ & $3,222,000$ & $35.4 \%$ & $7,115,000$ & $67.6 \%$ \\
\hline 2017 & 89,000 & $5.9 \%$ & $3,140,000$ & $35.6 \%$ & $7,093,000$ & $68.0 \%$ \\
\hline 2018 & 87,000 & $6.0 \%$ & $3,089,000$ & $35.9 \%$ & $7,044,000$ & $68.5 \%$ \\
\hline 2019 & 85,000 & $6.1 \%$ & $3,036,000$ & $36.2 \%$ & $6,990,000$ & $69.0 \%$ \\
\hline 2020 & 84,000 & $6.2 \%$ & $3,007,000$ & $36.5 \%$ & $6,897,000$ & $69.5 \%$ \\
\hline 2021 & 82,000 & $6.3 \%$ & $2,973,000$ & $36.9 \%$ & $6,806,000$ & $70.0 \%$ \\
\hline 2022 & 81,000 & $6.4 \%$ & $2,928,000$ & $37.2 \%$ & $6,718,000$ & $70.4 \%$ \\
\hline 2023 & 80,000 & $6.5 \%$ & $2,881,000$ & $37.5 \%$ & $6,633,000$ & $70.8 \%$ \\
\hline 2024 & 78,000 & $6.6 \%$ & $2,830,000$ & $37.7 \%$ & $6,547,000$ & $71.2 \%$ \\
\hline
\end{tabular}

SOURCE: RAND analysis of DoD, Census, VA, and MEPS data. 
Table C-53

Projected Prevalence of Diagnosed Hypertension Among Veterans, by Race/Ethnicity, 2014-2024

\begin{tabular}{|c|c|c|c|c|c|c|c|c|}
\hline \multirow[b]{2}{*}{ Year } & \multicolumn{2}{|c|}{ White, Non-Hispanic } & \multicolumn{2}{|c|}{ Black, Non-Hispanic } & \multicolumn{2}{|c|}{ Hispanic } & \multicolumn{2}{|c|}{ Asian/Other } \\
\hline & Count & Prevalence & Count & Prevalence & Count & Prevalence & Count & Prevalence \\
\hline 2014 & $8,483,000$ & $49.4 \%$ & $1,310,000$ & $53.0 \%$ & 469,000 & $37.2 \%$ & 255,000 & $38.0 \%$ \\
\hline 2015 & $8,369,000$ & $49.9 \%$ & $1,320,000$ & $53.8 \%$ & 479,000 & $37.8 \%$ & 260,000 & $38.2 \%$ \\
\hline 2016 & $8,241,000$ & $50.3 \%$ & $1,328,000$ & $54.6 \%$ & 488,000 & $38.4 \%$ & 266,000 & $38.5 \%$ \\
\hline 2017 & $8,117,000$ & $50.8 \%$ & $1,334,000$ & $55.5 \%$ & 498,000 & $39.0 \%$ & 271,000 & $38.8 \%$ \\
\hline 2018 & $7,988,000$ & $51.3 \%$ & $1,340,000$ & $56.3 \%$ & 507,000 & $39.7 \%$ & 276,000 & $39.2 \%$ \\
\hline 2019 & $7,848,000$ & $51.7 \%$ & $1,344,000$ & $57.0 \%$ & 515,000 & $40.3 \%$ & 281,000 & $39.5 \%$ \\
\hline 2020 & $7,704,000$ & $52.1 \%$ & $1,347,000$ & $57.8 \%$ & 524,000 & $41.0 \%$ & 286,000 & $39.9 \%$ \\
\hline 2021 & $7,555,000$ & $52.5 \%$ & $1,349,000$ & $58.6 \%$ & 532,000 & $41.6 \%$ & 291,000 & $40.3 \%$ \\
\hline 2022 & $7,408,000$ & $52.9 \%$ & $1,350,000$ & $59.3 \%$ & 540,000 & $42.2 \%$ & 296,000 & $40.7 \%$ \\
\hline 2023 & $7,258,000$ & $53.2 \%$ & $1,349,000$ & $60.1 \%$ & 547,000 & $42.8 \%$ & 301,000 & $41.1 \%$ \\
\hline 2024 & $7,103,000$ & $53.5 \%$ & $1,346,000$ & $60.7 \%$ & 554,000 & $43.4 \%$ & 306,000 & $41.5 \%$ \\
\hline
\end{tabular}

SOURCE: RAND analysis of DoD, Census, VA, and MEPS data.

\section{C.4.1.8. Ischemic Heart Disease Among Veterans}

See also sensitivity analysis in Section C.4.5.

Table C-54

Projected Prevalence of Diagnosed Ischemic Heart Disease Among Veterans, by Sex, 2014-2024

\begin{tabular}{|c|c|c|c|c|c|c|}
\hline \multirow[b]{2}{*}{ Year } & \multicolumn{2}{|c|}{ Male } & \multicolumn{2}{|c|}{ Female } & \multicolumn{2}{|c|}{ Total } \\
\hline & Count & Prevalence & Count & Prevalence & Count & Prevalence \\
\hline 2014 & $3,154,000$ & $16.0 \%$ & 87,000 & $4.8 \%$ & $3,241,000$ & $15.0 \%$ \\
\hline 2015 & $3,016,000$ & $15.6 \%$ & 85,000 & $4.7 \%$ & $3,101,000$ & $14.6 \%$ \\
\hline 2016 & $2,875,000$ & $15.2 \%$ & 84,000 & $4.6 \%$ & $2,959,000$ & $14.3 \%$ \\
\hline 2017 & $2,749,000$ & $14.8 \%$ & 82,000 & $4.5 \%$ & $2,831,000$ & $13.9 \%$ \\
\hline 2018 & $2,627,000$ & $14.5 \%$ & 81,000 & $4.4 \%$ & $2,708,000$ & $13.6 \%$ \\
\hline 2019 & $2,502,000$ & $14.2 \%$ & 80,000 & $4.3 \%$ & $2,582,000$ & $13.2 \%$ \\
\hline 2020 & $2,379,000$ & $13.8 \%$ & 79,000 & $4.3 \%$ & $2,458,000$ & $12.9 \%$ \\
\hline 2021 & $2,256,000$ & $13.4 \%$ & 78,000 & $4.2 \%$ & $2,334,000$ & $12.5 \%$ \\
\hline 2022 & $2,143,000$ & $13.0 \%$ & 77,000 & $4.2 \%$ & $2,220,000$ & $12.1 \%$ \\
\hline 2023 & $2,036,000$ & $12.7 \%$ & 77,000 & $4.1 \%$ & $2,113,000$ & $11.8 \%$ \\
\hline 2024 & $1,929,000$ & $12.3 \%$ & 76,000 & $4.1 \%$ & $2,005,000$ & $11.5 \%$ \\
\hline
\end{tabular}

SOURCE: RAND analysis of DoD, Census, VA, and MEPS data. 
Table C-55

Projected Prevalence of Diagnosed Ischemic Heart Disease Among Veterans, by Age, 2014-2024

\begin{tabular}{|c|c|c|c|c|c|c|}
\hline \multirow[b]{2}{*}{ Year } & \multicolumn{2}{|c|}{$<35$} & \multicolumn{2}{|c|}{$35-64$} & \multicolumn{2}{|c|}{$65+$} \\
\hline & Count & Prevalence & Count & Prevalence & Count & Prevalence \\
\hline 2014 & 5,000 & $0.3 \%$ & 555,000 & $5.9 \%$ & $2,681,000$ & $25.5 \%$ \\
\hline 2015 & 5,000 & $0.3 \%$ & 500,000 & $5.5 \%$ & $2,597,000$ & $24.7 \%$ \\
\hline 2016 & 5,000 & $0.3 \%$ & 460,000 & $5.2 \%$ & $2,494,000$ & $23.9 \%$ \\
\hline 2017 & 4,000 & $0.3 \%$ & 429,000 & $5.0 \%$ & $2,397,000$ & $23.3 \%$ \\
\hline 2018 & 4,000 & $0.3 \%$ & 401,000 & $4.8 \%$ & $2,303,000$ & $22.7 \%$ \\
\hline 2019 & 4,000 & $0.3 \%$ & 378,000 & $4.6 \%$ & $2,200,000$ & $22.2 \%$ \\
\hline 2020 & 3,000 & $0.3 \%$ & 356,000 & $4.4 \%$ & $2,098,000$ & $21.6 \%$ \\
\hline 2021 & 3,000 & $0.3 \%$ & 334,000 & $4.2 \%$ & $1,996,000$ & $20.9 \%$ \\
\hline 2022 & 3,000 & $0.2 \%$ & 314,000 & $4.1 \%$ & $1,904,000$ & $20.3 \%$ \\
\hline 2023 & 3,000 & $0.2 \%$ & 293,000 & $3.9 \%$ & $1,817,000$ & $19.8 \%$ \\
\hline 2024 & 3,000 & $0.2 \%$ & 275,000 & $3.8 \%$ & $1,727,000$ & $19.2 \%$ \\
\hline
\end{tabular}

SOURCE: RAND analysis of DoD, Census, VA, and MEPS data.

Table C-56

Projected Prevalence of Diagnosed Ischemic Heart Disease Among Veterans, by Race/Ethnicity, 2014-2024

\begin{tabular}{|c|c|c|c|c|c|c|c|c|}
\hline \multirow[b]{2}{*}{ Year } & \multicolumn{2}{|c|}{ White, Non-Hispanic } & \multicolumn{2}{|c|}{ Black, Non-Hispanic } & \multicolumn{2}{|c|}{ Hispanic } & \multicolumn{2}{|c|}{ Asian/Other } \\
\hline & Count & Prevalence & Count & Prevalence & Count & Prevalence & Count & Prevalence \\
\hline 2014 & $2,826,000$ & $16.5 \%$ & 248,000 & $10.0 \%$ & 108,000 & $8.6 \%$ & 59,000 & $8.8 \%$ \\
\hline 2015 & $2,695,000$ & $16.1 \%$ & 241,000 & $9.8 \%$ & 107,000 & $8.4 \%$ & 58,000 & $8.5 \%$ \\
\hline 2016 & $2,562,000$ & $15.6 \%$ & 235,000 & $9.7 \%$ & 105,000 & $8.3 \%$ & 57,000 & $8.3 \%$ \\
\hline 2017 & $2,443,000$ & $15.3 \%$ & 229,000 & $9.5 \%$ & 103,000 & $8.1 \%$ & 56,000 & $8.1 \%$ \\
\hline 2018 & $2,328,000$ & $15.0 \%$ & 223,000 & $9.3 \%$ & 102,000 & $8.0 \%$ & 55,000 & $7.9 \%$ \\
\hline 2019 & $2,212,000$ & $14.6 \%$ & 216,000 & $9.2 \%$ & 100,000 & $7.8 \%$ & 54,000 & $7.7 \%$ \\
\hline 2020 & $2,096,000$ & $14.2 \%$ & 210,000 & $9.0 \%$ & 98,000 & $7.7 \%$ & 53,000 & $7.4 \%$ \\
\hline 2021 & $1,982,000$ & $13.8 \%$ & 204,000 & $8.9 \%$ & 96,000 & $7.5 \%$ & 52,000 & $7.2 \%$ \\
\hline 2022 & $1,878,000$ & $13.4 \%$ & 198,000 & $8.7 \%$ & 94,000 & $7.3 \%$ & 51,000 & $7.0 \%$ \\
\hline 2023 & $1,779,000$ & $13.0 \%$ & 192,000 & $8.5 \%$ & 92,000 & $7.2 \%$ & 50,000 & $6.8 \%$ \\
\hline 2024 & $1,680,000$ & $12.7 \%$ & 186,000 & $8.4 \%$ & 90,000 & $7.0 \%$ & 49,000 & $6.7 \%$ \\
\hline
\end{tabular}

SOURCE: RAND analysis of DoD, Census, VA, and MEPS data. 


\section{C.4.1.9. Lipid Disorder Among Veterans}

Table C-57

Projected Prevalence of Diagnosed Lipid Disorder Among Veterans, by Sex, 2014-2024

\begin{tabular}{cccccccc}
\hline & \multicolumn{2}{c}{ Male } & & \multicolumn{2}{c}{ Female } & \multicolumn{2}{c}{ Total } \\
\cline { 2 - 5 } \cline { 7 - 8 } \cline { 7 - 8 } Year & Count & Prevalence & Count & Prevalence & Count & Prevalence \\
\hline 2014 & $8,182,000$ & $41.4 \%$ & 434,000 & $24.0 \%$ & $8,616,000$ & $39.9 \%$ \\
\hline 2015 & $8,002,000$ & $41.3 \%$ & 441,000 & $24.2 \%$ & $8,443,000$ & $39.9 \%$ \\
\hline 2016 & $7,813,000$ & $41.2 \%$ & 448,000 & $24.6 \%$ & $8,261,000$ & $39.8 \%$ \\
\hline 2017 & $7,629,000$ & $41.2 \%$ & 456,000 & $24.9 \%$ & $8,085,000$ & $39.7 \%$ \\
\hline 2018 & $7,443,000$ & $41.1 \%$ & 464,000 & $25.3 \%$ & $7,907,000$ & $39.7 \%$ \\
\hline 2019 & $7,249,000$ & $41.0 \%$ & 471,000 & $25.6 \%$ & $7,720,000$ & $39.6 \%$ \\
\hline 2020 & $7,056,000$ & $40.9 \%$ & 480,000 & $26.0 \%$ & $7,536,000$ & $39.5 \%$ \\
\hline 2021 & $6,864,000$ & $40.8 \%$ & 488,000 & $26.4 \%$ & $7,352,000$ & $39.3 \%$ \\
\hline 2023 & $6,673,000$ & $40.6 \%$ & 496,000 & $26.8 \%$ & $7,169,000$ & $39.2 \%$ \\
\hline 2024 & $6,481,000$ & $40.4 \%$ & 504,000 & $27.2 \%$ & $6,985,000$ & $39.0 \%$ \\
\hline
\end{tabular}

SOURCE: RAND analysis of DoD, Census, VA, and MEPS data.

Table C-58

Projected Prevalence of Diagnosed Lipid Disorder Among Veterans, by Age,2014-2024

\begin{tabular}{lccccccc}
\hline & \multicolumn{2}{c}{$<35$} & \multicolumn{2}{c}{$35-64$} & \multicolumn{2}{c}{$65+$} \\
\cline { 2 - 4 } \cline { 7 - 8 } Year & Count & Prevalence & Count & Prevalence & Count & Prevalence \\
\hline 2014 & 53,000 & $3.3 \%$ & $2,654,000$ & $28.0 \%$ & $5,909,000$ & $56.3 \%$ \\
\hline 2015 & 51,000 & $3.3 \%$ & $2,500,000$ & $27.5 \%$ & $5,891,000$ & $56.0 \%$ \\
\hline 2016 & 49,000 & $3.3 \%$ & $2,394,000$ & $27.2 \%$ & $5,817,000$ & $55.8 \%$ \\
\hline 2017 & 47,000 & $3.2 \%$ & $2,318,000$ & $26.9 \%$ & $5,720,000$ & $55.6 \%$ \\
\hline 2018 & 45,000 & $3.2 \%$ & $2,242,000$ & $26.7 \%$ & $5,619,000$ & $55.5 \%$ \\
\hline 2019 & 44,000 & $3.2 \%$ & $2,189,000$ & $26.6 \%$ & $5,488,000$ & $55.3 \%$ \\
\hline 2020 & 42,000 & $3.2 \%$ & $2,135,000$ & $26.5 \%$ & $5,359,000$ & $55.1 \%$ \\
\hline 2022 & 41,000 & $3.2 \%$ & $2,074,000$ & $26.3 \%$ & $5,237,000$ & $54.9 \%$ \\
\hline 2023 & 39,000 & $3.2 \%$ & $2,013,000$ & $26.2 \%$ & $5,118,000$ & $54.6 \%$ \\
\hline 2024 & 36,000 & $3.1 \%$ & $1,950,000$ & $26.0 \%$ & $4,998,000$ & $54.4 \%$ \\
\hline
\end{tabular}

SOURCE: RAND analysis of DoD, Census, VA, and MEPS data. 
Table C-59

Projected Prevalence of Diagnosed Lipid Disorder Among Veterans, by Race/Ethnicity, 2014-2024

\begin{tabular}{|c|c|c|c|c|c|c|c|c|}
\hline \multirow[b]{2}{*}{ Year } & \multicolumn{2}{|c|}{ White, Non-Hispanic } & \multicolumn{2}{|c|}{ Black, Non-Hispanic } & \multicolumn{2}{|c|}{ Hispanic } & \multicolumn{2}{|c|}{ Asian/Other } \\
\hline & Count & Prevalence & Count & Prevalence & Count & Prevalence & Count & Prevalence \\
\hline 2014 & $7,312,000$ & $42.6 \%$ & 735,000 & $29.7 \%$ & 359,000 & $28.5 \%$ & 209,000 & $31.1 \%$ \\
\hline 2015 & $7,134,000$ & $42.5 \%$ & 735,000 & $30.0 \%$ & 363,000 & $28.6 \%$ & 211,000 & $30.9 \%$ \\
\hline 2016 & $6,948,000$ & $42.4 \%$ & 735,000 & $30.2 \%$ & 365,000 & $28.8 \%$ & 212,000 & $30.8 \%$ \\
\hline 2017 & $6,770,000$ & $42.4 \%$ & 733,000 & $30.5 \%$ & 368,000 & $28.9 \%$ & 214,000 & $30.6 \%$ \\
\hline 2018 & $6,589,000$ & $42.3 \%$ & 731,000 & $30.7 \%$ & 371,000 & $29.0 \%$ & 215,000 & $30.5 \%$ \\
\hline 2019 & $6,403,000$ & $42.2 \%$ & 729,000 & $30.9 \%$ & 373,000 & $29.2 \%$ & 216,000 & $30.5 \%$ \\
\hline 2020 & $6,218,000$ & $42.1 \%$ & 726,000 & $31.2 \%$ & 375,000 & $29.3 \%$ & 218,000 & $30.4 \%$ \\
\hline 2021 & $6,034,000$ & $41.9 \%$ & 723,000 & $31.4 \%$ & 376,000 & $29.4 \%$ & 219,000 & $30.3 \%$ \\
\hline 2022 & $5,853,000$ & $41.8 \%$ & 719,000 & $31.6 \%$ & 377,000 & $29.5 \%$ & 220,000 & $30.2 \%$ \\
\hline 2023 & $5,672,000$ & $41.6 \%$ & 714,000 & $31.8 \%$ & 378,000 & $29.6 \%$ & 221,000 & $30.1 \%$ \\
\hline 2024 & $5,488,000$ & $41.4 \%$ & 708,000 & $32.0 \%$ & 379,000 & $29.7 \%$ & 221,000 & $30.1 \%$ \\
\hline
\end{tabular}

SOURCE: RAND analysis of DoD, Census, VA, and MEPS data.

\section{C.4.1.10. Lower Back Pain Among Veterans}

Table C-60

Projected Prevalence of Diagnosed Lower Back Pain Among Veterans, by Sex, 2014-2024

\begin{tabular}{lccccccc}
\hline \multirow{2}{*}{ Year } & \multicolumn{2}{c}{ Male } & \multicolumn{2}{c}{ Female } & & \multicolumn{2}{c}{ Total } \\
\cline { 2 - 4 } \cline { 7 - 8 } \cline { 7 - 8 } & Count & Prevalence & Count & Prevalence & Count & Prevalence \\
\hline 2014 & $2,674,000$ & $13.5 \%$ & 252,000 & $14.0 \%$ & $2,926,000$ & $13.6 \%$ \\
\hline 2015 & $2,664,000$ & $13.8 \%$ & 259,000 & $14.3 \%$ & $2,923,000$ & $13.8 \%$ \\
\hline 2016 & $2,653,000$ & $14.0 \%$ & 266,000 & $14.6 \%$ & $2,919,000$ & $14.1 \%$ \\
\hline 2018 & $2,639,000$ & $14.2 \%$ & 273,000 & $14.9 \%$ & $2,912,000$ & $14.3 \%$ \\
\hline 2019 & $2,623,000$ & $14.5 \%$ & 280,000 & $15.3 \%$ & $2,903,000$ & $14.6 \%$ \\
\hline 2020 & $2,606,000$ & $14.7 \%$ & 287,000 & $15.6 \%$ & $2,893,000$ & $14.8 \%$ \\
\hline 2021 & $2,589,000$ & $15.0 \%$ & 294,000 & $16.0 \%$ & $2,883,000$ & $15.1 \%$ \\
\hline 2022 & $2,572,000$ & $15.3 \%$ & 301,000 & $16.3 \%$ & $2,873,000$ & $15.4 \%$ \\
\hline 2023 & $2,556,000$ & $15.6 \%$ & 308,000 & $16.7 \%$ & $2,864,000$ & $15.7 \%$ \\
\hline 2024 & $2,537,000$ & $15.8 \%$ & 315,000 & $17.0 \%$ & $2,852,000$ & $15.9 \%$ \\
\hline & $2,516,000$ & $16.1 \%$ & 322,000 & $17.4 \%$ & $2,838,000$ & $16.2 \%$ \\
\hline
\end{tabular}

SOURCE: RAND analysis of DoD, Census, VA, and MEPS data. 
Table C-61

Projected Prevalence of Diagnosed Lower Back Pain Among Veterans, by Age, 2014-2024

\begin{tabular}{lcccccc}
\hline \multirow{2}{*}{ Year } & \multicolumn{2}{c}{$<35$} & \multicolumn{2}{c}{$35-64$} & \multicolumn{2}{c}{$65+$} \\
\cline { 2 - 4 } \cline { 7 - 8 } \cline { 7 - 8 } & Count & Prevalence & Count & Prevalence & Count & Prevalence \\
\hline 2014 & 143,000 & $8.9 \%$ & $1,270,000$ & $13.4 \%$ & $1,513,000$ & $14.4 \%$ \\
\hline 2015 & 143,000 & $9.1 \%$ & $1,239,000$ & $13.6 \%$ & $1,542,000$ & $14.7 \%$ \\
\hline 2016 & 141,000 & $9.3 \%$ & $1,222,000$ & $13.9 \%$ & $1,556,000$ & $14.9 \%$ \\
\hline 2017 & 139,000 & $9.5 \%$ & $1,214,000$ & $14.1 \%$ & $1,559,000$ & $15.2 \%$ \\
\hline 2019 & 136,000 & $9.7 \%$ & $1,205,000$ & $14.3 \%$ & $1,562,000$ & $15.4 \%$ \\
\hline 2020 & 135,000 & $10.0 \%$ & $1,203,000$ & $14.6 \%$ & $1,555,000$ & $15.7 \%$ \\
\hline 2021 & 133,000 & $10.2 \%$ & $1,200,000$ & $14.9 \%$ & $1,550,000$ & $15.9 \%$ \\
\hline 2022 & 132,000 & $10.4 \%$ & $1,194,000$ & $15.2 \%$ & $1,548,000$ & $16.2 \%$ \\
\hline 2023 & 130,000 & $10.6 \%$ & $1,186,000$ & $15.4 \%$ & $1,548,000$ & $16.5 \%$ \\
\hline 2024 & 128,000 & $10.8 \%$ & $1,178,000$ & $15.7 \%$ & $1,546,000$ & $16.8 \%$ \\
\hline
\end{tabular}

SOURCE: RAND analysis of DoD, Census, VA, and MEPS data.

Table C-62

Projected Prevalence of Diagnosed Lower Back Pain Among Veterans, by Race/Ethnicity, 2014-2024

\begin{tabular}{|c|c|c|c|c|c|c|c|c|}
\hline \multirow[b]{2}{*}{ Year } & \multicolumn{2}{|c|}{ White, Non-Hispanic } & \multicolumn{2}{|c|}{ Black, Non-Hispanic } & \multicolumn{2}{|c|}{ Hispanic } & \multicolumn{2}{|c|}{ Asian/Other } \\
\hline & Count & Prevalence & Count & Prevalence & Count & Prevalence & Count & Prevalence \\
\hline 2014 & $2,502,000$ & $14.6 \%$ & 237,000 & $9.6 \%$ & 118,000 & $9.4 \%$ & 69,000 & $10.2 \%$ \\
\hline 2015 & $2,490,000$ & $14.8 \%$ & 241,000 & $9.8 \%$ & 121,000 & $9.6 \%$ & 71,000 & $10.4 \%$ \\
\hline 2016 & $2,477,000$ & $15.1 \%$ & 244,000 & $10.0 \%$ & 124,000 & $9.8 \%$ & 74,000 & $10.7 \%$ \\
\hline 2017 & $2,461,000$ & $15.4 \%$ & 247,000 & $10.3 \%$ & 128,000 & $10.0 \%$ & 76,000 & $10.9 \%$ \\
\hline 2018 & $2,444,000$ & $15.7 \%$ & 250,000 & $10.5 \%$ & 131,000 & $10.2 \%$ & 79,000 & $11.2 \%$ \\
\hline 2019 & $2,425,000$ & $16.0 \%$ & 253,000 & $10.7 \%$ & 133,000 & $10.4 \%$ & 81,000 & $11.4 \%$ \\
\hline 2020 & $2,407,000$ & $16.3 \%$ & 256,000 & $11.0 \%$ & 136,000 & $10.7 \%$ & 84,000 & $11.7 \%$ \\
\hline 2021 & $2,389,000$ & $16.6 \%$ & 258,000 & $11.2 \%$ & 139,000 & $10.9 \%$ & 86,000 & $11.9 \%$ \\
\hline 2022 & $2,372,000$ & $16.9 \%$ & 261,000 & $11.5 \%$ & 142,000 & $11.1 \%$ & 89,000 & $12.2 \%$ \\
\hline 2023 & $2,352,000$ & $17.3 \%$ & 264,000 & $11.7 \%$ & 145,000 & $11.4 \%$ & 91,000 & $12.5 \%$ \\
\hline 2024 & $2,330,000$ & $17.6 \%$ & 266,000 & $12.0 \%$ & 148,000 & $11.6 \%$ & 94,000 & $12.8 \%$ \\
\hline
\end{tabular}

SOURCE: RAND analysis of DoD, Census, VA, and MEPS data. 


\section{C.4.1.11. Malignant Cancers Among Veterans}

Table C-63

Projected Prevalence of Diagnosed Malignant Cancers Among Veterans, by Sex, 2014-2024

\begin{tabular}{lccccccc}
\hline \multirow{2}{*}{ Year } & \multicolumn{2}{c}{ Male } & \multicolumn{2}{c}{ Female } & & \multicolumn{2}{c}{ Total } \\
\cline { 2 - 5 } \cline { 7 - 8 } \cline { 7 - 8 } & Count & Prevalence & Count & Prevalence & Count & Prevalence \\
\hline 2014 & $3,091,000$ & $15.6 \%$ & 159,000 & $8.8 \%$ & $3,250,000$ & $15.1 \%$ \\
\hline 2015 & $3,067,000$ & $15.8 \%$ & 163,000 & $9.0 \%$ & $3,230,000$ & $15.2 \%$ \\
\hline 2016 & $3,035,000$ & $16.0 \%$ & 167,000 & $9.2 \%$ & $3,202,000$ & $15.4 \%$ \\
\hline 2018 & $3,010,000$ & $16.3 \%$ & 172,000 & $9.4 \%$ & $3,182,000$ & $15.6 \%$ \\
\hline 2019 & $2,983,000$ & $16.5 \%$ & 178,000 & $9.7 \%$ & $3,161,000$ & $15.9 \%$ \\
\hline 2020 & $2,947,000$ & $16.7 \%$ & 183,000 & $10.0 \%$ & $3,130,000$ & $16.0 \%$ \\
\hline 2021 & $2,909,000$ & $16.9 \%$ & 189,000 & $10.3 \%$ & $3,098,000$ & $16.2 \%$ \\
\hline 2022 & $2,866,000$ & $17.0 \%$ & 195,000 & $10.6 \%$ & $3,061,000$ & $16.4 \%$ \\
\hline 2023 & $2,826,000$ & $17.2 \%$ & 202,000 & $10.9 \%$ & $3,028,000$ & $16.6 \%$ \\
\hline 2024 & $2,783,000$ & $17.4 \%$ & 208,000 & $11.3 \%$ & $2,991,000$ & $16.7 \%$ \\
\hline
\end{tabular}

SOURCE: RAND analysis of DoD, Census, VA, and MEPS data.

Table C-64

Projected Prevalence of Diagnosed Malignant Cancers Among Veterans, by Age, 2014-2024

\begin{tabular}{lccccccc}
\hline \multirow{2}{*}{ Year } & \multicolumn{2}{c}{$<$} & \multicolumn{2}{c}{$35-64$} & \multicolumn{2}{c}{$65+$} \\
\cline { 2 - 5 } \cline { 7 - 8 } \cline { 7 - 8 } & Count & Prevalence & Count & Prevalence & Count & Prevalence \\
\hline 2014 & 19,000 & $1.2 \%$ & 697,000 & $7.4 \%$ & $2,534,000$ & $24.1 \%$ \\
\hline 2015 & 19,000 & $1.2 \%$ & 661,000 & $7.3 \%$ & $2,550,000$ & $24.2 \%$ \\
\hline 2016 & 18,000 & $1.2 \%$ & 640,000 & $7.3 \%$ & $2,544,000$ & $24.4 \%$ \\
\hline 2018 & 18,000 & $1.2 \%$ & 628,000 & $7.3 \%$ & $2,536,000$ & $24.7 \%$ \\
\hline 2019 & 17,000 & $1.2 \%$ & 617,000 & $7.3 \%$ & $2,526,000$ & $24.9 \%$ \\
\hline 2020 & 17,000 & $1.2 \%$ & 612,000 & $7.4 \%$ & $2,502,000$ & $25.2 \%$ \\
\hline 2021 & 16,000 & $1.3 \%$ & 606,000 & $7.5 \%$ & $2,476,000$ & $25.5 \%$ \\
\hline 2022 & 16,000 & $1.3 \%$ & 598,000 & $7.6 \%$ & $2,448,000$ & $25.6 \%$ \\
\hline 2023 & 16,000 & $1.3 \%$ & 588,000 & $7.7 \%$ & $2,423,000$ & $25.9 \%$ \\
\hline 2024 & 15,000 & $1.3 \%$ & 578,000 & $7.7 \%$ & $2,398,000$ & $26.1 \%$ \\
\hline & 15,000 & $1.3 \%$ & 570,000 & $7.8 \%$ & $2,365,000$ & $26.3 \%$ \\
\hline
\end{tabular}

SOURCE: RAND analysis of DoD, Census, VA, and MEPS data. 
Table C-65

Projected Prevalence of Diagnosed Malignant Cancers Among Veterans, by Race/Ethnicity, 2014-2024

\begin{tabular}{|c|c|c|c|c|c|c|c|c|}
\hline \multirow[b]{2}{*}{ Year } & \multicolumn{2}{|c|}{ White, Non-Hispanic } & \multicolumn{2}{|c|}{ Black, Non-Hispanic } & \multicolumn{2}{|c|}{ Hispanic } & \multicolumn{2}{|c|}{ Asian/Other } \\
\hline & Count & Prevalence & Count & Prevalence & Count & Prevalence & Count & Prevalence \\
\hline 2014 & $2,977,000$ & $17.3 \%$ & 167,000 & $6.8 \%$ & 67,000 & $5.3 \%$ & 39,000 & $5.7 \%$ \\
\hline 2015 & $2,951,000$ & $17.6 \%$ & 170,000 & $6.9 \%$ & 69,000 & $5.5 \%$ & 40,000 & $5.8 \%$ \\
\hline 2016 & $2,918,000$ & $17.8 \%$ & 173,000 & $7.1 \%$ & 71,000 & $5.6 \%$ & 41,000 & $5.9 \%$ \\
\hline 2017 & $2,891,000$ & $18.1 \%$ & 176,000 & $7.3 \%$ & 73,000 & $5.7 \%$ & 42,000 & $6.0 \%$ \\
\hline 2018 & $2,864,000$ & $18.4 \%$ & 179,000 & $7.5 \%$ & 75,000 & $5.9 \%$ & 43,000 & $6.1 \%$ \\
\hline 2019 & $2,828,000$ & $18.6 \%$ & 182,000 & $7.7 \%$ & 77,000 & $6.0 \%$ & 44,000 & $6.1 \%$ \\
\hline 2020 & $2,790,000$ & $18.9 \%$ & 185,000 & $7.9 \%$ & 78,000 & $6.1 \%$ & 45,000 & $6.2 \%$ \\
\hline 2021 & $2,747,000$ & $19.1 \%$ & 188,000 & $8.2 \%$ & 80,000 & $6.3 \%$ & 46,000 & $6.3 \%$ \\
\hline 2022 & $2,708,000$ & $19.3 \%$ & 191,000 & $8.4 \%$ & 82,000 & $6.4 \%$ & 47,000 & $6.4 \%$ \\
\hline 2023 & $2,667,000$ & $19.6 \%$ & 193,000 & $8.6 \%$ & 84,000 & $6.5 \%$ & 48,000 & $6.5 \%$ \\
\hline 2024 & $2,621,000$ & $19.8 \%$ & 195,000 & $8.8 \%$ & 85,000 & $6.7 \%$ & 49,000 & $6.6 \%$ \\
\hline
\end{tabular}

SOURCE: RAND analysis of DoD, Census, VA, and MEPS data.

\section{C.4.2. Projections for VA Patients}

\section{C.4.2.1. Asthma Among VA Patients}

Table C-66

Projected Prevalence of Diagnosed Asthma Among VA Patients, by Sex, 2014-2024

\begin{tabular}{|c|c|c|c|c|c|c|}
\hline \multirow[b]{2}{*}{ Year } & \multicolumn{2}{|c|}{ Male } & \multicolumn{2}{|c|}{ Female } & \multicolumn{2}{|c|}{ Total } \\
\hline & Count & Prevalence & Count & Prevalence & Count & Prevalence \\
\hline 2014 & 423,000 & $7.7 \%$ & 52,000 & $12.2 \%$ & 475,000 & $8.0 \%$ \\
\hline 2015 & 437,000 & $7.9 \%$ & 56,000 & $12.5 \%$ & 493,000 & $8.2 \%$ \\
\hline 2016 & 450,000 & $8.0 \%$ & 60,000 & $12.9 \%$ & 510,000 & $8.4 \%$ \\
\hline 2017 & 461,000 & $8.2 \%$ & 64,000 & $13.2 \%$ & 525,000 & $8.6 \%$ \\
\hline 2018 & 472,000 & $8.4 \%$ & 68,000 & $13.6 \%$ & 540,000 & $8.8 \%$ \\
\hline 2019 & 482,000 & $8.6 \%$ & 73,000 & $14.0 \%$ & 555,000 & $9.0 \%$ \\
\hline 2020 & 490,000 & $8.7 \%$ & 77,000 & $14.4 \%$ & 567,000 & $9.2 \%$ \\
\hline 2021 & 498,000 & $8.9 \%$ & 82,000 & $14.8 \%$ & 580,000 & $9.4 \%$ \\
\hline 2022 & 505,000 & $9.1 \%$ & 86,000 & $15.2 \%$ & 591,000 & $9.7 \%$ \\
\hline 2023 & 513,000 & $9.3 \%$ & 91,000 & $15.6 \%$ & 604,000 & $9.9 \%$ \\
\hline 2024 & 520,000 & $9.5 \%$ & 95,000 & $16.0 \%$ & 615,000 & $10.1 \%$ \\
\hline
\end{tabular}

SOURCE: RAND analysis of DoD, Census, VA, and MEPS data. 
Table C-67

Projected Prevalence of Diagnosed Asthma Among VA Patients, by Age, 2014-2024

\begin{tabular}{lccccccc}
\hline \multirow{2}{*}{ Year } & \multicolumn{2}{c}{$<35$} & \multicolumn{2}{c}{$35-64$} & \multicolumn{2}{c}{$65+$} \\
\cline { 2 - 5 } \cline { 7 - 8 } \cline { 7 - 8 } & Count & Prevalence & Count & Prevalence & Count & Prevalence \\
\hline 2014 & 32,000 & $6.0 \%$ & 186,000 & $8.1 \%$ & 257,000 & $8.3 \%$ \\
\hline 2015 & 33,000 & $6.2 \%$ & 193,000 & $8.3 \%$ & 266,000 & $8.5 \%$ \\
\hline 2016 & 33,000 & $6.3 \%$ & 200,000 & $8.5 \%$ & 276,000 & $8.7 \%$ \\
\hline 2018 & 33,000 & $6.5 \%$ & 208,000 & $8.7 \%$ & 285,000 & $8.9 \%$ \\
\hline 2019 & 32,000 & $6.6 \%$ & 215,000 & $8.9 \%$ & 294,000 & $9.1 \%$ \\
\hline 2020 & 31,000 & $6.8 \%$ & 222,000 & $9.1 \%$ & 302,000 & $9.3 \%$ \\
\hline 2021 & 31,000 & $7.0 \%$ & 229,000 & $9.3 \%$ & 308,000 & $9.5 \%$ \\
\hline 2023 & 29,000 & $7.1 \%$ & 236,000 & $9.5 \%$ & 314,000 & $9.7 \%$ \\
\hline 2024 & 28,000 & $7.3 \%$ & 243,000 & $9.8 \%$ & 321,000 & $9.9 \%$ \\
\hline & 26,000 & $7.5 \%$ & 250,000 & $10.0 \%$ & 327,000 & $10.1 \%$ \\
\hline
\end{tabular}

SOURCE: RAND analysis of DoD, Census, VA, and MEPS data.

Table C-68

Projected Prevalence of Diagnosed Asthma Among VA Patients, by Race/Ethnicity, 2014-2024

\begin{tabular}{|c|c|c|c|c|c|c|c|c|}
\hline \multirow[b]{2}{*}{ Year } & \multicolumn{2}{|c|}{ White, Non-Hispanic } & \multicolumn{2}{|c|}{ Black, Non-Hispanic } & \multicolumn{2}{|c|}{ Hispanic } & \multicolumn{2}{|c|}{ Asian/Other } \\
\hline & Count & Prevalence & Count & Prevalence & Count & Prevalence & Count & Prevalence \\
\hline 2014 & 387,000 & $8.2 \%$ & 53,000 & $8.2 \%$ & 20,000 & $6.0 \%$ & 15,000 & $7.6 \%$ \\
\hline 2015 & 399,000 & $8.4 \%$ & 56,000 & $8.4 \%$ & 22,000 & $6.1 \%$ & 16,000 & $7.8 \%$ \\
\hline 2016 & 410,000 & $8.6 \%$ & 60,000 & $8.6 \%$ & 23,000 & $6.3 \%$ & 17,000 & $8.0 \%$ \\
\hline 2017 & 420,000 & $8.8 \%$ & 63,000 & $8.8 \%$ & 24,000 & $6.5 \%$ & 18,000 & $8.3 \%$ \\
\hline 2018 & 430,000 & $9.0 \%$ & 66,000 & $9.1 \%$ & 26,000 & $6.6 \%$ & 19,000 & $8.5 \%$ \\
\hline 2019 & 438,000 & $9.2 \%$ & 69,000 & $9.4 \%$ & 27,000 & $6.8 \%$ & 21,000 & $8.8 \%$ \\
\hline 2020 & 445,000 & $9.4 \%$ & 72,000 & $9.6 \%$ & 29,000 & $7.0 \%$ & 22,000 & $9.0 \%$ \\
\hline 2021 & 452,000 & $9.6 \%$ & 75,000 & $9.9 \%$ & 30,000 & $7.2 \%$ & 23,000 & $9.2 \%$ \\
\hline 2022 & 458,000 & $9.8 \%$ & 78,000 & $10.2 \%$ & 31,000 & $7.4 \%$ & 24,000 & $9.5 \%$ \\
\hline 2023 & 465,000 & $10.0 \%$ & 80,000 & $10.4 \%$ & 33,000 & $7.6 \%$ & 25,000 & $9.8 \%$ \\
\hline 2024 & 471,000 & $10.3 \%$ & 83,000 & $10.7 \%$ & 34,000 & $7.8 \%$ & 27,000 & $10.0 \%$ \\
\hline
\end{tabular}

SOURCE: RAND analysis of DoD, Census, VA, and MEPS data. 


\section{C.4.2.2. Congestive Heart Failure Among VA Patients}

See also related sensitivity analysis in Section C.4.5.

Table C-69

Projected Prevalence of Diagnosed CHF Among VA Patients, by Sex, 2014-2024

\begin{tabular}{cccccccc}
\hline & \multicolumn{2}{c}{ Male } & & \multicolumn{2}{c}{ Female } & & \multicolumn{2}{c}{ Total } \\
\cline { 2 - 5 } \cline { 7 - 8 } Year & Count & Prevalence & & Count & Prevalence & Count & Prevalence \\
\hline 2014 & 102,000 & $1.9 \%$ & & 3,000 & $0.7 \%$ & 105,000 & $1.8 \%$ \\
\hline 2015 & 93,000 & $1.7 \%$ & 3,000 & $0.6 \%$ & 96,000 & $1.6 \%$ \\
\hline 2016 & 86,000 & $1.5 \%$ & 3,000 & $0.6 \%$ & 89,000 & $1.5 \%$ \\
\hline 2017 & 78,000 & $1.4 \%$ & 3,000 & $0.5 \%$ & 81,000 & $1.3 \%$ \\
\hline 2018 & 71,000 & $1.3 \%$ & 2,000 & $0.5 \%$ & 73,000 & $1.2 \%$ \\
\hline 2019 & 64,000 & $1.1 \%$ & 2,000 & $0.4 \%$ & 66,000 & $1.1 \%$ \\
\hline 2020 & 58,000 & $1.0 \%$ & 2,000 & $0.4 \%$ & 60,000 & $1.0 \%$ \\
\hline 2021 & 52,000 & $0.9 \%$ & 2,000 & $0.4 \%$ & 54,000 & $0.9 \%$ \\
\hline 2022 & 47,000 & $0.8 \%$ & 2,000 & $0.3 \%$ & 49,000 & $0.8 \%$ \\
\hline 2023 & 42,000 & $0.8 \%$ & 2,000 & $0.3 \%$ & 44,000 & $0.7 \%$ \\
\hline 2024 & 38,000 & $0.7 \%$ & 2,000 & $0.3 \%$ & 40,000 & $0.6 \%$ \\
\hline
\end{tabular}

SOURCE: RAND analysis of DoD, Census, VA, and MEPS data.

Table C-70

Projected Prevalence of Diagnosed CHF Among VA Patients, by Age, 2014-2024

\begin{tabular}{|c|c|c|c|c|c|c|}
\hline \multirow[b]{2}{*}{ Year } & \multicolumn{2}{|c|}{$<35$} & \multicolumn{2}{|c|}{$35-64$} & \multicolumn{2}{|c|}{$65+$} \\
\hline & Count & Prevalence & Count & Prevalence & Count & Prevalence \\
\hline 2014 & $<1,000$ & $<0.1 \%$ & 16,000 & $0.7 \%$ & 88,000 & $2.9 \%$ \\
\hline 2015 & $<1,000$ & $<0.1 \%$ & 14,000 & $0.6 \%$ & 82,000 & $2.6 \%$ \\
\hline 2016 & $<1,000$ & $<0.1 \%$ & 13,000 & $0.5 \%$ & 75,000 & $2.4 \%$ \\
\hline 2017 & $<1,000$ & $<0.1 \%$ & 12,000 & $0.5 \%$ & 69,000 & $2.1 \%$ \\
\hline 2018 & $<1,000$ & $<0.1 \%$ & 10,000 & $0.4 \%$ & 63,000 & $1.9 \%$ \\
\hline 2019 & $<1,000$ & $<0.1 \%$ & 9,000 & $0.4 \%$ & 57,000 & $1.8 \%$ \\
\hline 2020 & $<1,000$ & $<0.1 \%$ & 8,000 & $0.3 \%$ & 52,000 & $1.6 \%$ \\
\hline 2021 & $<1,000$ & $<0.1 \%$ & 7,000 & $0.3 \%$ & 46,000 & $1.4 \%$ \\
\hline 2022 & $<1,000$ & $<0.1 \%$ & 7,000 & $0.3 \%$ & 42,000 & $1.3 \%$ \\
\hline 2023 & $<1,000$ & $<0.1 \%$ & 6,000 & $0.2 \%$ & 38,000 & $1.2 \%$ \\
\hline 2024 & $<1,000$ & $<0.1 \%$ & 5,000 & $0.2 \%$ & 34,000 & $1.0 \%$ \\
\hline
\end{tabular}

SOURCE: RAND analysis of DoD, Census, VA, and MEPS data. 
Table C-71

Projected Prevalence of Diagnosed CHF Among VA Patients, by Race/Ethnicity, 2014-2024

\begin{tabular}{|c|c|c|c|c|c|c|c|c|}
\hline \multirow[b]{2}{*}{ Year } & \multicolumn{2}{|c|}{ White, Non-Hispanic } & \multicolumn{2}{|c|}{ Black, Non-Hispanic } & \multicolumn{2}{|c|}{ Hispanic } & \multicolumn{2}{|c|}{ Asian/Other } \\
\hline & Count & Prevalence & Count & Prevalence & Count & Prevalence & Count & Prevalence \\
\hline 2014 & 90,000 & $1.9 \%$ & 11,000 & $1.7 \%$ & 2,000 & $0.7 \%$ & 1,000 & $0.8 \%$ \\
\hline 2015 & 82,000 & $1.7 \%$ & 10,000 & $1.5 \%$ & 2,000 & $0.7 \%$ & 1,000 & $0.7 \%$ \\
\hline 2016 & 75,000 & $1.6 \%$ & 10,000 & $1.4 \%$ & 2,000 & $0.6 \%$ & 1,000 & $0.6 \%$ \\
\hline 2017 & 68,000 & $1.4 \%$ & 9,000 & $1.2 \%$ & 2,000 & $0.6 \%$ & 1,000 & $0.6 \%$ \\
\hline 2018 & 62,000 & $1.3 \%$ & 8,000 & $1.1 \%$ & 2,000 & $0.5 \%$ & 1,000 & $0.5 \%$ \\
\hline 2019 & 56,000 & $1.2 \%$ & 8,000 & $1.0 \%$ & 2,000 & $0.5 \%$ & 1,000 & $0.5 \%$ \\
\hline 2020 & 50,000 & $1.1 \%$ & 7,000 & $0.9 \%$ & 2,000 & $0.4 \%$ & 1,000 & $0.4 \%$ \\
\hline 2021 & 45,000 & $1.0 \%$ & 6,000 & $0.9 \%$ & 2,000 & $0.4 \%$ & 1,000 & $0.4 \%$ \\
\hline 2022 & 40,000 & $0.9 \%$ & 6,000 & $0.8 \%$ & 1,000 & $0.3 \%$ & 1,000 & $0.4 \%$ \\
\hline 2023 & 36,000 & $0.8 \%$ & 5,000 & $0.7 \%$ & 1,000 & $0.3 \%$ & 1,000 & $0.3 \%$ \\
\hline 2024 & 32,000 & $0.7 \%$ & 5,000 & $0.6 \%$ & 1,000 & $0.3 \%$ & 1,000 & $0.3 \%$ \\
\hline
\end{tabular}

SOURCE: RAND analysis of DoD, Census, VA, and MEPS data.

\section{C.4.2.3. Chronic Obstructive Pulmonary Disease Among VA Patients}

Table C-72

Projected Prevalence of Diagnosed COPD Among VA Patients, by Sex, 2014-2024

\begin{tabular}{|c|c|c|c|c|c|c|}
\hline \multirow[b]{2}{*}{ Year } & \multicolumn{2}{|c|}{ Male } & \multicolumn{2}{|c|}{ Female } & \multicolumn{2}{|c|}{ Total } \\
\hline & Count & Prevalence & Count & Prevalence & Count & Prevalence \\
\hline 2014 & 563,000 & $10.3 \%$ & 35,000 & $8.4 \%$ & 598,000 & $10.1 \%$ \\
\hline 2015 & 560,000 & $10.1 \%$ & 37,000 & $8.4 \%$ & 597,000 & $10.0 \%$ \\
\hline 2016 & 556,000 & $9.9 \%$ & 39,000 & $8.4 \%$ & 595,000 & $9.8 \%$ \\
\hline 2017 & 551,000 & $9.8 \%$ & 41,000 & $8.4 \%$ & 592,000 & $9.7 \%$ \\
\hline 2018 & 545,000 & $9.7 \%$ & 43,000 & $8.5 \%$ & 588,000 & $9.6 \%$ \\
\hline 2019 & 538,000 & $9.5 \%$ & 45,000 & $8.5 \%$ & 583,000 & $9.5 \%$ \\
\hline 2020 & 529,000 & $9.4 \%$ & 46,000 & $8.6 \%$ & 575,000 & $9.4 \%$ \\
\hline 2021 & 520,000 & $9.3 \%$ & 48,000 & $8.7 \%$ & 568,000 & $9.3 \%$ \\
\hline 2022 & 512,000 & $9.2 \%$ & 50,000 & $8.7 \%$ & 562,000 & $9.2 \%$ \\
\hline 2023 & 503,000 & $9.1 \%$ & 51,000 & $8.8 \%$ & 554,000 & $9.1 \%$ \\
\hline 2024 & 495,000 & $9.0 \%$ & 53,000 & $8.9 \%$ & 548,000 & $9.0 \%$ \\
\hline
\end{tabular}

SOURCE: RAND analysis of DoD, Census, VA, and MEPS data. 
Table C-73

Projected Prevalence of Diagnosed COPD Among VA Patients, by Age, 2014-2024

\begin{tabular}{lccccccc}
\hline & \multicolumn{2}{c}{$<35$} & \multicolumn{2}{c}{$35-64$} & & \multicolumn{2}{c}{$65+$} \\
\cline { 2 - 5 } \cline { 7 - 8 } Year & Count & Prevalence & Count & Prevalence & Count & Prevalence \\
\hline 2014 & 9,000 & $1.6 \%$ & 164,000 & $7.1 \%$ & & 426,000 & $13.8 \%$ \\
\hline 2015 & 9,000 & $1.6 \%$ & 162,000 & $7.0 \%$ & 426,000 & $13.6 \%$ \\
\hline 2016 & 9,000 & $1.6 \%$ & 160,000 & $6.8 \%$ & 426,000 & $13.5 \%$ \\
\hline 2017 & 8,000 & $1.6 \%$ & 158,000 & $6.6 \%$ & 426,000 & $13.3 \%$ \\
\hline 2018 & 8,000 & $1.6 \%$ & 156,000 & $6.5 \%$ & 424,000 & $13.1 \%$ \\
\hline 2019 & 7,000 & $1.6 \%$ & 154,000 & $6.3 \%$ & 422,000 & $12.9 \%$ \\
\hline 2020 & 7,000 & $1.6 \%$ & 152,000 & $6.2 \%$ & 417,000 & $12.8 \%$ \\
\hline 2021 & 6,000 & $1.6 \%$ & 150,000 & $6.1 \%$ & 412,000 & $12.7 \%$ \\
\hline 2022 & 6,000 & $1.5 \%$ & 148,000 & $5.9 \%$ & 407,000 & $12.5 \%$ \\
\hline 2023 & 5,000 & $1.5 \%$ & 146,000 & $5.8 \%$ & 403,000 & $12.4 \%$ \\
\hline 2024 & 5,000 & $1.5 \%$ & 144,000 & $5.8 \%$ & 399,000 & $12.3 \%$ \\
\hline
\end{tabular}

SOURCE: RAND analysis of DoD, Census, VA, and MEPS data.

Table C-74

Projected Prevalence of Diagnosed COPD Among VA Patients, by Race/Ethnicity, 2014-2024

\begin{tabular}{|c|c|c|c|c|c|c|c|c|}
\hline \multirow[b]{2}{*}{ Year } & \multicolumn{2}{|c|}{ White, Non-Hispanic } & \multicolumn{2}{|c|}{ Black, Non-Hispanic } & \multicolumn{2}{|c|}{ Hispanic } & \multicolumn{2}{|c|}{ Asian/Other } \\
\hline & Count & Prevalence & Count & Prevalence & Count & Prevalence & Count & Prevalence \\
\hline 2014 & 526,000 & $11.1 \%$ & 44,000 & $6.7 \%$ & 17,000 & $5.1 \%$ & 11,000 & $5.9 \%$ \\
\hline 2015 & 523,000 & $11.0 \%$ & 45,000 & $6.7 \%$ & 18,000 & $5.0 \%$ & 12,000 & $5.8 \%$ \\
\hline 2016 & 519,000 & $10.8 \%$ & 46,000 & $6.7 \%$ & 18,000 & $5.0 \%$ & 12,000 & $5.7 \%$ \\
\hline 2017 & 514,000 & $10.7 \%$ & 47,000 & $6.6 \%$ & 19,000 & $4.9 \%$ & 13,000 & $5.7 \%$ \\
\hline 2018 & 508,000 & $10.6 \%$ & 48,000 & $6.6 \%$ & 19,000 & $4.9 \%$ & 13,000 & $5.7 \%$ \\
\hline 2019 & 501,000 & $10.5 \%$ & 49,000 & $6.6 \%$ & 19,000 & $4.9 \%$ & 13,000 & $5.7 \%$ \\
\hline 2020 & 492,000 & $10.4 \%$ & 50,000 & $6.6 \%$ & 20,000 & $4.9 \%$ & 14,000 & $5.7 \%$ \\
\hline 2021 & 484,000 & $10.3 \%$ & 50,000 & $6.6 \%$ & 20,000 & $4.8 \%$ & 14,000 & $5.6 \%$ \\
\hline 2022 & 476,000 & $10.2 \%$ & 51,000 & $6.6 \%$ & 21,000 & $4.8 \%$ & 14,000 & $5.7 \%$ \\
\hline 2023 & 468,000 & $10.1 \%$ & 51,000 & $6.6 \%$ & 21,000 & $4.8 \%$ & 15,000 & $5.7 \%$ \\
\hline 2024 & 460,000 & $10.0 \%$ & 51,000 & $6.6 \%$ & 21,000 & $4.8 \%$ & 15,000 & $5.7 \%$ \\
\hline
\end{tabular}

SOURCE: RAND analysis of DoD, Census, VA, and MEPS data. 


\section{C.4.2.4. Diabetes Among VA Patients}

Table C-75

Projected Prevalence of Diagnosed Diabetes Among VA Patients, by Sex, 2014-2024

\begin{tabular}{cccccccc}
\hline & \multicolumn{2}{c}{ Male } & & \multicolumn{2}{c}{ Female } & & \multicolumn{2}{c}{ Total } \\
\cline { 2 - 5 } \cline { 7 - 8 } Year & Count & Prevalence & & Count & Prevalence & Count & Prevalence \\
\hline 2014 & $1,707,000$ & $31.2 \%$ & & 81,000 & $19.3 \%$ & $1,788,000$ & $30.3 \%$ \\
\hline 2015 & $1,741,000$ & $31.4 \%$ & 88,000 & $19.8 \%$ & $1,829,000$ & $30.5 \%$ \\
\hline 2016 & $1,776,000$ & $31.7 \%$ & 95,000 & $20.4 \%$ & $1,871,000$ & $30.9 \%$ \\
\hline 2017 & $1,807,000$ & $32.1 \%$ & 102,000 & $21.1 \%$ & $1,909,000$ & $31.3 \%$ \\
\hline 2018 & $1,834,000$ & $32.5 \%$ & 110,000 & $21.9 \%$ & $1,944,000$ & $31.7 \%$ \\
\hline 2019 & $1,857,000$ & $32.9 \%$ & 118,000 & $22.6 \%$ & $1,975,000$ & $32.1 \%$ \\
\hline 2020 & $1,872,000$ & $33.4 \%$ & 126,000 & $23.4 \%$ & $1,998,000$ & $32.5 \%$ \\
\hline 2021 & $1,887,000$ & $33.8 \%$ & 134,000 & $24.2 \%$ & $2,021,000$ & $32.9 \%$ \\
\hline 2022 & $1,902,000$ & $34.3 \%$ & 142,000 & $25.1 \%$ & $2,044,000$ & $33.4 \%$ \\
\hline 2023 & $1,916,000$ & $34.8 \%$ & 151,000 & $26.0 \%$ & $2,067,000$ & $33.9 \%$ \\
\hline 2024 & $1,930,000$ & $35.3 \%$ & 160,000 & $26.9 \%$ & $2,090,000$ & $34.4 \%$ \\
\hline
\end{tabular}

SOURCE: RAND analysis of DoD, Census, VA, and MEPS data.

Table C-76

Projected Prevalence of Diagnosed Diabetes Among VA Patients, by Age, 2014-2024

\begin{tabular}{cccccccc}
\hline & \multicolumn{2}{c}{$<35$} & \multicolumn{2}{c}{$35-64$} & \multicolumn{2}{c}{$65+$} \\
\cline { 2 - 5 } \cline { 7 - 8 } \cline { 7 - 8 } Year & Count & Prevalence & Count & Prevalence & Count & Prevalence \\
\hline 2014 & 19,000 & $3.7 \%$ & 487,000 & $21.2 \%$ & $1,281,000$ & $41.6 \%$ \\
\hline 2015 & 20,000 & $3.8 \%$ & 496,000 & $21.3 \%$ & $1,313,000$ & $42.1 \%$ \\
\hline 2016 & 20,000 & $3.9 \%$ & 503,000 & $21.3 \%$ & $1,347,000$ & $42.5 \%$ \\
\hline 2017 & 20,000 & $4.0 \%$ & 510,000 & $21.4 \%$ & $1,378,000$ & $43.0 \%$ \\
\hline 2018 & 20,000 & $4.1 \%$ & 517,000 & $21.4 \%$ & $1,407,000$ & $43.5 \%$ \\
\hline 2019 & 20,000 & $4.2 \%$ & 522,000 & $21.4 \%$ & $1,433,000$ & $44.0 \%$ \\
\hline 2020 & 19,000 & $4.3 \%$ & 528,000 & $21.5 \%$ & $1,451,000$ & $44.5 \%$ \\
\hline 2021 & 18,000 & $4.4 \%$ & 534,000 & $21.6 \%$ & $1,468,000$ & $45.1 \%$ \\
\hline 2022 & 17,000 & $4.5 \%$ & 542,000 & $21.8 \%$ & $1,485,000$ & $45.7 \%$ \\
\hline 2023 & 16,000 & $4.5 \%$ & 549,000 & $22.0 \%$ & $1,503,000$ & $46.3 \%$ \\
\hline 2024 & 15,000 & $4.5 \%$ & 555,000 & $22.2 \%$ & $1,520,000$ & $46.9 \%$ \\
\hline
\end{tabular}

SOURCE: RAND analysis of DoD, Census, VA, and MEPS data. 
Table C-77

Projected Prevalence of Diagnosed Diabetes Among VA Patients, by Race/Ethnicity, 2014-2024

\begin{tabular}{|c|c|c|c|c|c|c|c|c|}
\hline \multirow[b]{2}{*}{ Year } & \multicolumn{2}{|c|}{ White, Non-Hispanic } & \multicolumn{2}{|c|}{ Black, Non-Hispanic } & \multicolumn{2}{|c|}{ Hispanic } & \multicolumn{2}{|c|}{ Asian/Other } \\
\hline & Count & Prevalence & Count & Prevalence & Count & Prevalence & Count & Prevalence \\
\hline 2014 & $1,433,000$ & $30.3 \%$ & 205,000 & $31.6 \%$ & 100,000 & $29.8 \%$ & 50,000 & $26.2 \%$ \\
\hline 2015 & $1,455,000$ & $30.6 \%$ & 215,000 & $32.0 \%$ & 106,000 & $30.0 \%$ & 53,000 & $26.2 \%$ \\
\hline 2016 & $1,478,000$ & $30.9 \%$ & 226,000 & $32.6 \%$ & 111,000 & $30.5 \%$ & 56,000 & $26.5 \%$ \\
\hline 2017 & $1,497,000$ & $31.2 \%$ & 236,000 & $33.2 \%$ & 117,000 & $31.0 \%$ & 59,000 & $26.8 \%$ \\
\hline 2018 & $1,513,000$ & $31.6 \%$ & 246,000 & $33.9 \%$ & 123,000 & $31.5 \%$ & 62,000 & $27.2 \%$ \\
\hline 2019 & $1,527,000$ & $31.9 \%$ & 255,000 & $34.6 \%$ & 128,000 & $32.1 \%$ & 65,000 & $27.6 \%$ \\
\hline 2020 & $1,532,000$ & $32.3 \%$ & 264,000 & $35.3 \%$ & 133,000 & $32.6 \%$ & 68,000 & $28.0 \%$ \\
\hline 2021 & $1,538,000$ & $32.6 \%$ & 272,000 & $36.0 \%$ & 139,000 & $33.2 \%$ & 71,000 & $28.5 \%$ \\
\hline 2022 & $1,546,000$ & $33.1 \%$ & 280,000 & $36.7 \%$ & 144,000 & $33.9 \%$ & 74,000 & $29.1 \%$ \\
\hline 2023 & $1,552,000$ & $33.5 \%$ & 289,000 & $37.5 \%$ & 149,000 & $34.6 \%$ & 77,000 & $29.6 \%$ \\
\hline 2024 & $1,559,000$ & $33.9 \%$ & 296,000 & $38.3 \%$ & 155,000 & $35.3 \%$ & 80,000 & $30.2 \%$ \\
\hline
\end{tabular}

SOURCE: RAND analysis of DoD, Census, VA, and MEPS data.

\section{C.4.2.5. Gastroesophageal Reflux Disease Among VA Patients}

Table C-78

Projected Prevalence of Diagnosed Gastroesophageal Reflux Disease Among VA Patients, by Sex, 2014-2024

\begin{tabular}{|c|c|c|c|c|c|c|}
\hline \multirow[b]{2}{*}{ Year } & \multicolumn{2}{|c|}{ Male } & \multicolumn{2}{|c|}{ Female } & \multicolumn{2}{|c|}{ Total } \\
\hline & Count & Prevalence & Count & Prevalence & Count & Prevalence \\
\hline 2014 & 947,000 & $17.3 \%$ & 64,000 & $15.3 \%$ & $1,011,000$ & $17.1 \%$ \\
\hline 2015 & 965,000 & $17.4 \%$ & 69,000 & $15.6 \%$ & $1,034,000$ & $17.3 \%$ \\
\hline 2016 & 982,000 & $17.6 \%$ & 74,000 & $16.0 \%$ & $1,056,000$ & $17.4 \%$ \\
\hline 2017 & 998,000 & $17.8 \%$ & 80,000 & $16.4 \%$ & $1,078,000$ & $17.6 \%$ \\
\hline 2018 & $1,012,000$ & $18.0 \%$ & 85,000 & $16.9 \%$ & $1,097,000$ & $17.9 \%$ \\
\hline 2019 & $1,023,000$ & $18.2 \%$ & 90,000 & $17.3 \%$ & $1,113,000$ & $18.1 \%$ \\
\hline 2020 & $1,029,000$ & $18.3 \%$ & 96,000 & $17.8 \%$ & $1,125,000$ & $18.3 \%$ \\
\hline 2021 & $1,035,000$ & $18.5 \%$ & 101,000 & $18.3 \%$ & $1,136,000$ & $18.5 \%$ \\
\hline 2022 & $1,041,000$ & $18.8 \%$ & 107,000 & $18.8 \%$ & $1,148,000$ & $18.8 \%$ \\
\hline 2023 & $1,046,000$ & $19.0 \%$ & 112,000 & $19.3 \%$ & $1,158,000$ & $19.0 \%$ \\
\hline 2024 & $1,051,000$ & $19.2 \%$ & 118,000 & $19.8 \%$ & $1,169,000$ & $19.3 \%$ \\
\hline
\end{tabular}

SOURCE: RAND analysis of DoD, Census, VA, and MEPS data. 
Table C-79

Projected Prevalence of Diagnosed GaStroesophageal Reflux Disease Among VA Patients, by Age, 2014-2024

\begin{tabular}{lccccccc}
\hline \multirow{2}{*}{ Year } & \multicolumn{2}{c}{$<35$} & \multicolumn{2}{c}{$35-64$} & \multicolumn{2}{c}{$65+$} \\
\cline { 2 - 4 } \cline { 7 - 8 } \cline { 7 - 8 } & Count & Prevalence & Count & Prevalence & Count & Prevalence \\
\hline 2014 & 19,000 & $3.7 \%$ & 341,000 & $14.8 \%$ & 651,000 & $21.2 \%$ \\
\hline 2015 & 20,000 & $3.8 \%$ & 346,000 & $14.8 \%$ & 668,000 & $21.4 \%$ \\
\hline 2016 & 20,000 & $3.9 \%$ & 351,000 & $14.9 \%$ & 685,000 & $21.6 \%$ \\
\hline 2018 & 20,000 & $4.0 \%$ & 356,000 & $14.9 \%$ & 701,000 & $21.9 \%$ \\
\hline 2019 & 20,000 & $4.0 \%$ & 361,000 & $14.9 \%$ & 716,000 & $22.1 \%$ \\
\hline 2020 & 19,000 & $4.1 \%$ & 365,000 & $15.0 \%$ & 729,000 & $22.4 \%$ \\
\hline 2021 & 18,000 & $4.2 \%$ & 370,000 & $15.1 \%$ & 736,000 & $22.6 \%$ \\
\hline 2022 & 17,000 & $4.3 \%$ & 381,000 & $15.3 \%$ & 750,000 & $23.1 \%$ \\
\hline 2024 & 15,000 & $4.4 \%$ & 386,000 & $15.5 \%$ & 757,000 & $23.3 \%$ \\
\hline
\end{tabular}

SOURCE: RAND analysis of DoD, Census, VA, and MEPS data.

Table C-80

Projected Prevalence of Diagnosed Gastroesophageal Reflux Disease Among VA Patients, by Race/Ethnicity, 2014-2024

\begin{tabular}{|c|c|c|c|c|c|c|c|c|}
\hline \multirow[b]{2}{*}{ Year } & \multicolumn{2}{|c|}{ White, Non-Hispanic } & \multicolumn{2}{|c|}{ Black, Non-Hispanic } & \multicolumn{2}{|c|}{ Hispanic } & \multicolumn{2}{|c|}{ Asian/Other } \\
\hline & Count & Prevalence & Count & Prevalence & Count & Prevalence & Count & Prevalence \\
\hline 2014 & 883,000 & $18.7 \%$ & 86,000 & $13.2 \%$ & 26,000 & $7.7 \%$ & 16,000 & $8.6 \%$ \\
\hline 2015 & 898,000 & $18.9 \%$ & 91,000 & $13.5 \%$ & 27,000 & $7.8 \%$ & 18,000 & $8.7 \%$ \\
\hline 2016 & 914,000 & $19.1 \%$ & 95,000 & $13.7 \%$ & 29,000 & $7.9 \%$ & 19,000 & $8.8 \%$ \\
\hline 2017 & 928,000 & $19.3 \%$ & 100,000 & $14.0 \%$ & 31,000 & $8.1 \%$ & 20,000 & $9.0 \%$ \\
\hline 2018 & 939,000 & $19.6 \%$ & 104,000 & $14.3 \%$ & 32,000 & $8.3 \%$ & 21,000 & $9.2 \%$ \\
\hline 2019 & 949,000 & $19.8 \%$ & 108,000 & $14.7 \%$ & 34,000 & $8.5 \%$ & 22,000 & $9.4 \%$ \\
\hline 2020 & 953,000 & $20.1 \%$ & 112,000 & $15.0 \%$ & 36,000 & $8.7 \%$ & 23,000 & $9.6 \%$ \\
\hline 2021 & 958,000 & $20.3 \%$ & 116,000 & $15.3 \%$ & 37,000 & $8.9 \%$ & 25,000 & $9.9 \%$ \\
\hline 2022 & 963,000 & $20.6 \%$ & 119,000 & $15.7 \%$ & 39,000 & $9.1 \%$ & 26,000 & $10.1 \%$ \\
\hline 2023 & 968,000 & $20.9 \%$ & 123,000 & $16.0 \%$ & 40,000 & $9.3 \%$ & 27,000 & $10.3 \%$ \\
\hline 2024 & 972,000 & $21.2 \%$ & 127,000 & $16.3 \%$ & 42,000 & $9.6 \%$ & 28,000 & $10.6 \%$ \\
\hline
\end{tabular}

SOURCE: RAND analysis of DoD, Census, VA, and MEPS data. 


\section{C.4.2.6. Hearing Loss Among VA Patients}

Table C-81

Projected Prevalence of Diagnosed Hearing Loss Among VA Patients, by Sex, 2014-2024

\begin{tabular}{cccccccc}
\hline \multirow{2}{*}{ Year } & \multicolumn{2}{c}{ Male } & & \multicolumn{2}{c}{ Female } & & \multicolumn{2}{c}{ Total } \\
\cline { 2 - 5 } \cline { 7 - 8 } \cline { 7 - 8 } & Count & Prevalence & Count & Prevalence & Count & Prevalence \\
\hline 2014 & 379,000 & $6.9 \%$ & & 10,000 & $2.3 \%$ & 389,000 & $6.6 \%$ \\
\hline 2015 & 402,000 & $7.2 \%$ & 11,000 & $2.4 \%$ & 413,000 & $6.9 \%$ \\
\hline 2016 & 425,000 & $7.6 \%$ & 12,000 & $2.5 \%$ & 437,000 & $7.2 \%$ \\
\hline 2018 & 449,000 & $8.0 \%$ & 13,000 & $2.7 \%$ & 462,000 & $7.6 \%$ \\
\hline 2019 & 472,000 & $8.4 \%$ & 14,000 & $2.8 \%$ & 486,000 & $7.9 \%$ \\
\hline 2020 & 594,000 & $8.8 \%$ & 16,000 & $3.0 \%$ & 510,000 & $8.3 \%$ \\
\hline 2022 & 516,000 & $9.2 \%$ & 17,000 & $3.2 \%$ & 533,000 & $8.7 \%$ \\
\hline 2023 & 539,000 & $9.6 \%$ & 19,000 & $3.4 \%$ & 558,000 & $9.1 \%$ \\
\hline 2024 & 610,000 & $11.1 \%$ & 25,000 & $4.2 \%$ & 635,000 & $10.5 \%$ \\
\hline
\end{tabular}

SOURCE: RAND analysis of DoD, Census, VA, and MEPS data.

Table C-82

Projected Prevalence of Diagnosed Hearing Loss Among VA Patients, by Age, 2014-2024

\begin{tabular}{|c|c|c|c|c|c|c|}
\hline \multirow[b]{2}{*}{ Year } & \multicolumn{2}{|c|}{$<35$} & \multicolumn{2}{|c|}{$35-64$} & \multicolumn{2}{|c|}{$65+$} \\
\hline & Count & Prevalence & Count & Prevalence & Count & Prevalence \\
\hline 2014 & 2,000 & $0.4 \%$ & 57,000 & $2.5 \%$ & 329,000 & $10.7 \%$ \\
\hline 2015 & 3,000 & $0.5 \%$ & 59,000 & $2.5 \%$ & 351,000 & $11.2 \%$ \\
\hline 2016 & 3,000 & $0.5 \%$ & 61,000 & $2.6 \%$ & 373,000 & $11.8 \%$ \\
\hline 2017 & 3,000 & $0.5 \%$ & 64,000 & $2.7 \%$ & 395,000 & $12.3 \%$ \\
\hline 2018 & 3,000 & $0.6 \%$ & 66,000 & $2.7 \%$ & 417,000 & $12.9 \%$ \\
\hline 2019 & 3,000 & $0.6 \%$ & 69,000 & $2.8 \%$ & 439,000 & $13.5 \%$ \\
\hline 2020 & 3,000 & $0.7 \%$ & 71,000 & $2.9 \%$ & 459,000 & $14.1 \%$ \\
\hline 2021 & 3,000 & $0.7 \%$ & 75,000 & $3.0 \%$ & 480,000 & $14.8 \%$ \\
\hline 2022 & 3,000 & $0.7 \%$ & 78,000 & $3.1 \%$ & 502,000 & $15.4 \%$ \\
\hline 2023 & 3,000 & $0.8 \%$ & 82,000 & $3.3 \%$ & 524,000 & $16.2 \%$ \\
\hline 2024 & 3,000 & $0.8 \%$ & 85,000 & $3.4 \%$ & 547,000 & $16.9 \%$ \\
\hline
\end{tabular}

SOURCE: RAND analysis of DoD, Census, VA, and MEPS data. 
Table C-83

Projected Prevalence of Diagnosed Hearing Loss Among VA Patients, by Race/Ethnicity, 2014-2024

\begin{tabular}{|c|c|c|c|c|c|c|c|c|}
\hline \multirow[b]{2}{*}{ Year } & \multicolumn{2}{|c|}{ White, Non-Hispanic } & \multicolumn{2}{|c|}{ Black, Non-Hispanic } & \multicolumn{2}{|c|}{ Hispanic } & \multicolumn{2}{|c|}{ Asian/Other } \\
\hline & Count & Prevalence & Count & Prevalence & Count & Prevalence & Count & Prevalence \\
\hline 2014 & 360,000 & $7.6 \%$ & 14,000 & $2.2 \%$ & 8,000 & $2.4 \%$ & 6,000 & $3.1 \%$ \\
\hline 2015 & 381,000 & $8.0 \%$ & 16,000 & $2.4 \%$ & 9,000 & $2.5 \%$ & 7,000 & $3.3 \%$ \\
\hline 2016 & 403,000 & $8.4 \%$ & 17,000 & $2.5 \%$ & 10,000 & $2.7 \%$ & 7,000 & $3.4 \%$ \\
\hline 2017 & 424,000 & $8.8 \%$ & 19,000 & $2.6 \%$ & 11,000 & $2.8 \%$ & 8,000 & $3.6 \%$ \\
\hline 2018 & 445,000 & $9.3 \%$ & 20,000 & $2.8 \%$ & 12,000 & $3.0 \%$ & 9,000 & $3.8 \%$ \\
\hline 2019 & 466,000 & $9.7 \%$ & 22,000 & $3.0 \%$ & 13,000 & $3.2 \%$ & 9,000 & $4.0 \%$ \\
\hline 2020 & 486,000 & $10.2 \%$ & 24,000 & $3.2 \%$ & 14,000 & $3.4 \%$ & 10,000 & $4.2 \%$ \\
\hline 2021 & 506,000 & $10.7 \%$ & 25,000 & $3.4 \%$ & 15,000 & $3.6 \%$ & 11,000 & $4.4 \%$ \\
\hline 2022 & 527,000 & $11.3 \%$ & 27,000 & $3.6 \%$ & 16,000 & $3.8 \%$ & 12,000 & $4.7 \%$ \\
\hline 2023 & 548,000 & $11.8 \%$ & 29,000 & $3.8 \%$ & 17,000 & $4.0 \%$ & 13,000 & $5.0 \%$ \\
\hline 2024 & 570,000 & $12.4 \%$ & 31,000 & $4.1 \%$ & 19,000 & $4.3 \%$ & 14,000 & $5.3 \%$ \\
\hline
\end{tabular}

SOURCE: RAND analysis of DoD, Census, VA, and MEPS data.

\section{C.4.2.7. Hypertension Among VA Patients}

Table C-84

Projected Prevalence of Diagnosed Hypertension Among VA Patients, by Sex, 2014-2024

\begin{tabular}{|c|c|c|c|c|c|c|}
\hline \multirow[b]{2}{*}{ Year } & \multicolumn{2}{|c|}{ Male } & \multicolumn{2}{|c|}{ Female } & \multicolumn{2}{|c|}{ Total } \\
\hline & Count & Prevalence & Count & Prevalence & Count & Prevalence \\
\hline 2014 & $3,227,000$ & $58.9 \%$ & 162,000 & $38.4 \%$ & $3,389,000$ & $57.4 \%$ \\
\hline 2015 & $3,276,000$ & $59.1 \%$ & 174,000 & $39.1 \%$ & $3,450,000$ & $57.6 \%$ \\
\hline 2016 & $3,324,000$ & $59.4 \%$ & 186,000 & $40.1 \%$ & $3,510,000$ & $58.0 \%$ \\
\hline 2017 & $3,365,000$ & $59.9 \%$ & 199,000 & $41.1 \%$ & $3,564,000$ & $58.4 \%$ \\
\hline 2018 & $3,397,000$ & $60.3 \%$ & 213,000 & $42.2 \%$ & $3,610,000$ & $58.8 \%$ \\
\hline 2019 & $3,422,000$ & $60.7 \%$ & 226,000 & $43.3 \%$ & $3,648,000$ & $59.2 \%$ \\
\hline 2020 & $3,428,000$ & $61.1 \%$ & 239,000 & $44.4 \%$ & $3,667,000$ & $59.6 \%$ \\
\hline 2021 & $3,434,000$ & $61.5 \%$ & 252,000 & $45.6 \%$ & $3,686,000$ & $60.1 \%$ \\
\hline 2022 & $3,439,000$ & $62.0 \%$ & 266,000 & $46.8 \%$ & $3,705,000$ & $60.6 \%$ \\
\hline 2023 & $3,443,000$ & $62.5 \%$ & 280,000 & $48.1 \%$ & $3,723,000$ & $61.1 \%$ \\
\hline 2024 & $3,445,000$ & $62.9 \%$ & 293,000 & $49.3 \%$ & $3,738,000$ & $61.6 \%$ \\
\hline
\end{tabular}

SOURCE: RAND analysis of DoD, Census, VA, and MEPS data. 
Table C-85

Projected Prevalence of Diagnosed Hypertension Among VA Patients, by Age, 2014-2024

\begin{tabular}{cccccccc}
\hline & \multicolumn{2}{c}{$<35$} & \multicolumn{2}{c}{$35-64$} & \multicolumn{2}{c}{$65+$} \\
\cline { 2 - 5 } \cline { 7 - 8 } \cline { 7 - 8 } Year & Count & Prevalence & Count & Prevalence & Count & Prevalence \\
\hline 2014 & 41,000 & $7.9 \%$ & $1,058,000$ & $46.0 \%$ & $2,289,000$ & $74.4 \%$ \\
\hline 2015 & 43,000 & $8.0 \%$ & $1,072,000$ & $45.9 \%$ & $2,336,000$ & $74.8 \%$ \\
\hline 2016 & 44,000 & $8.3 \%$ & $1,083,000$ & $45.8 \%$ & $2,384,000$ & $75.2 \%$ \\
\hline 2017 & 43,000 & $8.5 \%$ & $1,094,000$ & $45.8 \%$ & $2,427,000$ & $75.7 \%$ \\
\hline 2018 & 43,000 & $8.8 \%$ & $1,103,000$ & $45.7 \%$ & $2,464,000$ & $76.1 \%$ \\
\hline 2019 & 42,000 & $9.0 \%$ & $1,110,000$ & $45.6 \%$ & $2,496,000$ & $76.5 \%$ \\
\hline 2020 & 40,000 & $9.2 \%$ & $1,120,000$ & $45.6 \%$ & $2,507,000$ & $77.0 \%$ \\
\hline 2021 & 38,000 & $9.4 \%$ & $1,130,000$ & $45.7 \%$ & $2,517,000$ & $77.4 \%$ \\
\hline 2023 & 36,000 & $9.5 \%$ & $1,141,000$ & $45.9 \%$ & $2,527,000$ & $77.8 \%$ \\
\hline 2024 & 34,000 & $9.6 \%$ & $1,152,000$ & $46.1 \%$ & $2,536,000$ & $78.2 \%$ \\
\hline
\end{tabular}

SOURCE: RAND analysis of DoD, Census, VA, and MEPS data.

Table C-86

Projected Prevalence of Diagnosed Hypertension Among VA Patients, by Race/Ethnicity, 2014-2024

\begin{tabular}{|c|c|c|c|c|c|c|c|c|}
\hline \multirow[b]{2}{*}{ Year } & \multicolumn{2}{|c|}{ White, Non-Hispanic } & \multicolumn{2}{|c|}{ Black, Non-Hispanic } & \multicolumn{2}{|c|}{ Hispanic } & \multicolumn{2}{|c|}{ Asian/Other } \\
\hline & Count & Prevalence & Count & Prevalence & Count & Prevalence & Count & Prevalence \\
\hline 2014 & $2,753,000$ & $58.3 \%$ & 398,000 & $61.2 \%$ & 152,000 & $45.2 \%$ & 86,000 & $44.7 \%$ \\
\hline 2015 & $2,785,000$ & $58.5 \%$ & 415,000 & $61.7 \%$ & 160,000 & $45.5 \%$ & 90,000 & $44.6 \%$ \\
\hline 2016 & $2,816,000$ & $58.8 \%$ & 432,000 & $62.4 \%$ & 168,000 & $46.0 \%$ & 95,000 & $44.9 \%$ \\
\hline 2017 & $2,841,000$ & $59.2 \%$ & 448,000 & $63.1 \%$ & 176,000 & $46.6 \%$ & 100,000 & $45.4 \%$ \\
\hline 2018 & $2,858,000$ & $59.6 \%$ & 463,000 & $63.9 \%$ & 184,000 & $47.4 \%$ & 105,000 & $45.9 \%$ \\
\hline 2019 & $2,870,000$ & $60.0 \%$ & 477,000 & $64.6 \%$ & 192,000 & $48.0 \%$ & 110,000 & $46.4 \%$ \\
\hline 2020 & $2,864,000$ & $60.3 \%$ & 489,000 & $65.4 \%$ & 199,000 & $48.7 \%$ & 114,000 & $47.0 \%$ \\
\hline 2021 & $2,860,000$ & $60.7 \%$ & 501,000 & $66.2 \%$ & 206,000 & $49.5 \%$ & 119,000 & $47.7 \%$ \\
\hline 2022 & $2,856,000$ & $61.1 \%$ & 512,000 & $67.0 \%$ & 214,000 & $50.3 \%$ & 124,000 & $48.4 \%$ \\
\hline 2023 & $2,851,000$ & $61.5 \%$ & 522,000 & $67.9 \%$ & 221,000 & $51.2 \%$ & 128,000 & $49.2 \%$ \\
\hline 2024 & $2,846,000$ & $62.0 \%$ & 531,000 & $68.6 \%$ & 228,000 & $52.0 \%$ & 133,000 & $50.0 \%$ \\
\hline
\end{tabular}

SOURCE: RAND analysis of DoD, Census, VA, and MEPS data. 


\section{C.4.2.8. Ischemic Heart Disease Among VA Patients}

See also related sensitivity analysis in Section C.4.5.

Table C-87

Projected Prevalence of Diagnosed Ischemic Heart Disease Among VA Patients, by Sex, 2014-2024

\begin{tabular}{|c|c|c|c|c|c|c|}
\hline \multirow[b]{2}{*}{ Year } & \multicolumn{2}{|c|}{ Male } & \multicolumn{2}{|c|}{ Female } & \multicolumn{2}{|c|}{ Total } \\
\hline & Count & Prevalence & Count & Prevalence & Count & Prevalence \\
\hline 2014 & $1,092,000$ & $19.9 \%$ & 23,000 & $5.3 \%$ & $1,115,000$ & $18.9 \%$ \\
\hline 2015 & $1,077,000$ & $19.4 \%$ & 23,000 & $5.2 \%$ & $1,100,000$ & $18.4 \%$ \\
\hline 2016 & $1,061,000$ & $19.0 \%$ & 24,000 & $5.1 \%$ & $1,085,000$ & $17.9 \%$ \\
\hline 2017 & $1,043,000$ & $18.5 \%$ & 24,000 & $5.0 \%$ & $1,067,000$ & $17.5 \%$ \\
\hline 2018 & $1,022,000$ & $18.1 \%$ & 25,000 & $5.0 \%$ & $1,047,000$ & $17.1 \%$ \\
\hline 2019 & 999,000 & $17.7 \%$ & 26,000 & $4.9 \%$ & $1,025,000$ & $16.6 \%$ \\
\hline 2020 & 972,000 & $17.3 \%$ & 26,000 & $4.9 \%$ & 998,000 & $16.2 \%$ \\
\hline 2021 & 945,000 & $16.9 \%$ & 27,000 & $4.9 \%$ & 972,000 & $15.8 \%$ \\
\hline 2022 & 919,000 & $16.6 \%$ & 28,000 & $4.9 \%$ & 947,000 & $15.5 \%$ \\
\hline 2023 & 893,000 & $16.2 \%$ & 28,000 & $4.9 \%$ & 921,000 & $15.1 \%$ \\
\hline 2024 & 868,000 & $15.9 \%$ & 29,000 & $4.9 \%$ & 897,000 & $14.8 \%$ \\
\hline
\end{tabular}

SOURCE: RAND analysis of DoD, Census, VA, and MEPS data.

Table C-88

Projected Prevalence of Diagnosed Ischemic Heart Disease Among VA Patients, by Age, 2014-2024

\begin{tabular}{cccccccc}
\hline & \multicolumn{2}{c}{$<35$} & \multicolumn{2}{c}{$35-64$} & & \multicolumn{2}{c}{$65+$} \\
\cline { 2 - 5 } \cline { 7 - 8 } Year & Count & Prevalence & Count & Prevalence & & Count & Prevalence \\
\hline 2014 & 2,000 & $0.4 \%$ & 191,000 & $8.3 \%$ & 921,000 & $29.9 \%$ \\
\hline 2015 & 2,000 & $0.4 \%$ & 184,000 & $7.9 \%$ & 914,000 & $29.3 \%$ \\
\hline 2016 & 2,000 & $0.4 \%$ & 176,000 & $7.4 \%$ & 907,000 & $28.6 \%$ \\
\hline 2017 & 2,000 & $0.4 \%$ & 168,000 & $7.0 \%$ & 897,000 & $28.0 \%$ \\
\hline 2018 & 2,000 & $0.4 \%$ & 161,000 & $6.7 \%$ & 884,000 & $27.3 \%$ \\
\hline 2019 & 2,000 & $0.4 \%$ & 154,000 & $6.3 \%$ & 870,000 & $26.7 \%$ \\
\hline 2021 & 1,000 & $0.4 \%$ & 141,000 & $5.7 \%$ & 829,000 & $25.5 \%$ \\
\hline 2022 & 1,000 & $0.3 \%$ & 136,000 & $5.5 \%$ & 809,000 & $24.9 \%$ \\
\hline 2023 & 1,000 & $0.3 \%$ & 131,000 & $5.2 \%$ & 790,000 & $24.3 \%$ \\
\hline 2024 & 1,000 & $0.3 \%$ & 126,000 & $5.0 \%$ & 770,000 & $23.8 \%$ \\
\hline
\end{tabular}

SOURCE: RAND analysis of DoD, Census, VA, and MEPS data. 
Table C-89

Projected Prevalence of Diagnosed Ischemic Heart Disease Among VA Patients, by Race/Ethnicity, 2014-2024

\begin{tabular}{|c|c|c|c|c|c|c|c|c|}
\hline \multirow[b]{2}{*}{ Year } & \multicolumn{2}{|c|}{ White, Non-Hispanic } & \multicolumn{2}{|c|}{ Black, Non-Hispanic } & \multicolumn{2}{|c|}{ Hispanic } & \multicolumn{2}{|c|}{ Asian/Other } \\
\hline & Count & Prevalence & Count & Prevalence & Count & Prevalence & Count & Prevalence \\
\hline 2014 & 972,000 & $20.6 \%$ & 84,000 & $13.0 \%$ & 38,000 & $11.2 \%$ & 21,000 & $10.9 \%$ \\
\hline 2015 & 955,000 & $20.1 \%$ & 85,000 & $12.7 \%$ & 38,000 & $10.9 \%$ & 21,000 & $10.5 \%$ \\
\hline 2016 & 938,000 & $19.6 \%$ & 86,000 & $12.4 \%$ & 39,000 & $10.7 \%$ & 22,000 & $10.2 \%$ \\
\hline 2017 & 919,000 & $19.2 \%$ & 86,000 & $12.2 \%$ & 40,000 & $10.5 \%$ & 22,000 & $10.0 \%$ \\
\hline 2018 & 898,000 & $18.7 \%$ & 87,000 & $12.0 \%$ & 40,000 & $10.3 \%$ & 22,000 & $9.7 \%$ \\
\hline 2019 & 875,000 & $18.3 \%$ & 87,000 & $11.8 \%$ & 40,000 & $10.1 \%$ & 23,000 & $9.5 \%$ \\
\hline 2020 & 848,000 & $17.9 \%$ & 87,000 & $11.6 \%$ & 41,000 & $9.9 \%$ & 23,000 & $9.3 \%$ \\
\hline 2021 & 822,000 & $17.4 \%$ & 86,000 & $11.4 \%$ & 41,000 & $9.8 \%$ & 23,000 & $9.2 \%$ \\
\hline 2022 & 797,000 & $17.0 \%$ & 86,000 & $11.2 \%$ & 41,000 & $9.6 \%$ & 23,000 & $9.0 \%$ \\
\hline 2023 & 772,000 & $16.7 \%$ & 85,000 & $11.1 \%$ & 41,000 & $9.5 \%$ & 23,000 & $8.9 \%$ \\
\hline 2024 & 748,000 & $16.3 \%$ & 85,000 & $10.9 \%$ & 41,000 & $9.4 \%$ & 23,000 & $8.7 \%$ \\
\hline
\end{tabular}

SOURCE: RAND analysis of DoD, Census, VA, and MEPS data.

\section{C.4.2.9. Lipid Disorder Among VA Patients}

Table C-90

Projected Prevalence of Diagnosed Lipid Disorder Among VA Patients, by Sex, 2014-2024

\begin{tabular}{|c|c|c|c|c|c|c|}
\hline \multirow[b]{2}{*}{ Year } & \multicolumn{2}{|c|}{ Male } & \multicolumn{2}{|c|}{ Female } & \multicolumn{2}{|c|}{ Total } \\
\hline & Count & Prevalence & Count & Prevalence & Count & Prevalence \\
\hline 2014 & $2,730,000$ & $49.8 \%$ & 116,000 & $27.4 \%$ & $2,846,000$ & $48.2 \%$ \\
\hline 2015 & $2,744,000$ & $49.5 \%$ & 123,000 & $27.6 \%$ & $2,867,000$ & $47.9 \%$ \\
\hline 2016 & $2,758,000$ & $49.3 \%$ & 130,000 & $28.1 \%$ & $2,888,000$ & $47.7 \%$ \\
\hline 2017 & $2,766,000$ & $49.2 \%$ & 138,000 & $28.5 \%$ & $2,904,000$ & $47.6 \%$ \\
\hline 2018 & $2,767,000$ & $49.1 \%$ & 146,000 & $29.1 \%$ & $2,913,000$ & $47.5 \%$ \\
\hline 2019 & $2,763,000$ & $49.0 \%$ & 154,000 & $29.6 \%$ & $2,917,000$ & $47.4 \%$ \\
\hline 2020 & $2,742,000$ & $48.9 \%$ & 162,000 & $30.2 \%$ & $2,904,000$ & $47.2 \%$ \\
\hline 2021 & $2,721,000$ & $48.7 \%$ & 170,000 & $30.8 \%$ & $2,891,000$ & $47.1 \%$ \\
\hline 2022 & $2,701,000$ & $48.7 \%$ & 178,000 & $31.4 \%$ & $2,879,000$ & $47.1 \%$ \\
\hline 2023 & $2,680,000$ & $48.6 \%$ & 186,000 & $32.0 \%$ & $2,866,000$ & $47.0 \%$ \\
\hline 2024 & $2,659,000$ & $48.6 \%$ & 194,000 & $32.6 \%$ & $2,853,000$ & $47.0 \%$ \\
\hline
\end{tabular}

SOURCE: RAND analysis of DoD, Census, VA, and MEPS data. 
Table C-91

Projected Prevalence of Diagnosed Lipid Disorder Among VA Patients, by Age, 2014-2024

\begin{tabular}{lccccccc}
\hline & \multicolumn{2}{c}{$<35$} & \multicolumn{2}{c}{$35-64$} & \multicolumn{2}{c}{$65+$} \\
\cline { 2 - 5 } \cline { 7 - 8 } Year & Count & Prevalence & Count & Prevalence & Count & Prevalence \\
\hline 2014 & 23,000 & $4.4 \%$ & 855,000 & $37.1 \%$ & $1,968,000$ & $63.9 \%$ \\
\hline 2015 & 23,000 & $4.4 \%$ & 852,000 & $36.5 \%$ & $1,991,000$ & $63.8 \%$ \\
\hline 2016 & 23,000 & $4.4 \%$ & 848,000 & $35.9 \%$ & $2,017,000$ & $63.7 \%$ \\
\hline 2017 & 23,000 & $4.5 \%$ & 844,000 & $35.3 \%$ & $2,037,000$ & $63.5 \%$ \\
\hline 2018 & 22,000 & $4.6 \%$ & 838,000 & $34.7 \%$ & $2,053,000$ & $63.4 \%$ \\
\hline 2019 & 22,000 & $4.6 \%$ & 831,000 & $34.1 \%$ & $2,064,000$ & $63.3 \%$ \\
\hline 2020 & 20,000 & $4.7 \%$ & 827,000 & $33.7 \%$ & $2,056,000$ & $63.1 \%$ \\
\hline 2021 & 19,000 & $4.7 \%$ & 824,000 & $33.3 \%$ & $2,049,000$ & $63.0 \%$ \\
\hline 2023 & 18,000 & $4.6 \%$ & 821,000 & $33.0 \%$ & $2,040,000$ & $62.8 \%$ \\
\hline 2024 & 16,000 & $4.5 \%$ & 819,000 & $32.8 \%$ & $2,032,000$ & $62.6 \%$ \\
\hline
\end{tabular}

SOURCE: RAND analysis of DoD, Census, VA, and MEPS data.

Table C-92

Projected Prevalence of Diagnosed Lipid Disorder Among VA Patients, by Race/Ethnicity, 2014-2024

\begin{tabular}{|c|c|c|c|c|c|c|c|c|}
\hline \multirow[b]{2}{*}{ Year } & \multicolumn{2}{|c|}{ White, Non-Hispanic } & \multicolumn{2}{|c|}{ Black, Non-Hispanic } & \multicolumn{2}{|c|}{ Hispanic } & \multicolumn{2}{|c|}{ Asian/Other } \\
\hline & Count & Prevalence & Count & Prevalence & Count & Prevalence & Count & Prevalence \\
\hline 2014 & $2,412,000$ & $51.1 \%$ & 243,000 & $37.3 \%$ & 120,000 & $35.6 \%$ & 71,000 & $37.1 \%$ \\
\hline 2015 & $2,417,000$ & $50.7 \%$ & 251,000 & $37.3 \%$ & 125,000 & $35.4 \%$ & 74,000 & $36.6 \%$ \\
\hline 2016 & $2,422,000$ & $50.6 \%$ & 260,000 & $37.5 \%$ & 130,000 & $35.5 \%$ & 77,000 & $36.5 \%$ \\
\hline 2017 & $2,421,000$ & $50.5 \%$ & 268,000 & $37.7 \%$ & 135,000 & $35.6 \%$ & 80,000 & $36.4 \%$ \\
\hline 2018 & $2,415,000$ & $50.4 \%$ & 276,000 & $38.0 \%$ & 139,000 & $35.8 \%$ & 83,000 & $36.5 \%$ \\
\hline 2019 & $2,405,000$ & $50.2 \%$ & 282,000 & $38.3 \%$ & 144,000 & $36.0 \%$ & 86,000 & $36.5 \%$ \\
\hline 2020 & $2,379,000$ & $50.1 \%$ & 288,000 & $38.5 \%$ & 148,000 & $36.2 \%$ & 89,000 & $36.6 \%$ \\
\hline 2021 & $2,354,000$ & $50.0 \%$ & 294,000 & $38.8 \%$ & 152,000 & $36.4 \%$ & 92,000 & $36.8 \%$ \\
\hline 2022 & $2,331,000$ & $49.9 \%$ & 299,000 & $39.1 \%$ & 156,000 & $36.6 \%$ & 94,000 & $37.0 \%$ \\
\hline 2023 & $2,307,000$ & $49.8 \%$ & 304,000 & $39.4 \%$ & 160,000 & $37.0 \%$ & 97,000 & $37.2 \%$ \\
\hline 2024 & $2,283,000$ & $49.7 \%$ & 307,000 & $39.7 \%$ & 163,000 & $37.2 \%$ & 100,000 & $37.5 \%$ \\
\hline
\end{tabular}

SOURCE: RAND analysis of DoD, Census, VA, and MEPS data. 


\section{C.4.2.10. Lower Back Pain Among VA Patients}

Table C-93

Projected Prevalence of Diagnosed Lower Back Pain Among VA Patients, by Sex, 2014-2024

\begin{tabular}{cccccccc}
\hline & \multicolumn{2}{c}{ Male } & & \multicolumn{2}{c}{ Female } & & \multicolumn{2}{c}{ Total } \\
\cline { 2 - 5 } \cline { 7 - 8 } Year & Count & Prevalence & & Count & Prevalence & Count & Prevalence \\
\hline 2014 & 986,000 & $18.0 \%$ & & 77,000 & $18.2 \%$ & $1,063,000$ & $18.0 \%$ \\
\hline 2015 & $1,013,000$ & $18.3 \%$ & & 82,000 & $18.6 \%$ & $1,095,000$ & $18.3 \%$ \\
\hline 2016 & $1,040,000$ & $18.6 \%$ & & 88,000 & $19.0 \%$ & $1,128,000$ & $18.6 \%$ \\
\hline 2017 & $1,065,000$ & $18.9 \%$ & & 95,000 & $19.5 \%$ & $1,160,000$ & $19.0 \%$ \\
\hline 2018 & $1,087,000$ & $19.3 \%$ & & 101,000 & $20.0 \%$ & $1,188,000$ & $19.3 \%$ \\
\hline 2019 & $1,107,000$ & $19.6 \%$ & & 107,000 & $20.5 \%$ & $1,214,000$ & $19.7 \%$ \\
\hline 2020 & $1,122,000$ & $20.0 \%$ & & 113,000 & $21.0 \%$ & $1,235,000$ & $20.1 \%$ \\
\hline 2021 & $1,137,000$ & $20.4 \%$ & & 119,000 & $21.5 \%$ & $1,256,000$ & $20.5 \%$ \\
\hline 2022 & $1,152,000$ & $20.8 \%$ & 125,000 & $22.0 \%$ & $1,277,000$ & $20.9 \%$ \\
\hline 2023 & $1,165,000$ & $21.1 \%$ & 131,000 & $22.5 \%$ & $1,296,000$ & $21.3 \%$ \\
\hline 2024 & $1,179,000$ & $21.5 \%$ & 137,000 & $23.0 \%$ & $1,316,000$ & $21.7 \%$ \\
\hline
\end{tabular}

SOURCE: RAND analysis of DoD, Census, VA, and MEPS data.

Table C-94

Projected Prevalence of Diagnosed Lower Back Pain Among VA Patients, by Age, 2014-2024

\begin{tabular}{lccccccc}
\hline \multirow{2}{*}{ Year } & \multicolumn{2}{c}{$<$} & \multicolumn{2}{c}{$35-64$} & \multicolumn{2}{c}{$65+$} \\
\cline { 2 - 4 } \cline { 7 - 8 } \cline { 7 - 8 } & Count & Prevalence & Count & Prevalence & Count & Prevalence \\
\hline 2014 & 63,000 & $12.0 \%$ & 417,000 & $18.1 \%$ & 583,000 & $18.9 \%$ \\
\hline 2015 & 66,000 & $12.3 \%$ & 429,000 & $18.4 \%$ & 602,000 & $19.3 \%$ \\
\hline 2016 & 67,000 & $12.6 \%$ & 441,000 & $18.7 \%$ & 621,000 & $19.6 \%$ \\
\hline 2018 & 66,000 & $13.0 \%$ & 453,000 & $19.0 \%$ & 640,000 & $20.0 \%$ \\
\hline 2019 & 65,000 & $13.4 \%$ & 465,000 & $19.3 \%$ & 657,000 & $20.3 \%$ \\
\hline 2020 & 62,000 & $14.1 \%$ & 489,000 & $19.9 \%$ & 684,000 & $21.0 \%$ \\
\hline 2021 & 59,000 & $14.4 \%$ & 501,000 & $20.3 \%$ & 695,000 & $21.4 \%$ \\
\hline 2022 & 56,000 & $14.7 \%$ & 514,000 & $20.6 \%$ & 707,000 & $21.8 \%$ \\
\hline 2023 & 53,000 & $14.9 \%$ & 525,000 & $21.0 \%$ & 719,000 & $22.2 \%$ \\
\hline 2024 & 49,000 & $15.1 \%$ & 536,000 & $21.4 \%$ & 731,000 & $22.6 \%$ \\
\hline
\end{tabular}

SOURCE: RAND analysis of DoD, Census, VA, and MEPS data. 
Table C-95

Projected Prevalence of Diagnosed Lower Back Pain Among VA Patients, by Race/Ethnicity, 2014-2024

\begin{tabular}{|c|c|c|c|c|c|c|c|c|}
\hline \multirow[b]{2}{*}{ Year } & \multicolumn{2}{|c|}{ White, Non-Hispanic } & \multicolumn{2}{|c|}{ Black, Non-Hispanic } & \multicolumn{2}{|c|}{ Hispanic } & \multicolumn{2}{|c|}{ Asian/Other } \\
\hline & Count & Prevalence & Count & Prevalence & Count & Prevalence & Count & Prevalence \\
\hline 2014 & 910,000 & $19.3 \%$ & 84,000 & $12.9 \%$ & 42,000 & $12.6 \%$ & 26,000 & $13.8 \%$ \\
\hline 2015 & 933,000 & $19.6 \%$ & 89,000 & $13.2 \%$ & 45,000 & $12.9 \%$ & 28,000 & $14.0 \%$ \\
\hline 2016 & 957,000 & $20.0 \%$ & 94,000 & $13.5 \%$ & 48,000 & $13.2 \%$ & 30,000 & $14.4 \%$ \\
\hline 2017 & 978,000 & $20.4 \%$ & 98,000 & $13.8 \%$ & 51,000 & $13.5 \%$ & 33,000 & $14.8 \%$ \\
\hline 2018 & 996,000 & $20.8 \%$ & 103,000 & $14.2 \%$ & 54,000 & $13.8 \%$ & 35,000 & $15.1 \%$ \\
\hline 2019 & $1,013,000$ & $21.2 \%$ & 107,000 & $14.5 \%$ & 57,000 & $14.2 \%$ & 37,000 & $15.5 \%$ \\
\hline 2020 & $1,026,000$ & $21.6 \%$ & 111,000 & $14.9 \%$ & 59,000 & $14.5 \%$ & 39,000 & $15.9 \%$ \\
\hline 2021 & $1,038,000$ & $22.0 \%$ & 115,000 & $15.2 \%$ & 62,000 & $14.8 \%$ & 41,000 & $16.3 \%$ \\
\hline 2022 & $1,051,000$ & $22.5 \%$ & 119,000 & $15.6 \%$ & 65,000 & $15.2 \%$ & 43,000 & $16.7 \%$ \\
\hline 2023 & $1,062,000$ & $22.9 \%$ & 123,000 & $16.0 \%$ & 67,000 & $15.6 \%$ & 45,000 & $17.1 \%$ \\
\hline 2024 & $1,073,000$ & $23.4 \%$ & 127,000 & $16.4 \%$ & 70,000 & $15.9 \%$ & 47,000 & $17.5 \%$ \\
\hline
\end{tabular}

SOURCE: RAND analysis of DoD, Census, VA, and MEPS data.

\section{C.4.2.11. Malignant Cancers Among VA Patients}

Table C-96

Projected Prevalence of Diagnosed Malignant Cancers Among VA Patients, by Sex, 2014-2024

\begin{tabular}{|c|c|c|c|c|c|c|}
\hline \multirow[b]{2}{*}{ Year } & \multicolumn{2}{|c|}{ Male } & \multicolumn{2}{|c|}{ Female } & \multicolumn{2}{|c|}{ Total } \\
\hline & Count & Prevalence & Count & Prevalence & Count & Prevalence \\
\hline 2014 & $1,080,000$ & $19.7 \%$ & 42,000 & $9.9 \%$ & $1,122,000$ & $19.0 \%$ \\
\hline 2015 & $1,101,000$ & $19.9 \%$ & 45,000 & $10.1 \%$ & $1,146,000$ & $19.1 \%$ \\
\hline 2016 & $1,122,000$ & $20.1 \%$ & 48,000 & $10.4 \%$ & $1,170,000$ & $19.3 \%$ \\
\hline 2017 & $1,141,000$ & $20.3 \%$ & 52,000 & $10.7 \%$ & $1,193,000$ & $19.5 \%$ \\
\hline 2018 & $1,158,000$ & $20.6 \%$ & 56,000 & $11.0 \%$ & $1,214,000$ & $19.8 \%$ \\
\hline 2019 & $1,172,000$ & $20.8 \%$ & 59,000 & $11.4 \%$ & $1,231,000$ & $20.0 \%$ \\
\hline 2020 & $1,179,000$ & $21.0 \%$ & 63,000 & $11.8 \%$ & $1,242,000$ & $20.2 \%$ \\
\hline 2021 & $1,186,000$ & $21.2 \%$ & 68,000 & $12.2 \%$ & $1,254,000$ & $20.4 \%$ \\
\hline 2022 & $1,193,000$ & $21.5 \%$ & 72,000 & $12.7 \%$ & $1,265,000$ & $20.7 \%$ \\
\hline 2023 & $1,200,000$ & $21.8 \%$ & 77,000 & $13.2 \%$ & $1,277,000$ & $20.9 \%$ \\
\hline 2024 & $1,206,000$ & $22.0 \%$ & 81,000 & $13.7 \%$ & $1,287,000$ & $21.2 \%$ \\
\hline
\end{tabular}

SOURCE: RAND analysis of DoD, Census, VA, and MEPS data. 
Table C-97

Projected Prevalence of Diagnosed Malignant Cancers Among VA Patients, by Age, 2014-2024

\begin{tabular}{lccccccc}
\hline & \multicolumn{2}{c}{$<$} & \multicolumn{2}{c}{$35-64$} & \multicolumn{2}{c}{$65+$} \\
\cline { 2 - 4 } \cline { 7 - 8 } Year & Count & Prevalence & Count & Prevalence & Count & Prevalence \\
\hline 2014 & 8,000 & $1.5 \%$ & 234,000 & $10.2 \%$ & 879,000 & $28.6 \%$ \\
\hline 2015 & 8,000 & $1.6 \%$ & 235,000 & $10.1 \%$ & 902,000 & $28.9 \%$ \\
\hline 2016 & 8,000 & $1.6 \%$ & 237,000 & $10.0 \%$ & 926,000 & $29.2 \%$ \\
\hline 2017 & 8,000 & $1.6 \%$ & 238,000 & $10.0 \%$ & 947,000 & $29.5 \%$ \\
\hline 2018 & 8,000 & $1.6 \%$ & 239,000 & $9.9 \%$ & 966,000 & $29.8 \%$ \\
\hline 2019 & 8,000 & $1.7 \%$ & 241,000 & $9.9 \%$ & 984,000 & $30.2 \%$ \\
\hline 2020 & 7,000 & $1.7 \%$ & 242,000 & $9.9 \%$ & 993,000 & $30.5 \%$ \\
\hline 2021 & 7,000 & $1.7 \%$ & 245,000 & $9.9 \%$ & $1,002,000$ & $30.8 \%$ \\
\hline 2022 & 7,000 & $1.7 \%$ & 248,000 & $10.0 \%$ & $1,011,000$ & $31.1 \%$ \\
\hline 2023 & 6,000 & $1.7 \%$ & 250,000 & $10.0 \%$ & $1,020,000$ & $31.4 \%$ \\
\hline 2024 & 6,000 & $1.7 \%$ & 253,000 & $10.1 \%$ & $1,029,000$ & $31.8 \%$ \\
\hline
\end{tabular}

SOURCE: RAND analysis of DoD, Census, VA, and MEPS data.

Table C-98

Projected Prevalence of Diagnosed Malignant Cancers Among VA Patients, by Race/Ethnicity, 2014-2024

\begin{tabular}{|c|c|c|c|c|c|c|c|c|}
\hline \multirow[b]{2}{*}{ Year } & \multicolumn{2}{|c|}{ White, Non-Hispanic } & \multicolumn{2}{|c|}{ Black, Non-Hispanic } & \multicolumn{2}{|c|}{ Hispanic } & \multicolumn{2}{|c|}{ Asian/Other } \\
\hline & Count & Prevalence & Count & Prevalence & Count & Prevalence & Count & Prevalence \\
\hline 2014 & $1,026,000$ & $21.7 \%$ & 58,000 & $8.9 \%$ & 24,000 & $7.1 \%$ & 14,000 & $7.3 \%$ \\
\hline 2015 & $1,045,000$ & $21.9 \%$ & 61,000 & $9.1 \%$ & 25,000 & $7.2 \%$ & 15,000 & $7.3 \%$ \\
\hline 2016 & $1,064,000$ & $22.2 \%$ & 64,000 & $9.3 \%$ & 27,000 & $7.3 \%$ & 16,000 & $7.4 \%$ \\
\hline 2017 & $1,081,000$ & $22.5 \%$ & 67,000 & $9.5 \%$ & 28,000 & $7.5 \%$ & 17,000 & $7.5 \%$ \\
\hline 2018 & $1,096,000$ & $22.8 \%$ & 71,000 & $9.7 \%$ & 30,000 & $7.7 \%$ & 17,000 & $7.6 \%$ \\
\hline 2019 & $1,108,000$ & $23.2 \%$ & 74,000 & $10.0 \%$ & 31,000 & $7.9 \%$ & 18,000 & $7.8 \%$ \\
\hline 2020 & $1,114,000$ & $23.4 \%$ & 77,000 & $10.3 \%$ & 33,000 & $8.0 \%$ & 19,000 & $7.9 \%$ \\
\hline 2021 & $1,119,000$ & $23.8 \%$ & 80,000 & $10.6 \%$ & 34,000 & $8.2 \%$ & 20,000 & $8.1 \%$ \\
\hline 2022 & $1,125,000$ & $24.1 \%$ & 83,000 & $10.9 \%$ & 36,000 & $8.4 \%$ & 21,000 & $8.3 \%$ \\
\hline 2023 & $1,131,000$ & $24.4 \%$ & 86,000 & $11.2 \%$ & 37,000 & $8.7 \%$ & 22,000 & $8.5 \%$ \\
\hline 2024 & $1,136,000$ & $24.7 \%$ & 89,000 & $11.5 \%$ & 39,000 & $8.9 \%$ & 23,000 & $8.7 \%$ \\
\hline
\end{tabular}

SOURCE: RAND analysis of DoD, Census, VA, and MEPS data. 


\section{C.4.3. Projections of Service-connected Conditions for Veterans and VA Patients C.4.3.1. Mental Health Conditions Among Veterans}

Table C-99

Projected Prevalence of Diagnosed Mental Health Conditions Among Veterans, 2015-2024

\begin{tabular}{|c|c|c|c|c|c|c|}
\hline \multirow[b]{2}{*}{ Year } & \multicolumn{2}{|c|}{$\begin{array}{l}\text { Service-Connected } \\
\text { With Remission }\end{array}$} & \multicolumn{2}{|c|}{$\begin{array}{l}\text { Service-Connected } \\
\text { Without Remission }\end{array}$} & \multicolumn{2}{|c|}{ Age-Based } \\
\hline & Count & Prevalence & Count & Prevalence & Count & Prevalence \\
\hline 2015 & $4,105,000$ & $19.4 \%$ & $4,126,000$ & $19.5 \%$ & $4,248,000$ & $22.8 \%$ \\
\hline 2016 & $4,053,000$ & $19.5 \%$ & $4,077,000$ & $19.6 \%$ & $4,296,000$ & $23.7 \%$ \\
\hline 2017 & $4,001,000$ & $19.7 \%$ & $4,028,000$ & $19.8 \%$ & $4,339,000$ & $24.6 \%$ \\
\hline 2018 & $3,947,000$ & $19.8 \%$ & $3,978,000$ & $20.0 \%$ & $4,380,000$ & $25.6 \%$ \\
\hline 2019 & $3,896,000$ & $20.0 \%$ & $3,930,000$ & $20.1 \%$ & $4,418,000$ & $26.6 \%$ \\
\hline 2020 & $3,839,000$ & $20.1 \%$ & $3,876,000$ & $20.3 \%$ & $4,455,000$ & $27.6 \%$ \\
\hline 2021 & $3,784,000$ & $20.2 \%$ & $3,824,000$ & $20.5 \%$ & $4,489,000$ & $28.7 \%$ \\
\hline 2022 & $3,729,000$ & $20.4 \%$ & $3,771,000$ & $20.6 \%$ & $4,520,000$ & $29.8 \%$ \\
\hline 2023 & $3,674,000$ & $20.5 \%$ & $3,719,000$ & $20.8 \%$ & $4,547,000$ & $30.9 \%$ \\
\hline 2024 & $3,622,000$ & $20.7 \%$ & $3,670,000$ & $21.0 \%$ & $4,573,000$ & $32.0 \%$ \\
\hline
\end{tabular}

SOURCE: RAND analysis of DoD, Census, VA, and MEPS data.

NOTES: Service-connected with remission is the baseline analysis presented in the report. Service-connected without remission uses the same projection method, but sets the first year remission rate to zero. The age-based projections use the same projection method as was used for the other conditions (e.g. diabetes, hypertension). 


\section{C.4.3.2. Posttraumatic Stress Disorder Among Veterans}

Table C-100

Projected Prevalence of Diagnosed Posttraumatic Stress Disorder Among Veterans, 2015-2024

\begin{tabular}{cccccc}
\hline \multirow{2}{*}{ Year } & \multicolumn{2}{c}{$\begin{array}{c}\text { Service-Connected } \\
\text { With Remission }\end{array}$} & & \multicolumn{2}{c}{$\begin{array}{c}\text { Service-Connected } \\
\text { Without Remission }\end{array}$} \\
\cline { 2 - 3 } \cline { 5 - 6 } & Count & Prevalence & & Count & Prevalence \\
\hline 2015 & 602,000 & $2.8 \%$ & & 619,000 & $2.9 \%$ \\
\hline 2016 & 604,000 & $2.9 \%$ & 624,000 & $3.0 \%$ \\
\hline 2017 & 601,000 & $3.0 \%$ & 625,000 & $3.1 \%$ \\
\hline 2018 & 597,000 & $3.0 \%$ & 624,000 & $3.1 \%$ \\
\hline 2019 & 592,000 & $3.0 \%$ & 623,000 & $3.2 \%$ \\
\hline 2021 & 591,000 & $3.1 \%$ & 624,000 & $3.3 \%$ \\
\hline 2022 & 591,000 & $3.2 \%$ & 628,000 & $3.4 \%$ \\
\hline 2023 & 588,000 & $3.2 \%$ & 628,000 & $3.4 \%$ \\
\hline 2024 & 584,000 & $3.3 \%$ & 626,000 & $3.5 \%$ \\
\hline $5 n n n n y y$ & 579,000 & $3.3 \%$ & 624,000 & $3.6 \%$ \\
\hline
\end{tabular}

SOURCE: RAND analysis of DoD, Census, VA, and MEPS data. NOTES: Service-connected with remission is the baseline analysis presented in the report. Service-connected without remission uses the same projection method, but sets the first year remission rate to zero. 


\section{C.4.3.3. Mental Health Conditions Among VA Patients}

Table C-101

Projected Prevalence of Diagnosed Mental Health Conditions Among VA Patients, 2015-2024

\begin{tabular}{|c|c|c|c|c|c|c|}
\hline \multirow[b]{2}{*}{ Year } & \multicolumn{2}{|c|}{$\begin{array}{l}\text { Service-Connected } \\
\text { With Remission }\end{array}$} & \multicolumn{2}{|c|}{$\begin{array}{l}\text { Service-Connected } \\
\text { Without Remission }\end{array}$} & \multicolumn{2}{|c|}{ Age-Based } \\
\hline & Count & Prevalence & Count & Prevalence & Count & Prevalence \\
\hline 2015 & $1,707,000$ & $28.5 \%$ & $1,720,000$ & $28.7 \%$ & $1,712,000$ & $28.6 \%$ \\
\hline 2016 & $1,775,000$ & $29.3 \%$ & $1,802,000$ & $29.7 \%$ & $1,782,000$ & $29.4 \%$ \\
\hline 2017 & $1,835,000$ & $30.0 \%$ & $1,874,000$ & $30.7 \%$ & $1,849,000$ & $30.3 \%$ \\
\hline 2018 & $1,886,000$ & $30.7 \%$ & $1,937,000$ & $31.6 \%$ & $1,912,000$ & $31.2 \%$ \\
\hline 2019 & $1,931,000$ & $31.4 \%$ & $1,993,000$ & $32.4 \%$ & $1,973,000$ & $32.0 \%$ \\
\hline 2020 & $1,961,000$ & $31.9 \%$ & $2,033,000$ & $33.1 \%$ & $2,025,000$ & $32.9 \%$ \\
\hline 2021 & $1,987,000$ & $32.4 \%$ & $2,068,000$ & $33.7 \%$ & $2,076,000$ & $33.8 \%$ \\
\hline 2022 & $2,011,000$ & $32.9 \%$ & $2,099,000$ & $34.3 \%$ & $2,126,000$ & $34.8 \%$ \\
\hline 2023 & $2,031,000$ & $33.3 \%$ & $2,127,000$ & $34.9 \%$ & $2,174,000$ & $35.7 \%$ \\
\hline 2024 & $2,049,000$ & $33.8 \%$ & $2,152,000$ & $35.5 \%$ & $2,223,000$ & $36.6 \%$ \\
\hline
\end{tabular}

SOURCE: RAND analysis of DoD, Census, VA, and VA encounter data.

NOTES: Service-connected with remission is the baseline analysis presented in the report. Service-connected without remission uses the same projection method, but sets the first year remission rate to zero. The age-based projections use the same projection method as was used for the other conditions (e.g. diabetes, hypertension). 


\section{C.4.3.4. Posttraumatic Stress Disorder Among VA Patients}

Table C-102

Projected Prevalence of Diagnosed Posttraumatic Stress Disorder Among VA Patients, 2015-2024

\begin{tabular}{cccccc}
\hline \multirow{2}{*}{ Year } & \multicolumn{2}{c}{$\begin{array}{c}\text { Service-Connected } \\
\text { With Remission }\end{array}$} & & \multicolumn{2}{c}{$\begin{array}{c}\text { Service-Connected } \\
\text { Without Remission }\end{array}$} \\
\cline { 2 - 3 } \cline { 5 - 6 } \cline { 5 - 6 } 2015 & Count & Prevalence & & Count & Prevalence \\
\hline 2016 & 470,000 & $7.8 \%$ & & 480,000 & $8.0 \%$ \\
\hline 2017 & 496,000 & $8.2 \%$ & & 522,000 & $8.6 \%$ \\
\hline 2018 & 520,000 & $8.5 \%$ & & 560,000 & $9.2 \%$ \\
\hline 2019 & 541,000 & $8.8 \%$ & & 594,000 & $9.7 \%$ \\
\hline 2020 & 561,000 & $9.1 \%$ & 625,000 & $10.1 \%$ \\
\hline 2021 & 575,000 & $9.4 \%$ & 650,000 & $10.6 \%$ \\
\hline 2022 & 588,000 & $9.6 \%$ & 673,000 & $11.0 \%$ \\
\hline 2023 & 600,000 & $9.8 \%$ & 694,000 & $11.3 \%$ \\
\hline 2024 & 611,000 & $10.0 \%$ & 712,000 & $11.7 \%$ \\
\hline & 621,000 & $10.2 \%$ & 730,000 & $12.0 \%$ \\
\hline
\end{tabular}

SOURCE: RAND analysis of DoD, Census, VA, and VA encounter data. Service-connected with remission is the baseline analysis presented in the report. Service-connected without remission uses the same projection method, but sets the first year remission rate to zero. 


\section{C.4.3.5. Traumatic Brain Injury Among VA Patients}

Table C-103

Projected Prevalence of Diagnosed Traumatic Brain Injury Among VA Patients, 2015-2024

\begin{tabular}{cccccc}
\hline \multirow{2}{*}{ Year } & \multicolumn{2}{c}{$\begin{array}{c}\text { Service-Connected } \\
\text { With Remission }\end{array}$} & & \multicolumn{2}{c}{$\begin{array}{c}\text { Service-Connected } \\
\text { Without Remission }\end{array}$} \\
\cline { 2 - 3 } \cline { 5 - 6 } \cline { 5 - 6 } 2015 & Count & Prevalence & & Count & Prevalence \\
\hline 2016 & 110,000 & $1.8 \%$ & & 117,000 & $2.0 \%$ \\
\hline 2017 & 108,000 & $1.8 \%$ & & 117,000 & $1.9 \%$ \\
\hline 2018 & 107,000 & $1.7 \%$ & & 116,000 & $1.9 \%$ \\
\hline 2019 & 105,000 & $1.7 \%$ & & 115,000 & $1.9 \%$ \\
\hline 2020 & 104,000 & $1.7 \%$ & & 115,000 & $1.9 \%$ \\
\hline 2021 & 104,000 & $1.7 \%$ & & 116,000 & $1.9 \%$ \\
\hline 2022 & 103,000 & $1.7 \%$ & & 116,000 & $1.9 \%$ \\
\hline 2023 & 102,000 & $1.7 \%$ & & 115,000 & $1.9 \%$ \\
\hline 2024 & 101,000 & $1.7 \%$ & 115,000 & $1.9 \%$ \\
\hline & 100,000 & $1.6 \%$ & 114,000 & $1.9 \%$ \\
\hline
\end{tabular}

SOURCE: RAND analysis of DoD, Census, VA, and VA encounter data.

NOTES: Service-connected with remission is the baseline analysis presented in the report. Service-connected without remission uses the same projection method, but sets the first year remission rate to zero. 


\section{C.4.3.6. Musculoskeletal Conditions with Chronic Pain Among VA Patients}

\begin{tabular}{|c|c|c|}
\hline \multirow{2}{*}{ Year } & \multicolumn{2}{|c|}{ Total } \\
\hline & Count & Prevalence \\
\hline 2015 & $1,106,000$ & $18.5 \%$ \\
\hline 2016 & $1,167,000$ & $19.3 \%$ \\
\hline 2017 & $1,230,000$ & $20.1 \%$ \\
\hline 2018 & $1,294,000$ & $21.1 \%$ \\
\hline 2019 & $1,361,000$ & $22.1 \%$ \\
\hline 2020 & $1,427,000$ & $23.2 \%$ \\
\hline 2021 & $1,493,000$ & $24.3 \%$ \\
\hline 2022 & $1,558,000$ & $25.5 \%$ \\
\hline 2023 & $1,621,000$ & $26.6 \%$ \\
\hline 2024 & $1,681,000$ & $27.7 \%$ \\
\hline
\end{tabular}

\section{C.4.4. Predicted Prevalence using VA Encounter Data: Goodness of Fit}

In this section, we provide additional detail about the models we use to predict the prevalence of TBI and musculoskeletal conditions. Predicted prevalence is based on projecting observed information forward, based on logistic regression models fit to VA encounter data. The model is the similar to that used to estimate prevalence with MEPS (model 4 in Section C.1.5.2). Our model describes prevalence as a function of Veteran age, sex, and state of residence, and also includes a continuous time effect that captures observed changes in prevalence that go beyond changes expected because of changes in population demographics. These secular changes capture cohort effects that are related to Veteran combat experience. Including these secular trends is important for accurate estimation of demographic effects that are used to project trends forward based on changing demographic characteristics in the projected VA patient population.

The accuracy of predictions, by their very nature, is unknown. However, we can examine how closely our model is able to capture observed trends. Close prediction of observed prevalence rates provides reassurance that our predictions are reasonable. As shown in Figure C-1, our models provided a good fit to the observed prevalence rates and trends. TBI is relatively rare, and, at the scale shown, the curvature from the logistic regression fit is evident. However, this reflects very small differences between the observed and estimated prevalence. In 2014, we estimate 1.97 percent TBI prevalence and observe 1.75 percent prevalence, a 0.22 percentage point difference. Estimated prevalence was very close to observed values musculoskeletal conditions. In 2014, we estimate 18.01 percent prevalence of musculoskeletal conditions and observe 17.99 percent prevalence, a 0.02 percentage point difference. 
Figure C-1

Predicted Prevalence Goodness of Fit
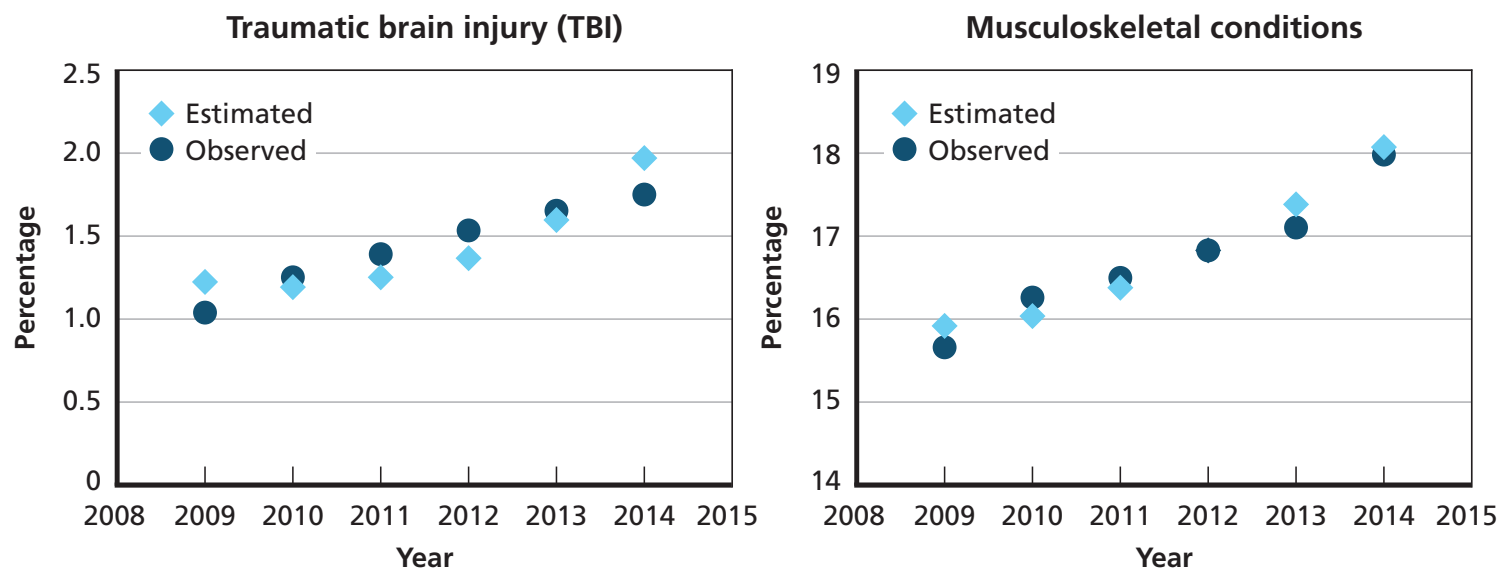

SOURCE: RAND analysis of VA encounter data (2009-2014).

RAND RR1165z1-C.1

\section{C.4.5. Sensitivity Analyses for Prevalence Projections}

Future prevalence often follows demographic trends because aging is a risk factor for many chronic conditions, including IHD. For example, several studies have projected increases in the prevalence of IHD in the overall U.S. population; this result follows from combining static estimates of IHD prevalence with future expected aging (Heidenreich et al., 2011). Similarly, we project that the aging trend for the VA patient population over this period (a one-year projected increase in mean age-see Chapter Three) will tend to increase the prevalence of IHD and other chronic conditions. However, prevalence trends often also depend on trends in other risk factors; for example, factors known to affect IHD prevalence (in addition to aging) include smoking, obesity, and usage of preventive medications such as aspirin. Decreases in the incidence of AMI suggest that better treatment and improved risk factor control have reduced the incidence of acute IHD, and may also mitigate or outweigh future increases in chronic acute IHD due to aging. A projection model that solely relies on aging to project future changes would miss such trends.

To better model future prevalence, we developed models that incorporated a nonlinear trend to account for unobservable trends in risk factors. (Data limitations precluded the use of more complex modeling.) The disadvantage of this approach is that a strictly linear trend may not be appropriate, and may exaggerate true changes when extrapolated for longer periods. For example, in projecting the prevalence of $\mathrm{CHF}$, the decreases in prevalence that were evident in MEPS in 2008-2012 translated into unrealistically sharp projected declines during 2015-2024.

To better demonstrate how our assumptions affect projections, we show projections that incorporate a linear trend in prevalence (solid blue lines) alongside "static" projections (dashed gray lines), in which future prevalence rates only reflect aging (Figure C-2). In most cases, the differences between the two projections were not large. However, the projected decline in the prevalence of IHD and CHF among Veterans are clearly attributable to the estimated time trend in the prevalence projections, rather than changes in the demographic composition of the population. As discussed in Chapter Five, the trends do have relatively wide confidence intervals, which suggests that there is significant uncertainty regarding the magnitude of these declines. 
Figure C-2

Veteran Prevalence Projections with and without Time Trends for Common Chronic Conditions
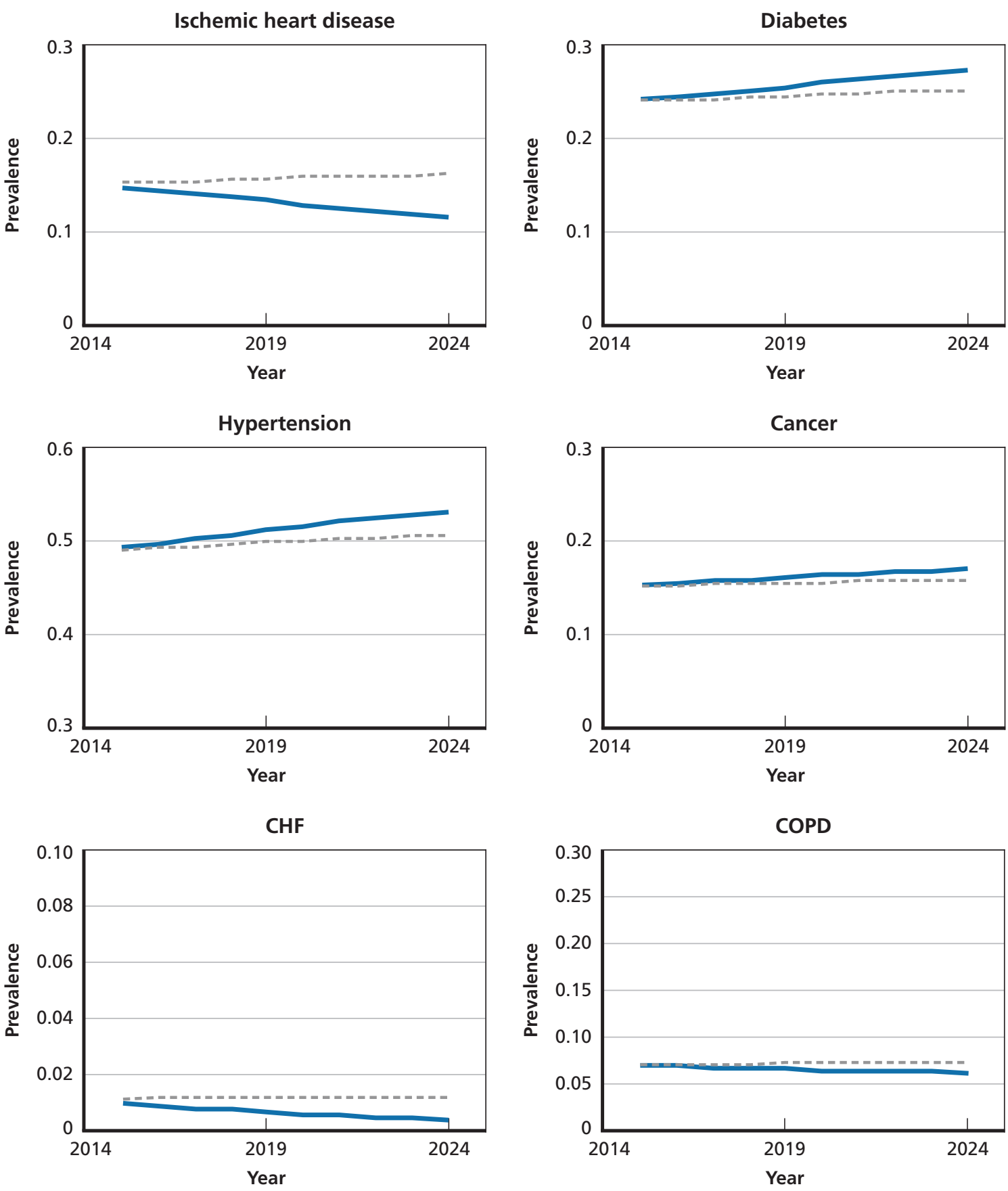

RAND RR1165z1-C.2 

APPENDIX D

\section{Scenario Development and Evaluation}

\section{D.1. Detailed Scenario Evaluation Methods}

\section{D.1.1. Changes to VHA Eligibility, by Priority Group D.1.1.1. Priority Group Sorting Algorithm}

Chapter Six developed an algorithm to sort Veterans in the 2013 ACS PUMS (see Section 2.6 for a description of these data) into the VA priority groups. Priority groups determine whether a Veteran is eligible for health services and whether that Veteran will pay co-pays. All Veterans discharged as Honorable, General, or Uncharacterized and who satisfy the two-year length of service requirement (unless this requirement is waived) are eligible for assignment to one of these priority groups, but eligibility for health services is limited to Veterans in priority groups 1 through $8 \mathrm{~d}$. Currently, Veterans in priority groups $8 \mathrm{e}$ and $8 \mathrm{~g}$ and not eligible to use VA services (although those in 8e are eligible for VA services related specifically to their service connected conditions).

Testing the scenarios described above requires not just measures of currently VA enrollees or VA users, but the entire population of Veterans and the corresponding priority group they would be assigned if they were enrolled. Therefore, we construct an algorithm to sort the entire Veteran population in the 2013 ACS PUMS into respective priority groups. We validate this sorting algorithm by comparing the subpopulation of Veterans reporting VA use with the corresponding subpopulation in VA administrative records, demonstrating a close fit. We then implement the scenarios described above, measuring the changes in priority group eligibility and estimating resulting VA users.

Before the algorithm begins, the ACS counts of Veterans are inflated to provide an overall count of the Veteran population in alignment with the RAND model described in the demography section (i.e., 21.9 million Veterans in 2013). The undercounting in the ACS arises from two sources: misreporting by respondents and limitation of the ACS in reaching individuals not living in housing units or group quarters. To address this undercounting, separate Veteran counts were calculated for cells defined by state, age band, sex, and race/ethnicity, then the frequency weights in the corresponding 2013 ACS PUMS cells were inflated to match these counts. Because the VA user counts in the ACS closely match administrative records, VA users were excluded from this process, so only non-patient Veteran cells had their weights inflated to match RAND model cells.

The sorting algorithm then proceeds in two broad steps:

1. Using the information available in the ACS on disability rating, household income, family size, and area of residence, it sequentially sorts Veterans into different priority groups, starting with the highest priority (priority group 1) and proceeding to priority 
group 5, sorting Veterans previously unassigned into higher groups into a given priority group based on qualifying factors. For example, an individual with service-connected disability rating of 100 percent (entitling this Veteran to priority group 1) and income below the National Means Test (entitling this Veteran to priority group 5) is first classified as priority group 1 , and by the time the algorithm is assigning individuals priority group 5 status, he is already assigned to priority group 1, so he is not reassigned to priority group 5. At each stage, the pool of Veterans eligible to be assigned to priority group under consideration excludes any Veterans already assigned to a higher priority.

2. After this first-round set of assignments is carried out, the algorithm then reassigns the remaining Veterans who report use of VA to priority groups $1-5$ in accordance with various actuarial adjustments to reflect actual differences in VA patient counts. The algorithm then assigns priority groups $6-8$ status to the remaining Veterans, using both self-reported data and actuarial adjustments. These actuarial adjustments are required for two reasons: item nonresponse or misresponse in fields vital to accurate priority group classification (e.g., service-connected disability ratings), and a lack of elicitations of criteria used by VA (e.g., date of initial enrollment, exact dates of service, possible exposure during service that qualifies Veterans for priority group 6 status). Finally, all Veterans unassigned at this stage who report being VA enrollees are assigned to priority group 8 status.

The ACS directly asks individuals their service-connected disability rating, allowing separate answers for 0 percent, $10-20$ percent, $30-40$ percent, 50-60 percent, and 70-100 percent. 50 percent or above entitles Veterans to priority group 1;30-40 percent entitles Veterans to priority group 2; and 10-20 percent entitles Veterans to priority group 3. The algorithm therefore assigns these Veterans accordingly.

Priority group 4-the catastrophically disabled and/or housebound-are assigned based on the presence of ambulatory, independent living, or self-care difficulty, or report a disability or receipt of a disability benefit (Social Security Income or SSDI) and household income that falls below the VA housebound threshold.

Priority group 5 eligibility is assigned if any of three criteria are met: the Veteran reports Medicaid coverage; the Veteran reports Social Security Income receipt (which carries with it Medicaid eligibility); or the Veteran's household satisfies the National Income Test, given how many dependents the Veteran has.

However, the count of VA patients in priority group 5 is substantially lower than the actual priority group 5 patients in 2013 from VA administrative records. This deviation may arise from a number of sources, be they non-response or misreporting of household income (or differences in how this income would be deemed by VA); to correct for this deviation, unassigned Veterans who report being VA users are sorted in ascending order according to household income and are sequentially assigned priority group 5 status until the ratio of priority group 5 users to overall VA users agrees with 2013 VA administrative records.

Priority group 6 status is assigned among the remaining Veterans according to service era (inclusively between Vietnam era to 8/2001) or report a 0 percent service-connected disability. The resulting priority group 6 size is substantially larger than in VA administrative records, since the true qualifying criteria for priority group 6 is a strict subset of these parameters (e.g., the 0 percent service-connected rating must be compensable). The number of priority group 6 
VA users is correspondingly reduced (randomly) such that the ratio of priority group 6 users to priority group 5 users in the ACS mirrors that observed in VA administrative records.

However, there remain a substantial portion of potentially eligible VA non-users assigned priority group 6 status; this population is randomly reduced to agree with the ratio of projected eligible priority group 6 Veterans to potentially eligible priority group 5 VA users from the EHCPM model.

Priority group 7 status is assigned according to whether a currently unassigned Veteran satisfies the higher threshold of the National Income Test or the GMT for the number of dependents in that household and the area of residence. Although the ACS provides information on each respondent's PUMA, the GMT is assessed at the county level. We reconcile these differences by assigning individuals in MSAs the corresponding GMT (for which there is no disconnect) and use the remaining state-level averages of non-MSA counties as the GMT for individuals residing in non-MSA PUMAs in that state.

The algorithm then revisits priority groups 1 through 3, which have previously been undercounted when comparing to VA administrative records. To rectify this issue, a logit model is fit to the likelihood of being previously assigned priority group 1 status (i.e., having reported a service-connected disability rating of at least 50 percent), where predictors include age, family size, race, ethnicity, family income, and era of service. Veterans who report being a VA user but who have not yet been assigned a priority group are then given a predicted probability of being priority group 1 using this logit model and their personal characteristics. They are then sequentially reassigned to priority group 1 by descending order of this probability (i.e., those most likely to be priority group 1 given their characteristics are the first to be assigned priority group 1) until the ratio of priority group 1 VA users to total VA users agrees with VA administrative records. This procedure is repeated sequentially for priority groups 2 and 3 on previously unassigned VA users.

Next, the count of VA patients in priority group 7 is substantially lower than the actual priority group 7 patients in 2013 from VA administrative records. This deviation may arise from a number of sources, be they nonresponse or misreporting of household income (or differences in how this income would be deemed by VA). To correct for this deviation, unassigned Veterans who report being VA users are sorted in ascending order according to household income and are sequentially assigned priority group 7 status until the ratio of priority group 7 users to overall VA users agrees with 2013 VA administrative records. A similar procedure is performed on VA non-users by ascending family income until the ratio of priority group 7 eligible Veterans to priority group 7 VA users matches Vet Pop Proxy predicted values.

Priority group $8 \mathrm{~b} / 8 \mathrm{~d}$ is determined by assigning all remaining Veterans with household income under their GMT+10 percent (see priority group 7 calculation for a discussion of how GMT is determined) for their dependent class to this set of groups. Priority group $8 \mathrm{a} / 8 \mathrm{c}$ is assigned to all Veterans reporting being VA users who are not otherwise assigned to a priority group and who do not report a 0 percent service-connected disability rating. Priority group $8 \mathrm{e}$ status is assigned to Veterans otherwise unassigned reporting being VA users and report a 0 percent service-connected disability rating.

Priority group $8 \mathrm{~g}$ status is then assigned to all previously unassigned Veterans. However, this group is reduced by the exclusion of two classes of Veterans: Veterans who were discharged as "other-than-honorable," as "bad conduct," or dishonorably. These discharge categories render the Veteran in question ineligible for VA health services. According to DoD, 5.6 percent of discharges from 2000 through 2013 fell into this category, so this percentage of the total number 
of Veterans in the ACS is subtracted from the priority group $8 \mathrm{~g}$ group. The second class of Veterans is those who do not satisfy the required two-year length of service requirement. The size of this population was estimated using the corresponding question in the 2000 Decennial Census, which asks respondents whether they served less than two years. Because this population may contain individuals injured due to service and thus eligible for VA services regardless of their less-than-two years of service, separate ratios were calculated for the disabled Veteran population and the non-disabled Veteran population, then these ratios were applied to the ACS Veteran population respectively, and the corresponding number of Veterans was subtracted from the priority group $8 \mathrm{~g}$ pool. It should be noted that because these groups are treated independently, the resulting estimates of the size of these two ineligible groups is mechanically larger than the actual population, leading to the estimated $8 \mathrm{~g}$ group being an underestimate or lower bound of this group of Veterans. This underestimate is even larger since the method accounting for those who do not satisfy their length-of-service requirement also includes some Veterans who will have received waivers for not satisfying this requirement and thus will be eligible for priority group assignment.

\section{D.1.2. Changes in Presumptive Eligibility \\ D.1.2.1. Detailed Methodology for Estimating the Impact in Changes in Presumptive Eligibility}

We estimated the maximum number of new VA patients that would utilize VA health care if VA decides that hypertension can be presumptively included as a service-connected condition for Veterans who served in the Vietnam theater. Table D-1 presents our approach.

Since VA data systems do not track whether a Veteran served in the Vietnam theater, we relied on estimates from NSV for the number of Veterans who served during the Vietnam era, as well as in the Vietnam theater (rows A and B). We use VA Business Intelligence data on Veterans to estimate the number of VA enrollees by priority group (rows A1 and A2). Given data limitations, we make the simplifying assumption that enrolled Veterans between the ages of 58 and 96 (in 2014) primarily served during the Vietnam era. Since it is likely that there are enrolled Veterans between those ages who did not serve during the Vietnam era, this assumption will likely over estimate of the number of Vietnam-era Veterans who are already enrolled (and under estimate the number who are not enrolled in row A3). The error introduced by this assumption is offset by the fact that it is unlikely that all newly eligible Veterans will enroll and become patients, though we use ratios that assume that they will. In row A4, we adjust for the discharge status of Veterans. Based on our analysis of ACS data, we estimate that 94.4 percent of Veterans are discharged with "other than dishonorable" discharges. This is a populationwide estimate for all Veterans. Due to data limitations, we were not able to produce age-specific estimates.

To estimate the prevalence of hypertension, we use the MEPS Veteran patients and nonpatients of VA and who, again by age, were likely to have served in the Vietnam era (rows D and F). We then apply the ratio of hypertension prevalence and the proportion of Veterans who served in theater to the number of non-enrolled Veterans to produce a high-end estimate of potential new enrollees (row E).

When VA presumes that a health condition is service connected, a Veteran is typically eligible for a priority group of 6 or higher (up to 1). As such, presumptive eligibility determinations also have the effect of "promoting" enrolled Veterans in lower priority groups (7 and 8) to a higher priority group. To produce our estimate of the maximum number of priority group 
Table D-1

Estimate for New VA Patients If Hypertension Becomes a Presumptively Service-Connected Condition for Vietnam Veterans (thousands)

\begin{tabular}{|c|c|c|c|c|}
\hline & \multicolumn{3}{|c|}{ Age of Veteran } & \multirow[b]{2}{*}{ Data Source } \\
\hline & $58-64$ & $65-74$ & $75-96$ & \\
\hline A. Vietnam-era (VNE) Veterans & 1,475 & 5,015 & 661 & NSV \\
\hline 1. Enrollees (1-6) & 742 & 1,794 & 342 & VA Business Intelligence Data \\
\hline 2. Enrollees (7-8) & 195 & 558 & 286 & VA Business Intelligence Data \\
\hline 3. Vietnam-era Veterans not enrolled & 538 & 2,663 & 32 & \\
\hline $\begin{array}{l}\text { 4. Non-enrollees with "other than } \\
\text { dishonorable" discharges }\end{array}$ & 508 & 2,513 & 30 & RAND analysis of ACS Data \\
\hline B. Vietnam theater Veterans & 319 & 2,441 & 322 & NSV \\
\hline C. Proportion of VNE Veterans in theater (B/A) & 0.22 & 0.49 & 0.49 & \\
\hline $\begin{array}{l}\text { D. Hypertension prevalence (non-user Vietnam- } \\
\text { era Veterans) }\end{array}$ & 0.52 & 0.64 & 0.61 & RAND analysis of MEPS Data \\
\hline E. Potential new enrollees $\left(A 4^{*} C^{*} D\right)$ & 57 & 779 & 9 & \\
\hline F. Hypertension prevalence in current VHA users & 0.67 & 0.71 & 0.71 & RAND analysis of MEPS Data \\
\hline G. Estimated number in theater $\left(A 2^{*} \mathrm{C}\right)$ & 42 & 272 & 140 & \\
\hline $\begin{array}{l}\text { H. Potential enrollees }(7-8) \text { moving up to a higher } \\
\text { priority group }\left(F^{*} G\right)\end{array}$ & 28 & 192 & 98 & \\
\hline I. Estimated use & 0.30 & 0.32 & 0.24 & RAND analysis of MEPS Data \\
\hline $\begin{array}{l}\text { J. Ceiling estimate for new users in priority groups } \\
1-6 \text { who served in theater and have hypertension } \\
((E+H) * I)\end{array}$ & 25 & 312 & 26 & \\
\hline Total & & 363 & & \\
\hline
\end{tabular}

SOURCE: RAND analysis of NSV, MEPS, ACS, and VA Business Intelligence (Enrollment) data.

promotions (row $\mathrm{H}$ ), we apply the hypertension prevalence and proportion of Veterans who served in theater to the number of Veterans enrolled in priority groups 7 and 8.

Up to this point, we have estimated the number of enrolled Veterans. To estimate the number who are likely to become new patients, we apply usage rates (by age) which we estimate using MEPS (row I).

Using the above procedure, we estimate that the maximum number of new VA patients in priority groups 1-6 for this presumptive eligibility scenario to be 363,000 new VA patients.

\section{D.1.3. Potential Impact of Future Conflict on VA Use \\ D.1.3.1. Maximum and Minimum End-Strengths in the Post-911 Period}

Figure D-1 reports the maximum and minimum number of non-civilian U.S. military personnel for each component of each service during the post-9/11 era. The top of each bar indicates the maximum, while the bottom of each bar denotes the minimum. The year to the right of each bar indicates the year in which that extreme occurred. Years before 2014 indicate that extreme value is derived from historical data, and years after 2014 derive from planning docu- 
ments. For example, the Army reached its largest number (just under 500,000) of enlisted reserve/National Guard personnel in 2014. For example, the active component of the Army will reach its smallest size in 2019 (around 350,000). Year labels omitted for segments that have not varied by more than 10,000 over the entire post-9/11 period.

Figure D-1 reveals wide variation in end-strengths, both over time and by service. Comparing components, reserve and National Guard components have experienced (or are expected to experience) far more variation in end-strength, compared with active components. However, the Air Force is a notable exception. Comparing services, the Army has both the largest size and the most variation in size. In all cases, officers compose a very small portion of total end-strength.

\section{D.1.3.2. Possible Range of Conflict Exposure, by Segment of the Military Personnel}

Figure D-2 reports the maximum and minimum percentage of separations in a given year that had been deployed into a combat environment. Following mostly peaceful late 1990s, less than 20 percent of separating service members had been deployed into a combat zone, as shown by the bottoms of the bars. During the latter years of the Afghanistan and Iraq missions, over 60 percent of active component separators had experienced at least one deployment. Several aspects of Figure D-2 are notable. First, officers in the active components all tended to experience higher rates of conflict exposure than the enlisted members of their service. Second, Marines and soldiers experienced significantly higher maximum rates of conflict exposure than airmen and sailors. Third, even among those separating before the start of the Iraq and Afghanistan missions and after serving during the mostly peaceful late 1990s, conflict exposure rates for active component separating service members hovered around 10 percent, not 0 percent.

Figure D-1

Maximum and Minimum Non-Civilian U.S. Military Personnel

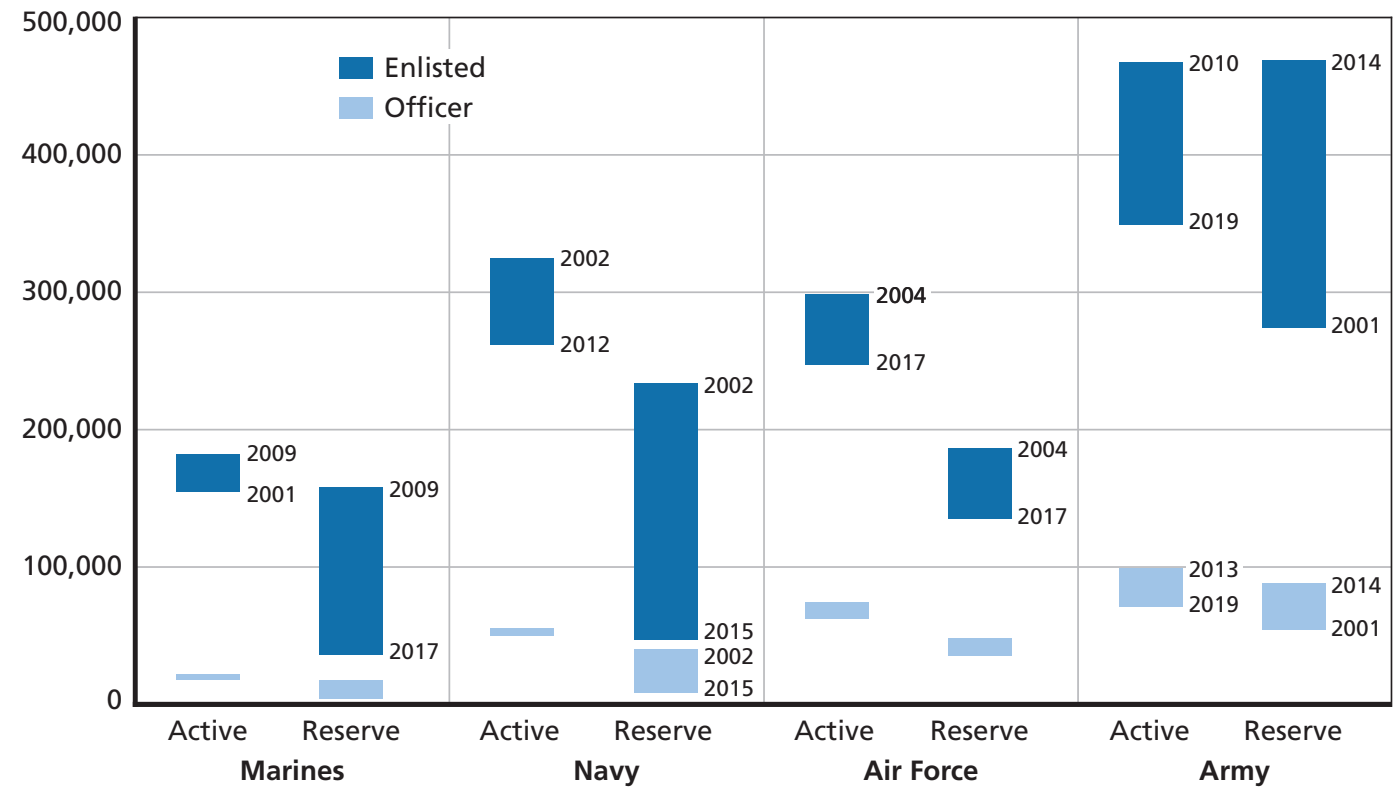

SOURCE: RAND analysis based on Census Bureau data (2012), Congressional Budget Office Analysis (2014), U.S. Army posture statements (2014), and DoD administrative data (2015). 
Figure D-2

Maximum and Minimum Rates of Hostile Deployment

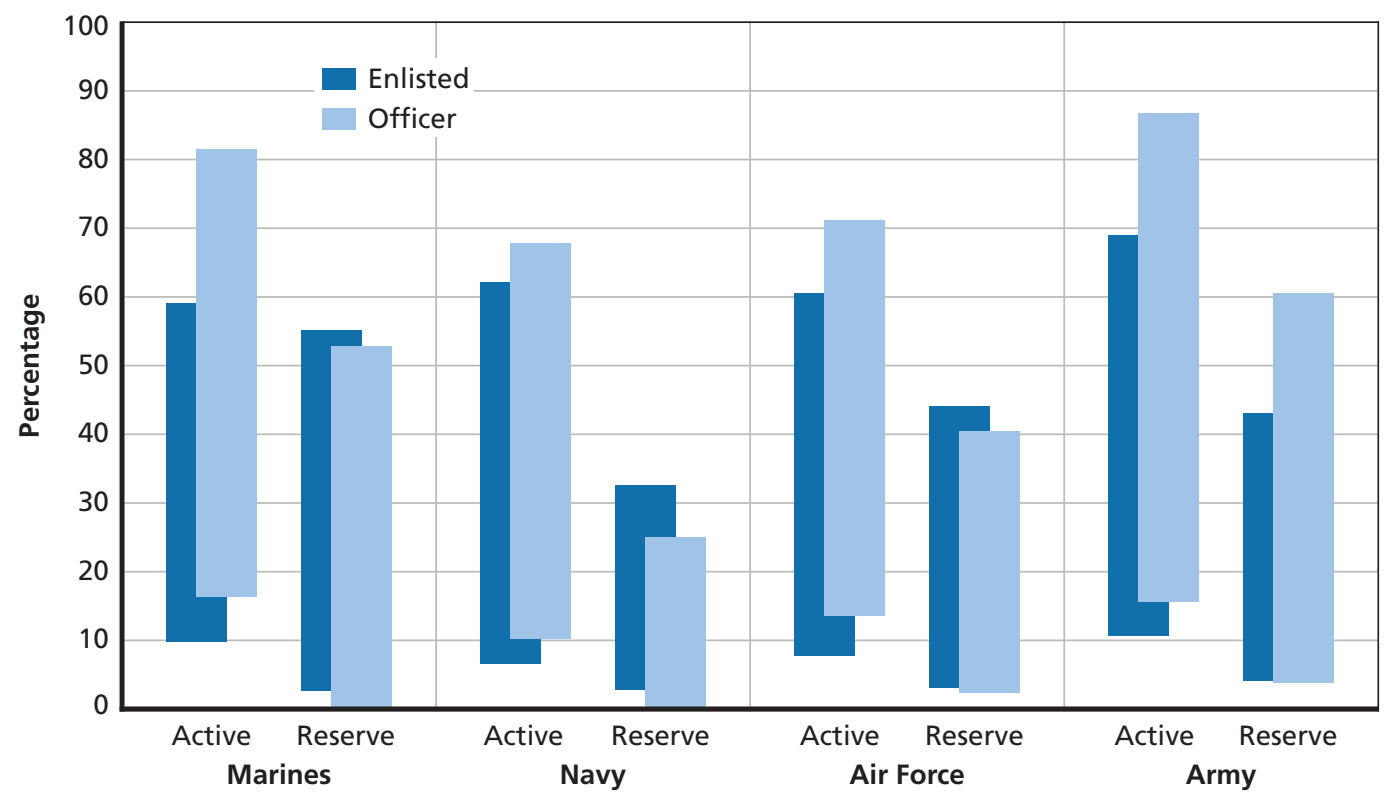

SOURCE: DoD administrative data (2014).

RAND RR1165z1-D.2

\section{D.1.3.3. Typical Length of Service}

Figure D-3 reports the percentile distribution of active officers/enlisted separating with a given number of years of service for each year between 2000 and 2014. The top panel reports years of service for enlisted members of the active component military. The bottom panel reports years of service for officers. In each figure, the shading indicates the percentile distribution of years of service among separating service members in that year. For example, in 2005, the bottom 3 percent of officers separated with a year or less of active duty service. 25 percent of officers separated with six years or less, 50 percent with 14 years or less, and so forth. In contrast, 50 percent of separating officers in 2000 had served 11 years-notably less. In essence, the darkest shading (50th percentile) plots the average years of service of separating service members, the next darkest shade (25th-75th percentile) reports the typical variation of years, and the lightest shade (3rd-97th percentile) provides some sense of the wider range of service years.

The top panel reports those figures for enlisted separations. The typical enlisted service member separates with three to 11 years of active service, with five or six years being most common. In general, the distribution of years of service is very skewed, with very few service members separating two years sooner than the average length of service, but many more than two years longer than the average. Despite stop loss, the surge, and the wars, the average years of service remained mostly steady throughout the 2000-2014 period.

The bottom panel reports years of service for officers. The average officer serves two to three times as long as average enlisted service member, with over a quarter spending more than 20 years in service. Iraq and Afghanistan may have affected the years of service among officers far more than enlisted. With the exception of 2006-2007, officers separating after 2003 averaged at least three more years in service, compared to those separating between 2000 and 2002. 
Figure D-3

Typical Years of Service for Active Component Service Members, Officers vs. Enlisted
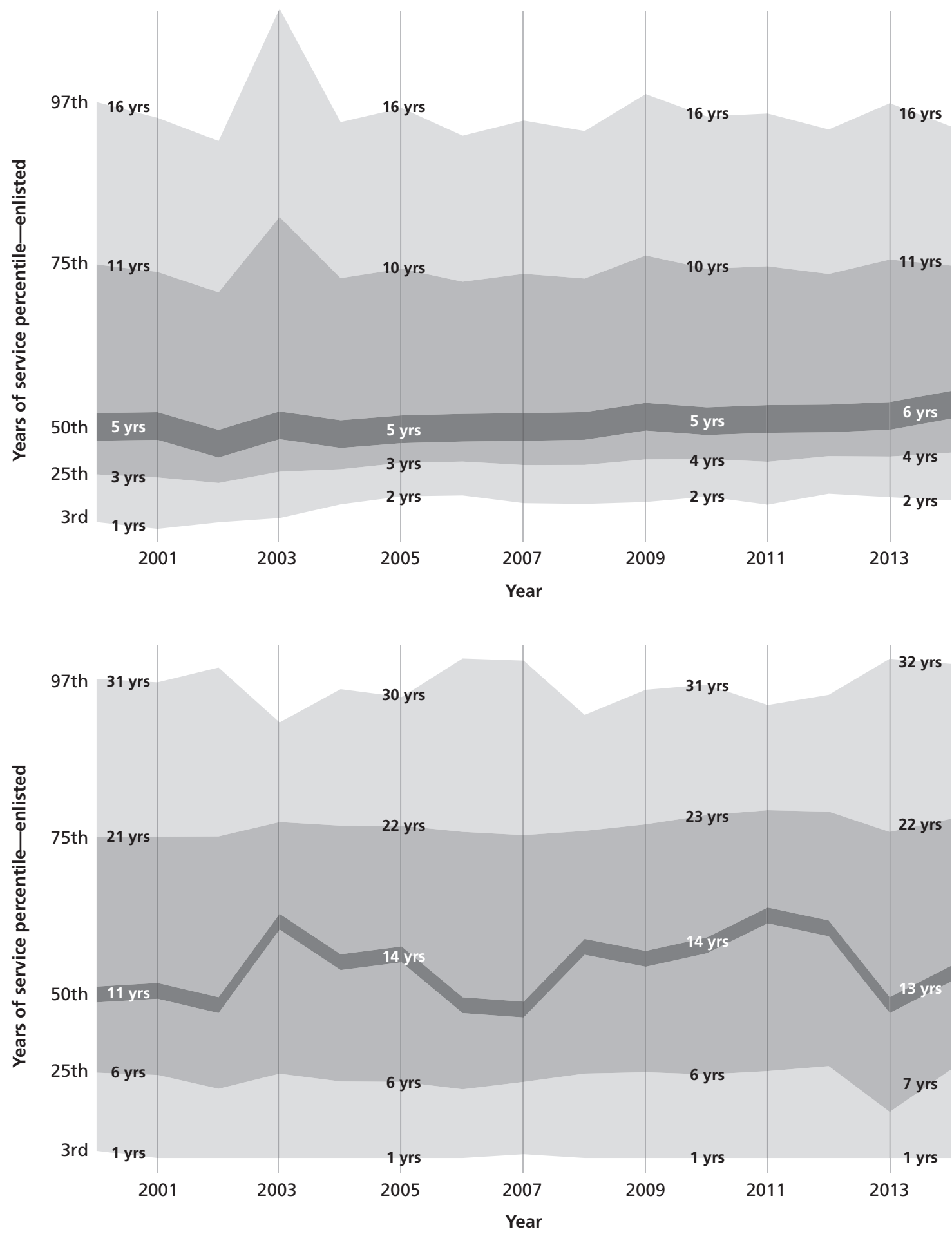

SOURCE: DoD administrative data (2014).

RAND RR1165z1-D.3 


\section{D.1.4. The ACA's Coverage Expansion}

Our approach to estimate the potential impact of the ACA coverage expansion combines two separate calculations: first, an estimate of the number of uninsured Veterans that could enroll in VA health care to obtain qualifying coverage under ACA and later use VA health care services; and second, an estimate of the number of current VA patients who will take advantage of new coverage opportunities and stop using VA services. Both calculations involve three related steps:

1. Estimate the starting population using the NSV.

2. Apply an estimate of how health insurance coverage will change over time from the RAND COMPARE microsimulation model, a tool designed to help policymakers understand the coverage impacts of health reform.

3. Apply enrollment and use rates to translate coverage changes into impacts measured in terms of the number of Veterans enrolled in VA health care and the number of VA patients. ${ }^{1}$

The fourth and final step is to sum the two separate impacts to arrive at an overall net impact.

This approach is intended to illustrate the potential impacts of the ACA coverage expansion. Evidence on the impacts of the ACA coverage expansion in general is developing but incomplete, and evidence on the impacts on Veterans specifically is sparse. In addition, key coverage expansion provisions may change in the future as a result of implementation decisions, such as states opting to expand their Medicaid programs. As a result of these uncertainties, in addition to reporting estimates from a set of base case assumptions we also discuss impacts under a wide range of other assumptions.

The following sections walk through our detailed methodology for each of the three steps, separately for the uninsured-to-enrollee and the patient-to-non-patient calculations.

\section{D.1.4.1. Estimating the Uninsured to VA Enrollee Impact}

As a first step, we used the NSV to calculate the number uninsured Veterans that were not VA patients. We used a "No" response to NSV item E2, "Have you ever used any VA health care benefits?" to identify Veterans that were not VA patients. We applied a hierarchical approach to categorize each respondent into a single coverage category. The hierarchical approach was necessary because Veterans often report multiple forms of coverage, for example, VA health care and Medicare. We used coverage information reported in NSV item E1. The specific criteria were as follows:

- Private coverage only: ("Insurance through a current or former employer or union (of yours or another family member)" OR "Insurance purchased directly from an insurance company (by you or another family member)") AND no other source of coverage reported.

- Medicare only: "Medicare, for people 65 and older, or people with certain disabilities" AND no other source of coverage reported.

- Medicaid only: "Medicaid, Medical Assistance, or any kind of government-assistance plan for those with low incomes or a disability" AND no other source of coverage reported.

1 Our analysis takes into account both the geographic distribution of Veterans and states' decisions to expand Medicaid. Veterans who live in non-expansion states are therefore less likely to gain a new source of insurance under the ACA than Veterans who live in expansion states. 
- TRICARE only: "TRICARE or other military health care" AND no other source of coverage reported.

- Other only: ("Indian Health Service" OR "Any other type of health insurance or health coverage plan") AND no other source of coverage reported.

- Private and Medicare: ("Insurance through a current or former employer or union (of yours or another family member)" OR "Insurance purchased directly from an insurance company (by you or another family member)") AND "Medicare, for people 65 and older, or people with certain disabilities" AND no other source of coverage reported.

- Medicare and TRICARE: "Medicare, for people 65 and older, or people with certain disabilities" AND "TRICARE or other military health care" AND no other source of coverage reported AND no other source of coverage reported.

- Any other combination: All other Veterans reporting health insurance coverage other than "CHAMPVA (Civilian Health and Medical Program of VA)"

- Uninsured or VA only: Veterans that did not satisfy any of the above criteria.

We also assigned each NSV respondent to a household income category using NSV item K2, "Which income range category represents your total combined income during the past 12 months?" For tractability and in order to ensure that we could link with other data, we used the single adult FPL thresholds and did not adjust for family size. The estimated numbers of uninsured, non-VA patient Veterans was 698,000 under 133 percent of the FPL, 227,000 between 133 and 400 percent of the FPL, and 81,000 above 400 percent of the FPL.

The next step involved applying estimates on the proportion of uninsured individuals that would gain coverage through some other source-for example, Medicaid or the health insurance exchanges. We used the RAND COMPARE ratios for the proportion of the uninsured population that would gain coverage by 2016 for the three income bands: 34 percent for $<133$ percent of the FPL, 69 percent for 133 to 400 percent of the FPL, and 63 percent above 400 percent of the FPL. We multiplied the starting population by these factors to determine how many Veterans would gain coverage and how many would not gain other coverage, assuming that Veterans had and took advantage of opportunities for other coverage at the same rates as the U.S. population by income band as estimated by COMPARE (see Table D-2).

We used our analysis in the ACS data (described earlier in Chapter Six) to estimate the share of Veterans that were not VA patients in different coverage and income categories that would be eligible to receive VA health care services. Some Veterans are not eligible to receive care due to a dishonorable discharge or insufficient length of service. Other Veterans would fall in priority groups $8 \mathrm{e}$ or $8 \mathrm{~g}$ which are not currently eligible to receive VA health care services. We estimated that virtually all Veterans under 133 percent of the FPL were potentially eligible. However, only 58 and 13 percent of Veterans in the 133 to 400 percent FPL and 400 percent FPL categories, respectively, were predicted to be eligible to receive care. We deflated the counts of Veterans in Table D-2 using these factors to estimate the number of uninsured Veterans that were not VA patients but that were predicted to be eligible to enroll to receive VA health care services (Table D-3).

We made different enrollment rate assumptions for different groups of Veterans. We applied a single enrollment rate (X percent) to uninsured, likely eligible Veterans that we predict would not gain other coverage regardless of income level. We used a more nuanced approach for uninsured, likely eligible Veterans that we predict would gain other coverage to account for the impacts of choices and Marketplace subsidies especially at lower income levels. 
Table D-2

Estimated Number of Uninsured Veterans Who Were Not VA Patients and Predicted to Gain or Not Gain Other Coverage by 2016

\begin{tabular}{lcccc}
\hline Poverty Level & $\begin{array}{c}\text { Uninsured, Non-VA } \\
\text { Patient Veterans }\end{array}$ & $\begin{array}{c}\text { RAND COMPARE Estimate, } \\
\text { Uninsured Gaining Coverage }\end{array}$ & $\begin{array}{c}\text { Gaining Other } \\
\text { Coverage }\end{array}$ & $\begin{array}{c}\text { Not Gaining Other } \\
\text { Coverage }\end{array}$ \\
\hline$<133 \% \mathrm{FPL}$ & 698,000 & $34.4 \%$ & 240,000 & 458,000 \\
$133-400 \% \mathrm{FPL}$ & 227,000 & $68.8 \%$ & 156,000 & 71,000 \\
$>400 \% \mathrm{FPL}$ & 81,000 & $62.7 \%$ & 51,000 & 30,000 \\
Total & $1,007,000$ & & 447,000 & 559,000 \\
\hline
\end{tabular}

Table D-3

Estimated Number of Uninsured Veterans Who Were Not VA Patients, Predicted to Be Eligible to Enroll in VA Health Care, and Predicted to Gain or Not Gain Other Coverage by 2016

\begin{tabular}{lccccc}
\hline Poverty Level & $\begin{array}{c}\text { Gaining Other } \\
\text { Coverage }\end{array}$ & $\begin{array}{c}\text { Not gaining } \\
\text { Other Coverage }\end{array}$ & $\begin{array}{c}\text { Percent Likely } \\
\text { Eligible To } \\
\text { Receive Care }\end{array}$ & $\begin{array}{c}\text { Gaining Other } \\
\text { Coverage and } \\
\text { Likely Eligible }\end{array}$ & $\begin{array}{c}\text { Not Gaining Other } \\
\text { Coverage and } \\
\text { Likely Eligible }\end{array}$ \\
\hline$<133 \%$ FPL & 240,000 & 458,000 & $100.0 \%$ & 240,000 & 458,000 \\
$133-400 \%$ FPL & 156,000 & 71,000 & $57.6 \%$ & 90,000 & 41,000 \\
$>400 \%$ FPL & 51,000 & 30,000 & $13.1 \%$ & 7,000 & 4,000 \\
Total & 447,000 & 559,000 & $89.9 \%$ & 337,000 & 503,000 \\
\hline
\end{tabular}

For these Veterans, we applied a base enrollment rate (Y percent) to the $>400$ percent FPL population. We applied half of this base enrollment rate to the 133-400 percent FPL population to account for the fact that Marketplace subsidies might make private coverage more appealing to Veterans. Finally, we applied a quarter of the base enrollment rate to the $<133$ percent FPL population because these Veterans can choose between Medicaid (in some states), Marketplace, and VA for low-cost coverage.

Table D-4 reports the number of enrollees at each 10-percent increment of the two key enrollment rates-X percent and $Y$ percent. We propose $X=50$ percent and $Y=50$ percent as a base case. The estimated number of new enrollees ranges from 0 in the upper left corner of Table D-4 to 614,000 in the lower right corner of Table D-4. The base case estimate is 307,000.

As a last step for this first of two calculations, we applied a use rate on top of the estimated number of newly enrolled Veterans to arrive at an estimate of new VA patients. The actual use rate for newly enrolled, previously uninsured Veterans is difficult to predict. Some newly insured Veterans might avail themselves of new coverage, although these Veterans had been eligible for coverage prior to ACA and opted not to enroll. Other Veterans might enroll to gain qualifying coverage but might not regularly interact with the VA health care system. Use rates for these Veterans may increase over time as they have more interaction and exposure to VA health care. In order to generate use rates for our analysis, we calculated the use rate across Veterans who have private coverage only using NSV data. The use rate was 14 percent for Veterans with incomes $>400$ percent of the FPL, 20 percent for Veterans with incomes between 134 and 400 percent of the FPL, and 25 percent for Veterans with incomes $<134$ percent of the FPL. Table D-5 reports estimates of new patients calculated by applying these use rates to the previous estimates of new enrollees. Under the base case assumptions, we estimate 74,000 new VA patients. 
Table D-4

Thousands of Estimated New VA Enrollees at Different Enrollment Rate Assumptions

\begin{tabular}{|c|c|c|c|c|c|c|c|c|c|c|c|}
\hline \multirow[b]{2}{*}{$\begin{array}{l}\text { Enrollment } \\
\text { Rate, No Other } \\
\text { Coverage }(X \%)\end{array}$} & \multicolumn{11}{|c|}{ Base Enrollment Rate, Other Coverage Options ( $Y \%$, which varies by income $\left.{ }^{a}\right)$} \\
\hline & $0 \%$ & $\begin{array}{c}10 \% \\
5 \% \\
2.5 \%\end{array}$ & $\begin{array}{c}20 \% \\
10 \% \\
5 \%\end{array}$ & $\begin{array}{l}30 \% \\
15 \% \\
7.5 \%\end{array}$ & $\begin{array}{l}40 \% \\
20 \% \\
10 \%\end{array}$ & $\begin{array}{c}50 \% \\
25 \% \\
12.5 \%\end{array}$ & $\begin{array}{l}60 \% \\
30 \% \\
15 \%\end{array}$ & $\begin{array}{c}70 \% \\
35 \% \\
17.5 \%\end{array}$ & $\begin{array}{l}80 \% \\
40 \% \\
20 \%\end{array}$ & $\begin{array}{c}90 \% \\
45 \% \\
22.5 \%\end{array}$ & $\begin{array}{c}100 \% \\
50 \% \\
25 \%\end{array}$ \\
\hline $0 \%$ & 0 & 11 & 22 & 34 & 45 & 56 & 67 & 78 & 89 & 101 & 112 \\
\hline $10 \%$ & 50 & 61 & 73 & 84 & 95 & 106 & 117 & 129 & 140 & 151 & 162 \\
\hline $20 \%$ & 101 & 112 & 123 & 134 & 145 & 156 & 168 & 179 & 190 & 201 & 212 \\
\hline $30 \%$ & 151 & 162 & 173 & 184 & 195 & 207 & 218 & 229 & 240 & 251 & 263 \\
\hline $40 \%$ & 201 & 212 & 223 & 235 & 246 & 257 & 268 & 279 & 290 & 302 & 313 \\
\hline $50 \%$ & 251 & 262 & 274 & 285 & 296 & 307 & 318 & 330 & 341 & 352 & 363 \\
\hline $60 \%$ & 302 & 313 & 324 & 335 & 346 & 357 & 369 & 380 & 391 & 402 & 413 \\
\hline $70 \%$ & 352 & 363 & 374 & 385 & 397 & 408 & 419 & 430 & 441 & 452 & 464 \\
\hline $80 \%$ & 402 & 413 & 424 & 436 & 447 & 458 & 469 & 480 & 491 & 503 & 514 \\
\hline $90 \%$ & 452 & 463 & 475 & 486 & 497 & 508 & 519 & 531 & 542 & 553 & 564 \\
\hline $100 \%$ & 503 & 514 & 525 & 536 & 547 & 558 & 570 & 581 & 592 & 603 & 614 \\
\hline
\end{tabular}

${ }^{a}$ For Veterans who gain other coverage options as a result of the ACA, we assume their enrollment rate varies depending on the degree of subsidy that they receive for these options. Veterans who gain access to unsubsidized employer coverage have a VA enrollment rate of $Y \%$, Veterans who gain access to Marketplace subsidies have an enrollment rate of $\mathrm{Y} / 2 \%$, and Veterans who gain access to Medicaid coverage have an enrollment rate of $\mathrm{Y} / 4 \%$.

\section{D.1.4.2. Estimating the Impact of Veterans Gaining Other Coverage}

For the second calculation, we started in the same way as the first calculation, by estimating the size of the relevant population using NSV. We used the same approach to defining coverage to estimate the number of Veterans in each income group that was enrolled in VA health care and did not have any other source of health care coverage. We also applied the same factors from RAND COMPARE to estimate the proportion of Veterans in each category that could gain another form of coverage by 2016. Table D-6 reports the number of Veterans in each income category overall and by whether or not they could gain another form of coverage.

We calculated a reduction in the number of VA patients assuming that the 727,000 Veterans would shift to a lower use rate than their current rates. Use rates for Veterans with no other source of health coverage are very high-85 percent for Veterans over 400 percent of the FPL and approximately 97 percent for Veterans at lower incomes. Use rates calculated across all Veterans with Medicaid or Private insurance are significantly lower (between 14 and 25 percent). In addition, Veterans eligible for exchange subsidies may have to dis-enroll in VA health care to qualify for subsidies. We decreased use rates by 25 percent to reflect lower utilization of VA health care services by Veterans with multiple coverage options.

If all Veterans that we predict will gain coverage do so and shift to the lower use rate immediately, we would expect 172,000 fewer VA patients in the following year. Different com- 
Table D-5

Thousands of Estimated New VA Patients at Different Enrollment Rate Assumptions

\begin{tabular}{|c|c|c|c|c|c|c|c|c|c|c|c|}
\hline \multirow[b]{2}{*}{$\begin{array}{l}\text { Enrollment } \\
\text { Rate, No Other } \\
\text { Coverage (X\%) }\end{array}$} & \multicolumn{11}{|c|}{ Base Enrollment Rate, Other Coverage Options ( $\mathrm{Y} \%$, which varies by income ${ }^{\mathrm{a}}$ ) } \\
\hline & $0 \%$ & $\begin{array}{c}10 \% \\
5 \% \\
2.5 \%\end{array}$ & $\begin{array}{c}20 \% \\
10 \% \\
5 \%\end{array}$ & $\begin{array}{l}30 \% \\
15 \% \\
7.5 \%\end{array}$ & $\begin{array}{l}40 \% \\
20 \% \\
10 \%\end{array}$ & $\begin{array}{c}50 \% \\
25 \% \\
12.5 \%\end{array}$ & $\begin{array}{l}60 \% \\
30 \% \\
15 \%\end{array}$ & $\begin{array}{c}70 \% \\
35 \% \\
17.5 \%\end{array}$ & $\begin{array}{l}80 \% \\
40 \% \\
20 \%\end{array}$ & $\begin{array}{c}90 \% \\
45 \% \\
22.5 \%\end{array}$ & $\begin{array}{r}100 \% \\
50 \% \\
25 \%\end{array}$ \\
\hline $0 \%$ & 0 & 2 & 5 & 7 & 10 & 12 & 15 & 17 & 20 & 22 & 25 \\
\hline $10 \%$ & 12 & 15 & 17 & 20 & 22 & 25 & 27 & 30 & 32 & 35 & 37 \\
\hline $20 \%$ & 24 & 27 & 29 & 32 & 34 & 37 & 39 & 42 & 44 & 47 & 49 \\
\hline $30 \%$ & 37 & 39 & 42 & 44 & 47 & 49 & 52 & 54 & 57 & 59 & 62 \\
\hline $40 \%$ & 49 & 51 & 54 & 56 & 59 & 61 & 64 & 66 & 69 & 71 & 74 \\
\hline $50 \%$ & 61 & 64 & 66 & 69 & 71 & 74 & 76 & 79 & 81 & 84 & 86 \\
\hline $60 \%$ & 73 & 76 & 78 & 81 & 83 & 86 & 88 & 91 & 93 & 96 & 98 \\
\hline $70 \%$ & 86 & 88 & 91 & 93 & 96 & 98 & 101 & 103 & 105 & 108 & 110 \\
\hline $80 \%$ & 98 & 100 & 103 & 105 & 108 & 110 & 113 & 115 & 118 & 120 & 123 \\
\hline $90 \%$ & 110 & 113 & 115 & 118 & 120 & 122 & 125 & 127 & 130 & 132 & 135 \\
\hline $100 \%$ & 122 & 125 & 127 & 130 & 132 & 135 & 137 & 140 & 142 & 145 & 147 \\
\hline
\end{tabular}

a We hold the use rate constant across all scenarios but allow the enrollment rate to vary. For Veterans who gain other coverage options as a result of the ACA, we assume their enrollment rate varies depending on the degree of subsidy that they receive for these options. Veterans who gain access to unsubsidized employer coverage have a VA enrollment rate of $Y \%$, Veterans who gain access to Marketplace subsidies have an enrollment rate of $Y / 2 \%$, and Veterans who gain access to Medicaid coverage have an enrollment rate of $Y / 4 \%$. The use rate is 14 percent for Veterans with incomes above 400 percent of FPL, 20 percent for Veterans with incomes between 134 and 400 percent of FPL, and 25 percent for Veterans with incomes below 134 percent of FPL.

Table D-6

Estimated Number of Veterans with Only VA Coverage and Predicted to Gain or Not Gain Other Coverage by 2016

\begin{tabular}{|c|c|c|c|c|}
\hline Poverty Level & $\begin{array}{l}\text { Uninsured, Non-VA } \\
\text { Patient Veterans }\end{array}$ & $\begin{array}{l}\text { RAND COMPARE Estimate, } \\
\text { Uninsured Gaining Coverage }\end{array}$ & $\begin{array}{l}\text { Gaining Other } \\
\text { Coverage }\end{array}$ & $\begin{array}{c}\text { Not Gaining } \\
\text { Other Coverage }\end{array}$ \\
\hline$<133 \% \mathrm{FPL}$ & 761,000 & $34.4 \%$ & 262,000 & 500,000 \\
\hline $133-400 \% \mathrm{FPL}$ & 479,000 & $68.8 \%$ & 329,000 & 149,000 \\
\hline$>400 \% \mathrm{FPL}$ & 208,000 & $62.7 \%$ & 130,000 & 77,000 \\
\hline Total & $1,447,000$ & & 721,000 & 726,000 \\
\hline
\end{tabular}

binations of these Veterans' current and potential future use rates result in estimates ranging from 0 fewer patients (assuming that these Veterans continue to use VA health care at the same rates that they do today despite gaining coverage) to 721,000 fewer patients if all Veterans predicted to gain other coverage immediately begin accessing care through their other coverage rather than VA. 


\section{D.1.4.3. Net Impact}

Under the base case, the net impact is the sum of 74,000 new, previously uninsured VA patients and 172,000 fewer VA patients due to Veterans gaining other coverage, or a net decrease of about 98,000 VA patients. Different combinations of assumptions at different points in the analysis yield significantly different net impacts_-and in some cases net increases in the number of VA patients, for example, if Veterans gaining new coverage continue to use VA health care services at high rates and significant shares of uninsured Veterans enroll to gain qualifying coverage and then use health care services. 


\section{References}

Aday, L. A., \& Andersen, R. (1974). A framework for the study of access to medical care. Health Services Research, 9(3), 208-220.

Agency for Healthcare Research and Quality. (undated). HCUPnet: A tool for identifying, tracking, and analyzing national hospital statistics. Rockville, MD. Retrieved from http://hcupnet.ahrq.gov/

. (2004). MEPS-MPC sample design and data collection process. Retrieved from

http://meps.ahrq.gov/mepsweb/survey_comp/mpc_data_collection.jsp

. (2014). 2013 national healthcare disparities report. Rockville, MD.

Asch, B. J., Heaton, P., Hosek, J. R., Martorell, F., Simon, C., \& Warner, J. T. (2010). Do bonuses affect enlistment and reenlistment? Santa Monica, CA: RAND Corporation, RB-9539-OSD. Retrieved from http://www.rand.org/pubs/research_briefs/RB9539.html

Asch, S. M., McGlynn, E. A., Hogan, M. M., Hayward, R. A., Shekelle, P., Rubenstein, L., Keesey, J., Adams, J., \& Kerr, E. A. (2004). Comparison of quality of care for patients in the Veterans Health Administration and patients in a national sample. Annals of Internal Medicine, 141(12).

Beaghen, M., \& Weidman, L. (2008). Statistical issues of interpretation of the American Community Survey's one-, three-, and five-year period estimates. U.S. Census Bureau. Retrieved from https://usa.ipums.org/usa/resources/MYE_Guidelines.pdf

Bedimo, R. J., McGinnis, K. A., Dunlap, M., Rodriguez-Barradas, M. C., \& Justice, A. C. (2009). Incidence of non-AIDS-defining malignancies in HIV-infected vs. non-infected patients in the HAART era: Impact of immunosuppression. Journal of Acquired Immune Deficiency Syndromes (1999), 52(2), 203.

Beehler, G. P., Rodrigues, A. E., Mercurio-Riley, D., \& Dunn, A. S. (2013). Primary care utilization among Veterans with chronic musculoskeletal pain: A retrospective chart review. Pain Medicine, 14(7), 1021-1031.

Benetsky, M. J., and Fields, A. (2015). Millennial migration: How has the Great Recession affected the migration of a cohort as it came of age? U.S. Census Bureau, SEHSD Working Paper Number 2015-1.

Beronio, K., Po, R., Skopec, L., \& Glied, S. (2013). Affordable Care Act expands mental health and substance use disorder benefits and federal parity protections for 62 million Americans. Office of the Assistant Secretary for Planning and Evaluation.

Bertakis, K. D., \& Azari, R. (2010). Patient gender differences in the prediction of medical expenditures. Journal of Women's Health, 19(10), 1925-1932.

Bertakis, K. D., Azari, R., Helms, L. J., Callahan, E. J., \& Robbins, J. A. (2000). Gender differences in the utilization of health care services. Journal of Family Practice, 49(2), 147-152.

Bhandari, A., \& Wagner, T. (2006). Self-reported utilization of health care services: Improving measurement and accuracy. Medical Care Research and Review, 63(2): 217-235.

Blanchard, M. S., Eisen, S. A., Alpern, R., Karlinsky, J., Toomey, R., Reda, D. J., Murphy, F. M., Jackson, L. W., \& Kang, H. K. (2006). Chronic multisymptom illness complex in Gulf War I Veterans 10 years later. American Journal of Epidemiology, 163(1), 66-75. 
Bohnert, A. S., Ilgen, M. A., Bossarte, R. M., Britton, P. C., Chermack, S. T., \& Blow, F. C. (2012). Veteran status and alcohol use in men in the United States. Military Medicine, 177(2), 198-203.

Boyle, A. M. (2014). Recruits, military breathe easier with relaxed asthma accession standards. U.S. Medicine. Retrieved from http://www.usmedicine.com/agencies/department-of-defense-dod/ recruits-military-breathe-easier-with-relaxed-asthma-accession-standards/

Brackley, M. E., Penning, M. J., \& Lesperance, M. L. (2006). In the absence of cancer registry data, is it sensible to assess incidence using hospital separation records? International Journal for Equity in Health, 5, 12. doi: $10.1186 / 1475-9276-5-12$

Brady, S. D. (2014). Assessing stop-loss policy options through personnel flow modeling. Santa Monica, CA: RAND Corporation, DB-573-OSD. Retrieved from http://www.rand.org/pubs/documented_briefings/DB573.html

Brauner, M. K., Jackson, T., \& Gayton, E. (2012). Medical readiness of the reserve component. Santa Monica, CA: RAND Corporation, MG-1105-OSD. Retrieved from http://www.rand.org/pubs/monographs/MG1105.html

Buckman, J. E. J., Sundin, J., Greene, T., Fear, N. T., Dandeker, C., Greenberg, N., \& Wessely, S. (2010). The impact of deployment length on the health and well-being of military personnel: A systematic review of the literature. Occupational and Environmental Medicine, 68(1), 69-76. oem. 2009.054692.

Bureau of Labor Statistics. (2015). News release: Employment situation of Veterans-2014. Washington, DC: U.S. Department of Labor.

Carlson, K. F., Barnes, J. E., Hagel, E. M., Taylor, B. C., Cifu, D. X., \& Sayer, N. A. (2013). Sensitivity and specificity of traumatic brain injury diagnosis codes in United States Department of Veterans Affairs administrative data. Brain Injury, 27(6), 640-650. doi: 10.3109/02699052.2013.771795

Carman, K. G., \& Eibner, C. (2014). Changes in health insurance enrollment since 2013: Evidence from the RAND Health Reform Opinion Study. Santa Monica, CA: RAND Corporation, RR-656-RC. Retrieved from http://www.rand.org/pubs/research_reports/RR656.html

Carter, Philip. (2013, November 10). The Vets we reject and ignore. The New York Times. Retrieved from http://www.nytimes.com/2013/11/11/opinion/the-vets-we-reject-and-ignore.html?_r=1

Centers for Disease Control and Prevention. (undated). Ambulatory health care data. Retrieved from http://www.cdc.gov/nchs/ahcd.htm

- (2009). Prevalence and most common causes of disability among adults-United States, 2005. Morbidity and Mortality Weekly Report, 58(16), 421-426.

_. (2012, June 13). About the National Health Interview Survey. Retrieved from http://www.cdc.gov/nchs/nhis/about_nhis.htm

. (2013). CDC health disparities and inequalities report-United States, 2013. Morbidity and Mortality Weekly Report Supplements, 62(3). Retrieved from http:/www.cdc.gov/mmwr/pdf/other/su6203.pdf

. (2014a). About BRFSS. Retrieved from http://www.cdc.gov/brfss/about/index.htm

. (2014b). QuickStats: Current smoking among men aged 25-64 years, by age group and Veteran status-National Health Interview Survey (NHIS), United States, 2007-2010. Morbidity and Mortality Weekly Report, 61, 929.

. (2014c). Rates of TBI-related emergency department visits by age group-United States, 2001-2010. Retrieved from http://www.cdc.gov/traumaticbraininjury/data/rates_ed_byage.html

. (2015). QuickStats: Percentage of men aged 25-74 years who consume $\geq 15$ alcoholic drinks per week, by age group and Veteran status. Morbidity and Mortality Weekly Report, 64(6), 160.

Centers for Medicare \& Medicaid Services. (undated). ACO \#9_Prevention Quality Indicator (PQI): Ambulatory sensitive conditions admissions for chronic obstructive pulmonary disease (COPD) or asthma in older adults.

(2015a). Medicaid \& CHIP: February 2015 monthly applications, eligibility determinations and enrollment report. Baltimore, MD: U.S. Department of Health and Human Services. 
(2015b). Physician quality reporting system measures codes. Retrieved from http://www.cms.gov/ Medicare/Quality-Initiatives-Patient-Assessment-Instruments/PQRS/MeasuresCodes.html

Chao, S. Y., Zarzabal, L. A., Walker, S. M., Herzog, C. M., Eilerman, P. A., Luce, B. K., \& Carnahan, D. H. (2013). Estimating diabetes prevalence in the Military Health System population from 2006 to 2010. Military Medicine, 178(9), 986-993. doi: 10.7205/milmed-d-13-00147

Charlson, M. E., Pompei, P., Ales, K. L., \& MacKenzie, C. R. (1987). A new method of classifying prognostic comorbidity in longitudinal studies: Development and validation. Journal of Chronic Diseases, 40(5), 373-383.

Congressional Budget Office. (2007). The health care system for Veterans: An interim report. Washington, DC.

. (2009). Quality initiatives undertaken by the Veterans Health Administration. Washington, DC.

. (2012). The Veterans Health Administration's treatment of PTSD and traumatic brain injury among recent combat Veterans. Washington, DC.

. (2014a). Long-term implications of the 2015 Future Years Defense Program. Washington, DC.

. (2014b). Veterans' disability compensation: Trends and policy options. Washington, DC.

Cordova, A., Girosi, F., Nowak, S., Eibner, C., \& Finegold, K. (2013). The COMPARE Microsimulation Model and the US Affordable Care Act. International Journal of Microsimulation, 6(3), 78-117.

Counseling and Treatment for Sexual Trauma, 38 U.S.C. $\$ 1720 D$ (2011). Retrieved from

http:/www.gpo.gov/fdsys/pkg/USCODE-2011-title38/pdf/

USCODE-2011-title38-partII-chap17-subchapII-sec1720D.pdf

Cowper, D. C., \& Longino, C. F., Jr. (1992). Veteran interstate migration and VA health service use. The Gerontologist, 32(1), 44-50.

De Haas, H. (2007). The myth of invasion: Irregular migration from West Africa to the Maghreb and the European Union. International Migration Institute, University of Oxford.

- (2009). Migration transitions: A theoretical and empirical inquiry into the developmental drivers of international migration. Paper presented at the International Population Conference, Marrakech, Morocco.

Defense Manpower Data Center. (2014). Active duty military personnel by rank/grade.

Definitions, 38 U.S.C. $\$ 101$ (2011). Retrieved from

http://www.gpo.gov/fdsys/pkg/USCODE-2011-title38/pdf/USCODE-2011-title38-partI-chap1-sec101.pdf

Disabled American Veterans. (2015). Women Veterans: The long journey home. West Haven, CT.

Dobkin, C., \& Shabani, R. (2009). The health effects of military service: Evidence from the Vietnam draft. Economic Inquiry, 47(1), 69-80.

Dursa, E. K., Reinhard, M. J., Barth, S. K., \& Schneiderman, A. I. (2014). Prevalence of a positive screen for PTSD among OEF/OIF and OEF/OIF-era Veterans in a large population-based cohort. Journal of Traumatic Stress, 27(5), 542-549.

Elbogen, E. B., Wagner, H. R., Johnson, S. C., Kinneer, P., Kang, H., Vasterling, J. J., Timko, C., \& Beckham, J. C. (2013). Are Iraq and Afghanistan Veterans using mental health services? New data from a national random-sample survey. Psychiatric Services 64(2), 134-141.

Elhai, J. D., Grubaugh, A. L., Richardson, J. D., Egede, L. E., \& Creamer, M. (2008). Outpatient medical and mental healthcare utilization models among military Veterans: Results from the 2001 National Survey of Veterans. Journal of Psychiatric Research, 42(10), 858-867. doi: 10.1016/j.jpsychires.2007.09.006

Enrollment-Provision of Hospital and Outpatient Care to Veterans Subpriorities of Priority Categories 7 and 8 and Annual Enrollment Level Decision, 68 C.F.R. $\$ 2670$ (2003). Retrieved from http://www.gpo.gov/fdsys/pkg/FR-2003-01-17/pdf/03-1201.pdf

Epidemiology Program Office of Public Health. (2015). Analysis of VA health care utilization among Operation Enduring Freedom (OEF), Operation Iraqi Freedom (OIF), and Operation New Dawn (OND) Veterans. Department of Veterans Affairs.

Expansion of Enrollment in the VA Health Care System, 74 Fed. Reg. 22832 (May 15, 2009). 
Expiring Authorities Act of 2014, Public Law 113-175 (2014).

Fahimi, M., Link, M., Mokdad, A., Schwartz, D.A., \& Levy, P. (2008). Tracking chronic disease and risk behavior prevalence as survey participation declines: Statistics from the behavioral risk factor surveillance system and other national surveys. Preventing Chronic Disease, 5(3), A80.

Fiest, K. M., Jette, N., Quan, H., St Germaine-Smith, C., Metcalfe, A., Patten, S. B., \& Beck, C. A. (2014). Systematic review and assessment of validated case definitions for depression in administrative data. $B M C$ Psychiatry, 14, 289. doi: 10.1186/s12888-014-0289-5

Fischer, H. (2010). U.S. Military Casualty Statistics: Operation New Dawn, Operation Iraqi Freedom, and Operation Enduring Freedom. Washington, DC.

Frayne, S. M., Parker, V. A., Christiansen, C. L., Loveland, S., Seaver, M. R., Kazis, L. E., \& Skinner, K. M. (2006). Health status among 28,000 women Veterans: The VA women's health program evaluation project. Journal of General Internal Medicine, 21, S40-S46. doi: 10.1111/j.1525-1497.2006.00373.x

Frayne, S. M., Phibbs, C. S., Saechao, F., Maisel, N. C., Friedman, S. A., Finlay, A., Berg, E., Balasubramanian, V., Dally, S. K., Ananth, L., Romodan, Y., Lee, J., Iqbal, S., Hayes, P. M., Zephyrin, L., Whitehead, A., Torgal, A., Katon, J. G., \& Haskell, S. (2014). Sourcebook: Women Veterans in the Veterans Health Administration. Volume 3. Sociodemographics, utilization, costs of care, and health profile. Washington, DC.

Frieden, T., \& Collins, F. (Eds.). (2013). Report to Congress on traumatic brain injury in the United States: Understanding the public health problem among current and former military personnel. Centers for Disease Control and Prevention, National Institutes of Health, U.S. Department of Defense, and U.S. Department of Veterans Affairs.

Garfield, R., Damico, A., Stephens, J., \& Rouhani, S. (2014). The coverage gap: Uninsured poor adults in states that do not expand Medicaid-An update. Kaiser Family Foundation.

Garvey Wilson, A. L., Messer, S. C., \& Hoge, C. W. (2009). U.S. military mental health care utilization and attrition prior to the wars in Iraq and Afghanistan. Social Psychiatry and Psychiatric Epidemiology, 44(6), 473-481. doi: 10.1007/s00127-008-0461-7

Goldberg, M. S. (2015). Testimony on comparing the costs of the Veterans' health care system with private-sector costs. Washington, DC: Congressional Budget Office. Retrieved from http:/www.cbo.gov/sites/default/files/cbofiles/attachments/49905-Testimony.pdf

Golding, H. L. W. (2011, July 27). Potential costs of health care for Veterans of recent and ongoing U.S. military operations. Testimony before the Committee on Veterans' Affairs, U.S. Senate. Washington, DC.

Greenwood, M. J., Hunt, G. L., Rickman, D. S., \& Treyz, G. I. (1991). Migration, regional equilibrium, and the estimation of compensating differentials. The American Economic Review, 81(5), 1382-1390. doi: $10.2307 / 2006927$

Hagel, C. (2014, February 24). Secretary of Defense speech: FY15 budget preview. Washington, DC: U.S. Department of Defense. Retrieved from http://www.defense.gov/Speeches/Speech.aspx?SpeechID=1831

Hagen, E. M., Rekand, T., Gilhus, N. E., \& Gronning, M. (2009). Diagnostic coding accuracy for traumatic spinal cord injuries. Spinal Cord, 47(5), 367-371. doi: 10.1038/sc.2008.118

Head, K. (2000). Gravity for beginners. Paper presented at the Rethinking the Line: The Canada-U.S. Border Conference, Vancouver, British Columbia, Canada.

Healthcare Cost and Utilization Project. (2015, June). Clinical Classifications Software (CCS) for ICD-9-CM. Agency for Healthcare Research and Quality. Rockville, MD. Retrieved from https://www.hcup-us.ahrq.gov/toolssoftware/ccs/ccs.jsp

Heidenreich, P. A., Trogdon, J. G., Khavjou, O. A., Butler, J., Dracup, K., Ezekowitz, M. D., Finkelstein, E. A., Hong, Y., Johnston, S. C., Khera, A., Lloyd-Jones, D. M., Nelson, S. A., Nichol, G., Orenstein, D., Wilson, P. W. F., \& Woo, Y. J. (2011). Forecasting the future of cardiovascular disease in the United States: A policy statement from the American Heart Association. Circulation, 123, 933-944. 
Hicks, J. (2014). House panel to examine VA's progress with backlog of disability claims. Washington Post. Retrieved from

http://www.washingtonpost.com/blogs/federal-eye/wp/2014/07/14/

house-panel-to-examine-vas-progress-with-backlog-of-disability-claims

Hirsch, A. G., \& Scheck McAlearney, A. (2013). Measuring diabetes care performance using electronic health record data: The impact of diabetes definitions on performance measure outcomes. American Journal of Medical Quality, 29(4), 292-299. doi: 10.1177/1062860613500808

Hoge, C. W., Auchterlonie, J. L., \& Milliken, C. S. (2006). Mental health problems, use of mental health services, and attrition from military service after returning from deployment to Iraq or Afghanistan. JAMA, 295(9), 1023-1032.

Hoge, C. W., Castro, C. A., Messer, S. C., McGurk, D., Cotting, D. I., \& Koffman, R. L. (2004). Combat duty in Iraq and Afghanistan, mental health problems, and barriers to care. New England Journal of Medicine, 351(1), 13-22.

Holder, K. (2014). Employment status and occupations of Gulf War-era Veterans. American Community Survey Briefs Number 13-22.

Holowka, D. W., Marx, B. P., Gates, M. A., Litman, H. J., Ranganathan, G., Rosen, R. C., \& Keane, T. M. (2014). PTSD diagnostic validity in Veterans Affairs electronic records of Iraq and Afghanistan Veterans. Journal of Consulting and Clinical Psychology, 82(4), 569-579. doi: 10.1037/a0036347

Holt, J. B., Huston, S. L., Heidari, K., Schwartz, R., Gollmar, C. W., Tran, A., Bryan, L., Liu, Y., \& Croft, J. B. (2015). Indicators for chronic disease surveillance-United States, 2013. In CDC (Ed.), Morbidity and Mortality Weekly Recommendations and Reports, 64, 1-246.

Hosek, J. R., \& Martorell, F. (2009). Military reenlistment and deployment during the war on terrorism. Santa Monica, CA: RAND Corporation, RB-9468-OSD. Retrieved from http://www.rand.org/pubs/research_briefs/RB9468.html

Hoyert, D. L., \& Xu, J. Q. (2012, October 10). Deaths: Preliminary data for 2011. National Vital Statistics Reports, 61(6).

Hsu, C., Ried, L. D., Bengtson, M. A., Garman, P. M., McConkey, J. R., \& Rahnavard, F. (2008). Metabolic monitoring in Veterans with schizophrenia-related disorders and treated with second-generation antipsychotics: Findings from a Veterans Affairs-based population. Journal of the American Pharmacists Association (2003), 48(3), 393-400. doi: 10.1331/JAPhA.2008.07007

Institute of Medicine of the National Academies. (2013). Veterans and Agent Orange: Update 2012. Committee to Review the Health Effects in Vietnam Veterans of Exposure to Herbicides (Ninth Biennial Update). Washington, DC: National Academies Press.

- (2014a). Chronic multisymptom illness in Gulf War Veterans: Case definitions reexamined. Washington, DC.

. (2014b). Treatment for posttraumatic stress disorder in military and Veteran populations: Final assessment. Washington, DC.

Integrated Public Use Microdata Series. (undated). MIGPUMA1. Retrieved from https://usa.ipums.org/usa-action/variables/MIGPUMA1\#description_section

Johnson, N. B., Hayes, L. D., Brown, K., Hoo, E. C., \& Ethier, K. A. (2014). CDC national health report: Leading causes of morbidity and mortality and associated behavioral risk and protective factors-United States, 2005-2013. Morbidity and Mortality Weekly Report Supplements, 63(4), 3-27.

Justice, A. C., Lasky, E., McGinnis, K. A., Skanderson, M., Conigliaro, J., Fultz, S. L., Crothers, K., Rabeneck, L., Rodriguez-Barradas, M., Weissman, S. B., \& Bryant, K. (2006). Medical disease and alcohol use among Veterans with human immunodeficiency infection: A comparison of disease measurement strategies. Medical Care, 44(8), S52-S60.

Kaiser Family Foundation. (2015). State health facts: Status of state action on the Medicaid expansion decision. Retrieved from http://kff.org/health-reform/state-indicator/

state-activity-around-expanding-medicaid-under-the-affordable-care-act 
Kaplan, M. S., Huguet, N., McFarland, B. H., \& Newsom, J. T. (2007). Suicide among male Veterans: A prospective population-based study. Journal of Epidemiology and Community Health, 61(7), 619-624. doi: 10.1136/jech.2006.054346

Kaplan, M. S., McFarland, B. H., Huguet, N., \& Valenstein, M. (2012). Suicide risk and precipitating circumstances among young, middle-aged, and older male Veterans. American Journal of Public Health, 102(1), S131-S137. doi: 10.2105/AJPH.2011.300445

Karney, B. R., Loughran, D. S., \& Pollard, M. S. (2012). Comparing marriage status and divorce status in civilian and military populations. Journal of Family Issues, 33(12) 1572-1594. Retrieved from http://jfi.sagepub.com/content/33/12/1572.full.pdf+html

Kavanagh, J. (2013). Are U.S. military interventions contagious: Intervention timing and its implications for force planning. Santa Monica, CA: RAND Corporation, RR-192-A. Retrieved from http://www.rand.org/pubs/research_reports/RR192.html

Kline, A., Falca-Dodson, M., Sussner, B., Ciccone, D. S., Chandler, H., Callahan, L., \& Losonczy, M. (2010). Effects of repeated deployment to Iraq and Afghanistan on the health of New Jersey Army National Guard troops: Implications for military readiness. American Journal of Public Health, 100(2), 276.

Kramarow, E. A., and Pastor, P. N. (2012). The health of male Veterans and nonveterans aged 25-64: United States, 2007-2010. National Center for Health Statistics Data Brief 101, Centers for Disease Control and Prevention. Retrieved from http://www.cdc.gov/nchs/data/databriefs/db101.pdf

Kramer, J. R., Davila, J. A., Miller, E. D., Richardson, P., Giordano, T. P., \& El-Serag, H. B. (2008). The validity of viral hepatitis and chronic liver disease diagnoses in Veterans Affairs administrative databases. Alimentary Pharmacology and Therapeutics, 27(3), 274-282. doi: 10.1111/j.1365-2036.2007.03572.x

Krumholz, H. M., Normand, S. T., \& Wang, Y. (2014). Trends in hospitalizations and outcomes for acute cardiovascular disease and stroke, 1999-2011. Circulation, 130(12), 966-975.

Lee, E. S. (1966). A theory of migration. Demography, 3(1), 47-57. doi: 10.2307/2060063

Lee, J.-H., \& Beckhusen, J. B. (2012). Veterans' racial and ethnic composition and place of birth: 2011. American Community Survey Briefs.

Lehavot, K., Hoerster, K. D., Nelson, K. M., Jakupcak, M., \& Simpson, T. L. (2012). Health indicators for military, Veteran, and civilian women. American Journal of Preventive Medicine, 42(5), 473-480. doi: 10.1016/j.amepre.2012.01.006

Li, C., Balluz, L. S., Ford, E. S., Okoro, C. A., Zhao, G., \& Pierannunzi, C. (2012). A comparison of prevalence estimates for selected health indicators and chronic diseases or conditions from the Behavioral Risk Factor Surveillance System, the National Health Interview Survey, and the National Health and Nutrition Examination Survey, 2007-2008. Preventive Medicine, 54(6): 381-387.

Liu, C. F., Bryson, C. L., Burgess, J. F., Jr., Sharp, N., Perkins, M., \& Maciejewski, M. L. (2012). Use of outpatient care in VA and Medicare among disability-eligible and age-eligible Veteran patients. BMC Health Services Research, 12, 51. doi: 10.1186/1472-6963-12-51

Long, S. K., Kenney, G. M., Zuckerman, S., Goin, D. E., Wissoker, D., Blavin, F., Blumber, L. J., ClemansCope, L., Holahan, J., \& Hempstead, K. (2013). The Health Reform Monitoring Survey: Addressing data gaps to provide timely insights into the Affordable Care Act. Health Affairs, 33(1), 161-167.

doi: $10.1377 /$ hlthaff.2013.0934

Lowry, I. S. (1966). Migration and metropolitan growth: two analytical models. San Francisco, CA: Chandler Pub. Co.

MacLean, A. (2010). The things they carry: Combat, disability, and unemployment among US men. American Sociological Review, 75(4), 563-585.

Martin, L. M., Leff, M., Calonge, N., Garrett, C., \& Nelson, D. E. (2000). Validation of self-reported chronic conditions and health services in a managed care population. American Journal of Preventive Medicine, 18(3), 215-218.

Mateyka, P. (2012). Who's dissatisfied? Race and ethnic differences in residential dissatisfaction and migration, 2010-2011. U.S. Census Bureau, SEHSD Working Paper Number 2015-2. 
McCarron, K. K., Reinhard, M. J., Bloeser, K. J., Mahan, C. M., \& Kang, H. K. (2014). PTSD diagnoses among Iraq and Afghanistan Veterans: Comparison of administrative data to chart review. Journal of Traumatic Stress, 27(5), 626-629. doi: 10.1002/jts.21961

McDowell, C., \& de Haan, A. (1997). Migration and sustainable livelihoods: A critical review of the literature. Brighton, England: University of Sussex Institute of Development Studies.

McGeary, M., Ford, M. A., McCutchen, S. R., \& Barnes, D. K. (2007). A 21st century system for evaluating Veterans for disability benefits. Washington, DC.

Medical Expenditure Panel Survey. (undated). Data overview. Rockville, MD. Retrieved from http://meps.ahrq.gov/mepsweb/data_stats/data_overview.jsp - (2014, September). MEPS HC-155: 2012 full year consolidated data file. Rockville, MD. Retrieved from http://meps.ahrq.gov/mepsweb/data_stats/download_data/pufs/h155/h155doc.shtml

Medicare Improvements for Patients and Providers Act, Public Law 110-275 (July 15, 2008).

Missouri Census Data Center. (2012). MABLE/Geocorr12: Geographic Correspondence Engine (Version 1.2). Retrieved from http://mcdc.missouri.edu/websas/geocorr12.html

Morina, N., Wicherts, J. M., Lobbrecht, J., \& Priebe, S. (2014). Remission from post-traumatic stress disorder in adults: A systematic review and meta-analysis of long term outcome studies. Clinical Psychology Review, 34(3), 249-255.

Muggah, E., Graves, E., Bennett, C., \& Manuel, D. G. (2013). Ascertainment of chronic diseases using population health data: A comparison of health administrative data and patient self-report. BMC Public Health, 13, 16. doi: 10.1186/1471-2458-13-16

National Center for Veterans Analysis and Statistics. (2011a). America's women Veterans: Military service history and VA benefit utilization statistics. Washington, DC: U.S. Department of Veterans Affairs.

Affairs.

. (2011b). Gulf War era Veterans report: Pre-9/11. Washington, DC: U.S. Department of Veterans

. (2011c). Health insurance coverage, poverty, and income of Veterans: 2000 to 2009. Washington, DC:

U.S. Department of Veterans Affairs.

Affairs.

. (2012a). Characteristics of rural Veterans: 2010. Washington, DC: U.S. Department of Veterans

. (2012b). Profile of sheltered homeless Veterans for fiscal years 2009 and 2010. Washington, DC:

U.S. Department of Veterans Affairs.

- (2014a). 2012 minority Veterans report. Washington, DC: U.S. Department of Veterans Affairs.

- (2014b). Department of Veterans Affairs: Statistics at a glance. Washington, DC: U.S. Department of Veterans Affairs.

—. (2014c). Profile of Veterans 2012. Washington, DC: U.S. Department of Veterans Affairs.

- (2014d). Profile of women Veterans: 2012: U.S. Department of Veterans Affairs.

- (2014e). Unique Veteran users report FY 2012. Washington, DC: U.S. Department of Veterans Affairs.

National Federation of Independent Business v. Sebelius, 132 S.Ct. 603 (2012).

Nelson, K. M., Starkebaum, G. A., \& Reiber, G. E. (2007). Veterans using and uninsured Veterans not using Veterans Affairs (VA) health care. Public Health Reports, 122(1), 93-100.

Niu, B., Forde, K. A., \& Goldberg, D. S. (2015). Coding algorithms for identifying patients with cirrhosis and hepatitis B or C virus using administrative data. Pharmacoepidemiology and Drug Safety, 24(1), 107-111. doi: $10.1002 /$ pds.3721

Office of Public Health and Environmental Hazards. (2010). State of care for Veterans with chronic hepatitis C. Washington, DC. 
Office of the Actuary. (2014). Veteran population projection model 2014. Washington, DC: U.S. Department of Veterans Affairs.

Office of the Assistant Secretary for Planning and Evaluation. (2015a). Health insurance coverage and the Affordable Care Act. Retrieved from http://aspe.hhs.gov/health/reports/2015/uninsured_change/ib_uninsured_change.pdf

- (2015b). Health insurance marketplaces 2015 open enrollment period: March enrollment report. U.S. Department of Health and Human Services.

Office of the Under Secretary of Defense (Comptroller). (2015). United States Department of Defense fiscal year 2016 budget request: Overview. Washington, DC: U.S. Department of Defense.

Office of the Assistant Secretary of Defense (Health Affairs). (2015). Evaluation of the TRICARE program: Access, cost, and quality, fiscal year 2015 report to Congress.

O'Hare, W. P., Pollard, K. M., \& Ritualo, A. R. (2004). Educational and economic characteristics, 211-251. In Jacob S. Siegel \& David A. Swanson (Eds.), The methods and materials of demography, 2nd ed. San Diego, CA: Elsevier Academic Press.

Okura, Y., Urban, L. H., Mahoney, D. W., Jacobsen, S. J., \& Rodeheffer, R. J. (2004). Agreement between self-report questionnaires and medical record data was substantial for diabetes, hypertension, myocardial infarction and stroke but not for heart failure. Journal of Clinical Epidemiology, 57(10), 1096-1103. doi: $10.1016 /$ j.jclinepi.2004.04.005

Park, L. S., Tate, J. P., Rodriguez-Barradas, M. C., Rimland, D., Goetz, M. B., Gibert, C., Brown, S. T., Kelley, M. J., Justice, A. C., \& Dubrow, R. (2014). Cancer incidence in HIV-infected versus uninfected Veterans: Comparison of cancer registry and ICD-9 code diagnoses. Journal of AIDS and Clinical Research, 5(7), 318. doi: 10.4172/2155-6113.1000318

Parrish, K. (2011). DOD directs Army, Marine drawdowns for 2015, 2016. DoD News. Retrieved from http://www.defense.gov/news/newsarticle.aspx?id=62355

Passaris, C. (1989). Immigration and the evolution of economic theory. International Migration, 27(4), 525-542. doi: 10.1111/j.1468-2435.1989.tb00469.x

Petersen, L. A., Wright, S., Normand, S. L., \& Daley, J. (1999). Positive predictive value of the diagnosis of acute myocardial infarction in an administrative database. Journal of General Internal Medicine, 14(9), $555-558$.

Philipps, Dave. (2013, May 19). Disposable: Surge in discharges includes wounded soldiers. The Gazette. Retrieved from http://cdn.csgazette.biz/soldiers/dayl.html

Pint, E. M., Lewis, M. W., Lippiatt, T. F., Hall-Partyka, P., Wong, J. P., \& Puharic, A. A. (2015). Active component responsibility in reserve component pre-and postmobilization training. Santa Monica, CA: RAND Corporation, RR-738-A. Retrieved from http://www.rand.org/pubs/research_reports/RR738.html

Pizer, S. D., \& Prentice, J. C. (2011). What are the consequences of waiting for health care in the Veteran population? Journal of General Internal Medicine, 26(2), 676-682. doi: 10.1007/s11606-011-1819-1

Possemato, K., Wade, M., Andersen, J., \& Ouimette, P. (2010). The impact of PTSD, depression, and substance use disorders on disease burden and health care utilization among OEF/OIF Veterans. Psychological Trauma: Theory, Research, Practice, and Policy, 2(3), 218-223. doi: 10.1037/a0019236

Pöyhönen, P. (1963). A tentative model for the volume of trade between countries. Weltwirtschaftliches Archives, 90, 93-100. doi: 10.2307/40436776

Preston, S. H., Heuveline, P., \& Guillot, M. (2001). Demography: Measuring and modeling population processes. Malden, MA: Blackwell Publishers, Inc.

Quan, H., Khan, N., Hemmelgarn, B. R., Tu, K., Chen, G., Campbell, N., Hill., M. D., Ghali, W. A., \& McAlister, F. A. (2009). Validation of a case definition to define hypertension using administrative data. Hypertension, 54(6), 1423-1428. doi: 10.1161/hypertensionaha.109.139279 
Ramchand, R., Rudavsky, R., Grant, S., Tanielian, T., \& Jaycox, L. H. (2015). Prevalence of, risk factors for, and consequences of posttraumatic stress disorder and other mental health problems in military populations deployed to Iraq and Afghanistan. Santa Monica, CA: RAND Corporation, EP-50677. Retrieved from http://www.rand.org/pubs/external_publications/EP50677.html

RAND Health. (2015). Resources and capabilities of the Department of Veterans Affairs to provide timely and accessible care to Veterans. Santa Monica, CA: RAND Corporation, RR-1165/2-VA. Retrieved from http://www.rand.org/pubs/research_reports/RR1165z2.html

Ravenstein, E. G. (1885). The laws of migration. Journal of the Statistical Society of London, 48(2), 167-235. doi: $10.2307 / 2979181$

. (1889). The laws of migration. Journal of the Royal Statistical Society, 52(2), 241-305. doi: $10.2307 / 2979333$

Raymer, J., \& Rogers, A. (2007). Using age and spatial flow structures in the indirect estimation of migration streams. Demography, 44(2), 199-223.

Richardson, C., \& Waldrop, J. (2003). Veterans: 2000. Census 2000 Brief. U.S. Census Bureau.

Roemer, M. (2012). Health and insurance status, health care use, and expenditures for male Veterans, 2008:

Estimates for the U.S. civilian noninstitutionalized population. Statistical Brief 370. Rockville, MD: Agency for Healthcare Research and Quality. Retrieved from

http://meps.ahrq.gov/data_files/publications/st370/stat370.pdf

Samet, J. M., and Bodurow, C. C., (Eds.). (2008). Improving the presumptive disability decision-making process for Veterans. Washington, DC: The National Academies Press.

Santiago, P. N., Ursano, R. J., Gray, C. L., Pynoos, R. S., Spiegel, D., Lewis-Fernandez, R., Friedman, M. J., \& Fullerton, C. S. (2013). A systematic review of PTSD prevalence and trajectories in DSM-5 defined trauma exposed populations: Intentional and non-intentional traumatic events. PLoS One, 8(4).

doi: 10.1371/journal.pone.0059236

Setoguchi, S., Solomon, D. H., Glynn, R. J., Cook, E. F., Levin, R., \& Schneeweiss, S. (2007). Agreement of diagnosis and its date for hematologic malignancies and solid tumors between medicare claims and cancer registry data. Cancer Causes Control, 18(5), 561-569. doi: 10.1007/s10552-007-0131-1

Shen, Y., Hendricks, A., Wang, F., Gardner, J., \& Kazis, L. E. (2008). The impact of private insurance coverage on Veterans' use of VA care: Insurance and selection effects. Health Services Research, 43(1 Pt 1), 267-286. doi: 10.1111/j.1475-6773.2007.00743.x

Shiner, B., Drake, R. E., Watts, B. V., Desai, R. A., \& Schnurr, P. P. (2012). Access to VA services for returning Veterans with PTSD. Military Medicine, 177(7), 814-822.

Singh, J. A. (2009). Accuracy of Veterans Affairs databases for diagnoses of chronic diseases. Preventing Chronic Disease, 6(4), A126.

Smith, D. L. (2014). The relationship between employment and Veteran status, disability and gender from 2004-2011 Behavioral Risk Factor Surveillance System (BRFSS). Work, 49(2), 325-334.

doi: $10.3233 /$ wor-131648

Sommers, B. D, Musco, T., Finegold, K., Gunja, M. Z., Burke, A., \& McDowell, A. M. (2014). Health reform and changes in health insurance coverage in 2014. New England Journal of Medicine, 371(9), 867-874.

Soni, A. (2011). Top 10 most costly conditions among men and women, 2008: Estimates for the U.S. civilian noninstitutionalized adult population, age 18 and older. Statistical Brief 331. Rockville, MD: Agency for Healthcare Research and Quality.

Stillwell, J. (2009). Inter-regional migration modelling: A review. In B. W. Jacques Poot \& Leo van Wissen (Eds.), Migration and human capital. Cheltenham, UK: Edward Elgar Publishing.

Stroupe, K. T., Hynes, D. M., Giobbie-Hurder, A., Oddone, E. Z., Weinberger, M., Reda, D. J., \& Henderson, W. G. (2005). Patient satisfaction and use of Veterans Affairs versus non-Veterans Affairs healthcare services by Veterans. Medical Care, 43(5), 453-460. 
Talbott, E. O., Rager, J. R., Brink, L. L., Benson, S. M., Bilonick, R. A., Wu, W. C., \& Han, Y.-Y. (2013). Trends in acute myocardial infarction hospitalization rates for US states in the CDC tracking network. doi: 10.1371 /journal.pone.0064457

Tan, Michelle. (2015, January 22). Army leaders now hope to slow the drawdown. Army Times. Retrieved from http://www.armytimes.com/story/military/pentagon/2015/01/22/

army-secretary-john-mchugh-slow-drawdown-sequestration-challenges/22162247/

Tanielian, T., \& Jaycox, L. (2008). Invisible wounds of war: Psychological and cognitive injuries, their consequences, and services to assist recovery. Santa Monica, CA: RAND Corporation, MG-720-CCF. Retrieved from http://www.rand.org/pubs/monographs/MG720.html

Taylor, B. (2012). Fiscal year 2011 VA utilization report for Iraq and Afghanistan War Veterans diagnosed with TBI. Minneapolis, MN: Quality Enhancement Research Initiative.

Taylor, B. C., Hagel, E. M., Carlson, K. F., Cifu, D. X., Cutting, A., Bidelspach, D. E., \& Sayer, N. A. (2012). Prevalence and costs of co-occurring traumatic brain injury with and without psychiatric disturbance and pain among Afghanistan and Iraq War Veteran V.A. users. Medical Care, 50(4), 342-346.

doi: 10.1097/MLR.0b013e318245a558

Tinbergen, J. (1962). Shaping the world economy: Suggestions for an international economic policy. New York: Twentieth Century Fund.

U.S. Census Bureau. (undated a). American Community Survey (ACS) data. Retrieved from http://www.census.gov/programs-surveys/acs/data.html

—. (undated b). Rural and Urban Projection (RUP) overview. Retrieved from

https://www.census.gov/population/international/software/rup/

from

. (undated c). Cartographic Boundary Shapefiles_Public Use Microdata Areas (PUMAs). Retrieved

https://www.census.gov/geo/maps-data/data/cbf/cbf_puma.html

. (undated d). TIGER/Line Shapefiles and TIGER/Line files. Retrieved from

https://www.census.gov/geo/maps-data/data/tiger-line.html

- (2012a, July). How do we know? A snapshot of our nation's Veterans. Retrieved from http://www.census.gov/library/infographics/veterans.html

- (2012b). Section 10: National security and Veterans affairs. Statistical Abstract of the United States: 2012. Retrieved from

https://www.census.gov/prod/2011pubs/12statab/defense.pdf

. (2014). Census data 2010. Retrieved from

http://www.census.gov/2010census/data/

_ (2015). What is the American Community Survey? Retrieved from

http://www.census.gov/acs/www/about_the_survey/american_community_survey/

U.S. Defense and Veterans Brain Injury Center. (2014). DoD numbers for traumatic brain injury.

U.S. Department of Agriculture. (2014, June 2). Rural-urban communting area codes. Economic Research Service. Retrieved from http://www.ers.usda.gov/data-products/rural-urban-commuting-area-codes.aspx

U.S. Department of Defense. (2010). Coding guidance for traumatic brain injury fact sheet. Washington, DC.

- (2014). Quadrennial defense review 2014. Washington, DC.

U.S. Department of Housing and Urban Development. (2014). The 2014 annual homeless assessment report (AHAR) to Congress. Washington, DC.

U.S. Department of Veterans Affairs. (2009a). Relaxation of priority group 8 restrictions. Washington, DC.

. (2009b). Veterans benefits timetable. Washington, DC.

. (2011). National HIVIAIDS strategy operational plan. Washington, DC.

. (2012). The state of care for Veterans with HIVIAIDS. Washington, DC. 
. (2014a, December 3). Health benefits: VA health care enrollment and eligibility. Retrieved from http:/www.va.gov/healthbenefits/resources/publications/hbco/hbco_enrollment_eligibility.asp

. (2014b, February 27). Not enrolled in VA health care? What you should know. Retrieved from http://www.va.gov/health/aca/NonEnrolledVeterans.asp

- (2014c). Sample Veterans health benefits handbook. Washington, DC.

—. (2015a, January). 2015 Copayment requirements at a glance. Veterans Health Administration.

Retrieved from

http://www.va.gov/healthbenefits/resources/publications/IB10-431_copay_requirements_at_a_glance.pdf

_. (2015b, March 10). Health benefits: Apply for VA health benefits. Retrieved from

http://www.va.gov/HEALTHBENEFITS/apply/index.asp

. (2015c, February 5). Health benefits: Veterans eligibility. Retrieved from

http://www.va.gov/healthbenefits/apply/veterans.asp

- (2015d). Progress report: Veterans Access, Choice, and Accountability Act 2014. Washington, DC:

Retrieved from

http://www.va.gov/opa/choiceact/documents/FactSheets/Progress-Report-March-2015-Fact-Sheet.pdf

—. (2015e, April 30). VA works to expand Choice Program eligibility. Retrieved from

http://www.va.gov/healthbenefits/

- (2015f). Veterans' diseases associated with Agent Orange. Washington, DC. Retrieved from

http://www.publichealth.va.gov/exposures/agentorange/conditions/index.asp

. (2015g). VHA handbook 1601A.02_Eligibility determination. Washington, DC.

U.S. Government Accountability Office. (2009). VA health care: Preliminary findings on VA's provision of health care services to women Veterans. Retrieved from

http://www.gao.gov/products/GAO-09-899T

. (2011). VA mental health: Number of Veterans receiving care, barriers faced, and efforts to increase access. Washington, DC. Retrieved from http:/www.gao.gov/assets/590/585743.pdf

- (2012). VA health care: Reliability of reported outpatient medical appointment wait times and scheduling oversight need improvement. Washington, DC.

. (2013a). VA health care: Appointment scheduling oversight and wait time measures need improvement. Washington, DC: Retrieved from http://www.gao.gov/assets/660/652795.pdf

. (2013b). VA health care: Improvements needed in processes used to address providers' actions that contribute to adverse events. Washington, DC. Retrieved from

http://www.gao.gov/assets/660/659378.pdf

- (2013c). Veterans' disability benefits: Challenges to timely processing persist. Washington, DC. Retrieved from

http://www.gao.gov/products/GAO-13-453T

. (2014a). VA health care: Further action needed to address weaknesses in management and oversight of non-VA medical care. Washington, DC. Retrieved from http://www.gao.gov/assets/670/664171.pdf

- (2014b). VA health care: Management and oversight of consult process need improvement to help ensure Veterans receive timely outpatient specialty care. Washington DC. Retrieved from http://www.gao.gov/assets/670/666248.pdf

van Asbeck, F. W., Post, M. W., \& Pangalila, R. F. (2000). An epidemiological description of spinal cord injuries in the Netherlands in 1994. Spinal Cord, 38(7), 420-424.

Varas-Lorenzo, C., Castellsague, J., Stang, M. R., Tomas, L., Aguado, J., \& Perez-Gutthann, S. (2008). Positive predictive value of ICD-9 codes 410 and 411 in the identification of cases of acute coronary syndromes in the Saskatchewan Hospital automated database. Pharmacoepidemiology and Drug Safety, 17(8), 842-852. doi: 10.1002/pds.1619

Veterans Access, Choice, and Accountability Act of 2014, Public Law 113-146 (August 7, 2014). 
Veterans Claims Assistance Act of 2000, Public Law 106-475 (November 9, 2000).

Veterans Health Administration. (2015). 2015 VA budget request.

Veterans' Health Care Eligibility Reform Act of 1996, Public Law 104-262 (1996).

Virgo, K. S., Price, R. K., Spitznagel, E. L., \& Ji, T. H. C. (1999). Substance abuse as a predictor of VA medical care utilization among Vietnam Veterans. Journal of Behavioral Health Services and Research, 26(2), 126-139. doi: 10.1007/bf02287486

Voigt, L. F., Koepsell, T. D., \& Daling, J. R. (2003). Characteristics of telephone survey respondents according to willingness to participate. American Journal of Epidemiology, 157(1), 66-73.

Waitzman, N. J., \& Smith, K. R. (1998). Phantom of the area: Poverty-area residence and mortality in the United States. American Journal of Public Health, 88(6), 973-976.

Wang, L., Haider, S., Nedrow, K., Chambers, R., Tawadrous, M., Baser, O., \& Simpson, K. N. (2015). HIV economic burden of illness in the Veterans Health Administration population. AIDS Care, 27(1), 123-131. doi: 10.1080/09540121.2014.947237

Washington, D. L., Bean-Mayberry, B., Hamilton, A. B., Cordasco, K. M., \& Yano, E. M. (2013). Women Veterans' healthcare delivery preferences and use by military service era: Findings from the National Survey of Women Veterans. Journal of General Internal Medicine, 28(2), S571-S576. doi: 10.1007/s11606-012-2323-y

Washington, D. L., Yano, E. M., McGuire, J., Hines, V., Lee, M., \& Gelberg, L. (2010). Risk factors for homelessness among women Veterans. Journal of Health Care for the Poor and Underserved, 21(1), 81-91.

Watkins, K. E., Pincus, H. A., Paddock, S., Smith, B., Woodroffe, A., Farmer, C., Sorbero, M. E., HorvitzLennon, M., Mannle, T., Jr., Hepner, K. A., Solomon, J., \& Call, C. (2011). Care for Veterans with mental and substance use disorders: Good performance, but room to improve on many measures. Health Affairs (Millwood), 30(11), 2194-2203. doi: 10.1377/hlthaff.2011.0509

Weeks, W. B., Kazis, L. E., Shen, Y. J., Cong, Z., Ren, X. S., Miller, D., Lee, A., \& Perlin, J. B. (2004). Differences in health-related quality of life in rural and urban Veterans. American Journal of Public Health, 94(10), 1762-1767. doi: 10.2105/ajph.94.10.1762

Weeks, W. B., \& Mills, P. D. (2003). Reduction in patient enrollment in the Veterans Health Administration after media coverage of adverse medical events. Joint Commission Journal on Quality and Safety, 29(12), $652-658$.

Wicker, Tom. (1991, January 26). In the nation; ghosts of Vietnam. New York Times. Retrieved from http://www.nytimes.com/1991/01/26/opinion/in-the-nation-ghosts-of-vietnam.html

Wilmoth, J. M., London, A. S, \& Parker, W. M. (2011). Sex differences in the relationship between military service status and functional limitations and disabilities. Population Research and Policy Review, 30(3), $333-354$.

Winkelmayer, W. C., Schneeweiss, S., Mogun, H., Patrick, A. R., Avorn, J., \& Solomon, D. H. (2005). Identification of individuals with CKD from Medicare claims data: A validation study. American Journal of Kidney Diseases, 46(2), 225-232. doi: 10.1053/j.ajkd.2005.04.029

Wittrock, S., Ono, S., Stewart, K., Reisinger, H. S., \& Charlton, M. (2015). Unclaimed health care benefits: A mixed-method analysis of rural Veterans. Journal of Rural Health, 31(1), 35-46. doi: 10.1111/jrh.12082

Zeber, J. E., Copeland, L. A., \& Grazier, K. L. (2006). Serious mental illness, aging, and utilization patterns among Veterans. Military Medicine, 171(7), 619-626.

Zoroya, G. (2014). Report cites VA struggles with benefits paid to Veterans. USA Today. Retrieved from http://www.usatoday.com/story/news/nation/2014/07/14/va-backlog-committee-hearing-veterans/12573043

Zuniga, I. A., Chen, J. J., Lane, D. S., Allmer, J., \& Jimenez-Lucho, V. E. (2006). Analysis of a hepatitis C screening programme for US Veterans. Epidemiology and Infection, 134(2), 249-257.

doi: $10.1017 / \mathrm{s} 095026880500498 \mathrm{x}$

Zuvekas, S. H., \& Olin, G. L. (2009). Validating household reports of health care use in the Medical Expenditure Panel Survey. Health Services Research, 44: 1679-1700. 